MEASURED TOTAL SEDIMENT LOADS

(SUSPENDED LOADS AND BEDLOADS)

FOR 93 UNITED STATES STREAMS

By Garnett P. Williams, U.S. Geological Survey, and David L. Rosgen, Wildland Hydrology Consultants

U.S. GEOLOGICAL SURVEY

Open-File Report 89-67 
DEPARTMENT OF THE INTERIOR

MANUEL LUJAN, JR., Secretary

U.S. GEOLOGICAL SURVEY

Dallas L. Peck, Director

For additional information write to:

Chief, Branch of Regional Research U.S. Geological Survey Box 25046, Mail Stop 418

Federa1 Center

Denver, CO 80225-0046
Copies of this report can be purchased from:

U.S. Geological Survey

Books and Open-File Reports Section Federal Center

Box 25425

Denver, CO 80225-0425

[Telephone: (303) 236-7476] 
Abstract-

Introduction-

Measurement techniques-_-

Suspended load-10

Bedload-_-_-_-

Hydraulic variables--

Particle sizes--

Errors in sediment-transport measurement-_.

Site criteria--

Explanation of tables-1..-

Acknowledgments-10

References-.---

\section{TABLES}

Table 1. Hydraulic and sediment-transport data-10

2. Suspended load particle-size distributions-_...... 56

3. Bedload particle-size distributions-_. 70

4. Bed-material particle-size distributions-_- 112 


\title{
MEASURED TOTAL SEDIMENT LOADS (SUSPENDED LOADS AND BEDLOADS) FOR 93 UNITED STATES STREAMS
}

By Garnett P. Williams and David L. Rosgen

\begin{abstract}
The report presents field data of suspended-sediment loads and approximately concurrently measured bedloads for 93 United States streams. Also included are the associated hydraulic variables and (where available) the particle-size analyses of the bed material and sediment loads. The data, many of which have not been published heretofore, were measured by various individuals and organizations. Suspended loads were measured with standard methods. Bedloads, except for one site, were measured with the Helley-Smith sampler, generally at a minimum of ten sampling points over the stream cross section. In spite of the well-known sampling problems, the data probably are the best available at the present (1986) stage of sampling capability. They are the first comprehensive collection of field-measured total sediment loads (bedloads plus suspended loads) in a variety of streams.
\end{abstract}

\section{INTRODUCTION}

The total sediment load of streams usually is considered to be the sum of two components, called for convenience the suspended load and bedload. Suspended loads have been measured routinely for many years, but bedload measurements have been scarce. In recent years, however, the U.S. Geological Survey, U.S. Forest Service and others have made a considerable number of approximately-concurrent bedload and suspended load measurements on various streams. Such data are valuable in that they represent the first extensive measurements of total sediment loads for a variety of streams. This report presents these data, along with associated hydraulic variables.

\section{MEASUREMENT TECHNIQUES}

\section{Suspended Load}

Suspended loads were determined with a common depth-integrating discharge-weighted sampler, generally the D-49, D-74, DH-48, P-61, or P-63 (Guy and Norman, 1970). These devices sample the water-sediment mixture from the water surface to within about 8 or $9 \mathrm{~cm}$ of the streambed. For the data reported here, such sampling was done at 3-20 "verticals" across the channel. The verticals in most cases were equally spaced across the stream, using a method known as equal transit rate or equal width increment. 
The separate samples for a channel cross section were composited in the analysis to yield a mean suspended-sediment concentration that is representative of the entire cross section. Results usually are published in milligrams per liter. Suspended load, in mass per unit time, is calculated as water discharge times sediment concentration times a units-conversion constant.

\section{Bedload}

Sediment moving within about $8 \mathrm{~cm}$ of the streambed, herein called bedload, was sampled with a pressure-difference sampler called the Helley-Smith (H-S) bedload sampler (Helley and Smith, 1971). Several sizes are available; the most commonly used one has a square orifice measuring $7.6 \times 7.6 \mathrm{~cm}$. One site reported here--Oak Creek near Corvallis, Oreg.--is an exception; this site used a slot or pit sampler whereby a flume placed diagonally across the streambed (with tops of flume sidewalls at streambed elevation) generated a vortex-type flow to route bedload to an off-channel trap.

Bedloads using the H-S sampler were obtained by sampling at a minimum of 10 equidistant lateral stations across the streambed. More than one traverse across the channel was made in many cases, especially if the channel was less than 1 or 2 meters wide.

The H-S sampler usually was placed on the streambed for periods of 30 to 60 seconds. For 52 Colorado sites, the total sampling time for an entire cross section totaled 10-20 min, and at least 20 samples were taken over each cross section.

All individual samples at a cross section were combined for an average stream-wide bedload rate. Inasmuch as both suspended load and bedload represent composited values for the entire stream width, they do not necessarily reflect the rates or proportions at any selected vertical. Hydraulic variables presented in this report also apply to the entire cross section rather than to any particular vertical.

\section{Hydraulic Variables}

Water discharges ( $Q$ ) were determined either by standard current-meter streamflow-gaging techniques or from a rating curve (plot of gage height versus discharge).

Mean flow velocities (V), water-surface widths (W), and mean flow depths (D) $(=\mathrm{A} / \mathrm{W}$, where $\mathrm{A}=$ cross-sectional flow area) were determined by one of three methods: (1) standard streamflow-gaging measurement; (2) a rating curve-hydraulic geometry technique, whereby discharge for a given gage height is read from a rating curve and $V, W$, and $D$ are estimated from previously prepared log plots relating these variables to instantaneous discharge; or (3) a rating curve-area method in which discharge is read from a rating curve, $A$ (and hence $W$ and $D$ ) are determined from the verticals of the bedload sampling, and $V=Q / A$. In some instances a value for width was measured during the bedload sampling, but $A, D$, and $V$ were not determined. 
The product VWD in this report may or may not equal the water discharge listed for bedload and (or) suspended load. Three situations where VWD does equal the listed discharge are: (1) Water discharge measured by current meter, and discharge remained (or was assumed to remain) essentially unchanged while the sediment loads were measured; (2) water discharge taken from rating curve, $A, W$ and $D$ given by the cross section as obtained during the bedload measurement, and $\mathrm{V}=\mathrm{Q} / \mathrm{A}$; and (3) water discharge taken from rating curve and, for this discharge, $V, W$ and $D$ estimated from least-squares-fitted hydraulic geometry relations. (All values of $\mathrm{V}, \mathrm{W}$, and $\mathrm{D}$ given by hydraulic geometry relations are estimates rather than measurements.)

The product VWD may not equal the listed water discharges at some stations where rating curves were available. When measured by current meter, water discharge (and hence $V, W$ and $D$ ) generally were the first features measured on a given day. By the time sediment loads were measured, the discharge may have changed. The investigator in such instances read a discharge from the rating curve for the time at which the particular sediment load was measured.

Water-surface slopes, when measured, generally were determined by reading water-surface elevations along the reach, either (a) by a direct level-andstadia rod survey or (b) from a series of precalibrated staff gages.

Normally, only one set of sediment and hydraulic measurements was made on a given day. The observations cover periods ranging from one runoff season (a few months) to several years.

\section{Particle Sizes}

Suspended loads generally were analyzed by visual-accumulation tube for particles $>0.062 \mathrm{~mm}$ and by pipette for particles $<0.062 \mathrm{~mm}$. Bedload usually was analyzed by dry sieving; the exceptions are nine Colorado sites (stations $35,39-44,51-52$ ), where bedload sediments were wet-sieved in the field and the data then adjusted by an empirically determined factor to equate approximately to dry weight.

Surficial bed material that did not include particles larger than about $128 \mathrm{~mm}$ in diameter was sampled in a variety of ways from one stream to another. The size distribution of such sediment generally was determined by dry-sieving and therefore is expressed in percent by weight. Where the streambed material included particles coarser than about $128 \mathrm{~mm}$, as in nearly all Colorado streams of this report, bed sediment (including even the sandsized particles, if present) was analyzed by the pebble-count method. The size distribution then is expressed in percent by number.

\section{ERRORS IN SEDIMENT-TRANSPORT MEASUREMENT}

Sampling efficiency is the ratio of quantity of sediment trapped in the sampler to the quantity of sediment the stream actually would transport at the same time and place had the sampler not been there. Due mainly to the extreme difficulty of calibrating them, suspended-load samplers generally are assumed to have an efficiency of 1.0 , that is, perfect efficiency. 
Inaccuracy in the measured suspended-sediment load can arise from sampler inefficiency, improper sampling in the vertical, and errors in measuring water discharge. Probably a more serious problem arises in determining the average transport rate for the entire cross section from a group of verticals. This problem is due to hydraulic causes and to natural fluctuations in sediment concentrations, both spatially and temporally. These fluctuations can vary by several orders of magnitude.

How efficiently the H-S device samples the true rate of "bedload" transport at a station has not been decisively established. Some tests (Emmett, 1980) indicate an efficiency of about 1.0 to 1.1 . Other tests (Hubbell and others, 1981; Hubbel1 and Stevens, 1986; Pitlick, 1988) suggest that the efficiency can vary considerably, depending on transport rate, size of bed particles, and dimensions and wall thickness of sampler orifice. At some sites included in this report, particles larger than the H-S orifice may have been in transport. For lack of sufficient evidence to the contrary, however, we made no adjustments to any of the reported bedload values.

For given streamflow conditions, wide variations in measured bedload transport rate can occur at a sampling spot because of sampling duration, rate of bedform travel, and natural cyclic variations in sediment transport (Hubbell and others, 1981). Variations also occur across the stream, due to irregular lateral distribution of bedload and to number and location of sampling sites. (Indeed, at some places along a traverse, no bedload at all may be moving. For this reason, caution is needed in applying stream-width mean hydraulic variables to stream-wide mean transport rates.) Sampling problems due to cross-channel variability can be reduced or nearly eliminated as the investigator systematically samples at many (for example, 10-20) sites across the stream (Emmett, 1984). Even so, it is probably not possible to specify precisely the extent to which a "measured" stream-wide bedload transport rate might vary from the true mean transport rate for the cross section.

On a larger scale, both bedload and suspended-load transport rate can be a nonunique function of water discharge, as with hysteresis loops. For a given discharge, sediment loads can vary with time, namely from rising to falling stages during floods, early to late stage of annual runoff season, and year to year (Nordin, 1980). The relations with time also can be affected by the location of the measuring section relative to a pool or riffle (Meade and others, 1981; Emmett and others, 1982).

\section{SITE CRITERIA}

Arbitrarily, we accepted for inclusion in this report only those stream sites at which at least 10 pairs of concurrent measurements of bedload and suspended load had been made. (The bedload measurement can take an hour or longer; the associated suspended load usually was measured just before or just after the bedload measurement. For practical purposes, such measurements herein are considered concurrent or simultaneous.)

Some data were measured on unstable streams, that is, streams on which the bed and possibly banks were (or may have been) aggrading or degrading. Examples are the two Mt. St. Helens streams (Toutle River at Tower Road near Silver Lake, Wash., and North Fork Toutle River near Kid Valley, Wash.) and possibly one or more of those in the Redwood Creek basin of California. 


\section{EXPLANATION OF TABLES}

Table 1 lists the hydraulic- and sediment-transport data. Hydraulic data include the instantaneous water discharge associated with the bedload (called water discharge--bedload), the instantaneous water discharge associated with the suspended load (called water discharge--suspended load), mean flow velocity, water-surface width, mean flow depth, water-surface slope, and water temperature. Sediment-transport data include suspended sediment concentration, suspended load, number of sampling points for bedload, and bedload. Where more than about 20 sampling points are indicated for bedload, the investigator made two or more trips across the channel. In some instances the number of bedload-sampling points was known only approximately, such as 16-25 or $\geqq 20$.

Water discharge in some cases remained essentially constant during the measurement of bedload and suspended load. (Such a constant discharge was assumed for table 1 unless information to the contrary was available.) In other instances, water discharge changed slightly during the measuring period, in which case the water discharge (bedload) and water discharge (suspended load) for a given date in table 1 have different values.

Tables 2,3 , and 4 give particle-size analyses of suspended loads, bedloads, and bed material, respectively. For many of the sediment loads of table 1 , size analyses of the trapped material were not made. This is especially true of suspended loads because of the typically small amounts of sediment in the sample. If no particle-size data are available for a date listed in table 1 under a particular station, no entry is made (that is, the date is not listed) in tables 2,3 , or 4 .

Whenever possible, bed-material size analyses are given for samples taken on the same day as the sediment transport measurements. For stations lacking such concurrent data, a bed-material size analysis is given for any available date.

Some of the data in the four tables have been published in Andrews (1977), Burrows and others (1981), Burrows and Harrold (1983), Elliott and others (1984), Emmett and Seitz (1973), Emmett and others (1980), Harrold and Burrows (1983), Jones and Seitz (1980), Knott and Lipscomb (1983; 1985), Knott and others (1986), Milhous (1973), and Williams and Krupin (1984). Much of the data also are available on WATSTORE and STORET, which are U.S. Government computer data bases.

\section{ACKNOWLEDGMENTS}

The data presented herein were collected by, for, or under the auspices of various individuals working on a variety of projects. Many of these people were assisted in the field by others. With all due apologies to those not listed here, we wish to give credit to the following individuals:

Alaska :

7 sites--James M. Knott, Stephen W. Lipscomb, Jack D. McKechnie, Gregory L. Pope

2 sites--Robert L. Burrows, Philip E. Harrold, Bruce Parks 
Washington:

2 sites--Steven A. Gustafson, Richard L. Kittleson, Kurt R. Spicer, Leonard L. Reed, Stephen E. Hammond

1 site--William W. Emmett, Harold R. Seitz, Michael L. Jones

Oregon:

1 site--Robert T. Milhous

California:

A11 sites--Larry F. Trujillo

5 sites--Richard J. Janda, K. Michael Nolan, Mary Ann Madej

1 site--Dallas Childers, Jr.

Idaho:

5 sites--Rhea $P$. Williams

3 sites--Walter F. Megahan, Rhea P. Williams

1 site--William W. Emmett, Harold R. Seitz, Michael L. Jones

Wyoming:

1 site--Edmund D. Andrews

1 site--William W. Emmett, Robert M. Myrick, Robert H. Meade

Colorado:

51 sites--David L. Rosgen, Hilton L. Silvey, Lela K. Chavez, Kathleen K. Zillich, Alice E. Johns, Mark T. Story, Kerry D. Sundeen

2 sites--David L. Butler

1 site--John G. Elliott, James E. Kircher, Paul B. von Guerard

Iowa :

2 sites--Wilbur J. Matthes, Jr.

Wisconsin:

5 sites--William J. Rose, Halward L. Hanson, Jack T. Freshwaters, Kenneth J. Hedmark, Thomas J. Popowski

A great deal of valuable assistance in processing and organizing the basic data was provided by Karen L. Pereira-Bentz, Peter L. Adams, and Michael J. Werito. We thank Randy S. Parker and Herbert H. Stevens for helpful comments on an early version of the manuscript. 


\section{REFERENCES}

Andrews, E.D., 1977, Hydraulic adjustment of an alluvial stream channel to the supply of sediment, western Wyoming [Ph.D. dissertation]: Berkeley, University of California, $152 \mathrm{p}$.

Burrows, R.L., Emmett, W.W., and Parks, B., 1981, Sediment transport in the Tanana River near Fairbanks, Alaska, 1977-79: U.S. Geological Survey Water-Resources Investigations Report 81-20, $56 \mathrm{p}$.

Burrows, R.L., and Harrold, P.E., 1983, Sediment transport in the Tanana River near Fairbanks, Alaska, 1980-81: U.S. Geological Survey WaterResources Investigations Report 83-4064, 116 p.

Elliott, J.G., Kircher, J.E., and von Guerard, P., 1984, Sediment transport in the lower Yampa River, northwestern Colorado: U.S. Geological Survey Water-Resources Investigations Report 84-4141, $44 \mathrm{p}$.

Emmett, W.W., 1980, A field calibration of the sediment-trapping characteristics of the Helley-Smith bedload sampler: U.S. Geological Survey Professional Paper 1139, 44 p. 1984, Measurement of bedload in rivers, in Hadley, R.F., and Walling, D.E. (eds.), Erosion and sediment yield, some methods of measurement and modelling: Norwich, England, Geo Books, p. 91-109.

Emmett, W.W., Myrick, R.M., and Meade, R.H., 1980, Field data describing the movement and storage of sediment in the East Fork River, Wyoming--Part I. River hydraulics and sediment transport, 1979: U.S. Geological Survey Open-File Report 80-1189, 43 p. 1982, Field data describing the movement and storage of sediment in the East Fork River, Wyoming--Part III. River hydraulics and sediment transport, 1980: U.S. Geological Survey Open-File Report 82-359, 289 p.

Emmett, W.W., and Seitz, H.R., 1973, Suspended and bedload sediment transport in the Snake and Clearwater Rivers in the vicinity of Lewiston, Idaho, March 1972 through June 1973: U.S. Geological Survey Basic-Data Report, $78 \mathrm{p}$.

Guy, H.P., and Norman, V.W., 1970, Field methods for measurement of fluvial sediment: U.S. Geological Survey Techniques of Water-Resources Investigations, bk. 3, chap. C2, 59 p.

Harrold, P.E., and Burrows, R.L., 1983, Sediment transport in the Tanana River near Fairbanks, Alaska, 1982: U.S. Geological Survey WaterResources Investigations Report 83-4213, 53 p.

Helley, E.J., and Smith, W., 1971, Development and calibration of a pressuredifference bedload sampler: U.S. Geological Survey Open-File Report, $18 \mathrm{p}$.

Hubbel1, D.W., and Stevens, H.H., Jr., 1986, Factors affecting accuracy of bedload sampling, in Proceedings of the Fourth Federal Interagency Sedimentation Conference, March 24-27, 1986, Las Vegas: Las Vegas, Subcommittee on Sedimentation, v. 1, p. 4-20-4-29.

Hubbell, D.W., Stevens, H.H., Jr., Skinner, J.V., and Beverage, J.P., 1981, Recent refinements in calibrating bedload samplers, in Proceedings of the specialty conference, water forum ' 81 , August 10-14, 1981, San Francisco: New York, American Society of Civil Engineers, p. 128-140.

Jones, M.L., and Seitz, H.R., 1980, Sediment transport in the Snake and Clearwater Rivers in the vicinity of Lewiston, Idaho: U.S. Geological Survey Open-File Report 80-690, 179 p. 
Knott, J.M., and Lipscomb, S.W., 1983, Sediment discharge data for selected sites in the Susitna River Basin, Alaska, 1981-82: U.S. Geological Survey Open-File Report 83-870, 45 p. 1985, Sediment discharge data for selected sites in the Susitna River Basin, Alaska, October 1982 to February 1984: U.S. Geological Survey Open-File Report 85-157, 68 p.

Knott, J.M., Lipscomb, S.W., and Lewis, T.W., 1986, Sediment transport characteristics of selected streams in the Susitna River Basin, Alaska, October 1983 to September 1984: U.S. Geological Survey Open-File Report $86-424 \mathrm{~W}, 73 \mathrm{p}$.

Meade, R.H., Emmett, W.W., and Myrick, R.M., 1981, Movement and storage of bed material during 1979 in East Fork River, Wyoming, USA: International Association of Hydrological Sciences Publication 132, p. 225-235.

Milhous, R.T., 1973, Sediment transport in a gravel-bottomed stream [ $\mathrm{PhD}$ dissertation]: Corvallis, Oregon State University, $232 \mathrm{p}$.

Nordin, C.F., Jr., 1980, Data collection and analyses, in Shen, H.W., and Kikkawa, H. (eds.), Application of stochastic processes in sediment transport: Littleton, Colorado, Water Resources Publications, p. $2-1-2-25$.

Pitlick, J., 1988, Variability of bed load measurement: Water Resources Research, v. 24, no. 1, p. 173-177.

Williams, R.P., and Krupin, P.J., 1984, Erosion, channel change, and sediment transport in the Big Lost River, Idaho: U.S. Geological Survey WaterResources Investigations Report 84-4147, 87 p. 
Table 1.--Hydraulic and sediment-transport data

$\left[\mathrm{m}^{3} / \mathrm{s}\right.$, cubic meters per second; $\mathrm{m} / \mathrm{s}$, meters per second; $\mathrm{m}$, meters; $\mathrm{m} / \mathrm{m}$, meters per meter; ${ }^{\circ} \mathrm{C}$, degrees Celsius; $\mathrm{mg} / \mathrm{L}$, milligrams per liter; kg/s, kilograms per second; --, no data available]

\begin{tabular}{|c|c|c|c|c|c|c|c|c|c|c|c|}
\hline Date & $\begin{array}{c}\text { Water } \\
\text { discharge } \\
\text { (bedload })^{1} \\
\left(\mathrm{~m}^{3} / \mathrm{s}\right)\end{array}$ & $\begin{array}{c}\text { Water } \\
\text { discharge } \\
\text { (suspended } \\
\text { load) } \\
\left(\mathrm{m}^{3} / \mathrm{s}\right)\end{array}$ & $\begin{array}{l}\text { Mean } \\
\text { flow } \\
\text { velocity } \\
(\mathrm{m} / \mathrm{s})\end{array}$ & $\begin{array}{l}\text { Water- } \\
\text { surface } \\
\text { width } \\
\text { (m) }\end{array}$ & $\begin{array}{l}\text { Mean } \\
\text { flow } \\
\text { depth } \\
(m)\end{array}$ & $\begin{array}{c}\text { Water- } \\
\text { surface } \\
\text { slope } \\
(\mathrm{m} / \mathrm{m})\end{array}$ & $\begin{array}{l}\text { Water } \\
\text { temp. } \\
\left({ }^{\circ} \mathrm{C}\right)\end{array}$ & $\begin{array}{l}\text { Suspended } \\
\text { sediment } \\
\text { concern- } \\
\text { tration } \\
(\mathrm{mg} / \mathrm{L})\end{array}$ & $\begin{array}{c}\text { Suspended } \\
\text { load } \\
(\mathrm{kg} / \mathrm{s})\end{array}$ & $\begin{array}{l}\text { Number of } \\
\text { sampling } \\
\text { points } \\
\text { for } \\
\text { bedload }\end{array}$ & $\begin{array}{r}\text { Bedload } \\
(\mathrm{kg} / \mathrm{s})\end{array}$ \\
\hline & \multicolumn{11}{|c|}{ 1. Susitna River near Talkeetna, Alaska ${ }^{3}$} \\
\hline $\begin{array}{l}06-03-82 \\
06-15-82 \\
06-22-82 \\
06-30-82 \\
07-08-82\end{array}$ & $\begin{array}{r}1,010 \\
685 \\
1,050 \\
855 \\
589\end{array}$ & $\begin{array}{r}1,010 \\
685 \\
1,050 \\
855 \\
586\end{array}$ & $\begin{array}{l}2.2 \\
2.3 \\
2.4 \\
2.3 \\
2.0\end{array}$ & $\begin{array}{l}191 \\
189 \\
197 \\
190 \\
182\end{array}$ & $\begin{array}{l}2.4 \\
1.6 \\
2.0 \\
2.0 \\
1.6\end{array}$ & $\begin{array}{c}.0 \\
0.0015 \\
.0018 \\
.0013\end{array}$ & $\begin{array}{r}6.0 \\
8.0 \\
10.0 \\
11.5 \\
14.5\end{array}$ & $\begin{array}{l}769 \\
181 \\
438 \\
438 \\
145\end{array}$ & $\begin{array}{r}777 \\
124 \\
460 \\
374 \\
85.0\end{array}$ & $\begin{array}{l}16-25 \\
16-25 \\
16-25 \\
16-25 \\
16-25\end{array}$ & $\begin{array}{c}29.8 \\
8.73 \\
10.4 \\
4.64 \\
3.40\end{array}$ \\
\hline $\begin{array}{l}07-14-82 \\
07-21-82 \\
07-28-82 \\
08-04-82 \\
08-10-82\end{array}$ & $\begin{array}{l}872 \\
705 \\
872 \\
646 \\
572\end{array}$ & $\begin{array}{l}872 \\
705 \\
872 \\
643 \\
566\end{array}$ & $\begin{array}{l}2.3 \\
2.1 \\
2.1 \\
2.1 \\
2.1\end{array}$ & $\begin{array}{l}190 \\
184 \\
188 \\
184 \\
182\end{array}$ & $\begin{array}{l}2.0 \\
1.8 \\
2.2 \\
1.7 \\
1.5\end{array}$ & $\begin{array}{l}.0014 \\
.0015 \\
.0016 \\
.0014 \\
.0013\end{array}$ & $\begin{array}{c}12.0 \\
13.5 \\
-0 \\
13.0 \\
10.0\end{array}$ & $\begin{array}{l}768 \\
383 \\
461 \\
341 \\
289\end{array}$ & $\begin{array}{l}670 \\
270 \\
402 \\
219 \\
164\end{array}$ & $\begin{array}{l}16-25 \\
16-25 \\
16-25 \\
16-25 \\
16-25\end{array}$ & $\begin{array}{l}9.51 \\
3.78 \\
6.30 \\
2.26 \\
2.96\end{array}$ \\
\hline $\begin{array}{l}08-18-82 \\
08-25-82 \\
08-31-82 \\
09-19-82 \\
05-19-83\end{array}$ & $\begin{array}{l}504 \\
479 \\
549 \\
818 \\
612\end{array}$ & $\begin{array}{l}501 \\
476 \\
547 \\
813 \\
612\end{array}$ & $\begin{array}{l}2.0 \\
2.0 \\
2.2 \\
2.4 \\
1.8\end{array}$ & $\begin{array}{l}170 \\
170 \\
178 \\
188 \\
188\end{array}$ & $\begin{array}{l}1.5 \\
1.4 \\
1.4 \\
1.8 \\
1.8\end{array}$ & $\begin{array}{l}.0014 \\
.0013 \\
.0013 \\
.0014 \\
.0013\end{array}$ & $\begin{array}{r}10.5 \\
12.0 \\
9.0 \\
6.5 \\
4.5\end{array}$ & $\begin{array}{l}285 \\
219 \\
251 \\
442 \\
386\end{array}$ & $\begin{array}{l}143 \\
104 \\
137 \\
359 \\
236\end{array}$ & $\begin{array}{l}16-25 \\
16-25 \\
16-25 \\
16-25 \\
16-25\end{array}$ & $\begin{array}{l}1.11 \\
1.16 \\
1.97 \\
3.91 \\
4.52\end{array}$ \\
\hline $\begin{array}{l}05-25-83 \\
06-01-83 \\
06-08-83 \\
06-23-83 \\
07-07-83\end{array}$ & $\begin{array}{r}547 \\
1,110 \\
685 \\
765 \\
776\end{array}$ & $\begin{array}{r}547 \\
1,080 \\
685 \\
784 \\
776\end{array}$ & $\begin{array}{l}1.8 \\
2.4 \\
2.0 \\
2.2 \\
2.1\end{array}$ & $\begin{array}{l}183 \\
202 \\
190 \\
187 \\
183\end{array}$ & $\begin{array}{l}1.7 \\
2.3 \\
1.8 \\
1.8 \\
2.0\end{array}$ & $\begin{array}{l}.0012 \\
.0016 \\
.0013 \\
.0014 \\
.0015\end{array}$ & $\begin{array}{r}6.5 \\
9.0 \\
10.5 \\
14.0 \\
-.\end{array}$ & $\begin{array}{l}164 \\
663 \\
287 \\
346 \\
958\end{array}$ & $\begin{array}{l}89.7 \\
716 \\
197 \\
271 \\
743\end{array}$ & $\begin{array}{l}16-25 \\
16-25 \\
16-25 \\
16-25 \\
16-25\end{array}$ & $\begin{array}{l}3.13 \\
2.36 \\
6.64 \\
8.82 \\
8.15\end{array}$ \\
\hline $\begin{array}{l}07-21-83 \\
08-02-83 \\
08-11-83 \\
08-31-83 \\
09-14-83\end{array}$ & $\begin{array}{l}544 \\
680 \\
932 \\
759 \\
320\end{array}$ & $\begin{array}{l}535 \\
674 \\
923 \\
759 \\
320\end{array}$ & $\begin{array}{l}1.9 \\
2.1 \\
2.7 \\
2.0 \\
1.5\end{array}$ & $\begin{array}{l}182 \\
183 \\
186 \\
194 \\
172\end{array}$ & $\begin{array}{l}1.6 \\
1.8 \\
1.9 \\
2.0 \\
1.2\end{array}$ & $\begin{array}{l}.0013 \\
.0014 \\
.0015 \\
.0014 \\
.0014\end{array}$ & $\begin{array}{r}13.0 \\
14.0 \\
11.0 \\
9.0 \\
6.0\end{array}$ & $\begin{array}{r}297 \\
521 \\
603 \\
297 \\
41\end{array}$ & $\begin{array}{r}159 \\
351 \\
557 \\
225 \\
13.1\end{array}$ & $\begin{array}{l}16-25 \\
16-25 \\
16-25 \\
16-25 \\
16-25\end{array}$ & $\begin{array}{c}3.17 \\
7.01 \\
8.97 \\
4.19 \\
.735\end{array}$ \\
\hline $\begin{array}{l}10-06-83 \\
06-13-84 \\
07-09-84 \\
07-30-84 \\
08-16-84\end{array}$ & $\begin{array}{l}303 \\
700 \\
632 \\
875 \\
430\end{array}$ & $\begin{array}{l}300 \\
733 \\
634 \\
875 \\
430\end{array}$ & $\begin{array}{l}1.5 \\
2.2 \\
2.0 \\
2.2 \\
1.7\end{array}$ & $\begin{array}{l}166 \\
187 \\
184 \\
191 \\
170\end{array}$ & $\begin{array}{l}1.2 \\
1.7 \\
1.7 \\
2.1 \\
1.5\end{array}$ & $\begin{array}{l}.0014 \\
.0014 \\
.0014 \\
-- \\
.0012\end{array}$ & $\begin{array}{c}.5 \\
10.5 \\
12.5 \\
-- \\
12.0\end{array}$ & $\begin{array}{r}23 \\
279 \\
323 \\
458 \\
220\end{array}$ & $\begin{array}{l}6.90 \\
205 \\
205 \\
401 \\
94.6\end{array}$ & $\begin{array}{l}16-25 \\
16-25 \\
16-25 \\
16-25 \\
16-25\end{array}$ & $\begin{array}{l}.284 \\
4.11 \\
2.50 \\
5.92 \\
2.54\end{array}$ \\
\hline $\begin{array}{l}08-26-84 \\
09-13-84 \\
09-25-84 \\
05-29-85 \\
06-26-85\end{array}$ & $\begin{array}{r}1,160 \\
265 \\
240 \\
1,310 \\
867\end{array}$ & $\begin{array}{r}1,160 \\
266 \\
238 \\
1,300 \\
875\end{array}$ & $\begin{array}{l}2.6 \\
1.2 \\
1.3 \\
2.7 \\
2.4\end{array}$ & $\begin{array}{l}194 \\
168 \\
165 \\
201 \\
189\end{array}$ & $\begin{array}{l}2.3 \\
1.2 \\
1.1 \\
2.4 \\
1.9\end{array}$ & $\begin{array}{l}.0014 \\
.0011 \\
.0012 \\
-- \\
-\end{array}$ & $\begin{array}{l}7.5 \\
7.5 \\
6.0 \\
4.0 \\
9.0\end{array}$ & $\begin{array}{r}732 \\
27 \\
14 \\
703 \\
251\end{array}$ & $\begin{array}{c}849 \\
7.18 \\
3.33 \\
914 \\
220\end{array}$ & $\begin{array}{l}16-25 \\
16-25 \\
16-25 \\
16-25 \\
16-25\end{array}$ & $\begin{array}{c}9.39 \\
.147 \\
.305 \\
6.20 \\
3.65\end{array}$ \\
\hline $\begin{array}{l}07-26-85 \\
08-13-85 \\
09-06-85 \\
09-19-85\end{array}$ & $\begin{array}{l}595 \\
833 \\
405 \\
535\end{array}$ & $\begin{array}{l}595 \\
850 \\
402 \\
535\end{array}$ & $\begin{array}{l}1.8 \\
2.3 \\
1.8 \\
1.9\end{array}$ & $\begin{array}{l}185 \\
190 \\
171 \\
181\end{array}$ & $\begin{array}{l}1.8 \\
1.9 \\
1.3 \\
1.6\end{array}$ & $\begin{array}{l}-- \\
-- \\
--\end{array}$ & $\begin{array}{r}12.5 \\
9.5 \\
7.5 \\
3.5\end{array}$ & $\begin{array}{r}310 \\
474 \\
69 \\
110\end{array}$ & $\begin{array}{r}184 \\
403 \\
27.7 \\
58.9\end{array}$ & $\begin{array}{l}16-25 \\
16-25 \\
16-25 \\
16-25\end{array}$ & $\begin{array}{l}2.23 \\
5.88 \\
.704 \\
2.23\end{array}$ \\
\hline
\end{tabular}


Table 1,--hydraulic and sediment-transpurt data--Continued

\begin{tabular}{|c|c|c|c|c|c|c|c|c|c|c|c|}
\hline Date & $\begin{array}{c}\text { Water } \\
\text { discharge } \\
\text { (bedload) } \\
\left(\mathrm{m}^{3} / \mathrm{s}\right)\end{array}$ & $\begin{array}{c}\text { Water } \\
\text { discharge } \\
\text { (suspended } \\
\text { load) } \\
\left(\mathrm{m}^{3} / \mathrm{s}\right)\end{array}$ & $\begin{array}{l}\text { Hean } \\
\text { flow } \\
\text { velocity } \\
(\mathrm{m} / \mathrm{s})\end{array}$ & $\begin{array}{l}\text { Water-. } \\
\text { surface } \\
\text { width } \\
(m)\end{array}$ & $\begin{array}{l}\text { Mean } \\
\text { flow } \\
\text { depth } \\
(m)\end{array}$ & $\begin{array}{l}\text { Water-- } \\
\text { surface } \\
\text { slope } \\
(\mathrm{m} / \mathrm{m})\end{array}$ & $\begin{array}{l}\text { Water } \\
\text { temp. } \\
\left({ }^{\circ} \mathrm{C}\right)\end{array}$ & $\begin{array}{c}\text { Suspended } \\
\text { sediment } \\
\text { concern- } \\
\text { tration } \\
(\mathrm{mg} / \mathrm{l})\end{array}$ & $\begin{array}{c}\text { Suspended } \\
\text { load } \\
(\mathrm{kg} / \mathrm{s})\end{array}$ & $\begin{array}{c}\text { Number of } \\
\text { sampling } \\
\text { points } \\
\text { for } \\
\text { bedload }\end{array}$ & $\begin{array}{r}\text { Bedload } \\
(\mathrm{kg} / \mathrm{s})\end{array}$ \\
\hline
\end{tabular}

2. Talkeetna River near Talkeetna, $\mathrm{Alaska}^{3}$

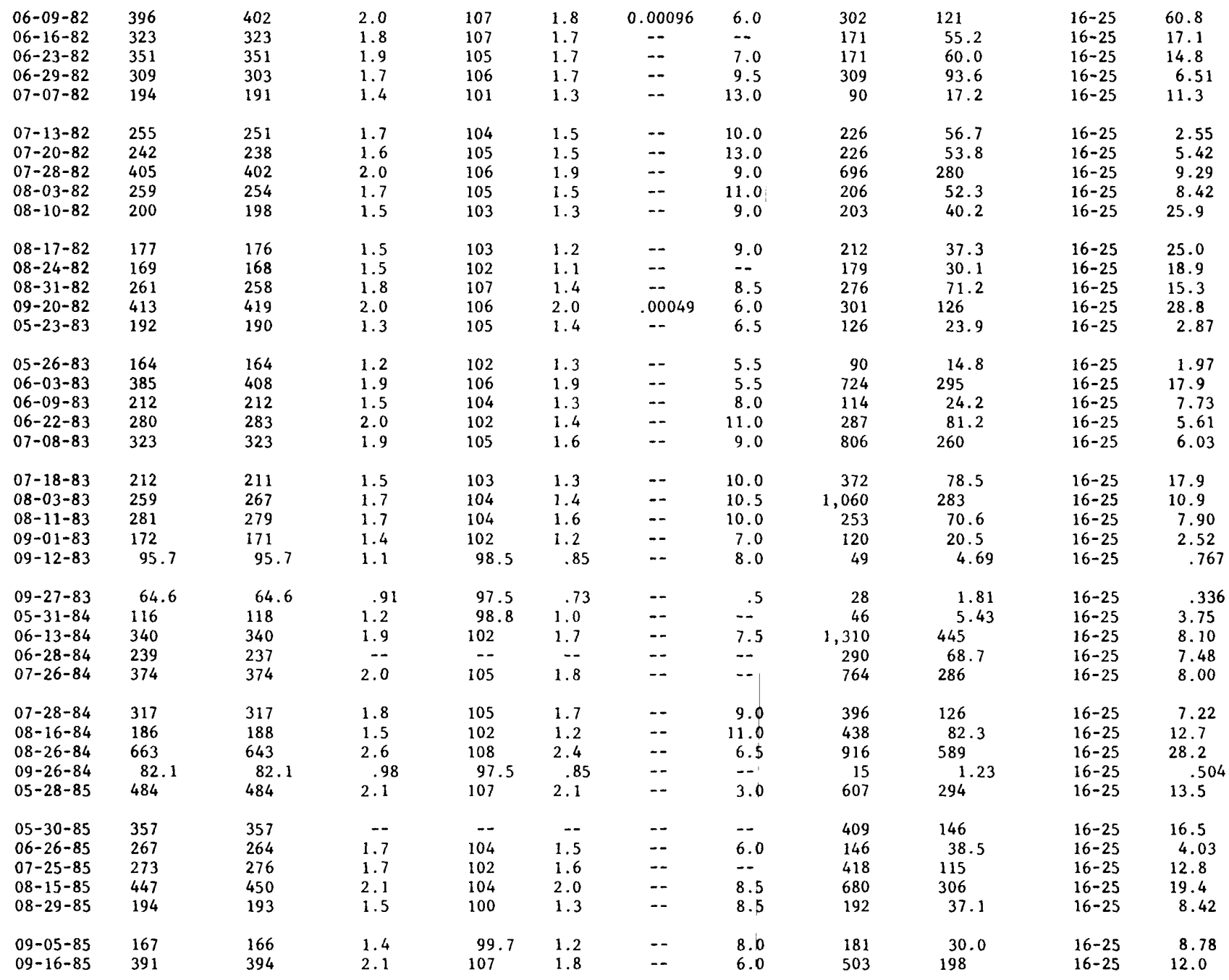


Table 1.--Hydraulic and sediment-transport data-Conlinued

\begin{tabular}{|c|c|c|c|c|c|c|c|c|c|c|c|}
\hline Date & $\begin{array}{c}\text { Water } \\
\text { discharge } \\
\text { (bedload) } \\
\left(\mathrm{m}^{3} / \mathrm{s}\right)\end{array}$ & $\begin{array}{c}\text { Water } \\
\text { discharge } \\
\text { (suspended } \\
\text { load) } \\
\left(\mathrm{m}^{3} / \mathrm{s}\right)\end{array}$ & $\begin{array}{l}\text { Mean } \\
\text { flow } \\
\text { velocity } \\
(\mathrm{m} / \mathrm{s})\end{array}$ & $\begin{array}{l}\text { Water- } \\
\text { surface } \\
\text { width } \\
\text { (m) }\end{array}$ & $\begin{array}{l}\text { Mean } \\
\text { flow } \\
\text { depth } \\
\text { (m) }\end{array}$ & $\begin{array}{l}\text { Water- } \\
\text { surface } \\
\text { slope } \\
(\mathrm{m} / \mathrm{m})\end{array}$ & $\begin{array}{l}\text { Water } \\
\text { temp. } \\
\left({ }^{\circ} \mathrm{C}\right)\end{array}$ & $\begin{array}{l}\text { Suspended } \\
\text { sediment } \\
\text { concern- } \\
\text { tration } \\
(\mathrm{mg} / \mathrm{L})\end{array}$ & $\begin{array}{c}\text { Suspended } \\
\text { load } \\
\left(\mathrm{kg}_{\mathrm{g}} / \mathrm{s}\right)\end{array}$ & $\begin{array}{c}\text { Number of } \\
\text { sampling } \\
\text { points } \\
\text { for } \\
\text { bedload }\end{array}$ & $\begin{array}{r}\text { Bedload } \\
(\mathrm{kg} / \mathrm{s})\end{array}$ \\
\hline
\end{tabular}

3. Chulitna River below Canyon near Talkeetna, Alaska

\begin{tabular}{|c|c|c|c|c|c|c|c|c|c|c|c|}
\hline $\begin{array}{l}06-04-82 \\
06-09-82 \\
06-16-82 \\
06-22-82 \\
06-29-82\end{array}$ & $\begin{array}{l}354 \\
487 \\
413 \\
549 \\
818\end{array}$ & $\begin{array}{l}326 \\
479 \\
411 \\
552 \\
821\end{array}$ & $\begin{array}{l}1.7 \\
1.9 \\
1.8 \\
2.1 \\
2.4\end{array}$ & $\begin{array}{l}105 \\
106 \\
105 \\
109 \\
119\end{array}$ & $\begin{array}{l}2.0 \\
2.4 \\
2.2 \\
2.5 \\
2.9\end{array}$ & $\begin{array}{c}0.00080 \\
.- \\
.00068 \\
.0012 \\
.0014\end{array}$ & $\begin{array}{l}6.0 \\
6.5 \\
4.5 \\
7.5 \\
7.0\end{array}$ & $\begin{array}{r}424 \\
760 \\
428 \\
880 \\
1,600\end{array}$ & $\begin{array}{r}138 \\
364 \\
176 \\
486 \\
1,310\end{array}$ & $\begin{array}{l}16-25 \\
16-25 \\
16-25 \\
16-25 \\
16-25\end{array}$ & $\begin{array}{l}120 \\
192 \\
120 \\
107 \\
137\end{array}$ \\
\hline $\begin{array}{l}07-07-82 \\
07-13-82 \\
07-20-82 \\
07-27-82 \\
08-03-82\end{array}$ & $\begin{array}{l}583 \\
646 \\
654 \\
946 \\
666\end{array}$ & $\begin{array}{l}586 \\
643 \\
654 \\
903 \\
660\end{array}$ & $\begin{array}{l}2.1 \\
2.1 \\
2.1 \\
2.5 \\
2.3\end{array}$ & $\begin{array}{l}109 \\
114 \\
112 \\
123 \\
115\end{array}$ & $\begin{array}{l}2.5 \\
2.6 \\
2.7 \\
3.1 \\
2.5\end{array}$ & $\begin{array}{l}.0012 \\
.0011 \\
.0012 \\
.0014 \\
.0014\end{array}$ & $\begin{array}{l}9.0 \\
6.5 \\
9.0 \\
6.0 \\
8.0\end{array}$ & $\begin{array}{r}1,000 \\
1,270 \\
1,140 \\
1,110 \\
803\end{array}$ & $\begin{array}{r}586 \\
817 \\
746 \\
1,000 \\
530\end{array}$ & $\begin{array}{l}16-25 \\
16-25 \\
16-25 \\
16-25 \\
16-25\end{array}$ & $\begin{array}{c}101 \\
95.7 \\
145 \\
72.5 \\
78.6\end{array}$ \\
\hline $\begin{array}{l}08-11-82 \\
08-17-82 \\
08-24-82 \\
09-01-82 \\
09-08-82\end{array}$ & $\begin{array}{l}615 \\
623 \\
507 \\
484 \\
838\end{array}$ & $\begin{array}{l}603 \\
620 \\
515 \\
490 \\
827\end{array}$ & $\begin{array}{l}2.2 \\
2.2 \\
1.9 \\
1.9 \\
2.5\end{array}$ & $\begin{array}{l}110 \\
110 \\
109 \\
108 \\
119\end{array}$ & $\begin{array}{l}2.5 \\
2.6 \\
2.4 \\
2.3 \\
2.8\end{array}$ & $\begin{array}{l}.0010 \\
.0012 \\
.0010 \\
.00092 \\
.0012\end{array}$ & $\begin{array}{l}6.0 \\
5.0 \\
5.5 \\
6.0 \\
5.0\end{array}$ & $\begin{array}{r}766 \\
1,180 \\
830 \\
506 \\
1,680\end{array}$ & $\begin{array}{r}462 \\
732 \\
427 \\
248 \\
1,390\end{array}$ & $\begin{array}{l}16-25 \\
16-25 \\
16-25 \\
16-25 \\
16-25\end{array}$ & $\begin{array}{r}102 \\
127 \\
79.4 \\
78.5 \\
26.9\end{array}$ \\
\hline $\begin{array}{l}05-19-83 \\
05-25-83 \\
05-31-83 \\
06-02-83 \\
06-09-83\end{array}$ & $\begin{array}{l}346 \\
323 \\
524 \\
504 \\
388\end{array}$ & $\begin{array}{l}348 \\
329 \\
524 \\
498 \\
388\end{array}$ & $\begin{array}{l}1.8 \\
1.6 \\
2.1 \\
2.0 \\
1.8\end{array}$ & $\begin{array}{l}98.5 \\
102 \\
108 \\
103 \\
108\end{array}$ & $\begin{array}{l}1.9 \\
2.0 \\
2.3 \\
2.4 \\
2.0\end{array}$ & $\begin{array}{l}.00068 \\
.00068 \\
.0010 \\
.0012 \\
.0010\end{array}$ & $\begin{array}{l}5.5 \\
-- \\
7.0 \\
7.5 \\
6.5\end{array}$ & $\begin{array}{r}347 \\
235 \\
1,080 \\
773 \\
443\end{array}$ & $\begin{array}{l}121 \\
77.3 \\
566 \\
385 \\
172\end{array}$ & $\begin{array}{l}16-25 \\
16-25 \\
16-25 \\
16-25 \\
16-25\end{array}$ & $\begin{array}{l}35.3 \\
74.0 \\
49.8 \\
86.5 \\
50.5\end{array}$ \\
\hline $\begin{array}{l}06-22-83 \\
07-06-83 \\
07-20-83 \\
08-02-83 \\
08-09-83\end{array}$ & $\begin{array}{r}666 \\
821 \\
566 \\
629 \\
1,350\end{array}$ & $\begin{array}{r}666 \\
830 \\
566 \\
634 \\
1,350\end{array}$ & $\begin{array}{l}2.2 \\
2.3 \\
2.0 \\
2.2 \\
2.7\end{array}$ & $\begin{array}{l}113 \\
118 \\
109 \\
113 \\
136\end{array}$ & $\begin{array}{l}2.7 \\
3.1 \\
2.6 \\
2.5 \\
3.6\end{array}$ & $\begin{array}{l}.0015 \\
.0015 \\
.0010 \\
.0013 \\
.0026\end{array}$ & $\begin{array}{r}10.0 \\
16.5 \\
6.0 \\
6.5 \\
6.0\end{array}$ & $\begin{array}{l}1,500 \\
2,040 \\
1,240 \\
1,770 \\
4,690\end{array}$ & $\begin{array}{r}1,600 \\
1,690 \\
702 \\
1,120 \\
6,330\end{array}$ & $\begin{array}{l}16-25 \\
16-25 \\
16-25 \\
16-25 \\
16-25\end{array}$ & $\begin{array}{c}71.8 \\
124 \\
68.3 \\
73.3 \\
83.8\end{array}$ \\
\hline $\begin{array}{l}08-31-83 \\
09-13-83 \\
10-05-83 \\
05-18-84 \\
06-11-84\end{array}$ & $\begin{array}{l}770 \\
279 \\
260 \\
261 \\
456\end{array}$ & $\begin{array}{l}765 \\
279 \\
260 \\
261 \\
456\end{array}$ & $\begin{array}{l}2.3 \\
1.5 \\
1.5 \\
1.5 \\
2.0\end{array}$ & $\begin{array}{l}118 \\
101 \\
101 \\
100 \\
105\end{array}$ & $\begin{array}{l}2.8 \\
1.8 \\
1.8 \\
1.7 \\
2.2\end{array}$ & $\begin{array}{l}.0012 \\
.00064 \\
.00044 \\
.00074 \\
.0010\end{array}$ & $\begin{array}{l}6.5 \\
5.5 \\
1.5 \\
4.0 \\
8.5\end{array}$ & $\begin{array}{r}1,500 \\
614 \\
200 \\
580 \\
571\end{array}$ & $\begin{array}{c}1,150 \\
171 \\
52.0 \\
151 \\
260\end{array}$ & $\begin{array}{l}16-25 \\
16-25 \\
16-25 \\
16-25 \\
16-25\end{array}$ & $\begin{array}{r}122 \\
60.7 \\
35.5 \\
29.9 \\
69.2\end{array}$ \\
\hline $\begin{array}{l}06-14-84 \\
07-11-84 \\
07-31-84 \\
08-17-84 \\
08-28-84\end{array}$ & $\begin{array}{l}549 \\
572 \\
660 \\
564 \\
507\end{array}$ & $\begin{array}{l}544 \\
572 \\
649 \\
575 \\
513\end{array}$ & $\begin{array}{l}2.2 \\
2.1 \\
2.2 \\
2.0 \\
2.0\end{array}$ & $\begin{array}{l}108 \\
109 \\
113 \\
108 \\
106\end{array}$ & $\begin{array}{l}2.3 \\
2.5 \\
2.7 \\
2.6 \\
2.4\end{array}$ & $\begin{array}{l}.0011 \\
.00098 \\
.0012 \\
.0012 \\
.00083\end{array}$ & $\begin{array}{l}6.5 \\
8.0 \\
6.0 \\
6.0 \\
4.0\end{array}$ & $\begin{array}{r}895 \\
1,010 \\
921 \\
931 \\
556\end{array}$ & $\begin{array}{l}487 \\
578 \\
598 \\
535 \\
285\end{array}$ & $\begin{array}{l}16-25 \\
16-25 \\
16-25 \\
16-25 \\
16-25\end{array}$ & $\begin{array}{l}54.3 \\
40.5 \\
54.5 \\
59.2 \\
43.1\end{array}$ \\
\hline $\begin{array}{l}09-14-84 \\
09-27-84 \\
05-31-85 \\
06-27-85 \\
07-24-85\end{array}$ & $\begin{array}{l}317 \\
212 \\
518 \\
632 \\
668\end{array}$ & $\begin{array}{l}314 \\
212 \\
510 \\
643 \\
697\end{array}$ & $\begin{array}{l}1.6 \\
1.2 \\
2.1 \\
2.3 \\
2.3\end{array}$ & $\begin{array}{l}103 \\
101 \\
104 \\
105 \\
105\end{array}$ & $\begin{array}{l}1.9 \\
1.7 \\
2.3 \\
2.6 \\
2.8\end{array}$ & $\begin{array}{l}.00057 \\
.00039 \\
.- \\
-- \\
--\end{array}$ & $\begin{array}{l}4.0 \\
4.0 \\
2.0 \\
6.5 \\
6.5\end{array}$ & $\begin{array}{r}388 \\
133 \\
594 \\
1,240 \\
985\end{array}$ & $\begin{array}{l}122 \\
28.2 \\
303 \\
797 \\
687\end{array}$ & $\begin{array}{l}16-25 \\
16-25 \\
16-25 \\
16-25 \\
16-25\end{array}$ & $\begin{array}{l}21.9 \\
22.3 \\
63.1 \\
73.4 \\
37.7\end{array}$ \\
\hline $\begin{array}{l}08-16-85 \\
09-05-85 \\
09-17-85\end{array}$ & $\begin{array}{r}1,104 \\
405 \\
515\end{array}$ & $\begin{array}{r}1,102 \\
391 \\
515\end{array}$ & $\begin{array}{l}2.7 \\
1.7 \\
2.0\end{array}$ & $\begin{array}{l}120 \\
103 \\
104\end{array}$ & $\begin{array}{l}3.4 \\
2.3 \\
2.5\end{array}$ & $\begin{array}{l}-- \\
--\end{array}$ & $\begin{array}{l}7.0 \\
4.5 \\
3.0\end{array}$ & $\begin{array}{r}1,920 \\
410 \\
544\end{array}$ & $\begin{array}{r}2,120 \\
160 \\
280\end{array}$ & $\begin{array}{l}16-25 \\
16-25 \\
16-25\end{array}$ & $\begin{array}{l}49.0 \\
41.0 \\
63.2\end{array}$ \\
\hline
\end{tabular}


Table 1.--Hydraulic and sediment-transport data--Continued

\begin{tabular}{|c|c|c|c|c|c|c|c|c|c|c|c|}
\hline Date & $\begin{array}{c}\text { Water } \\
\text { discharge } \\
\text { (bedload) } \\
\left(\mathrm{m}^{3} / \mathrm{s}\right)\end{array}$ & $\begin{array}{c}\text { Water } \\
\text { discharge } \\
\text { (suspended } \\
\text { load) } \\
\left(\mathrm{m}^{3} / \mathrm{s}\right)\end{array}$ & $\begin{array}{l}\text { Mean } \\
\text { flow } \\
\text { velocity } \\
(\mathrm{m} / \mathrm{s})\end{array}$ & $\begin{array}{l}\text { Water.- } \\
\text { surface } \\
\text { width } \\
\text { (m) }\end{array}$ & $\begin{array}{l}\text { Mean } \\
\text { flow } \\
\text { depth } \\
(m)\end{array}$ & $\begin{array}{l}\text { Water- } \\
\text { surface } \\
\text { slope } \\
(\mathrm{m} / \mathrm{m})\end{array}$ & $\begin{array}{l}\text { Water } \\
\text { temp. } \\
\left({ }^{\circ} \mathrm{C}\right)\end{array}$ & $\begin{array}{c}\text { Suspended } \\
\text { sediment } \\
\text { concern- } \\
\text { tration } \\
(m g / L)\end{array}$ & $\begin{array}{c}\text { Suspended } \\
\text { load } \\
(\mathrm{kg} / \mathrm{s})\end{array}$ & $\begin{array}{l}\text { Number of } \\
\text { sampling } \\
\text { points } \\
\text { for } \\
\text { bedload }\end{array}$ & $\begin{array}{r}\text { Bedload } \\
(\mathrm{kg} / \mathrm{s})\end{array}$ \\
\hline
\end{tabular}

4. Yentna River near Susitna Station, Alaska

\begin{tabular}{|c|c|c|c|c|c|c|c|c|c|c|c|}
\hline $\begin{array}{l}05-14-84 \\
06-12-84 \\
07-17-84 \\
09-19-84 \\
05-23-85\end{array}$ & $\begin{array}{r}705 \\
1,240 \\
1,300 \\
578 \\
660\end{array}$ & $\begin{array}{r}691 \\
1,260 \\
1,220 \\
581 \\
654\end{array}$ & $\begin{array}{l}1.0 \\
1.2 \\
1.3 \\
.94 \\
.82\end{array}$ & $\begin{array}{l}393 \\
390 \\
393 \\
387 \\
389\end{array}$ & $\begin{array}{l}1.7 \\
2.6 \\
2.6 \\
1.6 \\
2.1\end{array}$ & $\begin{array}{l}-- \\
-- \\
-- \\
-- \\
--\end{array}$ & $\begin{array}{l}-- \\
-- \\
-- \\
5.0\end{array}$ & $\begin{array}{l}227 \\
363 \\
684 \\
257 \\
494\end{array}$ & $\begin{array}{l}157 \\
457 \\
834 \\
149 \\
323\end{array}$ & $\begin{array}{l}16-25 \\
16-25 \\
16-25 \\
16-25 \\
16-25\end{array}$ & $\begin{array}{l}119 \\
105 \\
71.4 \\
86.3 \\
60.1\end{array}$ \\
\hline $\begin{array}{l}06-20-85 \\
07-17-85 \\
08-14-85 \\
08-19-85 \\
09-18-85\end{array}$ & $\begin{array}{r}1,210 \\
1,540 \\
1,500 \\
1,120 \\
640\end{array}$ & $\begin{array}{r}1,210 \\
1,520 \\
1,490 \\
1,130 \\
643\end{array}$ & $\begin{array}{l}1.2 \\
1.4 \\
1.4 \\
1.2 \\
1.0\end{array}$ & $\begin{array}{l}390 \\
393 \\
393 \\
392 \\
392\end{array}$ & $\begin{array}{l}2.6 \\
2.9 \\
2.7 \\
2.3 \\
1.7\end{array}$ & $\begin{array}{l}-- \\
-- \\
-- \\
--\end{array}$ & $\begin{array}{r}8.5 \\
10.5 \\
9.0 \\
8.0 \\
4.0\end{array}$ & $\begin{array}{l}322 \\
784 \\
657 \\
669 \\
355\end{array}$ & $\begin{array}{r}390 \\
1,190 \\
979 \\
756 \\
228\end{array}$ & $\begin{array}{l}16-25 \\
16-25 \\
16-25 \\
16-25 \\
16-25\end{array}$ & $\begin{array}{c}64.4 \\
84.7 \\
140 \\
45.8 \\
78.6\end{array}$ \\
\hline
\end{tabular}

5. Susitna River (Right Channel) below Chulitna River near Talkeetna, Alaska

\begin{tabular}{|c|c|c|c|c|c|c|c|c|c|c|c|}
\hline $\begin{array}{l}06-12-84 \\
07-10-84 \\
07-30-84 \\
08-27-84 \\
09-12-84\end{array}$ & $\begin{array}{r}668 \\
827 \\
1,010 \\
821 \\
473\end{array}$ & $\begin{array}{r}668 \\
827 \\
1,010 \\
821 \\
473\end{array}$ & $\begin{array}{l}2.2 \\
2.3 \\
2.5 \\
2.2 \\
1.9\end{array}$ & $\begin{array}{l}219 \\
230 \\
235 \\
390 \\
206\end{array}$ & $\begin{array}{l}1.4 \\
1.6 \\
1.7 \\
.94 \\
1.2\end{array}$ & $\begin{array}{l}-- \\
-- \\
-- \\
--\end{array}$ & $\begin{array}{r}10.5 \\
6.5 \\
6.5 \\
5.0 \\
6.5\end{array}$ & $\begin{array}{r}707 \\
1,070 \\
975 \\
831 \\
209\end{array}$ & $\begin{array}{r}472 \\
885 \\
985 \\
682 \\
98.9\end{array}$ & $\begin{array}{l}16 \\
16 \\
16 \\
16 \\
16\end{array}$ & $\begin{array}{c}73.3 \\
22.6 \\
57.4 \\
128 \\
6.85\end{array}$ \\
\hline $\begin{array}{l}09-26-84 \\
05-30-85 \\
06-25-85 \\
07-25-85 \\
08-15-85\end{array}$ & $\begin{array}{r}357 \\
963 \\
892 \\
954 \\
1,100\end{array}$ & $\begin{array}{r}357 \\
963 \\
892 \\
954 \\
1,100\end{array}$ & $\begin{array}{l}1.7 \\
2.6 \\
2.8 \\
2.2 \\
2.2\end{array}$ & $\begin{array}{l}183 \\
335 \\
247 \\
287 \\
506\end{array}$ & $\begin{array}{l}1.1 \\
1.1 \\
1.3 \\
1.5 \\
1.0\end{array}$ & $\begin{array}{l}-- \\
-- \\
-- \\
--\end{array}$ & $\begin{array}{r}6.0 \\
2.5 \\
7.5 \\
10.5 \\
7.5\end{array}$ & $\begin{array}{r}159 \\
912 \\
591 \\
1,120 \\
3,400\end{array}$ & $\begin{array}{r}56.8 \\
878 \\
527 \\
1.070 \\
3,740\end{array}$ & $\begin{array}{l}16 \\
16 \\
16 \\
16 \\
16\end{array}$ & $\begin{array}{l}24.5 \\
58.1 \\
37.9 \\
28.8 \\
25.6\end{array}$ \\
\hline $\begin{array}{l}09-04-85 \\
09-18-85\end{array}$ & $\begin{array}{l}736 \\
765\end{array}$ & $\begin{array}{l}736 \\
765\end{array}$ & $\begin{array}{l}2.1 \\
2.1\end{array}$ & $\begin{array}{l}250 \\
250\end{array}$ & $\begin{array}{l}1.4 \\
1.5\end{array}$ & -- & $\begin{array}{l}8.0 \\
3.5\end{array}$ & $\begin{array}{l}365 \\
388\end{array}$ & $\begin{array}{l}269 \\
297\end{array}$ & $\begin{array}{l}16 \\
16\end{array}$ & $\begin{array}{r}7.14 \\
24.4\end{array}$ \\
\hline
\end{tabular}

6. Susitna River (Left Channel) below Chulitna River near Talkeetna, Alaska ${ }^{5}$

\begin{tabular}{|c|c|c|c|c|c|c|c|c|c|c|c|}
\hline $\begin{array}{l}06-12-84 \\
07-10-84 \\
07-29-84 \\
09-12-84 \\
09-26-84\end{array}$ & $\begin{array}{c}459 \\
425 \\
558 \\
172 \\
76.5\end{array}$ & $\begin{array}{c}459 \\
453 \\
558 \\
172 \\
76.5\end{array}$ & $\begin{array}{l}1.8 \\
1.8 \\
1.6 \\
1.2 \\
1.2\end{array}$ & $\begin{array}{r}189 \\
177 \\
226 \\
152 \\
89.0\end{array}$ & $\begin{array}{l}1.4 \\
1.3 \\
1.5 \\
1.0 \\
.73\end{array}$ & $\begin{array}{l}-- \\
-- \\
-- \\
--\end{array}$ & $\begin{array}{r}11.5 \\
12.0 \\
12.0 \\
8.0 \\
7.0\end{array}$ & $\begin{array}{r}215 \\
314 \\
509 \\
36 \\
21\end{array}$ & $\begin{array}{r}98.7 \\
142 \\
284 \\
6.19 \\
1.61\end{array}$ & $\begin{array}{l}16 \\
16 \\
16 \\
16 \\
16\end{array}$ & $\begin{array}{l}3.78 \\
3.35 \\
8.30 \\
.263 \\
.189\end{array}$ \\
\hline $\begin{array}{l}05-30-85 \\
06-25-85 \\
07-25-85 \\
08-15-85 \\
09-04-85 \\
09-18-85\end{array}$ & $\begin{array}{l}680 \\
408 \\
385 \\
708 \\
278 \\
283\end{array}$ & $\begin{array}{l}680 \\
408 \\
385 \\
708 \\
278 \\
283\end{array}$ & $\begin{array}{l}1.6 \\
1.6 \\
1.6 \\
1.6 \\
1.7 \\
1.4\end{array}$ & $\begin{array}{l}469 \\
241 \\
226 \\
518 \\
165 \\
238\end{array}$ & $\begin{array}{l}.88 \\
1.1 \\
1.0 \\
.82 \\
1.0 \\
.85\end{array}$ & $\begin{array}{l}-- \\
-- \\
-- \\
-- \\
--\end{array}$ & $\begin{array}{r}3.5 \\
9.0 \\
12.0 \\
10.0 \\
10.0 \\
4.0\end{array}$ & $\begin{array}{l}554 \\
193 \\
390 \\
508 \\
126 \\
222\end{array}$ & $\begin{array}{l}377 \\
78.7 \\
150 \\
360 \\
35.0 \\
62.8\end{array}$ & $\begin{array}{l}16 \\
16 \\
16 \\
16 \\
16 \\
16\end{array}$ & $\begin{array}{c}3.05 \\
7.78 \\
6.01 \\
10.8 \\
1.42 \\
3.90\end{array}$ \\
\hline
\end{tabular}

\section{Susitna River at Sunshine, $\mathrm{Alaska}^{3}$}

\begin{tabular}{|c|c|c|c|c|c|c|c|c|c|c|c|}
\hline $\begin{array}{l}06-03-82 \\
06-10-82 \\
06-17-82 \\
06-21-82 \\
06-28-82\end{array}$ & $\begin{array}{l}2,010 \\
1,830 \\
1,440 \\
2,230 \\
2,140\end{array}$ & $\begin{array}{l}2,090 \\
1,830 \\
1,440 \\
2,220 \\
2,140\end{array}$ & $\begin{array}{l}2.1 \\
1.9 \\
1.8 \\
2.0 \\
2.1\end{array}$ & $\begin{array}{l}311 \\
311 \\
295 \\
308 \\
305\end{array}$ & $\begin{array}{l}3.1 \\
3.1 \\
2.7 \\
3.7 \\
3.4\end{array}$ & $\begin{array}{c}0.0015 \\
.0014 \\
.0018 \\
\ldots\end{array}$ & $\begin{array}{r}7.5 \\
7.0 \\
7.0 \\
11.0\end{array}$ & $\begin{array}{l}847 \\
414 \\
360 \\
683 \\
702\end{array}$ & $\begin{array}{r}1,770 \\
758 \\
518 \\
1,520 \\
1,500\end{array}$ & $\begin{array}{l}16 \\
16 \\
16 \\
16 \\
16\end{array}$ & $\begin{array}{c}63.8 \\
143 \\
19.6 \\
26.4 \\
67.1\end{array}$ \\
\hline $\begin{array}{l}07-06-82 \\
07-12-82 \\
07-19-82\end{array}$ & $\begin{array}{l}1,320 \\
1,680 \\
1,740\end{array}$ & $\begin{array}{l}1,320 \\
1,690 \\
1,720\end{array}$ & $\begin{array}{l}1.8 \\
2.0 \\
1.9\end{array}$ & $\begin{array}{l}274 \\
286 \\
305\end{array}$ & $\begin{array}{l}2.7 \\
2.9 \\
3.0\end{array}$ & $\begin{array}{l}.0014 \\
.0015 \\
.0022\end{array}$ & $\begin{array}{c}10.0 \\
-- \\
9.5\end{array}$ & $\begin{array}{l}503 \\
800 \\
548\end{array}$ & $\begin{array}{r}664 \\
1,350 \\
943\end{array}$ & $\begin{array}{l}16 \\
16 \\
16\end{array}$ & $\begin{array}{l}63.2 \\
39.9 \\
41.6\end{array}$ \\
\hline
\end{tabular}


Table 1.--Hydraulic and sediment-transport data--Continued

\begin{tabular}{|c|c|c|c|c|c|c|c|c|c|c|c|}
\hline Date & $\begin{array}{c}\text { Water } \\
\text { discharge } \\
\text { (bedload) } \\
\left(\mathrm{m}^{3} / \mathrm{s}\right)\end{array}$ & $\begin{array}{c}\text { Water } \\
\text { discharge } \\
\text { (suspended } \\
\text { load) } \\
\left(\mathrm{m}^{3} / \mathrm{s}\right)\end{array}$ & $\begin{array}{c}\text { Mean } \\
\text { flow } \\
\text { velocity } \\
(\mathrm{m} / \mathrm{s})\end{array}$ & $\begin{array}{l}\text { Water- } \\
\text { surface } \\
\text { width } \\
(\mathrm{m})\end{array}$ & $\begin{array}{l}\text { Mean } \\
\text { flow } \\
\text { depth } \\
\text { (m) }\end{array}$ & $\begin{array}{l}\text { Water- } \\
\text { surface } \\
\text { slope } \\
(\mathrm{m} / \mathrm{m})\end{array}$ & $\begin{array}{l}\text { Water } \\
\text { temp. } \\
\left({ }^{\circ} \mathrm{C}\right)\end{array}$ & $\begin{array}{c}\text { Suspended } \\
\text { sediment } \\
\text { concern- } \\
\text { tration } \\
(\mathrm{mg} / \mathrm{L})\end{array}$ & $\begin{array}{c}\text { Suspended } \\
\text { load } \\
(\mathrm{kg} / \mathrm{s})\end{array}$ & $\begin{array}{l}\text { Number of } \\
\text { sampling } \\
\text { points } \\
\text { for } \\
\text { bedload }\end{array}$ & $\begin{array}{r}\text { Bedload } \\
(\mathrm{kg} / \mathrm{s})\end{array}$ \\
\hline
\end{tabular}

7. Susitna River at Sunshine, Alaska--Continued

\begin{tabular}{|c|c|c|c|c|c|c|c|c|c|c|c|}
\hline $\begin{array}{l}07-26-82 \\
08-02-82 \\
08-09-82 \\
08-16-82 \\
08-23-82\end{array}$ & $\begin{array}{l}2,800 \\
1,800 \\
1,520 \\
1,360 \\
1,090\end{array}$ & $\begin{array}{l}2,740 \\
1,770 \\
1,530 \\
1,350 \\
1,090\end{array}$ & $\begin{array}{l}2.1 \\
1.9 \\
1.8 \\
1.8 \\
2.0\end{array}$ & $\begin{array}{l}308 \\
305 \\
290 \\
262 \\
209\end{array}$ & $\begin{array}{l}4.4 \\
3.1 \\
2.9 \\
2.9 \\
2.6\end{array}$ & $\begin{array}{r}0.0024 \\
.0022 \\
.0019 \\
.0016 \\
.0017\end{array}$ & $\begin{array}{r}9.5 \\
11.0 \\
10.5 \\
10.5 \\
10.0\end{array}$ & $\begin{array}{r}1,430 \\
704 \\
813 \\
726 \\
527\end{array}$ & $\begin{array}{r}3,920 \\
1,250 \\
1,240 \\
980 \\
574\end{array}$ & $\begin{array}{l}16 \\
16 \\
16 \\
16 \\
16\end{array}$ & $\begin{array}{l}91.9 \\
36.5 \\
54.8 \\
28.8 \\
11.0\end{array}$ \\
\hline $\begin{array}{l}08-30-82 \\
09-17-82 \\
05-18-83 \\
05-24-83 \\
06-01-83\end{array}$ & $\begin{array}{l}1,110 \\
2,480 \\
1,230 \\
1,110 \\
2,120\end{array}$ & $\begin{array}{l}1,130 \\
2,450 \\
1,230 \\
1,110 \\
2,130\end{array}$ & $\begin{array}{l}2.0 \\
2.0 \\
2.2 \\
2.0 \\
2.3\end{array}$ & $\begin{array}{l}206 \\
305 \\
194 \\
197 \\
290\end{array}$ & $\begin{array}{l}2.7 \\
4.1 \\
2.8 \\
2.8 \\
3.2\end{array}$ & $\begin{array}{l}.0015 \\
.0022 \\
-- \\
.0023 \\
.0023\end{array}$ & $\begin{array}{l}9.0 \\
6.5 \\
5.5 \\
6.5 \\
7.5\end{array}$ & $\begin{array}{r}424 \\
1,300 \\
396 \\
225 \\
871\end{array}$ & $\begin{array}{r}479 \\
3,190 \\
487 \\
250 \\
1,860\end{array}$ & $\begin{array}{l}16 \\
16 \\
16 \\
16 \\
16\end{array}$ & $\begin{array}{l}15.5 \\
85.3 \\
23.0 \\
27.1 \\
49.1\end{array}$ \\
\hline $\begin{array}{l}06-08-83 \\
06-23-83 \\
07-05-83 \\
07-19-83 \\
08-01-83\end{array}$ & $\begin{array}{l}1,330 \\
1,940 \\
1,880 \\
1,440 \\
1,680\end{array}$ & $\begin{array}{l}1,330 \\
1,920 \\
1,890 \\
1,440 \\
1,680\end{array}$ & $\begin{array}{l}2.3 \\
2.3 \\
2.1 \\
2.3 \\
2.2\end{array}$ & $\begin{array}{l}205 \\
275 \\
275 \\
206 \\
275\end{array}$ & $\begin{array}{l}2.9 \\
3.1 \\
3.2 \\
3.0 \\
2.8\end{array}$ & $\begin{array}{l}.0019 \\
.0021 \\
.0020 \\
.0020 \\
.0018\end{array}$ & $\begin{array}{l}11.0 \\
14.0 \\
14.0 \\
10.0 \\
13.0\end{array}$ & $\begin{array}{r}431 \\
850 \\
1,060 \\
753 \\
950\end{array}$ & $\begin{array}{r}573 \\
1,630 \\
2,000 \\
1,080 \\
1,600\end{array}$ & $\begin{array}{l}16 \\
16 \\
16 \\
16 \\
16\end{array}$ & $\begin{array}{l}46.6 \\
98.5 \\
58.9 \\
20.6 \\
30.5\end{array}$ \\
\hline $\begin{array}{l}08-03-83 \\
08-08-83 \\
08-29-83 \\
09-12-83 \\
10-04-83\end{array}$ & $\begin{array}{r}1,630 \\
2,090 \\
1,350 \\
714 \\
799\end{array}$ & $\begin{array}{r}1,630 \\
2,160 \\
1,350 \\
714 \\
793\end{array}$ & $\begin{array}{l}2.1 \\
2.3 \\
2.2 \\
1.7 \\
1.8\end{array}$ & $\begin{array}{l}275 \\
291 \\
204 \\
181 \\
186\end{array}$ & $\begin{array}{l}2.8 \\
3.2 \\
3.0 \\
2.3 \\
2.4\end{array}$ & $\begin{array}{l}.0 \\
.0021 \\
.0018 \\
.0012 \\
.0014\end{array}$ & $\begin{array}{r}10.5 \\
10.0 \\
8.5 \\
7.5 \\
2.0\end{array}$ & $\begin{array}{r}1,030 \\
2,840 \\
401 \\
167 \\
171\end{array}$ & $\begin{array}{r}1,680 \\
6,130 \\
541 \\
119 \\
136\end{array}$ & $\begin{array}{l}16 \\
16 \\
16 \\
16 \\
16\end{array}$ & $\begin{array}{l}20.4 \\
27.3 \\
25.2 \\
51.1 \\
13.9\end{array}$ \\
\hline $\begin{array}{l}05-16-84 \\
06-14-84 \\
07-13-84 \\
07-28-84 \\
08-14-84\end{array}$ & $\begin{array}{r}697 \\
1,930 \\
1,480 \\
2,210 \\
1,300\end{array}$ & $\begin{array}{r}697 \\
1,930 \\
1,480 \\
2,200 \\
1,300\end{array}$ & $\begin{array}{l}1.7 \\
2.3 \\
2.0 \\
2.3 \\
1.9\end{array}$ & $\begin{array}{l}181 \\
287 \\
288 \\
291 \\
260\end{array}$ & $\begin{array}{l}2.3 \\
3.0 \\
2.5 \\
3.3 \\
2.7\end{array}$ & $\begin{array}{l}-- \\
-- \\
-- \\
-- \\
--\end{array}$ & $\begin{array}{c}5.0 \\
-- \\
10.5 \\
9.0 \\
9.5\end{array}$ & $\begin{array}{l}440 \\
990 \\
638 \\
960 \\
748\end{array}$ & $\begin{array}{r}307 \\
1,910 \\
944 \\
2,110 \\
972\end{array}$ & $\begin{array}{l}16 \\
16 \\
16 \\
16 \\
16\end{array}$ & $\begin{array}{l}14.3 \\
30.7 \\
17.0 \\
34.9 \\
37.7\end{array}$ \\
\hline $\begin{array}{l}09-11-84 \\
09-21-84 \\
09-28-84 \\
05-31-85 \\
06-25-85\end{array}$ & $\begin{array}{r}660 \\
838 \\
504 \\
1,910 \\
1,580\end{array}$ & $\begin{array}{r}660 \\
838 \\
504 \\
1,890 \\
1,580\end{array}$ & $\begin{array}{l}1.6 \\
1.9 \\
1.4 \\
2.0 \\
2.0\end{array}$ & $\begin{array}{l}177 \\
181 \\
174 \\
288 \\
288\end{array}$ & $\begin{array}{l}2.3 \\
2.5 \\
2.1 \\
3.2 \\
2.8\end{array}$ & $\begin{array}{l}-- \\
-- \\
-- \\
-- \\
--\end{array}$ & $\begin{array}{l}7.0 \\
-- \\
5.0 \\
3.5 \\
--\end{array}$ & $\begin{array}{r}168 \\
284 \\
88 \\
560 \\
333\end{array}$ & $\begin{array}{c}111 \\
238 \\
44.4 \\
1,060 \\
526\end{array}$ & $\begin{array}{l}16 \\
16 \\
16 \\
16 \\
16\end{array}$ & $\begin{array}{l}12.5 \\
27.2 \\
12.5 \\
27.2 \\
25.0\end{array}$ \\
\hline $\begin{array}{l}07-23-85 \\
08-12-85 \\
09-03-85 \\
09-16-85\end{array}$ & $\begin{array}{l}2,070 \\
1,790 \\
1,330 \\
2,170\end{array}$ & $\begin{array}{l}2,050 \\
1,920 \\
1,310 \\
2,140\end{array}$ & $\begin{array}{l}-.- \\
2.6 \\
2.0 \\
2.2\end{array}$ & $\begin{array}{l}-- \\
291 \\
286 \\
290\end{array}$ & $\begin{array}{l}-- \\
3.1 \\
2.4 \\
3.4\end{array}$ & $\begin{array}{l}-- \\
-- \\
-- \\
--\end{array}$ & $\begin{array}{c}11.0 \\
8.5 \\
-- \\
6.5\end{array}$ & $\begin{array}{r}912 \\
1,680 \\
381 \\
710\end{array}$ & $\begin{array}{r}1,870 \\
3,230 \\
499 \\
1,520\end{array}$ & $\begin{array}{l}16 \\
16 \\
16 \\
16\end{array}$ & $\begin{array}{r}6.1 \\
13.2 \\
9.6 \\
15.0\end{array}$ \\
\hline
\end{tabular}

8. Tanana River at Upper End of Goose Island, $\mathrm{Alaska}^{3}$

\begin{tabular}{|c|c|c|c|c|c|c|c|c|c|c|c|}
\hline $\begin{array}{l}09-05-80 \\
09-17-80 \\
10-08-80 \\
03-06-81 \\
03-24-81\end{array}$ & $\begin{array}{l}666 \\
643 \\
581 \\
156 \\
161\end{array}$ & $\begin{array}{l}666 \\
643 \\
581 \\
156 \\
161\end{array}$ & $\begin{array}{c}1.9^{\circ} \\
1.8 \\
1.9 \\
-- \\
--\end{array}$ & $\begin{array}{r}140 \\
154 \\
129 \\
-- \\
--\end{array}$ & $\begin{array}{r}2.5 \\
2.3 \\
2.4 \\
-- \\
--\end{array}$ & $\begin{array}{l}-- \\
-- \\
-- \\
--\end{array}$ & $\begin{array}{l}-- \\
-- \\
-- \\
--\end{array}$ & $\begin{array}{r}597 \\
1,130 \\
374 \\
47 \\
51\end{array}$ & $\begin{array}{r}398 \\
727 \\
217 \\
7.33 \\
8.21\end{array}$ & $\begin{array}{l}18 \\
18 \\
18 \\
18 \\
18\end{array}$ & $\begin{array}{c}2.97 \\
1.65 \\
2.16 \\
3.91 \\
.270\end{array}$ \\
\hline $\begin{array}{l}06-19-81 \\
07-16-81 \\
07-28-81 \\
08-14-81 \\
10-07-81\end{array}$ & $\begin{array}{r}842 \\
1,480 \\
1,840 \\
1,650 \\
354\end{array}$ & $\begin{array}{r}842 \\
1,480 \\
1,840 \\
1,650 \\
354\end{array}$ & $\begin{array}{r}2.5 \\
-. \\
2.0 \\
1.9 \\
1.1\end{array}$ & $\begin{array}{r}179 \\
-- \\
311 \\
311 \\
152\end{array}$ & $\begin{array}{r}1.9 \\
-. \\
3.0 \\
2.7 \\
2.1\end{array}$ & $\begin{array}{l}-- \\
-- \\
-- \\
--\end{array}$ & $\begin{array}{l}-- \\
-- \\
-- \\
--\end{array}$ & $\begin{array}{r}1,250 \\
2,540 \\
3,120 \\
2,940 \\
166\end{array}$ & $\begin{array}{r}1,050 \\
3,760 \\
5,740 \\
4,850 \\
58.8\end{array}$ & $\begin{array}{l}18 \\
18 \\
18 \\
18 \\
18\end{array}$ & $\begin{array}{c}35.5 \\
40.8 \\
38.1 \\
31.9 \\
1.38\end{array}$ \\
\hline $\begin{array}{l}06-29-82 \\
07-21-82\end{array}$ & $\begin{array}{l}1,310 \\
1,760\end{array}$ & $\begin{array}{l}1,310 \\
1,760\end{array}$ & -- & -- & -- & -- & -- & $\begin{array}{l}2,600 \\
2,620\end{array}$ & $\begin{array}{l}3,410 \\
4,610\end{array}$ & $\begin{array}{l}18 \\
18\end{array}$ & $\begin{array}{l}13.4 \\
16.7\end{array}$ \\
\hline
\end{tabular}


Tahle 1.--Hydraulic and sediment-transport data--Continued

\begin{tabular}{|c|c|c|c|c|c|c|c|c|c|c|c|}
\hline Date & $\begin{array}{c}\text { Water } \\
\text { discharge } \\
\text { (bed load) } \\
\left(\mathrm{m}^{3} / \mathrm{s}\right)\end{array}$ & $\begin{array}{c}\text { Water } \\
\text { discharge } \\
\text { (suspended } \\
\text { load) } \\
\left(\mathrm{m}^{3} / \mathrm{s}\right)\end{array}$ & $\begin{array}{l}\text { Mean } \\
\text { flow } \\
\text { velocity } \\
(\mathrm{m} / \mathrm{s})\end{array}$ & $\begin{array}{l}\text { Water - } \\
\text { surface } \\
\text { width } \\
\text { (m) }\end{array}$ & $\begin{array}{l}\text { Mean } \\
\text { flow } \\
\text { depth } \\
(\mathrm{m})\end{array}$ & $\begin{array}{l}\text { Water- } \\
\text { surface } \\
\text { slope } \\
(\mathrm{m} / \mathrm{m})\end{array}$ & $\begin{array}{l}\text { Water } \\
\text { temp. } \\
\left({ }^{\circ} \mathrm{C}\right)\end{array}$ & $\begin{array}{c}\text { Suspended } \\
\text { sediment } \\
\text { concern- } \\
\text { tration } \\
(\mathrm{mg} / \mathrm{L})\end{array}$ & $\begin{array}{c}\text { Suspended } \\
\text { load } \\
(\mathrm{kg} / \mathrm{s})\end{array}$ & $\begin{array}{c}\text { Number of } \\
\text { sampling } \\
\text { points } \\
\text { for } \\
\text { bedload }\end{array}$ & Bed \\
\hline & \multicolumn{11}{|c|}{ 9. Tanana River at Fairbanks, Alaska } \\
\hline $06-07-77$ & 750 & 750 & 1.6 & 264 & 1.8 & 0.00047 & -- & 952 & 714 & 18 & 10.2 \\
\hline $06-29-77$ & 1,320 & 1,320 & 1.3 & 360 & 2.8 & .00050 & -- & 1,860 & 2,460 & 18 & 89.4 \\
\hline $07-06-77$ & 1,170 & 1,170 & 1.7 & 326 & 2.2 & .00050 & -- & 1,510 & 1,770 & 18 & 21.4 \\
\hline $07-12-77$ & 1,080 & 1,080 & 1.7 & 323 & 2.0 & .00049 & -- & 1,490 & 1,610 & 18 & 30.1 \\
\hline $07-20-77$ & 1,270 & 1,270 & 1.6 & 360 & 2.3 & .00050 & -- & 2,210 & 2,810 & 18 & 16.3 \\
\hline $08-03-77$ & 1,680 & 1,680 & 1.8 & 396 & 2.3 & .00052 & -- & 4,340 & 7,290 & 18 & 63.2 \\
\hline $08-11-77$ & 1,460 & 1,460 & 1.7 & 372 & 2.3 & .00041 & -- & 3,270 & 4,770 & 18 & 46.2 \\
\hline $08-18-77$ & 1,450 & 1,450 & 1.7 & 378 & 2.2 & .00051 & -- & 2,620 & 3,800 & 18 & 26.2 \\
\hline $08-31-77$ & 1,410 & 1,410 & 1.8 & 305 & 2.5 & .00051 & -- & 2,020 & 2,850 & 18 & 19.2 \\
\hline $10-03-77$ & 592 & 592 & 1.6 & 134 & 2.8 & .00046 & -- & 563 & 333 & 18 & 9.07 \\
\hline $05-18-78$ & 566 & 566 & 1.5 & 145 & 2.5 & .00046 & -- & 769 & 435 & 18 & 4.21 \\
\hline $05-30-78$ & 521 & 521 & 1.6 & 116 & 2.8 & .00045 & -- & 476 & 248 & 18 & 4.06 \\
\hline $06-20-78$ & 804 & 804 & 1.6 & 207 & 2.4 & .00048 & -- & 975 & 784 & 18 & 17.3 \\
\hline $07-10-78$ & 983 & 983 & 1.5 & 317 & 2.0 & .00049 & -- & 1,790 & 1,760 & 18 & 8.68 \\
\hline $07-17-78$ & 1,650 & 1,650 & 1.6 & 463 & 2.2 & .00052 & -- & 3,700 & 6,110 & 18 & 74.5 \\
\hline $07-31-78$ & 1,460 & 1,460 & 1.7 & 402 & 2.1 & .00051 & -- & 2,700 & 3,940 & 18 & 28.6 \\
\hline $08-08-78$ & 1,540 & 1,540 & 1.6 & 399 & 2.4 & .00051 & -- & 2,870 & 4,420 & 18 & 30.0 \\
\hline $08-14-78$ & 1,640 & 1,640 & 1.7 & 415 & 2.4 & .00052 & -- & 2,680 & 4,400 & 18 & 62.6 \\
\hline $08-25-78$ & 1,220 & 1,220 & 1.6 & 366 & 2.1 & .00050 & $-\cdot$ & 1,250 & 1,530 & 18 & 26.6 \\
\hline $09-07-78$ & 966 & 966 & 1.7 & 277 & 2.1 & .00049 & -- & 1,020 & 985 & 18 & 21.6 \\
\hline $10-04-78$ & 411 & 411 & 1.4 & 154 & 1.9 & .00044 & -- & 389 & 160 & 18 & 4.8 \\
\hline $05-23-79$ & 484 & 484 & 1.3 & 201 & 1.9 & .00045 & -- & 197 & 95.3 & 18 & \\
\hline $06-18-79$ & 1,000 & 1,000 & 1.5 & 375 & 1.8 & .00049 & -- & 863 & 863 & 18 & 33.5 \\
\hline $07-10-79$ & 1,490 & 1,490 & 1.7 & 408 & 2.1 & .00051 & -- & 2,100 & 3,130 & 18 & 35.0 \\
\hline $07-24-79$ & 1,950 & 1,950 & 1.7 & 454 & 2.5 & .00053 & -- & 3,180 & 6,200 & 18 & 38.8 \\
\hline $08-01-79$ & 2,020 & 2,020 & 1.6 & 469 & 2.6 & .00051 & - & 3,090 & 6,240 & 18 & 40.9 \\
\hline $08-08-79$ & 1,880 & 1,880 & 1.6 & 466 & 2.5 & .00053 & -- & 2,130 & 4,000 & 18 & 49.3 \\
\hline $08-29-79$ & 1,460 & 1,460 & 1.7 & 436 & 2.0 & .00053 & -- & 1,650 & 2,410 & 18 & 33.1 \\
\hline $09-06-79$ & 912 & 912 & 1.6 & 326 & 1.8 & .00048 & - & 788 & 719 & 18 & 16.7 \\
\hline $09-12-79$ & 895 & 895 & 1.6 & 290 & 1.9 & .00048 & -- & 634 & 567 & 18 & 17.5 \\
\hline $10-02-79$ & 487 & 487 & 1.6 & 143 & 2.1 & .00045 & - & 578 & 281 & 18 & 16.3 \\
\hline $06-18-80$ & 1,010 & 1,010 & 1.6 & 296 & 2.2 & -- & -. & 2,530 & 2,560 & 18 & 30.2 \\
\hline $06-25-80$ & 711 & 711 & 1.3 & 282 & 2.0 & -- & $\cdots$ & 790 & 562 & 18 & 16.5 \\
\hline $07-15-80$ & 1,400 & 1,400 & 1.7 & 320 & 2.5 & -- & -- & 2,520 & 3,530 & 18 & 17.7 \\
\hline $07-30-80$ & 1,640 & 1,640 & 1.8 & 313 & 2.9 & -- & -- & 3,500 & 5,740 & 18 & 53.1 \\
\hline $08-11-80$ & 1,340 & 1,340 & -- & -- & $\cdots$ & -- & -- & 2,620 & 3,510 & 18 & 39.3 \\
\hline $09-03-80$ & 714 & 714 & -- & - &.- & -- & -- & 679 & 485 & 18 & \\
\hline $09-16-80$ & 532 & 532 & -- & -- & -- & -- & -- & 452 & 240 & 18 & 4.1 \\
\hline $10-07-80$ & 586 & 586 & 1.5 & 169 & 2.3 & -- & -- & 470 & 275 & 18 & 15.5 \\
\hline $10-23-80$ & 345 & 345 & 1.6 & 107 & 2.0 & - & -- & 352 & 121 & 18 & 3.5 \\
\hline $05-22-81$ & 558 & 558 & 1.6 & 177 & 2.0 & -- & -- & 589 & 329 & 18 & 20.8 \\
\hline $06-18-81$ & 864 & 864 & 1.8 & 284 & 1.7 & -- & -- & 1,140 & 985 & 18 & 48.5 \\
\hline $06-30-81$ & 1,070 & 1,070 & 1.8 & 284 & 2.1 & -- & -- & 1,260 & 1,350 & 18 & 16.4 \\
\hline $07-13-81$ & 1,760 & 1,760 & 1.9 & 406 & 2.3 & -- & -- & 5,700 & 10,030 & 18 & 54.3 \\
\hline $07-29-81$ & 1,720 & 1,720 & 1.8 & 378 & 2.5 & -- & -- & 2,940 & 5,060 & 18 & 81.7 \\
\hline
\end{tabular}


Table 1.--Hydraulic and sediment-transport data--Continued

\begin{tabular}{|c|c|c|c|c|c|c|c|c|c|c|c|}
\hline Date & $\begin{array}{c}\text { Water } \\
\text { discharge } \\
\text { (bedload) } \\
\left(\mathrm{m}^{3} / \mathrm{s}\right)\end{array}$ & $\begin{array}{c}\text { Water } \\
\text { discharge } \\
\text { (suspended } \\
\text { load) } \\
\left(\mathrm{m}^{3} / \mathrm{s}\right)\end{array}$ & $\begin{array}{l}\text { Mean } \\
\text { flow } \\
\text { velocity } \\
(\mathrm{m} / \mathrm{s})\end{array}$ & $\begin{array}{l}\text { Water- } \\
\text { surface } \\
\text { width } \\
(\mathrm{m})\end{array}$ & $\begin{array}{l}\text { Mean } \\
\text { flow } \\
\text { depth } \\
\text { (m) }\end{array}$ & $\begin{array}{l}\text { Water- } \\
\text { surface } \\
\text { slope } \\
(\mathrm{nr} / \mathrm{m})\end{array}$ & $\begin{array}{l}\text { Water } \\
\text { temp. } \\
\left({ }^{\circ} \mathrm{C}\right)\end{array}$ & $\begin{array}{l}\text { Suspended } \\
\text { sediment } \\
\text { concern- } \\
\text { tration } \\
(\mathrm{mg} / \mathrm{L})\end{array}$ & $\begin{array}{c}\text { Suspended } \\
\text { load } \\
(\mathrm{kg} / \mathrm{s})\end{array}$ & $\begin{array}{l}\text { Number of } \\
\text { sampling } \\
\text { points } \\
\text { for } \\
\text { bedload }\end{array}$ & $\begin{array}{r}\text { Bedload } \\
(\mathrm{kg} / \mathrm{s})\end{array}$ \\
\hline
\end{tabular}

9. Tanana River at Fairbanks, Alaska--Continued

$\begin{array}{rrrr}08-13-81 & 1,530 & 1,530 & 1 . \\ 09-03-81 & 920 & 920 & 1.5 \\ 10-06-81 & 368 & 368 & 1 . \\ 06-04-82 & 1,310 & 1,310 & - \\ 06-28-82 & 1,160 & 1.160 & 1.6 \\ & & & \\ 07-20-82 & 1,880 & 1,880 & 1.8 \\ 08-09-82 & 1,290 & 1,290 & 1 . \\ 09-08-82 & 634 & 634 & 1 . \\ 09-28-82 & 688 & 688 & 1 .\end{array}$

$\begin{array}{ll}1.7 & 357 \\ 1.5 & 294 \\ 1.0 & 247 \\ -. & \\ 1.6 & 302 \\ 1.8 & 374 \\ 1.6 & 306 \\ 1.2 & 299 \\ 1.2 & 301\end{array}$

$\begin{array}{rrrrr}357 & 2.6 & - & -- & 3,250 \\ 294 & 2.0 & -- & -- & 1,060 \\ 247 & 1.4 & -- & -- & 164 \\ -- & -- & -- & -- & 1,940 \\ 302 & 2.4 & -- & -- & 1,940 \\ & & & & \\ 374 & 2.8 & -- & -- & 2,660 \\ 306 & 2.7 & -- & -- & 1,700 \\ 299 & 1.8 & -- & -- & 644 \\ 301 & 1.9 & -- & -- & 812\end{array}$

4,970
975
60.4
2,540
2,250
5,000
2,190
408
559

24.3

10. Snake River near Anatone, Wash. ${ }^{6}$

\begin{tabular}{|c|c|c|}
\hline $\begin{array}{l}05-10-72 \\
05-19-72 \\
06-02-72 \\
06-14-72 \\
05-01-73\end{array}$ & $\begin{array}{r}2,200 \\
2,920 \\
3,770 \\
2,600 \\
878\end{array}$ & $\begin{array}{r}2,200 \\
2,920 \\
3,770 \\
2,600 \\
878\end{array}$ \\
\hline $\begin{array}{l}05-17-73 \\
06-18-73 \\
04-11-74 \\
04-24-74 \\
05-07-74\end{array}$ & $\begin{array}{l}1,520 \\
1,020 \\
2,450 \\
2,510 \\
3,060\end{array}$ & $\begin{array}{l}1,440 \\
1,020 \\
2,380 \\
2,450 \\
2,950\end{array}$ \\
\hline $\begin{array}{l}05-16-74 \\
06-11-74 \\
04-15-75 \\
05-01-75 \\
05-14-75\end{array}$ & $\begin{array}{l}2,450 \\
3,140 \\
1,600 \\
2,140 \\
2,660\end{array}$ & $\begin{array}{l}2,370 \\
2,600 \\
1,600 \\
2,150 \\
2,660\end{array}$ \\
\hline $\begin{array}{l}05-20-75 \\
06-11-75 \\
06-26-75 \\
04-07-76 \\
04-13-76\end{array}$ & $\begin{array}{l}3,000 \\
2,750 \\
2,950 \\
1,750 \\
2,810\end{array}$ & $\begin{array}{l}3,000 \\
2,750 \\
2,950 \\
1,750 \\
2,790\end{array}$ \\
\hline $\begin{array}{l}04-15-76 \\
04-19-76 \\
04-21-76 \\
05-05-76 \\
05-13-76\end{array}$ & $\begin{array}{l}2,690 \\
2,320 \\
2,380 \\
2,630 \\
3,260\end{array}$ & $\begin{array}{l}2,690 \\
2,320 \\
2,380 \\
2,600 \\
3,260\end{array}$ \\
\hline $\begin{array}{l}05-25-76 \\
05-27-76 \\
06-08-76 \\
06-10-76 \\
04-04-78\end{array}$ & $\begin{array}{l}2,890 \\
2,890 \\
2,740 \\
2,700 \\
1,850\end{array}$ & $\begin{array}{l}2,890 \\
2,890 \\
2,740 \\
2,680 \\
1,780\end{array}$ \\
\hline $\begin{array}{l}04-05-78 \\
04-29-78 \\
05-02-78 \\
05-03-78 \\
05-16-78\end{array}$ & $\begin{array}{l}1,630 \\
2,270 \\
2,170 \\
2,290 \\
2,180\end{array}$ & $\begin{array}{l}1,640 \\
2,270 \\
2,170 \\
2,290 \\
2,170\end{array}$ \\
\hline
\end{tabular}

\begin{tabular}{|c|c|c|c|}
\hline 181 & 4.8 & 0.00094 & 11.0 \\
\hline 189 & 5.3 & .00109 & 10.0 \\
\hline 197 & 5.8 & .00124 & 13.0 \\
\hline 184 & 5.1 & .00103 & 13.5 \\
\hline 158 & 3.5 & .00059 & -- \\
\hline 171 & 4.2 & .00078 & 15.0 \\
\hline 162 & 3.7 & .00064 & 17.0 \\
\hline 186 & 5.0 & .00101 & 8.0 \\
\hline 187 & 5.1 & .00102 & 10.0 \\
\hline 192 & 5.4 & .00112 & 11.0 \\
\hline 186 & 5.0 & .00101 & 10.5 \\
\hline 194 & 5.5 & .00114 & 11.5 \\
\hline 175 & 4.3 & .00082 & 8.5 \\
\hline 183 & 4.8 & .00094 & 10.0 \\
\hline 189 & 5.2 & .00105 & 11.5 \\
\hline 192 & 5.4 & .00112 & 11.0 \\
\hline 189 & 5.2 & .00107 & 13.0 \\
\hline 192 & 5.3 & .00110 & 12.0 \\
\hline 177 & 4.5 & .00084 & 8.0 \\
\hline 191 & 5.2 & .00108 & 8.0 \\
\hline 189 & 5.2 & .00105 & 9.0 \\
\hline 184 & 4.9 & .00098 & 9.0 \\
\hline 186 & 5.0 & .00099 & 9.0 \\
\hline 189 & 5.4 & .00104 & 11.0 \\
\hline 195 & 5.5 & .00116 & 12.0 \\
\hline 191 & 5.3 & .00109 & 13.0 \\
\hline 191 & 5.3 & .00109 & 13.0 \\
\hline 189 & 5.2 & .00107 & 15.0 \\
\hline 189 & 5.2 & .00106 & -- \\
\hline 178 & 5.3 & .00087 & 8.5 \\
\hline 177 & 4.3 & .00082 & 8.5 \\
\hline 184 & 4.9 & .00098 & 12.0 \\
\hline 183 & 4.8 & .00094 & 12.0 \\
\hline 184 & 4.9 & .00098 & 12.0 \\
\hline 183 & 4.8 & .00094 & 11.0 \\
\hline
\end{tabular}

61.6
347
1,270
387
15.8

83.5

13.3

66.6

196

313

66.4 229

49.6

94.6

327

273

273
674
348

266

393

172

69.6
80.9

80.
166

65.2

275

272

211

206
39.

32.8

32.8
92
91.

115

143
32.0

5.35

5.35
7.97

10.4

$00094 \quad 11.0$

66

$$
\begin{aligned}
& \geq 20 \\
& \geq 20 \\
& >20
\end{aligned}
$$$$
\begin{aligned}
& >20 \\
& >20
\end{aligned}
$$$$
\geq 20
$$$$
2.72
$$$$
4.65
$$$$
1.53
$$$$
.062
$$$$
3.09
$$$$
.949
$$$$
28.5
$$$$
\begin{aligned}
& 28.5 \\
& 17.7
\end{aligned}
$$$$
\begin{aligned}
& 17.7 \\
& 17.9
\end{aligned}
$$$$
11.0
$$$$
6.82
$$$$
3.83
$$$$
9.40
$$$$
9.49
$$$$
15.0
$$$$
3.29
$$$$
\begin{aligned}
& 5.19 \\
& 4.00
\end{aligned}
$$$$
18.3
$$

58.7

51.9

14.5

9.69

7.78

9.15

6.73

10.8

26.3

16.5

27.7

20.6

20.0

8.93 
T.1ble 1.--Hydraulic and sediment-transport-data--Cont inued

\begin{tabular}{|c|c|c|c|c|c|c|c|c|c|c|c|}
\hline Date & $\begin{array}{c}\text { Water } \\
\text { discharge } \\
\text { (bedload) }^{1} \\
\left(\mathrm{~m}^{3} / \mathrm{s}\right)\end{array}$ & $\begin{array}{c}\text { Water } \\
\text { discharge } \\
\text { (suspended } \\
10 a d)^{2} \\
\left(\mathrm{~m}^{3} / \mathrm{s}\right)\end{array}$ & $\begin{array}{c}\text { Nean } \\
\text { flow } \\
\text { velocity } \\
(\mathrm{m} / \mathrm{s})\end{array}$ & $\begin{array}{l}\text { Water- } \\
\text { surface } \\
\text { width } \\
\text { (m) }\end{array}$ & $\begin{array}{l}\text { Mean } \\
\text { flow } \\
\text { depth } \\
(\mathrm{m})\end{array}$ & $\begin{array}{l}\text { Water- } \\
\text { surface } \\
\text { slope } \\
(\mathrm{m} / \mathrm{m})\end{array}$ & $\begin{array}{l}\text { Water } \\
\text { temp. } \\
\left({ }^{\circ} \mathrm{C}\right)\end{array}$ & $\begin{array}{c}\text { Suspended } \\
\text { sediment } \\
\text { concern- } \\
\text { tration } \\
(\mathrm{mg} / \mathrm{L})\end{array}$ & $\begin{array}{c}\text { Suspended } \\
\text { load } \\
(\mathrm{kg} / \mathrm{s})\end{array}$ & $\begin{array}{l}\text { Number of } \\
\text { sampling } \\
\text { points } \\
\text { for } \\
\text { bedload }\end{array}$ & $\begin{array}{r}\text { Bedloa } \\
(\mathrm{kg} / \mathrm{s}\end{array}$ \\
\hline & & & & Snake & iver ne & Anatone & Wash. & Continued & & & \\
\hline $05-17-78$ & 2,130 & 2,120 & 2.5 & 183 & 4.8 & 0.00094 & 11.0 & 125 & 265 & $\geq 20$ & 16.1 \\
\hline $06-07-78$ & 2,320 & 2,290 & 2.6 & 186 & 4.9 & .00098 & 16.0 & 89 & 204 & $>20$ & 3.26 \\
\hline $06-13-78$ & 2,070 & 2,040 & 2.4 & 183 & 4.7 & .00092 & 13.5 & 82 & 167 & $>20$ & 4.20 \\
\hline $06-20-78$ & 1,810 & 1,900 & 2.3 & 177 & 4.5 & .00086 & 16.5 & 29 & 55.1 & $>20$ & 5.78 \\
\hline $05-02-79$ & 1,510 & 1,400 & 2.1 & 172 & 4.2 & .00078 & 13.0 & 52 & 72.8 & $\geq 20$ & 1.91 \\
\hline $05-15-79$ & 1,220 & 1,180 & 1.9 & 169 & 3.9 & .00072 & 14.0 & 20 & 23.6 & $\geq 20$ & .431 \\
\hline $05-22-79$ & 1,710 & 1,720 & 2.2 & 177 & 4.4 & .00084 & 13.0 & 54 & 92.9 & $>20$ & 5.64 \\
\hline $05-24-79$ & 1,990 & 2,000 & 2.4 & 181 & 4.7 & .00090 & 14.0 & 82 & 164 & $>20$ & 3.92 \\
\hline $06-05-79$ & 1,650 & 1,590 & 2.2 & 177 & 4.4 & .00082 & 14.5 & 20 & 31.8 & $>20$ & 4.03 \\
\hline $06-07-79$ & 1,350 & 1,390 & 2.0 & 171 & 4.1 & .00074 & 13.5 & 17 & 23.6 & $\geq 20$ & 1.72 \\
\hline
\end{tabular}

11. Toutle River at Tower Road near Silver Lake, Wash.7

\begin{tabular}{|c|c|c|c|c|c|c|c|c|c|c|c|}
\hline $01-17-85$ & 37.9 & 38.2 & 1.6 & 20.0 & 1.2 & - & 5.0 & 3,000 & 115 & 18 & 17.6 \\
\hline $01-31-85$ & 29.2 & 29.2 & 1.2 & 22.5 & 1.0 & $\cdots$ & 3.0 & 2,080 & 60.7 & 17 & 11.9 \\
\hline $02-21-85$ & 47.1 & 46.7 & 1.7 & .28 .5 & .94 & -- & 5.5 & 2,430 & 113 & 22 & 53.9 \\
\hline $03-06-85$ & 41.9 & 41.9 & -- & -- & -- & -- & 7.5 & 2,280 & 95.5 & 21 & 6.39 \\
\hline $03-23-85$ & 112 & 90.9 & 1.7 & 61 & .77 & 0.0024 & 8.5 & 7,140 & 649 & 33 & 33.3 \\
\hline $04-02-85$ & 83.2 & 83.8 & 1.8 & 61 & .76 & .0024 & 10.5 & 3,050 & 256 & 32 & 67.9 \\
\hline $04-11-85$ & 94.1 & 92.6 & 1.9 & 63 & .76 & .0026 & 11.0 & 6,860 & 635 & 24 & 158 \\
\hline $04-30-85$ & 69.2 & 69.2 & - & - & $\ldots$ & .0026 & 9.5 & 2,340 & 162 & 24 & 37.5 \\
\hline $06-07-85$ & 248 & 253 & 2.5 & 69 & 1.5 & .0055 & 12.0 & 23,000 & 5,820 & 23 & 81.4 \\
\hline $06-08-85$ & 163 & 160 & 2.3 & 66 & 1.1 & -. & 13.0 & 10,400 & 1,660 & 25 & 95.0 \\
\hline $08-23-85$ & 15.6 & 15.5 & 1.5 & 17.5 & .58 & -- & 27.0 & 2,410 & 37.4 & 28 & 3.36 \\
\hline $10-25-85$ & 167 & 162 & $\ldots$ & -- & - & -- & 12.0 & 9,230 & 1,500 & 44 & 46.0 \\
\hline $11-06-85$ & 152 & 158 & 2.2 & 67 & 1.1 & .0032 & 8.5 & 5,720 & 904 & 22 & 232 \\
\hline $11-08-85$ & 148 & 151 & 2.3 & 67 & 1.0 & .0035 & 7.0 & 6,290 & 950 & 22 & 158 \\
\hline $12-09-85$ & 83.4 & 85.0 & 1.9 & 63 & .78 & - & 3.5 & 1,980 & 168 & 13 & 83.8 \\
\hline $01-03-86$ & 56.3 & 57.2 & 1.5 & 59 & .64 & .0021 & 4.5 & 1,240 & 70.9 & 12 & 6.57 \\
\hline $01-19-86$ & 226 & 235 & 2.4 & 67 & 1.6 & . & 7.5 & 9,780 & 2,300 & 20 & 18.9 \\
\hline $01-29-86$ & 56.7 & 64.6 & 1.6 & 57 & .73 & -- & 6.5 & 2,570 & 166 & 11 & 24.7 \\
\hline $02-11-86$ & 42.8 & 43.6 & 1.7 & 24.5 & 1.0 & .0020 & 4.0 & 1,480 & 64.5 & 15 . & 10.4 \\
\hline $02-16-86$ & 178 & 164 & 2.2 & 66 & 1.2 & .0029 & 4.5 & 4,240 & 695 & 25 & 72.6 \\
\hline $05-22-86$ & 65.0 & 67.4 & 1.5 & 62 & .72 & .0020 & 14.0 & 2,640 & 178 & 13 & $8_{2.09}$ \\
\hline $07-02-86$ & 17.6 & 18.0 & .89 & 41 & .49 & - & 19.5 & 1,020 & 18.4 & 27 & 1.92 \\
\hline $07-22-86$ & 16.5 & 16.5 & .95 & 41 & .42 & -- & 17.5 & 927 & 15.3 & 26 & 3.06 \\
\hline $08-07-86$ & 12.0 & 12.0 & .79 & 40.5 & .37 & - & 20.5 & 1,110 & 13.3 & 26 & 2.24 \\
\hline $10-31-86$ & 51.5 & 56.6 & 1.5 & 62 & .62 & .0021 & 12.0 & 5,410 & 306 & 12 & 89.04 \\
\hline $11-14-86$ & 34.8 & 34.6 & 1.4 & 53 & .46 & .0013 & 9.5 & 2,250 & 77.9 & 16 & ${ }^{8} 2.67$ \\
\hline $11-21-86$ & 191 & 189 & 2.3 & 66 & 1.2 & .0027 & 9.5 & 7,540 & 1,430 & 13 & ${ }^{8} 141$ \\
\hline $11-22-86$ & 247 & 260 & 2.7 & 67 & 1.5 & .0028 & 9.5 & 6,810 & 1,770 & 12 & ${ }^{8} 169$ \\
\hline $01-14-87$ & 90.3 & 94.7 & 1.7 & 63 & .86 & - & 6.5 & 2,195 & 208 & 11 & ${ }^{8} 45.1$ \\
\hline $01-28-87$ & 135 & 125 & 1.9 & 64 & 1.1 & .0019 & 7.5 & 4,300 & 538 & 12 & ${ }^{8} 121.8$ \\
\hline $02-01-87$ & 592 & 592 & 3.1 & 70 & 2.3 & $\ldots$ & 8.0 & 23,900 & 14,100 & 9 & ${ }^{8} 188$ \\
\hline
\end{tabular}


Table 1.--Hydraulic and sediment-transport data--Continued

\begin{tabular}{|c|c|c|c|c|c|c|c|c|c|c|c|}
\hline Date & $\begin{array}{c}\text { Water } \\
\text { discharge } \\
\text { (bedload })^{1} \\
\left(\mathrm{~m}^{3} / \mathrm{s}\right)\end{array}$ & $\begin{array}{c}\text { Water } \\
\text { discharge } \\
\text { (suspended } \\
\text { load) } \\
\left(\mathrm{m}^{3} / \mathrm{s}\right)\end{array}$ & $\begin{array}{l}\text { Mean } \\
\text { flow } \\
\text { velocity } \\
(\mathrm{m} / \mathrm{s})\end{array}$ & $\begin{array}{l}\text { Water- } \\
\text { surface } \\
\text { width } \\
(\mathrm{m})\end{array}$ & $\begin{array}{l}\text { Mean } \\
\text { flow } \\
\text { depth } \\
(m)\end{array}$ & $\begin{array}{l}\text { Water- } \\
\text { surface } \\
\text { slope } \\
(\mathrm{m} / \mathrm{m})\end{array}$ & $\begin{array}{l}\text { Water } \\
\text { temp. } \\
\left({ }^{\circ} \mathrm{C}\right)\end{array}$ & $\begin{array}{l}\text { Suspended } \\
\text { sediment } \\
\text { concern- } \\
\text { tration } \\
(\mathrm{mg} / \mathrm{L})\end{array}$ & $\begin{array}{c}\text { Suspended } \\
\text { load } \\
(\mathrm{kg} / \mathrm{s})\end{array}$ & $\begin{array}{l}\text { Number of } \\
\text { sampling } \\
\text { points } \\
\text { for } \\
\text { bedload }\end{array}$ & $\begin{array}{r}\text { Bedload } \\
(\mathrm{kg} / \mathrm{s})\end{array}$ \\
\hline
\end{tabular}

\begin{tabular}{|c|c|c|c|c|c|c|c|c|c|c|c|}
\hline $06-07-85$ & 185 & 171 & 2.8 & 56 & 1.1 & 0.0038 & -- & 29,100 & 4,980 & 18 & 110 \\
\hline $06-08-85$ & 110 & 113 & 2.4 & 56 & .90 & .0037 & -- & 15,200 & 1,720 & 20 & 163 \\
\hline $12-18-85$ & 23.3 & 23.4 & 1.6 & 24.5 & .59 & .0049 & -- & 2,440 & 57.1 & 19 & 39.5 \\
\hline $01-19-86$ & 123 & 124 & 2.6 & 56 & .85 & .0041 & 6.5 & 15,000 & 1,860 & 28 & 185 \\
\hline $02-13-86$ & 26.2 & 26.1 & 1.7 & 20.5 & .74 & -- & 2.0 & 2,980 & 77.8 & 11 & ${ }^{8} 24.4$ \\
\hline $02-26-86$ & 147 & 127 & 2.4 & 59 & .85 & .0037 & 8.5 & 12,500 & 1,590 & 11 & ${ }^{8} 124$ \\
\hline $08-07-86$ & 9.32 & 9.35 & 1.3 & 18.5 & .39 & .0047 & -- & 280 & 2.62 & 10 & ${ }^{8} 5.37$ \\
\hline $09-09-86$ & 8.95 & 8.86 & 1.2 & 18.5 & .41 & .0047 & -- & 432 & 3.83 & 11 & .884 \\
\hline $10-10-86$ & 8.95 & 8.95 & 1.2 & 18.0 & .42 & -- & -- & 384 & 3.44 & 11 & 1.82 \\
\hline $02-02-87$ & 153 & 132 & 2.4 & 59 & .98 & .0032 & -- & 12,400 & 1,640 & 9 & ${ }^{8} 338$ \\
\hline
\end{tabular}

12. No. Fork Toutle River near Kid Valley, Wash. 7

13. Oak Creek near Corvallis, Oreg.

\begin{tabular}{|c|c|c|c|c|c|c|c|c|c|c|c|}
\hline $\begin{array}{l}01-08-71 \\
01-10-71 \\
01-10-71 \\
01-11-71 \\
01-11-71\end{array}$ & $\begin{array}{r}0.153 \\
.337 \\
.391 \\
.447 \\
.382\end{array}$ & $\begin{array}{r}0.150 \\
.340 \\
.425 \\
.408 \\
.354\end{array}$ & $\begin{array}{l}-- \\
-- \\
-- \\
-- \\
--\end{array}$ & $\begin{array}{r}93.7 \\
3.7 \\
3.7 \\
3.7 \\
3.7\end{array}$ & $\begin{array}{r}100.11 \\
.15 \\
.17 \\
.18 \\
.16\end{array}$ & $\begin{array}{r}0.0083 \\
.0086 \\
.0083 \\
.0087 \\
.0086\end{array}$ & $\begin{array}{l}-- \\
\cdots \\
\cdots \\
5.0 \\
5.0\end{array}$ & $\begin{array}{r}2 \\
11 \\
14 \\
7 \\
5\end{array}$ & $\begin{array}{l}0.000300 \\
.00374 \\
.00595 \\
.00286 \\
.00177\end{array}$ & $\begin{array}{r}110 \\
0 \\
0 \\
0 \\
0 \\
0\end{array}$ & $\begin{array}{c}0.00000197 \\
.00000500 \\
.0000228 \\
.0000389 \\
.0000156\end{array}$ \\
\hline $\begin{array}{l}01-12-71 \\
01-13-71 \\
01-14-71 \\
01-16-71 \\
01-16-71\end{array}$ & $\begin{array}{l}.323 \\
.275 \\
.227 \\
2.61 \\
2.83\end{array}$ & $\begin{array}{l}.289 \\
.261 \\
.193 \\
2.69 \\
2.97\end{array}$ & $\begin{array}{l}-- \\
-- \\
-- \\
-- \\
--\end{array}$ & $\begin{array}{l}3.7 \\
3.7 \\
3.7 \\
3.7 \\
3.7\end{array}$ & $\begin{array}{l}.15 \\
.13 \\
.13 \\
.40 \\
.42\end{array}$ & $\begin{array}{l}.0086 \\
.0085 \\
.0084 \\
.0097 \\
.0098\end{array}$ & $\begin{array}{l}4.0 \\
3.5 \\
3.5 \\
5.0 \\
5.0\end{array}$ & $\begin{array}{r}1 \\
3 \\
5 \\
67 \\
110\end{array}$ & $\begin{array}{l}.000289 \\
.000783 \\
.000965 \\
.180 \\
.327\end{array}$ & $\begin{array}{l}0 \\
0 \\
0 \\
0 \\
0\end{array}$ & $\begin{array}{l}.00000500 \\
.00000256 \\
.00000119 \\
.156 \\
.107\end{array}$ \\
\hline $\begin{array}{l}01-18-71 \\
01-20-71 \\
01-21-71 \\
01-23-71 \\
01-25-71\end{array}$ & $\begin{array}{l}1.90 \\
.906 \\
.765 \\
.708 \\
1.70\end{array}$ & $\begin{array}{l}1.93 \\
.935 \\
.765 \\
.736 \\
1.76\end{array}$ & $\begin{array}{l}-- \\
-- \\
-- \\
-- \\
--\end{array}$ & $\begin{array}{l}3.7 \\
3.7 \\
3.7 \\
3.7 \\
3.7\end{array}$ & $\begin{array}{l}.34 \\
.24 \\
.23 \\
.22 \\
.32\end{array}$ & $\begin{array}{l}.0100 \\
.0098 \\
.0097 \\
.0097 \\
.0100\end{array}$ & $\begin{array}{l}6.5 \\
6.0 \\
6.0 \\
6.5 \\
7.5\end{array}$ & $\begin{array}{r}39 \\
13 \\
21 \\
8 \\
63\end{array}$ & $\begin{array}{l}.0753 \\
.0122 \\
.0161 \\
.00589 \\
.111\end{array}$ & $\begin{array}{l}0 \\
0 \\
0 \\
0 \\
0\end{array}$ & $\begin{array}{l}.0281 \\
.000669 \\
.0000917 \\
.0000694 \\
.00583\end{array}$ \\
\hline $\begin{array}{l}01-25-71 \\
01-26-71 \\
01-26-71 \\
01-27-71 \\
01-28-71\end{array}$ & $\begin{array}{l}1.53 \\
1.16 \\
.821 \\
.623 \\
.453\end{array}$ & $\begin{array}{l}1.33 \\
.906 \\
.736 \\
.538 \\
.411\end{array}$ & $\begin{array}{l}-- \\
-- \\
-- \\
-- \\
--\end{array}$ & $\begin{array}{l}3.7 \\
3.7 \\
3.7 \\
3.7 \\
3.7\end{array}$ & $\begin{array}{l}.31 \\
.27 \\
.23 \\
.21 \\
.19\end{array}$ & $\begin{array}{l}.0100 \\
.0099 \\
.0098 \\
.0097 \\
.0096\end{array}$ & $\begin{array}{l}7.5 \\
7.5 \\
7.5 \\
7.5 \\
6.5\end{array}$ & $\begin{array}{r}25 \\
8 \\
7 \\
7 \\
6\end{array}$ & $\begin{array}{l}.0333 \\
.00725 \\
.00515 \\
.00377 \\
.00247\end{array}$ & $\begin{array}{l}0 \\
0 \\
0 \\
0 \\
0\end{array}$ & $\begin{array}{l}.00333 \\
.00103 \\
.000164 \\
.0000361 \\
.0000133\end{array}$ \\
\hline $\begin{array}{l}02-04-71 \\
02-05-71 \\
02-09-71 \\
02-14-71 \\
02-15-71\end{array}$ & $\begin{array}{l}.651 \\
.453 \\
.221 \\
.193 \\
.198\end{array}$ & $\begin{array}{l}.609 \\
.354 \\
.295 \\
.204 \\
.235\end{array}$ & $\begin{array}{l}-- \\
-- \\
-- \\
-- \\
--\end{array}$ & $\begin{array}{l}3.7 \\
3.7 \\
3.7 \\
3.7 \\
3.7\end{array}$ & $\begin{array}{l}.22 \\
.19 \\
.15 \\
.14 \\
.14\end{array}$ & $\begin{array}{l}.0097 \\
.0096 \\
.0094 \\
.0094 \\
.0094\end{array}$ & $\begin{array}{l}5.5 \\
5.5 \\
6.5 \\
7.5 \\
8.0\end{array}$ & $\begin{array}{r}19 \\
8 \\
16 \\
4 \\
7\end{array}$ & $\begin{array}{l}.0116 \\
.00283 \\
.00472 \\
.000816 \\
.00165\end{array}$ & $\begin{array}{l}0 \\
0 \\
0 \\
0 \\
0\end{array}$ & $\begin{array}{l}.0000944 \\
.0000122 \\
.00000128 \\
.00000258 \\
.00000556\end{array}$ \\
\hline $\begin{array}{l}02-18-71 \\
02-22-71 \\
02-25-71 \\
02-26-71 \\
02-27-71\end{array}$ & $\begin{array}{l}.218 \\
.204 \\
.238 \\
.300 \\
.269\end{array}$ & $\begin{array}{l}.221 \\
.198 \\
.303 \\
.283 \\
.255\end{array}$ & $\begin{array}{l}-- \\
-- \\
-- \\
--\end{array}$ & $\begin{array}{l}3.7 \\
3.7 \\
3.7 \\
3.7 \\
3.7\end{array}$ & $\begin{array}{l}.15 \\
.14 \\
.15 \\
.16 \\
.16\end{array}$ & $\begin{array}{l}.0094 \\
.0094 \\
.0094 \\
.0096 \\
.0095\end{array}$ & $\begin{array}{l}6.5 \\
7.5 \\
5.5 \\
4.5 \\
4.0\end{array}$ & $\begin{array}{r}5 \\
13 \\
51 \\
3 \\
6\end{array}$ & $\begin{array}{l}.00111 \\
.00257 \\
.0155 \\
.000849 \\
.00153\end{array}$ & $\begin{array}{l}0 \\
0 \\
0 \\
0 \\
0\end{array}$ & $\begin{array}{l}.000000444 \\
.000000722 \\
.00000161 \\
.00000500 \\
.00000333\end{array}$ \\
\hline $\begin{array}{l}03-01-71 \\
03-03-71 \\
03-04-71 \\
03-05-71 \\
03-06-71\end{array}$ & $\begin{array}{l}.232 \\
.566 \\
.600 \\
.419 \\
.362\end{array}$ & $\begin{array}{l}.190 \\
.722 \\
.439 \\
.357 \\
.351\end{array}$ & $\begin{array}{l}-- \\
-- \\
-- \\
-- \\
--\end{array}$ & $\begin{array}{l}3.7 \\
3.7 \\
3.7 \\
3.7 \\
3.7\end{array}$ & $\begin{array}{l}.15 \\
.20 \\
.21 \\
.18 \\
.17\end{array}$ & $\begin{array}{l}.0094 \\
.0097 \\
.0097 \\
.0096 \\
.0096\end{array}$ & $\begin{array}{l}4.5 \\
4.5 \\
4.5 \\
5.0 \\
5.0\end{array}$ & $\begin{array}{r}9 \\
31 \\
4 \\
7 \\
7\end{array}$ & $\begin{array}{l}.00171 \\
.0224 \\
.00176 \\
.00250 \\
.00246\end{array}$ & $\begin{array}{l}0 \\
0 \\
0 \\
0 \\
0\end{array}$ & $\begin{array}{l}.00000333 \\
.000119 \\
.0000306 \\
.00000333 \\
.00000172\end{array}$ \\
\hline
\end{tabular}


Table 1.--Hydraulic and sediment-transport data--Continued

\begin{tabular}{|c|c|c|c|c|c|c|c|c|c|c|c|}
\hline Date & $\begin{array}{c}\text { Water } \\
\text { discharge } \\
\text { (bedload) } \\
\left(\mathrm{m}^{3} / \mathrm{s}\right)\end{array}$ & $\begin{array}{c}\text { Water } \\
\text { discharge } \\
\text { (suspended } \\
\text { load) } \\
\left(\mathrm{m}^{3} / \mathrm{s}\right)\end{array}$ & $\begin{array}{l}\text { Mean } \\
\text { flow } \\
\text { velocity } \\
(\mathrm{m} / \mathrm{s})\end{array}$ & $\begin{array}{l}\text { Water- } \\
\text { surface } \\
\text { width } \\
\text { (m) }\end{array}$ & $\begin{array}{l}\text { Mean } \\
\text { flow } \\
\text { depth } \\
(m)\end{array}$ & $\begin{array}{l}\text { Water- } \\
\text { surface } \\
\text { slope } \\
(\mathrm{m} / \mathrm{m})\end{array}$ & $\begin{array}{l}\text { Water } \\
\text { temp. } \\
\left({ }^{\circ} \mathrm{C}\right)\end{array}$ & $\begin{array}{l}\text { Suspended } \\
\text { sediment } \\
\text { concern- } \\
\text { tration } \\
(\mathrm{mg} / \mathrm{L})\end{array}$ & $\begin{array}{c}\text { Suspended } \\
\text { load } \\
(\mathrm{kg} / \mathrm{s})\end{array}$ & $\begin{array}{l}\text { Number of } \\
\text { sampling } \\
\text { points } \\
\text { for } \\
\text { bedload }\end{array}$ & $\begin{array}{r}\text { Bedload } \\
(\mathrm{kg} / \mathrm{s})\end{array}$ \\
\hline
\end{tabular}

13. Oak Creek near Corvallis, Oreg.--Continued

\begin{tabular}{|c|c|c|c|c|c|c|c|c|c|c|c|}
\hline $\begin{array}{l}03-10-71 \\
03-10-71 \\
03-10-71 \\
03-10-71 \\
03-11-71\end{array}$ & $\begin{array}{l}1.76 \\
2.04 \\
2.21 \\
1.53 \\
1.33\end{array}$ & $\begin{array}{l}1.78 \\
2.10 \\
2.12 \\
1.59 \\
.963\end{array}$ & $\begin{array}{l}-- \\
-- \\
-- \\
--\end{array}$ & $\begin{array}{l}3.7 \\
3.7 \\
3.7 \\
3.7 \\
3.7\end{array}$ & $\begin{array}{l}.36 \\
.37 \\
.37 \\
.34 \\
.33\end{array}$ & $\begin{array}{r}0.0100 \\
.0101 \\
.0108 \\
.0105 \\
.0104\end{array}$ & $\begin{array}{l}5.0 \\
5.5 \\
5.5 \\
5.5 \\
5.5\end{array}$ & $\begin{array}{r}24 \% \\
217 \\
649 \\
47 \\
19\end{array}$ & $\begin{array}{c}0.440 \\
.456 \\
1.38 \\
.0747 \\
.0183\end{array}$ & $\begin{array}{l}0 \\
0 \\
0 \\
0 \\
0\end{array}$ & $\begin{array}{c}0.0119 \\
.0250 \\
.406 \\
.0361 \\
.0175\end{array}$ \\
\hline $\begin{array}{l}03-11-71 \\
03-11-71 \\
03-11-71\end{array}$ & $\begin{array}{l}1.02 \\
1.44 \\
1.93\end{array}$ & $\begin{array}{l}1.30 \\
1.64 \\
1.95\end{array}$ & $\begin{array}{l}-- \\
-- \\
--\end{array}$ & $\begin{array}{l}3.7 \\
3.7 \\
3.7\end{array}$ & $\begin{array}{l}.31 \\
.34 \\
.36\end{array}$ & $\begin{array}{l}.0102 \\
.0100 \\
.0100\end{array}$ & $\begin{array}{l}5.5 \\
5.5 \\
5.5\end{array}$ & $\begin{array}{r}24 \\
50 \\
200\end{array}$ & $\begin{array}{l}.0312 \\
.0820 \\
.390\end{array}$ & $\begin{array}{l}0 \\
0 \\
0\end{array}$ & $\begin{array}{l}.00528 \\
.0217 \\
.109\end{array}$ \\
\hline
\end{tabular}

14. San Antonio River near Lockwood, Calif. ${ }^{7}$

\begin{tabular}{|c|c|c|c|c|c|c|c|c|c|c|c|}
\hline $\begin{array}{l}11-21-72 \\
01-17-73 \\
03-28-74 \\
12-10-74 \\
01-06-75\end{array}$ & $\begin{array}{c}1.81 \\
28.0 \\
17.8 \\
1.05 \\
.793\end{array}$ & $\begin{array}{c}1.81 \\
28.9 \\
17.8 \\
1.05 \\
.793\end{array}$ & $\begin{array}{l}0.50 \\
1.0 \\
1.0 \\
.50 \\
.45\end{array}$ & $\begin{array}{l}34.5 \\
55.0 \\
43.0 \\
16.0 \\
14.5\end{array}$ & $\begin{array}{r}0.11 \\
.49 \\
.40 \\
.13 \\
.12\end{array}$ & $\begin{array}{l}-- \\
-- \\
-- \\
-- \\
--\end{array}$ & $\begin{array}{r}12.0 \\
9.5 \\
14.0 \\
16.0 \\
7.5\end{array}$ & $\begin{array}{r}24 \\
1,350 \\
791 \\
19 \\
18\end{array}$ & $\begin{array}{l}0.0434 \\
39.0 \\
14.1 \\
.0200 \\
.0143\end{array}$ & $\begin{array}{l}47 \\
17 \\
27 \\
37 \\
23\end{array}$ & $\begin{array}{c}0.714 \\
12.4 \\
6.77 \\
.189 \\
.210\end{array}$ \\
\hline $\begin{array}{l}02-06-75 \\
03-06-75 \\
04-04-75 \\
05-07-75 \\
06-05-75\end{array}$ & $\begin{array}{l}8.84 \\
4.64 \\
5.78 \\
2.44 \\
.821\end{array}$ & $\begin{array}{l}8.84 \\
4.64 \\
5.78 \\
2.44 \\
.821\end{array}$ & $\begin{array}{l}.82 \\
.60 \\
.73 \\
.55 \\
.46\end{array}$ & $\begin{array}{l}36.0 \\
42.0 \\
38.0 \\
25.0 \\
15.0\end{array}$ & $\begin{array}{l}.30 \\
.18 \\
.21 \\
.18 \\
.12\end{array}$ & $\begin{array}{l}-- \\
-- \\
-- \\
-- \\
--\end{array}$ & $\begin{array}{l}13.5 \\
14.0 \\
11.5 \\
24.0 \\
28.0\end{array}$ & $\begin{array}{r}151 \\
118 \\
113 \\
26 \\
8\end{array}$ & $\begin{array}{l}1.33 \\
.548 \\
.653 \\
.0634 \\
.00657\end{array}$ & $\begin{array}{l}23 \\
26 \\
25 \\
19 \\
23\end{array}$ & $\begin{array}{l}6.24 \\
2.10 \\
2.83 \\
1.38 \\
.263\end{array}$ \\
\hline $\begin{array}{l}03-12-76 \\
04-09-76 \\
05-06-76 \\
01-18-78 \\
02-21-78\end{array}$ & $\begin{aligned} & .538 \\
& .368 \\
& .0680 \\
& 39.6 \\
& 13.5\end{aligned}$ & $\begin{aligned} & .538 \\
& .368 \\
& .0680 \\
& 39.6 \\
& 13.5\end{aligned}$ & $\begin{array}{l}.43 \\
.45 \\
.35 \\
-- \\
--\end{array}$ & $\begin{array}{r}15.0 \\
7.9 \\
2.9 \\
67.0 \\
55.0\end{array}$ & $\begin{array}{l}.091 \\
.11 \\
.066 \\
\ldots \\
\ldots\end{array}$ & $\begin{array}{l}-- \\
-- \\
-- \\
-- \\
--\end{array}$ & $\begin{array}{c}19.5 \\
19.0 \\
17.0 \\
-0 \\
-\end{array}$ & $\begin{array}{r}4 \\
4 \\
3 \\
1,030 \\
305\end{array}$ & $\begin{array}{l}.00215 \\
.00147 \\
.000204 \\
40.8 \\
4.12\end{array}$ & $\begin{array}{r}16 \\
12 \\
9 \\
42 \\
26\end{array}$ & $\begin{aligned} .263 \\
.147 \\
.0147 \\
11.6 \\
5.39\end{aligned}$ \\
\hline $\begin{array}{l}03-28-78 \\
05-06-78 \\
06-16-78 \\
07-11-78 \\
12-04-78\end{array}$ & $\begin{array}{l}10.7 \\
3.23 \\
1.56 \\
.425 \\
.396\end{array}$ & $\begin{array}{l}10.7 \\
3.23 \\
1.56 \\
.425 \\
.396\end{array}$ & $\begin{array}{l}-- \\
-- \\
-- \\
-- \\
--\end{array}$ & $\begin{array}{r}51.0 \\
23.0 \\
11.5 \\
9.1 \\
14.0\end{array}$ & $\begin{array}{l}-- \\
-- \\
-- \\
-- \\
--\end{array}$ & $\begin{array}{l}-- \\
-- \\
-- \\
--\end{array}$ & $\begin{array}{l}- \\
- \\
- \\
-\end{array}$ & $\begin{array}{r}168 \\
44 \\
42 \\
8 \\
9\end{array}$ & $\begin{array}{l}1.80 \\
.142 \\
.0655 \\
.00340 \\
.00356\end{array}$ & $\begin{array}{l}33 \\
15 \\
13 \\
16 \\
12\end{array}$ & $\begin{array}{l}6.59 \\
1.46 \\
.756 \\
.179 \\
.137\end{array}$ \\
\hline $\begin{array}{l}01-08-79 \\
02-06-79 \\
04-05-79 \\
05-21-79 \\
02-27-80\end{array}$ & $\begin{array}{c}.538 \\
2.97 \\
5.30 \\
.906 \\
19.0\end{array}$ & $\begin{array}{l}.538 \\
2.97 \\
5.30 \\
.906 \\
20.1\end{array}$ & $\begin{array}{l}-- \\
-- \\
-- \\
.53 \\
.76\end{array}$ & $\begin{array}{l}6.1 \\
32.5 \\
27.5 \\
11.5 \\
62\end{array}$ & $\begin{array}{l}-- \\
-. \\
\ldots \\
.15 \\
.42\end{array}$ & $\begin{array}{l}-- \\
-- \\
-- \\
--\end{array}$ & $\begin{array}{c}-- \\
-- \\
25.5 \\
\ldots\end{array}$ & $\begin{array}{r}12 \\
28 \\
65 \\
8 \\
377\end{array}$ & $\begin{array}{l}.00646 \\
.0832 \\
.345 \\
.00725 \\
7.58\end{array}$ & $\begin{array}{l}10 \\
25 \\
11 \\
10 \\
20\end{array}$ & $\begin{array}{l}.294 \\
2.65 \\
3.71 \\
.903 \\
6.42\end{array}$ \\
\hline $\begin{array}{l}04-17-80 \\
05-17-80 \\
06-07-80 \\
02-04-81 \\
03-18-81\end{array}$ & $\begin{array}{l}3.91 \\
2.04 \\
1.05 \\
2.95 \\
1.90\end{array}$ & $\begin{array}{l}3.91 \\
2.04 \\
1.05 \\
2.95 \\
1.90\end{array}$ & $\begin{array}{l}.53 \\
.51 \\
.41 \\
-. \\
-.\end{array}$ & $\begin{array}{l}36.0 \\
28.0 \\
18.0 \\
25.5 \\
23.0\end{array}$ & $\begin{array}{l}.20 \\
.14 \\
.14 \\
-- \\
--\end{array}$ & $\begin{array}{l}\cdots \\
-- \\
--\end{array}$ & $\begin{array}{c}23.5 \\
29.0 \\
30.0 \\
\ldots \\
\ldots\end{array}$ & $\begin{array}{r}40 \\
32 \\
14 \\
43 \\
9\end{array}$ & $\begin{array}{l}.156 \\
.0653 \\
.0147 \\
.127 \\
.0171\end{array}$ & $\begin{array}{l}23 \\
20 \\
20 \\
23 \\
20\end{array}$ & $\begin{array}{l}1.28 \\
.882 \\
.767 \\
.767 \\
.609\end{array}$ \\
\hline $\begin{array}{l}04-07-81 \\
11-16-81 \\
12-14-81 \\
02-10-82 \\
02-14-83\end{array}$ & $\begin{array}{c}2.75 \\
2.46 \\
.680 \\
2.46 \\
23.1\end{array}$ & $\begin{array}{c}2.69 \\
2.52 \\
.680 \\
2.46 \\
24.3\end{array}$ & $\begin{array}{l}-. \\
.44 \\
.45 \\
.49 \\
.84\end{array}$ & $\begin{array}{l}28.0 \\
42.5 \\
12.0 \\
21.0 \\
49.0\end{array}$ & $\begin{array}{l}-. \\
.14 \\
.13 \\
.24 \\
.61\end{array}$ & $\begin{array}{l}-- \\
-- \\
-- \\
-- \\
--\end{array}$ & $\begin{array}{c}-- \\
14.5 \\
17.0 \\
-- \\
--\end{array}$ & $\begin{array}{r}8 \\
71 \\
4 \\
32 \\
342\end{array}$ & $\begin{array}{l}.0215 \\
.179 \\
.00272 \\
.0787 \\
8.31\end{array}$ & $\begin{array}{l}20 \\
23 \\
26 \\
22 \\
19\end{array}$ & $\begin{array}{c}1.20 \\
1.22 \\
.294 \\
1.03 \\
31.1\end{array}$ \\
\hline $\begin{array}{l}03-01-83 \\
04-22-83 \\
12-06-83 \\
01-19-84\end{array}$ & $\begin{array}{c}156 \\
10.0 \\
4.73 \\
2.66\end{array}$ & $\begin{array}{c}157 \\
10.0 \\
4.73 \\
2.66\end{array}$ & $\begin{array}{l}1.7 \\
.66 \\
.55 \\
-.\end{array}$ & $\begin{array}{l}69 \\
36.5 \\
35.5 \\
26.0\end{array}$ & $\begin{array}{l}1.5 \\
.35 \\
.23 \\
--\end{array}$ & $\begin{array}{l}-- \\
-- \\
-- \\
--\end{array}$ & $\begin{array}{r}13.0 \\
15.0 \\
8.5 \\
13.5\end{array}$ & $\begin{array}{r}2,530 \\
109 \\
31 \\
33\end{array}$ & $\begin{array}{c}397 \\
1.09 \\
\quad .147 \\
.0878\end{array}$ & $\begin{array}{l}10 \\
23 \\
22 \\
18\end{array}$ & $\begin{array}{r}139 \\
6.25 \\
1.24 \\
1.91\end{array}$ \\
\hline
\end{tabular}


Table 1.--Hydraulic and sediment-transport data--Continued

\begin{tabular}{|c|c|c|c|c|c|c|c|c|c|c|c|}
\hline Date & $\begin{array}{c}\text { Water } \\
\text { discharge } \\
\text { (bedload })^{1} \\
\left(\mathrm{~m}^{3} / \mathrm{s}\right)\end{array}$ & $\begin{array}{c}\text { Water } \\
\text { discharge } \\
\text { (suspended } \\
\text { load) } \\
\left(\mathrm{m}^{3} / \mathrm{s}\right)\end{array}$ & $\begin{array}{l}\text { Mean } \\
\text { flow } \\
\text { velocity } \\
(\mathrm{m} / \mathrm{s})\end{array}$ & $\begin{array}{l}\text { Water- } \\
\text { surface } \\
\text { width } \\
\text { (m) }\end{array}$ & $\begin{array}{l}\text { Mean } \\
\text { flow } \\
\text { depth } \\
(m)\end{array}$ & $\begin{array}{l}\text { Water- } \\
\text { surface } \\
\text { slope } \\
(\mathrm{m} / \mathrm{m})\end{array}$ & $\begin{array}{l}\text { Water } \\
\text { temp. } \\
\left({ }^{\circ} \mathrm{C}\right)\end{array}$ & $\begin{array}{l}\text { Suspended } \\
\text { sediment } \\
\text { concern- } \\
\text { tration } \\
(\mathrm{mg} / \mathrm{L})\end{array}$ & $\begin{array}{c}\text { Suspended } \\
\text { load } \\
(\mathrm{kg} / \mathrm{s})\end{array}$ & $\begin{array}{l}\text { Number of } \\
\text { sampling } \\
\text { points } \\
\text { for } \\
\text { bedload }\end{array}$ & $\begin{array}{r}\text { Bedload } \\
(\mathrm{kg} / \mathrm{s})\end{array}$ \\
\hline
\end{tabular}

14. San Antonio River near Lockwood, Cal if.--Continued

\begin{tabular}{|c|c|c|c|c|c|c|c|c|c|c|c|}
\hline $04-13-84$ & 0.793 & 0.793 & 0.40 & 17.5 & 0.11 & - & 18.5 & 10 & 0.00793 & 23 & 0.210 \\
\hline $01-14-85$ & .878 & .878 & .42 & 20.5 & .10 & - & 7.5 & 1 & .000878 & 22 & .336 \\
\hline $02-07-85$ & .538 & .538 & .34 & 21.0 & .076 & - & 8.5 & 3 & .00161 & 23 & .884 \\
\hline $03-04-85$ & .878 & .878 & .41 & 21.0 & .10 & -- & 11.0 & 6 & .00527 & 22 & .462 \\
\hline $04-04-85$ & 2.18 & 2.18 & .49 & 34.0 & .12 & - & 15.5 & 11 & .0240 & 22 & 1.14 \\
\hline $04-29-85$ & .708 & .708 & .45 & 14.5 & .11 & -- & 24.5 & 5 & .00354 & 25 & .059 \\
\hline
\end{tabular}

15. Chamise Creck near Is land Mtn, Calif. ?

\begin{tabular}{|c|c|c|c|c|c|c|c|c|c|c|c|}
\hline $11-07-72$ & 1.56 & 1.56 & 0.47 & 8.2 & 0.40 & -- & 11.0 & 29 & 0.0452 & 13 & 0.0147 \\
\hline $02-28-73$ & 3.57 & 3.57 & 1.1 & 12.5 & .26 & -- & 10.5 & 78 & .278 & 19 & .0536 \\
\hline $04-05-73$ & 1.19 & 1.19 & .60 & 8.8 & .23 & -- & 11.0 & 12 & .0143 & -- & .00 \\
\hline $05-14-73$ & .187 & .187 & .28 & 5.1 & .13 & -- & 22.0 & 1 & .000187 & -- & .00 \\
\hline $11-14-73$ & 8.33 & 8.21 & 1.1 & 19.5 & .38 & -- & 12.0 & 373 & 3.06 & 21 & 2.69 \\
\hline $12-21-73$ & 12.7 & 12.7 & 1.4 & 20.0 & .46 & -- & 9.5 & 751 & 9.54 & 21 & 5.77 \\
\hline $03-05-74$ & 3.74 & 3.77 & .85 & 22.0 & .20 & -- & 10.0 & 161 & .607 & 19 & .441 \\
\hline $04-11-74$ & 1.76 & 1.76 & .60 & 17.5 & .16 & -- & 10.5 & 44 & .0774 & 10 & .0179 \\
\hline $11-07-75$ & .481 & .481 & .33 & 10.0 & .14 & -- & 13.5 & 16 & .00770 & - & .00 \\
\hline $12-12-75$ & .595 & .595 & .36 & 11.5 & .14 & -- & 7.5 & 6 & .00357 & -- & .00 \\
\hline $03-03-76$ & 3.77 & 3.77 & .81 & 19.5 & .24 & -- & 9.0 & 156 & .588 & 28 & .0557 \\
\hline
\end{tabular}

16. Redwood Creek near Blue Lake, Calif.?

\begin{tabular}{|c|c|c|c|c|c|c|c|c|c|c|c|}
\hline $01-12-74$ & 7.14 & 7.14 & 1.1 & 25.5 & 0.27 & -- & 5.0 & 70 & 0.500 & 13 & 1.40 \\
\hline $04-01-75$ & 13.6 & 13.6 & 1.4 & 24.0 & .42 & -- & 8.5 & 218 & 2.96 & 17 & 6.49 \\
\hline $03-10-78$ & 17.9 & 17.9 & 1.3 & 24.0 & .58 & -- & 8.5 & 116 & 2.08 & 14 & 6.14 \\
\hline $01-27-81$ & 57.8 & 54.7 & 2.0 & 28.5 & .89 & -- & 7.5 & 2,440 & 133 & 10 & 25.5 \\
\hline $04-02-81$ & 12.1 & 12.1 & 1.1 & 21.5 & .53 & -- & 9.0 & 34 & .411 & 10 & .357 \\
\hline $01-27-82$ & -- & 18.0 & 1.2 & 20.5 & .71 & - & 7.0 & 76 & 1.37 & 18 & 5.04 \\
\hline $11-30-82$ & 34.0 & 33.4 & 1.7 & 23.0 & .93 & -- & - & 464 & 15.5 & 17 & 9.57 \\
\hline $12-16-82$ & 84.1 & 86.2 & 2.1 & 30.0 & 1.2 & - & 8.0 & 1,765 & 152 & 10 & 12.8 \\
\hline $01-27-83$ & 76.2 & 77.0 & 2.3 & 29.5 & 1.0 & -- & 7.5 & 3,290 & 253 & 10 & 5.97 \\
\hline $02-28-83$ & 28.2 & 27.4 & 1.5 & 22.5 & .83 & - & 8.5 & 497 & 13.6 & 10 & 31.3 \\
\hline $11-17-83$ & 52.1 & 53.0 & 2.1 & 28.5 & .94 & - & 9.5 & 1,110 & 58.8 & 16 & 26.9 \\
\hline $12-14-83$ & 88.4 & 84.7 & 2.2 & 31.0 & 1.4 & -- & 9.5 & 1,420 & 120 & 15 & 48.4 \\
\hline $03-01-84$ & 13.8 & 13.6 & 1.1 & 20.0 & .63 & -- & 8.0 & 64 & .870 & 12 & .662 \\
\hline
\end{tabular}

17. Redwood Creek above Panther Creek near Orick, Calif.?

\begin{tabular}{|c|c|c|c|c|c|c|c|c|c|c|c|}
\hline $12-03-80$ & 47.3 & 46.7 & 1.7 & 32.0 & 0.90 & -- & 10.5 & 480 & 22.4 & 10 & 8.32 \\
\hline $01-23-81$ & 36.2 & 32.6 & 1.6 & 28.5 & .74 & -- & 9.5 & 306 & 9.98 & 10 & 7.42 \\
\hline $01-28-81$ & 112 & 112 & 2.1 & 31.0 & 1.6 & -- & 9.0 & 1,350 & 151 & 10 & 53.1 \\
\hline $02-05-81$ & 14.2 & 14.2 & 1.1 & 28.0 & .48 & -- & 8.5 & 23 & .327 & 10 & 1.05 \\
\hline $11-02-81$ & 4.02 & 4.02 & .68 & 22.0 & .27 & -- & 11.5 & 2 & .00804 & 21 & .0651 \\
\hline $12-09-81$ & 43.0 & 44.5 & 1.6 & 29.5 & .87 & -- & 10.5 & 272 & 12.1 & 10 & 4.98 \\
\hline $02-10-82$ & 12.5 & 12.5 & 1.0 & 27.0 & .45 & -- & 6.0 & 10 & .125 & 20 & 1.19 \\
\hline $03-19-82$ & 17.5 & 17.8 & 1.3 & 27.0 & .50 & -- & 8.5 & 74 & 1.32 & 17 & 2.27 \\
\hline $12-01-82$ & 70.0 & 72.8 & -- & 31.0 & -- & -- & - & 393 & 28.6 & 16 & 30.2 \\
\hline $12-22-82$ & 107 & 106 & -- & 32.5 & -- & -- & -- & 831 & 88.1 & 10 & 52.9 \\
\hline
\end{tabular}


Table 1.--Hydraulic and sediment-transport data--Continued

\begin{tabular}{|c|c|c|c|c|c|c|c|c|c|c|c|}
\hline Date & $\begin{array}{c}\text { Water } \\
\text { discharge } \\
\text { (bedload) } \\
\left(\mathrm{m}^{3} / \mathrm{s}\right)\end{array}$ & $\begin{array}{c}\text { Water } \\
\text { discharge } \\
\text { (suspended } \\
\text { load) } \\
\left(\mathrm{m}^{3} / \mathrm{s}\right)\end{array}$ & $\begin{array}{l}\text { Mean } \\
\text { flow } \\
\text { velocity } \\
(\mathrm{m} / \mathrm{s})\end{array}$ & $\begin{array}{l}\text { Waler- } \\
\text { surface } \\
\text { width } \\
\text { (m) }\end{array}$ & $\begin{array}{l}\text { Mean } \\
\text { flow } \\
\text { depth } \\
\text { (m) }\end{array}$ & $\begin{array}{l}\text { Water- } \\
\text { surface } \\
\text { slope } \\
(\mathrm{m} / \mathrm{m})\end{array}$ & $\begin{array}{l}\text { Water } \\
\text { temp. } \\
\left({ }^{\circ} \mathrm{C}\right)\end{array}$ & $\begin{array}{l}\text { Suspended } \\
\text { sediment } \\
\text { concern- } \\
\text { tration } \\
(m g / L)\end{array}$ & $\begin{array}{c}\text { Suspended } \\
\text { load } \\
(\mathrm{kg} / \mathrm{s})\end{array}$ & $\begin{array}{l}\text { Number of } \\
\text { sampling } \\
\text { points } \\
\text { for } \\
\text { bedload }\end{array}$ & $\begin{array}{r}\text { Bedload } \\
(\mathrm{kg} / \mathrm{s})\end{array}$ \\
\hline
\end{tabular}

\section{Redwood Creek above Panther Creek near Orick, Calif.--Continued}

\begin{tabular}{|c|c|c|c|c|c|c|c|c|c|c|c|}
\hline $\begin{array}{l}01-20-83 \\
01-26-83 \\
02-23-83 \\
04-05-83 \\
11-16-83\end{array}$ & $\begin{array}{c}30.0 \\
201 \\
54.9 \\
41.3 \\
33.4\end{array}$ & $\begin{array}{c}31.2 \\
172 \\
55.2 \\
41.6 \\
34.8\end{array}$ & $\begin{array}{l}-- \\
-- \\
-- \\
--\end{array}$ & $\begin{array}{l}30.0 \\
34.5 \\
30.0 \\
29.0 \\
28.0\end{array}$ & $\begin{array}{l}-- \\
-- \\
-- \\
--\end{array}$ & $\begin{array}{l}-- \\
-- \\
-- \\
--\end{array}$ & $\begin{array}{l}-- \\
\ldots \\
-- \\
-- \\
11.0\end{array}$ & $\begin{array}{r}129 \\
3,420 \\
415 \\
185 \\
240\end{array}$ & $\begin{array}{l}4.02 \\
588 \\
22.9 \\
7.70 \\
8.37\end{array}$ & $\begin{array}{l}13 \\
12 \\
14 \\
15 \\
16\end{array}$ & $\begin{array}{l}5.94 \\
39.3 \\
40.6 \\
12.6 \\
10.1\end{array}$ \\
\hline $\begin{array}{l}01-13-84 \\
03-08-84 \\
04-03-84\end{array}$ & $\begin{array}{l}13.6 \\
16.8 \\
18.3\end{array}$ & $\begin{array}{l}13.4 \\
16.6 \\
18.3\end{array}$ & $\begin{array}{l}-- \\
--\end{array}$ & $\begin{array}{l}23.5 \\
26.0 \\
28.5\end{array}$ & $\begin{array}{l}-- \\
--\end{array}$ & $\begin{array}{l}-- \\
--\end{array}$ & $\begin{array}{l}6.0 \\
9.5 \\
-.\end{array}$ & $\begin{array}{r}9 \\
19 \\
19\end{array}$ & $\begin{array}{l}.121 \\
.315 \\
.348\end{array}$ & $\begin{array}{l}12 \\
20 \\
16\end{array}$ & $\begin{array}{l}.315 \\
1.34 \\
.368\end{array}$ \\
\hline
\end{tabular}

\section{Redwood Creek at Orick, Calif. 7}

\begin{tabular}{|c|c|c|c|c|c|c|c|c|c|c|c|}
\hline $\begin{array}{l}11-14-79 \\
02-01-80 \\
02-21-80 \\
12-11-80 \\
01-29-81\end{array}$ & $\begin{array}{r}14.2 \\
19.9 \\
87.2 \\
14.9 \\
135\end{array}$ & $\begin{array}{r}14.2 \\
19.7 \\
85.8 \\
14.8 \\
131\end{array}$ & $\begin{array}{c}0.81 \\
.91 \\
1.2 \\
.72 \\
1.3\end{array}$ & $\begin{array}{l}44.0 \\
49.0 \\
39.0 \\
43.5 \\
58\end{array}$ & $\begin{array}{l}0.40 \\
.45 \\
1.9 \\
.48 \\
1.7\end{array}$ & $\begin{array}{l}\cdots \\
\cdots \\
\cdots \\
\cdots\end{array}$ & $\begin{array}{r}11.5 \\
8.5 \\
10.0 \\
7.5 \\
9.0\end{array}$ & $\begin{array}{r}11 \\
24 \\
350 \\
23 \\
444\end{array}$ & $\begin{aligned} 0 . & 156 \\
& .473 \\
30 . & 0 \\
& .340 \\
58 . & 2\end{aligned}$ & $\begin{array}{l}10 \\
19 \\
10 \\
10 \\
10\end{array}$ & $\begin{array}{c}3.68 \\
2.64 \\
24.4 \\
1.80 \\
93.7\end{array}$ \\
\hline $\begin{array}{l}02-24-81 \\
04-11-81 \\
11-13-81 \\
12-14-81 \\
12-23-81\end{array}$ & $\begin{array}{l}66.6 \\
26.5 \\
44.5 \\
153 \\
122\end{array}$ & $\begin{array}{l}62.9 \\
26.5 \\
44.5 \\
153 \\
119\end{array}$ & $\begin{array}{l}1.1 \\
-. \\
.90 \\
1.3 \\
1.3\end{array}$ & $\begin{array}{l}54 \\
33.5 \\
52 \\
61 \\
52\end{array}$ & $\begin{array}{l}1.1 \\
-. \\
.89 \\
2.0 \\
1.8\end{array}$ & $\begin{array}{l}-- \\
\cdots \\
-- \\
--\end{array}$ & $\begin{array}{r}9.0 \\
-0 \\
12.0 \\
9.5 \\
10.0\end{array}$ & $\begin{array}{r}157 \\
20 \\
332 \\
577 \\
502\end{array}$ & $\begin{array}{c}9.88 \\
\quad .530 \\
14.8 \\
88.3 \\
59.7\end{array}$ & $\begin{array}{l}10 \\
11 \\
19 \\
11 \\
21\end{array}$ & $\begin{array}{c}18.7 \\
6.53 \\
9.11 \\
24.5 \\
11.3\end{array}$ \\
\hline $\begin{array}{l}03-03-82 \\
11-22-82 \\
12-22-82 \\
01-24-83 \\
03-30-83\end{array}$ & $\begin{array}{l}133 \\
39.1 \\
190 \\
110 \\
374\end{array}$ & $\begin{array}{l}130 \\
39.6 \\
187 \\
113 \\
385\end{array}$ & $\begin{array}{c}1.2 \\
\ldots \\
\ldots \\
-- \\
--\end{array}$ & $\begin{array}{l}57 \\
50 \\
59 \\
53 \\
95\end{array}$ & $\begin{array}{c}1.8 \\
-- \\
-- \\
- \\
--\end{array}$ & $\begin{array}{l}\cdots \\
\cdots \\
\cdots \\
\cdots\end{array}$ & $\begin{array}{l}9.5 \\
-- \\
=- \\
-- \\
--\end{array}$ & $\begin{array}{r}498 \\
97 \\
859 \\
1,080 \\
1,940\end{array}$ & $\begin{array}{c}64.7 \\
3.84 \\
161 \\
122 \\
747\end{array}$ & $\begin{array}{l}19 \\
22 \\
15 \\
15 \\
29\end{array}$ & $\begin{array}{c}43.2 \\
6.58 \\
58.2 \\
49.0 \\
49.5\end{array}$ \\
\hline $\begin{array}{l}12-07-83 \\
02-29-84\end{array}$ & $\begin{array}{l}331 \\
57.8\end{array}$ & $\begin{array}{l}312 \\
58.6\end{array}$ & $\cdots$ & $\begin{array}{l}85 \\
49.5\end{array}$ & -- & -- & -- & $\begin{array}{r}1,150 \\
105\end{array}$ & $\begin{array}{r}359 \\
6.15\end{array}$ & $\begin{array}{l}15 \\
10\end{array}$ & $\begin{array}{r}153 \\
16.5\end{array}$ \\
\hline
\end{tabular}

19. Hayes Creek near Orick, Calif.?

\begin{tabular}{|c|c|c|c|c|c|c|c|c|c|c|c|}
\hline $12-14-77$ & 0.852 & 0.852 & 0.62 & 6.1 & 0.22 & -- & -- & $356^{\circ}$ & 0.303 & 12 & 0.116 \\
\hline $12-14-77$ & .708 & .708 & $=$ & 5.8 & - & -- & - & 190 & .135 & 12 & .0777 \\
\hline $01-11-79$ & .212 & .212 & .55 & 2.6 & .15 & -- & 11.5 & 254 & .0538 & 9 & .00483 \\
\hline $02-13-79$ & .276 & .276 & .51 & 4.3 & .13 & -- & 9.5 & 31 & .00856 & $\cdots$ & .00 \\
\hline $02-20-79$ & .133 & .133 & .44 & 3.0 & .10 & - & 8.5 & 19 & .00253 & $\cdots$ & .00 \\
\hline $02-26-79$ & .261 & .261 & .54 & 3.8 & .12 & -- & 9.5 & 34 & .00887 & -- & .00 \\
\hline $02-27-79$ & .232 & .232 & .56 & 4.0 & .10 & $\ldots$ & 8.5 & 20 & .00464 & $\ldots$ & .00 \\
\hline $05-08-79$ & .204 & .204 & .53 & 3.3 & .12 & 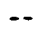 & 9.0 & 20 & .00408 & $\ldots$ & .00 \\
\hline $01-14-80$ & .481 & .481 & .66 & 4.0 & .18 & -- & 10.0 & 168 & .0808 & 13 & .00578 \\
\hline $01-15-80$ & .368 & .368 & .68 & 4.3 & .13 & -- & 10.0 & 49 & .0180 & 7 & .0189 \\
\hline $02-21-80$ & .144 & .144 & .56 & 1.9 & .14 & -- & 9.5 & 22 & .00317 & $\ldots$ & .00 \\
\hline $03-14-80$ & .623 & .595 & .71 & 4.0 & .22 & $\ldots$ & 9.5 & 412 & .245 & 12 & .0588 \\
\hline $04-09-80$ & .261 & .261 & $\cdots$ & - & -- & $\ldots$ & 9.5 & 5 & .00131 & $\ldots$ & .00 \\
\hline
\end{tabular}

20. Lacks Creek near Orick, Calif.?

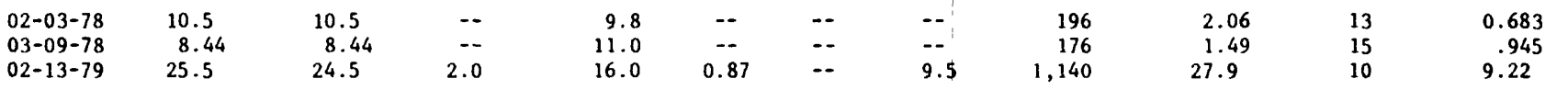


Table 1.--Hydraulic and sediment-transport data--Conlinued

\begin{tabular}{|c|c|c|c|c|c|c|c|c|c|c|c|}
\hline Date & $\begin{array}{c}\text { Water } \\
\text { discharge } \\
\text { (bedload) } \\
\left(\mathrm{m}^{3} / \mathrm{s}\right)\end{array}$ & $\begin{array}{c}\text { Water } \\
\text { discharge } \\
\text { (suspended } \\
\text { load })^{2} \\
\left(\mathrm{~m}^{3} / \mathrm{s}\right)\end{array}$ & $\begin{array}{l}\text { Mean } \\
\text { flow } \\
\text { velocity } \\
(\mathrm{m} / \mathrm{s})\end{array}$ & $\begin{array}{l}\text { Water- } \\
\text { surface } \\
\text { width } \\
\text { (m) }\end{array}$ & $\begin{array}{l}\text { Mean } \\
\text { flow } \\
\text { depth } \\
(\mathrm{m})\end{array}$ & $\begin{array}{l}\text { Water- } \\
\text { surface } \\
\text { slope } \\
(\mathrm{m} / \mathrm{m})\end{array}$ & $\begin{array}{l}\text { Water } \\
\text { temp. } \\
\left({ }^{\circ} \mathrm{C}\right)\end{array}$ & $\begin{array}{c}\text { Suspended } \\
\text { sediment } \\
\text { concern- } \\
\text { tration } \\
(\mathrm{mg} / \mathrm{L})\end{array}$ & $\begin{array}{c}\text { Suspended } \\
\text { load } \\
(\mathrm{kg} / \mathrm{s})\end{array}$ & $\begin{array}{c}\text { Number of } \\
\text { sampling } \\
\text { points } \\
\text { for } \\
\text { bedload }\end{array}$ & $\begin{array}{r}\text { Bedload } \\
\left(\mathrm{kg}_{\mathrm{g}} / \mathrm{s}\right)\end{array}$ \\
\hline
\end{tabular}

20. Lacks Creek near Orick, Calif.--Continued

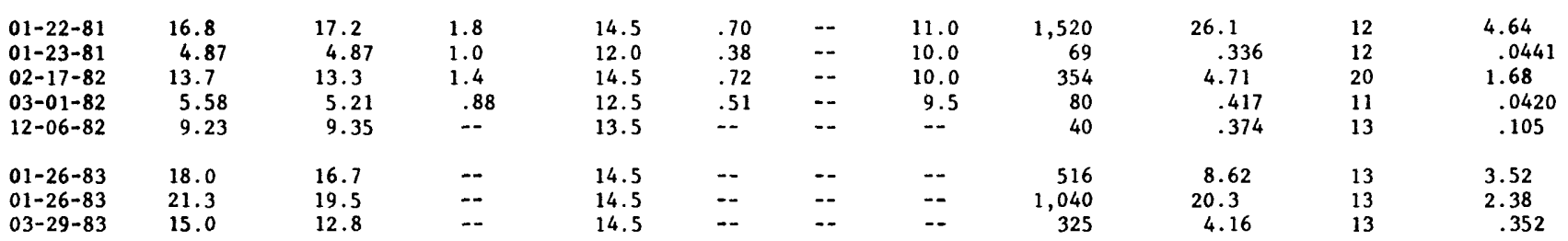

21. Sacramento River above Bend Bridge near Red Bluff, Calif. ${ }^{7}$

\begin{tabular}{|c|c|c|c|c|c|c|c|c|c|c|c|}
\hline $\begin{array}{l}12-01-77 \\
01-03-78 \\
02-02-78 \\
03-01-78 \\
05-01-78\end{array}$ & $\begin{array}{l}120 \\
242 \\
195 \\
202 \\
430\end{array}$ & $\begin{array}{l}120 \\
226 \\
204 \\
202 \\
429\end{array}$ & $\begin{array}{l}1.5 \\
1.6 \\
1.4 \\
1.4 \\
1.5\end{array}$ & $\begin{array}{l}104 \\
109 \\
110 \\
109 \\
112\end{array}$ & $\begin{array}{l}0.79 \\
1.4 \\
1.2 \\
1.3 \\
2.5\end{array}$ & $\begin{array}{l}-- \\
-- \\
-- \\
--\end{array}$ & $\begin{array}{r}10.5 \\
9.0 \\
9.0 \\
10.0 \\
10.5\end{array}$ & $\begin{array}{r}9 \\
118 \\
32 \\
24 \\
31\end{array}$ & $\begin{array}{c}1.08 \\
26.7 \\
6.53 \\
4.85 \\
13.3\end{array}$ & $\begin{array}{l}18 \\
20 \\
20 \\
19 \\
20\end{array}$ & $\begin{array}{c}0.0200 \\
.263 \\
.420 \\
.252 \\
.914\end{array}$ \\
\hline $\begin{array}{l}06-02-78 \\
07-05-78 \\
07-31-78 \\
11-07-78 \\
01-02-79\end{array}$ & $\begin{array}{l}234 \\
300 \\
317 \\
189 \\
198\end{array}$ & $\begin{array}{l}234 \\
300 \\
317 \\
185 \\
197\end{array}$ & $\begin{array}{r}1.4 \\
1.4 \\
1.5 \\
1.4 \\
. .\end{array}$ & $\begin{array}{l}110 \\
111 \\
111 \\
110 \\
110\end{array}$ & $\begin{array}{r}1.5 \\
1.9 \\
2.0 \\
1.2 \\
--\end{array}$ & $\begin{array}{l}-- \\
-- \\
-- \\
--\end{array}$ & $\begin{array}{r}13.5 \\
12.0 \\
12.0 \\
12.0 \\
8.5\end{array}$ & $\begin{array}{l}9 \\
7 \\
9 \\
3 \\
9\end{array}$ & $\begin{array}{l}2.11 \\
2.10 \\
2.85 \\
.555 \\
1.77\end{array}$ & $\begin{array}{l}19 \\
17 \\
18 \\
13 \\
17\end{array}$ & $\begin{array}{l}.242 \\
1.03 \\
.462 \\
.168 \\
.0861\end{array}$ \\
\hline $\begin{array}{l}03-01-79 \\
05-02-79 \\
12-03-79 \\
12-26-79 \\
01-02-80\end{array}$ & $\begin{array}{l}447 \\
261 \\
193 \\
374 \\
340\end{array}$ & $\begin{array}{l}459 \\
261 \\
193 \\
372 \\
338\end{array}$ & $\begin{array}{r}1.5 \\
-5 \\
1.5 \\
-. \\
1.5\end{array}$ & $\begin{array}{l}113 \\
110 \\
109 \\
110 \\
111\end{array}$ & $\begin{array}{r}2.9 \\
-- \\
1.7 \\
-- \\
2.0\end{array}$ & $\begin{array}{l}-- \\
-- \\
--\end{array}$ & $\begin{array}{r}8.0 \\
12.0 \\
11.0 \\
7.5 \\
9.0\end{array}$ & $\begin{array}{r}127 \\
16 \\
10 \\
69 \\
37\end{array}$ & $\begin{array}{c}58.3 \\
4.18 \\
1.93 \\
25.7 \\
12.5\end{array}$ & $\begin{array}{l}16 \\
17 \\
23 \\
23 \\
22\end{array}$ & $\begin{array}{c}2.31 \\
.336 \\
.536 \\
18.1 \\
14.8\end{array}$ \\
\hline $\begin{array}{l}02-01-80 \\
04-01-80 \\
05-01-80 \\
06-03-80\end{array}$ & $\begin{array}{l}368 \\
282 \\
242 \\
240\end{array}$ & $\begin{array}{l}368 \\
281 \\
242 \\
240\end{array}$ & $\begin{array}{l}1.4 \\
1.4 \\
1.4 \\
1.4\end{array}$ & $\begin{array}{l}113 \\
110 \\
108 \\
111\end{array}$ & $\begin{array}{l}2.3 \\
1.8 \\
1.6 \\
1.5\end{array}$ & $\begin{array}{l}-- \\
-- \\
--\end{array}$ & $\begin{array}{r}8.5 \\
10.0 \\
12.5 \\
13.0\end{array}$ & $\begin{array}{r}41 \\
20 \\
12 \\
9\end{array}$ & $\begin{array}{c}15.1 \\
5.62 \\
2.90 \\
2.16\end{array}$ & $\begin{array}{l}18 \\
23 \\
17 \\
17\end{array}$ & $\begin{array}{l}6.10 \\
.515 \\
.210 \\
.284\end{array}$ \\
\hline
\end{tabular}

22. Clearwater River at Spalding, Idaho ${ }^{6,12}$

\begin{tabular}{|c|c|c|c|c|c|c|c|c|c|c|c|}
\hline $\begin{array}{l}05-10-72 \\
06-01-72 \\
06-15-72 \\
04-30-73 \\
05-16-73\end{array}$ & $\begin{array}{r}1,590 \\
2,740 \\
1,510 \\
312 \\
736\end{array}$ & $\begin{array}{r}1,590 \\
2,740 \\
1,510 \\
312 \\
827\end{array}$ & $\begin{array}{l}2.3 \\
3.4 \\
2.2 \\
.70 \\
1.3\end{array}$ & $\begin{array}{l}140 \\
146 \\
140 \\
125 \\
133\end{array}$ & $\begin{array}{l}4.9 \\
5.5 \\
4.8 \\
3.5 \\
4.2\end{array}$ & $\begin{array}{c}0.00037 \\
.00056 \\
.00035 \\
.000098 \\
.000195\end{array}$ & $\begin{array}{l}10.0 \\
10.0 \\
11.0 \\
-- \\
13.5\end{array}$ & $\begin{array}{r}78 \\
209 \\
46 \\
26 \\
55\end{array}$ & $\begin{array}{c}124 \\
573 \\
69.5 \\
8.11 \\
45.5\end{array}$ & $\begin{array}{l}\geq 20 \\
\geq 20 \\
\geq 20 \\
\geq 20 \\
\geq 20\end{array}$ & $\begin{array}{l}2.42 \\
8.66 \\
2.88 \\
1.10 \\
2.65\end{array}$ \\
\hline $\begin{array}{l}06-05-73 \\
06-19-73 \\
04-10-74 \\
05-08-74 \\
05-15-74\end{array}$ & $\begin{array}{r}388 \\
496 \\
1,200 \\
1,810 \\
793\end{array}$ & $\begin{array}{r}374 \\
496 \\
1,230 \\
1,860 \\
810\end{array}$ & $\begin{array}{l}.82 \\
1.0 \\
1.9 \\
2.5 \\
1.4\end{array}$ & $\begin{array}{l}128 \\
130 \\
139 \\
143 \\
134\end{array}$ & $\begin{array}{l}3.6 \\
3.8 \\
4.6 \\
5.1 \\
4.2\end{array}$ & $\begin{array}{l}.000117 \\
.000142 \\
.00029 \\
.00040 \\
.00021\end{array}$ & $\begin{array}{l}-- \\
-- \\
7.0 \\
7.0 \\
7.0\end{array}$ & $\begin{array}{r}2 \\
2 \\
23 \\
55 \\
33\end{array}$ & $\begin{aligned} & .748 \\
& .992 \\
& 28.3 \\
& 102 \\
& 26.7\end{aligned}$ & $\begin{array}{l}\geq 20 \\
\geq 20 \\
\geq 20 \\
\geq 20 \\
\geq 20\end{array}$ & $\begin{array}{l}.0109 \\
.191 \\
.578 \\
5.51 \\
.391\end{array}$ \\
\hline $\begin{array}{l}04-16-75 \\
05-13-75 \\
05-21-75 \\
06-10-75 \\
06-12-75\end{array}$ & $\begin{array}{r}481 \\
1,290 \\
1,170 \\
1,670 \\
1,730\end{array}$ & $\begin{array}{r}481 \\
1,240 \\
1,170 \\
1,670 \\
1,730\end{array}$ & $\begin{array}{l}1.0 \\
2.0 \\
1.8 \\
2.4 \\
2.4\end{array}$ & $\begin{array}{l}130 \\
140 \\
139 \\
141 \\
142\end{array}$ & $\begin{array}{l}3.8 \\
4.7 \\
4.6 \\
5.0 \\
5.0\end{array}$ & $\begin{array}{l}.000139 \\
.000307 \\
.000283 \\
.000379 \\
.000388\end{array}$ & $\begin{array}{r}8.5 \\
10.5 \\
9.5 \\
11.0 \\
12.0\end{array}$ & $\begin{array}{l}29 \\
46 \\
25 \\
42 \\
41\end{array}$ & $\begin{array}{l}13.9 \\
57.0 \\
29.3 \\
70.1 \\
70.9\end{array}$ & $\begin{array}{l}\geq 20 \\
\geq 20 \\
\geq 20 \\
\geq 20 \\
>20\end{array}$ & $\begin{array}{l}.229 \\
1.62 \\
1.10 \\
4.53 \\
3.28\end{array}$ \\
\hline
\end{tabular}


Table 1.--Hydraulic and sediment-transport data--Continued

\begin{tabular}{|c|c|c|c|c|c|c|c|c|c|c|c|}
\hline Date & $\begin{array}{c}\text { Water } \\
\text { discharge } \\
\text { (bedload })^{1} \\
\left(\mathrm{~m}^{3} / \mathrm{s}\right)\end{array}$ & $\begin{array}{c}\text { Water } \\
\text { discharge } \\
\text { (suspended } \\
\text { load) } \\
\left(\mathrm{m}^{3} / \mathrm{s}\right)\end{array}$ & $\begin{array}{l}\text { Mean } \\
\text { flow } \\
\text { velocity } \\
(\mathrm{m} / \mathrm{s})\end{array}$ & $\begin{array}{l}\text { Water- } \\
\text { surface } \\
\text { width } \\
\text { (m) }\end{array}$ & $\begin{array}{l}\text { Mean } \\
\text { flow } \\
\text { depth } \\
\text { (m) }\end{array}$ & $\begin{array}{l}\text { Water- } \\
\text { surface } \\
\text { slope } \\
(\mathrm{m} / \mathrm{m})\end{array}$ & $\begin{array}{l}\text { Water } \\
\text { temp. } \\
\left({ }^{\circ} \mathrm{C}\right)\end{array}$ & $\begin{array}{c}\text { Suspended } \\
\text { sediment } \\
\text { concern- } \\
\text { tration } \\
\left(m_{g} / L\right)\end{array}$ & $\begin{array}{c}\text { Suspended } \\
\text { load } \\
(\mathrm{kg} / \mathrm{s})\end{array}$ & $\begin{array}{l}\text { Number of } \\
\text { sampling } \\
\text { points } \\
\text { for } \\
\text { bedload }\end{array}$ & $\begin{array}{r}\text { Bedload } \\
(\mathrm{kg} / \mathrm{s})\end{array}$ \\
\hline
\end{tabular}

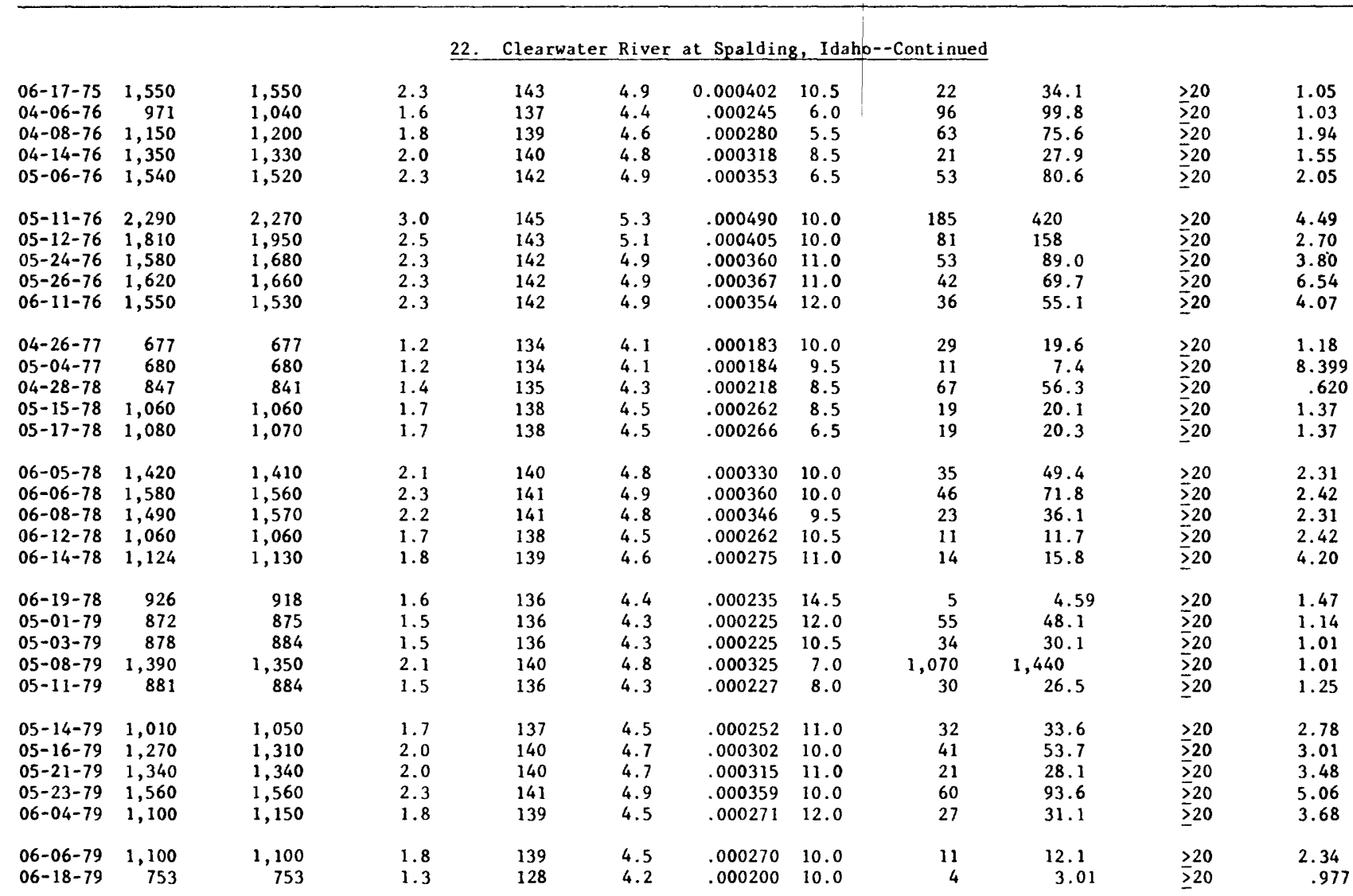

23. North Fork of Big Lost River at Wild Horse (near Chilly), Idaho ${ }^{5,13}$

\begin{tabular}{|c|c|c|c|c|c|c|c|c|c|c|c|}
\hline $\begin{array}{l}04-27-81 \\
04-30-81 \\
04-30-81 \\
05-07-81 \\
05-15-81\end{array}$ & $\begin{array}{l}4.93 \\
6.85 \\
6.85 \\
5.47 \\
6.77\end{array}$ & $\begin{array}{l}4.93 \\
6.85 \\
6.85 \\
5.47 \\
6.77\end{array}$ & $\begin{array}{r}0.77 \\
.88 \\
.88 \\
.93 \\
.88\end{array}$ & $\begin{array}{r}16.0 \\
16.0 \\
16.0 \\
7.6 \\
15.0\end{array}$ & $\begin{array}{r}0.41 \\
.49 \\
.49 \\
.77 \\
.50\end{array}$ & $\begin{array}{l}- \\
-- \\
-- \\
--\end{array}$ & $\begin{array}{l}-- \\
-- \\
-- \\
--\end{array}$ & $\begin{array}{l}19 \\
49 \\
49 \\
31 \\
22\end{array}$ & $\begin{array}{c}0.0937 \\
.336 \\
.336 \\
.170 \\
.149\end{array}$ & $\begin{array}{l}15-20 \\
15-20 \\
15-20 \\
15-20 \\
15-20\end{array}$ & $\begin{array}{c}0.0315 \\
.297 \\
.0609 \\
.116 \\
.161\end{array}$ \\
\hline $\begin{array}{l}05-27-81 \\
05-28-81 \\
05-30-81 \\
06-01-81 \\
06-03-81\end{array}$ & $\begin{array}{l}14.3 \\
15.2 \\
17.6 \\
16.9 \\
13.5\end{array}$ & $\begin{array}{l}14.3 \\
15.2 \\
17.6 \\
16.9 \\
13.5\end{array}$ & $\begin{array}{l}1.7 \\
1.7 \\
1.8 \\
1.8 \\
1.6\end{array}$ & $\begin{array}{l}7.6 \\
7.6 \\
7.6 \\
7.6 \\
7.6\end{array}$ & $\begin{array}{l}1.1 \\
1.2 \\
1.2 \\
1.2 \\
1.1\end{array}$ & $\begin{array}{l}-- \\
-- \\
-- \\
--\end{array}$ & $\begin{array}{l}-- \\
-- \\
-- \\
--\end{array}$ & $\begin{array}{l}137 \\
171 \\
244 \\
204 \\
121\end{array}$ & $\begin{array}{l}1.96 \\
2.60 \\
4.29 \\
3.45 \\
1.63\end{array}$ & $\begin{array}{l}15-20 \\
15-20 \\
15-20 \\
15-20 \\
15-20\end{array}$ & $\begin{aligned} .636 \\
.477 \\
13.1 \\
.414 \\
.208\end{aligned}$ \\
\hline $\begin{array}{l}06-05-81 \\
06-09-81 \\
06-10-81 \\
06-10-81 \\
06-11-81\end{array}$ & $\begin{array}{l}12.8 \\
22.4 \\
16.1 \\
16.1 \\
13.2\end{array}$ & $\begin{array}{l}12.8 \\
22.4 \\
16.1 \\
16.1 \\
13.2\end{array}$ & $\begin{array}{l}1.5 \\
2.0 \\
1.8 \\
1.8 \\
1.6\end{array}$ & $\begin{array}{l}7.6 \\
7.6 \\
7.6 \\
7.6 \\
7.6\end{array}$ & $\begin{array}{l}1.1 \\
1.4 \\
1.2 \\
1.2 \\
1.1\end{array}$ & $\begin{array}{l}-- \\
-- \\
-- \\
--\end{array}$ & $\begin{array}{l}-. \\
-- \\
4.0 \\
4.0 \\
9.0\end{array}$ & $\begin{array}{r}82 \\
236 \\
204 \\
196 \\
126\end{array}$ & $\begin{array}{l}1.05 \\
5.29 \\
3.28 \\
3.16 \\
1.66\end{array}$ & $\begin{array}{l}15-20 \\
15-20 \\
15-20 \\
15-20 \\
15-20\end{array}$ & $\begin{array}{l}.283 \\
.517 \\
2.51 \\
3.32 \\
.413\end{array}$ \\
\hline
\end{tabular}


Table 1.--Hydraulic and sediment-transport data--Cont inued

\begin{tabular}{|c|c|c|c|c|c|c|c|c|c|c|c|}
\hline Date & $\begin{array}{c}\text { Water } \\
\text { discharge } \\
\text { (bedload) } \\
\left(\mathrm{m}^{3} / \mathrm{s}\right)\end{array}$ & $\begin{array}{c}\text { Water } \\
\text { discharge } \\
\text { (suspended } \\
\text { load })^{2} \\
\left(\mathrm{~m}^{3} / \mathrm{s}\right)\end{array}$ & $\begin{array}{l}\text { Mean } \\
\text { flow } \\
\text { velocity } \\
(\mathrm{m} / \mathrm{s})\end{array}$ & $\begin{array}{l}\text { Water- } \\
\text { surface } \\
\text { width } \\
(\mathrm{m})\end{array}$ & $\begin{array}{l}\text { Mean } \\
\text { flow } \\
\text { depth } \\
\text { (m) }\end{array}$ & $\begin{array}{l}\text { Water- } \\
\text { surface } \\
\text { slope } \\
(\mathrm{m} / \mathrm{m})\end{array}$ & $\begin{array}{l}\text { Water } \\
\text { temp. } \\
\left({ }^{\circ} \mathrm{C}\right)\end{array}$ & $\begin{array}{c}\text { Suspended } \\
\text { sediment } \\
\text { concern- } \\
\text { tration } \\
(\mathrm{mg} / \mathrm{L})\end{array}$ & $\begin{array}{l}\text { Suspended } \\
\text { load } \\
\left(\mathrm{kg}_{\mathbf{g}} / \mathrm{s}\right)\end{array}$ & $\begin{array}{l}\text { Number of } \\
\text { sampling } \\
\text { points } \\
\text { for } \\
\text { bedload }\end{array}$ & $\begin{array}{r}\text { Bedload } \\
(\mathrm{kg} / \mathrm{s})\end{array}$ \\
\hline
\end{tabular}

23. North Fork of Big Lost River at Wild Horse (near Chilly), Idaho $5,13--$ Continued

\begin{tabular}{|c|c|c|c|c|c|c|c|c|c|c|c|}
\hline $06-16-81$ & 7.93 & 7.93 & 1.2 & 7.6 & 0.91 & -- & -- & 36 & 0.285 & $15-20$ & 0.116 \\
\hline $06-17-81$ & 11.2 & 11.2 & 1.4 & 7.6 & 1.0 & -- & 3.0 & 33 & .370 & $15-20$ & .0714 \\
\hline $06-18-81$ & 8.61 & 8.61 & 1.2 & 7.6 & .93 & -- & 4.0 & 24 & .207 & $15-20$ & .0273 \\
\hline $06-23-81$ & 10.2 & 10.2 & 1.3 & 7.6 & .99 & -- & 8.0 & 46 & .469 & $15-20$ & .170 \\
\hline $06-25-81$ & 9.63 & 9.63 & 1.3 & 7.6 & .96 & - & - & 27 & .260 & $15-20$ & .120 \\
\hline $07-01-81$ & 8.61 & 8.61 & 1.2 & 7.6 & .99 & $-\infty$ & 10.0 & 18 & .155 & $15-20$ & .117 \\
\hline $07-09-81$ & 5.18 & 5.18 & .90 & 7.6 & .76 & -- & - & 9 & .0466 & $15-20$ & .00210 \\
\hline $07-20-81$ & 3.29 & 3.29 & .61 & 14.5 & .37 & $\ldots$ & -- & 18 & .0592 & $15-20$ & .00105 \\
\hline
\end{tabular}

24. Big Lost River at Howell Ranch (near Chilly), Idaho 5,13

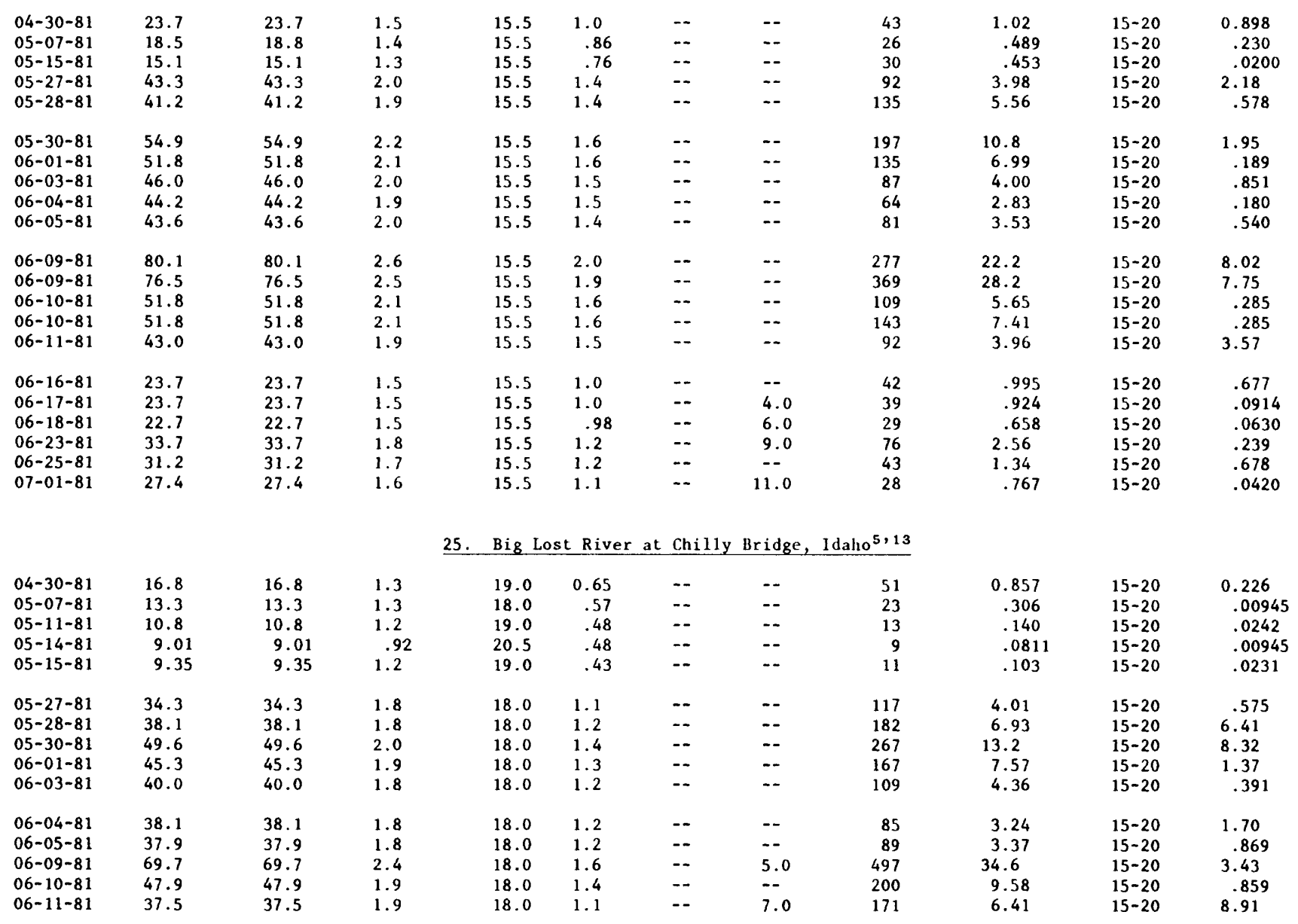


Tabli 1.--Hydraulic and sediment-transport data--Conlinued

\begin{tabular}{|c|c|c|c|c|c|c|c|c|c|c|c|}
\hline Date & $\begin{array}{c}\text { Water } \\
\text { discharge } \\
\text { (bedload })^{1} \\
\left(\mathrm{~m}^{3} / \mathrm{s}\right)\end{array}$ & $\begin{array}{c}\text { Water } \\
\text { discharge } \\
\text { (suspended } \\
\text { load })^{2} \\
\left(\mathrm{~m}^{3} / \mathrm{s}\right)\end{array}$ & $\begin{array}{l}\text { Mean } \\
\text { flow } \\
\text { velocity } \\
(\mathrm{m} / \mathrm{s})\end{array}$ & $\begin{array}{l}\text { Water- } \\
\text { surface } \\
\text { width } \\
\text { (m) }\end{array}$ & $\begin{array}{l}\text { Mean } \\
\text { flow } \\
\text { depth } \\
(\mathrm{m})\end{array}$ & $\begin{array}{l}\text { Water- } \\
\text { surface } \\
\text { slope } \\
(\mathrm{m} / \mathrm{m})\end{array}$ & $\begin{array}{l}\text { Water } \\
\text { temp. } \\
\left({ }^{\circ} \mathrm{C}\right)\end{array}$ & $\begin{array}{c}\text { Suspended } \\
\text { sediment } \\
\text { concern- } \\
\text { tration } \\
(\mathrm{mg} / \mathrm{L})\end{array}$ & $\begin{array}{c}\text { Suspended } \\
\text { load } \\
(\mathrm{kg} / \mathrm{s})\end{array}$ & $\begin{array}{l}\text { Number of } \\
\text { sampling } \\
\text { points } \\
\text { for } \\
\text { bedload }\end{array}$ & $\begin{array}{r}\text { Bedload } \\
(\mathrm{kg} / \mathrm{s})\end{array}$ \\
\hline
\end{tabular}

\section{Big Lost River at Chilly Bridge, Idaho 5,13 --Continued}

\begin{tabular}{|c|c|c|c|c|c|c|c|c|c|c|c|}
\hline $\begin{array}{l}06-11-81 \\
06-16-81 \\
06-16-81 \\
06-17-81 \\
06-18-81\end{array}$ & $\begin{array}{l}37.5 \\
16.7 \\
16.7 \\
16.4 \\
15.2\end{array}$ & $\begin{array}{l}37.5 \\
16.7 \\
16.7 \\
16.4 \\
15.2\end{array}$ & $\begin{array}{l}1.9 \\
1.4 \\
1.4 \\
1.4 \\
1.4\end{array}$ & $\begin{array}{l}18.0 \\
18.0 \\
18.0 \\
18.0 \\
18.0\end{array}$ & $\begin{array}{r}1.1 \\
.67 \\
.67 \\
.66 \\
.62\end{array}$ & $\begin{array}{l}-- \\
-- \\
-- \\
-- \\
--\end{array}$ & $\begin{array}{l}7.0 \\
-- \\
-- \\
6.0 \\
8.0\end{array}$ & $\begin{array}{r}171 \\
26 \\
26 \\
65 \\
19\end{array}$ & $\begin{array}{c}6.41 \\
.434 \\
.434 \\
1.07 \\
.289\end{array}$ & $\begin{array}{l}15-20 \\
15-20 \\
15-20 \\
15-20 \\
15-20\end{array}$ & $\begin{array}{l}7.92 \\
.0935 \\
.0998 \\
.148 \\
.137\end{array}$ \\
\hline $\begin{array}{l}06-23-81 \\
06-25-81 \\
07-01-81 \\
07-01-81 \\
07-09-81\end{array}$ & $\begin{array}{c}13.8 \\
21.8 \\
9.72 \\
9.72 \\
6.80\end{array}$ & $\begin{array}{l}13.8 \\
21.8 \\
9.72 \\
9.72 \\
6.80\end{array}$ & $\begin{array}{l}1.3 \\
1.5 \\
1.2 \\
1.2 \\
1.0\end{array}$ & $\begin{array}{l}18.0 \\
18.0 \\
18.0 \\
18.0 \\
17.0\end{array}$ & $\begin{array}{l}.59 \\
.80 \\
.45 \\
.45 \\
.40\end{array}$ & $\begin{array}{l}-- \\
-- \\
-- \\
--\end{array}$ & $\begin{array}{c}10.0 \\
-- \\
12.0 \\
-- \\
--\end{array}$ & $\begin{array}{r}101 \\
50 \\
22 \\
22 \\
11\end{array}$ & $\begin{array}{l}1.39 \\
1.09 \\
.214 \\
.214 \\
.0748\end{array}$ & $\begin{array}{l}15-20 \\
15-20 \\
15-20 \\
15-20 \\
15-20\end{array}$ & $\begin{array}{l}1.27 \\
.637 \\
.307 \\
.204 \\
.00525\end{array}$ \\
\hline
\end{tabular}

26. Big Lost River below Chilly Sinks, Idaho 5,13

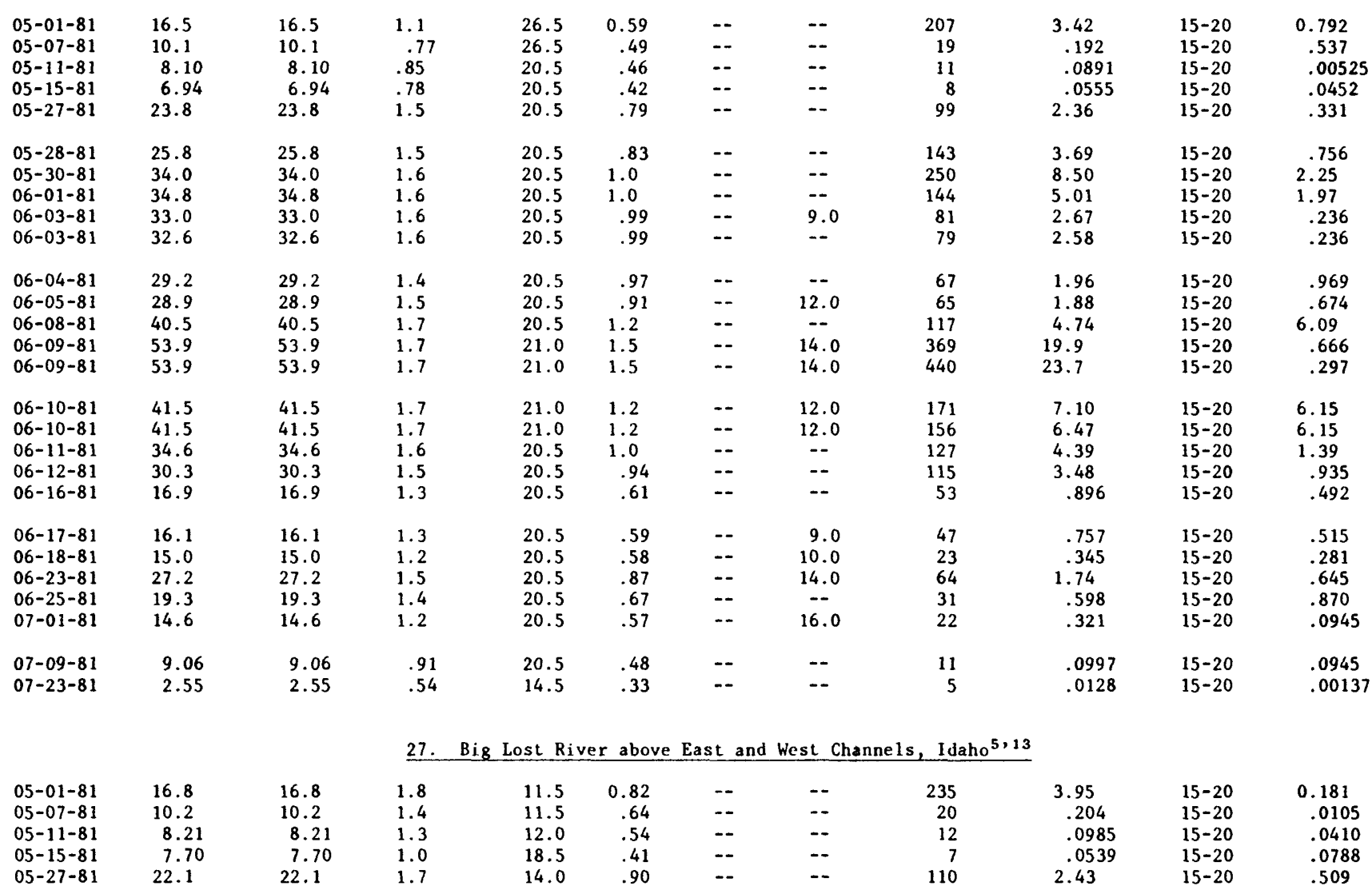


Table 1.--Hydraulic and sediment-transport data--Continued

\begin{tabular}{|c|c|c|c|c|c|c|c|c|c|c|c|}
\hline Date & $\begin{array}{c}\text { Water } \\
\text { discharge } \\
\text { (bedload) } \\
\left(\mathrm{m}^{3} / \mathrm{s}\right)\end{array}$ & $\begin{array}{c}\text { Water } \\
\text { discharge } \\
\text { (suspended } \\
\text { load) } \\
\left(\mathrm{m}^{3} / \mathrm{s}\right)\end{array}$ & $\begin{array}{l}\text { Mean } \\
\text { flow } \\
\text { velocity } \\
(\mathrm{m} / \mathrm{s})\end{array}$ & $\begin{array}{l}\text { Water } \\
\text { surface } \\
\text { width } \\
\text { (m) }\end{array}$ & $\begin{array}{l}\text { Mean } \\
\text { flow } \\
\text { depth } \\
\text { (m) }\end{array}$ & $\begin{array}{l}\text { Water } \\
\text { surface } \\
\text { slope } \\
(\mathrm{m} / \mathrm{m})\end{array}$ & $\begin{array}{l}\text { Water } \\
\text { temp. } \\
\left({ }^{\circ} \mathrm{C}\right)\end{array}$ & $\begin{array}{c}\text { Suspended } \\
\text { sediment } \\
\text { concern- } \\
\text { tration } \\
(\mathrm{mg} / \mathrm{L})\end{array}$ & $\begin{array}{c}\text { Suspended } \\
\text { load } \\
(\mathrm{kg} / \mathrm{s})\end{array}$ & $\begin{array}{l}\text { Number of } \\
\text { sampling } \\
\text { points } \\
\text { for } \\
\text { bedload }\end{array}$ & $\begin{array}{r}\text { Bedload } \\
\left(\mathrm{kg}_{\mathrm{g}} / \mathrm{s}\right)\end{array}$ \\
\hline
\end{tabular}

27. Big Lost River above East and West Channels, Idaho 5,13 --Continued

\begin{tabular}{|c|c|c|c|c|c|c|c|c|c|c|c|}
\hline $\begin{array}{l}05-28-81 \\
05-30-81 \\
06-01-81 \\
06-04-81 \\
06-05-81\end{array}$ & $\begin{array}{l}24.9 \\
32.0 \\
27.8 \\
30.3 \\
27.4\end{array}$ & $\begin{array}{l}24.9 \\
32.0 \\
27.8 \\
30.3 \\
27.4\end{array}$ & $\begin{array}{l}1.8 \\
2.0 \\
1.9 \\
1.8 \\
1.9\end{array}$ & $\begin{array}{l}14.0 \\
14.0 \\
14.0 \\
14.0 \\
13.5\end{array}$ & $\begin{array}{l}0.98 \\
1.1 \\
1.0 \\
1.2 \\
1.1\end{array}$ & $\begin{array}{l}-- \\
-- \\
-- \\
--\end{array}$ & $\begin{array}{l}-- \\
-- \\
-- \\
--\end{array}$ & $\begin{array}{l}167 \\
275 \\
196 \\
106 \\
107\end{array}$ & $\begin{array}{l}4.16 \\
8.80 \\
5.45 \\
3.21 \\
2.93\end{array}$ & $\begin{array}{l}15-20 \\
15-20 \\
15-20 \\
15-20 \\
15-20\end{array}$ & $\begin{array}{c}0.416 \\
5.80 \\
.851 \\
1.67 \\
1.17\end{array}$ \\
\hline $\begin{array}{l}06-10-81 \\
06-10-81 \\
06-11-81\end{array}$ & $\begin{array}{l}38.2 \\
38.2 \\
34.8\end{array}$ & $\begin{array}{l}38.2 \\
38.2 \\
34.8\end{array}$ & $\begin{array}{l}2.2 \\
2.2 \\
2.1\end{array}$ & $\begin{array}{l}14.0 \\
14.0 \\
14.0\end{array}$ & $\begin{array}{l}1.3 \\
1.3 \\
1.2\end{array}$ & $\begin{array}{l}-- \\
--\end{array}$ & $\begin{array}{l}12.0 \\
12.0 \\
11.0\end{array}$ & $\begin{array}{l}170 \\
173 \\
266\end{array}$ & $\begin{array}{l}6.49 \\
6.61 \\
9.26\end{array}$ & $\begin{array}{l}15-20 \\
15-20 \\
15-20\end{array}$ & $\begin{array}{l}5.37 \\
6.41 \\
.278\end{array}$ \\
\hline $\begin{array}{l}06-11-81 \\
06-16-81 \\
06-17-81 \\
06-18-81 \\
06-23-81\end{array}$ & $\begin{array}{l}34.8 \\
16.1 \\
15.0 \\
13.9 \\
26.1\end{array}$ & $\begin{array}{l}34.8 \\
16.1 \\
15.0 \\
13.9 \\
26.1\end{array}$ & $\begin{array}{l}2.1 \\
1.5 \\
1.5 \\
1.4 \\
1.8\end{array}$ & $\begin{array}{l}14.0 \\
14.0 \\
14.0 \\
14.0 \\
14.0\end{array}$ & $\begin{array}{r}1.2 \\
.75 \\
.72 \\
.69 \\
1.0\end{array}$ & $\begin{array}{l}-- \\
-- \\
-- \\
--\end{array}$ & $\begin{array}{c}11.0 \\
-- \\
9.0 \\
10.0 \\
14.0\end{array}$ & $\begin{array}{r}266 \\
58 \\
83 \\
49 \\
58\end{array}$ & $\begin{array}{l}9.26 \\
.934 \\
1.25 \\
.681 \\
1.51\end{array}$ & $\begin{array}{l}15-20 \\
15-20 \\
15-20 \\
15-20 \\
15-20\end{array}$ & $\begin{array}{l}.0483 \\
.108 \\
.925 \\
.105 \\
.0473\end{array}$ \\
\hline $\begin{array}{l}06-25-81 \\
07-01-81 \\
07-09-81 \\
07-22-81\end{array}$ & $\begin{array}{c}20.1 \\
14.4 \\
9.35 \\
2.89\end{array}$ & $\begin{array}{c}20.1 \\
14.4 \\
9.35 \\
2.89\end{array}$ & $\begin{array}{l}1.7 \\
1.5 \\
1.4 \\
.93\end{array}$ & $\begin{array}{r}14.0 \\
14.0 \\
9.8 \\
9.8\end{array}$ & $\begin{array}{l}.85 \\
.70 \\
.67 \\
.32\end{array}$ & $\begin{array}{l}-- \\
-- \\
--\end{array}$ & $\begin{array}{c}-- \\
15.0 \\
-- \\
--\end{array}$ & $\begin{array}{l}41 \\
32 \\
11 \\
37\end{array}$ & $\begin{array}{l}.824 \\
.461 \\
.103 \\
.107\end{array}$ & $\begin{array}{l}15-20 \\
15-20 \\
15-20 \\
15-20\end{array}$ & $\begin{array}{l}.0168 \\
.389 \\
.0536 \\
.00315\end{array}$ \\
\hline
\end{tabular}

28. North Fork of Lick Creek near Yellow Pine, Idaho ${ }^{13}$

\begin{tabular}{|c|c|c|c|c|c|c|c|c|c|c|c|}
\hline $\begin{array}{l}05-08-85 \\
05-15-85 \\
05-22-85 \\
05-29-85 \\
06-06-85\end{array}$ & $\begin{array}{l}2.12 \\
1.49 \\
2.77 \\
2.43 \\
2.59\end{array}$ & $\begin{array}{l}2.12 \\
1.49 \\
2.77 \\
2.43 \\
2.59\end{array}$ & $\begin{array}{r}0.63 \\
.56 \\
.80 \\
.75 \\
.80\end{array}$ & $\begin{array}{l}7.9 \\
7.6 \\
8.8 \\
8.5 \\
8.5\end{array}$ & $\begin{array}{r}0.42 \\
.35 \\
.39 \\
.37 \\
.39\end{array}$ & $\begin{array}{r}0.00500 \\
.00890 \\
.00500 \\
.00833 \\
.00810\end{array}$ & $\begin{array}{l}5.0 \\
-- \\
6.0 \\
5.0 \\
5.5\end{array}$ & $\begin{array}{r}4 \\
4 \\
9 \\
5 \\
10\end{array}$ & $\begin{array}{c}0.00848 \\
.00596 \\
.0249 \\
.0122 \\
.0259\end{array}$ & $\begin{array}{l}15-17 \\
15-17 \\
15-17 \\
15-17 \\
15-17\end{array}$ & $\begin{array}{l}0.00555 \\
.000500 \\
.0107 \\
.00405 \\
.00401\end{array}$ \\
\hline $\begin{array}{l}06-12-85 \\
06-18-85 \\
06-26-85 \\
07-10-85 \\
07-18-85\end{array}$ & $\begin{array}{r}1.78 \\
1.28 \\
.66 \\
.43 \\
.35\end{array}$ & $\begin{array}{r}1.78 \\
1.28 \\
.66 \\
.43 \\
.35\end{array}$ & $\begin{array}{l}.63 \\
.54 \\
.37 \\
.26 \\
.23\end{array}$ & $\begin{array}{l}8.2 \\
7.3 \\
7.0 \\
6.7 \\
6.4\end{array}$ & $\begin{array}{l}.34 \\
.32 \\
.28 \\
.25 \\
.23\end{array}$ & $\begin{array}{l}.00500 \\
.00578 \\
.00789 \\
.00731 \\
.00364\end{array}$ & $\begin{array}{c}10.0 \\
-- \\
9.5 \\
13.0 \\
10.5\end{array}$ & $\begin{array}{l}3 \\
3 \\
4 \\
2 \\
1\end{array}$ & $\begin{array}{l}.00534 \\
.00384 \\
.00264 \\
.000860 \\
.000350\end{array}$ & $\begin{array}{l}15-17 \\
15-17 \\
15-17 \\
15-17 \\
15-17\end{array}$ & $\begin{array}{l}.00261 \\
.000688 \\
.0000307 \\
.0000293 \\
.000000\end{array}$ \\
\hline $\begin{array}{l}07-23-85 \\
07-30-85 \\
08-07-85 \\
08-21-85 \\
04-23-86\end{array}$ & $\begin{array}{r}.35 \\
.27 \\
.22 \\
.10 \\
3.03\end{array}$ & $\begin{array}{r}.35 \\
.27 \\
.22 \\
.10 \\
3.03\end{array}$ & $\begin{array}{l}.23 \\
.22 \\
.18 \\
.12 \\
.90\end{array}$ & $\begin{array}{l}6.4 \\
6.1 \\
6.1 \\
5.5 \\
8.5\end{array}$ & $\begin{array}{l}.23 \\
.21 \\
.20 \\
.16 \\
.40\end{array}$ & $\begin{array}{l}.00714 \\
.00571 \\
.00600 \\
.00209 \\
.00552\end{array}$ & $\begin{array}{r}15.0 \\
13.0 \\
10.0 \\
9.0 \\
3.0\end{array}$ & $\begin{array}{r}4 \\
4 \\
2 \\
5 \\
12\end{array}$ & $\begin{array}{l}.00140 \\
.00108 \\
.000440 \\
.000500 \\
.0364\end{array}$ & $\begin{array}{l}15-17 \\
15-17 \\
15-17 \\
15-17 \\
15-17\end{array}$ & $\begin{array}{l}.0000140 \\
.0000133 \\
.000200 \\
.0000240 \\
.00926\end{array}$ \\
\hline $\begin{array}{l}04-25-86 \\
04-28-86 \\
05-21-86 \\
05-22-86\end{array}$ & $\begin{array}{l}2.29 \\
1.47 \\
2.97 \\
2.59\end{array}$ & $\begin{array}{l}2.29 \\
1.47 \\
2.97 \\
2.59\end{array}$ & $\begin{array}{l}.78 \\
.52 \\
.81 \\
.78\end{array}$ & $\begin{array}{l}7.9 \\
7.6 \\
8.5 \\
8.2\end{array}$ & $\begin{array}{l}.37 \\
.37 \\
.43 \\
.41\end{array}$ & $\begin{array}{l}.00552 \\
.00793 \\
.00621 \\
.00500\end{array}$ & $\begin{array}{l}3.0 \\
5.0 \\
4.0 \\
3.0\end{array}$ & $\begin{array}{r}8 \\
2 \\
18 \\
16\end{array}$ & $\begin{array}{l}.0183 \\
.00294 \\
.0535 \\
.0414\end{array}$ & $\begin{array}{l}15-17 \\
15-17 \\
15-17 \\
15-17\end{array}$ & $\begin{array}{l}.00196 \\
.000517 \\
.0243 \\
.0212\end{array}$ \\
\hline $\begin{array}{l}05-26-86 \\
06-03-86 \\
06-09-86\end{array}$ & $\begin{array}{l}3.14 \\
4.25 \\
3.03\end{array}$ & $\begin{array}{l}3.14 \\
4.25 \\
3.03\end{array}$ & $\begin{array}{l}.85 \\
.95 \\
.89\end{array}$ & $\begin{array}{l}8.5 \\
8.8 \\
8.2\end{array}$ & $\begin{array}{l}.43 \\
.51 \\
.41\end{array}$ & $\begin{array}{l}.00534 \\
.0124 \\
.00586\end{array}$ & $\begin{array}{l}5.5 \\
7.5 \\
7.5\end{array}$ & $\begin{array}{l}13 \\
23 \\
10\end{array}$ & $\begin{array}{l}.0408 \\
.0978 \\
.0303\end{array}$ & $\begin{array}{l}15-17 \\
15-17 \\
15-17\end{array}$ & $\begin{array}{l}.0109 \\
.0292 \\
.00463\end{array}$ \\
\hline
\end{tabular}


Table 1.--Hydraulic and sediment-transport data--Continued

\begin{tabular}{|c|c|c|c|c|c|c|c|c|c|c|c|}
\hline Date & $\begin{array}{c}\text { Water } \\
\text { discharge } \\
\text { (bedload) } \\
\left(\mathrm{m}^{3} / \mathrm{s}\right)\end{array}$ & $\begin{array}{c}\text { Water } \\
\text { discharge } \\
\text { (suspended } \\
1 \text { load) } \\
\left(\mathrm{m}^{3} / \mathrm{s}\right)\end{array}$ & $\begin{array}{l}\text { Mean } \\
\text { flow } \\
\text { velocity } \\
(m / s)\end{array}$ & $\begin{array}{l}\text { Water- } \\
\text { surface } \\
\text { width } \\
\text { (m) }\end{array}$ & $\begin{array}{l}\text { Mean } \\
\text { flow } \\
\text { depth } \\
\text { (m) }\end{array}$ & $\begin{array}{l}\text { Water- } \\
\text { surface } \\
\text { slope } \\
(\mathrm{m} / \mathrm{m})\end{array}$ & $\begin{array}{l}\text { Water } \\
\text { temp. } \\
\left({ }^{\circ} \mathrm{C}\right)\end{array}$ & $\begin{array}{c}\text { Suspended } \\
\text { sediment } \\
\text { concern- } \\
\text { tration } \\
(\mathrm{mg} / \mathrm{L})\end{array}$ & $\begin{array}{c}\text { Suspended } \\
\text { load } \\
(\mathrm{kg} / \mathrm{s})\end{array}$ & $\begin{array}{l}\text { Number of } \\
\text { sampling } \\
\text { points } \\
\text { for } \\
\text { bedload }\end{array}$ & $\begin{array}{r}\text { Bedload } \\
(\mathrm{kg} / \mathrm{s})\end{array}$ \\
\hline
\end{tabular}

29. Buckhorn Creek above Krassel Ranger Station near Cascade, Idaho ${ }^{13}$

\begin{tabular}{|c|c|c|c|c|c|c|c|c|c|c|c|}
\hline $\begin{array}{l}05-07-85 \\
05-16-85 \\
05-21-85 \\
05-30-85 \\
06-06-85\end{array}$ & $\begin{array}{l}4.28 \\
3.51 \\
6.12 \\
4.33 \\
4.87\end{array}$ & $\begin{array}{l}4.28 \\
3.51 \\
6.12 \\
4.33 \\
4.87\end{array}$ & $\begin{array}{l}1.1 \\
.94 \\
1.3 \\
1.1 \\
1.2\end{array}$ & $\begin{array}{r}10.5 \\
9.8 \\
9.8 \\
9.4 \\
9.1\end{array}$ & $\begin{array}{r}0.37 \\
.38 \\
.50 \\
.41 \\
.44\end{array}$ & $\begin{array}{r}0.00797 \\
.00944 \\
.00630 \\
.00714 \\
.00760\end{array}$ & $\begin{array}{l}-- \\
-- \\
6.5 \\
-- \\
--\end{array}$ & $\begin{array}{r}6 \\
2 \\
9 \\
57 \\
4\end{array}$ & $\begin{array}{l}0.0257 \\
.00702 \\
.0551 \\
.247 \\
.0195\end{array}$ & $\begin{array}{l}15-17 \\
15-17 \\
15-17 \\
15-17 \\
15-17\end{array}$ & $\begin{array}{l}0.00348 \\
.000960 \\
.0273 \\
.00591 \\
.00894\end{array}$ \\
\hline $\begin{array}{l}06-11-85 \\
06-17-85 \\
06-25-85 \\
07-09-85 \\
07-17-85\end{array}$ & $\begin{array}{r}4.30 \\
2.83 \\
1.73 \\
.91 \\
.71\end{array}$ & $\begin{array}{r}4.30 \\
2.83 \\
1.73 \\
.91 \\
.71\end{array}$ & $\begin{array}{r}1.1 \\
.92 \\
.55 \\
.33 \\
.29\end{array}$ & $\begin{array}{l}9.1 \\
9.1 \\
9.1 \\
9.1 \\
8.8\end{array}$ & $\begin{array}{l}.44 \\
.34 \\
.34 \\
.30 \\
.28\end{array}$ & $\begin{array}{l}.00960 \\
.00564 \\
.00559 \\
.00660 \\
.00681\end{array}$ & $\begin{array}{r}6.0 \\
10.0 \\
8.0 \\
14.5 \\
15.0\end{array}$ & $\begin{array}{l}3 \\
5 \\
2 \\
2 \\
1\end{array}$ & $\begin{array}{l}.0129 \\
.0142 \\
.00346 \\
.00182 \\
.000710\end{array}$ & $\begin{array}{l}15-17 \\
15-17 \\
15-17 \\
15-17 \\
15-17\end{array}$ & $\begin{array}{l}.00354 \\
.00132 \\
.000980 \\
.000160 \\
.000000\end{array}$ \\
\hline $\begin{array}{l}07-23-85 \\
07-30-85 \\
08-05-85 \\
08-20-85 \\
04-23-86\end{array}$ & $\begin{array}{r}.63 \\
.62 \\
.50 \\
.57 \\
6.71\end{array}$ & $\begin{array}{r}.63 \\
.62 \\
.50 \\
.57 \\
6.71\end{array}$ & $\begin{array}{l}.28 \\
.27 \\
.25 \\
.25 \\
1.3\end{array}$ & $\begin{array}{r}8.5 \\
8.5 \\
8.5 \\
8.8 \\
10.5\end{array}$ & $\begin{array}{l}.26 \\
.26 \\
.24 \\
.26 \\
.49\end{array}$ & $\begin{array}{l}.00574 \\
.00511 \\
.00553 \\
.00553 \\
.00850\end{array}$ & $\begin{array}{r}15.0 \\
14.0 \\
16.0 \\
13.0 \\
4.0\end{array}$ & $\begin{array}{r}2 \\
2 \\
2 \\
2 \\
17\end{array}$ & $\begin{array}{l}.00126 \\
.00124 \\
.00100 \\
.00114 \\
.114\end{array}$ & $\begin{array}{l}15-17 \\
15-17 \\
15-17 \\
15-17 \\
15-17\end{array}$ & $\begin{array}{l}.000466 \\
.000187 \\
.000000 \\
.000000 \\
.0137\end{array}$ \\
\hline $\begin{array}{l}04-24-86 \\
04-29-86 \\
05-21-86 \\
05-22-86 \\
05-26-86\end{array}$ & $\begin{array}{l}5.27 \\
3.09 \\
7.82 \\
5.66 \\
7.93\end{array}$ & $\begin{array}{l}5.27 \\
3.09 \\
7.82 \\
5.66 \\
7.93\end{array}$ & $\begin{array}{l}1.2 \\
.66 \\
1.5 \\
1.2 \\
1.7\end{array}$ & $\begin{array}{r}9.8 \\
10.5 \\
9.8 \\
9.8 \\
9.8\end{array}$ & $\begin{array}{l}.45 \\
.45 \\
.53 \\
.47 \\
.48\end{array}$ & $\begin{array}{l}.00978 \\
.00591 \\
.00760 \\
.00710 \\
.00839\end{array}$ & $\begin{array}{l}3.0 \\
5.0 \\
5.0 \\
3.0 \\
5.0\end{array}$ & $\begin{array}{r}9 \\
4 \\
34 \\
17 \\
20\end{array}$ & $\begin{array}{l}.0474 \\
.0124 \\
.266 \\
.0962 \\
.159\end{array}$ & $\begin{array}{l}15-17 \\
15-17 \\
15-17 \\
15-17 \\
15-17\end{array}$ & $\begin{array}{l}.00704 \\
.00191 \\
.0499 \\
.0281 \\
.00772\end{array}$ \\
\hline $\begin{array}{l}05-29-86 \\
05-30-86 \\
06-09-86\end{array}$ & $\begin{array}{c}23.5 \\
11.9 \\
6.34\end{array}$ & $\begin{array}{c}23.5 \\
11.9 \\
6.34\end{array}$ & $\begin{array}{l}2.9 \\
1.8 \\
1.2\end{array}$ & $\begin{array}{r}11.5 \\
11.5 \\
9.4\end{array}$ & $\begin{array}{l}.70 \\
.60 \\
.52\end{array}$ & $\begin{array}{l}.0200 \\
.00882 \\
.00745\end{array}$ & $\begin{array}{l}5.0 \\
8.0 \\
7.0\end{array}$ & $\begin{array}{r}716 \\
44 \\
18\end{array}$ & $\begin{array}{c}16.8 \\
.524 \\
.114\end{array}$ & $\begin{array}{l}15-17 \\
15-17 \\
15-17\end{array}$ & $\begin{array}{l}.970 \\
.256 \\
.0300\end{array}$ \\
\hline
\end{tabular}

30. South Fork of Salmon River near Cascade, Idaho 13

\begin{tabular}{|c|c|c|c|c|c|c|c|c|c|c|c|}
\hline $\begin{array}{l}04-18-85 \\
04-30-85 \\
05-01-85 \\
05-02-85 \\
05-07-85\end{array}$ & $\begin{array}{l}36.1 \\
22.0 \\
27.0 \\
37.5 \\
37.2\end{array}$ & $\begin{array}{l}36.1 \\
22.0 \\
27.0 \\
37.5 \\
37.2\end{array}$ & $\begin{array}{r}0.84 \\
.62 \\
.69 \\
.83 \\
.82\end{array}$ & $\begin{array}{l}32.5 \\
31.5 \\
31.5 \\
32.5 \\
33.0\end{array}$ & $\begin{array}{l}1.3 \\
1.1 \\
1.2 \\
1.4 \\
1.4\end{array}$ & $\begin{array}{r}0.00100 \\
.000833 \\
.000780 \\
.000722 \\
.000500\end{array}$ & $\begin{array}{l}7.0 \\
7.0 \\
5.5 \\
6.0 \\
7.0\end{array}$ & $\begin{array}{r}19 \\
6 \\
13 \\
14 \\
12\end{array}$ & $\begin{array}{r}0.686 \\
.132 \\
.351 \\
.525 \\
.446\end{array}$ & $\begin{array}{l}20 \\
20 \\
20 \\
20 \\
20\end{array}$ & $\begin{array}{c}0.0881 \\
.00632 \\
.00879 \\
.0582 \\
.128\end{array}$ \\
\hline $\begin{array}{l}05-08-85 \\
05-09-85 \\
05-15-85 \\
05-16-85 \\
05-17-85\end{array}$ & $\begin{array}{l}43.3 \\
41.1 \\
30.2 \\
29.3 \\
34.1\end{array}$ & $\begin{array}{l}43.3 \\
41.1 \\
30.2 \\
29.3 \\
34.1\end{array}$ & $\begin{array}{l}.91 \\
.87 \\
.75 \\
.73 \\
.79\end{array}$ & $\begin{array}{l}33.0 \\
33.0 \\
32.0 \\
32.5 \\
32.5\end{array}$ & $\begin{array}{l}1.4 \\
1.4 \\
1.3 \\
1.3 \\
1.3\end{array}$ & $\begin{array}{l}.000667 \\
.000667 \\
.000500 \\
.000722 \\
.000722\end{array}$ & $\begin{array}{l}6.0 \\
5.5 \\
5.5 \\
5.5 \\
6.0\end{array}$ & $\begin{array}{r}17 \\
14 \\
8 \\
6 \\
9\end{array}$ & $\begin{array}{l}.736 \\
.575 \\
.242 \\
.176 \\
.307\end{array}$ & $\begin{array}{l}20 \\
20 \\
20 \\
20 \\
20\end{array}$ & $\begin{array}{l}.0533 \\
.115 \\
.0620 \\
.00961 \\
.0557\end{array}$ \\
\hline $\begin{array}{l}05-21-85 \\
05-22-85 \\
05-23-85 \\
05-29-85 \\
05-30-85\end{array}$ & $\begin{array}{l}52.8 \\
57.8 \\
63.3 \\
51.7 \\
45.3\end{array}$ & $\begin{array}{l}52.8 \\
57.8 \\
63.3 \\
51.7 \\
45.3\end{array}$ & $\begin{array}{l}1.0 \\
1.1 \\
1.1 \\
1.0 \\
.95\end{array}$ & $\begin{array}{l}33.5 \\
34.0 \\
34.5 \\
33.0 \\
33.0\end{array}$ & $\begin{array}{l}1.6 \\
1.6 \\
1.7 \\
1.5 \\
1.4\end{array}$ & $\begin{array}{l}.00112 \\
.000556 \\
.000556 \\
.000556 \\
.000667\end{array}$ & $\begin{array}{l}8.0 \\
6.0 \\
7.0 \\
7.0 \\
5.0\end{array}$ & $\begin{array}{l}28 \\
38 \\
51 \\
17 \\
13\end{array}$ & $\begin{array}{l}1.48 \\
2.20 \\
3.23 \\
.879 \\
.589\end{array}$ & $\begin{array}{l}20 \\
20 \\
20 \\
20 \\
20\end{array}$ & $\begin{array}{l}.530 \\
1.48 \\
.842 \\
2.25 \\
1.46\end{array}$ \\
\hline $\begin{array}{l}05-31-85 \\
06-05-85 \\
06-06-85 \\
06-07-85 \\
06-11-85\end{array}$ & $\begin{array}{l}40.1 \\
38.4 \\
45.6 \\
46.4 \\
32.7\end{array}$ & $\begin{array}{l}40.1 \\
38.4 \\
45.6 \\
46.4 \\
32.7\end{array}$ & $\begin{array}{l}.89 \\
.86 \\
.94 \\
.95 \\
.79\end{array}$ & $\begin{array}{l}33.0 \\
33.0 \\
33.5 \\
33.5 \\
32.5\end{array}$ & $\begin{array}{l}1.4 \\
1.4 \\
1.4 \\
1.5 \\
1.3\end{array}$ & $\begin{array}{r}.000778 \\
.000722 \\
.000778 \\
.000833 \\
.000833\end{array}$ & $\begin{array}{r}5.0 \\
7.0 \\
8.0 \\
9.0 \\
11.0\end{array}$ & $\begin{array}{r}12 \\
10 \\
12 \\
22 \\
9\end{array}$ & $\begin{array}{c}.481 \\
.384 \\
.547 \\
1.02 \\
.294\end{array}$ & $\begin{array}{l}20 \\
20 \\
20 \\
20 \\
20\end{array}$ & $\begin{array}{l}1.40 \\
.414 \\
.990 \\
.254 \\
.0912\end{array}$ \\
\hline
\end{tabular}


T.ublo 1.--Hydraulic and sediment-transport data--Continued

\begin{tabular}{|c|c|c|c|c|c|c|c|c|c|c|c|}
\hline Date & $\begin{array}{c}\text { Water } \\
\text { discharge } \\
\text { (bedload) } \\
\left(\mathrm{m}^{3} / \mathrm{s}\right)\end{array}$ & $\begin{array}{c}\text { Water } \\
\text { discharge } \\
\text { (suspended } \\
\text { load })^{2} \\
\left(\mathrm{~m}^{3} / \mathrm{s}\right)\end{array}$ & $\begin{array}{l}\text { Mean } \\
\text { flow } \\
\text { velocity } \\
(\mathrm{m} / \mathrm{s})\end{array}$ & $\begin{array}{l}\text { Water- } \\
\text { surface } \\
\text { width } \\
\text { (m) }\end{array}$ & $\begin{array}{l}\text { Mean } \\
\text { flow } \\
\text { depth } \\
(m)\end{array}$ & $\begin{array}{l}\text { Water- } \\
\text { surface } \\
\text { slope } \\
(\mathrm{m} / \mathrm{m})\end{array}$ & $\begin{array}{l}\text { Water } \\
\text { temp. } \\
\left({ }^{\circ} \mathrm{C}\right)\end{array}$ & $\begin{array}{c}\text { Suspended } \\
\text { sediment } \\
\text { concern- } \\
\text { tration } \\
(\mathrm{mg} / \mathrm{L})\end{array}$ & $\begin{array}{c}\text { Suspended } \\
\text { load } \\
(\mathrm{kg} / \mathrm{s})\end{array}$ & $\begin{array}{l}\text { Number of } \\
\text { sampling } \\
\text { points } \\
\text { for } \\
\text { bedload }\end{array}$ & $\begin{array}{r}\text { Bedload } \\
(\mathrm{kg} / \mathrm{s})\end{array}$ \\
\hline
\end{tabular}

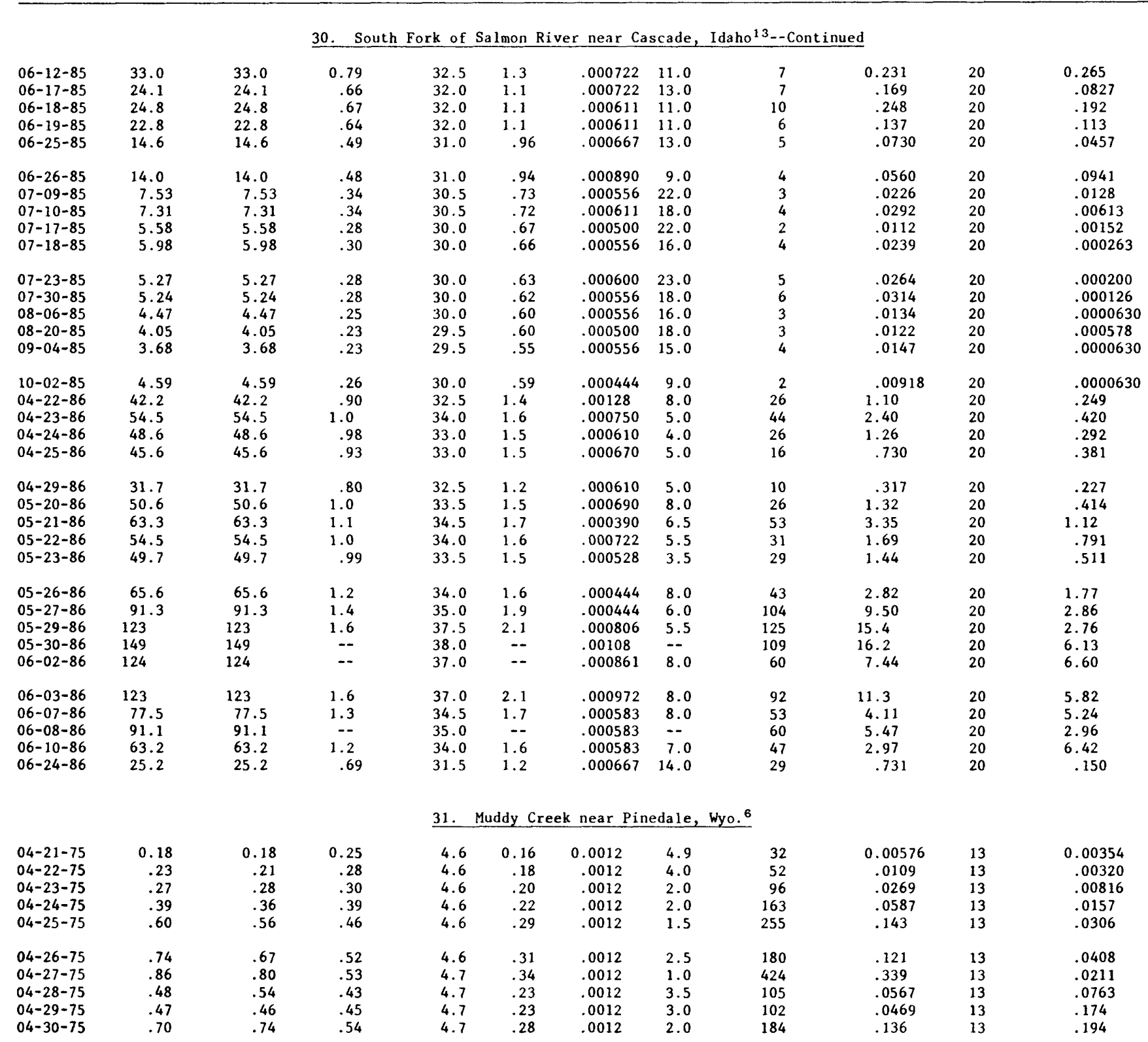


Table 1.--Hydraulic and sediment-transport data--Continued

\begin{tabular}{|c|c|c|c|c|c|c|c|c|c|c|c|}
\hline Date & $\begin{array}{c}\text { Water } \\
\text { discharge } \\
\text { (bedload) } \\
\left(\mathrm{m}^{3} / \mathrm{s}\right)\end{array}$ & $\begin{array}{c}\text { Water } \\
\text { discharge } \\
\text { (suspended } \\
\text { load) } \\
\left(\mathrm{m}^{3} / \mathrm{s}\right)\end{array}$ & $\begin{array}{l}\text { Mean } \\
\text { flow } \\
\text { velocity } \\
(\mathrm{m} / \mathrm{s})\end{array}$ & $\begin{array}{l}\text { Water- } \\
\text { surface } \\
\text { width } \\
(\mathrm{m})\end{array}$ & $\begin{array}{l}\text { Mean } \\
\text { flow } \\
\text { depth } \\
\text { (m) }\end{array}$ & $\begin{array}{l}\text { Water- } \\
\text { surface } \\
\text { slope } \\
(\mathrm{m} / \mathrm{m})\end{array}$ & $\begin{array}{l}\text { Water } \\
\text { temp. } \\
\left({ }^{\circ} \mathrm{C}\right)\end{array}$ & $\begin{array}{l}\text { Suspended } \\
\text { sediment } \\
\text { concern- } \\
\text { tration } \\
(m g / L)\end{array}$ & $\begin{array}{c}\text { Suspended } \\
\text { load } \\
(\mathrm{kg} / \mathrm{s})\end{array}$ & $\begin{array}{l}\text { Number of } \\
\text { sampling } \\
\text { points } \\
\text { for } \\
\text { bedload }\end{array}$ & $\begin{array}{r}\text { Bedload } \\
(\mathrm{kg} / \mathrm{s})\end{array}$ \\
\hline
\end{tabular}

31. Muddy Creek near Pinedale, Wyo. ${ }^{6}-$-Continued

$\begin{array}{rrrr}05-01-75 & 0.62 & 0.57 & 0.50 \\ 05-02-75 & .82 & .87 & .56 \\ 05-03-75 & .94 & .98 & .52 \\ 05-03-75 & 1.51 & 1.39 & .76 \\ 05-04-75 & 1.30 & 1.69 & .66 \\ 05-06-75 & 1.18 & 1.16 & .66 \\ 05-08-75 & .89 & .89 & .50 \\ 05-09-75 & .98 & .99 & .53 \\ 05-23-75 & .42 & .42 & .44 \\ 06-20-75 & 1.41 & 1.41 & .64 \\ 07-11-75 & 1.57 & 1.57 & .63\end{array}$

$\begin{array}{rrrrr}4.7 & 0.27 & 0.0012 & 4.0 & 149 \\ 4.7 & .31 & .0012 & 4.0 & 156 \\ 4.6 & .40 & .0012 & 3.5 & 32 \\ 4.7 & .42 & .0012 & 7.5 & 765 \\ 4.0 & .50 & .0012 & 2.5 & 252 \\ & & & & \\ 4.6 & .39 & .0012 & 1.5 & 434 \\ 4.6 & .39 & .0012 & 7.0 & 100 \\ 4.6 & .40 & .0012 & 4.5 & 78 \\ 4.6 & .21 & .0012 & 7.5 & 25 \\ 4.6 & .48 & .0012 & 12.5 & 92 \\ 4.7 & .52 & .0012 & -- & 69\end{array}$

$\begin{array}{cc}0.0849 & 13 \\ .136 & 13 \\ .0314 & 13 \\ 1.06 & 13 \\ .426 & 13 \\ .503 & 13 \\ .0890 & 13 \\ .0772 & 13 \\ .0105 & 13 \\ .130 & 13 \\ .108 & 13\end{array}$

0.361
.288
.245
.387
.578
.238
.170
.259
.184
.578
.598

32. East Fork River near Pinedale, Wyo. 6,14

\begin{tabular}{|c|c|c|c|c|c|c|c|c|c|c|c|}
\hline $\begin{array}{l}05-23-79 \\
05-24-79 \\
05-25-79 \\
05-26-79 \\
05-28-79\end{array}$ & $\begin{array}{l}18.6 \\
20.2 \\
20.1 \\
20.5 \\
22.4\end{array}$ & $\begin{array}{l}18.5 \\
20.3 \\
20.1 \\
20.5 \\
22.3\end{array}$ & $\begin{array}{r}0.86 \\
.88 \\
.87 \\
.88 \\
.90\end{array}$ & $\begin{array}{l}24.5 \\
24.5 \\
24.5 \\
24.5 \\
25.0\end{array}$ & $\begin{array}{r}0.89 \\
.94 \\
.93 \\
.94 \\
.99\end{array}$ & $\begin{array}{r}0.0007 \\
.0007 \\
.0007 \\
.0007 \\
.0007\end{array}$ & $\begin{array}{l}9.5 \\
7.0 \\
8.0 \\
8.5 \\
6.5\end{array}$ & $\begin{array}{l}88 \\
92 \\
78 \\
68 \\
64\end{array}$ & $\begin{array}{l}1.63 \\
1.87 \\
1.57 \\
1.39 \\
1.43\end{array}$ & $\begin{array}{l}20 \\
20 \\
20 \\
20 \\
20\end{array}$ & $\begin{array}{l}0.883 \\
1.30 \\
2.95 \\
2.16 \\
4.31\end{array}$ \\
\hline $\begin{array}{l}05-30-79 \\
06-01-79 \\
06-02-79 \\
06-03-79 \\
06-04-79\end{array}$ & $\begin{array}{c}16.8 \\
6.21 \\
4.54 \\
5.90 \\
9.35\end{array}$ & $\begin{array}{l}16.6 \\
5.74 \\
4.54 \\
5.74 \\
9.40\end{array}$ & $\begin{array}{l}.83 \\
.65 \\
.60 \\
.64 \\
.72\end{array}$ & $\begin{array}{l}24.0 \\
20.5 \\
19.5 \\
20.0 \\
21.5\end{array}$ & $\begin{array}{l}.84 \\
.47 \\
.40 \\
.46 \\
.60\end{array}$ & $\begin{array}{l}.0007 \\
.0007 \\
.0007 \\
.0007 \\
.0007\end{array}$ & $\begin{array}{r}4.0 \\
7.0 \\
9.0 \\
8.0 \\
11.0\end{array}$ & $\begin{array}{l}51 \\
31 \\
18 \\
15 \\
18\end{array}$ & $\begin{array}{l}.847 \\
.178 \\
.0817 \\
.0861 \\
.169\end{array}$ & $\begin{array}{l}20 \\
20 \\
20 \\
20 \\
20\end{array}$ & $\begin{array}{r}2.22 \\
.920 \\
.299 \\
.210 \\
.212\end{array}$ \\
\hline $\begin{array}{l}06-05-79 \\
06-06-79 \\
06-07-79 \\
06-08-79 \\
06-09-79\end{array}$ & $\begin{array}{c}14.6 \\
17.2 \\
14.2 \\
8.08 \\
4.49\end{array}$ & $\begin{array}{l}14.8 \\
17.0 \\
14.8 \\
8.32 \\
4.54\end{array}$ & $\begin{array}{l}.81 \\
.84 \\
.80 \\
.69 \\
.59\end{array}$ & $\begin{array}{l}23.5 \\
24.0 \\
23.0 \\
21.0 \\
19.5\end{array}$ & $\begin{array}{l}.78 \\
.85 \\
.76 \\
.55 \\
.39\end{array}$ & $\begin{array}{l}.0007 \\
.0007 \\
.0007 \\
.0007 \\
.0007\end{array}$ & $\begin{array}{l}8.5 \\
7.0 \\
5.5 \\
5.5 \\
4.5\end{array}$ & $\begin{array}{l}37 \\
72 \\
28 \\
35 \\
19\end{array}$ & $\begin{array}{l}.548 \\
1.22 \\
.414 \\
.291 \\
.0863\end{array}$ & $\begin{array}{l}20 \\
20 \\
20 \\
20 \\
20\end{array}$ & $\begin{array}{l}.442 \\
1.02 \\
1.90 \\
2.06 \\
1.22\end{array}$ \\
\hline $\begin{array}{l}06-10-79 \\
06-11-79 \\
06-13-79 \\
06-14-79 \\
06-15-79\end{array}$ & $\begin{array}{l}3.43 \\
6.70 \\
14.6 \\
17.3 \\
15.8\end{array}$ & $\begin{array}{l}3.36 \\
6.70 \\
14.5 \\
17.4 \\
15.7\end{array}$ & $\begin{array}{l}.55 \\
.66 \\
.81 \\
.84 \\
.82\end{array}$ & $\begin{array}{l}18.5 \\
20.5 \\
23.5 \\
24.0 \\
23.5\end{array}$ & $\begin{array}{l}.34 \\
.49 \\
.78 \\
.86 \\
.81\end{array}$ & $\begin{array}{l}.0007 \\
.0007 \\
.0007 \\
.0007 \\
.0007\end{array}$ & $\begin{array}{r}7.0 \\
9.0 \\
10.0 \\
11.0 \\
9.5\end{array}$ & $\begin{array}{r}6 \\
17 \\
43 \\
34 \\
23\end{array}$ & $\begin{array}{l}.0202 \\
.114 \\
.624 \\
.592 \\
.361\end{array}$ & $\begin{array}{l}20 \\
20 \\
20 \\
20 \\
20\end{array}$ & $\begin{array}{l}.250 \\
.153 \\
.681 \\
.688 \\
.648\end{array}$ \\
\hline $\begin{array}{l}06-16-79 \\
06-17-79 \\
06-18-79 \\
06-19-79 \\
06-20-79\end{array}$ & $\begin{array}{l}9.77 \\
7.99 \\
6.62 \\
3.97 \\
2.67\end{array}$ & $\begin{array}{l}9.68 \\
7.88 \\
6.54 \\
3.90 \\
2.54\end{array}$ & $\begin{array}{l}.73 \\
.69 \\
.66 \\
.58 \\
.52\end{array}$ & $\begin{array}{l}22.0 \\
21.0 \\
20.5 \\
19.0 \\
17.5\end{array}$ & $\begin{array}{l}.61 \\
.55 \\
.49 \\
.37 \\
.29\end{array}$ & $\begin{array}{l}.0007 \\
.0007 \\
.0007 \\
.0007 \\
.0007\end{array}$ & $\begin{array}{r}10.5 \\
10.5 \\
9.0 \\
7.0 \\
10.0\end{array}$ & $\begin{array}{l}15 \\
24 \\
13 \\
12 \\
15\end{array}$ & $\begin{array}{l}.145 \\
.189 \\
.0850 \\
.0468 \\
.0381\end{array}$ & $\begin{array}{l}20 \\
20 \\
20 \\
20 \\
20\end{array}$ & $\begin{array}{l}3.23 \\
1.86 \\
.444 \\
.253 \\
.589\end{array}$ \\
\hline
\end{tabular}

33. Yampa River at Deerlodge Park, Colo. 5

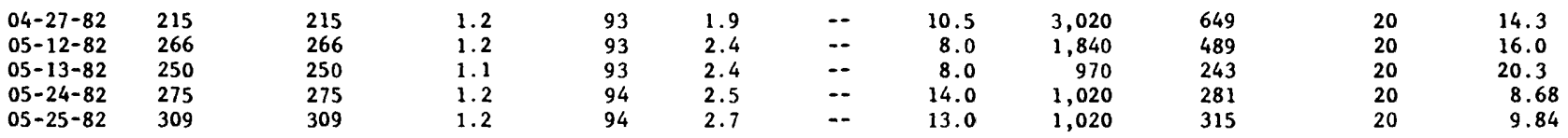


Table 1.--Hydraulic and sediment-transport data--Continued

\begin{tabular}{|c|c|c|c|c|c|c|c|c|c|c|c|}
\hline Date & $\begin{array}{c}\text { Water } \\
\text { discharge } \\
\text { (bedload })^{1} \\
\left(\mathrm{~m}^{3} / \mathrm{s}\right)\end{array}$ & $\begin{array}{c}\text { Water } \\
\text { discharge } \\
\text { (suspended } \\
10 a d)^{2} \\
\left(\mathrm{~m}^{3} / \mathrm{s}\right)\end{array}$ & $\begin{array}{l}\text { Mean } \\
\text { flow } \\
\text { velocity } \\
(\mathrm{m} / \mathrm{s})\end{array}$ & $\begin{array}{l}\text { Water- } \\
\text { surface } \\
\text { width } \\
\text { (m) }\end{array}$ & $\begin{array}{l}\text { Mean } \\
\text { flow } \\
\text { depth } \\
(m)\end{array}$ & $\begin{array}{l}\text { Water- } \\
\text { surface } \\
\text { slope } \\
(\mathrm{m} / \mathrm{m})\end{array}$ & $\begin{array}{l}\text { Water } \\
\text { temp. } \\
\left({ }^{\circ} \mathrm{C}\right)\end{array}$ & $\begin{array}{l}\text { Suspended } \\
\text { sediment } \\
\text { concern- } \\
\text { tration } \\
(\mathrm{mg} / \mathrm{L})\end{array}$ & $\begin{array}{c}\text { Suspended } \\
\text { load } \\
\left(\mathrm{kg}_{\mathrm{g}} / \mathrm{s}\right)\end{array}$ & $\begin{array}{l}\text { Number of } \\
\text { sampling } \\
\text { points } \\
\text { for } \\
\text { bedload }\end{array}$ & $\begin{array}{r}\text { Bedload } \\
(\mathrm{kg} / \mathrm{s})\end{array}$ \\
\hline
\end{tabular}

33. Yampa River at Deerlodge Park, Colo. ${ }^{5}$--Continued

\begin{tabular}{|c|c|c|c|c|c|c|c|c|c|c|c|}
\hline $\begin{array}{l}06-08-82 \\
06-24-82 \\
06-25-82 \\
07-07-82 \\
07-08-82\end{array}$ & $\begin{array}{l}266 \\
283 \\
272 \\
177 \\
177\end{array}$ & $\begin{array}{l}266 \\
283 \\
272 \\
177 \\
177\end{array}$ & $\begin{array}{l}1.1 \\
1.3 \\
1.3 \\
1.3 \\
1.3\end{array}$ & $\begin{array}{l}93 \\
93 \\
93 \\
91 \\
91\end{array}$ & $\begin{array}{l}2.5 \\
2.4 \\
2.3 \\
1.4 \\
1.4\end{array}$ & $\begin{array}{l}-- \\
-- \\
-- \\
--\end{array}$ & $\begin{array}{l}11.5 \\
15.5 \\
15.0 \\
17.5 \\
19.0\end{array}$ & $\begin{array}{r}1,040 \\
340 \\
939 \\
283 \\
1,200\end{array}$ & $\begin{array}{c}277 \\
96.2 \\
255 \\
50.1 \\
212\end{array}$ & $\begin{array}{l}20 \\
20 \\
20 \\
20 \\
20\end{array}$ & $\begin{array}{l}16.0 \\
19.5 \\
49.8 \\
23.1 \\
18.4\end{array}$ \\
\hline $\begin{array}{l}07-29-82 \\
04-07-83 \\
04-08-83 \\
04-19-83 \\
04-21-83\end{array}$ & $\begin{array}{l}66.6 \\
26.3 \\
24.3 \\
34.3 \\
103\end{array}$ & $\begin{array}{c}66.6 \\
26.3 \\
24.3 \\
34.3 \\
103\end{array}$ & $\begin{array}{l}.83 \\
.59 \\
.56 \\
.57 \\
.77\end{array}$ & $\begin{array}{l}89 \\
69 \\
69 \\
88 \\
89\end{array}$ & $\begin{array}{l}.91 \\
.65 \\
.63 \\
.68 \\
1.5\end{array}$ & $\begin{array}{l}-- \\
.00072 \\
-- \\
-- \\
--\end{array}$ & $\begin{array}{r}21.5 \\
5.5 \\
5.0 \\
9.5 \\
10.0\end{array}$ & $\begin{array}{r}706 \\
579 \\
322 \\
855 \\
2,480\end{array}$ & $\begin{array}{c}47.0 \\
15.2 \\
7.82 \\
29.3 \\
255\end{array}$ & $\begin{array}{l}20 \\
20 \\
20 \\
20 \\
20\end{array}$ & $\begin{array}{r}9.42 \\
.557 \\
.756 \\
6.52 \\
3.68\end{array}$ \\
\hline $\begin{array}{l}04-22-83 \\
05-07-83 \\
05-09-83 \\
05-12-83 \\
05-23-83\end{array}$ & $\begin{array}{l}108 \\
185 \\
172 \\
337 \\
244\end{array}$ & $\begin{array}{l}108 \\
185 \\
172 \\
337 \\
244\end{array}$ & $\begin{array}{l}.81 \\
1.1 \\
1.1 \\
1.2 \\
1.1\end{array}$ & $\begin{array}{l}90 \\
91 \\
91 \\
93 \\
92\end{array}$ & $\begin{array}{l}1.5 \\
1.8 \\
1.7 \\
2.9 \\
2.4\end{array}$ & $\begin{array}{l}.00047 \\
.00071 \\
-- \\
.00085 \\
.00060\end{array}$ & $\begin{array}{r}9.5 \\
5.0 \\
-- \\
9.5 \\
14.5\end{array}$ & $\begin{array}{r}2,620 \\
1,400 \\
812 \\
2,900 \\
1,480\end{array}$ & $\begin{array}{l}283 \\
259 \\
140 \\
977 \\
361\end{array}$ & $\begin{array}{l}20 \\
20 \\
20 \\
20 \\
20\end{array}$ & $\begin{array}{l}3.60 \\
12.2 \\
17.7 \\
13.3 \\
11.2\end{array}$ \\
\hline $\begin{array}{l}05-26-83 \\
05-27-83 \\
05-28-83 \\
06-08-83 \\
06-10-83\end{array}$ & $\begin{array}{l}430 \\
447 \\
498 \\
408 \\
425\end{array}$ & $\begin{array}{l}430 \\
447 \\
498 \\
408 \\
425\end{array}$ & $\begin{array}{l}1.3 \\
1.3 \\
1.3 \\
1.3 \\
1.3\end{array}$ & $\begin{array}{l}92 \\
92 \\
92 \\
92 \\
91\end{array}$ & $\begin{array}{l}3.6 \\
3.9 \\
4.1 \\
3.4 \\
3.5\end{array}$ & $\begin{array}{l}.00087 \\
.00078 \\
-- \\
.00067 \\
.00073\end{array}$ & $\begin{array}{l}14.5 \\
14.0 \\
12.5 \\
11.5 \\
13.0\end{array}$ & $\begin{array}{r}2,320 \\
2,060 \\
2,020 \\
1,030 \\
819\end{array}$ & $\begin{array}{r}998 \\
921 \\
1,010 \\
420 \\
348\end{array}$ & $\begin{array}{l}20 \\
20 \\
20 \\
20 \\
20\end{array}$ & $\begin{array}{c}10.4 \\
9.74 \\
10.4 \\
8.45 \\
5.63\end{array}$ \\
\hline $\begin{array}{l}06-21-83 \\
06-23-83 \\
07-12-83 \\
07-14-83\end{array}$ & $\begin{array}{l}447 \\
445 \\
203 \\
157\end{array}$ & $\begin{array}{l}447 \\
445 \\
203 \\
157\end{array}$ & $\begin{array}{l}1.3 \\
1.2 \\
1.0 \\
.90\end{array}$ & $\begin{array}{l}91 \\
91 \\
92 \\
91\end{array}$ & $\begin{array}{l}3.7 \\
3.9 \\
2.1 \\
1.9\end{array}$ & $\begin{array}{l}.00065 \\
- \\
.00040 \\
-\end{array}$ & $\begin{array}{l}16.5 \\
16.0 \\
20.0 \\
21.0\end{array}$ & $\begin{array}{l}634 \\
552 \\
559 \\
382\end{array}$ & $\begin{array}{r}283 \\
246 \\
113 \\
60.0\end{array}$ & $\begin{array}{l}20 \\
20 \\
20 \\
20\end{array}$ & $\begin{array}{r}13.2 \\
6.66 \\
9.26 \\
7.99\end{array}$ \\
\hline
\end{tabular}

34. Rock Creek near Toponas, Colo. 5

\begin{tabular}{|c|c|c|c|c|c|c|c|c|c|c|c|}
\hline $\begin{array}{l}05-02-85 \\
05-08-85 \\
05-14-85 \\
05-16-85 \\
05-20-85\end{array}$ & $\begin{array}{l}5.07 \\
9.26 \\
6.43 \\
5.81 \\
7.14\end{array}$ & $\begin{array}{l}5.07 \\
9.26 \\
6.43 \\
5.81 \\
7.14\end{array}$ & $\begin{array}{l}1.1 \\
1.3 \\
1.2 \\
1.2 \\
1.4\end{array}$ & $\begin{array}{l}8.1 \\
8.1 \\
8.4 \\
7.8 \\
8.0\end{array}$ & $\begin{array}{r}0.57 \\
.85 \\
.62 \\
.62 \\
.66\end{array}$ & $\begin{array}{l}-- \\
-- \\
-- \\
-- \\
--\end{array}$ & $\begin{array}{l}-- \\
-- \\
-- \\
--\end{array}$ & $\begin{array}{l}32 \\
17 \\
18 \\
11 \\
11\end{array}$ & $\begin{array}{c}0.162 \\
.157 \\
.116 \\
.0639 \\
.0785\end{array}$ & $\begin{array}{l}26 \\
24 \\
24 \\
24 \\
26\end{array}$ & $\begin{array}{c}0.0567 \\
.0872 \\
.0725 \\
.158 \\
.168\end{array}$ \\
\hline $\begin{array}{l}05-22-85 \\
05-29-85 \\
06-04-85 \\
06-10-85 \\
06-18-85\end{array}$ & $\begin{array}{l}6.40 \\
7.65 \\
4.90 \\
4.16 \\
2.04\end{array}$ & $\begin{array}{l}6.40 \\
7.65 \\
4.90 \\
4.16 \\
2.04\end{array}$ & $\begin{array}{l}1.3 \\
1.3 \\
1.1 \\
1.1 \\
.70\end{array}$ & $\begin{array}{l}8.4 \\
8.3 \\
8.2 \\
8.2 \\
7.9\end{array}$ & $\begin{array}{l}.60 \\
.71 \\
.52 \\
.47 \\
.37\end{array}$ & $\begin{array}{l}-- \\
-- \\
-- \\
--\end{array}$ & $\begin{array}{l}-- \\
-- \\
-- \\
--\end{array}$ & $\begin{array}{r}13 \\
12 \\
3 \\
6 \\
4\end{array}$ & $\begin{array}{l}.0832 \\
.0918 \\
.0147 \\
.0250 \\
.00816\end{array}$ & $\begin{array}{l}26 \\
26 \\
26 \\
13 \\
24\end{array}$ & $\begin{array}{l}.105 \\
.0714 \\
.0116 \\
.0179 \\
.00032\end{array}$ \\
\hline $\begin{array}{l}06-26-85 \\
07-10-85 \\
07-22-85 \\
04-24-86 \\
04-28-86\end{array}$ & $\begin{array}{r}1.59 \\
.48 \\
.65 \\
4.90 \\
2.95\end{array}$ & $\begin{array}{r}1.59 \\
.48 \\
.65 \\
4.90 \\
2.95\end{array}$ & $\begin{array}{l}.68 \\
.36 \\
.40 \\
1.2 \\
1.0\end{array}$ & $\begin{array}{l}8.1 \\
7.5 \\
8.1 \\
8.2 \\
7.9\end{array}$ & $\begin{array}{l}.29 \\
.18 \\
.20 \\
.50 \\
.37\end{array}$ & $\begin{array}{l}-- \\
-- \\
-- \\
--\end{array}$ & $\begin{array}{l}-- \\
-- \\
-- \\
2.5 \\
4.5\end{array}$ & $\begin{array}{r}4 \\
1 \\
9 \\
38 \\
17\end{array}$ & $\begin{array}{l}.00636 \\
.000480 \\
.00585 \\
.186 \\
.0502\end{array}$ & $\begin{array}{l}24 \\
11 \\
24 \\
24 \\
20\end{array}$ & $\begin{array}{l}.00053 \\
.00011 \\
.00158 \\
.165 \\
.0489\end{array}$ \\
\hline $\begin{array}{l}05-12-86 \\
05-15-86 \\
05-20-86 \\
05-21-86 \\
05-27-86\end{array}$ & $\begin{array}{l}5.16 \\
5.92 \\
6.39 \\
6.92 \\
7.42\end{array}$ & $\begin{array}{l}5.16 \\
5.92 \\
6.39 \\
6.92 \\
7.42\end{array}$ & $\begin{array}{l}1.2 \\
1.2 \\
1.3 \\
1.3 \\
1.4\end{array}$ & $\begin{array}{l}8.1 \\
8.2 \\
8.2 \\
8.2 \\
8.2\end{array}$ & $\begin{array}{l}.54 \\
.59 \\
.61 \\
.67 \\
.67\end{array}$ & $\begin{array}{l}-- \\
-- \\
-- \\
-- \\
--\end{array}$ & $\begin{array}{r}6.5 \\
4.5 \\
8.5 \\
6.0 \\
10.5\end{array}$ & $\begin{array}{l}15 \\
16 \\
17 \\
16 \\
14\end{array}$ & $\begin{array}{l}.0774 \\
.0947 \\
.109 \\
.111 \\
.104\end{array}$ & $\begin{array}{l}24 \\
24 \\
24 \\
24 \\
24\end{array}$ & $\begin{array}{l}.0659 \\
.0750 \\
.0725 \\
.122 \\
.152\end{array}$ \\
\hline $\begin{array}{l}06-09-86 \\
07-02-86\end{array}$ & $\begin{array}{l}6.21 \\
.840\end{array}$ & $\begin{array}{l}6.21 \\
.840\end{array}$ & $\begin{array}{c}1.2 \\
.44\end{array}$ & $\begin{array}{l}8.2 \\
7.9\end{array}$ & $\begin{array}{l}.61 \\
.24\end{array}$ & -- & $\begin{array}{r}9.5 \\
16.0\end{array}$ & $\begin{array}{r}29 \\
9\end{array}$ & $\begin{array}{l}.180 \\
.00756\end{array}$ & $\begin{array}{l}22 \\
22\end{array}$ & $\begin{array}{l}.0930 \\
.00189\end{array}$ \\
\hline
\end{tabular}


Table 1.--Hydraulic and sediment-transport data--Continued

\begin{tabular}{|c|c|c|c|c|c|c|c|c|c|c|c|}
\hline Date & $\begin{array}{c}\text { Water } \\
\text { discharge } \\
\text { (bedload })^{1} \\
\left(\mathrm{~m}^{3} / \mathrm{s}\right)\end{array}$ & $\begin{array}{c}\text { Water } \\
\text { discharge } \\
\text { (suspended } \\
\text { load) } \\
\left(\mathrm{m}^{3} / \mathrm{s}\right)\end{array}$ & $\begin{array}{c}\text { Mean } \\
\text { flow } \\
\text { velocity } \\
(\mathrm{m} / \mathrm{s})\end{array}$ & $\begin{array}{l}\text { Water- } \\
\text { surface } \\
\text { width } \\
(\mathrm{m})\end{array}$ & $\begin{array}{l}\text { Mean } \\
\text { flow } \\
\text { depth } \\
\text { (m) }\end{array}$ & $\begin{array}{l}\text { Water- } \\
\text { surface } \\
\text { slope } \\
(\mathrm{m} / \mathrm{m})\end{array}$ & $\begin{array}{l}\text { Water } \\
\text { temp. } \\
\left({ }^{\circ} \mathrm{C}\right)\end{array}$ & $\begin{array}{c}\text { Suspended } \\
\text { sediment } \\
\text { concern- } \\
\text { tration } \\
(\mathrm{mg} / \mathrm{L})\end{array}$ & $\begin{array}{c}\text { Suspended } \\
\text { load } \\
(\mathrm{kg} / \mathrm{s})\end{array}$ & $\begin{array}{l}\text { Number of } \\
\text { sampling } \\
\text { points } \\
\text { for } \\
\text { bedload }\end{array}$ & $\begin{array}{r}\text { Bedload } \\
(\mathrm{kg} / \mathrm{s})\end{array}$ \\
\hline
\end{tabular}

35. McIntyre Creek near Glendevey, Colo. 5

\begin{tabular}{|c|c|c|c|c|c|c|c|c|c|c|c|}
\hline $05-12-77$ & 1.39 & 1.39 & 0.64 & 7.5 & 0.29 & -- & -- & 6 & 0.00834 & $>20$ & 0.000095 \\
\hline $05-27-77$ & 1.50 & 1.50 & .63 & 7.5 & .32 & - & -. & 13 & .0195 & $>20$ & .00011 \\
\hline $05-31-77$ & 2.24 & 2.24 & .75 & 7.8 & .38 & -- & -. & 22 & .0493 & $>20$ & .00021 \\
\hline $06-06-77$ & 5.85 & 5.85 & 1.8 & 8.0 & .41 & -- & -. & 51 & .298 & $>20$ & .00200 \\
\hline $06-14-77$ & 2.04 & 2.04 & .77 & 7.5 & .35 & - & -- & 6 & .0122 & $\geq 20$ & .00084 \\
\hline $06-22-77$ & 1.10 & 1.10 & .54 & 7.6 & .27 & -- & - & 4 & .00440 & $\geq 20$ & .00032 \\
\hline $06-28-77$ & .74 & .74 & .47 & 6.9 & .23 & -- & -- & 8 & .00592 & $>20$ & .000095 \\
\hline $07-06-77$ & .76 & .76 & .46 & 6.7 & .25 & -- & -- & 4 & .00304 & $>20$ & .000095 \\
\hline $07-12-77$ & .45 & .45 & - & 6.4 & -- & - & -- & 3 & .00135 & $>20$ & .000095 \\
\hline $07-19-77$ & .34 & .34 & .83 & 6.1 & .22 & -- & - & 2 & .000680 & $>20$ & .000063 \\
\hline
\end{tabular}

36. Joe Wright Creek above Culvert \#1 near Chambers Lake, Colo. 3

\begin{tabular}{|c|c|c|c|c|c|c|c|c|c|c|c|}
\hline $\begin{array}{l}05-25-79 \\
05-25-79 \\
05-26-79 \\
05-27-79 \\
05-27-79\end{array}$ & $\begin{array}{r}0.42 \\
.25 \\
.31 \\
.42 \\
.37\end{array}$ & $\begin{array}{r}0.42 \\
.25 \\
.31 \\
.42 \\
.37\end{array}$ & $\begin{array}{l}-- \\
-- \\
-- \\
--\end{array}$ & $\begin{array}{l}6.1 \\
5.8 \\
5.8 \\
6.1 \\
6.1\end{array}$ & $\begin{array}{l}-- \\
-- \\
-- \\
--\end{array}$ & $\begin{array}{l}-- \\
-- \\
-- \\
-- \\
--\end{array}$ & $\begin{array}{l}-- \\
-- \\
-- \\
-- \\
--\end{array}$ & $\begin{array}{r}20 \\
16 \\
6 \\
13 \\
20\end{array}$ & $\begin{array}{r}0.00840 \\
.00400 \\
.00186 \\
.00546 \\
.00740\end{array}$ & $\begin{array}{l}\geq 20 \\
\geq 20 \\
\geq 20 \\
\geq 20 \\
\geq 20\end{array}$ & $\begin{array}{r}0.00053 \\
.00578 \\
.00189 \\
.00084 \\
.00095\end{array}$ \\
\hline $\begin{array}{l}05-28-79 \\
06-03-79 \\
06-03-79 \\
06-04-79 \\
06-12-79\end{array}$ & $\begin{array}{l}.51 \\
.25 \\
.37 \\
.31 \\
.37\end{array}$ & $\begin{array}{l}.51 \\
.25 \\
.37 \\
.31 \\
.37\end{array}$ & $\begin{array}{l}-- \\
-- \\
--\end{array}$ & $\begin{array}{l}6.1 \\
5.8 \\
6.1 \\
5.8 \\
6.1\end{array}$ & $\begin{array}{l}-- \\
-- \\
-- \\
--\end{array}$ & $\begin{array}{l}-- \\
-- \\
-- \\
--\end{array}$ & $\begin{array}{l}-- \\
-- \\
-- \\
--\end{array}$ & $\begin{array}{r}23 \\
8 \\
43 \\
5 \\
5\end{array}$ & $\begin{array}{l}.0117 \\
.00200 \\
.0159 \\
.00155 \\
.00185\end{array}$ & $\begin{array}{l}\geq 20 \\
\geq 20 \\
\geq 20 \\
\geq 20 \\
\geq 20\end{array}$ & $\begin{array}{l}.00137 \\
.000084 \\
.000032 \\
.00063 \\
.00021\end{array}$ \\
\hline $\begin{array}{l}06-12-79 \\
06-13-79 \\
06-13-79 \\
06-19-79\end{array}$ & $\begin{array}{r}.93 \\
.48 \\
1.08 \\
.65\end{array}$ & $\begin{array}{r}.93 \\
.48 \\
1.08 \\
.65\end{array}$ & $\begin{array}{l}-- \\
-- \\
--\end{array}$ & $\begin{array}{l}6.7 \\
6.1 \\
6.7 \\
6.4\end{array}$ & $\begin{array}{l}-- \\
-- \\
--\end{array}$ & $\begin{array}{l}-- \\
-- \\
-- \\
--\end{array}$ & $\begin{array}{l}-- \\
-- \\
--\end{array}$ & $\begin{array}{r}90 \\
9 \\
27 \\
16\end{array}$ & $\begin{array}{l}.0837 \\
.00432 \\
.0292 \\
.0104\end{array}$ & $\begin{array}{l}\geq 20 \\
\geq 20 \\
\geq 20 \\
>20\end{array}$ & $\begin{array}{l}.00074 \\
.00021 \\
.00063 \\
.00021\end{array}$ \\
\hline
\end{tabular}

37. Joe Wright Creek at Bridge \#4 near Chambers Lake, Colo. ${ }^{3}$

\begin{tabular}{|c|c|c|c|c|c|c|c|c|c|c|c|}
\hline $05-20-79$ & 1.98 & 1.98 & -- & 8.2 & -- & - & -- & 173 & 0.343 & $\geq 20$ & 0.00221 \\
\hline $05-20-79$ & 1.98 & 1.98 & -- & 8.2 & -- & - & -- & 166 & .329 & $>20$ & .00273 \\
\hline $05-21-79$ & 1.61 & 1.61 & -- & 8.2 & -- & -- & -- & 427 & .688 & $>20$ & .00105 \\
\hline $05-25-79$ & 1.98 & 1.98 & - & 8.2 & -- & -- & -- & 131 & .259 & $\geq 20$ & .00126 \\
\hline $05-25-79$ & 2.15 & 2.15 & -- & 8.2 & -- & -- & -- & 213 & .458 & $\geq 20$ & .00095 \\
\hline $05-26-79$ & 2.32 & 2.32 & -- & 8.5 & -- & -- & -- & 331 & .768 & $\geq 20$ & .00315 \\
\hline $05-27-79$ & 2.49 & 2.49 & - & 8.5 & -- & -- & -- & 243 & .605 & $>20$ & .00084 \\
\hline $05-27-79$ & 2.69 & 2.69 & -- & 8.5 & -- & -- & -- & 240 & .646 & $>20$ & .00063 \\
\hline $05-28-79$ & 2.83 & 2.83 & -- & 8.5 & -. & - & -- & 680 & 1.92 & $>20$ & .00683 \\
\hline $06-03-79$ & .48 & .48 & -- & 7.9 & -- & -- & -- & 45 & .0216 & $\geq 20$ & .00032 \\
\hline $06-03-79$ & 2.32 & 2. 32 & -- & 8.5 & -- & -- & -- & 298 & .691 & $\geq 20$ & .00126 \\
\hline $06-04-79$ & 2.32 & 2.32 & - & 8.5 & -- & -- & -- & 95 & .220 & $\geq 20$ & .00053 \\
\hline $06-12-79$ & 2.49 & 2.49 & -- & 8.5 & -- & -- & -- & 55 & .137 & $\geq 20$ & .00210 \\
\hline $06-12-79$ & 5.18 & 5.18 & - & 8.8 & -- & - & -- & 308 & 1.60 & $\geq 20$ & .0125 \\
\hline $06-13-79$ & 4.53 & 4.53 & -- & 8.8 & -- & -- & - & 31 & .140 & $\geq 20$ & .00168 \\
\hline $07-11-79$ & 3.12 & 3.12 & -- & 8.5 & -- & -- & -- & 144 & .449 & $\geq 20$ & .00042 \\
\hline $07-12-79$ & 2.32 & 2.32 & -- & 8.5 & -- & -. & -- & 380 & .882 & $\overline{20}$ & .00042 \\
\hline $07-12-79$ & 2.69 & 2.69 & -- & 8.5 & -- & -- & -- & 110 & .296 & $>20$ & .00042 \\
\hline $07-20-79$ & 1.73 & 1.73 & -- & 8.2 & -- & -- & -- & 93 & .161 & $>20$ & .00032 \\
\hline
\end{tabular}


Table 1.--Hydraulic and sediment-transport data--Continued

\begin{tabular}{|c|c|c|c|c|c|c|c|c|c|c|c|}
\hline Date & $\begin{array}{c}\text { Water } \\
\text { discharge } \\
\text { (bedload) }^{1} \\
\left(\mathrm{~m}^{3} / \mathrm{s}\right)\end{array}$ & $\begin{array}{c}\text { Water } \\
\text { discharge } \\
\text { (suspended } \\
\text { load) } \\
\left(\mathrm{m}^{3} / \mathrm{s}\right)\end{array}$ & $\begin{array}{c}\text { Mean } \\
\text { flow } \\
\text { velocity } \\
(\mathrm{m} / \mathrm{s})\end{array}$ & $\begin{array}{l}\text { Water- } \\
\text { surface } \\
\text { width } \\
\text { (m) }\end{array}$ & $\begin{array}{l}\text { Mean } \\
\text { flow } \\
\text { depth } \\
(\mathrm{m})\end{array}$ & $\begin{array}{l}\text { Water- } \\
\text { surface } \\
\text { slope } \\
(\mathrm{m} / \mathrm{m})\end{array}$ & $\begin{array}{l}\text { Water } \\
\text { temp. } \\
\left({ }^{\circ} \mathrm{C}\right)\end{array}$ & $\begin{array}{l}\text { Suspended } \\
\text { sediment } \\
\text { concern- } \\
\text { tration } \\
(\mathrm{mg} / \mathrm{L})\end{array}$ & $\begin{array}{c}\text { Suspended } \\
\text { load } \\
(\mathrm{kg} / \mathrm{s})\end{array}$ & $\begin{array}{l}\text { Number of } \\
\text { sampling } \\
\text { points } \\
\text { for } \\
\text { bedload }\end{array}$ & $\begin{array}{r}\text { Bedload } \\
(\mathrm{kg} / \mathrm{s})\end{array}$ \\
\hline
\end{tabular}

38. North Fork of Joe Wright Creek Lower Station near Chambers Lake, Colo. ${ }^{3}$

\begin{tabular}{|c|c|c|c|c|c|c|c|c|c|c|c|}
\hline $06-29-77$ & 0.198 & 0.198 & - & 4.6 & - & -- & - & 2 & 0.000396 & $\geq 20$ & 0.00011 \\
\hline $05-20-79$ & 1.53 & 1.53 & -. & 8.5 & -- & -- & -- & 795 & 1.22 & $\overline{20}$ & .00063 \\
\hline $05-20-79$ & 1.53 & 1.53 & - & 8.5 & - & - & -- & 290 & .444 & $\ulcorner 20$ & .00095 \\
\hline $05-21-79$ & 1.27 & 1.27 & - & 8.5 & -- & -- & -- & 112 & .142 & $>20$ & .00074 \\
\hline $05-25-79$ & 1.19 & 1.19 & - & 8.5 & -- & -- & $\cdots$ & 1,160 & 1.38 & $\geq 20$ & .00053 \\
\hline $05-25-79$ & 1.13 & 1.13 & - & 8.5 & -- & -- & -- & 370 & .418 & $\geq 20$ & .00084 \\
\hline $05-27-79$ & 1.30 & 1.30 & $\ldots$ & 8.5 & -. & -- & -- & 190 & .247 & $\overline{20}$ & .00063 \\
\hline $05-27-79$ & 1.47 & 1.47 & $\ldots$ & 8.5 & -- & -- & - & 357 & .525 & $\overline{20}$ & .00095 \\
\hline $05-28-79$ & 1.70 & 1.70 & -- & 8.5 & $\cdots$ & - & -- & 831 & 1.41 & $\overline{20}$ & .00200 \\
\hline $06-03-79$ & 1.22 & 1.22 & - & 8.5 & -- & - & - & 64 & .0781 & $\geq 20$ & .00189 \\
\hline $06-04-79$ & 1.42 & 1.42 & - & 8.5 & -. & -- & - & 140 & .199 & $\geq 20$ & .00053 \\
\hline $06-12-79$ & 1.59 & 1.59 & - & 8.5 & - & -- & -. & 63 & .100 & $>20$ & .00063 \\
\hline $06-12-79$ & 2.49 & 2.49 & -- & 8.5 & -- & -. & - & 294 & .732 & $>20$ & .0111 \\
\hline $06-13-79$ & 1.93 & 1.93 & - & 8.5 & -- & - & -- & 41 & .0791 & $\geq 20$ & .00137 \\
\hline $06-26-79$ & 1.08 & 1.08 & -- & 8.5 & - & -. & 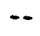 & 19 & .0205 & $\overline{2} 20$ & .00084 \\
\hline $07-11-79$ & 1.81 & 1.81 & $\cdots$ & 8.5 & -- & -- & $=$ & 236 & .427 & $\geq 20$ & .00063 \\
\hline $07-12-79$ & 1.42 & 1.42 & - & 8.5 & -- & - & -- & 814 & 1.16 & $\overline{20}$ & .00074 \\
\hline $07-12-79$ & 1.59 & 1.59 & - & 8.5 & -- & -- & -- & 180 & .286 & $>20$ & .00053 \\
\hline $07-20-79$ & 1.19 & 1.19 & $=$ & 8.5 & -. & -. & - & 136 & .162 & $>20$ & .00032 \\
\hline
\end{tabular}

39. Cabin Creek at Mouth near Granby, Colo. 5

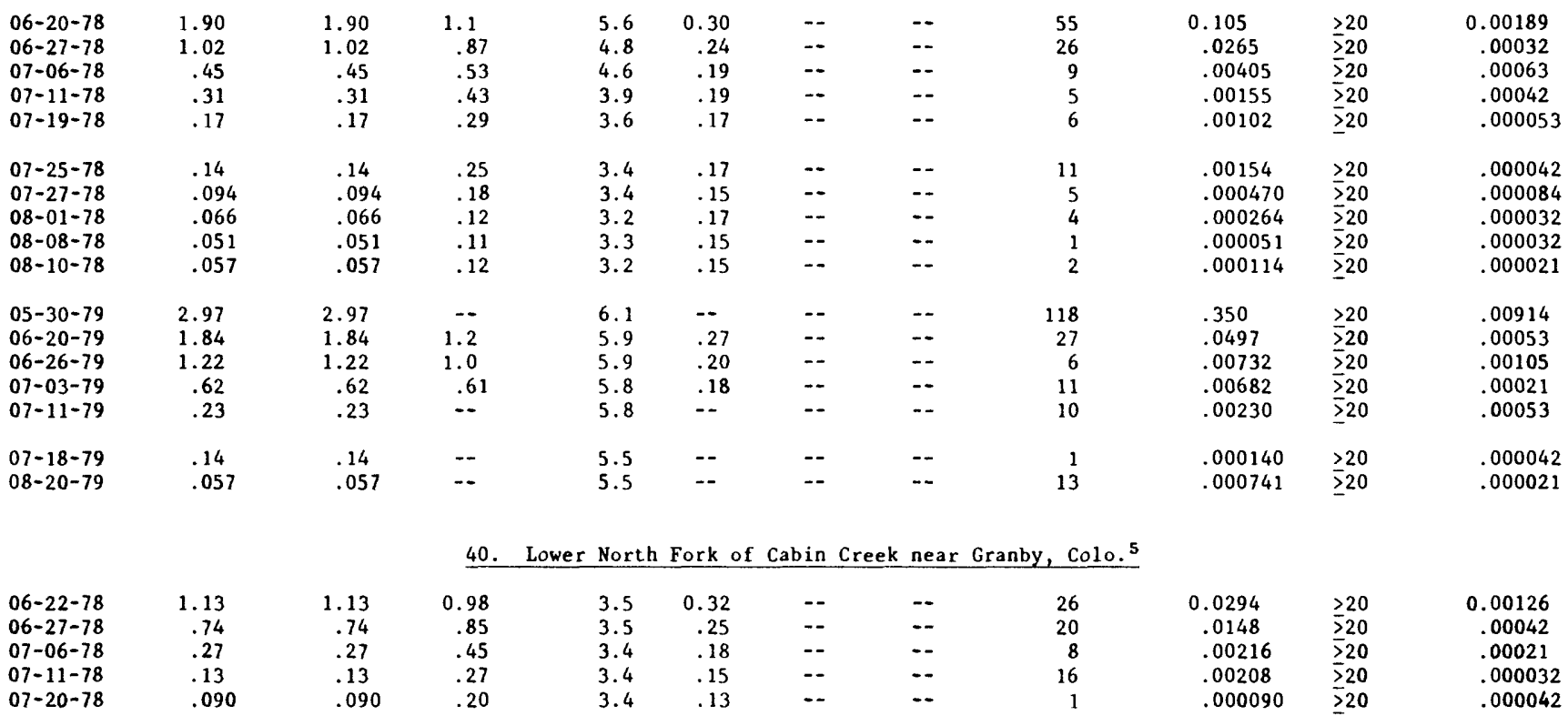


Table 1.--Hydraulic and sediment-transport data--Continued

\begin{tabular}{|c|c|c|c|c|c|c|c|c|c|c|c|}
\hline Date & $\begin{array}{c}\text { Water } \\
\text { discharge } \\
\text { (bedload) } \\
\left(m^{3} / s\right)\end{array}$ & $\begin{array}{c}\text { Water } \\
\text { discharge } \\
\text { (suspended } \\
\text { load) } \\
\left(\mathrm{m}^{3} / \mathrm{s}\right)\end{array}$ & $\begin{array}{c}\text { Mean } \\
\text { flow } \\
\text { velocity } \\
(\mathrm{m} / \mathrm{s})\end{array}$ & $\begin{array}{l}\text { Water- } \\
\text { surface } \\
\text { width } \\
\text { (m) }\end{array}$ & $\begin{array}{l}\text { Mean } \\
\text { flow } \\
\text { depth } \\
(m)\end{array}$ & $\begin{array}{l}\text { Water- } \\
\text { surface } \\
\text { slope } \\
(\mathrm{m} / \mathrm{m})\end{array}$ & $\begin{array}{l}\text { Water } \\
\text { temp. } \\
\left({ }^{\circ} \mathrm{C}\right)\end{array}$ & $\begin{array}{l}\text { Suspended } \\
\text { sediment } \\
\text { concern- } \\
\text { tration } \\
(\mathrm{mg} / \mathrm{L})\end{array}$ & $\begin{array}{c}\text { Suspended } \\
\text { load } \\
(\mathrm{kg} / \mathrm{s})\end{array}$ & $\begin{array}{l}\text { Number of } \\
\text { sampling } \\
\text { points } \\
\text { for } \\
\text { bedload }\end{array}$ & $\begin{array}{r}\text { Bedload } \\
(\mathrm{kg} / \mathrm{s})\end{array}$ \\
\hline
\end{tabular}

40. Lower North Fork of Cabin Creek near Granby, Colo. 5 --Continued

\begin{tabular}{|c|c|c|c|c|c|c|c|c|c|c|c|}
\hline $07-25-78$ & 0.066 & 0.066 & 0.17 & 3.4 & 0.11 & -- & - & 7 & 0.000462 & $\geq 20$ & 0.000032 \\
\hline $06-19-79$ & 1.16 & 1.16 & 1.1 & 3.7 & .28 & -- & - & 22 & .0255 & $\overline{20}$ & .00221 \\
\hline $06-26-79$ & .65 & .65 & .76 & 3.7 & .23 & - & $\cdots$ & 222 & .144 & $\geq 20$ & .00053 \\
\hline $07-03-79$ & .17 & .17 & -- & 3.7 & -- & -- & -- & 8 & .00136 & $\geq 20$ & .00011 \\
\hline $07-10-79$ & .057 & .057 & -- & 2.4 & -- & - & -- & 20 & .00114 & $\geq 20$ & .00011 \\
\hline $07-16-79$ & .025 & .025 & -- & 2.1 & -- & - & -- & 1 & .000025 & $\geq 20$ & .000032 \\
\hline
\end{tabular}

41. Upper North Fork of Cabin Creek near Granby, Colo. 5

\begin{tabular}{|c|c|c|c|c|c|c|c|c|c|c|c|}
\hline $06-20-78$ & 0.48 & 0.48 & 1.0 & 2.2 & 0.22 & -- & -- & 18 & 0.00864 & $\geq 20$ & 0.00032 \\
\hline $06-28-78$ & .23 & .23 & .68 & 1.8 & .18 & - & - & 5 & .00115 & $\geq 20$ & .00032 \\
\hline $07-06-78$ & .085 & .085 & .40 & 1.8 & .12 & -- & -- & 4 & .000340 & $\geq 20$ & .00011 \\
\hline $07-11-78$ & .066 & .066 & .37 & 1.7 & .12 & - & -- & 11 & .000726 & $>20$ & .000021 \\
\hline $07-20-78$ & .033 & .033 & .23 & 1.7 & .083 & -- & -- & 8 & .000264 & $\geq 20$ & .000011 \\
\hline $07-25-78$ & .017 & .017 & .14 & 1.5 & .081 & - & -- & 4 & .000068 & $>20$ & .000011 \\
\hline $07-27-78$ & .015 & .015 & .12 & 1.6 & .076 & - & -- & 3 & .000045 & 520 & .000011 \\
\hline $08-10-78$ & .010 & .010 & .087 & 1.4 & .079 & -- & -- & 4 & .000040 & 520 & .000011 \\
\hline $06-18-79$ & .45 & .45 & .86 & 2.4 & .22 & - & - & 17 & .00765 & $\geq 20$ & .00042 \\
\hline $06-25-79$ & .27 & .27 & .75 & 2.0 & .18 & -- & -- & 29 & .00783 & $\overline{2} 20$ & .00011 \\
\hline
\end{tabular}

42. Lower Left Fork of North Fork of Cabin Creek near Granby, Colo. 5

\begin{tabular}{|c|c|c|c|c|c|c|c|c|c|c|c|}
\hline $06-21-78$ & 0.62 & 0.62 & 1.1 & 1.8 & 0.31 & -- & -- & 17 & 0.0105 & $\geq 20$ & 0.00084 \\
\hline $06-28-78$ & .38 & .38 & .80 & 1.8 & .27 & -- & -- & 16 & .00608 & $\geq 20$ & .00084 \\
\hline $07-06-78$ & .16 & .16 & .46 & 1.7 & .21 & -- & -- & 13 & .00208 & $\geq 20$ & .00021 \\
\hline $07-20-78$ & .051 & .051 & .20 & 1.6 & .16 & -- & -- & 9 & .000459 & $\geq 20$ & .000032 \\
\hline $07-25-78$ & .028 & .028 & .12 & 1.5 & .15 & $=$ & - & 4 & .000112 & $\geq 20$ & .000021 \\
\hline $08-03-78$ & .019 & .019 & .090 & 1.5 & .14 & $=$ & -- & 2 & .000038 & $\geq 20$ & .000042 \\
\hline $08-08-78$ & .014 & .014 & -- & 1.5 & -- & - & -- & 6 & .000084 & $\overline{20}$ & .000042 \\
\hline $06-18-79$ & .68 & .68 & 1.3 & 2.1 & .25 & - & -- & 47 & .0320 & $\geq 20$ & .00021 \\
\hline $06-25-79$ & .40 & .40 & .97 & 1.9 & .22 & $-\infty$ & -- & 27 & .0108 & $\geq 20$ & .000074 \\
\hline $07-09-79$ & .028 & .028 & - & 1.8 & -- & -- & -- & 4 & .000112 & $\geq 20$ & .00021 \\
\hline $07-16-79$ & .020 & .020 & -- & 1.5 & -- & $\cdots$ & -- & 5 & .000100 & $\geq 20$ & .000011 \\
\hline
\end{tabular}

43. Upper Left Fork of North Fork of Cabin Creek near Granby, Colo. 5

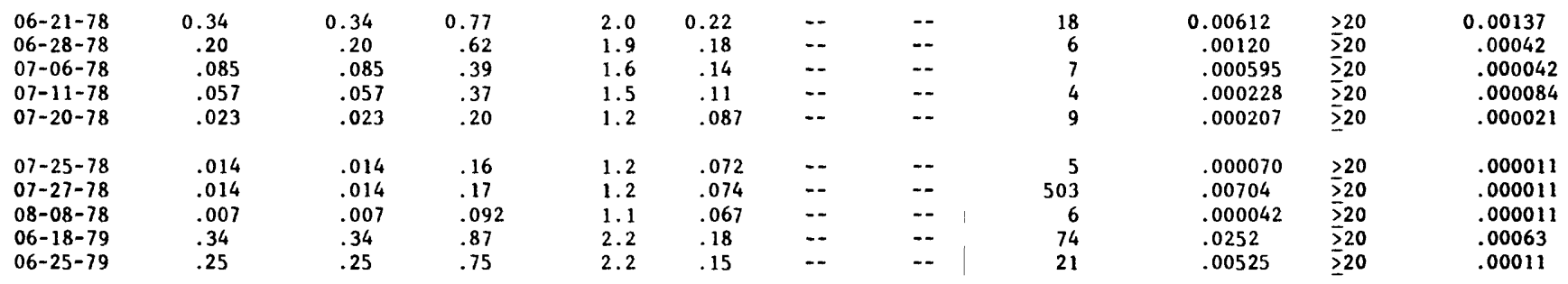


Table 1.--Hydraulic and sediment-transport data--Cont inued

\begin{tabular}{|c|c|c|c|c|c|c|c|c|c|c|c|}
\hline Date & $\begin{array}{c}\text { Water } \\
\text { discharge } \\
\text { (bedload) } \\
\left(\mathrm{m}^{3} / \mathrm{s}\right)\end{array}$ & $\begin{array}{c}\text { Water } \\
\text { discharge } \\
\text { (suspended } \\
\text { load })^{2} \\
\left(\mathrm{~m}^{3} / \mathrm{s}\right)\end{array}$ & $\begin{array}{l}\text { Mean } \\
\text { flow } \\
\text { velocity } \\
(\mathrm{m} / \mathrm{s})\end{array}$ & $\begin{array}{l}\text { Water- } \\
\text { surface } \\
\text { width } \\
\text { (m) }\end{array}$ & $\begin{array}{l}\text { Mean } \\
\text { flow } \\
\text { depth } \\
(m)\end{array}$ & $\begin{array}{l}\text { Water- } \\
\text { surface } \\
\text { slope } \\
(\mathrm{m} / \mathrm{m})\end{array}$ & $\begin{array}{l}\text { Water } \\
\text { temp. } \\
\left({ }^{\circ} \mathrm{C}\right)\end{array}$ & $\begin{array}{c}\text { Suspended } \\
\text { sediment } \\
\text { concern- } \\
\text { tration } \\
(\mathrm{mg} / \mathrm{L})\end{array}$ & $\begin{array}{c}\text { Suspended } \\
\text { load } \\
\left(\mathrm{kg}_{\mathrm{g}} / \mathrm{s}\right)\end{array}$ & $\begin{array}{l}\text { Number of } \\
\text { sampling } \\
\text { points } \\
\text { for } \\
\text { bedload }\end{array}$ & $\begin{array}{r}\text { Bedload } \\
(\mathrm{kg} / \mathrm{s})\end{array}$ \\
\hline
\end{tabular}

44. Upper E1k Creek near Granby, Colo. 5

\begin{tabular}{|c|c|c|c|c|c|c|c|c|c|c|c|}
\hline $06-19-78$ & 0.25 & 0.25 & 0.78 & 1.5 & 0.21 & -- & $\cdots$ & 16 & 0.00400 & $\geq 20$ & 0.00042 \\
\hline $06-26-78$ & .24 & .24 & .75 & 1.6 & .20 & $\cdots$ & $=-$ & 10 & .00240 & $\geq 20$ & .00021 \\
\hline $07-05-78$ & .17 & .17 & .67 & 1.5 & .17 & - & - & 6 & .00102 & $\geq 20$ & .00053 \\
\hline $07-10-78$ & .13 & .13 & .71 & 1.8 & .11 & - & - & 7 & .000910 & $\geq 20$ & .00021 \\
\hline $07-13-78$ & .10 & .10 & .58 & 1.4 & .12 & - & -- & 10 & .00100 & $\geq 20$ & .00021 \\
\hline $07-17-78$ & .10 & .10 & .67 & 1.4 & .11 & - & -- & 10 & .00100 & $\geq 20$ & .000095 \\
\hline $06-20-79$ & .16 & .16 & .80 & 1.6 & .13 & - & $\ldots$ & 15 & .00240 & $\geq 20$ & .00021 \\
\hline $06-26-79$ & .17 & .17 & .75 & 1.6 & .14 & - & - & 197 & .0335 & $\geq 20$ & .00011 \\
\hline $07-05-79$ & .34 & .34 & $=$ & 1.5 & - & -- & -- & 12 & .00408 & $\geq 20$ & .00011 \\
\hline $07-10-79$ & .25 & .25 & - & 1.5 & -- & - & - & 16 & .00400 & $\geq 20$ & .00011 \\
\hline $07-18-79$ & .20 & .20 & -- & 1.5 & -. & -- & - & 2 & .000400 & $\geq 20$ & .000063 \\
\hline
\end{tabular}

45. Upper Beaver Creek near llot Sulphur Springs, Co10. 5

\begin{tabular}{|c|c|c|c|c|c|c|c|c|c|c|c|}
\hline $\begin{array}{l}04-28-77 \\
05-04-77 \\
05-10-77 \\
05-12-77 \\
05-18-77\end{array}$ & $\begin{array}{r}0.30 \\
.37 \\
.88 \\
.96 \\
.51\end{array}$ & $\begin{array}{r}0.30 \\
.37 \\
.88 \\
.96 \\
.51\end{array}$ & $\begin{array}{r}0.36 \\
.45 \\
.59 \\
.69 \\
.47\end{array}$ & $\begin{array}{l}5.3 \\
4.9 \\
6.2 \\
6.1 \\
6.3\end{array}$ & $\begin{array}{r}0.15 \\
.17 \\
.24 \\
.23 \\
.17\end{array}$ & $\begin{array}{l}-- \\
-- \\
-- \\
--\end{array}$ & $\begin{array}{l}-- \\
-- \\
-- \\
--\end{array}$ & $\begin{array}{r}11 \\
24 \\
29 \\
19 \\
5\end{array}$ & $\begin{array}{c}0.00330 \\
.00888 \\
.0255 \\
.0182 \\
.00255\end{array}$ & $\begin{array}{l}\geq 20 \\
\geq 20 \\
\geq 20 \\
\geq 20 \\
\geq 20\end{array}$ & $\begin{array}{r}0.00011 \\
.00011 \\
.00042 \\
.00158 \\
.00032\end{array}$ \\
\hline $\begin{array}{l}05-24-77 \\
05-31-77 \\
06-07-77 \\
06-16-77 \\
06-20-77\end{array}$ & $\begin{array}{l}.45 \\
.34 \\
.27 \\
.17 \\
.14\end{array}$ & $\begin{array}{l}.45 \\
.34 \\
.27 \\
.17 \\
.14\end{array}$ & $\begin{array}{l}.43 \\
.39 \\
.34 \\
.31 \\
.28\end{array}$ & $\begin{array}{l}5.3 \\
5.5 \\
5.3 \\
4.3 \\
4.3\end{array}$ & $\begin{array}{l}.20 \\
.16 \\
.15 \\
.13 \\
.12\end{array}$ & $\begin{array}{l}\ldots \\
\ldots \\
\ldots \\
--\end{array}$ & $\begin{array}{l}-- \\
-- \\
-- \\
--\end{array}$ & $\begin{array}{l}5 \\
7 \\
8 \\
7 \\
2\end{array}$ & $\begin{array}{l}.00225 \\
.00238 \\
.00216 \\
.00119 \\
.00028\end{array}$ & $\begin{array}{l}\geq 20 \\
\geq 20 \\
\geq 20 \\
\geq 20 \\
\geq 20\end{array}$ & $\begin{array}{l}.00042 \\
.00021 \\
.00032 \\
.000063 \\
.000095\end{array}$ \\
\hline $\begin{array}{l}06-25-77 \\
06-28-77\end{array}$ & $\begin{array}{l}.082 \\
.071\end{array}$ & $\begin{array}{l}.082 \\
.071\end{array}$ & $\begin{array}{l}.20 \\
.21\end{array}$ & $\begin{array}{l}4.0 \\
4.0\end{array}$ & $\begin{array}{l}.10 \\
.087\end{array}$ & -- & -- & $\begin{array}{l}1 \\
1\end{array}$ & $\begin{array}{l}.000082 \\
.000071\end{array}$ & $\begin{array}{l}\geq 20 \\
\geq 20\end{array}$ & $\begin{array}{l}.000074 \\
.000063\end{array}$ \\
\hline
\end{tabular}

46. Upper Vasquez Creek near Winter Park, Colo. ${ }^{3}$

\begin{tabular}{|c|c|c|c|c|c|c|c|c|c|c|c|}
\hline $\begin{array}{l}05-25-77 \\
06-03-77\end{array}$ & $\begin{array}{r}0.27 \\
.48\end{array}$ & $\begin{array}{r}0.27 \\
.48\end{array}$ & $\begin{array}{l}1.5 \\
.60\end{array}$ & $\begin{array}{l}4.0 \\
4.0\end{array}$ & $\begin{array}{r}0.15 \\
.20\end{array}$ & -- & -- & $\begin{array}{r}14 \\
2\end{array}$ & $\begin{array}{c}0.00378 \\
.000960\end{array}$ & $\begin{array}{l}\geq 20 \\
\geq 20\end{array}$ & $\begin{array}{l}0.00011 \\
.000084\end{array}$ \\
\hline $06-08-77$ & .71 & .71 & .78 & 4.0 & .23 & -. & - & 4 & .00284 & $\Sigma 20$ & .00011 \\
\hline $06-15-77$ & .54 & .54 & .71 & 4.0 & .20 & -- & -- & 3 & .00162 & $\geq 20$ & .00011 \\
\hline $06-19-77$ & .48 & .48 & .61 & 3.8 & .21 & -- & -- & 2 & .000960 & $\geq 20$ & .00032 \\
\hline $06-26-77$ & .37 & .37 & .51 & 3.8 & .19 & - & -- & 2 & .000740 & $>20$ & .00011 \\
\hline $07-02-77$ & .31 & .31 & .47 & 3.9 & .17 & -- & -- & 2 & .000620 & $\geq 20$ & .00011 \\
\hline $07-09-77$ & .28 & .28 & .46 & 3.8 & .16 & -- & -- & 3 & .000840 & $>20$ & .00021 \\
\hline $07-26-77$ & .21 & .21 & .37 & 3.8 & .15 & -- & -. & 1 & .000210 & $\geq 20$ & .00021 \\
\hline $06-14-78$ & 1.27 & 1.27 & 1.1 & 3.9 & .31 & - & -. & 18 & .0229 & $>20$ & .00063 \\
\hline
\end{tabular}


Table 1.--Hydraulic and sediment-transport data--Continued

\begin{tabular}{|c|c|c|c|c|c|c|c|c|c|c|c|}
\hline Date & $\begin{array}{c}\text { Water } \\
\text { discharge } \\
\text { (bedload) }^{1} \\
\left(\mathrm{~m}^{3} / \mathrm{s}\right)\end{array}$ & $\begin{array}{c}\text { Water } \\
\text { discharge } \\
\text { (suspended } \\
\text { load) } \\
\left(\mathrm{m}^{3} / \mathrm{s}\right)\end{array}$ & $\begin{array}{l}\text { Mean } \\
\text { flow } \\
\text { velocity } \\
(\mathrm{m} / \mathrm{s})\end{array}$ & $\begin{array}{l}\text { Water- } \\
\text { surface } \\
\text { width } \\
(\mathrm{m})\end{array}$ & $\begin{array}{l}\text { Mean } \\
\text { flow } \\
\text { depth } \\
(m)\end{array}$ & $\begin{array}{l}\text { Water- } \\
\text { surface } \\
\text { slope } \\
(\mathrm{m} / \mathrm{m})\end{array}$ & $\begin{array}{l}\text { Water } \\
\text { temp. } \\
\left({ }^{\circ} \mathrm{C}\right)\end{array}$ & $\begin{array}{c}\text { Suspended } \\
\text { sediment } \\
\text { concern- } \\
\text { tration } \\
(m g / L)\end{array}$ & $\begin{array}{c}\text { Suspended } \\
\text { load } \\
(\mathrm{kg} / \mathrm{s})\end{array}$ & $\begin{array}{l}\text { Number of } \\
\text { sampling } \\
\text { points } \\
\text { for } \\
\text { bedload }\end{array}$ & $\begin{array}{r}\text { Bedload } \\
(\mathrm{kg} / \mathrm{s})\end{array}$ \\
\hline
\end{tabular}

\begin{tabular}{|c|c|c|c|c|c|c|c|c|c|c|c|}
\hline $06-22-78$ & 1.39 & 1.39 & 1.1 & 3.9 & 0.32 & $=-$ & - & 5 & 0.00695 & $\geq 20$ & 0.00105 \\
\hline $06-30-78$ & 1.67 & 1.67 & 1.3 & 4.0 & .33 & -- & -- & 3 & .00501 & $\overline{20}$ & .00231 \\
\hline $07-07-78$ & 1.13 & 1.13 & 1.0 & 3.9 & .28 & -- & -- & 1 & .00113 & $\geq 20$ & .000032 \\
\hline $07-20-78$ & .68 & .68 & .74 & 3.9 & .24 & -- & -- & 5 & .00340 & $\geq 20$ & .00074 \\
\hline $07-26-78$ & .54 & .54 & .66 & 3.9 & .21 & -- & -- & 4 & .00216 & $\geq 20$ & .00011 \\
\hline $08-02-78$ & .51 & .51 & .61 & 3.9 & .21 & $\ldots$ & -- & 2 & .00102 & $\geq 20$ & .000053 \\
\hline $08-09-78$ & .34 & .34 & .48 & 3.9 & .19 & -- & -- & 2 & .000680 & $>20$ & .000042 \\
\hline
\end{tabular}

\section{Fraser River at USGS Station near Winter Park, Colo. ${ }^{3}$}

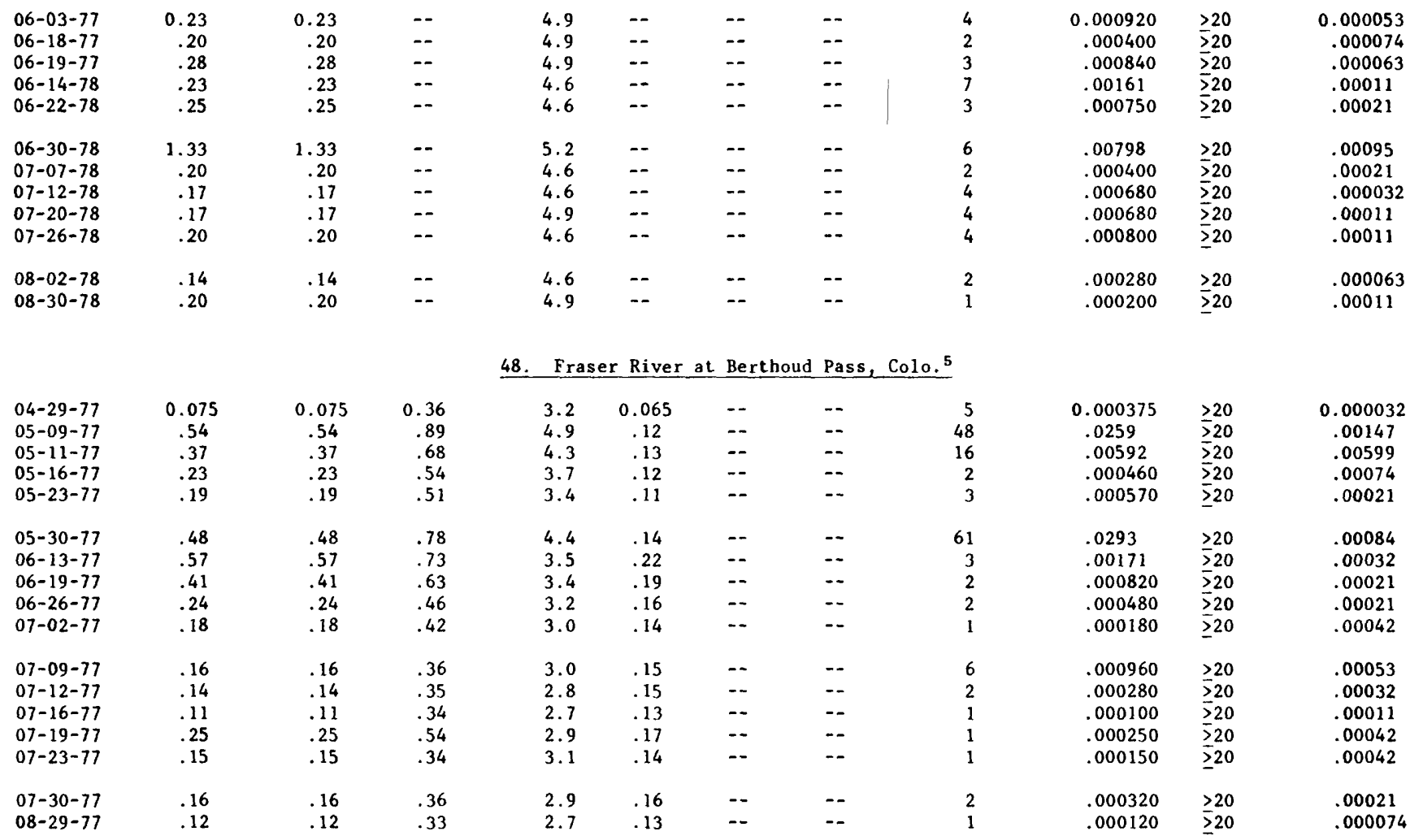


Table 1.--Hydraulic and sediment-transport data--Continued

\begin{tabular}{|c|c|c|c|c|c|c|c|c|c|c|c|}
\hline Date & $\begin{array}{c}\text { Water } \\
\text { discharge } \\
(\text { bedload)' } \\
\left(\mathrm{m}^{3} / \mathrm{s}\right)\end{array}$ & $\begin{array}{c}\text { Water } \\
\text { discharge } \\
\text { (suspended } \\
\text { load) } \\
\left(\mathrm{m}^{3} / \mathrm{s}\right)\end{array}$ & $\begin{array}{l}\text { Mean } \\
\text { flow } \\
\text { velocity } \\
(\mathrm{m} / \mathrm{s})\end{array}$ & $\begin{array}{l}\text { Water- } \\
\text { surface } \\
\text { width } \\
(\mathrm{m})\end{array}$ & $\begin{array}{l}\text { Mean } \\
\text { flow } \\
\text { depth } \\
(\mathrm{m})\end{array}$ & $\begin{array}{l}\text { Water- } \\
\text { surface } \\
\text { slope } \\
(\mathrm{m} / \mathrm{m})\end{array}$ & $\begin{array}{l}\text { Water } \\
\text { temp. } \\
\left({ }^{\circ} \mathrm{C}\right)\end{array}$ & $\begin{array}{l}\text { Suspended } \\
\text { sediment } \\
\text { concern- } \\
\text { tration } \\
(\mathrm{mg} / \mathrm{L})\end{array}$ & $\begin{array}{l}\text { Suspended } \\
\text { load } \\
(\mathrm{kg} / \mathrm{s})\end{array}$ & $\begin{array}{l}\text { Number of } \\
\text { sampling } \\
\text { points } \\
\text { for } \\
\text { bedload }\end{array}$ & $\begin{array}{r}\text { Bedload } \\
(\mathrm{kg} / \mathrm{s})\end{array}$ \\
\hline
\end{tabular}

49. Union Creek near Leadville, Colo. 5

\begin{tabular}{|c|c|c|c|c|c|c|c|c|c|c|c|}
\hline $05-24-78$ & 0.28 & 0.28 & $=$ & - & - & - & -- & 25 & 0.00700 & $20-40$ & 0.000021 \\
\hline $06-05-78$ & .54 & .54 & -- & -- & -- & -- & -- & 32 & .0173 & $20-40$ & .000042 \\
\hline $06-14-78$ & 1.10 & 1.10 & - & -- & -- & -- & - & 2 & .00220 & $20-40$ & .00053 \\
\hline $06-27-78$ & .79 & .79 & - & - & -. & - & - & 13 & .0103 & $20-40$ & .00011 \\
\hline $07-06-78$ & .68 & .68 & -- & - & -- & -- & -- & 10 & .00680 & $20-40$ & .000053 \\
\hline $08-08-78$ & .28 & .28 & -- & -- & -- & -2 & -- & 1 & .000280 & $20-40$ & .000032 \\
\hline $09-13-78$ & .17 & .17 & $=$ & - & -- & - & -- & 1 & .000170 & $20-40$ & .000021 \\
\hline $06-16-79$ & .57 & .57 & -- & -- & -- & - & -- & 28 & .0160 & $20-40$ & .00042 \\
\hline $07-11-79$ & .51 & .51 & $=$ & $=$ & -- & -- & -- & 13 & .00663 & $20-40$ & .00011 \\
\hline $05-21-80$ & .14 & .14 & -- & -- & -- & -- & -- & 8 & .00112 & $20-40$ & .00011 \\
\hline
\end{tabular}

50. Fourmile Creek near Fairplay, Colo. 5

\begin{tabular}{|c|c|c|c|c|c|c|c|c|c|c|c|}
\hline $\begin{array}{l}05-16-84 \\
05-24-84 \\
05-29-84 \\
05-31-84 \\
06-06-84\end{array}$ & $\begin{array}{r}0.44 \\
1.23 \\
1.24 \\
1.21 \\
.96\end{array}$ & $\begin{array}{r}0.44 \\
1.23 \\
1.24 \\
1.21 \\
.96\end{array}$ & $\begin{array}{l}0.66 \\
1.0 \\
1.1 \\
.94 \\
.88\end{array}$ & $\begin{array}{l}3.2 \\
3.4 \\
3.4 \\
3.4 \\
3.3\end{array}$ & $\begin{array}{r}0.21 \\
.36 \\
.34 \\
.38 \\
.33\end{array}$ & $\begin{array}{c}0.016 \\
-- \\
-- \\
-- \\
.016\end{array}$ & $\begin{array}{l}-- \\
-- \\
-- \\
--\end{array}$ & $\begin{array}{r}4 \\
31 \\
15 \\
14 \\
8\end{array}$ & $\begin{array}{c}0.00176 \\
.0381 \\
.0186 \\
.0169 \\
.00768\end{array}$ & $\begin{array}{l}40 \\
40 \\
40 \\
40 \\
40\end{array}$ & $\begin{array}{c}0.00102 \\
.0193 \\
.0109 \\
.00696 \\
.00093\end{array}$ \\
\hline $\begin{array}{l}06-12-84 \\
06-14-84 \\
06-18-84 \\
06-22-84 \\
06-26-84\end{array}$ & $\begin{array}{l}.79 \\
1.45 \\
1.39 \\
1.48 \\
1.40\end{array}$ & $\begin{array}{l}.79 \\
1.45 \\
1.39 \\
1.48 \\
1.40\end{array}$ & $\begin{array}{l}.75 \\
1.0 \\
1.1 \\
1.1 \\
.98\end{array}$ & $\begin{array}{l}3.2 \\
3.5 \\
3.4 \\
3.4 \\
3.5\end{array}$ & $\begin{array}{l}.33 \\
.40 \\
.38 \\
.39 \\
.41\end{array}$ & $\begin{array}{l}-- \\
-- \\
-- \\
-- \\
--\end{array}$ & $\begin{array}{l}-- \\
-- \\
-- \\
-- \\
--\end{array}$ & $\begin{array}{r}4 \\
20 \\
22 \\
22 \\
18\end{array}$ & $\begin{array}{l}.00316 \\
.0290 \\
.0306 \\
.0326 \\
.0252\end{array}$ & $\begin{array}{l}40 \\
40 \\
40 \\
40 \\
40\end{array}$ & $\begin{array}{l}.0129 \\
.0292 \\
.00423 \\
.00958 \\
.0386\end{array}$ \\
\hline $\begin{array}{l}06-29-84 \\
07-03-84 \\
07-06-84 \\
07-10-84 \\
07-17-84\end{array}$ & $\begin{array}{l}1.53 \\
1.66 \\
1.40 \\
2.18 \\
1.08\end{array}$ & $\begin{array}{l}1.53 \\
1.66 \\
1.40 \\
2.18 \\
1.08\end{array}$ & $\begin{array}{l}1.2 \\
1.2 \\
1.1 \\
1.3 \\
1.0\end{array}$ & $\begin{array}{l}3.5 \\
3.6 \\
3.4 \\
3.6 \\
3.3\end{array}$ & $\begin{array}{l}.38 \\
.40 \\
.38 \\
.45 \\
.32\end{array}$ & $\begin{array}{l}-- \\
-- \\
-- \\
.018 \\
--\end{array}$ & $\begin{array}{l}-- \\
-- \\
-- \\
--\end{array}$ & $\begin{array}{l}31 \\
46 \\
10 \\
29 \\
22\end{array}$ & $\begin{array}{l}.0474 \\
.0764 \\
.0140 \\
.0632 \\
.0238\end{array}$ & $\begin{array}{l}40 \\
40 \\
40 \\
40 \\
40\end{array}$ & $\begin{array}{l}.00913 \\
.00465 \\
.00288 \\
.00990 \\
.0321\end{array}$ \\
\hline $\begin{array}{l}07-24-84 \\
08-03-84 \\
08-08-84\end{array}$ & $\begin{array}{r}1.08 \\
.88 \\
.95\end{array}$ & $\begin{array}{r}1.08 \\
.88 \\
.95\end{array}$ & $\begin{array}{l}1.0 \\
.89 \\
.96\end{array}$ & $\begin{array}{l}3.3 \\
3.2 \\
3.3\end{array}$ & $\begin{array}{l}.32 \\
.31 \\
.30\end{array}$ & $\begin{array}{l}-- \\
-- \\
--\end{array}$ & $\begin{array}{l}-- \\
-- \\
-- \\
\text { Empir }\end{array}$ & $\begin{array}{l}10 \\
40 \\
39\end{array}$ & $\begin{array}{r}.0108 \\
.0352 \\
.0371\end{array}$ & $\begin{array}{l}40 \\
40 \\
40\end{array}$ & $\begin{array}{l}.0268 \\
.00326 \\
.00119\end{array}$ \\
\hline $\begin{array}{l}06-16-83 \\
07-14-83 \\
07-28-83 \\
05-22-84 \\
05-29-84\end{array}$ & $\begin{array}{l}0.10 \\
.31 \\
.25 \\
.029 \\
.11\end{array}$ & $\begin{array}{l}0.10 \\
.31 \\
.25 \\
.029 \\
.11\end{array}$ & $\begin{array}{l}-- \\
-- \\
-- \\
0.19 \\
.34\end{array}$ & $\begin{array}{l}-- \\
-- \\
-- \\
1.3 \\
1.8\end{array}$ & $\begin{array}{c}-- \\
-- \\
-- \\
0.12 \\
.18\end{array}$ & $\begin{array}{c}-- \\
-- \\
-- \\
0.188 \\
.188\end{array}$ & $\begin{array}{l}2.5 \\
5.0 \\
4.0 \\
2.0 \\
4.5\end{array}$ & $\begin{array}{l}3 \\
4 \\
2 \\
5 \\
3\end{array}$ & $\begin{array}{c}0.000300 \\
.00124 \\
.000500 \\
.000145 \\
.000330\end{array}$ & $\begin{array}{l}\geq 20 \\
\geq 20 \\
\geq 20 \\
\geq 20 \\
\geq 20\end{array}$ & $\begin{array}{l}0.000049 \\
.000038 \\
.0000084 \\
.000048 \\
.00147\end{array}$ \\
\hline $\begin{array}{l}06-06-84 \\
06-18-84 \\
06-25-84 \\
07-03-84 \\
07-12-84\end{array}$ & $\begin{array}{l}.15 \\
.19 \\
.21 \\
.24 \\
.14\end{array}$ & $\begin{array}{l}.15 \\
.19 \\
.21 \\
.24 \\
.14\end{array}$ & $\begin{array}{l}.42 \\
.45 \\
.46 \\
.57 \\
.37\end{array}$ & $\begin{array}{l}2.1 \\
2.2 \\
2.3 \\
1.9 \\
2.2\end{array}$ & $\begin{array}{l}.17 \\
.19 \\
.20 \\
.22 \\
.17\end{array}$ & $\begin{array}{l}.188 \\
.188 \\
.188 \\
.188 \\
.188\end{array}$ & $\begin{array}{l}3.0 \\
4.0 \\
3.5 \\
6.0 \\
7.0\end{array}$ & $\begin{array}{l}2 \\
8 \\
5 \\
3 \\
2\end{array}$ & $\begin{array}{l}.000300 \\
.00152 \\
.00105 \\
.000720 \\
.000280\end{array}$ & $\begin{array}{l}\geq 20 \\
\geq 20 \\
\geq 20 \\
\geq 20 \\
>20\end{array}$ & $\begin{array}{l}.000608 \\
.00336 \\
.00260 \\
.00234 \\
.000428\end{array}$ \\
\hline
\end{tabular}


Table 1.--Hydraulic and sediment-transport data--Continued

\begin{tabular}{|c|c|c|c|c|c|c|c|c|c|c|c|}
\hline Date & $\begin{array}{c}\text { Water } \\
\text { discharge } \\
\text { (bedload) } \\
\left(\mathrm{m}^{3} / \mathrm{s}\right)\end{array}$ & $\begin{array}{c}\text { Water } \\
\text { discharge } \\
\text { (suspended } \\
\text { load })^{2} \\
\left(\mathrm{~m}^{3} / \mathrm{s}\right)\end{array}$ & $\begin{array}{l}\text { Mean } \\
\text { flow } \\
\text { velocity } \\
(\mathrm{m} / \mathrm{s})\end{array}$ & $\begin{array}{l}\text { Water- } \\
\text { surface } \\
\text { width } \\
(\mathrm{m})\end{array}$ & $\begin{array}{l}\text { Mean } \\
\text { flow } \\
\text { depth } \\
\text { (m) }\end{array}$ & $\begin{array}{l}\text { Water- } \\
\text { surface } \\
\text { slope } \\
(\mathrm{m} / \mathrm{m})\end{array}$ & $\begin{array}{l}\text { Water } \\
\text { temp. } \\
\left({ }^{\circ} \mathrm{C}\right)\end{array}$ & $\begin{array}{c}\text { Suspended } \\
\text { sediment } \\
\text { concern- } \\
\text { tration } \\
(\mathrm{mg} / \mathrm{L})\end{array}$ & $\begin{array}{c}\text { Suspended } \\
\text { load } \\
(\mathrm{kg} / \mathrm{s})\end{array}$ & $\begin{array}{l}\text { Number of } \\
\text { sampling } \\
\text { points } \\
\text { for } \\
\text { bedload }\end{array}$ & $\begin{array}{r}\text { Bedload } \\
(\mathrm{kg} / \mathrm{s})\end{array}$ \\
\hline
\end{tabular}

52. Mad Creek (Site 3) near Empire, Colo. 5

\begin{tabular}{|c|c|c|c|c|c|c|c|c|c|c|c|}
\hline $\begin{array}{l}06-17-83 \\
07-14-83 \\
07-28-83 \\
08-04-83 \\
08-11-83\end{array}$ & $\begin{array}{r}0.17 \\
.43 \\
.42 \\
.22 \\
.18\end{array}$ & $\begin{array}{r}0.17 \\
.43 \\
.42 \\
.22 \\
.18\end{array}$ & $\begin{array}{l}-- \\
-- \\
-- \\
-- \\
--\end{array}$ & $\begin{array}{l}-- \\
-- \\
-- \\
-- \\
--\end{array}$ & $\begin{array}{l}-- \\
-- \\
-- \\
-- \\
--\end{array}$ & $\begin{array}{l}-- \\
-- \\
-- \\
-- \\
--\end{array}$ & $\begin{array}{l}4.0 \\
4.0 \\
6.0 \\
6.0 \\
7.0\end{array}$ & $\begin{array}{r}2 \\
11 \\
3 \\
5 \\
4\end{array}$ & $\begin{array}{c}0.000340 \\
.00473 \\
.00126 \\
.00110 \\
.000720\end{array}$ & $\begin{array}{l}\geq 20 \\
\geq 20 \\
\geq 20 \\
\geq 20 \\
\geq 20\end{array}$ & $\begin{array}{r}0.000079 \\
.000686 \\
.000077 \\
.000070 \\
.000044\end{array}$ \\
\hline $\begin{array}{l}05-29-84 \\
06-06-84 \\
06-11-84 \\
06-18-84 \\
06-25-84\end{array}$ & $\begin{array}{l}.19 \\
.24 \\
.12 \\
.35 \\
.46\end{array}$ & $\begin{array}{l}.19 \\
.24 \\
.12 \\
.35 \\
.46\end{array}$ & $\begin{array}{r}0.53 \\
.57 \\
.42 \\
.77 \\
.81\end{array}$ & $\begin{array}{l}1.9 \\
1.9 \\
1.8 \\
1.9 \\
1.9\end{array}$ & $\begin{array}{r}0.19 \\
.22 \\
.16 \\
.24 \\
.30\end{array}$ & $\begin{array}{l}-- \\
-- \\
-- \\
--\end{array}$ & $\begin{array}{l}3.5 \\
3.5 \\
3.5 \\
3.5 \\
3.5\end{array}$ & $\begin{array}{r}7 \\
3 \\
4 \\
10 \\
73\end{array}$ & $\begin{array}{l}.00133 \\
.000720 \\
.000480 \\
.00350 \\
.0336\end{array}$ & $\begin{array}{l}\geq 20 \\
\geq 20 \\
\geq 20 \\
\geq 20 \\
\geq 20\end{array}$ & $\begin{array}{l}.000590 \\
.00837 \\
.000366 \\
.0038 \\
.0113\end{array}$ \\
\hline $\begin{array}{l}07-03-84 \\
07-12-84 \\
07-20-84 \\
07-31-84 \\
08-16-84 \\
08-29-84\end{array}$ & $\begin{array}{l}.55 \\
.36 \\
.22 \\
.20 \\
.083 \\
.12\end{array}$ & $\begin{array}{l}.55 \\
.36 \\
.22 \\
.20 \\
.083 \\
.12\end{array}$ & $\begin{array}{l}1.1 \\
.78 \\
.64 \\
.66 \\
.42 \\
.48\end{array}$ & $\begin{array}{l}2.0 \\
2.0 \\
1.9 \\
1.9 \\
1.8 \\
1.8\end{array}$ & $\begin{array}{l}.25 \\
.23 \\
.18 \\
.16 \\
.11 \\
.14\end{array}$ & $\begin{array}{l}- \\
- \\
- \\
-- \\
0.0856 \\
-\end{array}$ & $\begin{array}{l}6.0 \\
7.0 \\
7.0 \\
6.0 \\
7.0 \\
6.5\end{array}$ & $\begin{array}{l}6 \\
2 \\
2 \\
1 \\
2 \\
4\end{array}$ & $\begin{array}{l}.00330 \\
.000720 \\
.000440 \\
.000200 \\
.000166 \\
.000480\end{array}$ & $\begin{array}{l}\geq 20 \\
\geq 20 \\
\geq 20 \\
\geq 20 \\
\geq 20 \\
>20\end{array}$ & $\begin{array}{l}.00918 \\
.00590 \\
.000662 \\
.000469 \\
.000614 \\
.000354\end{array}$ \\
\hline
\end{tabular}

53. Middle Fork of Boulder Creek at Nederland, Colo. 5

\begin{tabular}{|c|c|c|c|c|c|c|c|c|c|c|c|}
\hline $\begin{array}{l}05-14-84 \\
05-17-84 \\
05-23-84 \\
06-04-84 \\
06-08-84\end{array}$ & $\begin{array}{l}3.68 \\
5.01 \\
7.76 \\
6.77 \\
4.53\end{array}$ & $\begin{array}{l}3.68 \\
5.01 \\
7.76 \\
6.77 \\
4.53\end{array}$ & $\begin{array}{r}0.84 \\
.96 \\
1.2 \\
1.1 \\
.92\end{array}$ & $\begin{array}{l}14.0 \\
16.5 \\
20.0 \\
19.0 \\
15.5\end{array}$ & $\begin{array}{r}0.31 \\
.32 \\
.33 \\
.33 \\
.32\end{array}$ & $\begin{array}{r}0.0163 \\
.0163 \\
.0163 \\
.0163 \\
.0163\end{array}$ & $\begin{array}{l}-- \\
7.0 \\
5.5 \\
7.0 \\
8.5\end{array}$ & $\begin{array}{r}8 \\
13 \\
15 \\
4 \\
4\end{array}$ & $\begin{array}{c}0.0294 \\
.0651 \\
.116 \\
.0271 \\
.0181\end{array}$ & $\begin{array}{l}20-40 \\
20-40 \\
20-40 \\
20-40 \\
20-40\end{array}$ & $\begin{array}{c}0.0321 \\
.0119 \\
.0134 \\
.0134 \\
.00223\end{array}$ \\
\hline $\begin{array}{l}06-15-84 \\
06-20-84 \\
06-27-84 \\
07-02-84 \\
07-11-84\end{array}$ & $\begin{array}{l}7.84 \\
8.21 \\
8.21 \\
9.52 \\
6.26\end{array}$ & $\begin{array}{l}7.84 \\
8.21 \\
8.21 \\
9.52 \\
6.26\end{array}$ & $\begin{array}{l}1.2 \\
1.2 \\
1.2 \\
1.3 \\
1.1\end{array}$ & $\begin{array}{l}20.0 \\
20.5 \\
20.5 \\
22.0 \\
18.0\end{array}$ & $\begin{array}{l}.33 \\
.33 \\
.33 \\
.34 \\
.33\end{array}$ & $\begin{array}{l}.0163 \\
.0163 \\
.0163 \\
.0163 \\
.0163\end{array}$ & $\begin{array}{r}9.0 \\
7.0 \\
8.5 \\
13.0 \\
13.0\end{array}$ & $\begin{array}{l}7 \\
5 \\
6 \\
9 \\
5\end{array}$ & $\begin{array}{l}.0549 \\
.0411 \\
.0493 \\
.0857 \\
.0313\end{array}$ & $\begin{array}{l}20-40 \\
20-40 \\
20-40 \\
20-40 \\
20-40\end{array}$ & $\begin{array}{l}.0240 \\
.0161 \\
.0107 \\
.0612 \\
.0146\end{array}$ \\
\hline $\begin{array}{l}07-19-84 \\
07-27-84 \\
08-03-84 \\
08-15-84\end{array}$ & $\begin{array}{l}3.79 \\
4.45 \\
3.31 \\
2.58\end{array}$ & $\begin{array}{l}3.79 \\
4.45 \\
3.31 \\
2.58\end{array}$ & $\begin{array}{l}.85 \\
.91 \\
.80 \\
.72\end{array}$ & $\begin{array}{l}14.5 \\
15.5 \\
13.5 \\
12.0\end{array}$ & $\begin{array}{l}.31 \\
.32 \\
.31 \\
.30\end{array}$ & $\begin{array}{l}.0163 \\
.0163 \\
.0163 \\
.0163\end{array}$ & $\begin{array}{l}13.0 \\
13.0 \\
12.0 \\
14.0\end{array}$ & $\begin{array}{l}4 \\
3 \\
2 \\
2\end{array}$ & $\begin{array}{l}.0152 \\
.0134 \\
.00662 \\
.00516\end{array}$ & $\begin{array}{l}20-40 \\
20-40 \\
20-40 \\
20-40\end{array}$ & $\begin{array}{l}.0260 \\
.0237 \\
.00051 \\
.00130\end{array}$ \\
\hline & & & & Jef & son & ek near & Jefferson, & Colo. 5 & & & \\
\hline $\begin{array}{l}05-10-84 \\
05-16-84 \\
05-26-84 \\
06-07-84 \\
06-13-84\end{array}$ & $\begin{array}{c}0.093 \\
.42 \\
1.16 \\
.77 \\
.61\end{array}$ & $\begin{array}{c}0.093 \\
.42 \\
1.16 \\
.77 \\
.61\end{array}$ & $\begin{array}{c}0.26 \\
.58 \\
.86 \\
.75 \\
.68\end{array}$ & $\begin{array}{l}3.5 \\
3.6 \\
4.1 \\
4.1 \\
3.9\end{array}$ & $\begin{array}{r}0.10 \\
.20 \\
.33 \\
.25 \\
.23\end{array}$ & $\begin{array}{r}0.016 \\
.016 \\
.016 \\
.016 \\
.016\end{array}$ & $\begin{array}{l}-- \\
-- \\
9.0 \\
2.5 \\
8.0\end{array}$ & $\begin{array}{l}6 \\
5 \\
5 \\
4 \\
2\end{array}$ & $\begin{array}{c}0.000558 \\
.00210 \\
.00580 \\
.00308 \\
.00122\end{array}$ & $\begin{array}{l}20-40 \\
20-40 \\
20-40 \\
20-40 \\
20-40\end{array}$ & $\begin{array}{c}0.000137 \\
.00923 \\
.00973 \\
.00401 \\
.00414\end{array}$ \\
\hline $\begin{array}{l}06-21-84 \\
06-28-84 \\
07-10-84 \\
07-18-84 \\
07-25-84\end{array}$ & $\begin{array}{r}1.08 \\
1.05 \\
1.24 \\
.56 \\
1.37\end{array}$ & $\begin{array}{r}1.08 \\
1.05 \\
1.24 \\
.56 \\
1.37\end{array}$ & $\begin{array}{l}.79 \\
.80 \\
.88 \\
.62 \\
.93\end{array}$ & $\begin{array}{l}4.1 \\
4.0 \\
4.1 \\
4.1 \\
4.1\end{array}$ & $\begin{array}{l}.33 \\
.33 \\
.35 \\
.22 \\
.36\end{array}$ & $\begin{array}{l}.016 \\
.016 \\
.016 \\
.016 \\
.016\end{array}$ & $\begin{array}{r}6.0 \\
14.0 \\
14.5 \\
11.5 \\
10.0\end{array}$ & $\begin{array}{r}4 \\
4 \\
5 \\
284 \\
5\end{array}$ & $\begin{array}{l}.00432 \\
.00420 \\
.00620 \\
.159 \\
.00685\end{array}$ & $\begin{array}{l}20-40 \\
20-40 \\
20-40 \\
20-40 \\
20-40\end{array}$ & $\begin{array}{l}.00160 \\
.00517 \\
.00596 \\
.00180 \\
.0204\end{array}$ \\
\hline $\begin{array}{l}08-02-84 \\
09-19-84\end{array}$ & $\begin{array}{r}1.05 \\
.36\end{array}$ & $\begin{array}{r}1.05 \\
.36\end{array}$ & $\begin{array}{l}.91 \\
.57\end{array}$ & $\begin{array}{l}4.0 \\
3.6\end{array}$ & $\begin{array}{l}.29 \\
.18\end{array}$ & $\begin{array}{l}.016 \\
.016\end{array}$ & $\begin{array}{r}9.0 \\
10.0\end{array}$ & $\begin{array}{l}5 \\
4\end{array}$ & $\begin{array}{l}.00525 \\
.00144\end{array}$ & $\begin{array}{l}20-40 \\
20-40\end{array}$ & $\begin{array}{l}.00152 \\
.00207\end{array}$ \\
\hline
\end{tabular}


Table 1.--Hydraulic and sediment-transport data--Cont inued

\begin{tabular}{|c|c|c|c|c|c|c|c|c|c|c|c|}
\hline Date & $\begin{array}{c}\text { Water } \\
\text { discharge } \\
\text { (bedload })^{1} \\
\left(\mathrm{~m}^{3} / \mathrm{s}\right)\end{array}$ & $\begin{array}{c}\text { Water } \\
\text { discharge } \\
\text { (suspended } \\
\text { load) } \\
\left(\mathrm{m}^{3} / \mathrm{s}\right)\end{array}$ & $\begin{array}{l}\text { Mean } \\
\text { flow } \\
\text { velocity } \\
(\mathrm{m} / \mathrm{s})\end{array}$ & $\begin{array}{l}\text { Water- } \\
\text { surface } \\
\text { width } \\
\text { (m) }\end{array}$ & $\begin{array}{l}\text { Mean } \\
\text { flow } \\
\text { depth } \\
(m)\end{array}$ & $\begin{array}{l}\text { Water- } \\
\text { surface } \\
\text { slope } \\
(\mathrm{m} / \mathrm{m})\end{array}$ & $\begin{array}{l}\text { Water } \\
\text { temp. } \\
\left({ }^{\circ} \mathrm{C}\right)\end{array}$ & $\begin{array}{l}\text { Suspended } \\
\text { sediment } \\
\text { concern- } \\
\text { tration } \\
(\mathrm{mg} / \mathrm{L})\end{array}$ & $\begin{array}{c}\text { Suspended } \\
\text { load } \\
(\mathrm{kg} / \mathrm{s})\end{array}$ & $\begin{array}{l}\text { Number of } \\
\text { sampling } \\
\text { points } \\
\text { for } \\
\text { bedload }\end{array}$ & $\begin{array}{r}\text { Bedload } \\
\left(\mathrm{kg}_{\mathrm{g}} / \mathrm{s}\right)\end{array}$ \\
\hline
\end{tabular}

\begin{tabular}{lccc}
\hline & & & \\
$04-16-84$ & 0.051 & 0.051 & 0.047 \\
$04-26-84$ & .38 & .38 & .31 \\
$04-30-84$ & .35 & .35 & .35 \\
$05-03-84$ & .31 & .31 & .26 \\
$05-10-84$ & .69 & .69 & .44 \\
$05-15-84$ & 1.39 & 1.39 & .61 \\
$05-17-84$ & 1.65 & 1.65 & .68 \\
$05-24-84$ & 2.05 & 2.05 & .72 \\
$05-29-84$ & 2.25 & 2.25 & .86 \\
$05-31-84$ & 1.81 & 1.81 & .76 \\
$06-04-84$ & 1.77 & 1.77 & \\
$06-06-84$ & 1.38 & 1.38 & .66 \\
$06-11-84$ & 1.28 & 1.28 & .61 \\
$06-19-84$ & 1.25 & 1.25 & .62 \\
$06-26-84$ & .88 & .88 & .55 \\
$07-03-84$ & .66 & .66 & .40 \\
$07-12-84$ & .46 & .46 & .36 \\
$07-16-84$ & .48 & .48 & .37 \\
$08-01-84$ & .57 & .57 & .46 \\
$08-15-84$ & .49 & .49 & .44 \\
$08-21-84$ & 1.45 & 1.45 & .45 \\
$09-05-84$ & .74 & .74 & .51
\end{tabular}

\section{Craig Creek near Bailey, Colo. 5}

\begin{tabular}{|c|c|c|c|c|c|c|c|}
\hline $\begin{array}{l}4.3 \\
6.4 \\
6.2 \\
5.5 \\
6.6\end{array}$ & $\begin{array}{r}0.25 \\
.19 \\
.16 \\
.22 \\
.24\end{array}$ & $\begin{array}{r}0.0213 \\
.0213 \\
.0213 \\
.0213 \\
.0213\end{array}$ & $\begin{array}{l}2.0 \\
0.5 \\
4.0 \\
3.0 \\
3.0\end{array}$ & $\begin{array}{l}3 \\
2 \\
3 \\
6 \\
9\end{array}$ & $\begin{array}{c}0.000153 \\
.000760 \\
.00105 \\
.00186 \\
.00621\end{array}$ & $\begin{array}{l}20-40 \\
20-40 \\
20-40 \\
20-40 \\
20-40\end{array}$ & $\begin{array}{c}0.00106 \\
.00431 \\
.00653 \\
.00227 \\
.0112\end{array}$ \\
\hline $\begin{array}{l}6.9 \\
6.9 \\
6.9 \\
6.7 \\
6.8\end{array}$ & $\begin{array}{l}.33 \\
.35 \\
.42 \\
.39 \\
.35\end{array}$ & $\begin{array}{l}.0213 \\
.0213 \\
.0213 \\
.0213 \\
.0213\end{array}$ & $\begin{array}{l}4.0 \\
4.5 \\
-. \\
-- \\
-.\end{array}$ & $\begin{array}{l}12 \\
23 \\
23 \\
14 \\
13\end{array}$ & $\begin{array}{l}.0167 \\
.0380 \\
.0472 \\
.0315 \\
.0235\end{array}$ & $\begin{array}{l}20-40 \\
20-40 \\
20-40 \\
20-40 \\
20-40\end{array}$ & $\begin{array}{l}.0235 \\
.0138 \\
.0194 \\
.0181 \\
.00776\end{array}$ \\
\hline $\begin{array}{l}-. \\
6.8 \\
6.7 \\
6.7 \\
6.7\end{array}$ & $\begin{array}{l}-. \\
.31 \\
.31 \\
.30 \\
.24\end{array}$ & $\begin{array}{l}.0213 \\
.0213 \\
.0213 \\
.025 \\
.025\end{array}$ & $\begin{array}{l}-- \\
-- \\
9.0 \\
\therefore- \\
8.0\end{array}$ & $\begin{array}{r}9 \\
8 \\
12 \\
8 \\
1\end{array}$ & $\begin{array}{l}.0159 \\
.0110 \\
.0154 \\
.0100 \\
.000880\end{array}$ & $\begin{array}{l}20-40 \\
20-40 \\
20-40 \\
20-40 \\
20-40\end{array}$ & $\begin{array}{l}.00447 \\
.00180 \\
.00334 \\
.00218 \\
.0197\end{array}$ \\
\hline $\begin{array}{l}6.7 \\
6.6 \\
6.6 \\
6.6 \\
6.6\end{array}$ & $\begin{array}{l}.25 \\
.19 \\
.20 \\
.19 \\
.17\end{array}$ & $\begin{array}{l}.025 \\
.025 \\
.025 \\
.025 \\
.025\end{array}$ & $\begin{array}{c}11.0 \\
\ldots \\
13.0 \\
\ldots \\
13.0\end{array}$ & $\begin{array}{r}9 \\
17 \\
20 \\
5 \\
3\end{array}$ & $\begin{array}{r}.00594 \\
.00782 \\
.00960 \\
.00285 \\
.00147\end{array}$ & $\begin{array}{l}20-40 \\
20-40 \\
20-40 \\
20-40 \\
20-40\end{array}$ & $\begin{array}{l}.00532 \\
.00962 \\
.00136 \\
.0165 \\
.00472\end{array}$ \\
\hline $\begin{array}{l}6.7 \\
6.7\end{array}$ & $\begin{array}{l}.48 \\
.22\end{array}$ & $\begin{array}{l}.025 \\
.025\end{array}$ & $\begin{array}{c}-- \\
15.0\end{array}$ & $\begin{array}{r}34 \\
2\end{array}$ & $\begin{array}{l}.0493 \\
.00148\end{array}$ & $\begin{array}{l}20-40 \\
20-40\end{array}$ & $\begin{array}{l}.0121 \\
.00157\end{array}$ \\
\hline
\end{tabular}

\section{Geneva Creek near Grant, Colo. 5}

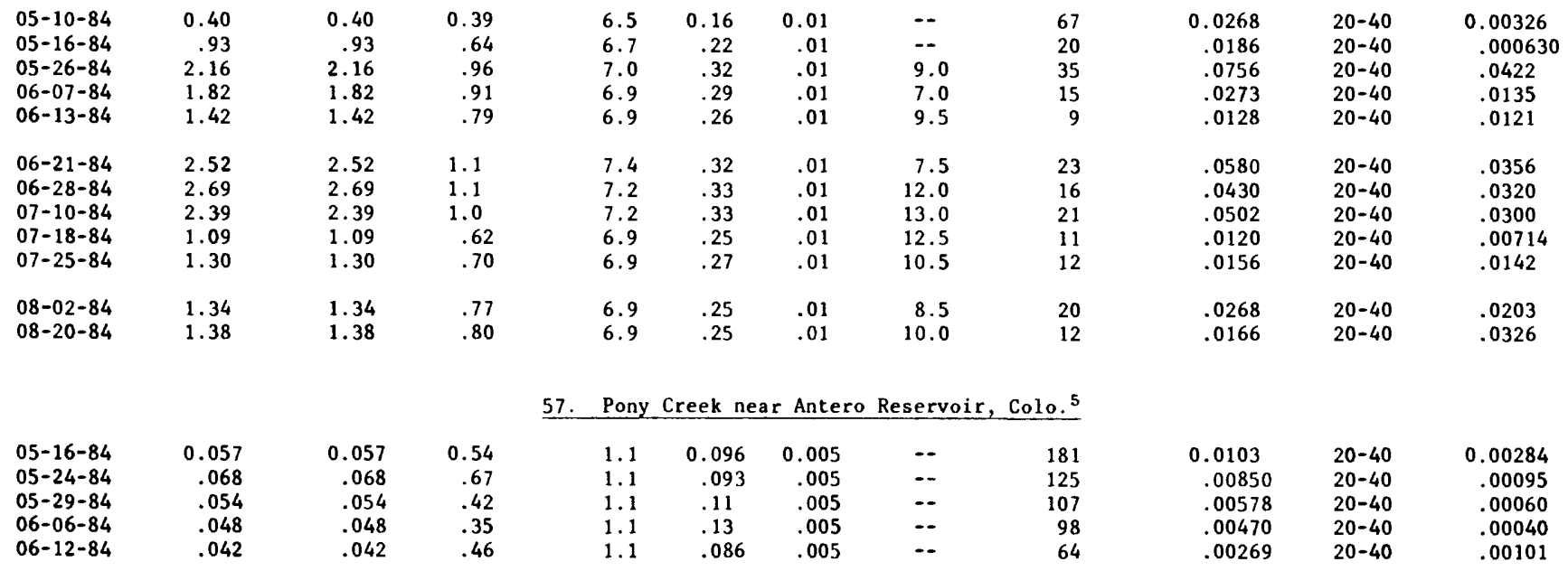


Table 1.--Hydraulic and sediment-transport data--Continued

\begin{tabular}{|c|c|c|c|c|c|c|c|c|c|c|c|}
\hline Date & $\begin{array}{c}\text { Water } \\
\text { discharge } \\
\text { (bedload) } \\
\left(\mathrm{m}^{3} / \mathrm{s}\right)\end{array}$ & $\begin{array}{c}\text { Water } \\
\text { discharge } \\
\text { (suspended } \\
\text { load) } \\
\left(\mathrm{m}^{3} / \mathrm{s}\right)\end{array}$ & $\begin{array}{l}\text { Mean } \\
\text { flow } \\
\text { velocity } \\
(\mathrm{m} / \mathrm{s})\end{array}$ & $\begin{array}{l}\text { Water- } \\
\text { surface } \\
\text { width } \\
(\mathrm{m})\end{array}$ & $\begin{array}{l}\text { Mean } \\
\text { flow } \\
\text { depth } \\
(m)\end{array}$ & $\begin{array}{l}\text { Water- } \\
\text { surface } \\
\text { slope } \\
(\mathrm{m} / \mathrm{m})\end{array}$ & $\begin{array}{l}\text { Water } \\
\text { temp. } \\
\left({ }^{\circ} \mathrm{C}\right)\end{array}$ & $\begin{array}{l}\text { Suspended } \\
\text { sediment } \\
\text { concern- } \\
\text { tration } \\
(\mathrm{mg} / \mathrm{L})\end{array}$ & $\begin{array}{c}\text { Suspended } \\
\text { load } \\
\left(\mathrm{kg}_{\mathrm{g}} / \mathrm{s}\right)\end{array}$ & $\begin{array}{l}\text { Number of } \\
\text { sampling } \\
\text { points } \\
\text { for } \\
\text { bedload }\end{array}$ & $\begin{array}{r}\text { Bedload } \\
\left(\mathrm{kg}_{\mathrm{g}} / \mathrm{s}\right)\end{array}$ \\
\hline
\end{tabular}

57. Pony Creek near Antero Reservoir, Colo. 5--Continued

\begin{tabular}{|c|c|c|c|c|c|c|c|c|c|c|c|}
\hline $\begin{array}{l}06-14-84 \\
06-19-84 \\
06-22-84 \\
06-26-84 \\
06-29-84\end{array}$ & $\begin{array}{r}0.040 \\
.037 \\
.037 \\
.031 \\
.028\end{array}$ & $\begin{array}{l}0.040 \\
.037 \\
.037 \\
.031 \\
.028\end{array}$ & $\begin{array}{r}0.34 \\
.33 \\
.36 \\
.29 \\
.27\end{array}$ & $\begin{array}{l}1.1 \\
1.1 \\
1.0 \\
1.1 \\
1.1\end{array}$ & $\begin{array}{l}0.11 \\
.10 \\
.10 \\
.098 \\
.096\end{array}$ & $\begin{array}{l}0.005 \\
.005 \\
.005 \\
.005 \\
.005\end{array}$ & $\begin{array}{l}-- \\
-- \\
-- \\
-- \\
--\end{array}$ & $\begin{array}{r}67 \\
82 \\
70 \\
58 \\
335\end{array}$ & $\begin{array}{r}0.00268 \\
.00303 \\
.00259 \\
.00180 \\
.00938\end{array}$ & $\begin{array}{l}20-40 \\
20-40 \\
20-40 \\
20-40 \\
20-40\end{array}$ & $\begin{array}{r}0.00102 \\
.00073 \\
.00050 \\
.00077 \\
.00039\end{array}$ \\
\hline $\begin{array}{l}07-03-84 \\
07-06-84 \\
07-10-84 \\
07-24-84\end{array}$ & $\begin{array}{l}.023 \\
.025 \\
.029 \\
.027\end{array}$ & $\begin{array}{l}.023 \\
.025 \\
.029 \\
.027\end{array}$ & $\begin{array}{l}.22 \\
.25 \\
.28 \\
.26\end{array}$ & $\begin{array}{l}1.1 \\
1.1 \\
1.1 \\
1.1\end{array}$ & $\begin{array}{l}.092 \\
.095 \\
.099 \\
.097\end{array}$ & $\begin{array}{l}.005 \\
.005 \\
.006 \\
.006\end{array}$ & $\begin{array}{l}-- \\
-- \\
-- \\
--\end{array}$ & $\begin{array}{l}59 \\
52 \\
45 \\
61\end{array}$ & $\begin{array}{l}.00136 \\
.00130 \\
.00131 \\
.00165\end{array}$ & $\begin{array}{l}20-40 \\
20-40 \\
20-40 \\
20-40\end{array}$ & $\begin{array}{l}.00053 \\
.00085 \\
.00198 \\
.00036\end{array}$ \\
\hline
\end{tabular}

58. North Fork of South Platte River at Shawnee, Colo. 5

\begin{tabular}{|c|c|c|c|c|c|c|c|c|c|c|c|}
\hline $\begin{array}{l}05-03-85 \\
05-06-85 \\
05-09-85 \\
05-14-85 \\
05-17-85\end{array}$ & $\begin{array}{l}3.12 \\
4.47 \\
5.81 \\
3.96 \\
4.50\end{array}$ & $\begin{array}{l}3.12 \\
4.47 \\
5.81 \\
3.96 \\
4.50\end{array}$ & $\begin{array}{r}0.70 \\
.85 \\
1.0 \\
.78 \\
.85\end{array}$ & $\begin{array}{l}15.0 \\
15.0 \\
15.5 \\
15.0 \\
15.0\end{array}$ & $\begin{array}{r}0.30 \\
.35 \\
.38 \\
.34 \\
.36\end{array}$ & $\begin{array}{l}-- \\
-- \\
-- \\
--\end{array}$ & $\begin{array}{l}-- \\
-- \\
-- \\
-- \\
--\end{array}$ & $\begin{array}{r}20 \\
17 \\
34 \\
12 \\
7\end{array}$ & $\begin{array}{c}0.0624 \\
.0760 \\
.198 \\
.0475 \\
.0315\end{array}$ & $\begin{array}{l}\geq 20 \\
\geq 20 \\
\geq 20 \\
\geq 20 \\
\geq 20\end{array}$ & $\begin{array}{c}0.0419 \\
.166 \\
.0385 \\
.0979 \\
.0634\end{array}$ \\
\hline $\begin{array}{l}05-21-85 \\
05-24-85 \\
05-28-85 \\
05-31-85 \\
06-10-85\end{array}$ & $\begin{array}{c}4.47 \\
5.81 \\
7.65 \\
7.62 \\
10.9\end{array}$ & $\begin{array}{c}4.47 \\
5.81 \\
7.65 \\
7.62 \\
10.9\end{array}$ & $\begin{array}{l}.87 \\
.96 \\
1.1 \\
1.1 \\
1.3\end{array}$ & $\begin{array}{l}15.0 \\
15.5 \\
15.5 \\
15.5 \\
16.0\end{array}$ & $\begin{array}{l}.34 \\
.40 \\
.43 \\
.43 \\
.52\end{array}$ & $\begin{array}{l}-- \\
-- \\
-- \\
-- \\
--\end{array}$ & $\begin{array}{l}-- \\
-- \\
-- \\
--\end{array}$ & $\begin{array}{r}7 \\
7 \\
22 \\
9 \\
23\end{array}$ & $\begin{array}{l}.0313 \\
.0407 \\
.168 \\
.0686 \\
.251\end{array}$ & $\begin{array}{l}\geq 20 \\
\geq 20 \\
\geq 20 \\
\geq 20 \\
\geq 20\end{array}$ & $\begin{array}{l}.0858 \\
.103 \\
.137 \\
.160 \\
.332\end{array}$ \\
\hline $\begin{array}{l}06-13-85 \\
06-17-85 \\
06-20-85 \\
06-25-85 \\
06-28-85\end{array}$ & $\begin{array}{l}9.94 \\
8.64 \\
8.35 \\
6.88 \\
5.75\end{array}$ & $\begin{array}{l}9.94 \\
8.64 \\
8.35 \\
6.88 \\
5.75\end{array}$ & $\begin{array}{l}1.2 \\
1.1 \\
1.1 \\
1.0 \\
.84\end{array}$ & $\begin{array}{l}16.0 \\
16.0 \\
16.0 \\
15.5 \\
15.5\end{array}$ & $\begin{array}{l}.51 \\
.48 \\
.47 \\
.44 \\
.44\end{array}$ & $\begin{array}{c}-- \\
-- \\
-- \\
0.0068 \\
\ldots\end{array}$ & $\begin{array}{r}8.5 \\
10.0 \\
10.0 \\
10.0 \\
9.0\end{array}$ & $\begin{array}{r}15 \\
6 \\
4 \\
10 \\
7\end{array}$ & $\begin{array}{l}.149 \\
.0518 \\
.0334 \\
.0688 \\
.0403\end{array}$ & $\begin{array}{l}\geq 20 \\
\geq 20 \\
\geq 20 \\
\geq 20 \\
\geq 20\end{array}$ & $\begin{array}{l}.167 \\
.145 \\
.345 \\
.185 \\
.0323\end{array}$ \\
\hline $\begin{array}{l}06-30-85 \\
07-03-85 \\
07-10-85 \\
07-16-85 \\
07-18-85 \\
07-23-85\end{array}$ & $\begin{array}{l}5.66 \\
5.07 \\
4.90 \\
3.94 \\
6.12 \\
4.87\end{array}$ & $\begin{array}{l}5.66 \\
5.07 \\
4.90 \\
3.94 \\
6.12 \\
4.87\end{array}$ & $\begin{array}{l}.87 \\
.84 \\
.89 \\
.80 \\
.92 \\
.86\end{array}$ & $\begin{array}{l}15.5 \\
15.0 \\
15.0 \\
15.0 \\
15.5 \\
15.0\end{array}$ & $\begin{array}{l}.42 \\
.40 \\
.36 \\
.33 \\
.43 \\
.38\end{array}$ & $\begin{array}{l}-- \\
-- \\
-- \\
-- \\
--\end{array}$ & $\begin{array}{r}8.5 \\
10.5 \\
11.0 \\
12.5 \\
12.5 \\
12.5\end{array}$ & $\begin{array}{r}6 \\
8 \\
26 \\
3 \\
372 \\
12\end{array}$ & $\begin{array}{c}.0340 \\
.0406 \\
.127 \\
.0118 \\
2.28 \\
.0584\end{array}$ & $\begin{array}{l}\geq 20 \\
\geq 20 \\
\geq 20 \\
\geq 20 \\
\geq 20 \\
\geq 20\end{array}$ & $\begin{array}{l}.190 \\
.0962 \\
.0664 \\
.0538 \\
.0701 \\
.0572\end{array}$ \\
\hline
\end{tabular}

59. North Fork of South Platte River at Crossons, Colo. 5

\begin{tabular}{|c|c|c|c|c|c|c|c|c|c|c|c|}
\hline $\begin{array}{l}05-14-85 \\
05-17-85 \\
05-21-85 \\
05-24-85 \\
05-28-85\end{array}$ & $\begin{array}{c}7.22 \\
7.36 \\
8.41 \\
9.26 \\
10.9\end{array}$ & $\begin{array}{c}7.22 \\
7.36 \\
8.41 \\
9.26 \\
10.9\end{array}$ & $\begin{array}{r}0.74 \\
.73 \\
.80 \\
.86 \\
.92\end{array}$ & $\begin{array}{l}19.5 \\
19.0 \\
19.5 \\
19.0 \\
19.0\end{array}$ & $\begin{array}{r}0.51 \\
.53 \\
.54 \\
.56 \\
.62\end{array}$ & $\begin{array}{l}-- \\
-- \\
-- \\
--\end{array}$ & $\begin{array}{l}-- \\
-- \\
-- \\
-- \\
--\end{array}$ & $\begin{array}{l}13 \\
16 \\
10 \\
20 \\
21\end{array}$ & $\begin{array}{l}0.0939 \\
.118 \\
.0841 \\
.185 \\
.229\end{array}$ & $\begin{array}{l}\geq 20 \\
\geq 20 \\
\geq 20 \\
\geq 20 \\
\geq 20\end{array}$ & $\begin{array}{c}0.164 \\
.0569 \\
.153 \\
.211 \\
.442\end{array}$ \\
\hline $\begin{array}{l}05-31-85 \\
06-13-85 \\
06-17-85 \\
06-20-85 \\
06-25-85\end{array}$ & $\begin{array}{c}11.0 \\
12.5 \\
12.3 \\
11.1 \\
9.63\end{array}$ & $\begin{array}{c}11.0 \\
12.5 \\
12.3 \\
11.1 \\
9.63\end{array}$ & $\begin{array}{l}.95 \\
.96 \\
.96 \\
.94 \\
.88\end{array}$ & $\begin{array}{l}19.0 \\
19.5 \\
19.5 \\
19.5 \\
19.0\end{array}$ & $\begin{array}{l}.61 \\
.67 \\
.66 \\
.61 \\
.57\end{array}$ & $\begin{array}{l}-- \\
-- \\
-- \\
-- \\
0.0047\end{array}$ & $\begin{array}{r}-- \\
9.0 \\
13.0 \\
9.0 \\
8.0\end{array}$ & $\begin{array}{r}13 \\
33 \\
11 \\
8 \\
21\end{array}$ & $\begin{array}{l}.143 \\
.413 \\
.135 \\
.0888 \\
.202\end{array}$ & $\begin{array}{l}\geq 20 \\
\geq 20 \\
\geq 20 \\
\geq 20 \\
\geq 20\end{array}$ & $\begin{array}{l}.343 \\
.292 \\
.224 \\
.221 \\
.0716\end{array}$ \\
\hline $\begin{array}{l}06-28-85 \\
06-30-85 \\
07-03-85 \\
07-10-85 \\
07-16-85 \\
07-23-85\end{array}$ & $\begin{array}{l}8.44 \\
7.33 \\
6.91 \\
6.23 \\
5.35 \\
6.74\end{array}$ & $\begin{array}{l}8.44 \\
7.33 \\
6.91 \\
6.23 \\
5.35 \\
6.74\end{array}$ & $\begin{array}{l}.78 \\
.75 \\
.69 \\
.67 \\
.65 \\
.74\end{array}$ & $\begin{array}{l}19.5 \\
19.5 \\
19.5 \\
19.0 \\
19.5 \\
19.5\end{array}$ & $\begin{array}{l}.56 \\
.50 \\
.52 \\
.48 \\
.42 \\
.47\end{array}$ & $\begin{array}{l}-- \\
-- \\
-- \\
-- \\
-- \\
--\end{array}$ & $\begin{array}{r}8.0 \\
12.5 \\
9.0 \\
14.5 \\
15.5 \\
15.5\end{array}$ & $\begin{array}{r}11 \\
10 \\
18 \\
5 \\
7 \\
7\end{array}$ & $\begin{array}{l}.0928 \\
.0733 \\
.124 \\
.0312 \\
.0375 \\
.0472\end{array}$ & $\begin{array}{l}\geq 20 \\
\geq 20 \\
\geq 20 \\
\geq 20 \\
\geq 20 \\
>20\end{array}$ & $\begin{array}{l}.202 \\
.0622 \\
.0512 \\
.0320 \\
.0434 \\
.103\end{array}$ \\
\hline
\end{tabular}


Table 1.--Hydraulic and sediment-transport data--Continued

\begin{tabular}{|c|c|c|c|c|c|c|c|c|c|c|c|}
\hline Date & $\begin{array}{c}\text { Water } \\
\text { discharge } \\
\text { (bedload) } \\
\left(\mathrm{m}^{3} / \mathrm{s}\right)\end{array}$ & $\begin{array}{c}\text { Water } \\
\text { discharge } \\
\text { (suspended } \\
\text { load) } \\
\left(\mathrm{m}^{3} / \mathrm{s}\right)\end{array}$ & $\begin{array}{l}\text { Mean } \\
\text { flow } \\
\text { velocity } \\
(\mathrm{m} / \mathrm{s})\end{array}$ & $\begin{array}{l}\text { Water- } \\
\text { surface } \\
\text { width } \\
(\mathrm{m})\end{array}$ & $\begin{array}{l}\text { Mean } \\
\text { flow } \\
\text { depth. } \\
\text { (m) }\end{array}$ & $\begin{array}{l}\text { Water- } \\
\text { surface } \\
\text { slope } \\
(\mathrm{m} / \mathrm{m})\end{array}$ & $\begin{array}{l}\text { Water } \\
\text { temp. } \\
\left({ }^{\circ} \mathrm{C}\right)\end{array}$ & $\begin{array}{l}\text { Suspended } \\
\text { sediment } \\
\text { concern- } \\
\text { tration } \\
(\mathrm{mg} / \mathrm{L})\end{array}$ & $\begin{array}{c}\text { Suspended } \\
\text { load } \\
(\mathrm{kg} / \mathrm{s})\end{array}$ & $\begin{array}{l}\text { Number of } \\
\text { sampling } \\
\text { points } \\
\text { for } \\
\text { bedload }\end{array}$ & $\begin{array}{r}\text { Bedload } \\
(\mathrm{kg} / \mathrm{s})\end{array}$ \\
\hline
\end{tabular}

60. North Fork of South Platte River at Buffalo, Colo. 5

\begin{tabular}{|c|c|c|c|c|c|c|c|c|c|c|c|}
\hline $\begin{array}{l}05-03-85 \\
05-06-85 \\
05-09-85 \\
05-15-85 \\
05-17-85\end{array}$ & $\begin{array}{c}10.6 \\
11.9 \\
13.7 \\
9.32 \\
8.98\end{array}$ & $\begin{array}{c}10.6 \\
11.9 \\
13.7 \\
9.32 \\
8.98\end{array}$ & $\begin{array}{l}1.1 \\
1.1 \\
1.2 \\
1.1 \\
1.0\end{array}$ & $\begin{array}{l}14.0 \\
14.5 \\
15.0 \\
14.0 \\
13.5\end{array}$ & $\begin{array}{r}0.66 \\
.73 \\
.74 \\
.62 \\
.64\end{array}$ & $\begin{array}{l}-- \\
-- \\
-- \\
-- \\
--\end{array}$ & $\begin{array}{l}-- \\
-- \\
-- \\
-- \\
--\end{array}$ & $\begin{array}{r}94 \\
85 \\
127 \\
34 \\
25\end{array}$ & $\begin{array}{l}0.996 \\
1.01 \\
1.74 \\
.317 \\
.225\end{array}$ & $\begin{array}{l}\geq 20 \\
\geq 20 \\
\geq 20 \\
\geq 20 \\
\geq 20\end{array}$ & $\begin{array}{l}1.36 \\
.623 \\
1.98 \\
2.41 \\
1.48\end{array}$ \\
\hline $\begin{array}{l}05-21-85 \\
05-23-85 \\
05-28-85 \\
05-30-85 \\
06-10-85\end{array}$ & $\begin{array}{r}9.46 \\
9.32 \\
11.9 \\
12.5 \\
18.0\end{array}$ & $\begin{array}{l}9.46 \\
9.32 \\
11.9 \\
12.5 \\
18.0\end{array}$ & $\begin{array}{l}1.0 \\
1.0 \\
1.2 \\
1.2 \\
1.4\end{array}$ & $\begin{array}{l}14.0 \\
14.0 \\
14.5 \\
15.0 \\
15.5\end{array}$ & $\begin{array}{l}.66 \\
.67 \\
.70 \\
.72 \\
.81\end{array}$ & $\begin{array}{l}-- \\
-- \\
-- \\
-- \\
--\end{array}$ & $\begin{array}{l}-- \\
-- \\
=- \\
-- \\
8.0\end{array}$ & $\begin{array}{l}21 \\
23 \\
31 \\
26 \\
38\end{array}$ & $\begin{array}{l}.199 \\
.214 \\
.369 \\
.325 \\
.684\end{array}$ & $\begin{array}{l}>20 \\
\geq 20 \\
\geq 20 \\
>20 \\
\geq 20\end{array}$ & $\begin{array}{l}.0753 \\
.107 \\
.401 \\
.457 \\
.705\end{array}$ \\
\hline $\begin{array}{l}06-12-85 \\
06-17-85 \\
06-19-85 \\
06-26-85 \\
06-27-85\end{array}$ & $\begin{array}{l}13.8 \\
12.7 \\
11.6 \\
11.2 \\
11.0\end{array}$ & $\begin{array}{l}13.8 \\
12.7 \\
11.6 \\
11.2 \\
11.0\end{array}$ & $\begin{array}{l}1.2 \\
1.2 \\
1.2 \\
1.1 \\
1.0\end{array}$ & $\begin{array}{l}15.0 \\
14.5 \\
14.5 \\
14.0 \\
14.0\end{array}$ & $\begin{array}{l}.74 \\
.70 \\
.70 \\
.69 \\
.74\end{array}$ & $\begin{array}{l}-- \\
-- \\
-- \\
0.0107 \\
--\end{array}$ & $\begin{array}{l}15.0 \\
14.0 \\
15.0 \\
12.5 \\
13.5\end{array}$ & $\begin{array}{l}15 \\
20 \\
13 \\
23 \\
17\end{array}$ & $\begin{array}{l}.207 \\
.254 \\
.151 \\
.258 \\
.187\end{array}$ & $\begin{array}{l}\geq 20 \\
\geq 20 \\
\geq 20 \\
\geq 20 \\
\geq 20\end{array}$ & $\begin{array}{l}.558 \\
.149 \\
.182 \\
.122 \\
.0502\end{array}$ \\
\hline $\begin{array}{l}06-30-85 \\
07-02-85 \\
07-11-85 \\
07-16-85 \\
07-24-85\end{array}$ & $\begin{array}{l}7.67 \\
6.88 \\
5.95 \\
5.38 \\
6.23\end{array}$ & $\begin{array}{l}7.67 \\
6.88 \\
5.95 \\
5.38 \\
6.23\end{array}$ & $\begin{array}{l}.94 \\
.83 \\
.75 \\
.71 \\
.81\end{array}$ & $\begin{array}{l}13.0 \\
13.0 \\
13.0 \\
13.0 \\
13.0\end{array}$ & $\begin{array}{l}.62 \\
.63 \\
.62 \\
.59 \\
.59\end{array}$ & $\begin{array}{l}-- \\
-- \\
-- \\
--\end{array}$ & $\begin{array}{l}13.5 \\
14.0 \\
12.5 \\
12.5 \\
13.0\end{array}$ & $\begin{array}{r}11 \\
12 \\
2 \\
11 \\
15\end{array}$ & $\begin{array}{l}.0844 \\
.0826 \\
.0119 \\
.0592 \\
.0935\end{array}$ & $\begin{array}{l}>20 \\
\geq 20 \\
\geq 20 \\
\geq 20 \\
\geq 20\end{array}$ & $\begin{array}{l}.0119 \\
.0729 \\
.0138 \\
.0262 \\
.0309\end{array}$ \\
\hline
\end{tabular}

61. South Fork of South Platte River above Vermillion Creek, Colo. 5

\begin{tabular}{|c|c|c|c|c|c|c|c|c|c|c|c|}
\hline $\begin{array}{l}05-15-85 \\
05-16-85 \\
05-21-85 \\
05-23-85 \\
05-29-85\end{array}$ & $\begin{array}{c}9.88 \\
9.63 \\
10.5 \\
10.4 \\
10.6\end{array}$ & $\begin{array}{c}9.88 \\
9.63 \\
10.5 \\
10.4 \\
10.6\end{array}$ & $\begin{array}{r}0.63 \\
.61 \\
.67 \\
.66 \\
.68\end{array}$ & $\begin{array}{l}25.5 \\
25.5 \\
25.0 \\
25.5 \\
25.0\end{array}$ & $\begin{array}{r}0.61 \\
.62 \\
.63 \\
.62 \\
.62\end{array}$ & $\begin{array}{l}-- \\
-- \\
-- \\
-- \\
--\end{array}$ & $\begin{array}{l}-- \\
-- \\
-- \\
-- \\
--\end{array}$ & $\begin{array}{l}68 \\
52 \\
50 \\
72 \\
47\end{array}$ & $\begin{array}{r}0.672 \\
.501 \\
.525 \\
.749 \\
.498\end{array}$ & $\begin{array}{l}\geq 20 \\
\geq 20 \\
\geq 20 \\
\geq 20 \\
\geq 20\end{array}$ & $\begin{array}{l}1.31 \\
1.32 \\
1.02 \\
1.29 \\
1.13\end{array}$ \\
\hline $\begin{array}{l}05-30-85 \\
06-11-85 \\
06-12-85 \\
06-18-85 \\
06-19-85\end{array}$ & $\begin{array}{l}10.3 \\
14.0 \\
14.8 \\
14.7 \\
14.9\end{array}$ & $\begin{array}{l}10.3 \\
14.0 \\
14.8 \\
14.7 \\
14.9\end{array}$ & $\begin{array}{l}.66 \\
.73 \\
.74 \\
.81 \\
.80\end{array}$ & $\begin{array}{l}25.0 \\
25.5 \\
25.5 \\
25.5 \\
25.5\end{array}$ & $\begin{array}{l}.62 \\
.75 \\
.78 \\
.72 \\
.73\end{array}$ & $\begin{array}{l}-- \\
-- \\
-- \\
--\end{array}$ & $\begin{array}{l}17.5 \\
12.5 \\
18.0 \\
14.0\end{array}$ & $\begin{array}{l}36 \\
55 \\
54 \\
37 \\
30\end{array}$ & $\begin{array}{l}.371 \\
.770 \\
.799 \\
.544 \\
.447\end{array}$ & $\begin{array}{l}\geq 20 \\
\geq 20 \\
\geq 20 \\
\geq 20 \\
\geq 20\end{array}$ & $\begin{array}{l}1.40 \\
.890 \\
.719 \\
.782 \\
.932\end{array}$ \\
\hline $\begin{array}{l}06-27-85 \\
07-01-85 \\
07-02-85 \\
07-12-85 \\
07-17-85\end{array}$ & $\begin{array}{c}10.7 \\
9.43 \\
8.41 \\
7.96 \\
8.47\end{array}$ & $\begin{array}{c}10.7 \\
9.43 \\
8.41 \\
7.96 \\
8.47\end{array}$ & $\begin{array}{l}.67 \\
.66 \\
.61 \\
.59 \\
.61\end{array}$ & $\begin{array}{l}25.5 \\
25.5 \\
25.5 \\
25.5 \\
25.5\end{array}$ & $\begin{array}{l}.62 \\
.56 \\
.55 \\
.53 \\
.54\end{array}$ & $\begin{array}{c}0.0004 \\
-- \\
-- \\
-- \\
--\end{array}$ & $\begin{array}{l}15.5 \\
17.0 \\
12.5 \\
15.5 \\
21.5\end{array}$ & $\begin{array}{l}15 \\
17 \\
10 \\
29 \\
33\end{array}$ & $\begin{array}{l}.161 \\
.160 \\
.0841 \\
.231 \\
.280\end{array}$ & $\begin{array}{l}\geq 20 \\
\geq 20 \\
\geq 20 \\
\geq 20 \\
\geq 20\end{array}$ & $\begin{array}{l}.550 \\
.622 \\
.0825 \\
.167 \\
.189\end{array}$ \\
\hline $\begin{array}{l}07-18-85 \\
07-25-85\end{array}$ & $\begin{array}{l}8.44 \\
14.8\end{array}$ & $\begin{array}{r}8.44 \\
14.8\end{array}$ & $\begin{array}{l}.58 \\
.79\end{array}$ & $\begin{array}{l}25.5 \\
26.0\end{array}$ & $\begin{array}{l}.57 \\
.73\end{array}$ & -- & $\begin{array}{l}15.5 \\
16.0\end{array}$ & $\begin{array}{l}31 \\
35\end{array}$ & $\begin{array}{l}.262 \\
.518\end{array}$ & $\begin{array}{l}\geq 20 \\
\geq 20\end{array}$ & $\begin{array}{l}.284 \\
1.37\end{array}$ \\
\hline
\end{tabular}

62. South Fork of South Platte River at Trumbul 1 , Colo. 5

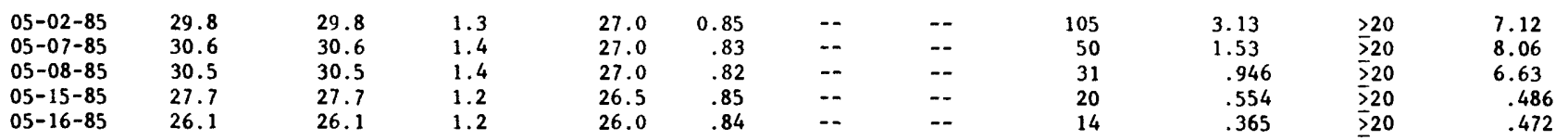


Table 1.--Hydraulic and sediment-transport data--Cont inued

\begin{tabular}{|c|c|c|c|c|c|c|c|c|c|c|c|}
\hline Date & $\begin{array}{c}\text { Water } \\
\text { discharge } \\
\text { (bedload) } \\
\left(\mathrm{m}^{3} / \mathrm{s}\right)\end{array}$ & $\begin{array}{c}\text { Water } \\
\text { discharge } \\
\text { (suspended } \\
\text { load) } \\
\left(\mathrm{m}^{3} / \mathrm{s}\right)\end{array}$ & $\begin{array}{l}\text { Mean } \\
\text { flow } \\
\text { velocity } \\
(\mathrm{m} / \mathrm{s})\end{array}$ & $\begin{array}{l}\text { Water- } \\
\text { surface } \\
\text { width } \\
\text { (m) }\end{array}$ & $\begin{array}{l}\text { Mean } \\
\text { flow } \\
\text { depth } \\
\text { (m) }\end{array}$ & $\begin{array}{l}\text { Water- } \\
\text { surface } \\
\text { slope } \\
(\mathrm{m} / \mathrm{m})\end{array}$ & $\begin{array}{l}\text { Water } \\
\text { temp. } \\
\left({ }^{\circ} \mathrm{C}\right)\end{array}$ & $\begin{array}{c}\text { Suspended } \\
\text { sediment } \\
\text { concern- } \\
\text { tration } \\
(\mathrm{mg} / \mathrm{L})\end{array}$ & $\begin{array}{c}\text { Suspended } \\
\text { load } \\
(\mathrm{kg} / \mathrm{s})\end{array}$ & $\begin{array}{c}\text { Number of } \\
\text { sampling } \\
\text { points } \\
\text { for } \\
\text { bedload }\end{array}$ & $\begin{array}{r}\text { Bedload } \\
(\mathrm{kg} / \mathrm{s})\end{array}$ \\
\hline
\end{tabular}

62. South Fork of South Platte River at Trumbul1, Colo.5--Continued

\begin{tabular}{|c|c|c|c|c|c|c|c|c|c|c|c|}
\hline $\begin{array}{l}05-22-85 \\
05-23-85 \\
05-29-85 \\
05-30-85 \\
06-11-85\end{array}$ & $\begin{array}{l}26.9 \\
24.7 \\
22.8 \\
24.7 \\
29.3\end{array}$ & $\begin{array}{l}26.9 \\
24.7 \\
22.8 \\
24.7 \\
29.3\end{array}$ & $\begin{array}{l}1.2 \\
1.1 \\
1.1 \\
1.2 \\
1.3\end{array}$ & $\begin{array}{l}25.5 \\
26.5 \\
25.5 \\
26.5 \\
26.5\end{array}$ & $\begin{array}{r}0.86 \\
.82 \\
.79 \\
.80 \\
.87\end{array}$ & $\begin{array}{l}-- \\
-- \\
-- \\
--\end{array}$ & $\begin{array}{l}-- \\
-- \\
-- \\
-- \\
15.5\end{array}$ & $\begin{array}{r}23 \\
24 \\
11 \\
11 \\
8\end{array}$ & $\begin{array}{r}0.619 \\
.593 \\
.251 \\
.272 \\
.234\end{array}$ & $\begin{array}{l}\geq 20 \\
\geq 20 \\
\geq 20 \\
\geq 20 \\
\geq 20\end{array}$ & $\begin{array}{r}0.271 \\
.219 \\
.356 \\
.600 \\
.173\end{array}$ \\
\hline $\begin{array}{l}06-12-85 \\
06-18-85 \\
06-26-85 \\
07-01-85 \\
07-02-85\end{array}$ & $\begin{array}{l}30.4 \\
26.7 \\
19.4 \\
12.8 \\
12.8\end{array}$ & $\begin{array}{l}30.4 \\
26.7 \\
19.4 \\
12.8 \\
12.8\end{array}$ & $\begin{array}{l}1.3 \\
1.3 \\
1.1 \\
.81 \\
.85\end{array}$ & $\begin{array}{l}26.5 \\
25.5 \\
25.5 \\
26.0 \\
25.0\end{array}$ & $\begin{array}{l}.91 \\
.83 \\
.71 \\
.60 \\
.61\end{array}$ & $\begin{array}{l}-- \\
-- \\
0.0035 \\
-- \\
--\end{array}$ & $\begin{array}{l}16.0 \\
17.5 \\
18.5 \\
18.0 \\
18.0\end{array}$ & $\begin{array}{l}5 \\
3 \\
4 \\
2 \\
8\end{array}$ & $\begin{array}{l}.152 \\
.0801 \\
.0776 \\
.0256 \\
.102\end{array}$ & $\begin{array}{l}\geq 20 \\
\geq 20 \\
\geq 20 \\
\geq 20 \\
\geq 20\end{array}$ & $\begin{array}{l}.170 \\
.0866 \\
.115 \\
.0102 \\
.0240\end{array}$ \\
\hline $\begin{array}{l}07-11-85 \\
07-17-85 \\
07-24-85\end{array}$ & $\begin{array}{l}22.3 \\
19.3 \\
13.7\end{array}$ & $\begin{array}{l}22.3 \\
19.3 \\
13.7\end{array}$ & $\begin{array}{l}1.0 \\
1.0 \\
.86\end{array}$ & $\begin{array}{l}26.5 \\
26.0 \\
25.5\end{array}$ & $\begin{array}{l}.80 \\
.74 \\
.63\end{array}$ & $\begin{array}{l}-- \\
--\end{array}$ & $\begin{array}{r}8.0 \\
7.5 \\
14.0\end{array}$ & $\begin{array}{r}10 \\
4 \\
55\end{array}$ & $\begin{array}{l}.223 \\
.0772 \\
.754\end{array}$ & $\begin{array}{l}\geq 20 \\
\geq 20 \\
>20\end{array}$ & $\begin{array}{l}.493 \\
.0892 \\
.0278\end{array}$ \\
\hline
\end{tabular}

63. Buffalo Creek at Buffalo, Colo. 5

$\begin{array}{lrrr}05-03-85 & 1.56 & 1.56 & 0.71 \\ 05-06-85 & 1.30 & 1.30 & .63 \\ 05-08-85 & 1.30 & 1.30 & .71 \\ 05-14-85 & 1.10 & 1.10 & .61 \\ 05-17-85 & .99 & .99 & .56 \\ & & & \\ 05-21-85 & 1.30 & 1.30 & .64 \\ 05-23-85 & 1.22 & 1.22 & .62 \\ 05-28-85 & 1.16 & 1.16 & .64 \\ 05-30-85 & 1.10 & 1.10 & .62 \\ 06-10-85 & 1.16 & 1.16 & .64 \\ & & & \\ 06-12-85 & 1.02 & 1.02 & .59 \\ 06-17-85 & .93 & .93 & .58 \\ 06-19-85 & .88 & .88 & .56 \\ 06-26-85 & .91 & .91 & .59 \\ 06-30-85 & .68 & .68 & .52 \\ & & & \\ 07-02-85 & .59 & .59 & .50 \\ 07-11-85 & .79 & .79 & .52 \\ 07-16-85 & .85 & .85 & .53 \\ 07-19-85 & .96 & .96 & .54 \\ 07-23-85 & .42 & .42 & .35\end{array}$

$\begin{array}{lllll}5.7 & 0.39 & -- & -- & 76 \\ 5.5 & .37 & -- & -- & 67 \\ 5.5 & .33 & -- & -- & 65 \\ 5.5 & .33 & -- & -- & 29 \\ 5.4 & .33 & -- & -- & 27 \\ & & & & \\ 5.5 & .37 & -- & -- & 33 \\ 5.5 & .36 & -- & -- & 34 \\ 5.5 & .33 & -- & -- & 20 \\ 5.4 & .33 & -- & 12.5 & 16 \\ 5.4 & .33 & -- & 15.5 & 23 \\ & & & & \\ 5.4 & .32 & -- & 15.0 & 13 \\ 5.3 & .30 & -- & 17.0 & 16 \\ 5.3 & .30 & -- & 10.0 & 9 \\ 5.3 & .29 & 0.0009 & 13.5 & 32 \\ 5.2 & .25 & -- & 13.5 & 15 \\ & & & & \\ 5.2 & .23 & -- & 13.5 & 13 \\ 5.3 & .29 & -- & 13.5 & 15 \\ 5.3 & .31 & -- & 15.5 & 15 \\ 5.4 & .33 & -- & 15.5 & 19 \\ 5.2 & .23 & -- & 15.5 & 16\end{array}$

0.119
.0871
.0845
.0319
.0267

.0429
.0415
.0232
.0176
.0267
.0133
.0149
.00792
.0291
.0102
.00767
.0119
.0128
.0182
.00672

1.36

$\begin{array}{ll}\geq 20 & 1.36 \\ \geq 20 & .412 \\ \geq 20 & .545 \\ \geq 20 & .590\end{array}$

$>20$

$>20 \quad .301$

$\geq 20 \quad .0852$

$>20 \quad .384$

$\geq 20 \quad .288$

$\begin{array}{ll}>20 & .377\end{array}$

.59

.56

.52

.50

.53

.35

64. Trail Creek near Westcreek, Colo. 5

$\begin{array}{rrrrrrrr}2.6 & 0.18 & 0.018 & -- & 38 & 0.00494 & 40 & 0.00540 \\ 2.8 & .20 & .018 & -- & 57 & .0188 & 40 & .0120 \\ 3.0 & .25 & .018 & -- & 242 & .119 & 40 & .00968 \\ 7.2 & .16 & .018 & -- & 308 & .182 & 40 & .0122 \\ 2.2 & .23 & .018 & -- & 66 & .0191 & 40 & .00251 \\ 2.5 & .17 & .018 & -- & 53 & .0122 & 40 & .00386 \\ 2.4 & .16 & .018 & -- & 57 & .0120 & 40 & .00370 \\ 2.4 & .14 & .018 & -- & 23 & .00368 & 40 & .00318 \\ 2.4 & .15 & .018 & -- & 56 & .00952 & 40 & .00392 \\ 2.1 & .12 & .018 & -- & 22 & .00286 & 40 & .00105\end{array}$


Table 1.--Hydraulic and sediment-transport data--Continued

\begin{tabular}{|c|c|c|c|c|c|c|c|c|c|c|c|}
\hline Date & $\begin{array}{c}\text { Water } \\
\text { discharge } \\
\text { (bedload) } 1 \\
\left(\mathrm{~m}^{3} / \mathrm{s}\right)\end{array}$ & $\begin{array}{c}\text { Water } \\
\text { discharge } \\
\text { (suspended } \\
\text { load })^{2} \\
\left(\mathrm{~m}^{3} / \mathrm{s}\right)\end{array}$ & $\begin{array}{l}\text { Mean } \\
\text { flow } \\
\text { velocity } \\
(\mathrm{m} / \mathrm{s})\end{array}$ & $\begin{array}{l}\text { Water- } \\
\text { surface } \\
\text { width } \\
\text { (m) }\end{array}$ & $\begin{array}{l}\text { Mean } \\
\text { flow } \\
\text { depth } \\
(m)\end{array}$ & $\begin{array}{l}\text { Water- } \\
\text { surface } \\
\text { slope } \\
(\mathrm{m} / \mathrm{m})\end{array}$ & $\begin{array}{l}\text { Water } \\
\text { temp. } \\
\left({ }^{\circ} \mathrm{C}\right)\end{array}$ & $\begin{array}{c}\text { Suspended } \\
\text { sediment } \\
\text { concern- } \\
\text { tration } \\
\left(m_{g} / L\right)\end{array}$ & $\begin{array}{c}\text { Suspended } \\
\text { load } \\
(\mathrm{kg} / \mathrm{s})\end{array}$ & $\begin{array}{l}\text { Number of } \\
\text { sampling } \\
\text { points } \\
\text { for } \\
\text { bedload }\end{array}$ & $\begin{array}{r}\text { Bedload } \\
(\mathrm{kg} / \mathrm{s})\end{array}$ \\
\hline
\end{tabular}

64. Trail Creek near Westcreek, Colo. ${ }^{5}$--Continued

$\begin{array}{cccccccccccc}07-27-84 & 0.071 & 0.071 & 0.37 & 2.0 & 0.098 & 0.018 & -2 & 16 & 0.00114 & 40 & 0.00138 \\ 08-13-84 & .20 & .20 & .44 & 2.5 & .18 & .018 & -. & 193 & .0386 & 40 & .0271 \\ 09-04-84 & .31 & .31 & .69 & 2.5 & .18 & .018 & -. & 106 & .0329 & 40 & .258\end{array}$

\section{Horse Creek near Westcreek, Colo. 5}

\begin{tabular}{|c|c|c|c|c|c|c|c|c|c|c|c|}
\hline $\begin{array}{l}05-02-85 \\
05-07-85 \\
05-08-85 \\
05-15-85 \\
05-16-85\end{array}$ & $\begin{array}{l}7.84 \\
5.98 \\
5.61 \\
4.90 \\
4.47\end{array}$ & $\begin{array}{l}7.84 \\
5.98 \\
5.61 \\
4.90 \\
4.47\end{array}$ & $\begin{array}{l}1.6 \\
1.4 \\
1.3 \\
1.2 \\
1.2\end{array}$ & $\begin{array}{l}9.3 \\
8.3 \\
8.4 \\
8.1 \\
7.8\end{array}$ & $\begin{array}{r}0.54 \\
.50 \\
.51 \\
.50 \\
.50\end{array}$ & $\begin{array}{l}-- \\
-- \\
-- \\
--\end{array}$ & $\begin{array}{l}- \\
-- \\
-- \\
--\end{array}$ & $\begin{array}{r}229 \\
138 \\
106 \\
79 \\
64\end{array}$ & $\begin{array}{l}1.80 \\
.825 \\
.595 \\
.387 \\
.286\end{array}$ & $\begin{array}{l}\geq 20 \\
\geq 20 \\
\geq 20 \\
\geq 20 \\
\geq 20\end{array}$ & $\begin{array}{r}10.0 \\
.555 \\
.522 \\
.227 \\
.157\end{array}$ \\
\hline $\begin{array}{l}05-22-85 \\
05-23-85 \\
05-29-85 \\
05-30-85 \\
06-11-85\end{array}$ & $\begin{array}{l}5.64 \\
5.13 \\
4.05 \\
3.51 \\
2.69\end{array}$ & $\begin{array}{l}5.64 \\
5.13 \\
4.05 \\
3.51 \\
2.69\end{array}$ & $\begin{array}{l}1.3 \\
1.2 \\
1.1 \\
.99 \\
.85\end{array}$ & $\begin{array}{l}8.0 \\
8.2 \\
7.9 \\
7.7 \\
7.7\end{array}$ & $\begin{array}{l}.56 \\
.54 \\
.48 \\
.46 \\
.41\end{array}$ & $\begin{array}{l}-- \\
-- \\
--\end{array}$ & $\begin{array}{c}-- \\
-- \\
-- \\
-- \\
14.5\end{array}$ & $\begin{array}{l}88 \\
48 \\
34 \\
32 \\
31\end{array}$ & $\begin{array}{l}.496 \\
.246 \\
.138 \\
.112 \\
.0834\end{array}$ & $\begin{array}{l}\geq 20 \\
\geq 20 \\
\geq 20 \\
\geq 20 \\
\geq 20\end{array}$ & $\begin{array}{l}1.30 \\
.547 \\
.307 \\
.419 \\
.0309\end{array}$ \\
\hline $\begin{array}{l}06-12-85 \\
06-18-85 \\
06-19-85 \\
06-27-85 \\
07-01-85\end{array}$ & $\begin{array}{l}2.49 \\
2.04 \\
1.98 \\
1.30 \\
1.36\end{array}$ & $\begin{array}{l}2.49 \\
2.04 \\
1.98 \\
1.30 \\
1.36\end{array}$ & $\begin{array}{l}.88 \\
.72 \\
.70 \\
.58 \\
.63\end{array}$ & $\begin{array}{l}7.6 \\
7.3 \\
7.3 \\
7.0 \\
7.1\end{array}$ & $\begin{array}{l}.38 \\
.38 \\
.39 \\
.32 \\
.30\end{array}$ & $\begin{array}{c}-- \\
- \\
0 \\
0.0039 \\
--\end{array}$ & $\begin{array}{l}15.5 \\
17.5 \\
17.0 \\
10.0 \\
18.0\end{array}$ & $\begin{array}{l}29 \\
13 \\
11 \\
19 \\
15\end{array}$ & $\begin{array}{l}.0722 \\
.0265 \\
.0218 \\
.0247 \\
.0204\end{array}$ & $\begin{array}{l}\geq 20 \\
\geq 20 \\
\geq 20 \\
\geq 20 \\
\geq 20\end{array}$ & $\begin{array}{l}.568 \\
.139 \\
.0569 \\
.0147 \\
.0305\end{array}$ \\
\hline $\begin{array}{l}07-02-85 \\
07-12-85 \\
07-17-85 \\
07-19-85 \\
07-24-85 \\
07-25-85\end{array}$ & $\begin{array}{l}1.27 \\
1.10 \\
1.33 \\
1.30 \\
2.29 \\
2.38\end{array}$ & $\begin{array}{l}1.27 \\
1.10 \\
1.33 \\
1.30 \\
2.29 \\
2.38\end{array}$ & $\begin{array}{l}.54 \\
.54 \\
.60 \\
.61 \\
.94 \\
1.0\end{array}$ & $\begin{array}{l}7.2 \\
6.9 \\
7.1 \\
7.1 \\
7.4 \\
7.7\end{array}$ & $\begin{array}{l}.33 \\
.29 \\
.31 \\
.30 \\
.33 \\
.31\end{array}$ & $\begin{array}{l}-- \\
-- \\
-- \\
-- \\
--\end{array}$ & $\begin{array}{l}15.5 \\
18.5 \\
21.5 \\
18.5 \\
17.0 \\
16.0\end{array}$ & $\begin{array}{l}14 \\
12 \\
19 \\
14 \\
37 \\
41\end{array}$ & $\begin{array}{l}.0178 \\
.0132 \\
.0253 \\
.0182 \\
.0847 \\
.0976\end{array}$ & $\begin{array}{l}\geq 20 \\
\geq 20 \\
\geq 20 \\
\geq 20 \\
\geq 20 \\
\geq 20\end{array}$ & $\begin{array}{l}.0218 \\
.00729 \\
.0347 \\
.0324 \\
1.79 \\
1.82\end{array}$ \\
\hline
\end{tabular}

66. Blue River below Green Mountain Reservoir, Colo. 5

\begin{tabular}{|c|c|c|c|c|c|c|c|c|c|c|c|}
\hline $\begin{array}{l}05-30-85 \\
06-04-85 \\
06-06-85 \\
06-10-85 \\
06-17-85\end{array}$ & $\begin{array}{l}25.1 \\
21.5 \\
21.3 \\
31.7 \\
36.4\end{array}$ & $\begin{array}{l}25.1 \\
21.5 \\
21.3 \\
31.7 \\
36.4\end{array}$ & $\begin{array}{r}0.96 \\
.83 \\
.85 \\
1.1 \\
1.1\end{array}$ & $\begin{array}{l}33.5 \\
33.0 \\
33.5 \\
34.0 \\
34.0\end{array}$ & $\begin{array}{r}0.79 \\
.78 \\
.74 \\
.88 \\
.94\end{array}$ & $\begin{array}{l}-- \\
-- \\
-- \\
-- \\
--\end{array}$ & $\begin{array}{c}-- \\
-- \\
-- \\
-- \\
12.5\end{array}$ & $\begin{array}{r}8 \\
4 \\
4 \\
31 \\
11\end{array}$ & $\begin{array}{l}0.201 \\
.0860 \\
.0852 \\
.983 \\
.400\end{array}$ & $\begin{array}{l}\geq 20 \\
\geq 20 \\
\geq 20 \\
\geq 20 \\
\geq 20\end{array}$ & $\begin{array}{r}0.0439 \\
.0518 \\
.0188 \\
.0918 \\
.0726\end{array}$ \\
\hline $\begin{array}{l}06-18-85 \\
06-25-85 \\
06-26-85 \\
06-30-85 \\
07-10-85\end{array}$ & $\begin{array}{l}43.8 \\
43.6 \\
46.1 \\
37.2 \\
18.0\end{array}$ & $\begin{array}{l}43.8 \\
43.6 \\
46.1 \\
37.2 \\
18.0\end{array}$ & $\begin{array}{l}1.3 \\
1.2 \\
1.3 \\
1.2 \\
.81\end{array}$ & $\begin{array}{l}34.5 \\
34.5 \\
34.5 \\
34.0 \\
32.5\end{array}$ & $\begin{array}{l}1.0 \\
1.0 \\
1.0 \\
.94 \\
.68\end{array}$ & $\begin{array}{l}-- \\
-- \\
0 \\
0.0026 \\
--\end{array}$ & $\begin{array}{l}11.0 \\
12.5 \\
10.0 \\
13.5 \\
14.5\end{array}$ & $\begin{array}{r}19 \\
15 \\
10 \\
10 \\
5\end{array}$ & $\begin{array}{l}.832 \\
.654 \\
.461 \\
.372 \\
.0900\end{array}$ & $\begin{array}{l}\geq 20 \\
\geq 20 \\
\geq 20 \\
\geq 20 \\
\geq 20\end{array}$ & $\begin{array}{l}.143 \\
.0443 \\
.102 \\
.0677 \\
.00188\end{array}$ \\
\hline $\begin{array}{l}07-17-85 \\
07-31-85\end{array}$ & $\begin{array}{l}31.0 \\
28.1\end{array}$ & $\begin{array}{l}31.0 \\
28.1\end{array}$ & $\begin{array}{c}1.0 \\
.90\end{array}$ & $\begin{array}{l}34.0 \\
33.5\end{array}$ & $\begin{array}{l}.91 \\
.93\end{array}$ & $\cdots$ & $\begin{array}{l}13.5 \\
15.5\end{array}$ & $\begin{array}{l}6 \\
7\end{array}$ & $\begin{array}{l}.186 \\
.197\end{array}$ & $\begin{array}{l}\geq 20 \\
\geq 20\end{array}$ & $\begin{array}{l}.106 \\
.126\end{array}$ \\
\hline
\end{tabular}

67. Williams Fork near Leal, Colo. 5

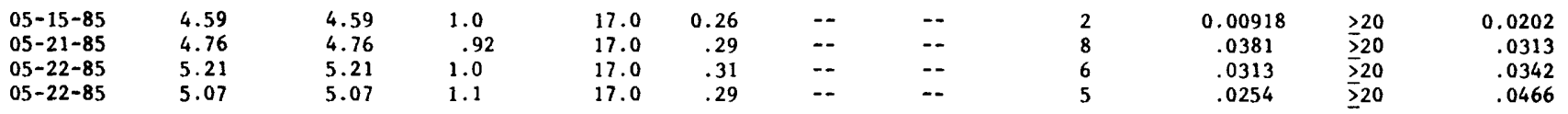


Table 1.--Hydraulic and sediment-transport data--Continued

\begin{tabular}{|c|c|c|c|c|c|c|c|c|c|c|c|}
\hline Date & $\begin{array}{c}\text { Water } \\
\text { discharge } \\
\text { (bedload) } \\
\left(\mathrm{m}^{3} / \mathrm{s}\right)\end{array}$ & $\begin{array}{c}\text { Water } \\
\text { discharge } \\
\text { (suspended } \\
\text { load) } \\
\left(\mathrm{m}^{3} / \mathrm{s}\right)\end{array}$ & $\begin{array}{l}\text { Mean } \\
\text { flow } \\
\text { velocity } \\
(\mathrm{m} / \mathrm{s})\end{array}$ & $\begin{array}{l}\text { Water- } \\
\text { surface } \\
\text { width } \\
\text { (m) }\end{array}$ & $\begin{array}{l}\text { Mean } \\
\text { flow } \\
\text { depth } \\
(\mathrm{m})\end{array}$ & $\begin{array}{l}\text { Water- } \\
\text { surface } \\
\text { slope } \\
(\mathrm{m} / \mathrm{m})\end{array}$ & $\begin{array}{l}\text { Water } \\
\text { temp. } \\
\left({ }^{\circ} \mathrm{C}\right)\end{array}$ & $\begin{array}{l}\text { Suspended } \\
\text { sediment } \\
\text { concern- } \\
\text { tration } \\
(\mathrm{mg} / \mathrm{L})\end{array}$ & $\begin{array}{c}\text { Suspended } \\
\text { load } \\
(\mathrm{kg} / \mathrm{s})\end{array}$ & $\begin{array}{l}\text { Number of } \\
\text { sampling } \\
\text { points } \\
\text { for } \\
\text { bedload }\end{array}$ & $\begin{array}{r}\text { Bedload } \\
(\mathrm{kg} / \mathrm{s})\end{array}$ \\
\hline
\end{tabular}

67. Williams Fork near Leal, Colo. ${ }^{5}$--Cont inued

\begin{tabular}{|c|c|c|c|c|c|c|c|c|c|c|c|}
\hline $\begin{array}{l}05-28-85 \\
05-29-85 \\
06-05-85 \\
06-06-85 \\
06-10-85\end{array}$ & $\begin{array}{l}9.71 \\
9.88 \\
14.2 \\
15.3 \\
22.7\end{array}$ & $\begin{array}{l}9.71 \\
9.88 \\
14.2 \\
15.3 \\
22.7\end{array}$ & $\begin{array}{l}1.1 \\
1.2 \\
1.4 \\
1.4 \\
1.8\end{array}$ & $\begin{array}{l}17.5 \\
17.5 \\
17.5 \\
18.0 \\
21.0\end{array}$ & $\begin{array}{r}0.49 \\
.49 \\
.59 \\
.60 \\
.62\end{array}$ & $\begin{array}{l}-- \\
-- \\
-- \\
--\end{array}$ & $\begin{array}{l}-- \\
-- \\
8.0 \\
-- \\
--\end{array}$ & $\begin{array}{r}15 \\
15 \\
9 \\
10 \\
12\end{array}$ & $\begin{array}{r}0.146 \\
.148 \\
.128 \\
.153 \\
.272\end{array}$ & $\begin{array}{l}\geq 20 \\
\geq 20 \\
\geq 20 \\
\geq 20 \\
\geq 20\end{array}$ & $\begin{array}{c}0.0812 \\
.722 \\
.334 \\
.104 \\
.0473\end{array}$ \\
\hline $\begin{array}{l}06-11-85 \\
06-18-85 \\
06-18-85 \\
06-26-85 \\
06-26-85\end{array}$ & $\begin{array}{l}20.3 \\
18.4 \\
17.9 \\
13.5 \\
13.7\end{array}$ & $\begin{array}{l}20.3 \\
18.4 \\
17.9 \\
13.5 \\
13.7\end{array}$ & $\begin{array}{l}1.7 \\
1.6 \\
1.6 \\
1.5 \\
1.3\end{array}$ & $\begin{array}{l}19.5 \\
18.5 \\
18.5 \\
17.5 \\
17.5\end{array}$ & $\begin{array}{l}.63 \\
.63 \\
.62 \\
.53 \\
.58\end{array}$ & $\begin{array}{l}-- \\
-- \\
-- \\
--\end{array}$ & $\begin{array}{l}-- \\
6.5 \\
-- \\
5.0 \\
4.5\end{array}$ & $\begin{array}{r}7 \\
9 \\
5 \\
11 \\
11\end{array}$ & $\begin{array}{l}.142 \\
.166 \\
.0895 \\
.149 \\
.151\end{array}$ & $\begin{array}{l}\geq 20 \\
\geq 20 \\
\geq 20 \\
\geq 20 \\
\geq 20\end{array}$ & $\begin{array}{l}.0660 \\
.0423 \\
.0487 \\
.0609 \\
.0504\end{array}$ \\
\hline $\begin{array}{l}07-01-85 \\
07-01-85 \\
07-11-85 \\
07-18-85 \\
08-01-85\end{array}$ & $\begin{array}{l}8.69 \\
7.96 \\
6.60 \\
5.72 \\
4.08\end{array}$ & $\begin{array}{l}8.69 \\
7.96 \\
6.60 \\
5.72 \\
4.08\end{array}$ & $\begin{array}{l}1.1 \\
1.1 \\
1.0 \\
1.0 \\
.90\end{array}$ & $\begin{array}{l}17.5 \\
17.5 \\
17.5 \\
17.5 \\
17.0\end{array}$ & $\begin{array}{l}.45 \\
.42 \\
.37 \\
.33 \\
.26\end{array}$ & $\begin{array}{c}0.0058 \\
\ldots \\
\ldots \\
\ldots \\
\ldots\end{array}$ & $\begin{array}{l}10.0 \\
10.0 \\
12.5 \\
12.5 \\
12.5\end{array}$ & $\begin{array}{r}10 \\
10 \\
4 \\
7 \\
7\end{array}$ & $\begin{array}{l}.0869 \\
.0796 \\
.0264 \\
.0400 \\
.0286\end{array}$ & $\begin{array}{l}\geq 20 \\
\geq 20 \\
\geq 20 \\
\geq 20 \\
>20\end{array}$ & $\begin{array}{l}.0276 \\
.0532 \\
.114 \\
.0261 \\
.0134\end{array}$ \\
\hline
\end{tabular}

68. Upper South Fork of Williams Fork near Leal, Colo. 5

\begin{tabular}{|c|c|c|c|c|c|c|c|c|c|c|c|}
\hline $05-16-85$ & 0.74 & 0.74 & 0.82 & 5.3 & 0.17 & $\cdots$ & -- & 1 & 0.000740 & $\geq 20$ & 0.0342 \\
\hline $05-23-85$ & .99 & .99 & .73 & 6.4 & .21 & -- & -- & 3 & .00297 & $\overline{>} 20$ & .0435 \\
\hline $05-30-85$ & 2.80 & 2.80 & .93 & 11.0 & .28 & -- & -- & 5 & .0140 & $>20$ & .0295 \\
\hline $06-04-85$ & 2.80 & 2.80 & .88 & 10.5 & .30 & -- & -- & 6 & .0168 & $>20$ & .152 \\
\hline $06-05-85$ & 2.80 & 2.80 & .93 & 10.5 & .29 & -- & 5.0 & 3 & .00840 & $\geq 20$ & .113 \\
\hline $06-10-85$ & 5.27 & 5.27 & 1.2 & 11.5 & .39 & -- & - & 19 & .100 & $>20$ & .353 \\
\hline $06-11-85$ & 4.50 & 4.50 & 1.1 & 11.5 & .36 & -- & -- & 5 & .0225 & $>20$ & .201 \\
\hline $06-17-85$ & 5.15 & 5.15 & 1.1 & 11.5 & .41 & -- & 9.0 & 19 & .0979 & $\geq 20$ & .165 \\
\hline $06-18-85$ & 3.71 & 3.71 & .97 & 11.0 & .35 & -- & 8.5 & 6 & .0223 & $\geq 20$ & .189 \\
\hline $07-01-85$ & 1.44 & 1.44 & .81 & 8.8 & .20 & 0.0096 & 5.0 & 3 & .00432 & $>20$ & .0175 \\
\hline $07-18-85$ & .74 & .74 & .71 & 6.7 & .15 & - & 9.0 & 8 & .00592 & $>20$ & .0236 \\
\hline
\end{tabular}

69. Lower South Fork of Williams Fork near Leal, Colo. ${ }^{5}$

\begin{tabular}{|c|c|c|c|c|c|c|c|c|c|c|c|}
\hline $\begin{array}{l}05-15-85 \\
05-28-85 \\
05-29-85 \\
06-05-85 \\
06-05-85\end{array}$ & $\begin{array}{l}1.44 \\
4.13 \\
3.96 \\
4.13 \\
6.34\end{array}$ & $\begin{array}{l}1.44 \\
4.13 \\
3.96 \\
4.13 \\
6.34\end{array}$ & $\begin{array}{r}0.44 \\
.96 \\
.94 \\
.96 \\
1.3\end{array}$ & $\begin{array}{l}8.8 \\
9.0 \\
9.0 \\
9.0 \\
9.0\end{array}$ & $\begin{array}{r}0.37 \\
.48 \\
.47 \\
.48 \\
.54\end{array}$ & $\begin{array}{l}-- \\
-- \\
-- \\
--\end{array}$ & $\begin{array}{l}-- \\
-- \\
-- \\
--\end{array}$ & $\begin{array}{r}1 \\
22 \\
9 \\
7 \\
35\end{array}$ & $\begin{array}{c}0.00144 \\
.0909 \\
.0356 \\
.0289 \\
.222\end{array}$ & $\begin{array}{l}\geq 20 \\
\geq 20 \\
\geq 20 \\
\geq 20 \\
\geq 20\end{array}$ & $\begin{array}{c}0.00652 \\
.185 \\
.123 \\
.0247 \\
.0593\end{array}$ \\
\hline $\begin{array}{l}06-06-85 \\
06-11-85 \\
06-11-85 \\
06-17-85 \\
06-18-85\end{array}$ & $\begin{array}{l}4.70 \\
6.80 \\
7.05 \\
7.05 \\
5.86\end{array}$ & $\begin{array}{l}4.70 \\
6.80 \\
7.05 \\
7.05 \\
5.86\end{array}$ & $\begin{array}{l}1.1 \\
1.4 \\
1.4 \\
1.4 \\
1.2\end{array}$ & $\begin{array}{l}9.0 \\
9.0 \\
9.0 \\
9.0 \\
9.0\end{array}$ & $\begin{array}{l}.48 \\
.55 \\
.56 \\
.56 \\
.53\end{array}$ & $\begin{array}{l}-- \\
-- \\
-- \\
-- \\
--\end{array}$ & $\begin{array}{l}-- \\
-- \\
-- \\
9.0 \\
6.5\end{array}$ & $\begin{array}{r}8 \\
11 \\
7 \\
11 \\
4\end{array}$ & $\begin{array}{l}.0376 \\
.0748 \\
.0494 \\
.0776 \\
.0234\end{array}$ & $\begin{array}{l}\geq 20 \\
\geq 20 \\
\geq 20 \\
\geq 20 \\
\geq 20\end{array}$ & $\begin{array}{l}.0955 \\
.104 \\
.197 \\
.0223 \\
.0298\end{array}$ \\
\hline $\begin{array}{l}06-25-85 \\
06-26-85 \\
07-01-85 \\
07-03-85 \\
07-18-85 \\
08-01-85\end{array}$ & $\begin{array}{l}4.13 \\
3.85 \\
2.32 \\
2.12 \\
1.22 \\
1.16\end{array}$ & $\begin{array}{l}4.13 \\
3.85 \\
2.32 \\
2.12 \\
1.22 \\
1.16\end{array}$ & $\begin{array}{l}.96 \\
.92 \\
.63 \\
.59 \\
.39 \\
.39\end{array}$ & $\begin{array}{l}9.0 \\
9.0 \\
8.9 \\
8.9 \\
8.8 \\
8.7\end{array}$ & $\begin{array}{l}.48 \\
.47 \\
.41 \\
.41 \\
.36 \\
.34\end{array}$ & $\begin{array}{c}-- \\
-- \\
0.016 \\
-- \\
-- \\
--\end{array}$ & $\begin{array}{r}9.0 \\
3.5 \\
9.5 \\
10.5 \\
9.5 \\
10.5\end{array}$ & $\begin{array}{l}13 \\
32 \\
33 \\
14 \\
37 \\
12\end{array}$ & $\begin{array}{l}.0537 \\
.123 \\
.0766 \\
.0297 \\
.0451 \\
.0139\end{array}$ & $\begin{array}{l}\geq 20 \\
\geq 20 \\
\geq 20 \\
. \geq 20 \\
\geq 20 \\
\geq 20\end{array}$ & $\begin{array}{l}.0171 \\
.0307 \\
.0117 \\
.00144 \\
.00376 \\
.00139\end{array}$ \\
\hline
\end{tabular}


Table 1.--Hydraulic and sediment-transport data--Continued

\begin{tabular}{|c|c|c|c|c|c|c|c|c|c|c|c|}
\hline Date & $\begin{array}{c}\text { Water } \\
\text { discharge } \\
\text { (bedload) } \\
\left(\mathrm{m}^{3} / \mathrm{s}\right)\end{array}$ & $\begin{array}{c}\text { Water } \\
\text { discharge } \\
\text { (suspended } \\
\text { load) } \\
\left(\mathrm{m}^{3} / \mathrm{s}\right)\end{array}$ & $\begin{array}{l}\text { Mean } \\
\text { flow } \\
\text { velocity } \\
(\mathbf{m} / \mathbf{s})\end{array}$ & $\begin{array}{l}\text { Water- } \\
\text { surface } \\
\text { width } \\
(\mathrm{m})\end{array}$ & $\begin{array}{l}\text { Mean } \\
\text { flow } \\
\text { depth } \\
(m)\end{array}$ & $\begin{array}{l}\text { Water- } \\
\text { surface } \\
\text { slope } \\
(\mathrm{m} / \mathrm{m})\end{array}$ & $\begin{array}{l}\text { Water } \\
\text { temp. } \\
\left({ }^{\circ} \mathrm{C}\right)\end{array}$ & $\begin{array}{l}\text { Suspended } \\
\text { sediment } \\
\text { concern- } \\
\text { tration } \\
(m g / L)\end{array}$ & $\begin{array}{c}\text { Suspended } \\
\text { load } \\
(\mathrm{kg} / \mathrm{s})\end{array}$ & $\begin{array}{l}\text { Number of } \\
\text { sampling } \\
\text { points } \\
\text { for } \\
\text { bedload }\end{array}$ & $\begin{array}{r}\text { Bedload } \\
(\mathrm{kg} / \mathrm{s})\end{array}$ \\
\hline
\end{tabular}

70. Williams Fork below Steelman Creek near Leal, Colo. 5

\begin{tabular}{|c|c|c|c|c|c|c|c|c|c|c|c|}
\hline $05-16-85$ & 0.68 & 0.68 & 0.58 & 4.4 & 0.27 & -- & -- & 4 & 0.00272 & $\geq 20$ & 0.000331 \\
\hline $05-23-85$ & .71 & .71 & .60 & 4.4 & .27 & - & -- & 3 & .00213 & $\geq 20$ & .00118 \\
\hline $05-23-85$ & .74 & .74 & .62 & 4.4 & .27 & - & -- & 7 & .00518 & $\geq 20$ & .00189 \\
\hline $06-03-85$ & 3.71 & 3.71 & 1.3 & 7.9 & .37 & - & -- & 3 & .0111 & $\geq 20$ & .0991 \\
\hline $06-03-85$ & 3.99 & 3.99 & 1.3 & 7.9 & .39 & -- & 5.5 & 10 & .0399 & $\geq 20$ & .0924 \\
\hline $06-03-85$ & 4.28 & 4.28 & 1.3 & 8.0 & .40 & -- & 3.5 & 2 & .00856 & $>20$ & .146 \\
\hline $06-13-85$ & 5.86 & 5.86 & 1.5 & 8.1 & .47 & -- & -- & 3 & .0176 & $\geq 20$ & .0912 \\
\hline $06-13-85$ & 6.06 & 6.06 & 1.6 & 8.1 & .48 & -- & -- & 1 & .00606 & $\geq 20$ & .113 \\
\hline $06-20-85$ & 5.07 & 5.07 & 1.4 & 8.0 & .45 & -- & 6.0 & 4 & .0203 & $\geq 20$ & .0448 \\
\hline $06-20-85$ & 5.30 & 5.30 & 1.5 & 8.0 & .45 & -- & 6.0 & 4 & .0212 & $\geq 20$ & .0998 \\
\hline $06-27-85$ & 3.74 & 3.74 & 1.3 & 7.9 & .37 & -- & 5.5 & 10 & .0374 & $\geq 20$ & .0496 \\
\hline $06-27-85$ & 4.16 & 4.16 & 1.3 & 7.9 & .40 & - & 6.5 & 15 & .0624 & $\geq 20$ & .0428 \\
\hline $07-02-85$ & 2.78 & 2.78 & 1.1 & 7.7 & .32 & - & 8.0 & 15 & .0417 & $\geq 20$ & .00154 \\
\hline $07-16-85$ & 2.21 & 2.21 & 1.1 & 7.4 & .28 & - & 6.0 & 8 & .0177 & $\geq 20$ & .000632 \\
\hline $07-30-85$ & 1.78 & 1.78 & .98 & 7.3 & .25 & -- & 8.5 & 9 & .0160 & $\geq 20$ & .00115 \\
\hline
\end{tabular}

\begin{tabular}{|c|c|c|c|c|c|c|c|c|c|c|c|}
\hline $05-15-85$ & 0.54 & 0.54 & 0.49 & 4.6 & 0.23 & -- & - & 4 & 0.00216 & $\geq 20$ & 0.00159 \\
\hline $05-16-85$ & .57 & .57 & .52 & 4.7 & .24 & -- & -- & 2 & .00114 & $\geq 20$ & .000703 \\
\hline $05-22-85$ & .57 & .57 & .52 & 4.7 & .23 & - & -- & 9 & .00513 & $\geq 20$ & .00278 \\
\hline $05-28-85$ & 1.22 & 1.22 & .79 & 4.9 & .31 & - & -- & 20 & .0244 & $>20$ & .0202 \\
\hline $05-29-85$ & 1.19 & 1.19 & .79 & 5.0 & .30 & -- & -- & 8 & .00952 & $\geq 20$ & .00361 \\
\hline $06-05-85$ & 1.33 & 1.33 & .80 & 4.9 & .34 & -- & 5.5 & 11 & .0146 & $\geq 20$ & .0607 \\
\hline $06-06-85$ & 1.27 & 1.27 & .78 & 5.0 & .33 & -- & 3.5 & 7 & .00889 & $>20$ & .0284 \\
\hline $06-11-85$ & 2.41 & 2.41 & 1.1 & 5.1 & .42 & -- & -- & 4 & .00964 & $>20$ & .0139 \\
\hline $06-11-85$ & 2.52 & 2.52 & 1.2 & 5.1 & .42 & $\cdots$ & $\cdots$ & 11 & .0277 & $\overline{20}$ & .00730 \\
\hline $06-18-85$ & 1.76 & 1.76 & 1.0 & 5.0 & .35 & -- & 4.5 & 7 & .0123 & $\geq 20$ & .0251 \\
\hline $06-26-85$ & 1.30 & 1.30 & .76 & 5.0 & .33 & -- & 6.5 & 10 & .0130 & $\geq 20$ & .00175 \\
\hline $06-26-85$ & 1.33 & 1.33 & .84 & 4.9 & .32 & -- & 3.5 & 11 & .0146 & $\geq 20$ & .00119 \\
\hline $07-01-85$ & .68 & .68 & .55 & 4.9 & .25 & 0.019 & 3.5 & 14 & .00952 & $>20$ & .000355 \\
\hline $07-03-85$ & .59 & .59 & .52 & 4.7 & .25 & -- & 7.5 & 19 & .0112 & $\geq 20$ & .000414 \\
\hline $07-11-85$ & .54 & .54 & .51 & 4.6 & .23 & $\cdots$ & 11.0 & 8 & .00432 & $>20$ & .000598 \\
\hline $08-01-85$ & .48 & .48 & .50 & 4.6 & .21 & -- & 5.5 & 19 & .00912 & $\overline{20}$ & .000584 \\
\hline
\end{tabular}

72. West Fork of San Juan River near Pagosa Springs, Colo. 5,15

\begin{tabular}{|c|c|c|c|c|c|c|c|c|c|c|c|}
\hline $\begin{array}{l}04-17-85 \\
04-23-85 \\
05-02-85 \\
05-05-85 \\
05-08-85\end{array}$ & $\begin{array}{c}16.6 \\
6.80 \\
9.91 \\
24.1 \\
24.0\end{array}$ & $\begin{array}{c}16.6 \\
6.80 \\
9.91 \\
24.1 \\
24.0\end{array}$ & $\begin{array}{r}1.15 \\
.68 \\
.80 \\
. . \\
1.42\end{array}$ & $\begin{array}{l}25.5 \\
24.0 \\
24.5 \\
26.0 \\
26.0\end{array}$ & $\begin{array}{r}0.57 \\
.41 \\
.47 \\
-- \\
.65\end{array}$ & $\begin{array}{l}-- \\
-- \\
-- \\
--\end{array}$ & $\begin{array}{r}8.5 \\
4.0 \\
13.0 \\
9.0 \\
9.0\end{array}$ & $\begin{array}{r}132 \\
265 \\
43 \\
215 \\
144\end{array}$ & $\begin{array}{l}2.19 \\
1.80 \\
.426 \\
5.18 \\
3.46\end{array}$ & $\begin{array}{l}20 \\
80 \\
40 \\
42 \\
20\end{array}$ & $\begin{array}{r}0.158 \\
.0105 \\
.0777 \\
.0273 \\
.0399\end{array}$ \\
\hline $\begin{array}{l}05-12-85 \\
05-15-85 \\
05-20-85 \\
05-26-85 \\
05-30-85\end{array}$ & $\begin{array}{l}17.2 \\
13.4 \\
12.7 \\
33.4 \\
30.6\end{array}$ & $\begin{array}{l}17.2 \\
13.4 \\
12.7 \\
33.4 \\
30.6\end{array}$ & $\begin{array}{l}-- \\
-- \\
-- \\
--\end{array}$ & $\begin{array}{l}24.5 \\
24.5 \\
24.5 \\
26.0 \\
26.0\end{array}$ & $\begin{array}{l}-- \\
-- \\
-- \\
--\end{array}$ & $\begin{array}{l}-- \\
-- \\
-- \\
--\end{array}$ & $\begin{array}{r}5.5 \\
11.0 \\
7.0 \\
8.0 \\
7.5\end{array}$ & $\begin{array}{r}37 \\
201 \\
26 \\
475 \\
98\end{array}$ & $\begin{array}{c}.636 \\
2.69 \\
.330 \\
15.9 \\
3.00\end{array}$ & $\begin{array}{l}20 \\
20 \\
20 \\
21 \\
21\end{array}$ & $\begin{array}{l}.126 \\
.0168 \\
.0609 \\
.168 \\
.683\end{array}$ \\
\hline $\begin{array}{l}06-03-85 \\
06-06-85 \\
06-18-85 \\
07-18-85\end{array}$ & $\begin{array}{c}32.9 \\
30.3 \\
31.7 \\
5.52\end{array}$ & $\begin{array}{c}32.9 \\
30.3 \\
31.7 \\
5.52\end{array}$ & $\begin{array}{l}-- \\
-- \\
-- \\
.54\end{array}$ & $\begin{array}{l}26.0 \\
26.0 \\
27.5 \\
23.5\end{array}$ & $\begin{array}{l}-- \\
-- \\
-- \\
.44\end{array}$ & $\begin{array}{l}-- \\
-- \\
--\end{array}$ & $\begin{array}{r}7.0 \\
8.0 \\
9.0 \\
11.0\end{array}$ & $\begin{array}{r}103 \\
85 \\
63 \\
6\end{array}$ & $\begin{array}{l}3.39 \\
2.58 \\
2.00 \\
.0331\end{array}$ & $\begin{array}{l}21 \\
21 \\
22 \\
50\end{array}$ & $\begin{array}{l}.515 \\
.210 \\
.294 \\
.00284\end{array}$ \\
\hline
\end{tabular}


Table 1.--Hydraulic and sediment-transport data--Continued

\begin{tabular}{|c|c|c|c|c|c|c|c|c|c|c|c|}
\hline Date & $\begin{array}{c}\text { Water } \\
\text { discharge } \\
\text { (bedload) } \\
\left(\mathrm{m}^{3} / \mathrm{s}\right)\end{array}$ & $\begin{array}{c}\text { Water } \\
\text { discharge } \\
\text { (suspended } \\
\text { load) } \\
\left(\mathrm{m}^{3} / \mathrm{s}\right)\end{array}$ & $\begin{array}{c}\text { Mean } \\
\text { flow } \\
\text { velocity } \\
(\mathrm{m} / \mathrm{s})\end{array}$ & $\begin{array}{l}\text { Water- } \\
\text { surface } \\
\text { width } \\
(\mathrm{m})\end{array}$ & $\begin{array}{l}\text { Mean } \\
\text { flow } \\
\text { depth } \\
\text { (m) }\end{array}$ & $\begin{array}{l}\text { Water- } \\
\text { surface } \\
\text { slope } \\
(\mathrm{m} / \mathrm{m})\end{array}$ & $\begin{array}{l}\text { Water } \\
\text { temp. } \\
\left({ }^{\circ} \mathrm{C}\right)\end{array}$ & $\begin{array}{c}\text { Suspended } \\
\text { sediment } \\
\text { concern- } \\
\text { tration } \\
(m g / L)\end{array}$ & $\begin{array}{c}\text { Suspended } \\
\text { load } \\
(\mathrm{kg} / \mathrm{s})\end{array}$ & $\begin{array}{l}\text { Number of } \\
\text { sampling } \\
\text { points } \\
\text { for } \\
\text { bedload }\end{array}$ & $\begin{array}{r}\text { Bedload } \\
(\mathrm{kg} / \mathrm{s})\end{array}$ \\
\hline
\end{tabular}

73. Rich Creek near Weston Pass, Colo. 5

$\begin{array}{lccc}05-16-84 & 0.29 & 0.29 & 0.59 \\ 05-24-84 & 1.51 & 1.51 & 1.2 \\ 05-29-84 & 1.25 & 1.25 & .94 \\ 05-31-84 & 1.46 & 1.46 & 1.1 \\ 06-06-84 & 1.13 & 1.13 & .98 \\ 06-12-84 & .73 & .73 & .76 \\ 06-14-84 & .93 & .93 & .79 \\ 06-18-84 & 1.13 & 1.13 & 1.0 \\ 06-23-84 & 1.04 & 1.04 & .87 \\ 06-26-84 & 1.13 & 1.13 & .97 \\ 06-29-84 & .73 & .73 & .71 \\ 07-03-84 & .62 & .62 & .64 \\ 07-06-84 & .69 & .69 & .76 \\ 07-10-84 & .60 & .60 & .57 \\ 07-17-84 & .38 & .38 & .52 \\ 08-08-84 & .18 & .18 & .32\end{array}$

0.59
1.2
.94
1.1
.98
.76
.79
1.0
.87
.97
.71
.64
.76
.57
.52
.32

$\begin{array}{ll}3.0 & 0.16 \\ 4.2 & .30 \\ 4.9 & .27 \\ 5.0 & .28 \\ 4.5 & .26 \\ 4.4 & .22 \\ 4.5 & .26 \\ 4.6 & .24 \\ 4.6 & .26 \\ 4.6 & .25\end{array}$

0.039

.039

.039

.039

$--$

$-$

$--$

16
24
24

24
17

.039

.039
.039

.039

.039

$--$

$--$

$-$

.039
.039

.039

.039

.039

.039

$\begin{array}{lr}-- & 8 \\ -- & 28 \\ -- & 15 \\ -- & 6 \\ -- & 23 \\ & 36\end{array}$
74. Trout Creek near Bayfield, Colo. 5

$\begin{array}{llll}05-02-84 & 0.037 & 0.037 & 0.26 \\ 05-03-84 & .038 & .038 & .25 \\ 05-07-84 & .11 & .11 & .41 \\ 05-08-84 & .10 & .10 & .47 \\ 05-15-84 & .35 & .35 & .72 \\ 05-16-84 & .28 & .28 & .67 \\ 05-21-84 & .26 & .26 & .61 \\ 05-22-84 & .29 & .29 & .64 \\ 05-30-84 & .068 & .068 & .26 \\ 05-31-84 & .061 & .061 & .27 \\ 06-05-84 & .051 & .051 & .26 \\ 06-11-84 & .030 & .030 & .19 \\ 06-12-84 & .031 & .031 & .29 \\ 04-23-85 & .10 & .10 & .56 \\ 04-29-85 & .11 & .11 & .50 \\ & & & \\ 04-30-85 & .11 & .11 & .50 \\ 05-06-85 & .40 & .40 & .86 \\ 05-07-85 & .33 & .33 & .80 \\ 05-20-85 & .13 & .13 & .53 \\ 05-21-85 & .11 & .11 & .46 \\ 05-21-85 & .11 & .11 & .44 \\ 05-22-85 & .11 & .11 & .41 \\ 05-29-85 & .18 & .18 & .62 \\ 05-30-85 & .13 & .13 & .41 \\ 06-04-85 & .12 & .12 & .50 \\ 06-05-85 & .10 & .10 & .46 \\ 06-13-85 & .050 & .050 & .29\end{array}$

\begin{tabular}{|c|c|c|c|}
\hline 0.82 & 0.17 & -- & 2.0 \\
\hline .85 & .11 & -- & 0.5 \\
\hline .94 & .28 & $\cdots$ & 1.5 \\
\hline 1.0 & .21 & -- & 1.0 \\
\hline 1.6 & .30 & -- & 3.0 \\
\hline 1.3 & .31 & -- & 3.0 \\
\hline 1.2 & .35 & -- & 7.5 \\
\hline 1.3 & .37 & -- & 3.5 \\
\hline .94 & .27 & -- & 9.5 \\
\hline .98 & .23 & $\cdots$ & 5.5 \\
\hline .91 & .21 & -- & - \\
\hline .85 & .19 & $\cdots$ & 5.0 \\
\hline .64 & .16 & $\cdots$ & 5.0 \\
\hline .88 & .20 & $\cdots$ & -- \\
\hline 1.0 & .21 & -- & -- \\
\hline .98 & .24 & $\cdots$ & -- \\
\hline 1.2 & .38 & -- & -. \\
\hline 1.2 & .34 & $\cdots$ & -- \\
\hline .91 & .27 & - & - \\
\hline .94 & .26 & - & $\cdots$ \\
\hline .91 & .28 & - & -. \\
\hline .98 & .26 & -- & - \\
\hline .94 & .31 & -. & -- \\
\hline 1.0 & .31 & -- & - \\
\hline .94 & .26 & $\cdots$ & - \\
\hline .91 & .24 & $\cdots$ & $=$ \\
\hline .91 & .19 & -- & -. \\
\hline
\end{tabular}

26

41

72

67

.61

26
27

26

29

.56

50

.86

53

46

44

41

50

46
.29
0.00464

$$
.0362
$$

.0300

.0248

.0102

.00730

.00744

.0283

.0260

.0203

.00584

.0174

.0104

.00360

.00874

.00648

$\begin{array}{ll}40 & 0.00325 \\ 40 & .0282 \\ 40 & .0135 \\ 40 & .00701 \\ 40 & .0267 \\ & \\ 40 & .00786 \\ 40 & .0126 \\ 40 & .00290 \\ 40 & .00298 \\ 40 & .00259 \\ & \\ 40 & .00120 \\ 40 & .00830 \\ 40 & .00359 \\ 40 & .00154 \\ 40 & .00393 \\ 40 & .000287\end{array}$

.000287

17
15
28
14
51

25
29
13
5

19
7

7

3
25
15$$
8
$$

8
36
14

2
8

8
5

5
4
8
9
4
7
7

$\begin{array}{lll}0.000629 & 10 & 0.0000063 \\ .000570 & 10 & .0000063 \\ .00308 & 10 & .000084 \\ .00140 & 10 & .000176 \\ .0179 & 10 & .000567 \\ .00700 & 10 & .000227 \\ .00754 & 10 & .000233 \\ .00377 & 10 & .000337 \\ .000340 & 10 & .000017 \\ .000244 & 10 & .0000053 \\ .000969 & 10 & .000018 \\ .000210 & 10 & .0000011 \\ .000093 & 10 & .0000011 \\ .00250 & 10 & .000032 \\ .00165 & 10 & .000081 \\ .000880 & 10 & .000021 \\ .0144 & 10 & .000389 \\ .00462 & 10 & .000166 \\ .000260 & 10 & .000076 \\ .000880 & 10 & .000017 \\ .000550 & 10 & .000046 \\ .000440 & 10 & .000031 \\ .00144 & 10 & .000042 \\ .00117 & 10 & .000056 \\ .000480 & 10 & .000020 \\ .000700 & 10 & .000049 \\ .000350 & 10 & \\ & & \\ & & \\ & & \\ .000047\end{array}$


Table 1.--Hydraulic and sediment-transport data--Continued

\begin{tabular}{|c|c|c|c|c|c|c|c|c|c|c|c|}
\hline Date & $\begin{array}{c}\text { Water } \\
\text { discharge } \\
\text { (bedload) } \\
\left(\mathrm{m}^{3} / \mathrm{s}\right)\end{array}$ & $\begin{array}{c}\text { Water } \\
\text { discharge } \\
\text { (suspended } \\
\text { load })^{2} \\
\left(\mathrm{~m}^{3} / \mathrm{s}\right)\end{array}$ & $\begin{array}{c}\text { Mean } \\
\text { flow } \\
\text { velocity } \\
(\mathrm{m} / \mathrm{s})\end{array}$ & $\begin{array}{l}\text { Water- } \\
\text { surface } \\
\text { width } \\
\text { (m) }\end{array}$ & $\begin{array}{l}\text { Mean } \\
\text { flow } \\
\text { depth } \\
(m)\end{array}$ & $\begin{array}{l}\text { Water- } \\
\text { surface } \\
\text { slope } \\
(\mathrm{m} / \mathrm{m})\end{array}$ & $\begin{array}{l}\text { Water } \\
\text { temp. } \\
\left({ }^{\circ} \mathrm{C}\right)\end{array}$ & $\begin{array}{l}\text { Suspended } \\
\text { sediment } \\
\text { concern- } \\
\text { tration } \\
\left(m_{g} / L\right)\end{array}$ & $\begin{array}{c}\text { Suspended } \\
\text { load } \\
(\mathrm{kg} / \mathrm{s})\end{array}$ & $\begin{array}{l}\text { Number of } \\
\text { sampling } \\
\text { points } \\
\text { for } \\
\text { bedload }\end{array}$ & $\begin{array}{r}\text { Bedload } \\
\left(k_{8} / \mathrm{s}\right)\end{array}$ \\
\hline
\end{tabular}

75. Clear Creek near Bayfield, Colo. 5

\begin{tabular}{|c|c|c|c|c|c|c|c|c|c|c|c|}
\hline $\begin{array}{l}05-07-84 \\
05-08-84 \\
05-15-84 \\
05-16-84 \\
05-21-84\end{array}$ & $\begin{array}{l}0.056 \\
.040 \\
.18 \\
.15 \\
.13\end{array}$ & $\begin{array}{l}0.056 \\
.040 \\
.18 \\
.15 \\
.13\end{array}$ & $\begin{array}{r}0.55 \\
.37 \\
.89 \\
.80 \\
.78\end{array}$ & $\begin{array}{l}1.2 \\
1.2 \\
1.5 \\
1.4 \\
1.3\end{array}$ & $\begin{array}{c}0.084 \\
.088 \\
.13 \\
.14 \\
.12\end{array}$ & $\begin{array}{l}-- \\
-- \\
-- \\
-- \\
--\end{array}$ & $\begin{array}{l}3.5 \\
1.0 \\
3.0 \\
3.5 \\
9.0\end{array}$ & $\begin{array}{r}59 \\
18 \\
157 \\
41 \\
23\end{array}$ & $\begin{array}{l}0.00330 \\
.000720 \\
.0283 \\
.00615 \\
.00299\end{array}$ & $\begin{array}{l}10 \\
10 \\
10 \\
10 \\
10\end{array}$ & $\begin{array}{r}0.000049 \\
.000014 \\
.000616 \\
.000452 \\
.000285\end{array}$ \\
\hline $\begin{array}{l}05-22-84 \\
05-30-84 \\
05-31-84 \\
06-05-84 \\
04-23-85\end{array}$ & $\begin{array}{l}.14 \\
.038 \\
.036 \\
.037 \\
.049\end{array}$ & $\begin{array}{l}.14 \\
.038 \\
.036 \\
.037 \\
.049\end{array}$ & $\begin{array}{l}.84 \\
.34 \\
.29 \\
-- \\
.33\end{array}$ & $\begin{array}{l}1.2 \\
1.4 \\
1.3 \\
1.2 \\
1.2\end{array}$ & $\begin{array}{l}.13 \\
.083 \\
.099 \\
-. \\
.12\end{array}$ & $\begin{array}{l}-- \\
-- \\
-- \\
-- \\
--\end{array}$ & $\begin{array}{r}5.0 \\
10.0 \\
6.5 \\
-- \\
--\end{array}$ & $\begin{array}{r}52 \\
9 \\
5 \\
26 \\
32\end{array}$ & $\begin{array}{l}.00728 \\
.000342 \\
.000180 \\
.000962 \\
.00157\end{array}$ & $\begin{array}{l}10 \\
10 \\
10 \\
10 \\
10\end{array}$ & $\begin{array}{l}.000231 \\
.0000053 \\
.000069 \\
.000187 \\
.000097\end{array}$ \\
\hline $\begin{array}{l}04-29-85 \\
04-30-85 \\
05-06-85 \\
05-07-85 \\
05-20-85\end{array}$ & $\begin{array}{l}.062 \\
.052 \\
.15 \\
.14 \\
.056\end{array}$ & $\begin{array}{l}.062 \\
.052 \\
.15 \\
.14 \\
.056\end{array}$ & $\begin{array}{l}.44 \\
.35 \\
.78 \\
.68 \\
.52\end{array}$ & $\begin{array}{l}1.3 \\
1.3 \\
1.5 \\
1.7 \\
1.2\end{array}$ & $\begin{array}{l}.11 \\
.11 \\
.13 \\
.12 \\
.093\end{array}$ & $\begin{array}{l}-- \\
-- \\
-- \\
-- \\
--\end{array}$ & $\begin{array}{l}-- \\
-- \\
-- \\
--\end{array}$ & $\begin{array}{r}35 \\
10 \\
72 \\
33 \\
8\end{array}$ & $\begin{array}{l}.00217 \\
.000520 \\
.0108 \\
.00462 \\
.000448\end{array}$ & $\begin{array}{l}10 \\
10 \\
10 \\
10 \\
10\end{array}$ & $\begin{array}{l}.000309 \\
.000048 \\
.00127 \\
.000017 \\
.000142\end{array}$ \\
\hline $\begin{array}{l}05-21-85 \\
05-21-85 \\
05-22-85 \\
05-29-85 \\
05-30-85\end{array}$ & $\begin{array}{l}.062 \\
.060 \\
.047 \\
.092 \\
.10\end{array}$ & $\begin{array}{l}.062 \\
.060 \\
.047 \\
.092 \\
.10\end{array}$ & $\begin{array}{l}.68 \\
.54 \\
.43 \\
.55 \\
.62\end{array}$ & $\begin{array}{l}1.1 \\
1.1 \\
1.1 \\
1.3 \\
1.3\end{array}$ & $\begin{array}{l}.085 \\
.10 \\
.099 \\
.13 \\
.13\end{array}$ & $\begin{array}{l}-- \\
-- \\
-- \\
--\end{array}$ & $\begin{array}{l}-- \\
-- \\
-- \\
-- \\
--\end{array}$ & $\begin{array}{r}9 \\
7 \\
6 \\
12 \\
10\end{array}$ & $\begin{array}{l}.000558 \\
.000420 \\
.000282 \\
.00110 \\
.00100\end{array}$ & $\begin{array}{l}10 \\
10 \\
10 \\
10 \\
10\end{array}$ & $\begin{array}{l}.000054 \\
.000055 \\
.000020 \\
.000199 \\
.000953\end{array}$ \\
\hline $\begin{array}{l}06-04-85 \\
06-05-85\end{array}$ & $\begin{array}{l}.058 \\
.044\end{array}$ & $\begin{array}{l}.058 \\
.044\end{array}$ & $\begin{array}{l}.55 \\
.50\end{array}$ & $\begin{array}{l}1.2 \\
1.2\end{array}$ & $\begin{array}{l}.090 \\
.078\end{array}$ & -- & -- & $\begin{array}{r}6 \\
11\end{array}$ & $\begin{array}{l}.000348 \\
.000484\end{array}$ & $\begin{array}{l}10 \\
10\end{array}$ & $\begin{array}{l}.000028 \\
.000050\end{array}$ \\
\hline
\end{tabular}

76. West Prong Creek near Bayfield, Colo. 5

\begin{tabular}{|c|c|c|c|c|c|c|c|c|c|c|c|}
\hline $\begin{array}{l}05-02-84 \\
05-03-84 \\
05-07-84 \\
05-08-84 \\
05-15-84\end{array}$ & $\begin{array}{r}0.27 \\
.24 \\
.63 \\
.61 \\
1.25\end{array}$ & $\begin{array}{r}0.27 \\
.24 \\
.63 \\
.61 \\
1.25\end{array}$ & $\begin{array}{r}0.93 \\
.71 \\
.95 \\
.96 \\
1.1\end{array}$ & $\begin{array}{l}1.80 \\
1.8 \\
2.7 \\
2.7 \\
3.7\end{array}$ & $\begin{array}{l}.16 \\
.18 \\
.25 \\
.23 \\
.30\end{array}$ & $\begin{array}{l}-- \\
-- \\
-- \\
--\end{array}$ & $\begin{array}{l}2.0 \\
2.0 \\
5.5 \\
2.0 \\
4.5\end{array}$ & $\begin{array}{r}21 \\
14 \\
27 \\
16 \\
145\end{array}$ & $\begin{array}{c}0.00567 \\
.00336 \\
.0170 \\
.00976 \\
.181\end{array}$ & $\begin{array}{l}10 \\
10 \\
10 \\
10 \\
10\end{array}$ & $\begin{array}{l}0.000026 \\
.000042 \\
.000492 \\
.000126 \\
.00217\end{array}$ \\
\hline $\begin{array}{l}05-16-84 \\
05-21-84 \\
05-22-84 \\
05-30-84 \\
05-31-84\end{array}$ & $\begin{array}{l}.91 \\
.72 \\
.76 \\
.26 \\
.17\end{array}$ & $\begin{array}{l}.91 \\
.72 \\
.76 \\
.26 \\
.17\end{array}$ & $\begin{array}{l}.97 \\
.93 \\
.93 \\
.56 \\
.46\end{array}$ & $\begin{array}{l}3.4 \\
3.2 \\
3.2 \\
2.6 \\
2.4\end{array}$ & $\begin{array}{l}.27 \\
.24 \\
.26 \\
.18 \\
.15\end{array}$ & $\begin{array}{l}-- \\
-- \\
-- \\
--\end{array}$ & $\begin{array}{l}4.5 \\
6.5 \\
8.0 \\
6.5 \\
7.5\end{array}$ & $\begin{array}{r}94 \\
18 \\
21 \\
5 \\
2\end{array}$ & $\begin{array}{l}.0855 \\
.0130 \\
.0160 \\
.00130 \\
.000340\end{array}$ & $\begin{array}{l}10 \\
10 \\
10 \\
10 \\
10\end{array}$ & $\begin{array}{l}.000419 \\
.000227 \\
.000219 \\
.000548 \\
.000029\end{array}$ \\
\hline $\begin{array}{l}06-05-84 \\
06-11-84 \\
06-12-84 \\
04-23-85 \\
04-29-85\end{array}$ & $\begin{array}{l}.16 \\
.14 \\
.16 \\
.39 \\
.50\end{array}$ & $\begin{array}{l}.16 \\
.14 \\
.16 \\
.39 \\
.50\end{array}$ & $\begin{array}{l}-. \\
.50 . \\
.53 \\
.79 \\
.86\end{array}$ & $\begin{array}{l}2.6 \\
2.3 \\
2.5 \\
2.7 \\
2.9\end{array}$ & $\begin{array}{l}-. \\
.13 \\
.12 \\
.18 \\
.20\end{array}$ & $\begin{array}{l}-- \\
-- \\
-- \\
--\end{array}$ & $\begin{array}{c}-- \\
11.0 \\
6.5 \\
-- \\
--\end{array}$ & $\begin{array}{r}14 \\
4 \\
3 \\
27 \\
16\end{array}$ & $\begin{array}{l}.00224 \\
.000560 \\
.000480 \\
.0105 \\
.00800\end{array}$ & $\begin{array}{l}10 \\
10 \\
10 \\
10 \\
10\end{array}$ & $\begin{array}{l}.000077 \\
.000020 \\
.0000042 \\
.000126 \\
.000302\end{array}$ \\
\hline
\end{tabular}


Table 1.--Hydraulic and sediment-transport data--Continued

\begin{tabular}{|c|c|c|c|c|c|c|c|c|c|c|c|}
\hline Date & $\begin{array}{c}\text { Water } \\
\text { discharge } \\
\text { (bedload) } \\
\left(\mathrm{m}^{3} / \mathrm{s}\right)\end{array}$ & $\begin{array}{c}\text { Water } \\
\text { discharge } \\
\text { (suspended } \\
\text { load) } \\
\left(\mathrm{m}^{3} / \mathrm{s}\right)\end{array}$ & $\begin{array}{l}\text { Mean } \\
\text { flow } \\
\text { velocity } \\
(\mathrm{m} / \mathrm{s})\end{array}$ & $\begin{array}{l}\text { Water- } \\
\text { surface } \\
\text { width } \\
\text { (m) }\end{array}$ & $\begin{array}{l}\text { Mean } \\
\text { flow } \\
\text { depth } \\
(\mathrm{m})\end{array}$ & $\begin{array}{l}\text { Water- } \\
\text { surface } \\
\text { slope } \\
(\mathrm{m} / \mathrm{m})\end{array}$ & $\begin{array}{l}\text { Water } \\
\text { temp. } \\
\left({ }^{\circ} \mathrm{C}\right)\end{array}$ & $\begin{array}{c}\text { Suspended } \\
\text { sediment } \\
\text { concern- } \\
\text { tration } \\
(\mathrm{mg} / \mathrm{L})\end{array}$ & $\begin{array}{c}\text { Suspended } \\
\text { load } \\
(\mathrm{kg} / \mathrm{s})\end{array}$ & $\begin{array}{l}\text { Number of } \\
\text { sampling } \\
\text { points } \\
\text { for } \\
\text { bedload }\end{array}$ & $\begin{array}{r}\text { Bedload } \\
(\mathrm{kg} / \mathrm{s})\end{array}$ \\
\hline
\end{tabular}

76. West Prong Creek near Bayfield, Colo. ${ }^{5}-$-Cont inued

\begin{tabular}{|c|c|c|c|c|c|c|c|c|c|c|c|}
\hline $\begin{array}{l}04-30-85 \\
05-06-85 \\
05-07-85 \\
05-20-85 \\
05-21-85\end{array}$ & $\begin{array}{l}0.54 \\
1.73 \\
1.24 \\
.60 \\
.55\end{array}$ & $\begin{array}{l}0.54 \\
1.73 \\
1.24 \\
.60 \\
.55\end{array}$ & $\begin{array}{l}0.89 \\
1.5 \\
1.1 \\
.94 \\
.88\end{array}$ & $\begin{array}{l}2.9 \\
3.4 \\
3.3 \\
2.7 \\
2.7\end{array}$ & $\begin{array}{r}0.21 \\
.33 \\
.33 \\
.23 \\
.23\end{array}$ & $\begin{array}{l}-- \\
-- \\
-- \\
-2\end{array}$ & $\begin{array}{l}-- \\
-- \\
-- \\
--\end{array}$ & $\begin{array}{r}7 \\
41 \\
29 \\
3 \\
6\end{array}$ & $\begin{array}{c}0.00378 \\
.0709 \\
.0360 \\
.00180 \\
.00330\end{array}$ & $\begin{array}{l}10 \\
10 \\
10 \\
10 \\
10\end{array}$ & $\begin{array}{c}0.000105 \\
.00273 \\
.00582 \\
.000103 \\
.000382\end{array}$ \\
\hline $\begin{array}{l}05-22-85 \\
05-22-85 \\
05-29-85 \\
05-30-85 \\
06-04-85\end{array}$ & $\begin{array}{l}.45 \\
.45 \\
.49 \\
.49 \\
.32\end{array}$ & $\begin{array}{l}.45 \\
.45 \\
.49 \\
.49 \\
.32\end{array}$ & $\begin{array}{l}.71 \\
-. \\
.81 \\
.84 \\
.70\end{array}$ & $\begin{array}{l}2.9 \\
2.9 \\
2.7 \\
2.8 \\
2.7\end{array}$ & $\begin{array}{l}.22 \\
-- \\
.22 \\
.21 \\
.17\end{array}$ & $\begin{array}{l}-- \\
- \\
\cdots \\
-\end{array}$ & $\begin{array}{l}-- \\
-- \\
-- \\
--\end{array}$ & $\begin{array}{l}6 \\
5 \\
7 \\
5 \\
4\end{array}$ & $\begin{array}{l}.00270 \\
.00225 \\
.00343 \\
.00245 \\
.00128\end{array}$ & $\begin{array}{l}10 \\
10 \\
10 \\
10 \\
10\end{array}$ & $\begin{array}{l}.000109 \\
.000114 \\
.000038 \\
.000044 \\
.000063\end{array}$ \\
\hline $\begin{array}{l}06-05-85 \\
06-14-85\end{array}$ & $\begin{array}{l}.33 \\
.11\end{array}$ & $\begin{array}{l}.33 \\
.11\end{array}$ & $\begin{array}{l}.88 \\
.39\end{array}$ & $\begin{array}{l}2.6 \\
2.0\end{array}$ & $\begin{array}{l}.15 \\
.13\end{array}$ & -- & -- & $\begin{array}{r}13 \\
3\end{array}$ & $\begin{array}{l}.00429 \\
.000330\end{array}$ & $\begin{array}{l}10 \\
10\end{array}$ & $\begin{array}{l}.00433 \\
.0000011\end{array}$ \\
\hline
\end{tabular}

77. Red Creek near Bayfield, Colo. 5

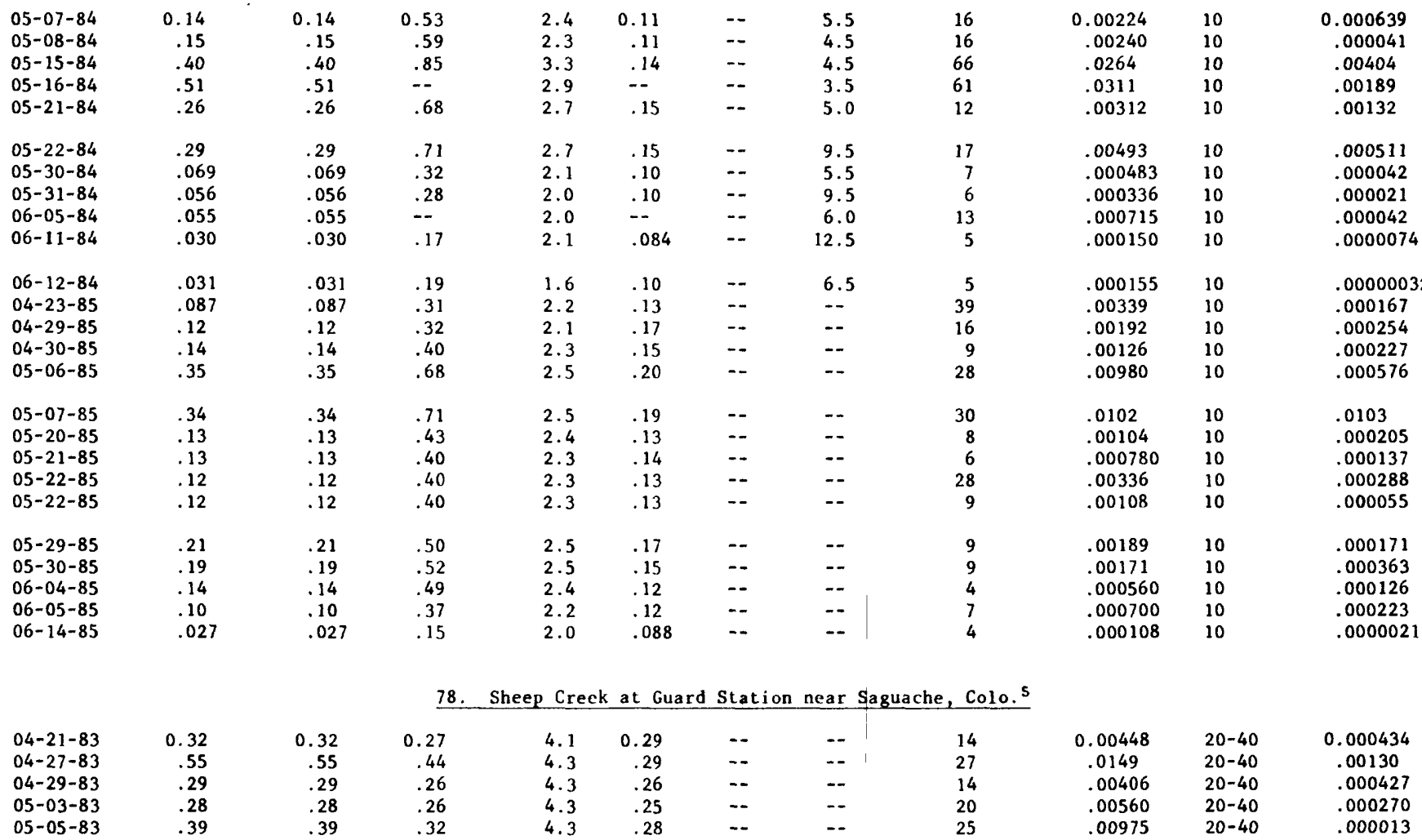


Table 1.--Hydraulic and sediment-transport data--Continued

\begin{tabular}{|c|c|c|c|c|c|c|c|c|c|c|c|}
\hline Date & $\begin{array}{c}\text { Water } \\
\text { discharge } \\
\text { (bedload) } \\
\left(\mathrm{m}^{3} / \mathrm{s}\right)\end{array}$ & $\begin{array}{c}\text { Water } \\
\text { discharge } \\
\text { (suspended } \\
\text { load) } \\
\left(\mathrm{m}^{3} / \mathrm{s}\right)\end{array}$ & $\begin{array}{l}\text { Mean } \\
\text { flow } \\
\text { velocity } \\
(\mathrm{m} / \mathrm{s})\end{array}$ & $\begin{array}{l}\text { Water- } \\
\text { surface } \\
\text { width } \\
\text { (m) }\end{array}$ & $\begin{array}{l}\text { Mean } \\
\text { flow } \\
\text { depth } \\
(\mathrm{m})\end{array}$ & $\begin{array}{l}\text { Water- } \\
\text { surface } \\
\text { slope } \\
(\mathrm{m} / \mathrm{m})\end{array}$ & $\begin{array}{l}\text { Water } \\
\text { temp. } \\
\left({ }^{\circ} \mathrm{C}\right)\end{array}$ & $\begin{array}{l}\text { Suspended } \\
\text { sediment } \\
\text { concern- } \\
\text { tration } \\
(\mathrm{mg} / \mathrm{L})\end{array}$ & $\begin{array}{c}\text { Suspended } \\
\text { load } \\
(\mathrm{kg} / \mathrm{s})\end{array}$ & $\begin{array}{l}\text { Number of } \\
\text { sampling } \\
\text { points } \\
\text { for } \\
\text { bedload }\end{array}$ & $\begin{array}{r}\text { Bedload } \\
(\mathrm{kg} / \mathrm{s})\end{array}$ \\
\hline
\end{tabular}

78. Sheep Creek at Guard Station near Saguache, Colo. ${ }^{5}$--Cont inued

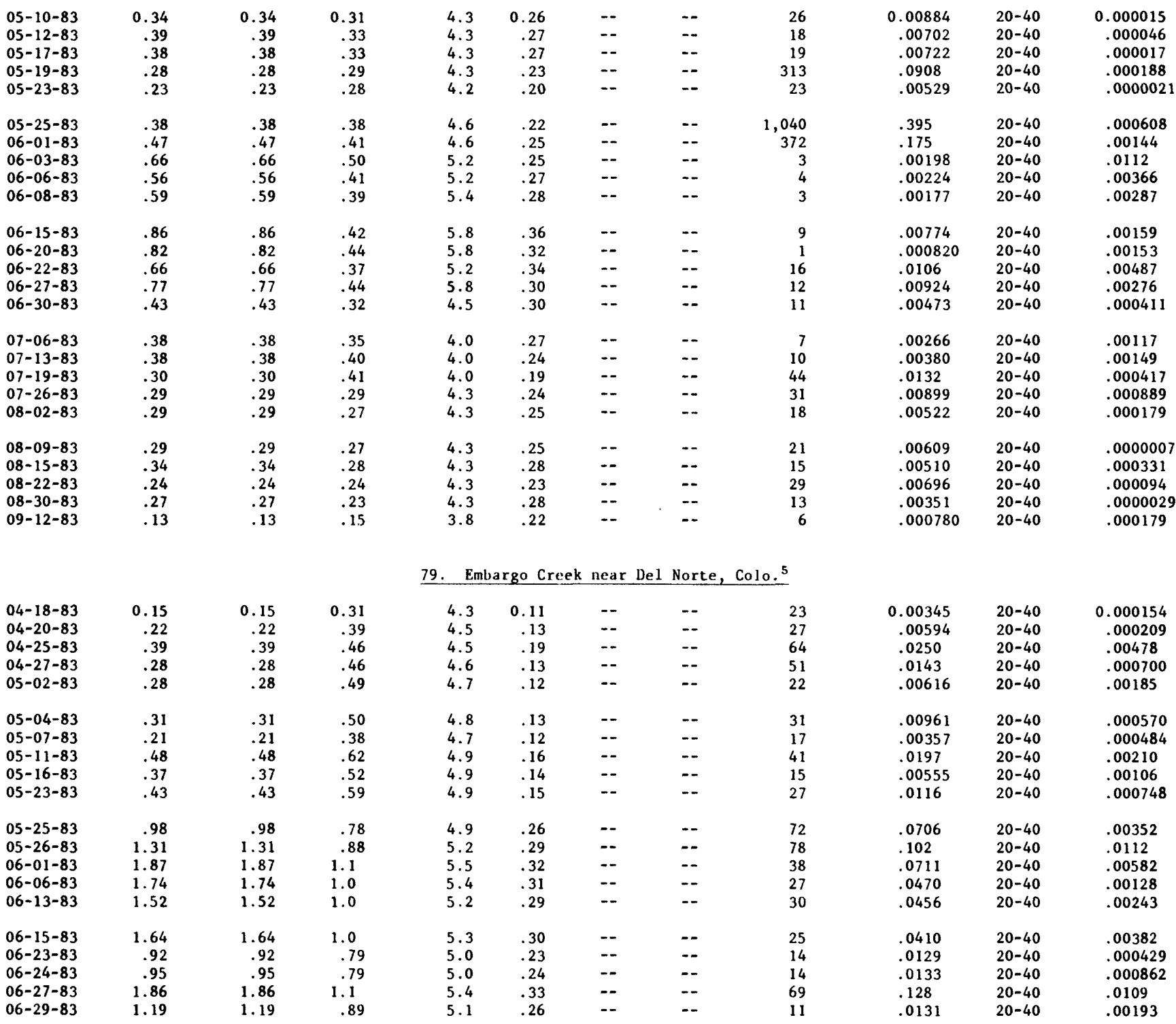


Talble 1.--Hydraulic and sediment-transport data--Continued

\begin{tabular}{|c|c|c|c|c|c|c|c|c|c|c|c|}
\hline Date & $\begin{array}{c}\text { Water } \\
\text { discharge } \\
\text { (bedload) } \\
\left(\mathrm{m}^{3} / \mathrm{s}\right)\end{array}$ & $\begin{array}{c}\text { Water } \\
\text { discharge } \\
\text { (suspended } \\
10 a d)^{2} \\
\left(\mathrm{~m}^{3} / \mathrm{s}\right)\end{array}$ & $\begin{array}{l}\text { Mean } \\
\text { flow } \\
\text { velocity } \\
(\mathrm{m} / \mathrm{s})\end{array}$ & $\begin{array}{l}\text { Water- } \\
\text { surface } \\
\text { width } \\
(\mathrm{m})\end{array}$ & $\begin{array}{l}\text { Mean } \\
\text { flow } \\
\text { depth } \\
(m)\end{array}$ & $\begin{array}{l}\text { Water- } \\
\text { surface } \\
\text { slope } \\
(\mathrm{m} / \mathrm{m})\end{array}$ & $\begin{array}{l}\text { Water } \\
\text { temp. } \\
\left({ }^{\circ} \mathrm{C}\right)\end{array}$ & $\begin{array}{c}\text { Suspended } \\
\text { sediment } \\
\text { concern- } \\
\text { tration } \\
(\mathrm{mg} / \mathrm{L})\end{array}$ & $\begin{array}{c}\text { Suspended } \\
\text { load } \\
\left(\mathrm{kg}_{\mathrm{g}} / \mathrm{s}\right)\end{array}$ & $\begin{array}{l}\text { Number of } \\
\text { sampling } \\
\text { points } \\
\text { for } \\
\text { bedload }\end{array}$ & $\begin{array}{r}\text { Bedload } \\
(\mathrm{kg} / \mathrm{s})\end{array}$ \\
\hline
\end{tabular}

79. Embargo Creek near De1 Norte, Colo. ${ }^{5}$-Continued

\begin{tabular}{|c|c|c|c|c|c|c|c|c|c|c|c|}
\hline $\begin{array}{l}07-08-83 \\
07-13-83 \\
07-18-83 \\
07-27-83 \\
08-03-83\end{array}$ & $\begin{array}{r}0.38 \\
.42 \\
.24 \\
.45 \\
.23\end{array}$ & $\begin{array}{r}0.38 \\
.42 \\
.24 \\
.45 \\
.23\end{array}$ & $\begin{array}{r}0.35 \\
.57 \\
.39 \\
.55 \\
.36\end{array}$ & $\begin{array}{l}4.9 \\
4.8 \\
4.2 \\
4.8 \\
4.3\end{array}$ & $\begin{array}{r}0.23 \\
.16 \\
.15 \\
.17 \\
.15\end{array}$ & $\begin{array}{l}-- \\
-- \\
-- \\
-- \\
--\end{array}$ & $\begin{array}{l}-- \\
-- \\
-- \\
--\end{array}$ & $\begin{array}{r}25 \\
10 \\
8 \\
14 \\
12\end{array}$ & $\begin{array}{r}0.00950 \\
.00420 \\
.00192 \\
.00630 \\
.00276\end{array}$ & $\begin{array}{l}20-40 \\
20-40 \\
20-40 \\
20-40 \\
20-40\end{array}$ & $\begin{array}{r}0.000418 \\
.000289 \\
.000744 \\
.000280 \\
.000190\end{array}$ \\
\hline $\begin{array}{l}08-10-83 \\
08-18-83 \\
08-24-83 \\
08-29-83\end{array}$ & $\begin{array}{l}.26 \\
.18 \\
.24 \\
.17\end{array}$ & $\begin{array}{l}.26 \\
.18 \\
.24 \\
.17\end{array}$ & $\begin{array}{l}.38 \\
.30 \\
.36 \\
.30\end{array}$ & $\begin{array}{l}4.0 \\
3.8 \\
4.2 \\
3.8\end{array}$ & $\begin{array}{l}.17 \\
.15 \\
.16 \\
.15\end{array}$ & $\begin{array}{l}-- \\
-- \\
--\end{array}$ & $\begin{array}{l}-- \\
-- \\
--\end{array}$ & $\begin{array}{l}14 \\
11 \\
16 \\
11\end{array}$ & $\begin{array}{l}.00364 \\
.00198 \\
.00384 \\
.00187\end{array}$ & $\begin{array}{l}20-40 \\
20-40 \\
20-40 \\
20-40\end{array}$ & $\begin{array}{l}.000090 \\
.0000080 \\
.000586 \\
.000142\end{array}$ \\
\hline
\end{tabular}

80. Pinos Creek near De1 Norte, Colo. 5

\begin{tabular}{|c|c|c|c|c|c|c|c|c|c|c|c|}
\hline $\begin{array}{l}04-20-83 \\
04-25-83 \\
04-27-83 \\
05-02-83 \\
05-04-83\end{array}$ & $\begin{array}{r}0.58 \\
.99 \\
1.04 \\
.98 \\
.86\end{array}$ & $\begin{array}{r}0.58 \\
.99 \\
1.04 \\
.98 \\
.86\end{array}$ & $\begin{array}{r}0.54 \\
.66 \\
.68 \\
.66 \\
.56\end{array}$ & $\begin{array}{l}7.9 \\
8.1 \\
7.8 \\
8.1 \\
7.8\end{array}$ & $\begin{array}{r}0.13 \\
.19 \\
.20 \\
.18 \\
.20\end{array}$ & $\begin{array}{l}-- \\
-- \\
-- \\
--\end{array}$ & $\begin{array}{l}-- \\
-- \\
-- \\
-- \\
--\end{array}$ & $\begin{array}{l}27 \\
46 \\
23 \\
23 \\
22\end{array}$ & $\begin{array}{r}0.0157 \\
.0455 \\
.0239 \\
.0225 \\
.0189\end{array}$ & $\begin{array}{l}20-40 \\
20-40 \\
20-40 \\
20-40 \\
20-40\end{array}$ & $\begin{array}{c}0.000537 \\
.00579 \\
.00450 \\
.00177 \\
.00189\end{array}$ \\
\hline $\begin{array}{l}05-07-83 \\
05-11-83 \\
05-16-83 \\
05-18-83 \\
05-23-83\end{array}$ & $\begin{array}{l}1.16 \\
1.91 \\
1.31 \\
1.23 \\
2.06\end{array}$ & $\begin{array}{l}1.16 \\
1.91 \\
1.31 \\
1.23 \\
2.06\end{array}$ & $\begin{array}{l}.66 \\
.75 \\
.64 \\
.62 \\
.77\end{array}$ & $\begin{array}{l}7.8 \\
8.3 \\
8.2 \\
8.1 \\
8.4\end{array}$ & $\begin{array}{l}.22 \\
.31 \\
.25 \\
.25 \\
.32\end{array}$ & $\begin{array}{l}-- \\
-- \\
-- \\
--\end{array}$ & $\begin{array}{l}-- \\
-- \\
-- \\
--\end{array}$ & $\begin{array}{l}35 \\
66 \\
20 \\
34 \\
47\end{array}$ & $\begin{array}{l}.0406 \\
.126 \\
.0262 \\
.0418 \\
.0968\end{array}$ & $\begin{array}{l}20-40 \\
20-40 \\
20-40 \\
20-40 \\
20-40\end{array}$ & $\begin{array}{l}.00543 \\
.00614 \\
.00261 \\
.000734 \\
.00555\end{array}$ \\
\hline $\begin{array}{l}05-25-83 \\
07-08-83 \\
07-13-83 \\
07-18-83 \\
07-27-83\end{array}$ & $\begin{array}{l}2.83 \\
2.25 \\
1.73 \\
1.26 \\
1.06\end{array}$ & $\begin{array}{l}2.83 \\
2.25 \\
1.73 \\
1.26 \\
1.06\end{array}$ & $\begin{array}{l}-. \\
.85 \\
.71 \\
.64 \\
.59\end{array}$ & $\begin{array}{l}-. \\
8.5 \\
8.3 \\
8.1 \\
7.9\end{array}$ & $\begin{array}{l}-. \\
.31 \\
.29 \\
.25 \\
.23\end{array}$ & $\begin{array}{l}-- \\
-- \\
-- \\
--\end{array}$ & $\begin{array}{l}-- \\
-- \\
-- \\
-- \\
--\end{array}$ & $\begin{array}{r}104 \\
27 \\
19 \\
12 \\
13\end{array}$ & $\begin{array}{l}.294 \\
.0608 \\
.0329 \\
.0151 \\
.0138\end{array}$ & $\begin{array}{l}20-40 \\
20-40 \\
20-40 \\
20-40 \\
20-40\end{array}$ & $\begin{array}{l}.00633 \\
.00190 \\
.00125 \\
.000354 \\
.000153\end{array}$ \\
\hline $\begin{array}{l}08-03-83 \\
08-10-83 \\
08-18-83 \\
08-24-83 \\
08-29-83 \\
09-13-83\end{array}$ & $\begin{array}{r}1.04 \\
1.23 \\
1.01 \\
.86 \\
.80 \\
.39\end{array}$ & $\begin{array}{l}1.04 \\
1.23 \\
1.01 \\
.86 \\
.80 \\
.39\end{array}$ & $\begin{array}{l}.60 \\
.68 \\
.62 \\
.58 \\
.56 \\
.39\end{array}$ & $\begin{array}{l}7.9 \\
8.0 \\
8.0 \\
7.9 \\
7.9 \\
7.4\end{array}$ & $\begin{array}{l}.22 \\
.23 \\
.20 \\
.19 \\
.18 \\
.14\end{array}$ & $\begin{array}{l}-- \\
-- \\
-- \\
--\end{array}$ & $\begin{array}{l}-- \\
-- \\
-- \\
-- \\
--\end{array}$ & $\begin{array}{l}62 \\
36 \\
19 \\
12 \\
17 \\
10\end{array}$ & $\begin{array}{l}.0645 \\
.0443 \\
.0192 \\
.0103 \\
.0136 \\
.00390\end{array}$ & $\begin{array}{l}20-40 \\
20-40 \\
20-40 \\
20-40 \\
20-40 \\
20-40\end{array}$ & $\begin{array}{l}.000833 \\
.00398 \\
.00104 \\
.000181 \\
.000130 \\
.000073\end{array}$ \\
\hline
\end{tabular}

81. Cross Creek near South Fork, Colo. 5

\begin{tabular}{|c|c|c|c|c|c|c|c|c|c|c|c|}
\hline $\begin{array}{l}04-20-83 \\
04-25-83 \\
04-27-83 \\
05-03-83 \\
05-05-83\end{array}$ & $\begin{array}{r}0.11 \\
.16 \\
.22 \\
.20 \\
.23\end{array}$ & $\begin{array}{r}0.11 \\
.16 \\
.22 \\
.20 \\
.23\end{array}$ & $\begin{array}{r}0.21 \\
.28 \\
.34 \\
.35 \\
.33\end{array}$ & $\begin{array}{l}4.1 \\
4.2 \\
4.2 \\
4.2 \\
4.2\end{array}$ & $\begin{array}{r}0.13 \\
.14 \\
.15 \\
.13 \\
.16\end{array}$ & $\begin{array}{l}-- \\
-- \\
-- \\
--\end{array}$ & $\begin{array}{l}-- \\
-- \\
-- \\
--\end{array}$ & $\begin{array}{r}4 \\
11 \\
11 \\
6 \\
13\end{array}$ & $\begin{array}{c}0.000440 \\
.00176 \\
.00242 \\
.00120 \\
.00299\end{array}$ & $\begin{array}{l}20-40 \\
20-40 \\
20-40 \\
20-40 \\
20-40\end{array}$ & $\begin{array}{r}0.000192 \\
.000355 \\
.000245 \\
.000103 \\
.000546\end{array}$ \\
\hline $\begin{array}{l}05-10-83 \\
05-13-83 \\
05-20-83 \\
05-24-83 \\
05-29-83\end{array}$ & $\begin{array}{l}.33 \\
.30 \\
.24 \\
.40 \\
.84\end{array}$ & $\begin{array}{l}.33 \\
.30 \\
.24 \\
.40 \\
.84\end{array}$ & $\begin{array}{l}.40 \\
.40 \\
.39 \\
.48 \\
.83\end{array}$ & $\begin{array}{l}4.2 \\
4.1 \\
4.2 \\
4.1 \\
4.1\end{array}$ & $\begin{array}{l}.20 \\
.18 \\
.15 \\
.20 \\
.25\end{array}$ & $\begin{array}{l}- \\
- \\
- \\
-\end{array}$ & $\begin{array}{l}-- \\
-- \\
-- \\
--\end{array}$ & $\begin{array}{r}17 \\
10 \\
4 \\
10 \\
29\end{array}$ & $\begin{array}{l}.00561 \\
.00300 \\
.000960 \\
.00400 \\
.0244\end{array}$ & $\begin{array}{l}20-40 \\
20-40 \\
20-40 \\
20-40 \\
20-40\end{array}$ & $\begin{array}{l}.00296 \\
.00296 \\
.000645 \\
.000540 \\
.00586\end{array}$ \\
\hline $\begin{array}{l}05-31-83 \\
06-03-83 \\
06-07-83 \\
06-09-83 \\
06-14-83\end{array}$ & $\begin{array}{r}1.26 \\
.98 \\
.83 \\
.93 \\
.80\end{array}$ & $\begin{array}{r}1.26 \\
.98 \\
.83 \\
.93 \\
.80\end{array}$ & $\begin{array}{l}.98 \\
.82 \\
.73 \\
.89 \\
-.\end{array}$ & $\begin{array}{l}4.1 \\
4.2 \\
4.1 \\
4.1 \\
4.1\end{array}$ & $\begin{array}{l}.31 \\
.29 \\
.27 \\
.26 \\
-.\end{array}$ & $\begin{array}{l}-- \\
-- \\
--\end{array}$ & $\begin{array}{l}-- \\
-- \\
-- \\
--\end{array}$ & $\begin{array}{l}70 \\
60 \\
17 \\
14 \\
11\end{array}$ & $\begin{array}{l}.0882 \\
.0588 \\
.0141 \\
.0130 \\
.00880\end{array}$ & $\begin{array}{l}20-40 \\
20-40 \\
20-40 \\
20-40 \\
20-40\end{array}$ & $\begin{array}{l}.00472 \\
.00313 \\
.000997 \\
.000404 \\
.000248\end{array}$ \\
\hline
\end{tabular}


Table 1.--Hydraulic and sediment-transport data--Continued

\begin{tabular}{|c|c|c|c|c|c|c|c|c|c|c|c|}
\hline Date & $\begin{array}{c}\text { Water } \\
\text { discharge } \\
\text { (bedload) } \\
\left(\mathrm{m}^{3} / \mathrm{s}\right)\end{array}$ & $\begin{array}{c}\text { Water } \\
\text { discharge } \\
\text { (suspended } \\
\text { load) } \\
\left(\mathrm{m}^{3} / \mathrm{s}\right)\end{array}$ & $\begin{array}{l}\text { Mean } \\
\text { flow } \\
\text { velocity } \\
(\mathrm{m} / \mathrm{s})\end{array}$ & $\begin{array}{l}\text { Water- } \\
\text { surface } \\
\text { width } \\
(\mathrm{m})\end{array}$ & $\begin{array}{l}\text { Mean } \\
\text { flow } \\
\text { depth } \\
(m)\end{array}$ & $\begin{array}{l}\text { Water- } \\
\text { surface } \\
\text { slope } \\
(\mathrm{m} / \mathrm{m})\end{array}$ & $\begin{array}{l}\text { Water } \\
\text { temp. } \\
\left({ }^{\circ} \mathrm{C}\right)\end{array}$ & $\begin{array}{c}\text { Suspended } \\
\text { sediment } \\
\text { concern- } \\
\text { tration } \\
(\mathrm{mg} / \mathrm{L})\end{array}$ & $\begin{array}{c}\text { Suspended } \\
\text { load } \\
(\mathrm{kg} / \mathrm{s})\end{array}$ & $\begin{array}{c}\text { Number of } \\
\text { sampling } \\
\text { points } \\
\text { for } \\
\text { bedload }\end{array}$ & $\begin{array}{r}\text { Bedload } \\
(\mathrm{kg} / \mathrm{s})\end{array}$ \\
\hline
\end{tabular}

81. Cross Creek near South Fork, Colo. 5 --Continued

\begin{tabular}{|c|c|c|c|c|c|c|c|c|c|c|c|}
\hline $\begin{array}{l}06-16-83 \\
06-20-83 \\
06-22-83 \\
06-25-83 \\
06-28-83\end{array}$ & $\begin{array}{r}0.76 \\
.59 \\
.74 \\
.93 \\
.74\end{array}$ & $\begin{array}{r}0.76 \\
.59 \\
.74 \\
.93 \\
.74\end{array}$ & $\begin{array}{r}0.74 \\
.63 \\
.82 \\
-- \\
.71\end{array}$ & $\begin{array}{l}4.1 \\
4.1 \\
4.1 \\
4.1 \\
4.3\end{array}$ & $\begin{array}{r}0.25 \\
.23 \\
.22 \\
-- \\
.24\end{array}$ & $\begin{array}{l}-- \\
-- \\
-- \\
--\end{array}$ & $\begin{array}{l}-- \\
-- \\
-- \\
--\end{array}$ & $\begin{array}{r}9 \\
16 \\
11 \\
51 \\
14\end{array}$ & $\begin{array}{c}0.00684 \\
.00944 \\
.00814 \\
.0474 \\
.0104\end{array}$ & $\begin{array}{l}20-40 \\
20-40 \\
20-40 \\
20-40 \\
20-40\end{array}$ & $\begin{array}{c}0.000608 \\
.000908 \\
.00127 \\
.00780 \\
.00197\end{array}$ \\
\hline $\begin{array}{l}06-30-83 \\
07-07-83 \\
07-12-83 \\
07-19-83 \\
07-21-83\end{array}$ & $\begin{array}{l}.55 \\
.24 \\
.26 \\
.22 \\
.35\end{array}$ & $\begin{array}{l}.55 \\
.24 \\
.26 \\
.22 \\
.35\end{array}$ & $\begin{array}{l}.55 \\
.31 \\
.38 \\
.32 \\
.46\end{array}$ & $\begin{array}{l}4.1 \\
4.0 \\
4.1 \\
4.1 \\
4.1\end{array}$ & $\begin{array}{l}.25 \\
.19 \\
.16 \\
.17 \\
.19\end{array}$ & $\begin{array}{l}-- \\
\ldots \\
\ldots \\
--\end{array}$ & $\begin{array}{l}-- \\
-- \\
-- \\
-- \\
--\end{array}$ & $\begin{array}{r}8 \\
5 \\
2 \\
4 \\
34\end{array}$ & $\begin{array}{l}.00220 \\
.00120 \\
.000520 \\
.000880 \\
.0119\end{array}$ & $\begin{array}{l}20-40 \\
20-40 \\
20-40 \\
20-40 \\
20-40\end{array}$ & $\begin{array}{l}.000197 \\
.000050 \\
.000198 \\
.000188 \\
.000211\end{array}$ \\
\hline $\begin{array}{l}07-26-83 \\
08-02-83 \\
08-11-83 \\
08-16-83 \\
08-23-83 \\
08-29-83\end{array}$ & $\begin{array}{l}.22 \\
.18 \\
.29 \\
.24 \\
.19 \\
.20\end{array}$ & $\begin{array}{l}.22 \\
.18 \\
.29 \\
.24 \\
.19 \\
.20\end{array}$ & $\begin{array}{l}.34 \\
.26 \\
.38 \\
.33 \\
.28 \\
.35\end{array}$ & $\begin{array}{l}4.0 \\
4.1 \\
4.1 \\
4.1 \\
4.1 \\
2.8\end{array}$ & $\begin{array}{l}.16 \\
.17 \\
.19 \\
.17 \\
.16 \\
.21\end{array}$ & $\begin{array}{l}-- \\
\ldots \\
-- \\
-- \\
-\end{array}$ & $\begin{array}{l}-- \\
-- \\
-- \\
-- \\
--\end{array}$ & $\begin{array}{l}3 \\
3 \\
7 \\
3 \\
2 \\
3\end{array}$ & $\begin{array}{l}.000660 \\
.000540 \\
.00203 \\
.000720 \\
.000380 \\
.000600\end{array}$ & $\begin{array}{l}20-40 \\
20-40 \\
20-40 \\
20-40 \\
20-40 \\
20-40\end{array}$ & $\begin{array}{l}.000143 \\
.000010 \\
.000032 \\
.000020 \\
.0000091 \\
.0000024\end{array}$ \\
\hline
\end{tabular}

82. La Garita Creek near La Garita, Colo. 5

\begin{tabular}{|c|c|c|c|c|c|c|c|c|c|c|c|}
\hline $\begin{array}{l}04-22-83 \\
04-26-83 \\
04-28-83 \\
05-02-83 \\
05-04-83\end{array}$ & $\begin{array}{r}0.46 \\
.75 \\
.53 \\
.44 \\
.47\end{array}$ & $\begin{array}{r}0.46 \\
.75 \\
.53 \\
.44 \\
.47\end{array}$ & $\begin{array}{r}0.44 \\
.58 \\
.52 \\
.44 \\
.48\end{array}$ & $\begin{array}{l}5.2 \\
5.2 \\
5.0 \\
5.2 \\
4.9\end{array}$ & $\begin{array}{r}0.20 \\
.25 \\
.20 \\
.19 \\
.20\end{array}$ & $\begin{array}{l}-- \\
-- \\
-- \\
--\end{array}$ & $\begin{array}{l}-- \\
-- \\
-- \\
--\end{array}$ & $\begin{array}{r}57 \\
211 \\
72 \\
36 \\
35\end{array}$ & $\begin{array}{c}0.0262 \\
.158 \\
.0382 \\
.0158 \\
.0158\end{array}$ & $\begin{array}{l}20-40 \\
20-40 \\
20-40 \\
20-40 \\
20-40\end{array}$ & $\begin{array}{r}0.00182 \\
.00673 \\
.00365 \\
.00457 \\
.00431\end{array}$ \\
\hline $\begin{array}{l}05-07-83 \\
05-11-83 \\
05-16-83 \\
05-23-83 \\
05-25-83\end{array}$ & $\begin{array}{r}.60 \\
1.48 \\
.69 \\
1.19 \\
1.95\end{array}$ & $\begin{array}{r}.60 \\
1.48 \\
.69 \\
1.19 \\
1.95\end{array}$ & $\begin{array}{l}.52 \\
.82 \\
.59 \\
.72 \\
.95\end{array}$ & $\begin{array}{l}5.1 \\
5.0 \\
4.7 \\
5.5 \\
5.5\end{array}$ & $\begin{array}{l}.23 \\
.36 \\
.25 \\
.30 \\
.38\end{array}$ & $\begin{array}{l}-- \\
-- \\
-- \\
--\end{array}$ & $\begin{array}{l}-- \\
-- \\
-- \\
--\end{array}$ & $\begin{array}{r}84 \\
243 \\
36 \\
86 \\
280\end{array}$ & $\begin{array}{l}.0504 \\
.360 \\
.0248 \\
.102 \\
.546\end{array}$ & $\begin{array}{l}20-40 \\
20-40 \\
20-40 \\
20-40 \\
20-40\end{array}$ & $\begin{array}{l}.00865 \\
.0185 \\
.00318 \\
.0159 \\
.0342\end{array}$ \\
\hline $\begin{array}{l}06-01-83 \\
06-03-83 \\
06-08-83 \\
06-13-83 \\
06-15-83\end{array}$ & $\begin{array}{l}3.40 \\
3.00 \\
2.46 \\
2.08 \\
1.97\end{array}$ & $\begin{array}{l}3.40 \\
3.00 \\
2.46 \\
2.08 \\
1.97\end{array}$ & $\begin{array}{l}1.3 \\
1.1 \\
.99 \\
.88 \\
.84\end{array}$ & $\begin{array}{l}6.6 \\
6.1 \\
5.9 \\
5.8 \\
5.7\end{array}$ & $\begin{array}{l}.41 \\
.45 \\
.42 \\
.41 \\
.41\end{array}$ & $\begin{array}{l}-- \\
-- \\
-- \\
--\end{array}$ & $\begin{array}{l}-- \\
-- \\
-- \\
--\end{array}$ & $\begin{array}{r}193 \\
110 \\
62 \\
51 \\
49\end{array}$ & $\begin{array}{l}.656 \\
.330 \\
.153 \\
.106 \\
.0965\end{array}$ & $\begin{array}{l}20-40 \\
20-40 \\
20-40 \\
20-40 \\
20-40\end{array}$ & $\begin{array}{l}.0107 \\
.0852 \\
.0105 \\
.00943 \\
.00748\end{array}$ \\
\hline $\begin{array}{l}06-21-83 \\
06-22-83 \\
06-26-83 \\
06-27-83 \\
06-29-83\end{array}$ & $\begin{array}{l}1.63 \\
1.78 \\
2.36 \\
2.34 \\
1.62\end{array}$ & $\begin{array}{l}1.63 \\
1.78 \\
2.36 \\
2.34 \\
1.62\end{array}$ & $\begin{array}{l}.79 \\
.85 \\
-. \\
.95 \\
.77\end{array}$ & $\begin{array}{l}5.7 \\
5.7 \\
6.0 \\
6.0 \\
5.6\end{array}$ & $\begin{array}{l}.37 \\
.37 \\
-- \\
.41 \\
.37\end{array}$ & $\begin{array}{l}-- \\
-- \\
-- \\
--\end{array}$ & $\begin{array}{l}-- \\
-- \\
-- \\
--\end{array}$ & $\begin{array}{r}41 \\
49 \\
120 \\
83 \\
31\end{array}$ & $\begin{array}{l}.0668 \\
.0872 \\
.283 \\
.194 \\
.0502\end{array}$ & $\begin{array}{l}20-40 \\
20-40 \\
20-40 \\
20-40 \\
20-40\end{array}$ & $\begin{array}{l}.00368 \\
.00475 \\
.0179 \\
.0235 \\
.0307\end{array}$ \\
\hline $\begin{array}{l}07-06-83 \\
07-13-83 \\
07-27-83 \\
08-03-83 \\
08-04-83\end{array}$ & $\begin{array}{r}1.10 \\
.71 \\
.65 \\
.44 \\
.91\end{array}$ & $\begin{array}{r}1.10 \\
.71 \\
.65 \\
.44 \\
.91\end{array}$ & $\begin{array}{l}.55 \\
.47 \\
.44 \\
.35 \\
.54\end{array}$ & $\begin{array}{l}5.5 \\
5.1 \\
5.0 \\
4.8 \\
5.2\end{array}$ & $\begin{array}{l}.37 \\
.30 \\
.29 \\
.26 \\
.32\end{array}$ & $\begin{array}{l}-- \\
-- \\
-- \\
--\end{array}$ & $\begin{array}{l}-- \\
-- \\
-- \\
--\end{array}$ & $\begin{array}{r}18 \\
3 \\
48 \\
15 \\
89\end{array}$ & $\begin{array}{l}.0198 \\
.00213 \\
.0312 \\
.00660 \\
.0810\end{array}$ & $\begin{array}{l}20-40 \\
20-40 \\
20-40 \\
20-40 \\
20-40\end{array}$ & $\begin{array}{l}.00527 \\
.00140 \\
.00129 \\
.000071 \\
.00591\end{array}$ \\
\hline $\begin{array}{l}08-10-83 \\
08-15-83 \\
08-24-83 \\
08-29-83 \\
09-08-83\end{array}$ & $\begin{array}{l}.37 \\
.38 \\
.44 \\
.28 \\
.37\end{array}$ & $\begin{array}{l}.37 \\
.38 \\
.44 \\
.28 \\
.37\end{array}$ & $\begin{array}{l}.37 \\
.34 \\
.39 \\
.29 \\
.34\end{array}$ & $\begin{array}{l}4.6 \\
4.6 \\
4.8 \\
4.6 \\
4.7\end{array}$ & $\begin{array}{l}.22 \\
.24 \\
.24 \\
.21 \\
.23\end{array}$ & $\begin{array}{l}-- \\
-- \\
-- \\
--\end{array}$ & $\begin{array}{l}-- \\
-- \\
-- \\
--\end{array}$ & $\begin{array}{r}15 \\
7 \\
16 \\
9 \\
10\end{array}$ & $\begin{array}{l}.00555 \\
.00266 \\
.00704 \\
.00252 \\
.00370\end{array}$ & $\begin{array}{l}20-40 \\
20-40 \\
20-40 \\
20-40 \\
20-40\end{array}$ & $\begin{array}{l}.00102 \\
.00202 \\
.000525 \\
.00143 \\
.000497\end{array}$ \\
\hline
\end{tabular}


Table 1.--Hydraulic and sediment-transport data--Continued

\begin{tabular}{|c|c|c|c|c|c|c|c|c|c|c|c|}
\hline Date & $\begin{array}{c}\text { Water } \\
\text { discharge } \\
\text { (bedload) } \\
\left(\mathrm{m}^{3} / \mathrm{s}\right)\end{array}$ & $\begin{array}{c}\text { Water } \\
\text { discharge } \\
\text { (suspended } \\
\text { load) } \\
\left(\mathrm{m}^{3} / \mathrm{s}\right)\end{array}$ & $\begin{array}{l}\text { Mean } \\
\text { flow } \\
\text { velocity } \\
(\mathrm{m} / \mathrm{s})\end{array}$ & $\begin{array}{l}\text { Water- } \\
\text { surface } \\
\text { width } \\
\text { (m) }\end{array}$ & $\begin{array}{l}\text { Mean } \\
\text { flow } \\
\text { depth } \\
(m)\end{array}$ & $\begin{array}{l}\text { Water- } \\
\text { surface } \\
\text { slope } \\
(\mathrm{m} / \mathrm{m})\end{array}$ & $\begin{array}{l}\text { Water } \\
\text { temp. } \\
\left({ }^{\circ} \mathrm{C}\right)\end{array}$ & $\begin{array}{l}\text { Suspended } \\
\text { sediment } \\
\text { concern- } \\
\text { tration } \\
(\mathrm{mg} / \mathrm{L})\end{array}$ & $\begin{array}{c}\text { Suspended } \\
\text { load } \\
(\mathrm{kg} / \mathrm{s})\end{array}$ & $\begin{array}{c}\text { Number of } \\
\text { sampling } \\
\text { points } \\
\text { for } \\
\text { bedload }\end{array}$ & $\begin{array}{r}\text { Bedload } \\
(\mathrm{kg} / \mathrm{s})\end{array}$ \\
\hline
\end{tabular}

$\begin{array}{llll}04-26-83 & 0.058 & 0.058 & 0.56 \\ 04-28-83 & .033 & .033 & .48 \\ 05-03-83 & .033 & .033 & .42 \\ 05-05-83 & .043 & .043 & .46 \\ 05-10-83 & .059 & .059 & .43 \\ & & & \\ 05-12-83 & .078 & .078 & .52 \\ 05-17-83 & .099 & .099 & .62 \\ 05-19-83 & .10 & .10 & .54 \\ 05-24-83 & .12 & .12 & .67 \\ 05-26-83 & .12 & .12 & .63 \\ 05-31-83 & .14 & .14 & .64 \\ 06-02-83 & .15 & .15 & .68 \\ 06-07-83 & .13 & .13 & .65 \\ 06-09-83 & .11 & .11 & .58 \\ 06-14-83 & .080 & .080 & .49 \\ 06-16-83 & .067 & .067 & .41 \\ 06-21-83 & .061 & .061 & .41 \\ 06-23-83 & .060 & .060 & .44 \\ 06-28-83 & .048 & .048 & .36 \\ 06-30-83 & .043 & .043 & .34 \\ 07-07-83 & .042 & .042 & .34 \\ 07-12-83 & .038 & .038 & .32 \\ 07-19-83 & .030 & .030 & .29 \\ 07-26-83 & .028 & .028 & .29 \\ 08-02-83 & .029 & .029 & .33 \\ & & & \\ 08-11-83 & .022 & .022 & .24 \\ 08-16-83 & .025 & .025 & .28 \\ 08-23-83 & .018 & .018 & .11 \\ 09-01-83 & .016 & .016 & .18\end{array}$

83. House Canyon Creek near Creede, Colo. 5

\begin{tabular}{|c|c|c|c|c|c|c|c|}
\hline 0.97 & 0.11 & $=-$ & -- & 157 & 0.00911 & $20-40$ & 0.000100 \\
\hline .99 & .068 & -- & -- & 14 & .000462 & $20-40$ & .000029 \\
\hline 1.0 & .076 & -- & -- & 12 & .000396 & $20-40$ & .000044 \\
\hline 1.0 & .092 & -- & $\cdots$ & 22 & .000946 & $20-40$ & .000088 \\
\hline 1.1 & .13 & -- & -- & 19 & .00112 & $20-40$ & .000072 \\
\hline 1.1 & .13 & -- & $\ldots$ & 37 & .00289 & $20-40$ & .000626 \\
\hline 1.1 & .14 & -- & -- & 347 & .0344 & $20-40$ & .000219 \\
\hline 1.2 & .16 & -- & $=-$ & 17 & .00170 & $20-40$ & .000570 \\
\hline 1.2 & .15 & -- & - & 26 & .00312 & $20-40$ & .00119 \\
\hline 1.2 & .17 & -- & -- & 37 & .00444 & $20-40$ & .00139 \\
\hline 1.2 & .18 & -- & -- & 31 & .00434 & $20-40$ & .00108 \\
\hline 1.2 & .18 & - & -- & 25 & .00375 & $20-40$ & .00161 \\
\hline 1.2 & .16 & -- & -- & 18 & .00234 & $20-40$ & .000435 \\
\hline 1.2 & .16 & + & $\ldots$ & 21 & .00231 & $20-40$ & .000433 \\
\hline 1.2 & .14 & -- & -- & 28 & .00224 & $20-40$ & .000365 \\
\hline 1.2 & .14 & - & - & 18 & .00121 & $20-40$ & .000152 \\
\hline 1.2 & .12 & -- & -- & 18 & .00110 & $20-40$ & .000022 \\
\hline 1.2 & .12 & -- & -- & 19 & .00114 & $20-40$ & .000086 \\
\hline 1.1 & .12 & - & -- & 10 & .000480 & $20-40$ & .000079 \\
\hline 1.1 & .11 & $m$ & -- & 13 & .000559 & $20-40$ & .000196 \\
\hline 1.1 & .12 & -- & - & 23 & .000966 & $20-40$ & .000086 \\
\hline 1.1 & .11 & $\cdots$ & -- & 12 & .000456 & $20-40$ & .000094 \\
\hline 1.1 & .092 & $-\infty$ & $-\infty$ & 11 & .000330 & $20-40$ & .000039 \\
\hline 1.1 & .086 & - & $=$ & 6 & .000168 & $20-40$ & .000196 \\
\hline 1.1 & .080 & - & -- & 31 & .000899 & $20-40$ & .000061 \\
\hline 1.1 & .080 & - & - & 10 & .000220 & $20-40$ & .0000076 \\
\hline 1.2 & .076 & - & -. & 6 & .000150 & $20-40$ & .000010 \\
\hline 1.2 & .14 & -- & -- & 6 & .000108 & $20-40$ & .000018 \\
\hline 1.1 & .078 & -- & - & 3 & .000048 & $20-40$ & .0000017 \\
\hline
\end{tabular}

84. Crooked Canyon Creek near Creede, Colo. 5

\begin{tabular}{|c|c|c|c|c|c|c|c|c|c|c|c|}
\hline $04-19-83$ & 0.064 & 0.064 & 0.23 & 2.5 & 0.11 & -- & -- & 82 & 0.00525 & $20-40$ & 0.000059 \\
\hline $04-26-83$ & .18 & .18 & .45 & 2.6 & .15 & -- & - & 19 & .00342 & $20-40$ & .000120 \\
\hline $04-28-83$ & .18 & .18 & .46 & 2.8 & .14 & -- & -- & 26 & .00468 & $20-40$ & .000030 \\
\hline $05-03-83$ & .22 & .22 & .55 & 2.8 & .14 & -- & -- & 12 & .00264 & $20-40$ & .000033 \\
\hline $05-05-83$ & .26 & .26 & .64 & 2.8 & .15 & -- & $\rightarrow$ & 17 & .00442 & $20-40$ & .000026 \\
\hline $05-10-83$ & .60 & .60 & .82 & 3.2 & .23 & -- & -- & 63 & .0378 & $20-40$ & .000925 \\
\hline $05-12-83$ & .62 & .62 & .75 & 3.3 & .25 & -- & -- & 40 & .0248 & $20-40$ & .000407 \\
\hline $05-17-83$ & .44 & .44 & .73 & 3.1 & .19 & -- & - & 24 & .0106 & $20-40$ & .000112 \\
\hline $05-19-83$ & .41 & .41 & .63 & 3.0 & .22 & -- & -- & 24 & .00984 & $20-40$ & .000034 \\
\hline $05-24-83$ & .87 & .87 & .96 & 3.4 & .27 & -- & -- & 91 & .0792 & $20-40$ & .00136 \\
\hline
\end{tabular}


Table 1.--Hydraulic and sediment-transport data--Continued

\begin{tabular}{|c|c|c|c|c|c|c|c|c|c|c|c|}
\hline Date & $\begin{array}{c}\text { Water } \\
\text { discharge } \\
\text { (bedload) } \\
\left(\mathrm{s}^{3} / \mathrm{s}\right)\end{array}$ & $\begin{array}{c}\text { Water } \\
\text { discharge } \\
\text { (suspended } \\
\text { load) } \\
\left(\mathrm{m}^{3} / \mathrm{s}\right)\end{array}$ & $\begin{array}{l}\text { Mean } \\
\text { flow } \\
\text { velocity } \\
(\mathrm{m} / \mathrm{s})\end{array}$ & $\begin{array}{l}\text { Water- } \\
\text { surface } \\
\text { width } \\
\text { (m) }\end{array}$ & $\begin{array}{l}\text { Mean } \\
\text { flow } \\
\text { depth } \\
(m)\end{array}$ & $\begin{array}{l}\text { Water- } \\
\text { surface } \\
\text { slope } \\
(\mathrm{m} / \mathrm{m})\end{array}$ & $\begin{array}{l}\text { Water } \\
\text { temp. } \\
\left({ }^{\circ} \mathrm{C}\right)\end{array}$ & $\begin{array}{l}\text { Suspended } \\
\text { sediment } \\
\text { concern- } \\
\text { tration } \\
\left(\mathrm{mg}_{\mathrm{g}} \mathrm{L}\right)\end{array}$ & $\begin{array}{c}\text { Suspended } \\
\text { load } \\
\left(\mathrm{kg}_{\mathrm{g}} / \mathrm{s}\right)\end{array}$ & $\begin{array}{l}\text { Number of } \\
\text { sampling } \\
\text { points } \\
\text { for } \\
\text { bedload }\end{array}$ & $\begin{array}{r}\text { Bedload } \\
\left(\mathrm{kg}_{\mathrm{g}} / \mathrm{s}\right)\end{array}$ \\
\hline
\end{tabular}

\section{Crooked Canyon Creek near Creede, Colo. ${ }^{5}-$ - Cont inued}

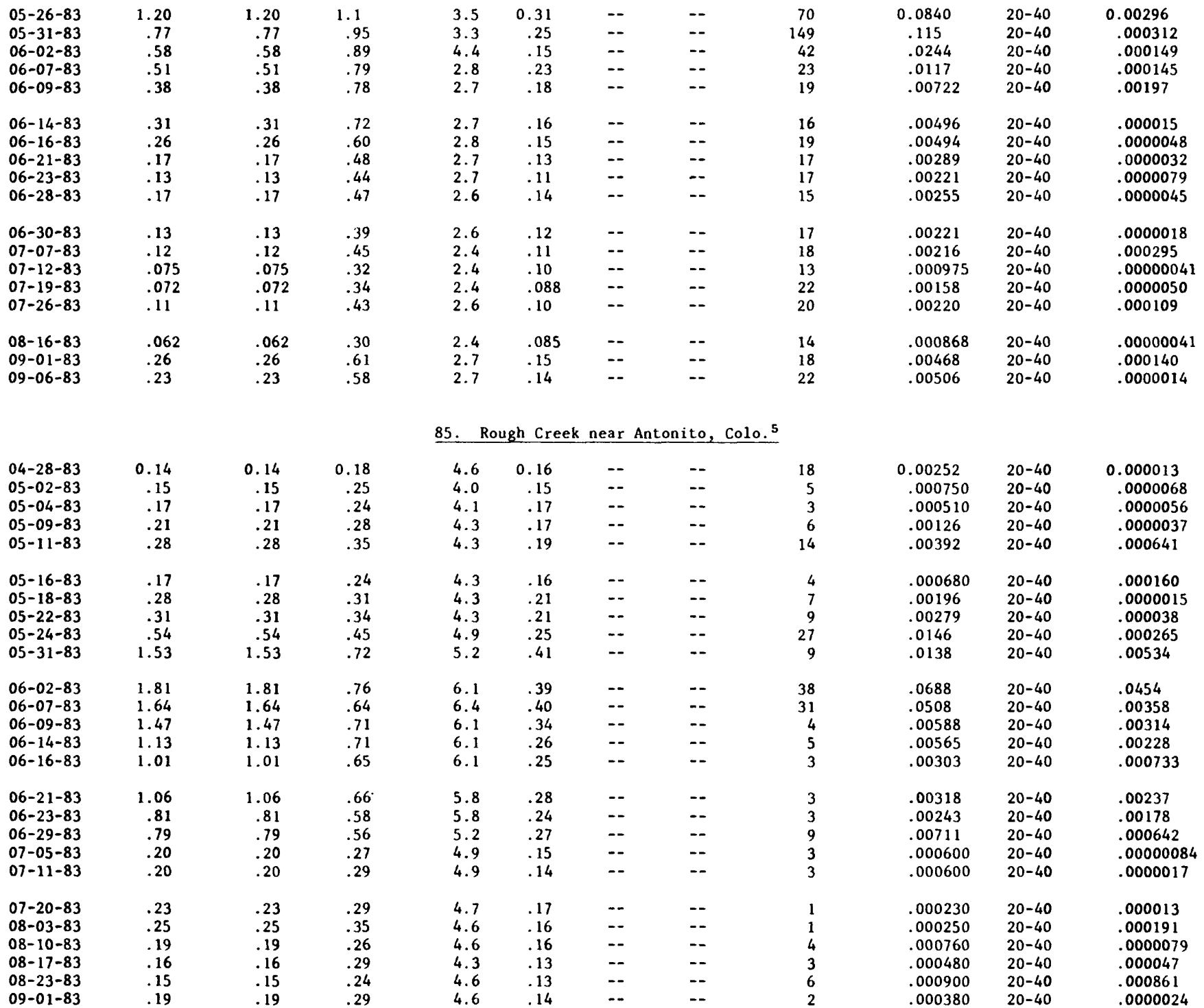


Table 1.--Hydraulic and sediment-transport data--Continued

\begin{tabular}{|c|c|c|c|c|c|c|c|c|c|c|c|}
\hline Date & $\begin{array}{c}\text { Water } \\
\text { discharge } \\
\text { (bedload) } \\
\left(\mathrm{m}^{3} / \mathrm{s}\right)\end{array}$ & $\begin{array}{c}\text { Water } \\
\text { discharge } \\
\text { (suspended } \\
\text { load) } \\
\left(\mathrm{m}^{3} / \mathrm{s}\right)\end{array}$ & $\begin{array}{l}\text { Mean } \\
\text { flow } \\
\text { velocity } \\
(\mathrm{m} / \mathrm{s})\end{array}$ & $\begin{array}{l}\text { Water- } \\
\text { surface } \\
\text { width } \\
\text { (m) }\end{array}$ & $\begin{array}{l}\text { Mean } \\
\text { flow } \\
\text { depth } \\
\text { (m) }\end{array}$ & $\begin{array}{l}\text { Water- } \\
\text { surface } \\
\text { slope } \\
(\mathrm{m} / \mathrm{m})\end{array}$ & $\begin{array}{l}\text { Water } \\
\text { temp. } \\
\left({ }^{\circ} \mathrm{C}\right)\end{array}$ & $\begin{array}{l}\text { Suspended } \\
\text { sediment } \\
\text { concern- } \\
\text { tration } \\
\text { (mg/L) }\end{array}$ & $\begin{array}{c}\text { Suspended } \\
\text { load } \\
(\mathrm{kg} / \mathrm{s})\end{array}$ & $\begin{array}{l}\text { Number of } \\
\text { sampling } \\
\text { points } \\
\text { for } \\
\text { bedload }\end{array}$ & $\begin{array}{r}\text { Bedload } \\
\left(\mathrm{kg}_{\mathrm{g}} / \mathrm{s}\right)\end{array}$ \\
\hline
\end{tabular}

\begin{tabular}{|c|c|c|c|c|c|c|c|c|c|c|c|}
\hline $\begin{array}{l}05-04-83 \\
05-09-83 \\
05-11-83 \\
05-16-83 \\
05-18-83\end{array}$ & $\begin{array}{r}0.10 \\
.16 \\
.18 \\
.10 \\
.11\end{array}$ & $\begin{array}{r}0.10 \\
.16 \\
.18 \\
.10 \\
.11\end{array}$ & $\begin{array}{r}0.31 \\
.46 \\
.45 \\
.29 \\
.34\end{array}$ & $\begin{array}{l}3.0 \\
3.0 \\
3.2 \\
3.2 \\
3.4\end{array}$ & $\begin{array}{l}0.11 \\
.11 \\
.13 \\
.11 \\
.094\end{array}$ & $\begin{array}{l}-- \\
-- \\
-- \\
--\end{array}$ & $\begin{array}{l}-- \\
-- \\
-- \\
--\end{array}$ & $\begin{array}{r}7 \\
12 \\
13 \\
5 \\
13\end{array}$ & $\begin{array}{l}0.000700 \\
.00192 \\
.00234 \\
.000500 \\
.00143\end{array}$ & $\begin{array}{l}20-40 \\
20-40 \\
20-40 \\
20-40 \\
20-40\end{array}$ & $\begin{array}{c}0.000020 \\
.000018 \\
.000229 \\
.0000028 \\
.000027\end{array}$ \\
\hline $\begin{array}{l}05-22-83 \\
05-24-83 \\
05-31-83 \\
06-02-83 \\
06-07-83\end{array}$ & $\begin{array}{l}.082 \\
.12 \\
.51 \\
.53 \\
.29\end{array}$ & $\begin{array}{l}.082 \\
.12 \\
.51 \\
.53 \\
.29\end{array}$ & $\begin{array}{l}.32 \\
.36 \\
.57 \\
.56 \\
.33\end{array}$ & $\begin{array}{l}3.4 \\
3.4 \\
4.3 \\
4.3 \\
4.3\end{array}$ & $\begin{array}{l}.078 \\
.10 \\
.21 \\
.22 \\
.21\end{array}$ & $\begin{array}{l}-- \\
-- \\
--\end{array}$ & $\begin{array}{l}-- \\
-- \\
-- \\
--\end{array}$ & $\begin{array}{r}1 \\
13 \\
14 \\
15 \\
1\end{array}$ & $\begin{array}{l}.000082 \\
.00156 \\
.00714 \\
.00795 \\
.000290\end{array}$ & $\begin{array}{l}20-40 \\
20-40 \\
20-40 \\
20-40 \\
20-40\end{array}$ & $\begin{array}{l}.000051 \\
.000203 \\
.00281 \\
.00188 \\
.000818\end{array}$ \\
\hline $\begin{array}{l}06-09-83 \\
06-14-83 \\
06-16-83 \\
06-21-83 \\
06-23-83\end{array}$ & $\begin{array}{l}.78 \\
.57 \\
.56 \\
.57 \\
.72\end{array}$ & $\begin{array}{l}.78 \\
.57 \\
.56 \\
.57 \\
.72\end{array}$ & $\begin{array}{l}.86 \\
.74 \\
.74 \\
.73 \\
.83\end{array}$ & $\begin{array}{l}3.6 \\
3.0 \\
3.0 \\
3.0 \\
3.4\end{array}$ & $\begin{array}{l}.25 \\
.25 \\
.25 \\
.26 \\
.26\end{array}$ & $\begin{array}{l}m \\
\cdots \\
\cdots \\
\cdots\end{array}$ & $\begin{array}{l}-- \\
-- \\
-- \\
--\end{array}$ & $\begin{array}{r}8 \\
6 \\
7 \\
12 \\
12\end{array}$ & $\begin{array}{l}.00624 \\
.00342 \\
.00392 \\
.00684 \\
.00864\end{array}$ & $\begin{array}{l}20-40 \\
20-40 \\
20-40 \\
20-40 \\
20-40\end{array}$ & $\begin{array}{l}.00570 \\
.000286 \\
.000426 \\
.00315 \\
.000601\end{array}$ \\
\hline $\begin{array}{l}06-29-83 \\
07-05-83 \\
07-11-83 \\
07-27-83 \\
08-03-83\end{array}$ & $\begin{array}{l}.69 \\
.46 \\
.45 \\
.10 \\
.11\end{array}$ & $\begin{array}{l}.69 \\
.46 \\
.45 \\
.10 \\
.11\end{array}$ & $\begin{array}{l}.81 \\
.66 \\
.74 \\
.29 \\
.31\end{array}$ & $\begin{array}{l}3.4 \\
3.0 \\
3.0 \\
2.7 \\
2.9\end{array}$ & $\begin{array}{l}.25 \\
.23 \\
.20 \\
.13 \\
.13\end{array}$ & $\begin{array}{l}-- \\
-- \\
-- \\
-\end{array}$ & $\begin{array}{l}-- \\
-- \\
-- \\
--\end{array}$ & $\begin{array}{r}11 \\
9 \\
114 \\
2 \\
7\end{array}$ & $\begin{array}{l}.00759 \\
.00414 \\
.0513 \\
.000200 \\
.000770\end{array}$ & $\begin{array}{l}20-40 \\
20-40 \\
20-40 \\
20-40 \\
20-40\end{array}$ & $\begin{array}{l}.000113 \\
.000765 \\
.000011 \\
.00000047 \\
.0000040\end{array}$ \\
\hline $\begin{array}{l}08-10-83 \\
08-23-83 \\
09-01-83\end{array}$ & $\begin{array}{l}.083 \\
.075 \\
.073\end{array}$ & $\begin{array}{l}.083 \\
.075 \\
.073\end{array}$ & $\begin{array}{l}.24 \\
.23 \\
.25\end{array}$ & $\begin{array}{l}2.9 \\
3.6 \\
2.4\end{array}$ & $\begin{array}{l}.12 \\
.13 \\
.12\end{array}$ & $\begin{array}{l}-- \\
-- \\
--\end{array}$ & $\begin{array}{l}-- \\
--\end{array}$ & $\begin{array}{l}4 \\
2 \\
2\end{array}$ & $\begin{array}{l}.000332 \\
.000150 \\
.000146\end{array}$ & $\begin{array}{l}20-40 \\
20-40 \\
20-40\end{array}$ & $\begin{array}{l}.00000050 \\
.0000013 \\
.0000034\end{array}$ \\
\hline
\end{tabular}

\section{Wapsipinicon River near Dewitt, Iowa 16}

\begin{tabular}{|c|c|c|c|c|c|c|c|c|c|c|c|}
\hline $\begin{array}{l}04-13-78 \\
06-22-78 \\
03-21-79 \\
04-26-79 \\
08-22-79\end{array}$ & $\begin{array}{l}173 \\
61.7 \\
277 \\
169 \\
171\end{array}$ & $\begin{array}{c}173 \\
61.7 \\
277 \\
169 \\
171\end{array}$ & $\begin{array}{r}0.95 \\
.73 \\
1.1 \\
.94 \\
.95\end{array}$ & $\begin{array}{l}91 \\
72 \\
94 \\
93 \\
79\end{array}$ & $\begin{array}{l}2.0 \\
1.2 \\
2.7 \\
1.9 \\
2.3\end{array}$ & $\begin{array}{l}-- \\
-- \\
-- \\
--\end{array}$ & $\begin{array}{c}10.5 \\
-- \\
-- \\
=- \\
=-\end{array}$ & $\begin{array}{l}189 \\
234 \\
538 \\
218 \\
292\end{array}$ & $\begin{array}{c}32.7 \\
14.4 \\
149 \\
36.8 \\
49.9\end{array}$ & $\begin{array}{l}19 \\
20 \\
20 \\
20 \\
17\end{array}$ & $\begin{array}{c}10.2 \\
1.17 \\
14.4 \\
1.47 \\
4.40\end{array}$ \\
\hline $\begin{array}{l}07-02-80 \\
08-25-80 \\
04-14-81 \\
06-17-81 \\
09-04-81\end{array}$ & $\begin{array}{c}66.3 \\
210 \\
90.1 \\
137 \\
154\end{array}$ & $\begin{array}{c}68.0 \\
208 \\
90.1 \\
137 \\
154\end{array}$ & $\begin{array}{c}.63 \\
1.1 \\
.82 \\
.97 \\
.85\end{array}$ & $\begin{array}{l}61 \\
94 \\
66 \\
66 \\
76\end{array}$ & $\begin{array}{l}1.8 \\
2.0 \\
1.7 \\
2.2 \\
2.4\end{array}$ & $\begin{array}{l}-- \\
-- \\
-- \\
--\end{array}$ & $\begin{array}{c}26.0 \\
24.5 \\
-- \\
21.5 \\
19.0\end{array}$ & $\begin{array}{r}423 \\
196 \\
565 \\
1,680 \\
308\end{array}$ & $\begin{array}{c}28.8 \\
40.8 \\
50.9 \\
230 \\
47.4\end{array}$ & $\begin{array}{l}19 \\
17 \\
14 \\
14 \\
15\end{array}$ & $\begin{array}{l}1.79 \\
8.22 \\
4.28 \\
5.12 \\
2.36\end{array}$ \\
\hline
\end{tabular}

88. Iowa River at Wapello, Iowa 17

\begin{tabular}{|c|c|c|c|c|c|c|c|c|c|c|c|}
\hline $\begin{array}{l}03-28-78 \\
04-25-78 \\
06-21-78 \\
07-25-78 \\
06-13-79\end{array}$ & $\begin{array}{l}623 \\
578 \\
368 \\
564 \\
300\end{array}$ & $\begin{array}{l}578 \\
578 \\
368 \\
564 \\
267\end{array}$ & $\begin{array}{c}1.2 \\
1.2 \\
.93 \\
1.1 \\
.67\end{array}$ & $\begin{array}{l}144 \\
144 \\
141 \\
155 \\
137\end{array}$ & $\begin{array}{l}3.4 \\
3.4 \\
2.8 \\
3.3 \\
2.9\end{array}$ & $\begin{array}{l}-- \\
-- \\
-- \\
=-\end{array}$ & $\begin{array}{c}10.0 \\
-- \\
24.0 \\
26.0 \\
23.0\end{array}$ & $\begin{array}{l}423 \\
633 \\
700 \\
370 \\
207\end{array}$ & $\begin{array}{r}244 \\
366 \\
258 \\
209 \\
55.3\end{array}$ & $\begin{array}{l}19 \\
19 \\
21 \\
25 \\
18\end{array}$ & $\begin{array}{c}20.2 \\
20.2 \\
11.7 \\
16.7 \\
1.93\end{array}$ \\
\hline $\begin{array}{l}04-03-80 \\
06-02-80 \\
09-03-80 \\
06-18-81 \\
07-02-81\end{array}$ & $\begin{array}{l}184 \\
280 \\
297 \\
343 \\
518\end{array}$ & $\begin{array}{l}184 \\
280 \\
323 \\
343 \\
518\end{array}$ & $\begin{array}{l}.70 \\
.92 \\
.95 \\
.95 \\
1.1\end{array}$ & $\begin{array}{l}136 \\
137 \\
137 \\
140 \\
146\end{array}$ & $\begin{array}{l}1.9 \\
2.2 \\
2.5 \\
2.6 \\
3.1\end{array}$ & $\begin{array}{l}-- \\
-- \\
-- \\
--\end{array}$ & $\begin{array}{r}9.0 \\
27.0 \\
23.0 \\
23.5 \\
25.0\end{array}$ & $\begin{array}{r}74 \\
720 \\
198 \\
1,160 \\
665\end{array}$ & $\begin{array}{l}13.6 \\
202 \\
64.0 \\
398 \\
344\end{array}$ & $\begin{array}{l}19 \\
13 \\
17 \\
13 \\
21\end{array}$ & $\begin{array}{l}4.57 \\
14.1 \\
16.5 \\
24.7 \\
20.0\end{array}$ \\
\hline
\end{tabular}


Table 1.--Hydraulic and sediment-transport data--Continued

\begin{tabular}{|c|c|c|c|c|c|c|c|c|c|c|c|}
\hline Date & $\begin{array}{c}\text { Water } \\
\text { discharge } \\
\text { (bedload) } \\
\left(\mathrm{m}^{3} / \mathrm{s}\right)\end{array}$ & $\begin{array}{c}\text { Water } \\
\text { discharge } \\
\text { (suspended } \\
\text { load })^{2} \\
\left(\mathrm{~m}^{3} / \mathrm{s}\right)\end{array}$ & $\begin{array}{c}\text { Mean } \\
\text { flow } \\
\text { velocity } \\
(\mathrm{m} / \mathrm{s})\end{array}$ & $\begin{array}{l}\text { Water- } \\
\text { surface } \\
\text { width } \\
(m)\end{array}$ & $\begin{array}{l}\text { Mean } \\
\text { flow } \\
\text { depth } \\
(m)\end{array}$ & $\begin{array}{l}\text { Water- } \\
\text { surface } \\
\text { slope } \\
(\mathrm{m} / \mathrm{m})\end{array}$ & $\begin{array}{l}\text { Water } \\
\text { temp. } \\
\left({ }^{\circ} \mathrm{C}\right)\end{array}$ & $\begin{array}{l}\text { Suspended } \\
\text { sediment } \\
\text { concern- } \\
\text { tration } \\
(m g / L)\end{array}$ & $\begin{array}{c}\text { Suspended } \\
\text { load } \\
\left(\mathrm{kg}_{\mathbf{g}} / \mathrm{s}\right)\end{array}$ & $\begin{array}{l}\text { Number of } \\
\text { sampling } \\
\text { points } \\
\text { for } \\
\text { bedload }\end{array}$ & $\begin{array}{r}\text { Bedload } \\
(\mathrm{kg} / \mathrm{s})\end{array}$ \\
\hline
\end{tabular}

\begin{tabular}{|c|c|c|c|c|c|c|c|c|c|c|c|}
\hline & & & & 89. & consin & iver at $M$ & scoda, & Wis. 18 & & & \\
\hline $\begin{array}{l}03-24-77 \\
04-19-77 \\
05-16-77 \\
06-15-77 \\
07-20-77\end{array}$ & $\begin{array}{l}149 \\
210 \\
118 \\
193 \\
114\end{array}$ & $\begin{array}{l}149 \\
210 \\
118 \\
193 \\
114\end{array}$ & $\begin{array}{r}0.60 \\
.63 \\
.52 \\
.64 \\
.58\end{array}$ & $\begin{array}{r}283 \\
-- \\
278 \\
292 \\
278\end{array}$ & $\begin{array}{l}0.88 \\
1.2 \\
.82 \\
1.0 \\
.71\end{array}$ & $\begin{array}{c}0.00022 \\
.00031 \\
.00041 \\
.00031 \\
.\end{array}$ & $\begin{array}{r}7.5 \\
18.5 \\
25.0 \\
21.0 \\
28.0\end{array}$ & $\begin{array}{l}12 \\
32 \\
12 \\
18 \\
59\end{array}$ & $\begin{array}{l}1.79 \\
6.72 \\
1.42 \\
3.47 \\
6.73\end{array}$ & $\begin{array}{l}20 \\
20 \\
20 \\
20 \\
20\end{array}$ & $\begin{array}{l}14.6 \\
2.78 \\
1.76 \\
3.99 \\
5.16\end{array}$ \\
\hline $\begin{array}{l}08-24-77 \\
09-27-77 \\
10-18-77 \\
11-14-77 \\
04-17-78\end{array}$ & $\begin{array}{l}86.9 \\
183 \\
289 \\
379 \\
456\end{array}$ & $\begin{array}{l}86.9 \\
183 \\
289 \\
379 \\
456\end{array}$ & $\begin{array}{l}.47 \\
.73 \\
.71 \\
.76 \\
.75\end{array}$ & $\begin{array}{r}219 \\
306 \\
302 \\
-- \\
309\end{array}$ & $\begin{array}{l}.85 \\
.82 \\
1.3 \\
1.7 \\
2.0\end{array}$ & $\begin{array}{l}.00028 \\
-. \\
.00031 \\
.00030 \\
.00031\end{array}$ & $\begin{array}{r}22.0 \\
15.0 \\
10.0 \\
6.0 \\
9.0\end{array}$ & $\begin{array}{r}7 \\
19 \\
23 \\
21 \\
10\end{array}$ & $\begin{array}{l}1.48 \\
3.48 \\
6.65 \\
7.96 \\
4.56\end{array}$ & $\begin{array}{l}20 \\
20 \\
20 \\
20 \\
20\end{array}$ & $\begin{array}{l}1.05 \\
10.8 \\
17.3 \\
23.3 \\
5.59\end{array}$ \\
\hline $\begin{array}{l}05-08-78 \\
06-06-78 \\
07-11-78 \\
08-15-78 \\
10-24-78\end{array}$ & $\begin{array}{l}195 \\
368 \\
714 \\
145 \\
210\end{array}$ & $\begin{array}{l}195 \\
368 \\
714 \\
145 \\
210\end{array}$ & $\begin{array}{l}.53 \\
.75 \\
.88 \\
.49 \\
.64\end{array}$ & $\begin{array}{r}299 \\
306 \\
310 \\
292 \\
--\end{array}$ & $\begin{array}{l}1.2 \\
1.6 \\
2.6 \\
1.0 \\
1.2\end{array}$ & $\begin{array}{l}.00033 \\
.00035 \\
.00037 \\
.00029 \\
.00028\end{array}$ & $\begin{array}{r}13.5 \\
22.5 \\
22.0 \\
26.0 \\
9.5\end{array}$ & $\begin{array}{l}28 \\
15 \\
28 \\
36 \\
13\end{array}$ & $\begin{array}{c}5.46 \\
5.52 \\
20.0 \\
5.22 \\
2.73\end{array}$ & $\begin{array}{l}20 \\
20 \\
20 \\
20 \\
20\end{array}$ & $\begin{array}{c}5.52 \\
12.9 \\
14.7 \\
5.59 \\
4.75\end{array}$ \\
\hline $\begin{array}{l}11-14-78 \\
03-28-79 \\
04-24-79 \\
07-05-79 \\
08-25-79\end{array}$ & $\begin{array}{r}196 \\
1,240 \\
413 \\
178 \\
229\end{array}$ & $\begin{array}{r}196 \\
1,240 \\
413 \\
178 \\
229\end{array}$ & $\begin{array}{l}.62 \\
1.2 \\
.78 \\
.60 \\
.65\end{array}$ & $\begin{array}{l}-- \\
-- \\
-- \\
--\end{array}$ & $\begin{array}{l}1.1 \\
3.4 \\
2.0 \\
1.3 \\
1.4\end{array}$ & $\begin{array}{l}.00025 \\
.00052 \\
.00025 \\
.00033 \\
.00036\end{array}$ & $\begin{array}{r}5.5 \\
1.0 \\
13.0 \\
22.0 \\
20.5\end{array}$ & $\begin{array}{l}27 \\
67 \\
15 \\
27 \\
20\end{array}$ & $\begin{array}{c}5.29 \\
83.1 \\
6.20 \\
4.81 \\
4.58\end{array}$ & $\begin{array}{l}20 \\
20 \\
20 \\
20 \\
20\end{array}$ & $\begin{array}{l}6.66 \\
49.0 \\
10.0 \\
4.02 \\
5.47\end{array}$ \\
\hline
\end{tabular}

\begin{tabular}{|c|c|c|c|c|c|c|c|c|c|c|c|}
\hline $04-23-77$ & 65.1 & 65.1 & -- & -- & -- & 0.00021 & 18.0 & 65 & 4.23 & 20 & 1.22 \\
\hline $05-27-77$ & 19.3 & 19.3 & -- & -- & -- & -- & 25.0 & 46 & .888 & 20 & .504 \\
\hline $06-29-77$ & 16.7 & 16.7 & -- & -- & - & .00028 & 26.0 & 41 & .685 & 20 & 1.32 \\
\hline $07-12-77$ & 20.7 & 20.7 & -- & -- & - & .00029 & 27.0 & 40 & .828 & 20 & 1.05 \\
\hline $08-02-77$ & 13.0 & 13.0 & -- & - & -- & .00035 & 24.0 & 51 & .663 & 20 & .840 \\
\hline $09-27-77$ & 67.1 & 67.1 & 0.50 & 117 & 1.2 & -- & 15.5 & 82 & 5.50 & 20 & 1.68 \\
\hline $10-19-77$ & 36.3 & 36.3 & .49 & -. & - & .00023 & 12.0 & 18 & .653 & 20 & 2.06 \\
\hline $11-08-77$ & 32.3 & 32.3 & .45 & 94 & .77 & .00023 & 10.0 & 16 & .517 & 20 & 1.20 \\
\hline $03-31-78$ & 163 & 163 & 1.0 & 84 & 1.9 & .00018 & 5.5 & 100 & 16.3 & 20 & 3.75 \\
\hline $04-10-78$ & 256 & 256 & -- & -- & - & .00020 & 6.0 & 160 & 41.0 & 20 & 3.00 \\
\hline $05-10-78$ & 28.9 & 28.9 & .54 & 94 & .57 & .00024 & 15.5 & 19 & .549 & 20 & 4.09 \\
\hline $06-13-78$ & 26.1 & 26.1 & .45 & 77 & .75 & .00029 & 21.0 & 24 & .626 & 20 & 2.11 \\
\hline $08-09-78$ & 20.1 & 20.1 & .51 & 72 & .55 & .00027 & 16.0 & 21 & .422 & 20 & 1.60 \\
\hline $10-28-78$ & 24.2 & 24.2 & .44 & 96 & .58 & .00018 & 8.0 & 20 & .484 & 20 & 1.28 \\
\hline $04-25-79$ & 80.7 & 80.7 & .48 & 122 & 1.4 & -- & 14.0 & 13 & 1.05 & 20 & 2.46 \\
\hline $05-31-79$ & 92.9 & 92.9 & .50 & 122 & 1.5 & .00011 & 18.0 & 1,130 & 105 & 20 & 1.60 \\
\hline $07-23-79$ & 17.3 & 17.3 & - & -- & - & .00017 & 24.5 & 35 & .606 & 20 & .189 \\
\hline
\end{tabular}

\section{Chippewa River near Caryville, Wis. 18}

\begin{tabular}{|c|c|c|c|c|c|c|c|c|c|c|c|}
\hline $08-30-76$ & 31.4 & 31.4 & 0.23 & 157 & 0.89 & - & 23.5 & 5 & 0.157 & 20 & 0 \\
\hline $09-20-76$ & 32.6 & 32.6 & .22 & 159 & .92 & -- & 19.5 & 7 & .228 & 20 & 0 \\
\hline $10-18-76$ & 30.6 & 30.6 & .23 & 124 & 1.1 & 0.00011 & 7.0 & 4 & .122 & 20 & 0 \\
\hline $11-17-76$ & 32.0 & 32.0 & .23 & 132 & 1.0 & .000093 & 4.5 & 5 & .160 & 20 & 0 \\
\hline $03-15-77$ & 149 & 149 & .57 & 185 & 1.4 & - & 5.5 & 4 & .596 & 20 & 0 \\
\hline
\end{tabular}


Table 1.--Hydraulic and sediment-transport data--Continued

\begin{tabular}{|c|c|c|c|c|c|c|c|c|c|c|c|}
\hline Date & $\begin{array}{c}\text { Water } \\
\text { discharge } \\
\text { (bedload) } \\
\left(\mathrm{m}^{3} / \mathrm{s}\right)\end{array}$ & $\begin{array}{c}\text { Water } \\
\text { discharge } \\
\text { (suspended } \\
\text { load) } \\
\left(\mathrm{m}^{3} / \mathrm{s}\right)\end{array}$ & $\begin{array}{l}\text { Mean } \\
\text { flow } \\
\text { velocity } \\
(\mathrm{m} / \mathrm{s})\end{array}$ & $\begin{array}{l}\text { Water- } \\
\text { surface } \\
\text { width } \\
\text { (m) }\end{array}$ & $\begin{array}{l}\text { Mean } \\
\text { flow } \\
\text { depth } \\
(m)\end{array}$ & $\begin{array}{l}\text { Water- } \\
\text { surface } \\
\text { slope } \\
(\mathrm{m} / \mathrm{m})\end{array}$ & $\begin{array}{l}\text { Water } \\
\text { temp. } \\
\left({ }^{\circ} \mathrm{C}\right)\end{array}$ & $\begin{array}{l}\text { Suspended } \\
\text { sediment } \\
\text { concern- } \\
\text { tration } \\
(\mathrm{mg} / \mathrm{L})\end{array}$ & $\begin{array}{c}\text { Suspended } \\
\text { load } \\
(\mathrm{kg} / \mathrm{s})\end{array}$ & $\begin{array}{l}\text { Number of } \\
\text { sampling } \\
\text { points } \\
\text { for } \\
\text { bedload }\end{array}$ & $\begin{array}{r}\text { Bedload } \\
\left(\mathrm{kg}_{\mathrm{g}} / \mathrm{s}\right)\end{array}$ \\
\hline
\end{tabular}

91. Chippewa River near Caryville, Wis. ${ }^{18}$--Continued

\begin{tabular}{|c|c|c|c|c|c|c|c|c|c|c|c|}
\hline $\begin{array}{l}04-13-77 \\
05-10-77 \\
07-06-77 \\
09-13-77 \\
10-12-77\end{array}$ & $\begin{array}{l}117 \\
51.8 \\
337 \\
255 \\
433\end{array}$ & $\begin{array}{l}117 \\
51.8 \\
337 \\
255 \\
433\end{array}$ & $\begin{array}{r}0.45 \\
.30 \\
.75 \\
.70 \\
.87\end{array}$ & $\begin{array}{l}185 \\
176 \\
209 \\
204 \\
231\end{array}$ & $\begin{array}{l}1.4 \\
.99 \\
2.2 \\
1.8 \\
2.2\end{array}$ & $\begin{array}{r}0.00013 \\
.00011 \\
.00023 \\
.00021 \\
.00025\end{array}$ & $\begin{array}{l}11.5 \\
17.0 \\
26.0 \\
19.0 \\
13.0\end{array}$ & $\begin{array}{r}8 \\
6 \\
23 \\
11 \\
10\end{array}$ & $\begin{array}{l}0.936 \\
.311 \\
7.75 \\
2.81 \\
4.33\end{array}$ & $\begin{array}{l}20 \\
20 \\
20 \\
20 \\
20\end{array}$ & $\begin{array}{l}0 \\
0 \\
0 \\
0 \\
5.29\end{array}$ \\
\hline $\begin{array}{l}11-16-77 \\
04-05-78 \\
04-14-78 \\
05-15-78 \\
06-06-78\end{array}$ & $\begin{array}{c}141 \\
320 \\
374 \\
94.0 \\
172\end{array}$ & $\begin{array}{c}141 \\
320 \\
374 \\
94.0 \\
172\end{array}$ & $\begin{array}{l}.53 \\
.78 \\
.80 \\
-. \\
.59\end{array}$ & $\begin{array}{r}185 \\
225 \\
231 \\
-- \\
200\end{array}$ & $\begin{array}{r}1.4 \\
1.8 \\
2.0 \\
-. \\
1.5\end{array}$ & $\begin{array}{l}.00015 \\
.00023 \\
.00025 \\
-0 \\
.00019\end{array}$ & $\begin{array}{r}8.5 \\
4.5 \\
6.5 \\
13.0 \\
22.0\end{array}$ & $\begin{array}{r}3 \\
16 \\
7 \\
9 \\
8\end{array}$ & $\begin{array}{l}.423 \\
5.12 \\
2.62 \\
.846 \\
1.38\end{array}$ & $\begin{array}{l}20 \\
20 \\
20 \\
20 \\
20\end{array}$ & $\begin{array}{l}0 \\
1.04 \\
7.51 \\
.0525 \\
.788\end{array}$ \\
\hline $\begin{array}{l}07-06-78 \\
09-19-78 \\
10-31-78 \\
04-17-79 \\
04-23-79\end{array}$ & $\begin{array}{l}348 \\
273 \\
123 \\
620 \\
779\end{array}$ & $\begin{array}{l}348 \\
273 \\
123 \\
620 \\
779\end{array}$ & $\begin{array}{l}.80 \\
-= \\
.51 \\
1.0 \\
1.1\end{array}$ & $\begin{array}{r}226 \\
-- \\
190 \\
245 \\
247\end{array}$ & $\begin{array}{r}1.9 \\
-. \\
1.3 \\
2.5 \\
2.8\end{array}$ & $\begin{array}{l}.00025 \\
.00021 \\
.00017 \\
.00025 \\
.00025\end{array}$ & $\begin{array}{r}21.0 \\
19.5 \\
10.5 \\
4.5 \\
8.0\end{array}$ & $\begin{array}{r}12 \\
4 \\
5 \\
14 \\
21\end{array}$ & $\begin{array}{c}4.18 \\
1.09 \\
.615 \\
8.68 \\
16.4\end{array}$ & $\begin{array}{l}20 \\
20 \\
20 \\
20 \\
20\end{array}$ & $\begin{array}{c}2.50 \\
.830 \\
0 \\
1.90 \\
13.5\end{array}$ \\
\hline $\begin{array}{l}05-29-79 \\
07-24-79 \\
09-10-79\end{array}$ & $\begin{array}{r}118 \\
55.2 \\
41.3\end{array}$ & $\begin{array}{r}118 \\
55.2 \\
41.3\end{array}$ & $\begin{array}{l}-- \\
-- \\
--\end{array}$ & $\begin{array}{l}-- \\
-- \\
--\end{array}$ & $\begin{array}{l}-- \\
-- \\
--\end{array}$ & $\begin{array}{l}.00016 \\
.00013 \\
.00013\end{array}$ & $\begin{array}{l}18.5 \\
25.5 \\
18.5\end{array}$ & $\begin{array}{r}11 \\
4 \\
7\end{array}$ & $\begin{array}{l}1.30 \\
.221 \\
.289\end{array}$ & $\begin{array}{l}20 \\
20 \\
20\end{array}$ & $\begin{array}{l}0 \\
0 \\
0\end{array}$ \\
\hline
\end{tabular}

92. Chippewa River at Durand, Wis. ${ }^{18}$

\begin{tabular}{|c|c|c|c|c|c|c|c|c|c|c|c|}
\hline $\begin{array}{l}11-11-75 \\
04-06-76 \\
04-14-76 \\
06-03-76 \\
07-01-76\end{array}$ & $\begin{array}{l}320 \\
833 \\
513 \\
167 \\
146\end{array}$ & $\begin{array}{l}320 \\
833 \\
513 \\
167 \\
146\end{array}$ & $\begin{array}{c}0.90 \\
-- \\
-- \\
-- \\
.68\end{array}$ & $\begin{array}{r}224 \\
-- \\
-- \\
-- \\
204\end{array}$ & $\begin{array}{c}1.6 \\
-- \\
-- \\
-- \\
1.0\end{array}$ & $\begin{array}{l}-- \\
-- \\
-- \\
--\end{array}$ & $\begin{array}{r}7.0 \\
5.5 \\
10.0 \\
23.0 \\
20.0\end{array}$ & $\begin{array}{r}119 \\
36 \\
57 \\
8 \\
14\end{array}$ & $\begin{array}{l}38.1 \\
30.0 \\
29.2 \\
1.34 \\
2.04\end{array}$ & $\begin{array}{l}20 \\
20 \\
20 \\
20 \\
20\end{array}$ & $\begin{array}{c}17.9 \\
28.2 \\
8.69 \\
5.60 \\
.924\end{array}$ \\
\hline $\begin{array}{l}08-31-76 \\
10-18-76 \\
11-16-76 \\
02-02-77 \\
03-17-77\end{array}$ & $\begin{array}{r}91.2 \\
50.7 \\
51.5 \\
67.1 \\
219\end{array}$ & $\begin{array}{l}91.2 \\
50.7 \\
51.5 \\
67.1 \\
219\end{array}$ & $\begin{array}{l}.65 \\
.52 \\
.55 \\
.59 \\
.77\end{array}$ & $\begin{array}{l}178 \\
160 \\
153 \\
164 \\
215\end{array}$ & $\begin{array}{l}.79 \\
.61 \\
.62 \\
.69 \\
1.3\end{array}$ & $\begin{array}{l}-- \\
0.00029 \\
.00032 \\
-- \\
.00032\end{array}$ & $\begin{array}{r}22.5 \\
6.0 \\
3.0 \\
.0 \\
3.0\end{array}$ & $\begin{array}{r}11 \\
6 \\
5 \\
7 \\
53\end{array}$ & $\begin{aligned} 1.00 \\
.304 \\
.258 \\
.470 \\
11.6\end{aligned}$ & $\begin{array}{l}20 \\
20 \\
20 \\
20 \\
20\end{array}$ & $\begin{array}{l}2.06 \\
1.11 \\
.588 \\
1.12 \\
3.35\end{array}$ \\
\hline $\begin{array}{l}04-13-77 \\
05-10-77 \\
06-15-77 \\
07-06-77 \\
08-24-77\end{array}$ & $\begin{array}{c}139 \\
132 \\
73.6 \\
377 \\
53.8\end{array}$ & $\begin{array}{c}139 \\
132 \\
73.6 \\
377 \\
53.8\end{array}$ & $\begin{array}{l}.69 \\
.69 \\
.58 \\
.94 \\
.50\end{array}$ & $\begin{array}{l}187 \\
188 \\
170 \\
225 \\
160\end{array}$ & $\begin{array}{c}1.1 \\
1.0 \\
.74 \\
1.8 \\
.68\end{array}$ & $\begin{array}{l}.00034 \\
.00033 \\
\ldots \\
.00036 \\
.00032\end{array}$ & $\begin{array}{l}15.0 \\
17.5 \\
22.0 \\
26.0 \\
20.0\end{array}$ & $\begin{array}{r}20 \\
22 \\
15 \\
60 \\
4\end{array}$ & $\begin{array}{c}2.78 \\
2.90 \\
1.10 \\
22.6 \\
.215\end{array}$ & $\begin{array}{l}20 \\
20 \\
20 \\
20 \\
20\end{array}$ & $\begin{array}{c}5.52 \\
3.33 \\
3.31 \\
15.4 \\
2.39\end{array}$ \\
\hline $\begin{array}{l}09-13-77 \\
10-12-77 \\
11-16-77 \\
04-05-78 \\
06-06-78\end{array}$ & $\begin{array}{l}279 \\
479 \\
198 \\
382 \\
247\end{array}$ & $\begin{array}{l}279 \\
479 \\
198 \\
382 \\
247\end{array}$ & $\begin{array}{l}.85 \\
.95 \\
.78 \\
-- \\
.71\end{array}$ & $\begin{array}{r}223 \\
227 \\
199 \\
-- \\
224\end{array}$ & $\begin{array}{l}1.5 \\
2.2 \\
1.3 \\
1.8 \\
1.6\end{array}$ & $\begin{array}{l}.00034 \\
.00032 \\
.00031 \\
-- \\
.00035\end{array}$ & $\begin{array}{r}19.0 \\
13.0 \\
5.0 \\
4.5 \\
22.0\end{array}$ & $\begin{array}{r}144 \\
52 \\
22 \\
146 \\
12\end{array}$ & $\begin{array}{c}40.2 \\
24.9 \\
4.36 \\
55.8 \\
2.96\end{array}$ & $\begin{array}{l}20 \\
20 \\
20 \\
20 \\
20\end{array}$ & $\begin{array}{c}8.96 \\
8.55 \\
4.94 \\
10.1 \\
1.69\end{array}$ \\
\hline $\begin{array}{l}07-06-78 \\
10-31-78 \\
04-17-79 \\
04-23-79 \\
05-29-79\end{array}$ & $\begin{array}{l}473 \\
187 \\
739 \\
884 \\
170\end{array}$ & $\begin{array}{l}473 \\
187 \\
739 \\
884 \\
170\end{array}$ & $\begin{array}{c}.99 \\
.- \\
1.1 \\
1.1 \\
.-\end{array}$ & $\begin{array}{r}233 \\
-- \\
239 \\
244 \\
--\end{array}$ & $\begin{array}{r}2.0 \\
-. \\
2.9 \\
3.2 \\
--\end{array}$ & $\begin{array}{l}.00029 \\
.00034 \\
.00031 \\
.00032 \\
.00023\end{array}$ & $\begin{array}{r}24.0 \\
10.0 \\
5.0 \\
9.5 \\
18.5\end{array}$ & $\begin{array}{l}48 \\
15 \\
41 \\
73 \\
52\end{array}$ & $\begin{array}{c}22.7 \\
2.81 \\
30.3 \\
64.5 \\
8.84\end{array}$ & $\begin{array}{l}20 \\
20 \\
20 \\
20 \\
20\end{array}$ & $\begin{array}{c}13.8 \\
7.72 \\
25.1 \\
23.3 \\
10.2\end{array}$ \\
\hline
\end{tabular}


Table 1.--Hydraulic and sediment-transport data--Continued

\begin{tabular}{|c|c|c|c|c|c|c|c|c|c|c|c|}
\hline Date & $\begin{array}{c}\text { Water } \\
\text { discharge } \\
{\text { (bedload })^{1}}_{\left(\mathrm{m}^{3} / \mathrm{s}\right)}\end{array}$ & $\begin{array}{c}\text { Water } \\
\text { discharge } \\
\text { (suspended } \\
\text { load })^{2} \\
\left(\mathrm{~m}^{3} / \mathrm{s}\right)\end{array}$ & $\begin{array}{l}\text { Mean } \\
\text { flow } \\
\text { velocity } \\
(\mathrm{m} / \mathrm{s})\end{array}$ & $\begin{array}{l}\text { Water- } \\
\text { surface } \\
\text { width } \\
(\mathrm{m})\end{array}$ & $\begin{array}{l}\text { Mean } \\
\text { flow } \\
\text { depth } \\
\text { (m) }\end{array}$ & $\begin{array}{l}\text { Waler- } \\
\text { surface } \\
\text { slope } \\
(\mathrm{m} / \mathrm{m})\end{array}$ & $\begin{array}{l}\text { Water } \\
\text { temp. } \\
\left({ }^{\circ} \mathrm{C}\right)\end{array}$ & $\begin{array}{l}\text { Suspended } \\
\text { sediment } \\
\text { concern- } \\
\text { tration } \\
(\mathrm{mg} / \mathrm{L})\end{array}$ & $\begin{array}{c}\text { Suspended } \\
\text { load } \\
(\mathrm{kg} / \mathrm{s})\end{array}$ & $\begin{array}{l}\text { Number of } \\
\text { sampling } \\
\text { points } \\
\text { for } \\
\text { bedload }\end{array}$ & $\begin{array}{r}\text { Bedload } \\
(\mathrm{kg} / \mathrm{s})\end{array}$ \\
\hline
\end{tabular}

$\begin{array}{lllllllr}09-01-76 & 76.2 & 76.2 & 0.45 & 195 & 0.88 & 0.00039 & 19.0 \\ 09-20-76 & 70.5 & 70.5 & .52 & 171 & .80 & .00039 & 16.0 \\ 10-19-76 & 72.5 & 72.5 & .57 & 171 & .75 & .00036 & 6.0 \\ 11-17-76 & 70.0 & 70.0 & .54 & 171 & .76 & .00058 & 4.0 \\ 03-16-77 & 257 & 257 & .66 & 265 & 1.5 & .00045 & 4.5 \\ 04-14-77 & 155 & 155 & .57 & 241 & 1.1 & .00041 & 15.0 \\ 05-11-77 & 110 & 110 & .48 & 243 & .95 & .00032 & 18.5 \\ 07-07-77 & 399 & 399 & .90 & 273 & 1.6 & .00037 & 25.5 \\ 09-14-77 & 295 & 295 & .86 & 274 & 1.3 & .00037 & 18.5 \\ 11-17-77 & 210 & 210 & .58 & 270 & 1.4 & .00036 & 3.5 \\ & & & & & & & \\ 04-06-78 & 391 & 391 & .80 & 276 & 1.8 & .00028 & 5.0 \\ 05-16-78 & 170 & 170 & .67 & 261 & .97 & .00025 & 16.5 \\ 06-07-78 & 217 & 217 & .73 & 264 & 1.1 & .00024 & 22.5 \\ 09-20-78 & 320 & 320 & .79 & 277 & 1.5 & .00033 & 19.0 \\ 11-01-78 & 187 & 187 & .64 & 246 & 1.2 & .00029 & 7.5 \\ 05-30-79 & 219 & 219 & .66 & 242 & 1.4 & .00017 & 20.5 \\ 07-24-79 & 140 & 140 & .66 & 247 & .85 & .00029 & 24.5 \\ 09-11-79 & 118 & 118 & .68 & 229 & .76 & .00025 & 18.5\end{array}$

$\begin{array}{rccc}14 & 1.07 & 20 & 2.06 \\ 18 & 1.27 & 20 & 5.94 \\ 10 & .725 & 20 & 3.93 \\ 13 & .910 & 20 & 4.20 \\ 178 & 45.7 & 20 & 5.85 \\ & & & \\ 162 & 25.1 & 20 & 4.99 \\ 15 & 1.65 & 20 & 2.00 \\ 324 & 129 & 20 & 13.4 \\ 39 & 11.5 & 20 & 13.5 \\ 20 & 4.20 & 20 & 10.4 \\ & & & \\ 84 & 32.8 & 20 & 5.80 \\ 42 & 7.14 & 20 & 2.89 \\ 18 & 3.91 & 20 & 9.84 \\ 35 & 11.2 & 20 & 14.7 \\ 17 & 3.18 & 20 & 7.61 \\ & & & \\ 24 & 5.26 & 20 & 7.44 \\ 21 & 2.94 & 20 & 1.69 \\ 19 & 2.24 & 20 & 5.88\end{array}$

${ }^{1}$ Water discharge at time of bedload measurement.

${ }^{2}$ Water discharge at time of suspended-load measurement.

${ }^{3}$ Water discharges (bedload and suspended load) determined from rating curve; velocities, widths and depths, where given, determined by the rating curve-area technique.

WWater discharges (bedload and suspended load) determined in some cases from rating curve (with velocities, widths and depths by the rating curve-area technique) and in other cases by a direct discharge measurement (latter also providing velocity, width and depth).

SWater discharges (bedload and suspended load), velocities, widths and depths determined from a direct (current-meter) discharge measurement.

6Water discharges (bedload and suspended load) determined from rating curve; velocities, widths and depths estimated by the rating curve-hydraulic geometry method.

${ }^{7}$ Water discharges (bedload and suspended load) determined from rating curve; velocities, widths and depths determined by a direct discharge measurement.

${ }^{8}$ Helley-Smith-type sampler, with orifice six inches high and 12 inches wide, used.

${ }^{9}$ Bed width (3.7 meters) only data available, for all dates and measurements.

${ }^{10}$ Hydraulic radius, for all dates and measurements.

${ }^{11}$ Bedload sampled by vortex flume and pit apparatus (see Milhous, 1973), on all dates.

${ }^{12}$ Six-inchxsix-inch-orifice Helley-Smith sampler used for 1973-79 bedload measurements.

${ }^{13}$ Data measured at somewhat constricted cross section (bridge).

${ }^{14}$ Data for section 3256 of Emmett and others (1980).

15 Six-inchxsix-inch-orifice Helley-Smith sampler used.

${ }^{16}$ Water discharges (bedload and suspended load) measured directly (with $V, W$ and $D$ as byproducts) except for $4 / 13 / 78$, $7 / 2 / 80$ and $8 / 25 / 80$, for which dates $Q$ taken from rating curve, $W$ measured directly, and $A$ estimated ( $V=Q / A$ and $D=A / W$ ).

${ }^{17}$ Water discharges (bedload and suspended load) measured directly (with $V, W$ and $D$ as byproducts) except for $3 / 28 / 78$, $9 / 3 / 80$ and $6 / 18 / 81$, for which dates $Q$ taken from rating curve, $W$ measured directly, and $A$ estimated ( $V=Q / A$ and $D=A / W$ ).

${ }^{18}$ Water discharges (bedload and suspended load) determined in some cases from rating curve (V and $D$ from hydraulic geometry, $W$ from bedload measurement) and in other cases by a direct discharge measurement (latter also providing $V$, W, D). 
Table 2.--Suspended load particle-size distributions

$$
\text { [--, no data available] }
$$

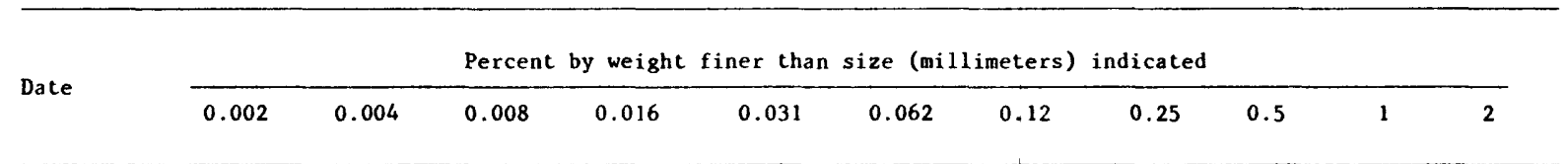

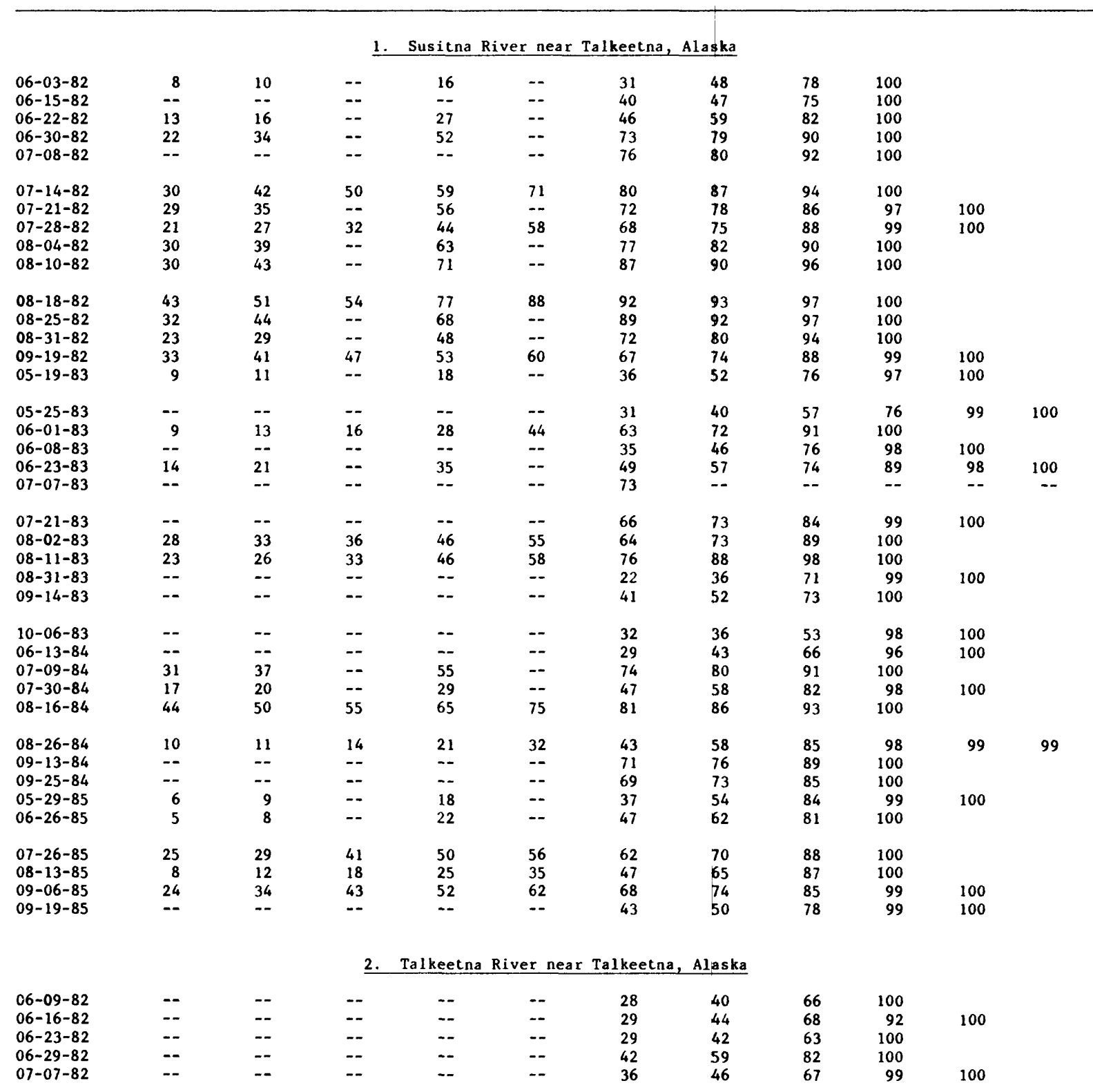


Table 2.--Suspended load particle-size distributions--Continued

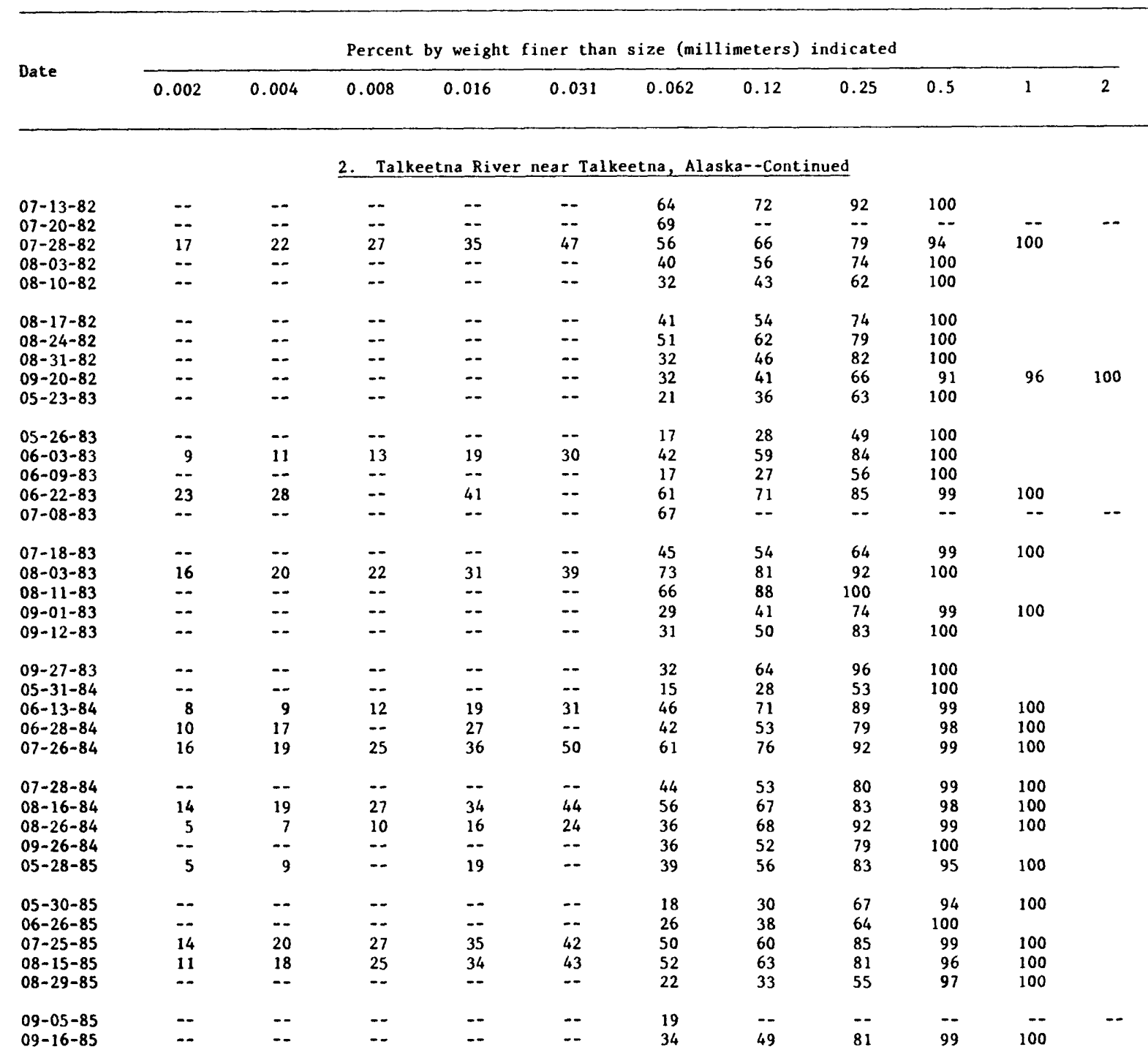

3. Chulitna River below Canyon near Talkeetna, Alaska

\begin{tabular}{|c|c|c|c|c|c|c|c|c|c|c|}
\hline $\begin{array}{l}06-04-82 \\
06-09-82 \\
06-16-82 \\
06-22-82 \\
06-29-82\end{array}$ & $\begin{array}{l}22 \\
19 \\
24 \\
19 \\
34\end{array}$ & $\begin{array}{l}32 \\
27 \\
36 \\
25 \\
45\end{array}$ & $\begin{array}{l}37 \\
-- \\
-- \\
32 \\
56\end{array}$ & $\begin{array}{l}46 \\
41 \\
48 \\
39 \\
62\end{array}$ & $\begin{array}{l}54 \\
-- \\
-- \\
47 \\
70\end{array}$ & $\begin{array}{l}59 \\
77 \\
62 \\
58 \\
77\end{array}$ & $\begin{array}{l}68 \\
83 \\
68 \\
64 \\
83\end{array}$ & $\begin{array}{l}88 \\
96 \\
84 \\
75 \\
94\end{array}$ & $\begin{array}{r}99 \\
99 \\
100 \\
98 \\
100\end{array}$ & $\begin{array}{l}100 \\
100\end{array}$ \\
\hline $\begin{array}{l}07-07-82 \\
07-13-82 \\
07-20-82 \\
07-27-82 \\
08-03-82\end{array}$ & $\begin{array}{l}26 \\
-- \\
30 \\
16 \\
24\end{array}$ & $\begin{array}{l}36 \\
-- \\
44 \\
25 \\
33\end{array}$ & $\begin{array}{l}51 \\
-- \\
54 \\
30 \\
42\end{array}$ & $\begin{array}{l}60 \\
-- \\
65 \\
42 \\
55\end{array}$ & $\begin{array}{l}69 \\
-- \\
77 \\
51 \\
67\end{array}$ & $\begin{array}{l}78 \\
71 \\
78 \\
60 \\
73\end{array}$ & $\begin{array}{l}84 \\
76 \\
84 \\
70 \\
77\end{array}$ & $\begin{array}{l}93 \\
83 \\
92 \\
85 \\
87\end{array}$ & $\begin{array}{r}100 \\
99 \\
100 \\
98 \\
99\end{array}$ & $\begin{array}{r}100 \\
99 \\
100\end{array}$ \\
\hline
\end{tabular}


Table 2.--Suspended load particle-size distributidns--Continued

\begin{tabular}{|c|c|c|c|c|c|c|c|c|c|c|c|}
\hline \multirow{2}{*}{ Date } & & & Percent & y weight & finer than & size (mi) & meters) & indicated & & & \\
\hline & 0.002 & 0.004 & 0.008 & 0.016 & 0.031 & 0.062 & 0.12 & 0.25 & 0.5 & 1 & 2 \\
\hline
\end{tabular}

\section{Chulitna River below Canyon near Talkeetna, Alaska--Continued}

\begin{tabular}{|c|c|c|c|c|c|c|c|c|c|c|}
\hline $\begin{array}{l}08-11-82 \\
08-17-82 \\
08-24-82 \\
09-01-82 \\
09-18-82\end{array}$ & $\begin{array}{l}23 \\
25 \\
24 \\
17 \\
33\end{array}$ & $\begin{array}{l}34 \\
37 \\
34 \\
26 \\
43\end{array}$ & $\begin{array}{l}40 \\
48 \\
42 \\
-- \\
52\end{array}$ & $\begin{array}{l}51 \\
59 \\
54 \\
42 \\
58\end{array}$ & $\begin{array}{l}60 \\
68 \\
65 \\
-- \\
62\end{array}$ & $\begin{array}{l}68 \\
75 \\
75 \\
64 \\
68\end{array}$ & $\begin{array}{l}75 \\
80 \\
81 \\
68 \\
74\end{array}$ & $\begin{array}{l}85 \\
87 \\
93 \\
84 \\
86\end{array}$ & $\begin{array}{r}99 \\
97 \\
100 \\
100 \\
96\end{array}$ & $\begin{array}{l}100 \\
100\end{array}$ \\
\hline $\begin{array}{l}05-19-83 \\
05-25-83 \\
05-31-83 \\
06-02-83 \\
06-09-83\end{array}$ & $\begin{array}{l}11 \\
25 \\
17 \\
22 \\
29\end{array}$ & $\begin{array}{l}18 \\
33 \\
19 \\
31 \\
40\end{array}$ & $\begin{array}{l}-- \\
-- \\
27 \\
37 \\
44\end{array}$ & $\begin{array}{l}28 \\
50 \\
35 \\
43 \\
55\end{array}$ & $\begin{array}{l}-- \\
-- \\
44 \\
50 \\
61\end{array}$ & $\begin{array}{l}42 \\
63 \\
53 \\
54 \\
66\end{array}$ & $\begin{array}{l}51 \\
70 \\
66 \\
61 \\
70\end{array}$ & $\begin{array}{l}67 \\
86 \\
84 \\
75 \\
79\end{array}$ & $\begin{array}{r}97 \\
100 \\
99 \\
100 \\
100\end{array}$ & 100 \\
\hline $\begin{array}{l}06-22-83 \\
07-06-83 \\
07-20-83 \\
08-02-83 \\
08-09-83\end{array}$ & $\begin{array}{l}33 \\
31 \\
-- \\
23 \\
19\end{array}$ & $\begin{array}{l}34 \\
33 \\
-- \\
32 \\
25\end{array}$ & $\begin{array}{l}44 \\
34 \\
-- \\
42 \\
28\end{array}$ & $\begin{array}{l}54 \\
35 \\
-- \\
51 \\
38\end{array}$ & $\begin{array}{l}62 \\
36 \\
-- \\
61 \\
50\end{array}$ & $\begin{array}{l}67 \\
75 \\
73 \\
70 \\
60\end{array}$ & $\begin{array}{l}77 \\
82 \\
-- \\
79 \\
73\end{array}$ & $\begin{array}{l}87 \\
91 \\
-- \\
92 \\
87\end{array}$ & $\begin{array}{r}97 \\
100 \\
-- \\
100 \\
97\end{array}$ & 100 \\
\hline $\begin{array}{l}06-14-84 \\
07-11-84 \\
07-31-84 \\
08-17-84 \\
08-28-84\end{array}$ & $\begin{array}{l}23 \\
30 \\
29 \\
30 \\
20\end{array}$ & $\begin{array}{l}27 \\
42 \\
35 \\
35 \\
22\end{array}$ & $\begin{array}{l}34 \\
47 \\
41 \\
42 \\
29\end{array}$ & $\begin{array}{l}42 \\
58 \\
49 \\
52 \\
37\end{array}$ & $\begin{array}{l}49 \\
65 \\
58 \\
63 \\
46\end{array}$ & $\begin{array}{l}56 \\
70 \\
64 \\
70 \\
56\end{array}$ & $\begin{array}{l}69 \\
75 \\
71 \\
77 \\
64\end{array}$ & $\begin{array}{l}78 \\
83 \\
82 \\
87 \\
80\end{array}$ & $\begin{array}{l}96 \\
94 \\
98 \\
97 \\
94\end{array}$ & $\begin{array}{r}99 \\
100 \\
100 \\
100 \\
97\end{array}$ \\
\hline $\begin{array}{l}09-14-84 \\
09-27-84 \\
05-31-85 \\
06-27-85 \\
07-24-85\end{array}$ & $\begin{array}{l}18 \\
32 \\
12 \\
19 \\
28\end{array}$ & $\begin{array}{l}23 \\
38 \\
18 \\
29 \\
40\end{array}$ & $\begin{array}{l}34 \\
44 \\
25 \\
40 \\
52\end{array}$ & $\begin{array}{l}43 \\
50 \\
33 \\
53 \\
64\end{array}$ & $\begin{array}{l}53 \\
60 \\
43 \\
62 \\
72\end{array}$ & $\begin{array}{l}62 \\
66 \\
53 \\
71 \\
79\end{array}$ & $\begin{array}{l}70 \\
69 \\
64 \\
80 \\
84\end{array}$ & $\begin{array}{l}83 \\
77 \\
82 \\
91 \\
92\end{array}$ & $\begin{array}{l}99 \\
98 \\
97 \\
99 \\
98\end{array}$ & $\begin{array}{r}100 \\
100 \\
100 \\
100 \\
98\end{array}$ \\
\hline $\begin{array}{l}05-14-84 \\
06-12-84 \\
07-17-84 \\
09-19-84 \\
05-23-85\end{array}$ & $\begin{array}{c}-- \\
20 \\
28 \\
\cdots \\
6\end{array}$ & $\begin{array}{r}-- \\
23 \\
36 \\
-- \\
9\end{array}$ & $\begin{array}{l}-- \\
27 \\
46 \\
-- \\
--\end{array}$ & $\begin{array}{l}\cdots \\
32 \\
54 \\
-- \\
22\end{array}$ & $\begin{array}{l}-- \\
42 \\
63 \\
-- \\
--\end{array}$ & $\begin{array}{l}30 \\
50 \\
68 \\
45 \\
52\end{array}$ & $\begin{array}{l}53 \\
64 \\
76 \\
58 \\
76\end{array}$ & $\begin{array}{l}85 \\
91 \\
89 \\
77 \\
98\end{array}$ & $\begin{array}{r}98 \\
100 \\
99 \\
97 \\
100\end{array}$ & $\begin{array}{l}100 \\
100 \\
100\end{array}$ \\
\hline $\begin{array}{l}06-20-85 \\
07-17-85 \\
08-14-85 \\
08-19-85 \\
09-18-85\end{array}$ & $\begin{array}{l}2- \\
21 \\
17 \\
20 \\
14\end{array}$ & $\begin{array}{l}-- \\
30 \\
25 \\
29 \\
19\end{array}$ & $\begin{array}{l}-- \\
40 \\
-- \\
38 \\
23\end{array}$ & $\begin{array}{l}\cdots \\
49 \\
42 \\
47 \\
26\end{array}$ & $\begin{array}{l}-- \\
57 \\
-- \\
55 \\
30\end{array}$ & $\begin{array}{l}34 \\
64 \\
61 \\
63 \\
35\end{array}$ & $\begin{array}{r}48 \\
73 \\
12 \\
73 \\
44\end{array}$ & $\begin{array}{l}83 \\
87 \\
90 \\
89 \\
72\end{array}$ & $\begin{array}{r}100 \\
100 \\
100 \\
100 \\
99\end{array}$ & 100 \\
\hline
\end{tabular}


Table 2,--Suspended load particle-size distributions--Continued

\begin{tabular}{|c|c|c|c|c|c|c|c|c|c|c|c|}
\hline \multirow{2}{*}{ Date } & \multicolumn{4}{|c|}{ Percent by weight } & finer than & \multicolumn{2}{|c|}{ size (millimeters) } & \multicolumn{2}{|l|}{ indicated } & \multirow[b]{2}{*}{1} & \\
\hline & 0.002 & 0.004 & 0.008 & 0.016 & 0.031 & 0.062 & 0.12 & 0.25 & 0.5 & & 2 \\
\hline
\end{tabular}

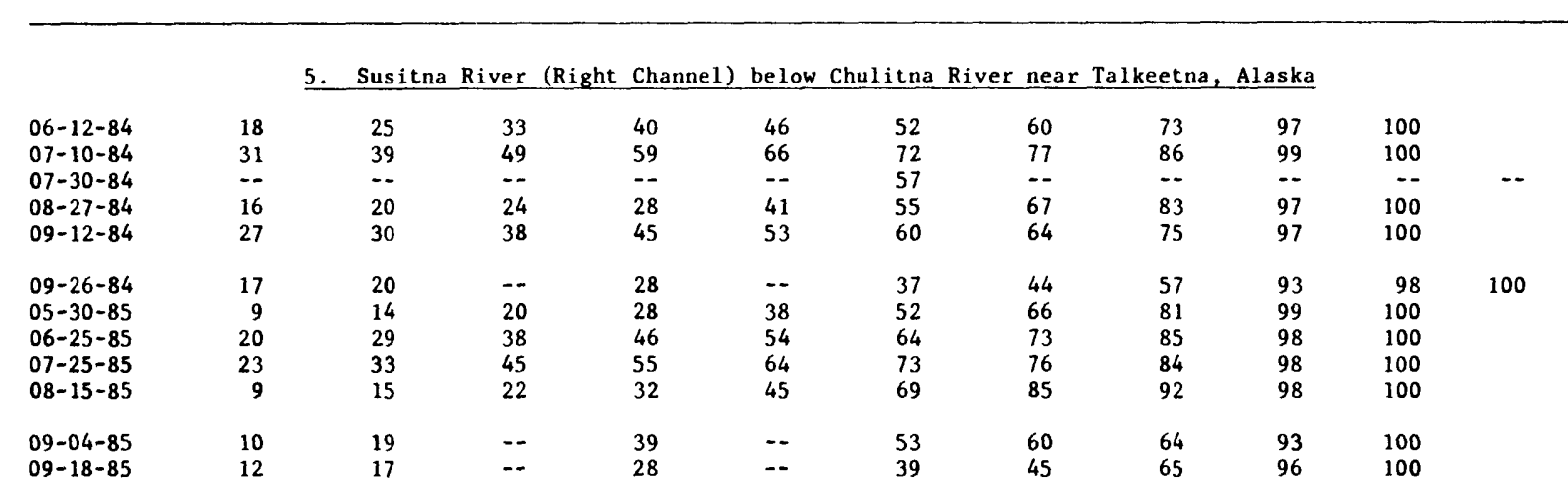

6. Susitna River (Left Channel) below Chulitna River near Talkeetna, Alaska

\begin{tabular}{|c|c|c|c|c|c|c|c|c|c|c|}
\hline $06-12-84$ & -- & - & - & -- & $\cdots$ & 30 & 40 & 69 & 97 & 100 \\
\hline $07-10-84$ & 33 & 41 & $\cdots$ & 56 & $\cdots$ & 74 & 80 & 90 & 99 & 100 \\
\hline $09-12-84$ & - & $\ldots$ & - & $\cdots$ & - & 67 & 74 & 84 & 99 & 100 \\
\hline $05-30-85$ & - & - & - & -- & -- & 37 & 50 & 80 & 100 & \\
\hline $06-25-85$ & 7 & 13 & -. & 26 & - & 40 & 47 & 74 & 100 & \\
\hline $07-25-85$ & 22 & 31 & 41 & 49 & 58 & 63 & 72 & 91 & 100 & \\
\hline $09-18-85$ & $\ldots$ & $\ldots$ & - & - & -. & 40 & 48 & 83 & 100 & \\
\hline
\end{tabular}

7. Susitna River at Sunshine, Alaska

\begin{tabular}{|c|c|c|c|c|c|c|c|c|c|c|c|}
\hline $\begin{array}{l}06-03-82 \\
06-10-82 \\
06-17-82 \\
06-21-82 \\
06-28-82\end{array}$ & $\begin{array}{l}-2 \\
16 \\
-- \\
17 \\
25\end{array}$ & $\begin{array}{l}-- \\
20 \\
-- \\
20 \\
33\end{array}$ & $\begin{array}{l}\ldots \\
\ldots \\
-- \\
27 \\
43\end{array}$ & $\begin{array}{l}\cdots \\
32 \\
\cdots \\
37 \\
53\end{array}$ & $\begin{array}{l}-- \\
-- \\
-- \\
48 \\
62\end{array}$ & $\begin{array}{l}42 \\
52 \\
35 \\
60 \\
73\end{array}$ & $\begin{array}{l}62 \\
62 \\
42 \\
76 \\
82\end{array}$ & $\begin{array}{l}85 \\
95 \\
62 \\
93 \\
92\end{array}$ & $\begin{array}{r}97 \\
100 \\
100 \\
100 \\
100\end{array}$ & 99 & 100 \\
\hline $\begin{array}{l}07-06-82 \\
07-12-82 \\
07-19-82 \\
07-26-82 \\
08-02-82\end{array}$ & $\begin{array}{l}25 \\
\ldots \\
27 \\
13 \\
\ldots\end{array}$ & $\begin{array}{l}40 \\
-- \\
39 \\
18 \\
\ldots\end{array}$ & $\begin{array}{l}45 \\
2- \\
47 \\
27 \\
\ldots\end{array}$ & $\begin{array}{l}54 \\
\cdots \\
60 \\
36 \\
\cdots\end{array}$ & $\begin{array}{l}62 \\
2- \\
69 \\
47 \\
\cdots\end{array}$ & $\begin{array}{l}67 \\
75 \\
78 \\
59 \\
61\end{array}$ & $\begin{array}{l}72 \\
82 \\
85 \\
74 \\
--\end{array}$ & $\begin{array}{l}84 \\
90 \\
93 \\
90 \\
--\end{array}$ & $\begin{array}{r}100 \\
100 \\
99 \\
99 \\
--\end{array}$ & $\begin{array}{r}100 \\
100 \\
-\ldots\end{array}$ & - \\
\hline $\begin{array}{l}08-09-82 \\
08-16-82 \\
08-23-82 \\
08-30-82 \\
09-17-82\end{array}$ & $\begin{array}{l}28 \\
37 \\
27 \\
19 \\
28\end{array}$ & $\begin{array}{l}33 \\
42 \\
41 \\
25 \\
38\end{array}$ & $\begin{array}{l}43 \\
55 \\
50 \\
34 \\
46\end{array}$ & $\begin{array}{l}55 \\
67 \\
62 \\
49 \\
54\end{array}$ & $\begin{array}{l}66 \\
77 \\
73 \\
62 \\
65\end{array}$ & $\begin{array}{l}75 \\
83 \\
81 \\
72 \\
72\end{array}$ & $\begin{array}{l}81 \\
88 \\
86 \\
80 \\
82\end{array}$ & $\begin{array}{l}89 \\
93 \\
94 \\
90 \\
94\end{array}$ & $\begin{array}{r}100 \\
100 \\
100 \\
99 \\
99\end{array}$ & $\begin{array}{r}- \\
100 \\
100\end{array}$ & \\
\hline $\begin{array}{l}05-18-83 \\
05-24-83 \\
06-01-83 \\
06-08-83 \\
06-23-83\end{array}$ & $\begin{array}{l}13 \\
-2 \\
10 \\
-2 \\
25\end{array}$ & $\begin{array}{l}16 \\
-- \\
12 \\
-2 \\
33\end{array}$ & $\begin{array}{l}\ldots \\
17 \\
17 \\
43\end{array}$ & $\begin{array}{l}26 \\
-- \\
24 \\
\overline{52}\end{array}$ & $\begin{array}{l}\cdots \\
34 \\
61\end{array}$ & $\begin{array}{l}47 \\
35 \\
41 \\
46 \\
69\end{array}$ & $\begin{array}{l}56 \\
43 \\
57 \\
-- \\
78\end{array}$ & $\begin{array}{l}72 \\
65 \\
83 \\
-- \\
89\end{array}$ & $\begin{array}{r}85 \\
98 \\
98 \\
-- \\
100\end{array}$ & $\begin{array}{r}97 \\
99 \\
100 \\
--\end{array}$ & $\begin{array}{l}100 \\
100\end{array}$ \\
\hline
\end{tabular}


Table 2.--Suspended load particle-size distributions--Continued

\begin{tabular}{|c|c|c|c|c|c|c|c|c|c|c|c|}
\hline \multirow{2}{*}{ Date } & \multicolumn{11}{|c|}{ Percent by weight finer than size (millimeters) indicated } \\
\hline & 0.002 & 0.004 & 0.008 & 0.016 & 0.031 & 0.062 & 0.12 & 0.25 & 0.5 & 1 & 2 \\
\hline & \multicolumn{11}{|c|}{ 7. Susitna River at Sunshine, Alaska--Continued } \\
\hline $07-05-83$ & -- & -- & -- & -- & -- & 80 & +- & -- & -- & -- & - \\
\hline $07-19-83$ & -- & -- & - & -- & -- & 71 & +- & -- & -- & - & $\ldots$ \\
\hline $08-01-83$ & 20 & 29 & 41 & 49 & 59 & 70 & 79 & 90 & 100 & & \\
\hline $08-03-83$ & 22 & 31 & 42 & 54 & 66 & 73 & 80 & 91 & 100 & & \\
\hline $08-08-83$ & 13 & 22 & 23 & 35 & 51 & 71 & 85 & 95 & 99 & 100 & \\
\hline $08-29-83$ & -- & -- & -- & -- & -- & 59 & -- & -- & -- & -- & $\cdots$ \\
\hline $09-12-83$ & 20 & 27 & -- & 34 & -- & 41 & 47 & 66 & 100 & & \\
\hline $10-04-83$ & 12 & 15 & -- & 19 & -- & 29 & 37 & 68 & 99 & 100 & \\
\hline $05-16-84$ & 10 & 13 & -- & 22 & $\cdots$ & 43 & 56 & 72 & 99 & 100 & \\
\hline $06-14-84$ & 15 & 17 & 20 & 26 & 34 & 48 & 70 & 87 & 99 & 100 & \\
\hline $07-13-84$ & 21 & 31 & 37 & 47 & 55 & 60 & 66 & 82 & 98 & 100 & \\
\hline $07-28-84$ & 27 & 31 & 36 & 45 & 55 & 64 & 74 & 87 & 96 & 99 & 100 \\
\hline $08-14-84$ & 22 & 27 & 40 & 50 & 57 & 63 & 70 & 84 & 100 & & \\
\hline $09-11-84$ & 23 & 29 & $\cdots$ & 46 & $\ldots$ & 57 & 63 & 76 & 100 & & \\
\hline $09-21-84$ & 13 & 16 & -- & 25 & -- & 35 & 41 & 63 & 96 & 100 & \\
\hline $09-28-84$ & 22 & 27 & -- & 34 & $=$ & 46 & 51 & 67 & 100 & & \\
\hline $05-31-85$ & 6 & 9 & -- & 19 & $=-$ & 35 & 52 & 83 & 99 & 100 & \\
\hline $06-25-85$ & 15 & 23 & 32 & 39 & 48 & 57 & 65 & 80 & 100 & & \\
\hline $07-23-85$ & 18 & 27 & 36 & 46 & 53 & 64 & 72 & 88 & 100 & & \\
\hline $08-12-85$ & 8 & 13 & 20 & 32 & 52 & 73 & 88 & 96 & 100 & & \\
\hline $09-03-85$ & 16 & 25 & 33 & 41 & 49 & 57 & 66 & 83 & 99 & 100 & \\
\hline $09-16-85$ & 11 & 17 & 23 & 32 & 42 & 56 & 73 & 93 & 99 & 100 & \\
\hline
\end{tabular}

\section{Tanana River at Upper End of Goose Island, Alaska}

\begin{tabular}{|c|c|c|c|c|c|c|c|c|c|c|c|}
\hline $09-05-80$ & -- & $\cdots$ & -- & $\cdots$ & -- & 33 & -- & -- & $\cdots$ & -- & -- \\
\hline $09-17-80$ & - & - & -- & -- & -- & 42 & -- & -- & -- & -- & - \\
\hline $10-08-80$ & -- & -- & -- & -- & -- & 41 & -- & -- & -- & - & -. \\
\hline $03-06-81$ & -- & - & -- & -- & -- & 41 & -- & -- & $-\infty$ & - & - \\
\hline $03-24-81$ & - & $\cdots$ & -- & -- & -- & 51 & -- & -- & - & - & -- \\
\hline $06-19-81$ & 13 & 20 & 30 & 42 & 53 & 63 & 79 & 100 & & & \\
\hline $07-02-81$ & 14 & 22 & 28 & 35 & 42 & 54 & 75 & 98 & 100 & & \\
\hline $07-16-81$ & 12 & 18 & 27 & 39 & 49 & 60 & 85 & 100 & & & \\
\hline $07-28-81$ & 16 & 23 & 33 & 45 & 58 & 75 & 94 & 100 & & & \\
\hline $08-14-81$ & 16 & 25 & 36 & 50 & 63 & 78 & 94 & 100 & & & \\
\hline $09-04-81$ & 12 & 18 & 28 & 38 & 44 & 50 & 66 & 98 & 100 & & \\
\hline $10-07-81$ & -- & - & -- & -- & - & 37 & - & - & - & $\cdots$ & $\cdots$ \\
\hline $06-03-82$ & $\cdots$ & -- & -- & -- & -- & 54 & 76 & 100 & & & \\
\hline $06-29-82$ & 20 & 28 & 36 & 50 & 67 & 81 & 95 & 100 & & & \\
\hline $07-21-82$ & 15 & 19 & 24 & 31 & 44 & 62 & 83 & 100 & & & \\
\hline $08-10-82$ & 21 & 30 & 37 & 46 & 55 & 66 & 80 & 96 & 100 & & \\
\hline $09-10-82$ & -- & -- & $-\infty$ & -- & - & 45 & 60 & 94 & 100 & & \\
\hline $09-29-82$ & 38 & 43 & -- & 55 & -- & 71 & 80 & 99 & 100 & & \\
\hline
\end{tabular}


Table 2.--Suspended load particle-size distributions--Continued

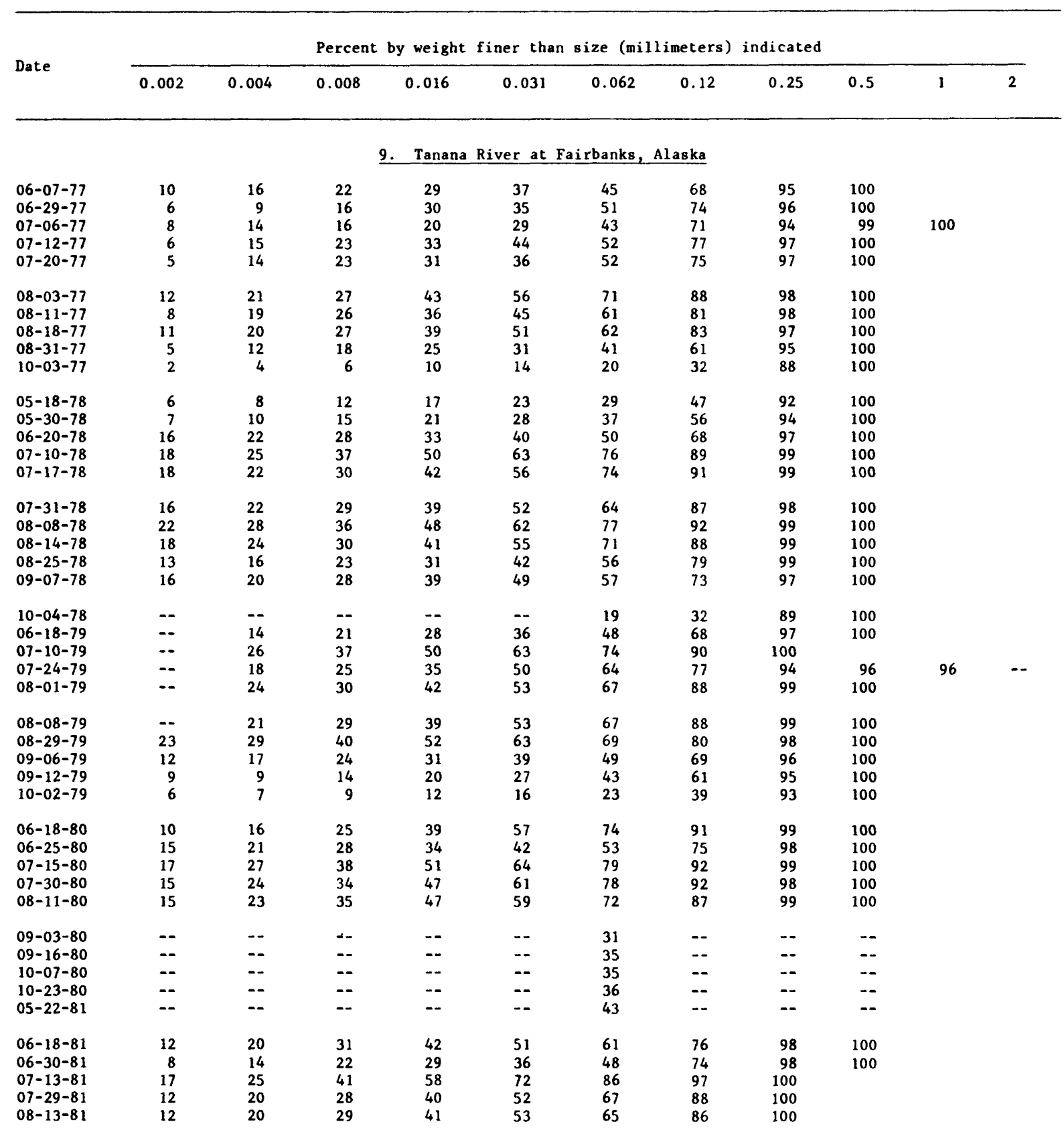


Table 2.--Suspended load particle-size distributions--Continued

\begin{tabular}{|c|c|c|c|c|c|c|c|c|c|c|c|}
\hline \multirow{2}{*}{ Date } & \multicolumn{11}{|c|}{ Percent by weight finer than size (millimeters) indicated } \\
\hline & 0.002 & 0.004 & 0.008 & 0.016 & 0.031 & 0.062 & 0.12 & 0.25 & 0.5 & 1 & 2 \\
\hline
\end{tabular}

\section{Tanana River at Fairbanks, Alaska--Continued}

\begin{tabular}{|c|c|c|c|c|c|c|c|c|c|}
\hline $09-03-81$ & 18 & 26 & 28 & 45 & 51 & 59 & 75 & 99 & 100 \\
\hline $10-06-81$ & $\cdots$ & -- & - & -- & $\ldots$ & 40 & -- & -- & $\cdots$ \\
\hline $06-04-82$ & 16 & 18 & 21 & 23 & 38 & 59 & 81 & 97 & 100 \\
\hline $06-28-82$ & 15 & 19 & 28 & 41 & 53 & 65 & 80 & 95 & 100 \\
\hline $07-20-82$ & 12 & 16 & 21 & 32 & 50 & 72 & 92 & 99 & 100 \\
\hline $08-09-82$ & 28 & 32 & 38 & 45 & 54 & 64 & 78 & 99 & 100 \\
\hline $09-08-82$ & $\therefore$ & 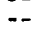 & $\ldots$ & -- & - & 36 & 54 & 97 & 100 \\
\hline $09-28-82$ & 29 & 34 & -- & 43 & -- & 57 & 69 & 97 & 100 \\
\hline
\end{tabular}

10.--Snake River near Anatone, Wash.

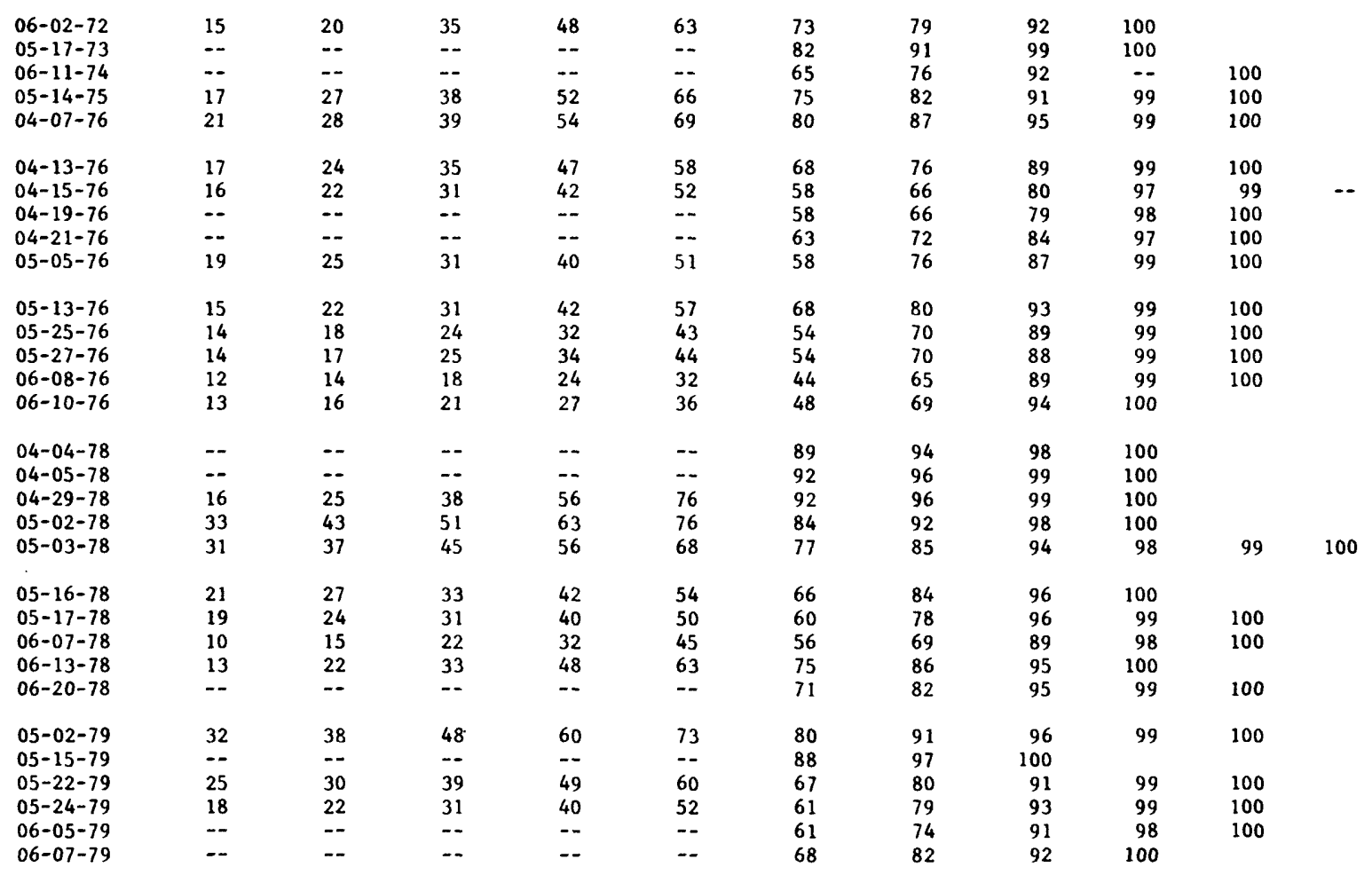

14. San Antonio River near Lockwood, Calif.

$\begin{array}{rrrrrrrrrrrr}11-21-72 & -- & -- & -- & -- & -- & 68 & 70 & 77 & 100 & 71 & 100 \\ 01-17-73 & -- & -- & -- & -- & -- & 06 & 08 & 18 & 42 & 71 \\ 03-28-74 & -- & -- & 16 & 21 & 28 & 36 & 44 & 64 & 90 & 100 & 53 \\ 02-06-75 & -- & -- & -- & -- & -- & 13 & 20 & 59 \\ 03-06-75 & -- & -- & -- & -- & -- & 27 & 33 & 50 & 90 & 98 & 100\end{array}$


Table 2.--Suspended load particle-size distributions--Continued

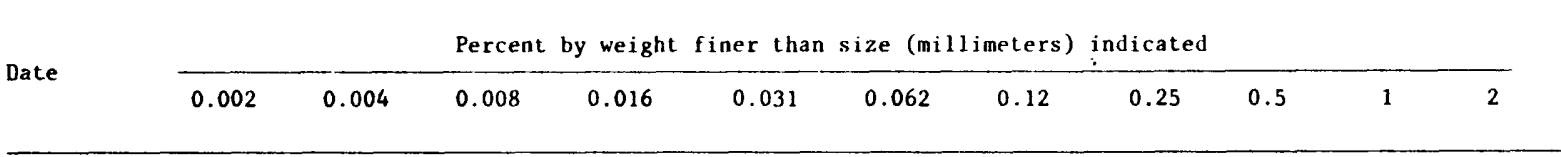

14. San Antonio River near Lockwood, Calif.--Cont inued

\begin{tabular}{|c|c|c|c|c|c|c|c|c|c|c|c|}
\hline $04-04-75$ & $=-$ & -- & -- & -- & -- & 10 & 15 & 38 & 73 & 96 & 100 \\
\hline $01-18-78$ & - & - & 8 & 10 & 13 & 16 & 28 & 57 & 74 & 87 & 95 \\
\hline $02-21-78$ & $n$ & - & $=$ & $\ldots$ & -- & 11 & 16 & 46 & 89 & 97 & 100 \\
\hline $03-28-78$ & - & -- & - & - & - & 16 & 23 & 62 & 94 & 100 & \\
\hline $01-08-79$ & - & -- & - & - & -- & 54 & -- & -- & -- & - & -- \\
\hline $02-06-79$ & - & -- & - & -- & -- & 39 & -- & -- & $\sim$ & $\approx$ & $\approx$ \\
\hline $04-05-79$ & $\approx$ & -- & $\cdots$ & -- & - & 27 & -- & $=$ & -- & -- & $=$ \\
\hline $11-16-81$ & -- & -- & -- & -- & -- & 8 & -- & -- & -- & $=$ & $=-$ \\
\hline $12-14-81$ & -- & 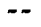 & $\cdots$ & -- & -- & 17 & -- & $\cdots$ & 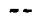 & -- & $\approx$ \\
\hline $02-14-83$ & $-\infty$ & - & - & -- & -- & 12 & 16 & 41 & 76 & 95 & 100 \\
\hline $03-01-83$ & -- & -- & $\cdots$ & - & -- & 23 & 34 & 51 & 69 & 88 & 97 \\
\hline $04-22-83$ & 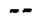 & 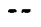 & -- & $\cdots$ & -- & 26 & 29 & 48 & 88 & 96 & 100 \\
\hline $12-06-83$ & 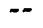 & $\approx$ & $=-$ & -- & $\cdots$ & 25 & $\cdots$ & $\cdots$ & $\cdots$ & 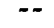 & -- \\
\hline $01-19-84$ & -- & -- & -- & - & -- & 16 & -- & -- & -- & $\cdots$ & $\cdots$ \\
\hline
\end{tabular}

15. Chamise Creek near Island Mountain, Calif.

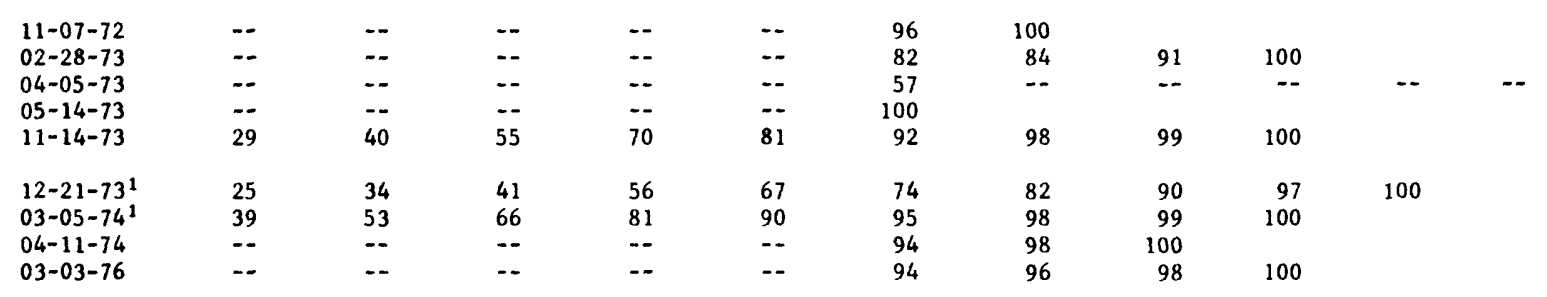

16. Redwood Creek near Blue Lake, Calif.

\begin{tabular}{|c|c|c|c|c|c|c|c|c|c|c|c|}
\hline $\begin{array}{l}01-12-74 \\
04-01-75 \\
03-10-78 \\
01-27-81 \\
04-02-81\end{array}$ & $\begin{array}{l}= \\
-- \\
-- \\
--\end{array}$ & $\begin{array}{l}-- \\
-- \\
-- \\
20 \\
--\end{array}$ & $\begin{array}{l}-- \\
-- \\
26 \\
--\end{array}$ & $\begin{array}{l}-- \\
-- \\
-- \\
35 \\
--\end{array}$ & $\begin{array}{l}\ldots \\
\cdots \\
43 \\
\cdots\end{array}$ & $\begin{array}{l}83 \\
73 \\
61 \\
53 \\
69\end{array}$ & $\begin{array}{l}87 \\
76 \\
67 \\
64 \\
75\end{array}$ & $\begin{array}{l}91 \\
80 \\
75 \\
79 \\
84\end{array}$ & $\begin{array}{l}96 \\
85 \\
86 \\
93 \\
96\end{array}$ & $\begin{array}{r}100 \\
94 \\
99 \\
99 \\
100\end{array}$ & $\begin{array}{r}100 \\
100 \\
99\end{array}$ \\
\hline $\begin{array}{l}01-27-82 \\
11-30-82 \\
12-16-82 \\
01-27-83 \\
02-28-83\end{array}$ & $\begin{array}{l}20 \\
20 \\
-- \\
18\end{array}$ & $\begin{array}{l}-- \\
27 \\
17 \\
23 \\
26\end{array}$ & $\begin{array}{l}-- \\
36 \\
25 \\
26 \\
34\end{array}$ & $\begin{array}{l}-- \\
45 \\
35 \\
37 \\
43\end{array}$ & $\begin{array}{l}-- \\
52 \\
45 \\
46 \\
52\end{array}$ & $\begin{array}{l}57 \\
58 \\
54 \\
54 \\
58\end{array}$ & $\begin{array}{l}62 \\
66 \\
66 \\
65 \\
67\end{array}$ & $\begin{array}{l}71 \\
78 \\
81 \\
78 \\
73\end{array}$ & $\begin{array}{l}85 \\
91 \\
93 \\
91 \\
83\end{array}$ & $\begin{array}{l}97 \\
99 \\
98 \\
97 \\
94\end{array}$ & $\begin{array}{r}100 \\
100 \\
99 \\
99 \\
100\end{array}$ \\
\hline $\begin{array}{l}11-17-83 \\
12-14-83 \\
03-01-84\end{array}$ & $\begin{array}{l}19 \\
16 \\
--\end{array}$ & $\begin{array}{l}21 \\
22 \\
--\end{array}$ & $\begin{array}{l}27 \\
31 \\
--\end{array}$ & $\begin{array}{l}37 \\
41 \\
--\end{array}$ & $\begin{array}{l}48 \\
50 \\
--\end{array}$ & $\begin{array}{l}56 \\
59 \\
80\end{array}$ & $\begin{array}{l}66 \\
68 \\
84\end{array}$ & $\begin{array}{l}78 \\
80 \\
88\end{array}$ & $\begin{array}{l}92 \\
93 \\
92\end{array}$ & $\begin{array}{l}98 \\
99 \\
97\end{array}$ & $\begin{array}{l}100 \\
100 \\
100\end{array}$ \\
\hline
\end{tabular}

17. Redwood Creek above Panther Creek near Orick, Calif.

\begin{tabular}{|c|c|c|c|c|c|c|c|c|c|c|c|}
\hline $12-03-80$ & 30 & 40 & 51 & 61 & 69 & 75 & 81 & 90 & 98 & 100 & \\
\hline $01-23-81$ & $\sim$ & $\approx$ & $\ldots$ & $=$ & - & 69 & - & - & - & -- & -- \\
\hline $01-28-81$ & - & 18 & 33 & 44 & 54 & 62 & 74 & 83 & 91 & 96 & 99 \\
\hline $02-05-81$ & - & $\therefore$ & $\therefore$ & $\approx$ & $\therefore$ & 80 & 85 & 93 & 98 & 100 & \\
\hline $11-02-81$ & -- & - & - & - & $\ldots$ & 91 & $\approx$ & $\because$ & - & - & -- \\
\hline
\end{tabular}


Table 2.--Suspended load particle-size distributions--Continued

\begin{tabular}{|c|c|c|c|c|c|c|c|c|c|c|c|}
\hline \multirow{2}{*}{ Date } & \multicolumn{3}{|r|}{ Percent } & by weight. & finer than & \multicolumn{2}{|c|}{ size (millimeters) } & \multicolumn{2}{|l|}{ indicated } & \multirow[b]{2}{*}{1} & \multirow[b]{2}{*}{2} \\
\hline & 0.002 & 0.004 & 0.008 & 0.016 & 0.031 & 0.062 & 0.12 & 0.25 & 0.5 & & \\
\hline
\end{tabular}

\section{Redwood Creek above Panther Creek near Orick, Calif.--Continued}

\begin{tabular}{|c|c|c|c|c|c|c|c|c|c|c|c|}
\hline $12-09-81$ & -- & -- & -- & -- & -- & 54 & -- & -- & -- & -- & -- \\
\hline $02-10-82$ & $\cdots$ & -- & $\cdots$ & +- & -- & 70 & -- & -- & $=-$ & -- & -- \\
\hline $03-19-82$ & -- & -- & $\cdots$ & -- & $\ldots$ & 55 & -- & -- & $\sim$ & -- & -- \\
\hline $12-01-82$ & 17 & 24 & 30 & 38 & 46 & 53 & 60 & 71 & 84 & 95 & 100 \\
\hline $12-22-82$ & 16 & 23 & 32 & 41 & 50 & 58 & 67 & 79 & 90 & 97 & 100 \\
\hline $01-20-83$ & -- & -- & -- & -- & -- & 61 & 66 & 79 & 91 & 99 & 100 \\
\hline $01-26-83$ & $-\infty$ & 20 & 26 & 42 & 52 & 62 & 75 & 88 & 96 & 99 & 100 \\
\hline $02-23-83$ & 24 & 26 & 32 & 38 & 43 & 46 & 53 & 63 & 80 & 93 & 100 \\
\hline $04-05-83$ & -- & -- & -- & -- & -- & 59 & -- & -- & -- & -- & -- \\
\hline $11-16-83$ & 27 & 35 & 44 & 52 & 56 & 59 & 63 & 68 & 76 & 84 & 100 \\
\hline $01-13-84$ & -- & -- & -- & -- & - & 46 & -- & -- & -- & -- & -- \\
\hline $03-08-84$ & - & -- & -- & -- & -- & 68 & -- & -- & -- & $-\infty$ & -- \\
\hline $04-03-84$ & - & -- & - & $\cdots$ & -- & 77 & - & - & -- & -- & -- \\
\hline
\end{tabular}

18. Redwood Creek at Orick, Calif.

\begin{tabular}{|c|c|c|c|c|c|c|c|c|c|c|c|}
\hline $11-14-79$ & -- & -- & -- & $-\infty$ & $-\cdot$ & 75 & -- & - & -- & -- & - \\
\hline $02-01-80$ & -- & -- & -- & - & -- & 64 & -- & -- & $\ldots$ & -- & -- \\
\hline $02-21-80$ & 29 & 36 & 46 & 55 & 66 & 72 & 79 & 86 & 98 & 99 & 100 \\
\hline $12-11-80$ & - & - & -- & - & - & 84 & 87 & 91 & 96 & 100 & \\
\hline $01-29-81$ & 24 & 33 & 43 & 55 & 64 & 71 & 77 & 87 & 95 & 96 & 100 \\
\hline $02-24-81$ & -- & -- & -- & -- & -- & 63 & 67 & 77 & 93 & 95 & 100 \\
\hline $04-11-81$ & -- & -- & - & - & -- & 78 & 82 & 89 & 98 & 100 & \\
\hline $11-13-81$ & 25 & 32 & 43 & 54 & 65 & 71 & 80 & 87 & 97 & 100 & \\
\hline $12-14-81$ & 15 & 21 & 29 & 38 & 46 & 53 & 59 & 70 & 88 & 96 & 97 \\
\hline $12-23-81$ & 24 & 33 & 43 & 55 & 65 & 73 & 81 & 90 & 99 & 100 & \\
\hline $03-03-82$ & 20 & 26 & 35 & 45 & 54 & 61 & 71 & 82 & 92 & 97 & 100 \\
\hline $11-22-82$ & - & - & - & - & - & 64 & 72 & 82 & 96 & 100 & \\
\hline $12-22-82$ & -- & 23 & 32 & 43 & 55 & 64 & 73 & 84 & 94 & 97 & 100 \\
\hline $01-24-83$ & - & 27 & 35 & 46 & 59 & 68 & 75 & 83 & 88 & 89 & 95 \\
\hline $03-30-83$ & -- & 26 & 35 & 49 & 59 & 69 & 80 & 92 & 98 & 99 & 100 \\
\hline $12-07-83$ & -- & -- & -- & - & - & 68 & -- & -- & -- & - & -- \\
\hline $02-29-84$ & -- & -- & - & -- & -- & 61 & -- & -- & -- & - & -- \\
\hline
\end{tabular}

19. Hayes Creek near Orick, Calif.

\begin{tabular}{|c|c|c|c|c|c|c|c|c|c|c|c|}
\hline $12-14-77$ & -- & -- & -- & -- & -- & 59 & - & -- & -- & - & -- \\
\hline $12-14-77$ & -- & -- & -- & -- & - & 71 & 78 & 87 & 96 & 100 & \\
\hline $01-11-79$ & - & -- & -- & -- & -- & 72 & -- & $=$ & -- & -- & -- \\
\hline $02-13-79$ & -- & -- & -- & -- & -- & 72 & -- & - & -- & - & - \\
\hline $02-20-79$ & -- & -- & $-\infty$ & -- & - & 67 & -- & -- & -- & - & - \\
\hline $02-26-79$ & $\cdots$ & $=$ & -- & -- & - & 68 & -- & - & -- & - & -- \\
\hline $02-27-79$ & -- & -. & -- & -- & - & 60 & -- & - & -- & - & - \\
\hline $05-08-79$ & -- & -- & -- & -- & $\cdots$ & 62 & -- & - & -- & - & $-\infty$ \\
\hline $01-14-80$ & -- & -- & -- & -- & - & 74 & -- & -- & -- & - & - \\
\hline $01-15-80$ & -- & - & -- & -- & -- & 82 & $-\infty$ & - & -- & $\ldots$ & $\cdots$ \\
\hline $02-21-80$ & -- & -- & -- & -- & - & 78 & -- & -- & -- & -- & - \\
\hline $03-14-80$ & -- & -- & - & -- & -- & 64 & -- & -- & -- & - & - \\
\hline $04-09-80$ & -- & -- & -- & -- & -- & 93 & -- & -- & -- & -- & $=$ \\
\hline
\end{tabular}


Table 2.--Suspended load particle-size distributions--Continued

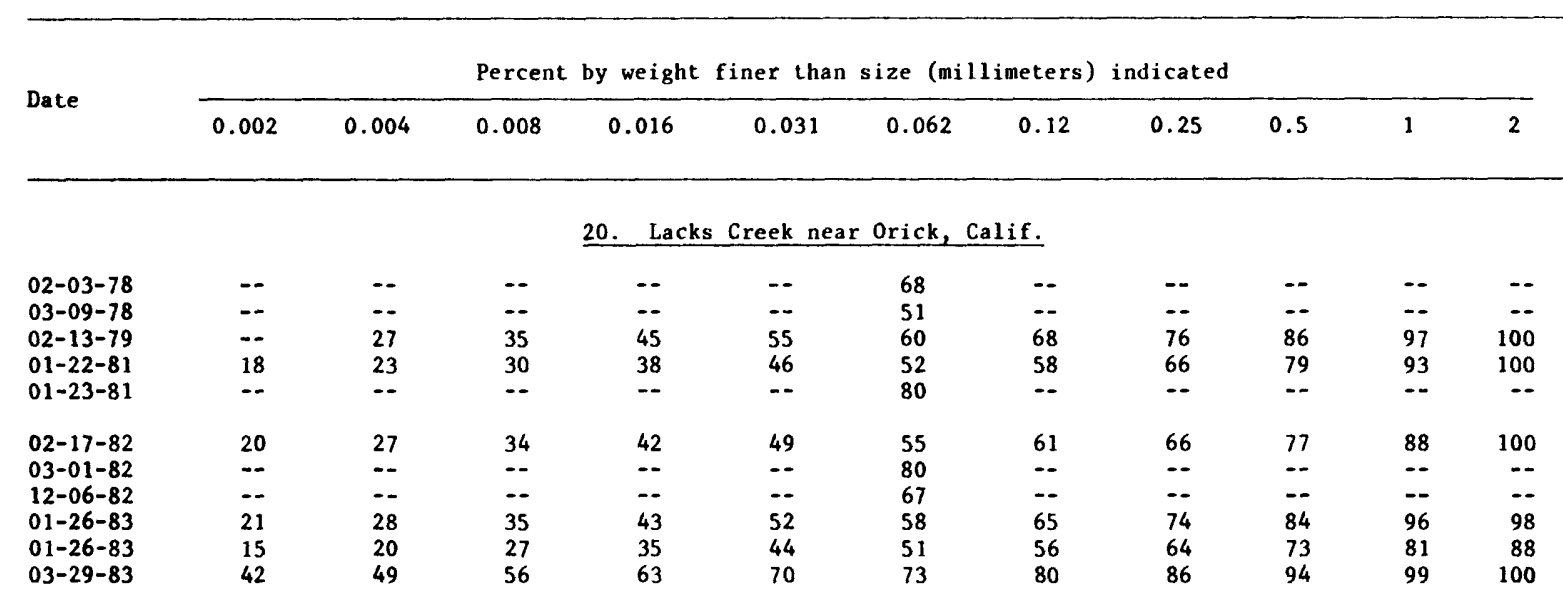

21. Sacramento River above Bend Bridge near Red Bluff, Calif.

\begin{tabular}{|c|c|c|c|c|c|c|c|c|c|c|}
\hline $01-03-78$ & 36 & 51 & 68 & 84 & 92 & 96 & 97 & 98 & 99 & 100 \\
\hline $03-01-78$ & - & $\ldots$ & $\ldots$ & -- & $\ldots$ & 73 & 80 & 90 & 98 & 100 \\
\hline $05-01-78$ & $=$ & $\cdots$ & - & -- & -- & 49 & 58 & 78 & 97 & 100 \\
\hline $03-01-79$ & -- & $\cdots$ & $\cdots$ & -- & -- & 68 & 78 & 88 & 98 & 100 \\
\hline $01-02-80$ & -- & -- & -- & -- & -- & 52 & 58 & 66 & 84 & 100 \\
\hline $02-01-80$ & -- & -- & -- & -- & - & 59 & 63 & 72 & 95 & 100 \\
\hline
\end{tabular}

22. Clearwater River at Spalding, Idaho

\begin{tabular}{|c|c|c|c|c|c|c|c|c|c|c|}
\hline $\begin{array}{l}06-01-72 \\
05-16-73 \\
06-10-75 \\
06-17-75 \\
04-06-76\end{array}$ & $\begin{array}{l}10 \\
=- \\
-- \\
-- \\
24\end{array}$ & $\begin{array}{l}11 \\
-- \\
-- \\
-- \\
33\end{array}$ & $\begin{array}{l}23 \\
\ldots \\
\ldots- \\
-- \\
45\end{array}$ & $\begin{array}{l}33 \\
-- \\
-- \\
-- \\
62\end{array}$ & $\begin{array}{l}46 \\
-- \\
-- \\
-- \\
80\end{array}$ & $\begin{array}{l}56 \\
57 \\
31 \\
41 \\
91\end{array}$ & $\begin{array}{l}69 \\
68 \\
47 \\
48 \\
94\end{array}$ & $\begin{array}{l}86 \\
82 \\
70 \\
62 \\
96\end{array}$ & $\begin{array}{l}99 \\
99 \\
98 \\
90 \\
99\end{array}$ & $\begin{array}{l}100 \\
100 \\
100 \\
100 \\
100\end{array}$ \\
\hline $\begin{array}{l}04-08-76 \\
04-14-76 \\
05-06-76 \\
05-11-76 \\
05-12-76\end{array}$ & $\begin{array}{c}21 \\
-- \\
-- \\
-- \\
8\end{array}$ & $\begin{array}{l}30 \\
-- \\
-- \\
-- \\
12\end{array}$ & $\begin{array}{l}39 \\
-- \\
-- \\
-- \\
17\end{array}$ & $\begin{array}{l}53 \\
-- \\
-- \\
-- \\
23\end{array}$ & $\begin{array}{l}68 \\
\ldots \\
\ldots- \\
-- \\
33\end{array}$ & $\begin{array}{l}80 \\
73 \\
47 \\
49 \\
36\end{array}$ & $\begin{array}{l}83 \\
80 \\
64 \\
65 \\
52\end{array}$ & $\begin{array}{l}88 \\
89 \\
84 \\
88 \\
74\end{array}$ & $\begin{array}{r}98 \\
98 \\
99 \\
100 \\
97\end{array}$ & $\begin{array}{l}100 \\
100 \\
100\end{array}$ \\
\hline $\begin{array}{l}05-24-76 \\
05-26-76 \\
06-11-76 \\
04-26-77 \\
05-04-77\end{array}$ & $\begin{array}{l}-- \\
-- \\
\cdots \\
--\end{array}$ & $\begin{array}{l}-- \\
-- \\
\cdots \\
--\end{array}$ & $\begin{array}{l}-- \\
-- \\
-- \\
--\end{array}$ & $\begin{array}{l}-- \\
-- \\
-- \\
--\end{array}$ & $\begin{array}{l}-- \\
-- \\
-- \\
--\end{array}$ & $\begin{array}{l}24 \\
30 \\
22 \\
64 \\
68\end{array}$ & $\begin{array}{l}42 \\
47 \\
34 \\
74 \\
76\end{array}$ & $\begin{array}{l}67 \\
74 \\
63 \\
85 \\
84\end{array}$ & $\begin{array}{r}100 \\
99 \\
100 \\
98 \\
96\end{array}$ & $\begin{array}{l}100 \\
100\end{array}$ \\
\hline $\begin{array}{l}04-28-78 \\
05-15-78 \\
05-17-78 \\
06-05-78 \\
06-06-78\end{array}$ & $\begin{array}{l}39 \\
-- \\
-- \\
-- \\
--\end{array}$ & $\begin{array}{l}48 \\
-- \\
-- \\
-- \\
--\end{array}$ & $\begin{array}{l}58 \\
\ldots \\
\ldots \\
\ldots \\
\ldots\end{array}$ & $\begin{array}{l}73 \\
\ldots \\
\ldots \\
\ldots \\
\ldots\end{array}$ & $\begin{array}{l}88 \\
-- \\
-- \\
-- \\
--\end{array}$ & $\begin{array}{l}96 \\
54 \\
67 \\
43 \\
42\end{array}$ & $\begin{array}{l}98 \\
68 \\
76 \\
54 \\
49\end{array}$ & $\begin{array}{r}100 \\
88 \\
87 \\
75 \\
72\end{array}$ & $\begin{array}{l}98 \\
96 \\
95 \\
94\end{array}$ & $\begin{array}{l}100 \\
100 \\
100 \\
100\end{array}$ \\
\hline $\begin{array}{l}06-08-78 \\
06-12-78 \\
06-14-78 \\
06-19-78 \\
05-01-79\end{array}$ & $\begin{array}{l}-- \\
-- \\
-- \\
--\end{array}$ & $\begin{array}{l}-- \\
-- \\
-- \\
--\end{array}$ & $\begin{array}{l}-- \\
-- \\
-- \\
--\end{array}$ & $\begin{array}{l}-- \\
\ldots \\
-- \\
--\end{array}$ & $\begin{array}{l}-- \\
-- \\
-- \\
--\end{array}$ & $\begin{array}{l}43 \\
39 \\
29 \\
58 \\
52\end{array}$ & $\begin{array}{l}53 \\
47 \\
34 \\
70 \\
69\end{array}$ & $\begin{array}{l}72 \\
66 \\
52 \\
83 \\
83\end{array}$ & $\begin{array}{r}93 \\
94 \\
86 \\
100 \\
94\end{array}$ & $\begin{array}{r}99 \\
100 \\
100\end{array}$ \\
\hline
\end{tabular}


Table 2.--Suspended load particle-size distributions--Continued

\begin{tabular}{|c|c|c|c|c|c|c|c|c|c|c|c|}
\hline \multirow{2}{*}{ Date } & \multicolumn{4}{|c|}{ Percent by weight } & finer than & \multicolumn{2}{|c|}{ size (millimeters) } & \multicolumn{2}{|l|}{ indicated } & \multirow[b]{2}{*}{1} & \multirow[b]{2}{*}{2} \\
\hline & 0.002 & 0.004 & 0.008 & 0.016 & 0.031 & 0.062 & 0.12 & 0.25 & 0.5 & & \\
\hline
\end{tabular}

\section{Clearwater River at Spalding, Idaho--Continued}

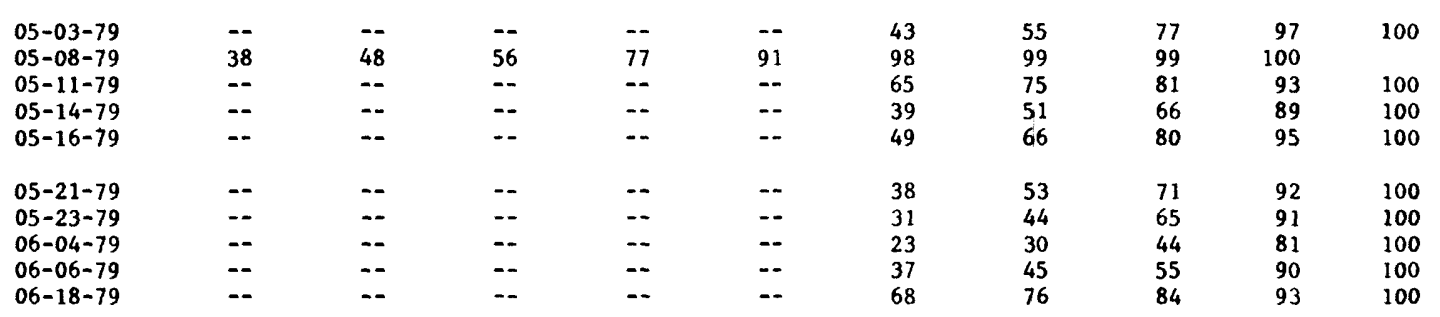

\section{Big Lost River at Howell Ranch, Idaho}

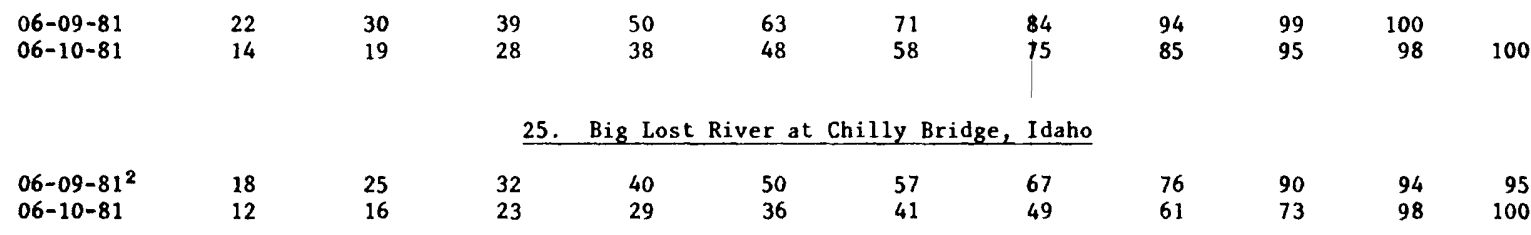

\section{Big Lost River below Chilly Sinks, Idaho}

$\begin{array}{rrrrrrrrrrrrr}06-09-81 & 24 & 34 & 43 & 53 & 64 & 73 & 84 & 94 & 98 & 100 & 94 & 94 \\ 06-10-81 & 20 & 28 & 33 & 41 & 49 & 59 & 64 & 99\end{array}$

27. Big Lost River above East and West Channels, Idaho

$\begin{array}{lllllllllllllll}06-10-81 & 16 & 24 & 31 & 40 & 48 & 54 & 64 & 72 & 91 & 98 & 100\end{array}$

32. East Fork River near Pinedale, Wyo.

\begin{tabular}{|c|c|c|c|c|c|c|c|c|c|c|c|}
\hline $05-23-79$ & -- & -- & -- & -- & - & 37 & -- & -- & -- & -- & -- \\
\hline $05-24-79$ & -- & -- & -- & -- & - & 34 & -- & -- & -- & -- & - \\
\hline $05-25-79$ & -- & 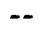 & -+ & $=$ & -- & 22 & $\cdots$ & - & $\cdots$ & $\ldots$ & - \\
\hline $05-26-79$ & -- & -- & -- & - & -- & 27 & -. & -- & $\ldots$ & -- & -- \\
\hline $05-28-79$ & -- & -- & -- & -- & -- & 17 & -- & $=-$ & -- & -- & -- \\
\hline $05-30-79$ & -- & -- & -- & -- & -- & 41 & -- & -- & -- & -- & -- \\
\hline $06-01-79$ & -- & -- & -- & -- & - & 24 & -- & -- & -. & -- & -. \\
\hline $06-02-79$ & -- & -- & -- & -- & - & 30 & -- &.- & -- & -- & -- \\
\hline $06-03-79$ & -- & -- & -- & -- & -- & 62 & -. & -- & -- & -- & - \\
\hline $06-04-79$ & -- & -- & - & $=-$ & - & 67 & -- & -- & - & -- & - \\
\hline $06-05-79$ & -- & -- & -- & -- & - & 62 & -- & -- & -- & -. & - \\
\hline $06-10-79$ & $=-$ & -- & -- & -- & - & 64 & - & $=$ & -- & -. & $\ldots$ \\
\hline $06-11-79$ & -- & -- & -- & -- & -. & 67 & -. & - & - & -. & $\ldots$ \\
\hline $06-13-79$ & -- & -- & -- & -- & $\ldots$ & 64 & -. & -. & -- & -. & - \\
\hline $06-14-79$ & -- & -- & -- & -- & - & 57 & -- & -- & -- & -. & - \\
\hline $06-15-79$ & -- & -- & -- & -- & - & 54 & -. & -- & -- & -- & -- \\
\hline
\end{tabular}


Table 2.--Suspended load particle-size distributions--Continued

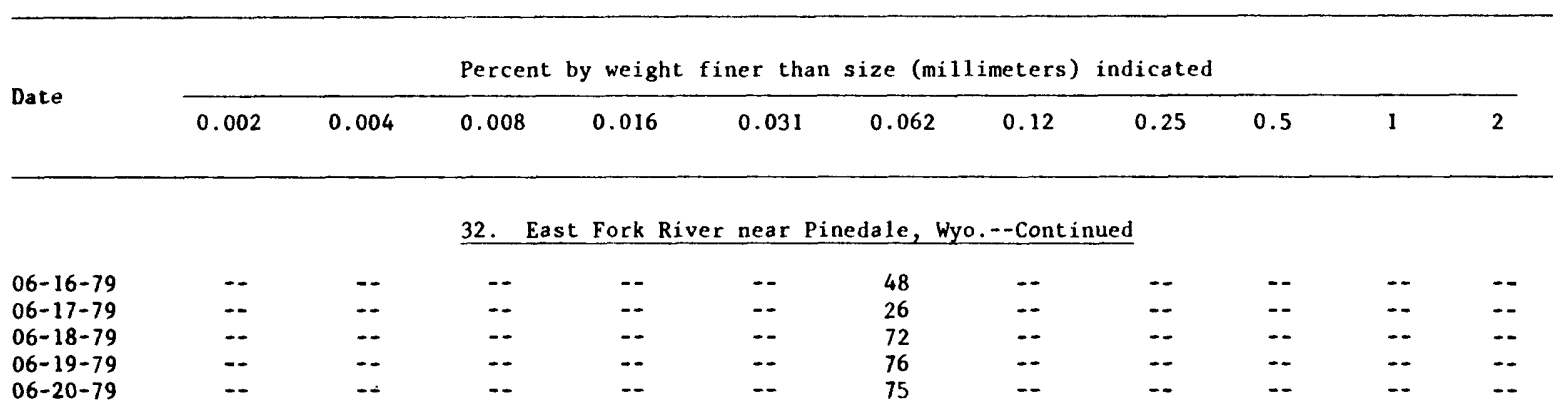

33. Yampa River at Deerlodge Park, Colo.

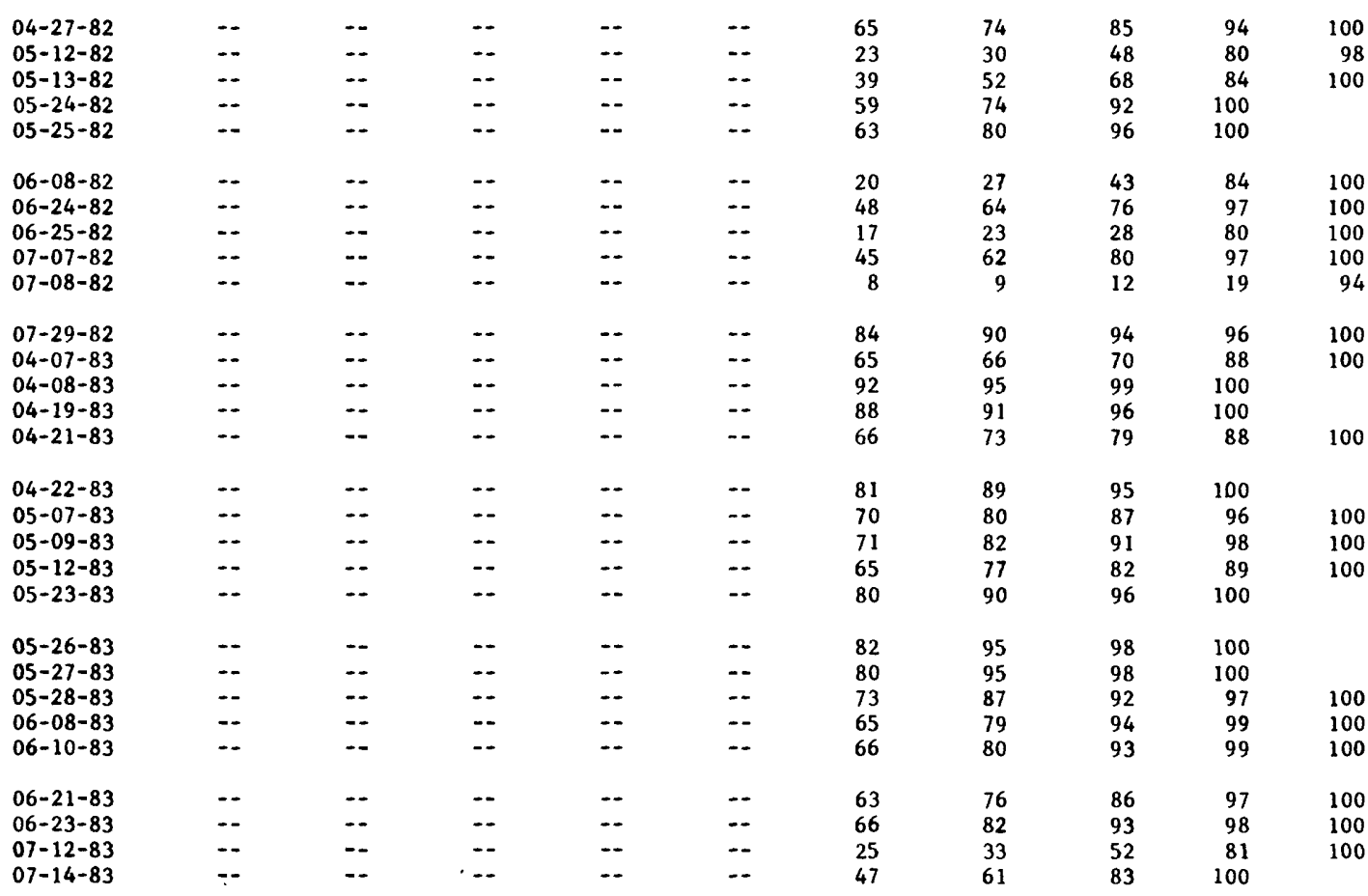

34. Rock Creek near Toponas, Colo.

\begin{tabular}{|c|c|c|c|c|c|c|c|c|c|c|c|}
\hline $05-22-85$ & $=$ & $=$ & $\cdots$ & $\cdots$ & -- & 47 & -- & $\cdots$ & $\cdots$ & - & - \\
\hline $05-29-85$ & $\cdots$ & $=$ & - & - & -- & 56 & $\cdots$ & $\cdots$ & - & - & - \\
\hline $06-10-85$ & -- & -- & -- & -- & - & 95 & $\cdots$ & $\cdots$ & $\cdots$ & $\cdots$ & $\cdots$ \\
\hline $07-22-85$ & $\cdots$ & $\cdots$ & $=$ & $\cdots$ & $\cdots$ & 23 & -- & $\cdots$ & $\cdots$ & - & $\cdots$ \\
\hline
\end{tabular}


Table 2.--Suspended load particle-size distributions--Continued

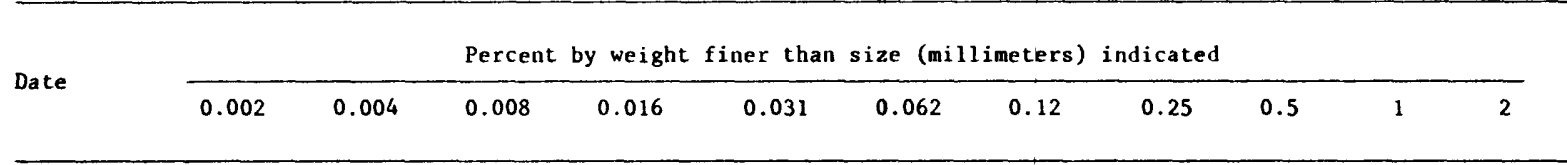

72. West Fork of San Juan River near Pagosa Springs, Colo.

\begin{tabular}{|c|c|c|c|c|c|c|c|c|c|c|c|}
\hline $04-23-85$ & -- & -- & $=-$ & -- & -- & 5 & -- & -- & $\cdots$ & -- & -- \\
\hline $05-05-85$ & -- & -- & -- & - & -- & 67 & -- & - & -- & -- & -- \\
\hline $05-08-85$ & -- & -- & $\cdots$ & -- & -- & 76 & -- & -- & $\cdots$ & -- & $\cdots$ \\
\hline $05-12-85$ & -- & -- & $-\infty$ & -- & -- & 68 & -- & - & $\cdots$ & -- & $=$ \\
\hline $05-15-85$ & -- & -- & -- & -- & -- & 88 & -- & -- & - & -- & -- \\
\hline $05-20-85$ & $\cdots$ & -- & - & -- & -- & 81 & -- & -- & -- & -- & -- \\
\hline $05-26-85$ & -- & -- & -- & -- & -- & 48 & -- & $\cdots$ & $\cdots$ & $\cdots$ & $=$ \\
\hline $05-30-85$ & -. & -- & $\cdots$ & -- & -- & 53 & -- & -- & -- & -- & -- \\
\hline $06-03-85$ & -- & -- & -- & -- & -- & 47 & -- & - & $=$ & -- & - \\
\hline $06-06-85$ & -- & -- & -- & -- & -- & 44 & $\rightarrow$ & -- & -- & -- & -- \\
\hline $06-18-85$ & - & -- & -- & -- & -- & 44 & -- & -- & -- & -- & -- \\
\hline $07-18-85$ & $\cdots$ & $\cdots$ & - & -- & -- & 57 & - & - & $=-$ & -- & -- \\
\hline
\end{tabular}

87. Wapsipinicon River near DeWitt, lowa

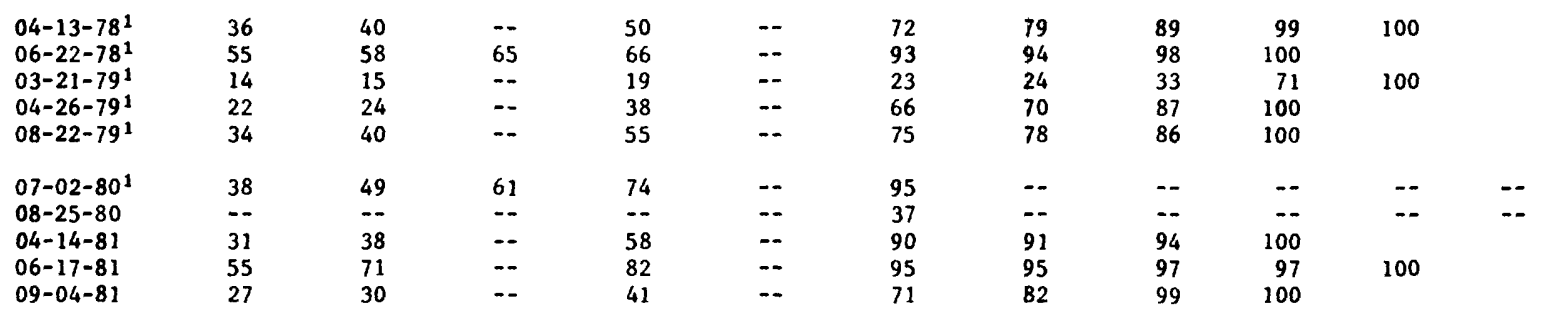

88. Iowa River at Wapello, Iowa

\begin{tabular}{|c|c|c|c|c|c|c|c|c|c|c|c|}
\hline $\begin{array}{l}03-28-78^{1} \\
04-25-78^{1} \\
06-21-78^{1} \\
07-25-78^{1} \\
06-13-79^{1}\end{array}$ & $\begin{array}{l}34 \\
40 \\
50 \\
51 \\
39\end{array}$ & $\begin{array}{l}38 \\
42 \\
59 \\
56 \\
52\end{array}$ & $\begin{array}{l}-- \\
43 \\
68 \\
61 \\
--\end{array}$ & $\begin{array}{l}47 \\
55 \\
78 \\
71 \\
75\end{array}$ & $\begin{array}{l}-- \\
-- \\
-- \\
--\end{array}$ & $\begin{array}{l}70 \\
88 \\
97 \\
96 \\
98\end{array}$ & $\begin{array}{l}73 \\
89 \\
98 \\
98 \\
99\end{array}$ & $\begin{array}{l}78 \\
92 \\
99 \\
100 \\
100\end{array}$ & $\begin{array}{r}95 \\
98 \\
100\end{array}$ & $\begin{array}{l}100 \\
100\end{array}$ & \\
\hline $\begin{array}{l}04-03-80^{1} \\
06-02-80^{1} \\
09-03-80^{1} \\
06-18-81^{1} \\
07-02-81^{1}\end{array}$ & $\begin{array}{l}-- \\
39 \\
-- \\
51 \\
48\end{array}$ & $\begin{array}{l}-- \\
47 \\
-- \\
67 \\
55\end{array}$ & $\begin{array}{l}-- \\
56 \\
-- \\
75 \\
66\end{array}$ & $\begin{array}{l}-- \\
68 \\
-- \\
80 \\
77\end{array}$ & $\begin{array}{l}-- \\
-- \\
-- \\
--\end{array}$ & $\begin{array}{l}91 \\
92 \\
79 \\
97 \\
92\end{array}$ & $\begin{array}{l}-- \\
-- \\
-- \\
-- \\
93\end{array}$ & $\begin{array}{l}=- \\
-- \\
-- \\
=-\end{array}$ & $\begin{array}{l}-- \\
-- \\
-- \\
-- \\
98\end{array}$ & $\begin{array}{l}-- \\
-- \\
-- \\
--\end{array}$ & $\begin{array}{l}-- \\
-- \\
100\end{array}$ \\
\hline
\end{tabular}

89. Wisconsin River at Muscoda, Wis.

\begin{tabular}{|c|c|c|c|c|c|c|c|c|c|c|c|}
\hline $07-20-77$ & -- & -- & - & $\cdots$ & -- & 94 & -- & -- & -- & -- & -- \\
\hline $08-24-77$ & - & -- & -- & -- & -- & 90 & -- & -- & -- & -- & - \\
\hline $10-18-77$ & -- & -- & -- & -- & -- & 51 & - & - & -- & -. & - \\
\hline $11-14-77$ & -- & -- & -- & -- & -- & 54 & -- & -- & -- & -- & -- \\
\hline $05-08-78$ & -- & -- & - & -- & - & 50 & $=$ & -- & -- & -- & - \\
\hline $06-06-78$ & -- & - & - & -- & -- & 80 & -- & -- & -- & -- & -- \\
\hline $10-24-78$ & -- & - & -- & - & - & 80 & -- & -- & -- & - & - \\
\hline $11-14-78$ & -- & -. & -- & -. & -- & 45 & - & -- & -- & - & - \\
\hline $03-28-79$ & -. & -- & -- & -- & -- & 29 & 34 & 59 & 100 & & \\
\hline $07-05-79$ & -- & -- & -- & -- & -- & 90 & -. & $\ldots$ & -- & -- & -- \\
\hline $08-25-79$ & $-\cdot$ & -- & -- & -. & - & 87 & -- & -- & -. & -. & - \\
\hline
\end{tabular}


Table 2.--Suspended load particle-size distributions--Continued

Percent by weight finer than size (millimeters) indicated

Date

$\begin{array}{llllllllll}0.002 & 0.004 & 0.008 & 0.016 & 0.031 & 0.062 & 0.12 & 0.25 & 0.5 & 1\end{array}$

90. Black River near Galesville, Wis.

\begin{tabular}{|c|c|c|c|c|c|c|c|c|c|c|c|}
\hline $04-23-77$ & - & $\cdots$ & - & $\cdots$ & -- & 75 & -- & - & -- & -- & -- \\
\hline $05-27-77$ & - & -- & $\cdots$ & -- & - & 95 & - & -- & -- & -- & - \\
\hline $06-29-77$ & -- & -- & -- & -- & -- & 86 & -- & $\cdots$ & -- & - & -- \\
\hline $07-12-77$ & - & $\cdots$ & -- & -- & - & 90 & - & -- & -- & -- & - \\
\hline $09-27-77$ & -- & -- & - & - & - & 83 & - & - & -- & -- & - \\
\hline $10-19-77$ & -- & -- & -- & -- & -- & 52 & - & 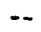 & -- & -- & -. \\
\hline $06-13-78$ & -- & -- & -- & -- & -- & 67 & -- & -- & -- & -- & -- \\
\hline $04-25-79$ & $\cdots$ & -- & -- & -- & -- & 82 & - & -- & -- & -- & - \\
\hline $05-31-79$ & $\cdots$ & $\cdots$ & $\cdots$ & -- & - & 94 & - & - & -- & $\cdots$ & $\cdots$ \\
\hline $07-23-79$ & -- & $\cdots$ & -- & $\cdots$ & - & 94 & $\cdots$ & -- & $\cdots$ & -- & - \\
\hline
\end{tabular}

91. Chippewa River near Caryville, Wis.

\begin{tabular}{|c|c|c|c|c|c|c|c|c|c|c|c|}
\hline $08-30-76$ & -- & -- & -- & -- & -- & 63 & -- & $\cdots$ & $-\infty$ & - & -- \\
\hline $09-20-76$ & $=$ & - & -- & -- & - & 71 & -- & - & -. & - & -. \\
\hline $04-17-79$ & - & - & -- & -- & - & 85 & 100 & & & & \\
\hline $04-23-79$ & -- & -. & - & -- & - & 54 & 100 & & & & \\
\hline $05-29-79$ & $\cdots$ & -- & - & -- & - & 82 & - & - & -- & -- & -- \\
\hline $07-24-79$ & - & - & -- & -- & -- & 74 & -- & - & -- & - & - \\
\hline $09-10-79$ & - & - & -- & - & - & 90 & - & - & -- & - & \\
\hline
\end{tabular}

92. Chippewa River at Durand, Wis.

\begin{tabular}{|c|c|c|c|c|c|c|c|c|c|c|c|}
\hline $11-11-75$ & 17 & 21 & 25 & 30 & 46 & 52 & 55 & 73 & 83 & 100 & \\
\hline $04-14-76$ & - & - & - & - & - & 17 & - & -- & -- & - & - \\
\hline $08-31-76$ & - & - & -- & - & - & 81 & -- & $\cdots$ & -- & - & - \\
\hline $02-02-77$ & -- & - & -- & -- & -- & 57 & -- & - & -- & - & -. \\
\hline $05-10-77$ & $\cdots$ & - & -- & - & - & 42 & -- & $\cdots$ & -- & - & - \\
\hline $06-15-77$ & - & - & -- & - & -- & 74 & -- & - & -- & -- & - \\
\hline $07-06-77$ & -- & - & - & - & -- & 70 & -- & - & -- & -- & - \\
\hline $11-16-77$ & - & - & - & -- & -- & 13 & -- & $\cdots$ & $\ldots$ & - & - \\
\hline $06-06-78$ & -- & $\cdots$ & - & - & -- & 50 & -- & - & -- & -- & - \\
\hline $04-17-79$ & - & -- & - & -- & - & 41 & 56 & 58 & 100 & & \\
\hline $04-23-79$ & - & - & -- & - & - & 22 & 27 & 100 & & & \\
\hline
\end{tabular}

93. Chippewa River near Pepin, Wis.

\begin{tabular}{|c|c|c|c|c|c|c|c|c|c|c|c|}
\hline $07-07-77$ & - & $\cdots$ & $\therefore$ & -- & -- & 38 & -- & -- & -- & - & -- \\
\hline $06-07-78$ & $\cdots$ & -- & $\cdots$ & -- & -- & 61 & -- & -- & -- & - & - \\
\hline $09-20-78$ & -- & -. & - & -- & -- & 5 & -- & -- & -- & - & - \\
\hline $05-30-79$ & - & -- & -- & -- & -- & 53 & -- & -- & - & - & -- \\
\hline $07-24-79$ & - & -- & -- & - & - & 63 & -- & -- & -- & -- & -- \\
\hline $09-11-79$ & - & $\cdots$ & $\cdots$ & -- & - & 81 & -- & -- & -- & - & -- \\
\hline
\end{tabular}

${ }^{1}$ All values are in terms of fall diameter.

2100 percent is less than 4 millimeters. 
Table 3.-Bedload particle-size distributions

[--, no data available]

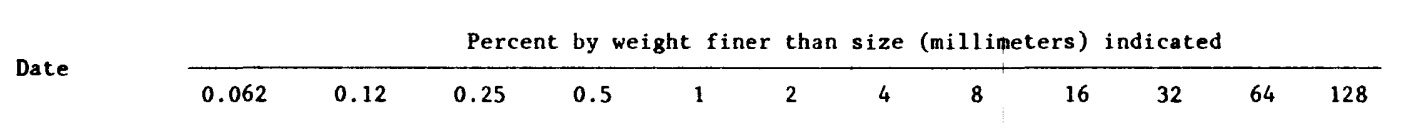

\section{Susitna River near Talkeetna, Alaska}

\begin{tabular}{|c|c|c|c|c|c|c|c|c|c|c|c|c|}
\hline $\begin{array}{l}06-03-82^{1} \\
06-15-82^{1} \\
06-22-82^{1} \\
06-30-82 \\
07-08-82\end{array}$ & $\begin{array}{l}-- \\
-- \\
--\end{array}$ & $\begin{array}{l}-- \\
-- \\
-- \\
--\end{array}$ & $\begin{array}{r}3 \\
-- \\
2 \\
1 \\
--\end{array}$ & $\begin{array}{l}37 \\
24 \\
47 \\
33 \\
65\end{array}$ & $\begin{array}{l}47 \\
32 \\
58 \\
39 \\
94\end{array}$ & $\begin{array}{l}48 \\
32 \\
60 \\
40 \\
96\end{array}$ & $\begin{array}{l}49 \\
33 \\
60 \\
41 \\
97\end{array}$ & $\begin{array}{l}52 \\
35 \\
61 \\
43 \\
99\end{array}$ & $\begin{array}{l}54 \\
38 \\
61 \\
46 \\
99\end{array}$ & $\begin{array}{r}58 \\
44 \\
62 \\
84 \\
100\end{array}$ & $\begin{array}{r}74 \\
76 \\
64 \\
100\end{array}$ & $\begin{array}{l}-- \\
--\end{array}$ \\
\hline $\begin{array}{l}07-14-82 \\
07-21-82 \\
07-28-82 \\
08-04-82 \\
08-10-82\end{array}$ & $\begin{array}{l}-- \\
-- \\
-- \\
--\end{array}$ & $\begin{array}{l}-- \\
-- \\
-- \\
--\end{array}$ & $\begin{array}{l}1 \\
1 \\
1 \\
2 \\
1\end{array}$ & $\begin{array}{l}51 \\
65 \\
70 \\
78 \\
66\end{array}$ & $\begin{array}{l}71 \\
90 \\
85 \\
98 \\
94\end{array}$ & $\begin{array}{l}74 \\
92 \\
86 \\
99 \\
96\end{array}$ & $\begin{array}{l}75 \\
93 \\
88 \\
99 \\
96\end{array}$ & $\begin{array}{l}77 \\
94 \\
91 \\
99 \\
96\end{array}$ & $\begin{array}{r}81 \\
96 \\
93 \\
100 \\
97\end{array}$ & $\begin{array}{r}90 \\
100 \\
100 \\
100\end{array}$ & 100 & \\
\hline $\begin{array}{l}08-18-82 \\
08-25-82 \\
08-31-82 \\
09-19-82 \\
05-19-83\end{array}$ & $\begin{array}{l}-- \\
-- \\
--\end{array}$ & $\begin{array}{r}-- \\
-- \\
-- \\
0\end{array}$ & $\begin{array}{l}1 \\
1 \\
1 \\
2 \\
1\end{array}$ & $\begin{array}{l}68 \\
69 \\
73 \\
63 \\
76\end{array}$ & $\begin{array}{l}92 \\
97 \\
95 \\
78 \\
98\end{array}$ & $\begin{array}{r}94 \\
99 \\
97 \\
80 \\
100\end{array}$ & $\begin{array}{r}96 \\
100 \\
97 \\
80\end{array}$ & $\begin{array}{l}99 \\
98 \\
82\end{array}$ & $\begin{array}{l}98 \\
84\end{array}$ & $\begin{array}{r}100 \\
91\end{array}$ & 100 & \\
\hline $\begin{array}{l}05-25-83 \\
06-01-83 \\
06-08-83 \\
06-23-83 \\
07-07-83\end{array}$ & & $\begin{array}{l}0 \\
0 \\
0 \\
0 \\
0\end{array}$ & $\begin{array}{l}1 \\
1 \\
1 \\
1 \\
1\end{array}$ & $\begin{array}{l}64 \\
19 \\
62 \\
60 \\
65\end{array}$ & $\begin{array}{l}87 \\
21 \\
80 \\
80 \\
87\end{array}$ & $\begin{array}{l}88 \\
21 \\
83 \\
82 \\
90\end{array}$ & $\begin{array}{l}89 \\
21 \\
83 \\
82 \\
91\end{array}$ & $\begin{array}{l}89 \\
23 \\
86 \\
83 \\
92\end{array}$ & $\begin{array}{l}90 \\
27 \\
88 \\
85 \\
94\end{array}$ & $\begin{array}{l}92 \\
29 \\
91 \\
86 \\
95\end{array}$ & $\begin{array}{l}100 \\
100 \\
100 \\
100 \\
100\end{array}$ & \\
\hline $\begin{array}{l}07-21-83 \\
08-02-83 \\
08-11-83 \\
08-31-83 \\
09-14-83\end{array}$ & & $\begin{array}{l}0 \\
0 \\
0 \\
0\end{array}$ & $\begin{array}{r}1 \\
1 \\
13 \\
1 \\
0\end{array}$ & $\begin{array}{l}87 \\
63 \\
44 \\
80 \\
76\end{array}$ & $\begin{array}{l}94 \\
78 \\
96 \\
98 \\
88\end{array}$ & $\begin{array}{l}95 \\
80 \\
98 \\
99 \\
92\end{array}$ & $\begin{array}{l}95 \\
81 \\
98 \\
99 \\
93\end{array}$ & $\begin{array}{r}95 \\
83 \\
98 \\
100 \\
93\end{array}$ & $\begin{array}{l}96 \\
85 \\
99 \\
93\end{array}$ & $\begin{array}{r}100 \\
89 \\
100 \\
100\end{array}$ & 100 & \\
\hline $\begin{array}{l}10-06-83 \\
06-13-84 \\
07-09-84 \\
07-30-84 \\
08-16-84\end{array}$ & & $\begin{array}{l}0 \\
0 \\
0\end{array}$ & $\begin{array}{l}0 \\
0 \\
1 \\
1 \\
1\end{array}$ & $\begin{array}{l}84 \\
63 \\
67 \\
61 \\
72\end{array}$ & $\begin{array}{l}98 \\
95 \\
88 \\
80 \\
96\end{array}$ & $\begin{array}{l}99 \\
96 \\
89 \\
81 \\
97\end{array}$ & $\begin{array}{l}99 \\
97 \\
90 \\
83 \\
98\end{array}$ & $\begin{array}{l}99 \\
97 \\
90 \\
86 \\
98\end{array}$ & $\begin{array}{r}100 \\
99 \\
91 \\
89 \\
98\end{array}$ & $\begin{array}{r}100 \\
94 \\
90 \\
100\end{array}$ & $\begin{array}{l}100 \\
100\end{array}$ & \\
\hline $\begin{array}{l}08-26-84 \\
09-13-84 \\
09-25-84 \\
05-29-85 \\
06-26-85\end{array}$ & & $\begin{array}{l}0 \\
0\end{array}$ & $\begin{array}{l}2 \\
0 \\
0 \\
2 \\
1\end{array}$ & $\begin{array}{l}74 \\
73 \\
77 \\
65 \\
72\end{array}$ & $\begin{array}{r}94 \\
95 \\
100 \\
82 \\
94\end{array}$ & $\begin{array}{l}95 \\
95 \\
84 \\
95\end{array}$ & $\begin{array}{l}96 \\
98\end{array}$ & $\begin{array}{l}98 \\
99 \\
88 \\
98\end{array}$ & $\begin{array}{r}91 \\
100\end{array}$ & 100 & & \\
\hline $\begin{array}{l}07-26-85 \\
08-13-85 \\
09-06-85 \\
09-19-85\end{array}$ & & $\begin{array}{l}0 \\
0 \\
0\end{array}$ & $\begin{array}{l}1 \\
1 \\
0 \\
1\end{array}$ & $\begin{array}{l}82 \\
68 \\
70 \\
75\end{array}$ & $\begin{array}{l}99 \\
88 \\
98 \\
94\end{array}$ & $\begin{array}{r}100 \\
89 \\
99 \\
95\end{array}$ & $\begin{array}{l}90 \\
99 \\
96\end{array}$ & $\begin{array}{r}90 \\
100 \\
96\end{array}$ & $\begin{array}{r}91 \\
100\end{array}$ & 91 & 100 & \\
\hline
\end{tabular}


Table 3.--Bedload particle-size distributions--Continued

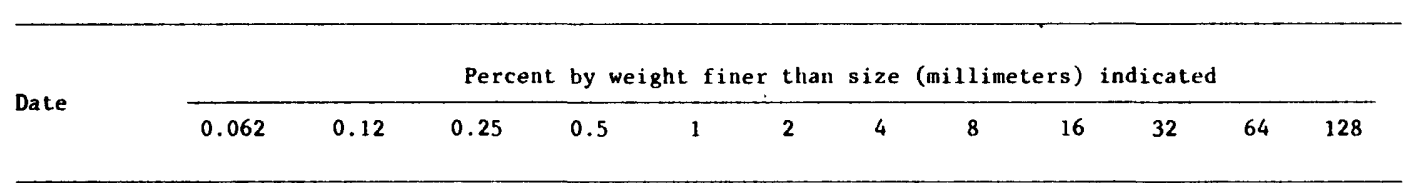

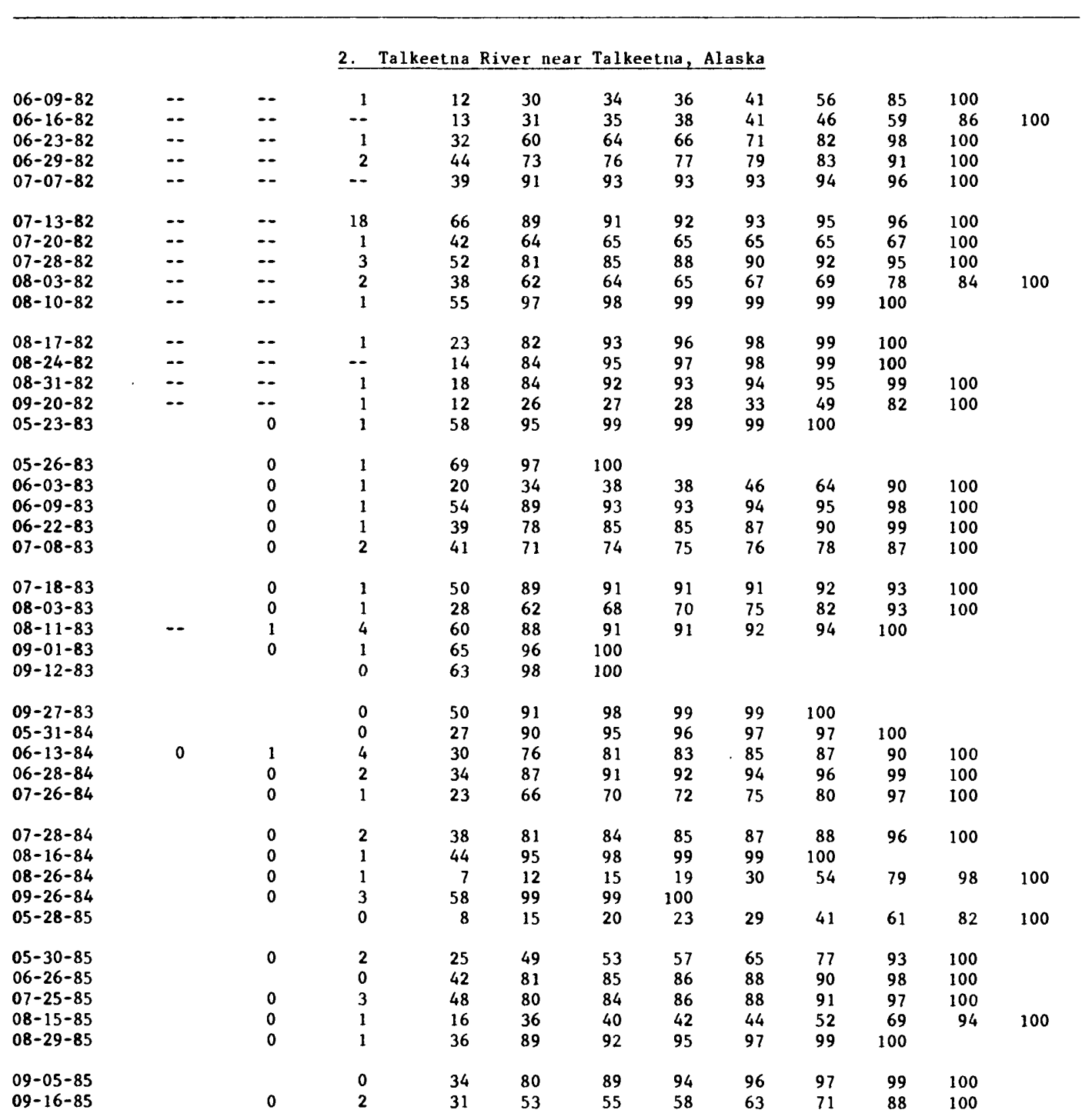


Table 3.--Bedload particle-size distributions--Continued

\begin{tabular}{|c|c|c|c|c|c|c|c|c|c|c|c|c|}
\hline \multirow{2}{*}{ Date } & \multicolumn{3}{|r|}{ Percent } & by weight & $t$ finer & \multicolumn{2}{|c|}{ than size } & \multicolumn{2}{|c|}{ (millimeters) } & \multicolumn{2}{|l|}{ indicated } & \multirow[b]{2}{*}{128} \\
\hline & 0.062 & 0.12 & 0.25 & 0.5 & 1 & 2 & 4 & 8 & 16 & 32 & 64 & \\
\hline
\end{tabular}

3. Chulitna River below Canyon near Talkeetna, Alaska

\begin{tabular}{|c|c|c|c|c|c|c|c|c|c|c|c|c|}
\hline $\begin{array}{l}06-04-82 \\
06-09-82 \\
06-16-82 \\
06-22-82 \\
06-29-82\end{array}$ & $\begin{array}{l}-- \\
-- \\
-- \\
--\end{array}$ & $\begin{array}{l}-- \\
-- \\
--\end{array}$ & $\begin{array}{l}1 \\
1 \\
1 \\
1 \\
2\end{array}$ & $\begin{array}{l}14 \\
15 \\
11 \\
28 \\
26\end{array}$ & $\begin{array}{l}28 \\
38 \\
40 \\
53 \\
61\end{array}$ & $\begin{array}{l}35 \\
47 \\
52 \\
58 \\
65\end{array}$ & $\begin{array}{l}54 \\
54 \\
63 \\
64 \\
70\end{array}$ & $\begin{array}{l}74 \\
67 \\
74 \\
71 \\
77\end{array}$ & $\begin{array}{l}90 \\
82 \\
83 \\
79 \\
84\end{array}$ & $\begin{array}{l}99 \\
95 \\
93 \\
91 \\
91\end{array}$ & $\begin{array}{l}100 \\
100 \\
100 \\
100 \\
100\end{array}$ & \\
\hline $\begin{array}{l}07-07-82 \\
07-13-82 \\
07-20-82 \\
07-27-82 \\
08-03-82\end{array}$ & $\begin{array}{l}-- \\
-- \\
-- \\
--\end{array}$ & $\begin{array}{l}-- \\
-- \\
-- \\
-\end{array}$ & $\begin{array}{r}1 \\
-- \\
1 \\
1 \\
1\end{array}$ & $\begin{array}{l}16 \\
11 \\
12 \\
15 \\
16\end{array}$ & $\begin{array}{l}43 \\
20 \\
35 \\
28 \\
38\end{array}$ & $\begin{array}{l}49 \\
24 \\
40 \\
35 \\
46\end{array}$ & $\begin{array}{l}58 \\
34 \\
45 \\
42 \\
53\end{array}$ & $\begin{array}{l}71 \\
50 \\
57 \\
53 \\
62\end{array}$ & $\begin{array}{l}84 \\
69 \\
67 \\
63 \\
75\end{array}$ & $\begin{array}{l}96 \\
88 \\
85 \\
84 \\
90\end{array}$ & $\begin{array}{r}100 \\
99 \\
100 \\
100 \\
98\end{array}$ & 100 \\
\hline $\begin{array}{l}08-11-82 \\
08-17-82 \\
08-24-82 \\
09-01-82 \\
09-18-82\end{array}$ & $\begin{array}{l}-- \\
-- \\
-- \\
--\end{array}$ & $\begin{array}{l}-- \\
-- \\
-- \\
--\end{array}$ & $\begin{array}{r}-- \\
1 \\
1 \\
1 \\
1\end{array}$ & $\begin{array}{l}13 \\
12 \\
12 \\
17 \\
22\end{array}$ & $\begin{array}{l}30 \\
39 \\
25 \\
40 \\
36\end{array}$ & $\begin{array}{l}35 \\
46 \\
29 \\
56 \\
41\end{array}$ & $\begin{array}{l}41 \\
54 \\
37 \\
64 \\
45\end{array}$ & $\begin{array}{l}51 \\
66 \\
52 \\
75 \\
53\end{array}$ & $\begin{array}{l}67 \\
80 \\
70 \\
86 \\
64\end{array}$ & $\begin{array}{l}90 \\
93 \\
91 \\
95 \\
82\end{array}$ & $\begin{array}{l}100 \\
100 \\
100 \\
100 \\
100\end{array}$ & \\
\hline $\begin{array}{l}05-19-83 \\
05-25-83 \\
05-31-83 \\
06-02-83 \\
06-09-83\end{array}$ & & $\begin{array}{l}0 \\
0\end{array}$ & $\begin{array}{l}1 \\
0 \\
2 \\
1 \\
0\end{array}$ & $\begin{array}{l}31 \\
12 \\
18 \\
20 \\
20\end{array}$ & $\begin{array}{l}70 \\
40 \\
38 \\
39 \\
42\end{array}$ & $\begin{array}{l}79 \\
60 \\
59 \\
51 \\
56\end{array}$ & $\begin{array}{l}79 \\
64 \\
60 \\
52 \\
57\end{array}$ & $\begin{array}{l}85 \\
78 \\
71 \\
66 \\
71\end{array}$ & $\begin{array}{l}93 \\
90 \\
82 \\
76 \\
86\end{array}$ & $\begin{array}{l}99 \\
99 \\
92 \\
87 \\
96\end{array}$ & $\begin{array}{l}100 \\
100 \\
100 \\
100 \\
100\end{array}$ & \\
\hline $\begin{array}{l}06-22-83 \\
07-06-83 \\
07-20-83 \\
08-02-83 \\
08-09-83\end{array}$ & & $\begin{array}{l}0 \\
0 \\
0 \\
0 \\
0\end{array}$ & $\begin{array}{l}1 \\
1 \\
1 \\
1 \\
1\end{array}$ & $\begin{array}{l}29 \\
21 \\
25 \\
21 \\
11\end{array}$ & $\begin{array}{l}50 \\
45 \\
51 \\
42 \\
19\end{array}$ & $\begin{array}{l}63 \\
57 \\
58 \\
46 \\
22\end{array}$ & $\begin{array}{l}64 \\
58 \\
61 \\
47 \\
27\end{array}$ & $\begin{array}{l}70 \\
67 \\
70 \\
53 \\
41\end{array}$ & $\begin{array}{l}78 \\
76 \\
79 \\
65 \\
63\end{array}$ & $\begin{array}{l}90 \\
88 \\
93 \\
80 \\
86\end{array}$ & $\begin{array}{r}100 \\
100 \\
100 \\
97 \\
100\end{array}$ & \\
\hline $\begin{array}{l}08-31-83 \\
09-13-83 \\
10-05-83 \\
05-18-84 \\
06-11-84\end{array}$ & & $\begin{array}{l}0 \\
0 \\
0\end{array}$ & $\begin{array}{l}1 \\
0 \\
1 \\
1 \\
1\end{array}$ & $\begin{array}{l}19 \\
31 \\
30 \\
26 \\
13\end{array}$ & $\begin{array}{l}30 \\
50 \\
52 \\
54 \\
42\end{array}$ & $\begin{array}{l}36 \\
71 \\
61 \\
63 \\
48\end{array}$ & $\begin{array}{l}41 \\
77 \\
68 \\
74 \\
55\end{array}$ & $\begin{array}{l}54 \\
87 \\
81 \\
84 \\
66\end{array}$ & $\begin{array}{l}69 \\
94 \\
90 \\
91 \\
83\end{array}$ & $\begin{array}{r}84 \\
100 \\
98 \\
98 \\
94\end{array}$ & $\begin{array}{l}100 \\
100 \\
100\end{array}$ & \\
\hline $\begin{array}{l}06-14-84 \\
07-11-84 \\
07-31-84 \\
08-17-84 \\
08-28-84\end{array}$ & & $\begin{array}{l}0 \\
0 \\
0 \\
0\end{array}$ & $\begin{array}{l}1 \\
1 \\
1 \\
1 \\
0\end{array}$ & $\begin{array}{r}12 \\
12 \\
12 \\
14 \\
8\end{array}$ & $\begin{array}{l}25 \\
35 \\
26 \\
37 \\
33\end{array}$ & $\begin{array}{l}31 \\
38 \\
31 \\
41 \\
37\end{array}$ & $\begin{array}{l}38 \\
42 \\
38 \\
47 \\
43\end{array}$ & $\begin{array}{l}47 \\
53 \\
54 \\
57 \\
55\end{array}$ & $\begin{array}{l}64 \\
69 \\
70 \\
73 \\
73\end{array}$ & $\begin{array}{l}90 \\
86 \\
88 \\
91 \\
92\end{array}$ & $\begin{array}{l}100 \\
100 \\
100 \\
100 \\
100\end{array}$ & \\
\hline $\begin{array}{l}09-14-84 \\
09-27-84 \\
05-31-85 \\
06-27-85 \\
07-24-85\end{array}$ & & $\begin{array}{l}0 \\
0 \\
0\end{array}$ & $\begin{array}{l}1 \\
0 \\
1 \\
1 \\
1\end{array}$ & $\begin{array}{l}24 \\
26 \\
12 \\
15 \\
13\end{array}$ & $\begin{array}{l}61 \\
74 \\
35 \\
33 \\
31\end{array}$ & $\begin{array}{l}71 \\
87 \\
42 \\
37 \\
39\end{array}$ & $\begin{array}{l}78 \\
94 \\
53 \\
43 \\
46\end{array}$ & $\begin{array}{l}84 \\
97 \\
71 \\
50 \\
55\end{array}$ & $\begin{array}{l}92 \\
99 \\
82 \\
65 \\
71\end{array}$ & $\begin{array}{r}98 \\
100 \\
95 \\
83 \\
92\end{array}$ & $\begin{array}{r}100 \\
98 \\
100\end{array}$ & 100 \\
\hline $\begin{array}{l}08-16-85 \\
09-05-85 \\
09-17-85\end{array}$ & & $\begin{array}{l}0 \\
0 \\
0\end{array}$ & $\begin{array}{l}1 \\
1 \\
1\end{array}$ & $\begin{array}{l}12 \\
20 \\
18\end{array}$ & $\begin{array}{l}24 \\
50 \\
45\end{array}$ & $\begin{array}{l}27 \\
66 \\
51\end{array}$ & $\begin{array}{l}34 \\
73 \\
56\end{array}$ & $\begin{array}{l}46 \\
79 \\
63\end{array}$ & $\begin{array}{l}61 \\
88 \\
76\end{array}$ & $\begin{array}{l}80 \\
97 \\
92\end{array}$ & $\begin{array}{l}100 \\
100 \\
100\end{array}$ & \\
\hline
\end{tabular}


Table 3.--Bedload particle-size distributions--Continued

Date

Percent by weight finer than size (millimeters) indicated

\begin{tabular}{llllllllllll}
\hline 0.062 & 0.12 & 0.25 & 0.5 & 1 & 2 & 4 & 8 & 16 & 32 & 64 & 128
\end{tabular}

\section{Yentna River near Susitna Station, Alaska}

$05-14-84$

$06-12-84$

07-17-84

$09-19-84$

$05-23-85$

06-20-85

$07-17-85$

$08-14-85$

$08-19-85$

09-18-85
$06-12-84$

$07-10-84$

07-30-84

$08-27-84$

09-12-84

09-26-84

05-30-85

$06-25-85$

$07-25-85$

08-15-85

09-04-85

09-18-85
0

0
0
0
0
1

0
0
0
0

5
5
2
2
14
3
3
2
3

$\begin{array}{lll}65 & 77 & 8 \\ 46 & 79 & 8 \\ 43 & 88 & 9 \\ 48 & 82 & 85 \\ 66 & 82 & 85\end{array}$

$\begin{array}{ll}84 & 90 \\ 84 & 88 \\ 91 & 94 \\ 85 & 90 \\ 85 & 86\end{array}$

$\begin{array}{rrrr}96 & 100 & & \\ 92 & 96 & 100 & \\ 96 & 99 & 100 & \\ 93 & 97 & 100 & \\ 88 & 94 & 99 & 100\end{array}$

5. Susitna River (Right Channel) below Chulitna River near Talkeetna, Alaska

$\begin{array}{rrrrrrr}70 & 91 & 94 & 96 & 98 & 99 & 100 \\ 60 & 90 & 93 & 95 & 98 & 99 & 100 \\ 47 & 84 & 88 & 92 & 96 & 99 & 100 \\ 57 & 92 & 96 & 97 & 99 & 100 & \\ 47 & 84 & 91 & 95 & 98 & 100 & \end{array}$

\begin{tabular}{|c|c|c|c|c|c|c|c|c|c|}
\hline & 0 & 9 & 20 & 22 & 25 & 33 & 50 & 76 & 100 \\
\hline 0 & 1 & 25 & 52 & 57 & 61 & 67 & 75 & 88 & 100 \\
\hline \multirow[t]{7}{*}{0} & 1 & 20 & 40 & 46 & 55 & 63 & 72 & 85 & 100 \\
\hline & 0 & 6 & 16 & 19 & 25 & 38 & 58 & 83 & 99 \\
\hline & 0 & 32 & 62 & 64 & 67 & 73 & 80 & 92 & 100 \\
\hline & 0 & 27 & 61 & 63 & 67 & 72 & 80 & 90 & 100 \\
\hline & 0 & 9 & 18 & 21 & 25 & 40 & 67 & 92 & 100 \\
\hline & 0 & 7 & 11 & 12 & 15 & 22 & 40 & 75 & 100 \\
\hline & 0 & 13 & 34 & 38 & 43 & 53 & 71 & 93 & 100 \\
\hline 0 & 1 & 22 & 41 & 45 & 48 & 55 & 70 & 87 & 100 \\
\hline 0 & 1 & 45 & 79 & 83 & 86 & 89 & 92 & 94 & 100 \\
\hline 0 & 1 & 25 & 43 & 46 & 52 & 59 & 72 & 96 & 100 \\
\hline
\end{tabular}

6. Susitna River (Left Channel) below Chulitna River near Talkeetna, Alaska

\begin{tabular}{|c|c|c|c|c|c|c|c|c|c|c|}
\hline $\begin{array}{l}06-12-84 \\
07-10-84 \\
07-29-84 \\
09-12-84 \\
09-26-84\end{array}$ & & $\begin{array}{l}0 \\
0 \\
0\end{array}$ & $\begin{array}{l}1 \\
1 \\
4 \\
0 \\
1\end{array}$ & $\begin{array}{l}44 \\
64 \\
77 \\
52 \\
46\end{array}$ & $\begin{array}{l}81 \\
94 \\
94 \\
72 \\
82\end{array}$ & $\begin{array}{l}83 \\
94 \\
95 \\
73 \\
83\end{array}$ & $\begin{array}{l}84 \\
95 \\
95 \\
74 \\
85\end{array}$ & $\begin{array}{l}86 \\
95 \\
96 \\
75 \\
85\end{array}$ & $\begin{array}{l}89 \\
97 \\
97 \\
80 \\
90\end{array}$ & $\begin{array}{l}100 \\
100 \\
100 \\
100 \\
100\end{array}$ \\
\hline $\begin{array}{l}05-30-85 \\
06-25-85 \\
07-25-85 \\
08-15-85 \\
09-04-85 \\
09-18-85\end{array}$ & 0 & $\begin{array}{l}1 \\
0 \\
0 \\
0 \\
0 \\
0\end{array}$ & $\begin{array}{l}5 \\
6 \\
2 \\
2 \\
1 \\
1\end{array}$ & $\begin{array}{l}59 \\
80 \\
62 \\
58 \\
63 \\
75\end{array}$ & $\begin{array}{l}76 \\
90 \\
78 \\
74 \\
84 \\
85\end{array}$ & $\begin{array}{l}78 \\
90 \\
79 \\
75 \\
86 \\
85\end{array}$ & $\begin{array}{l}80 \\
92 \\
80 \\
76 \\
88 \\
86\end{array}$ & $\begin{array}{l}82 \\
92 \\
82 \\
77 \\
90 \\
87\end{array}$ & $\begin{array}{l}88 \\
93 \\
86 \\
80 \\
98 \\
88\end{array}$ & $\begin{array}{r}93 \\
96 \\
96 \\
85 \\
100 \\
97\end{array}$ \\
\hline
\end{tabular}

\section{Susitna River at Sunshine, Alaska}

\begin{tabular}{|c|c|c|c|c|c|c|c|c|c|c|c|c|}
\hline $06-03-82$ & - & -- & 2 & 15 & 22 & 26 & 27 & 30 & 38 & 64 & 100 & \\
\hline $06-10-82$ & - & -- & 2 & 12 & 17 & 17 & 18 & 20 & 29 & 54 & 96 & 100 \\
\hline $06-17-82$ & -- & -- & 2 & 47 & 65 & 65 & 66 & 66 & 69 & 75 & 100 & \\
\hline $06-21-82$ & - & 1 & 12 & 45 & 50 & 51 & 53 & 57 & 62 & 70 & 95 & 100 \\
\hline $06-28-82$ & - & -- & 3 & 17 & 22 & 23 & 25 & 32 & 46 & 64 & 100 & \\
\hline
\end{tabular}


Table 3.--Bedload particle-size distributions--Continued

Date

Percent by weight finer than size (millimeters) indicated

$\begin{array}{lllllllllllll}0.062 & 0.12 & 0.25 & 0.5 & 1 & 2 & 4 & 8 & 16 & 32 & 64 & 128\end{array}$

\section{Susitna River at Sunshine, Alaska--Continued}

\begin{tabular}{|c|c|c|c|c|c|c|c|c|c|c|c|c|}
\hline $\begin{array}{l}07-06-82 \\
07-12-82 \\
07-19-82 \\
07-26-82 \\
08-02-82\end{array}$ & $\begin{array}{l}-- \\
-- \\
-- \\
--\end{array}$ & $\begin{array}{l}-- \\
-- \\
-- \\
--\end{array}$ & $\begin{array}{l}2 \\
3 \\
2 \\
2 \\
4\end{array}$ & $\begin{array}{l}35 \\
52 \\
40 \\
18 \\
60\end{array}$ & $\begin{array}{l}46 \\
75 \\
54 \\
28 \\
73\end{array}$ & $\begin{array}{l}47 \\
77 \\
58 \\
30 \\
74\end{array}$ & $\begin{array}{l}49 \\
80 \\
62 \\
33 \\
74\end{array}$ & $\begin{array}{l}57 \\
83 \\
69 \\
39 \\
75\end{array}$ & $\begin{array}{l}71 \\
88 \\
75 \\
53 \\
78\end{array}$ & $\begin{array}{l}86 \\
96 \\
84 \\
77 \\
93\end{array}$ & $\begin{array}{r}100 \\
100 \\
87 \\
97 \\
97\end{array}$ & $\begin{array}{l}100 \\
100 \\
100\end{array}$ \\
\hline $\begin{array}{l}08-09-82 \\
08-16-82 \\
08-23-82 \\
08-30-82 \\
09-17-82\end{array}$ & $\begin{array}{r}1 \\
-- \\
-- \\
1 \\
--\end{array}$ & $\begin{array}{r}1 \\
-- \\
-- \\
--\end{array}$ & $\begin{array}{l}5 \\
2 \\
1 \\
4 \\
1\end{array}$ & $\begin{array}{l}62 \\
61 \\
55 \\
44 \\
12\end{array}$ & $\begin{array}{l}81 \\
83 \\
85 \\
63 \\
20\end{array}$ & $\begin{array}{l}82 \\
84 \\
88 \\
64 \\
23\end{array}$ & $\begin{array}{l}83 \\
85 \\
89 \\
64 \\
26\end{array}$ & $\begin{array}{l}85 \\
86 \\
90 \\
65 \\
37\end{array}$ & $\begin{array}{l}89 \\
92 \\
92 \\
66 \\
60\end{array}$ & $\begin{array}{l}94 \\
98 \\
92 \\
70 \\
78\end{array}$ & $\begin{array}{l}100 \\
100 \\
100 \\
100 \\
100\end{array}$ & \\
\hline $\begin{array}{l}05-18-83 \\
05-24-83 \\
06-01-83 \\
06-08-83 \\
06-23-83\end{array}$ & & $\begin{array}{l}0 \\
0 \\
0 \\
0 \\
0\end{array}$ & $\begin{array}{l}1 \\
1 \\
1 \\
1 \\
1\end{array}$ & $\begin{array}{l}18 \\
33 \\
19 \\
27 \\
27\end{array}$ & $\begin{array}{l}31 \\
54 \\
26 \\
38 \\
39\end{array}$ & $\begin{array}{l}36 \\
57 \\
28 \\
44 \\
45\end{array}$ & $\begin{array}{l}36 \\
57 \\
29 \\
44 \\
46\end{array}$ & $\begin{array}{l}42 \\
60 \\
34 \\
51 \\
56\end{array}$ & $\begin{array}{l}53 \\
68 \\
49 \\
62 \\
75\end{array}$ & $\begin{array}{l}75 \\
83 \\
75 \\
80 \\
93\end{array}$ & $\begin{array}{r}100 \\
98 \\
96 \\
100 \\
100\end{array}$ & $\begin{array}{l}100 \\
100\end{array}$ \\
\hline $\begin{array}{l}07-05-83 \\
07-19-83 \\
08-01-83 \\
08-03-83 \\
08-08-83\end{array}$ & & $\begin{array}{l}0 \\
0 \\
0 \\
0 \\
0\end{array}$ & $\begin{array}{l}9 \\
1 \\
2 \\
1 \\
2\end{array}$ & $\begin{array}{l}14 \\
46 \\
31 \\
67 \\
26\end{array}$ & $\begin{array}{l}17 \\
66 \\
44 \\
86 \\
34\end{array}$ & $\begin{array}{l}17 \\
70 \\
48 \\
89 \\
37\end{array}$ & $\begin{array}{l}27 \\
73 \\
52 \\
92 \\
42\end{array}$ & $\begin{array}{l}53 \\
77 \\
60 \\
95 \\
50\end{array}$ & $\begin{array}{l}68 \\
85 \\
72 \\
98 \\
59\end{array}$ & $\begin{array}{r}89 \\
96 \\
89 \\
100 \\
82\end{array}$ & $\begin{array}{l}100 \\
100 \\
100 \\
100\end{array}$ & \\
\hline $\begin{array}{l}08-29-83 \\
09-12-83 \\
10-04-83 \\
05-16-84 \\
06-14-84\end{array}$ & 0 & $\begin{array}{l}0 \\
0 \\
1\end{array}$ & $\begin{array}{l}1 \\
0 \\
1 \\
2 \\
4\end{array}$ & $\begin{array}{l}37 \\
31 \\
40 \\
46 \\
45\end{array}$ & $\begin{array}{l}48 \\
44 \\
66 \\
80 \\
58\end{array}$ & $\begin{array}{l}52 \\
49 \\
71 \\
82 \\
60\end{array}$ & $\begin{array}{l}56 \\
52 \\
72 \\
83 \\
61\end{array}$ & $\begin{array}{l}64 \\
63 \\
77 \\
86 \\
65\end{array}$ & $\begin{array}{l}73 \\
78 \\
85 \\
90 \\
72\end{array}$ & $\begin{array}{l}89 \\
93 \\
96 \\
96 \\
84\end{array}$ & $\begin{array}{r}96 \\
100 \\
100 \\
100 \\
100\end{array}$ & 100 \\
\hline $\begin{array}{l}07-13-84 \\
07-28-84 \\
08-14-84 \\
09-11-84 \\
09-21-84\end{array}$ & & $\begin{array}{l}0 \\
0 \\
0 \\
0 \\
0\end{array}$ & $\begin{array}{l}1 \\
1 \\
2 \\
1 \\
2\end{array}$ & $\begin{array}{l}28 \\
32 \\
33 \\
36 \\
28\end{array}$ & $\begin{array}{l}55 \\
41 \\
56 \\
56 \\
45\end{array}$ & $\begin{array}{l}58 \\
43 \\
58 \\
58 \\
47\end{array}$ & $\begin{array}{l}60 \\
45 \\
60 \\
62 \\
53\end{array}$ & $\begin{array}{l}65 \\
52 \\
63 \\
71 \\
64\end{array}$ & $\begin{array}{l}71 \\
65 \\
72 \\
87 \\
78\end{array}$ & $\begin{array}{l}82 \\
79 \\
88 \\
98 \\
92\end{array}$ & $\begin{array}{l}100 \\
100 \\
100 \\
100 \\
100\end{array}$ & \\
\hline $\begin{array}{l}09-28-84 \\
05-31-85 \\
06-25-85 \\
07-23-85 \\
08-12-85\end{array}$ & $\begin{array}{l}0 \\
0\end{array}$ & $\begin{array}{l}0 \\
0 \\
0 \\
1 \\
1\end{array}$ & $\begin{array}{l}1 \\
3 \\
1 \\
6 \\
2\end{array}$ & $\begin{array}{l}59 \\
22 \\
44 \\
72 \\
48\end{array}$ & $\begin{array}{l}90 \\
30 \\
70 \\
87 \\
60\end{array}$ & $\begin{array}{l}91 \\
31 \\
71 \\
88 \\
62\end{array}$ & $\begin{array}{l}92 \\
34 \\
73 \\
89 \\
64\end{array}$ & $\begin{array}{l}94 \\
52 \\
75 \\
90 \\
69\end{array}$ & $\begin{array}{l}97 \\
72 \\
80 \\
92 \\
81\end{array}$ & $\begin{array}{r}100 \\
90 \\
92 \\
95 \\
99\end{array}$ & $\begin{array}{l}100 \\
100 \\
100 \\
100\end{array}$ & \\
\hline $\begin{array}{l}09-03-85 \\
09-16-85\end{array}$ & & $\begin{array}{l}0 \\
0\end{array}$ & $\begin{array}{l}2 \\
1\end{array}$ & $\begin{array}{l}52 \\
27\end{array}$ & $\begin{array}{l}68 \\
40\end{array}$ & $\begin{array}{l}70 \\
44\end{array}$ & $\begin{array}{l}72 \\
48\end{array}$ & $\begin{array}{l}76 \\
57\end{array}$ & $\begin{array}{l}87 \\
78\end{array}$ & $\begin{array}{l}97 \\
92\end{array}$ & $\begin{array}{l}100 \\
100\end{array}$ & \\
\hline
\end{tabular}

8. Tanana River at Upper End of Goose Island, Alaska

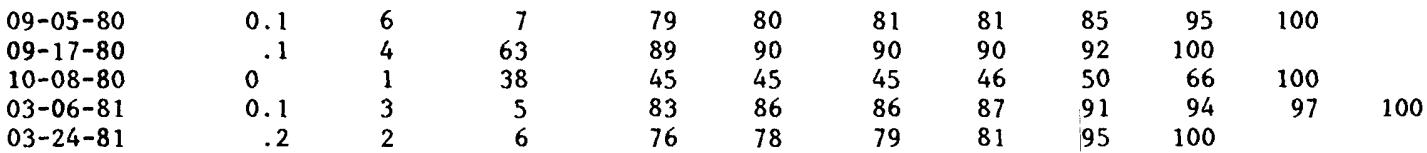


Table 3.--Bedload particle-size distributions--Continued

Percent by weight finer than size (millimeters) indicated

Date

\begin{tabular}{llllllllllll}
\hline 0.062 & 0.12 & 0.25 & 0.5 & 1 & 2 & 4 & 8 & 16 & 32 & 64 & 128
\end{tabular}

8. Tanana River at Upper End of Goose Island, Alaska--Continued

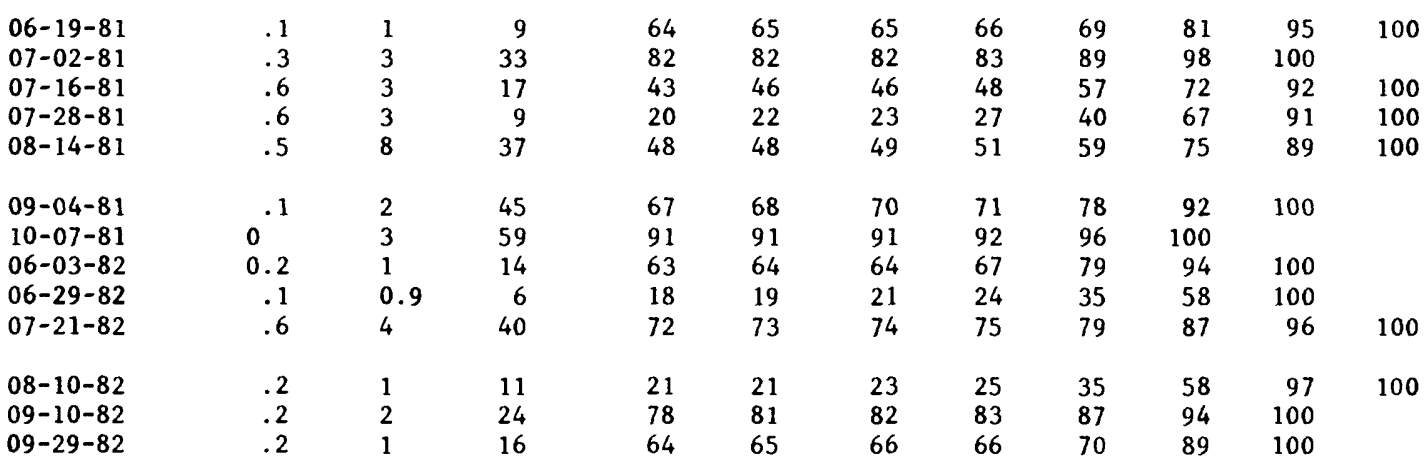

9. Tanana River at Fairbanks, Alaska

\begin{tabular}{|c|c|c|c|c|c|c|c|c|c|c|c|}
\hline $06-07-77$ & 0.5 & 3 & 16 & 35 & 35 & 35 & 36 & 47 & 77 & 99 & 100 \\
\hline $06-29-77$ & .7 & 2 & 13 & 25 & 25 & 26 & 27 & 42 & 74 & 95 & 100 \\
\hline $07-06-77$ & .1 & 0.4 & 5 & 22 & 40 & 30 & 31 & 35 & 45 & 84 & 98 \\
\hline $07-12-77$ & .5 & 2 & 21 & 48 & 49 & 50 & 52 & 60 & 74 & 94 & 100 \\
\hline $07-20-77$ & .5 & 2 & 10 & 25 & 27 & 28 & 29 & 35 & 50 & 70 & 99 \\
\hline $08-03-77$ & .4 & 1 & 8 & 25 & 25 & 25 & 27 & 32 & 55 & 83 & 100 \\
\hline $08-11-77$ & 1 & 3 & 15 & 33 & 34 & 35 & 35 & 41 & 48 & 82 & 96 \\
\hline $08-18-77$ & 0.9 & 2 & 30 & 54 & 61 & 61 & 63 & 71 & 88 & 98 & 100 \\
\hline $08-31-77$ & .7 & 2 & 33 & 66 & 67 & 67 & 68 & 71 & 81 & 99 & 100 \\
\hline $10-03-77$ & 0.3 & 8 & 33 & 97 & 100 & & & & & & \\
\hline $05-18-78$ & 1 & 9 & 72 & 91 & 92 & 92 & 92 & 96 & 99 & 100 & \\
\hline $05-30-78$ & 2 & 9 & 72 & 89 & 89 & 89 & 90 & 90 & 93 & 100 & \\
\hline $06-20-78$ & 2 & 7 & 48 & 78 & 79 & 79 & 80 & 90 & 100 & & \\
\hline $07-10-78$ & 5 & 19 & 74 & 89 & 89 & 89 & 90 & 91 & 93 & 93 & 100 \\
\hline $07-17-78$ & 2 & 7 & 32 & 82 & 84 & 84 & 86 & 88 & 92 & 99 & 100 \\
\hline $07-31-78$ & 1 & 4 & 37 & 84 & 85 & 85 & 86 & 89 & 95 & 98 & 100 \\
\hline $08-08-78$ & 2 & 8 & 45 & 77 & 80 & 81 & 82 & 85 & 91 & 100 & \\
\hline $08-14-78$ & 1 & 4 & 26 & 53 & 53 & 54 & 55 & 60 & 68 & 80 & 100 \\
\hline $08-25-78$ & 0.5 & 3 & 30 & 85 & 86 & 86 & 87 & 89 & 93 & 98 & 100 \\
\hline $09-07-78$ & .4 & 3 & 32 & 84 & 85 & 86 & 86 & 89 & 94 & 100 & \\
\hline $10-04-78$ & .2 & 2 & 39 & 57 & 57 & 58 & 59 & 61 & 64 & 71 & 100 \\
\hline $05-23-79$ & 1 & 9 & 46 & 83 & 83 & 84 & 84 & 88 & 96 & 100 & \\
\hline $06-18-79$ & 1 & 6 & 34 & 96 & 97 & 97 & 98 & 98 & 100 & & \\
\hline $07-10-79$ & 0.4 & 1 & 8 & 79 & 80 & 80 & 81 & 84 & 89 & 96 & 100 \\
\hline $07-24-79$ & 1 & 5 & 19 & 46 & 46 & 47 & 49 & 59 & 84 & 96 & 100 \\
\hline $08-01-79$ & 1 & 4 & 15 & 60 & 61 & 61 & 62 & 68 & 82 & 100 & \\
\hline $08-08-79$ & 0.9 & 3 & 13 & 34 & 37 & 39 & 44 & 58 & 81 & 99 & 100 \\
\hline $08-29-79$ & .4 & 2 & 8 & 64 & 65 & 65 & 67 & 73 & 86 & 100 & \\
\hline $09-06-79$ & .1 & 1 & 9 & 54 & 55 & 56 & 57 & 65 & 86 & 100 & \\
\hline $09-12-79$ & .2 & 2 & 17 & 89 & 89 & 90 & 90 & 92 & 97 & 100 & \\
\hline
\end{tabular}


Table 3.--Bedload particle-size distributions--Continued

Percent by weight finer than size (millimeters) indicated

Date

\begin{tabular}{llllllllllll}
\hline 0.062 & 0.12 & 0.25 & 0.5 & 1 & 2 & 4 & 8 & 16 & 32 & 64 & 128
\end{tabular}

\section{Tanana River at Fairbanks, Alaska--Continued}

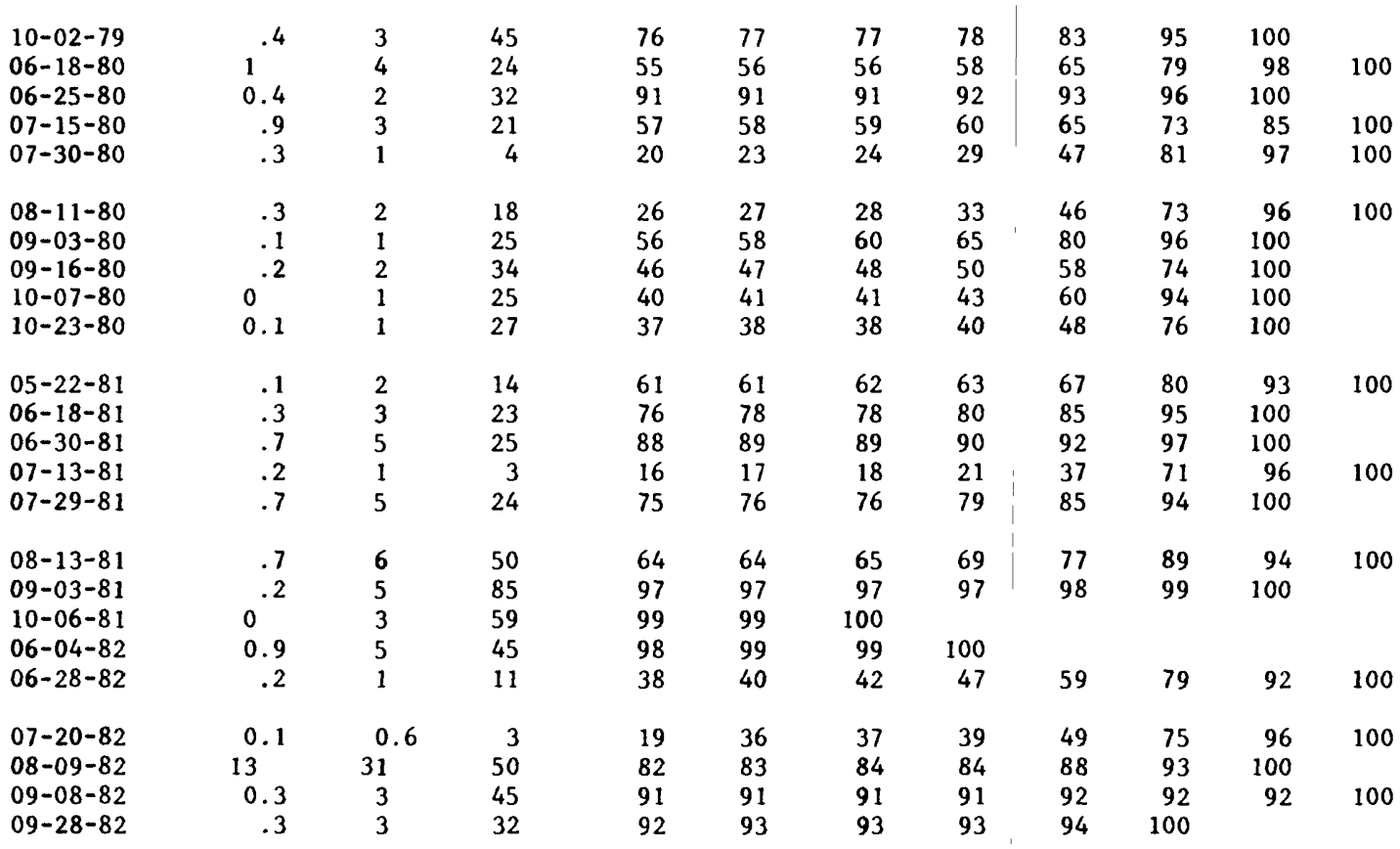

10. Snake River near Anatone, Wash.

\begin{tabular}{|c|c|c|c|c|c|c|c|c|c|c|c|c|}
\hline $\begin{array}{l}05-10-72 \\
05-19-72 \\
06-02-72 \\
06-14-72 \\
05-01-73\end{array}$ & $\begin{array}{l}-- \\
0 \\
0.1 \\
.2 \\
0\end{array}$ & $\begin{array}{r}0.2 \\
.3 \\
.8 \\
.9 \\
.2\end{array}$ & $\begin{array}{l}5 \\
6 \\
8 \\
8 \\
9\end{array}$ & $\begin{array}{l}41 \\
54 \\
50 \\
51 \\
60\end{array}$ & $\begin{array}{l}61 \\
86 \\
71 \\
94 \\
98\end{array}$ & $\begin{array}{r}69 \\
90 \\
75 \\
99 \\
100\end{array}$ & $\begin{array}{r}74 \\
94 \\
77 \\
100\end{array}$ & $\begin{array}{l}81 \\
97 \\
77\end{array}$ & $\begin{array}{c}100 \\
100 \\
78\end{array}$ & 81 & 100 & \\
\hline $\begin{array}{l}05-17-73 \\
06-18-73 \\
04-11-74 \\
04-24-74 \\
05-07-74\end{array}$ & $\begin{array}{l}0 \\
0.1 \\
0 \\
0 \\
0\end{array}$ & $\begin{array}{l}.2 \\
.3 \\
0 \\
0.1 \\
.1\end{array}$ & $\begin{array}{l}6 \\
4 \\
0.5 \\
1 \\
2\end{array}$ & $\begin{array}{r}44 \\
37 \\
6 \\
13 \\
13\end{array}$ & $\begin{array}{l}75 \\
94 \\
10 \\
30 \\
22\end{array}$ & $\begin{array}{l}79 \\
99 \\
14 \\
35 \\
25\end{array}$ & $\begin{array}{l}80 \\
99 \\
17 \\
38 \\
29\end{array}$ & $\begin{array}{r}80 \\
100 \\
21 \\
42 \\
32\end{array}$ & $\begin{array}{l}28 \\
44 \\
35\end{array}$ & $\begin{array}{l}43 \\
49 \\
40\end{array}$ & $\begin{array}{l}74 \\
61 \\
58\end{array}$ & $\begin{array}{l}100 \\
100 \\
100\end{array}$ \\
\hline $\begin{array}{l}05-16-74 \\
06-11-74 \\
04-15-75 \\
05-01-75^{2} \\
05-14-75^{2}\end{array}$ & $\begin{array}{l}0 \\
0.1 \\
0 \\
0.1 \\
0.1\end{array}$ & $\begin{array}{l}.1 \\
.4 \\
.1 \\
.1 \\
.2\end{array}$ & $\begin{array}{l}3 \\
9 \\
5 \\
3 \\
4\end{array}$ & $\begin{array}{l}28 \\
41 \\
51 \\
38 \\
35\end{array}$ & $\begin{array}{l}64 \\
71 \\
95 \\
58 \\
55\end{array}$ & $\begin{array}{l}69 \\
77 \\
98 \\
62 \\
59\end{array}$ & $\begin{array}{l}72 \\
80 \\
99 \\
62 \\
59\end{array}$ & $\begin{array}{l}75 \\
84 \\
99 \\
63 \\
60\end{array}$ & $\begin{array}{r}79 \\
89 \\
100 \\
67 \\
62\end{array}$ & $\begin{array}{l}82 \\
70\end{array}$ & $\begin{array}{l}100 \\
100\end{array}$ & $\begin{array}{l}100 \\
100\end{array}$ \\
\hline $\begin{array}{l}05-20-75^{2} \\
06-11-75 \\
06-26-75 \\
04-07-76 \\
04-13-76\end{array}$ & $\begin{array}{l}0 \\
0.2 \\
.2 \\
0 \\
0\end{array}$ & $\begin{array}{l}.1 \\
.9 \\
.6 \\
.1 \\
.1\end{array}$ & $\begin{array}{r}3 \\
11 \\
12 \\
3 \\
4\end{array}$ & $\begin{array}{l}25 \\
66 \\
65 \\
49 \\
34\end{array}$ & $\begin{array}{l}36 \\
93 \\
84 \\
74 \\
42\end{array}$ & $\begin{array}{l}38 \\
97 \\
87 \\
78 \\
43\end{array}$ & $\begin{array}{l}39 \\
99 \\
88 \\
81 \\
44\end{array}$ & $\begin{array}{l}42 \\
99 \\
89 \\
81 \\
45\end{array}$ & $\begin{array}{r}46 \\
100 \\
92 \\
82 \\
46\end{array}$ & $\begin{array}{l}95 \\
89 \\
52\end{array}$ & $\begin{array}{r}100 \\
100 \\
71\end{array}$ & 100 \\
\hline
\end{tabular}


Table 3.--Bedload particle-size distributions--Continued

Date

Percent by weight finer than size (millimeters) indicated

\begin{tabular}{lllllllllllll}
\hline 0.062 & 0.12 & 0.25 & 0.5 & 1 & 2 & 4 & 8 & 16 & 32 & 64 & 128
\end{tabular}

\section{Snake River near Anatone, Wash.--Continued}

\begin{tabular}{|c|c|c|c|c|c|c|c|c|c|c|c|c|}
\hline $04-15-76$ & 0 & .1 & 1 & 9 & 14 & 14 & 14 & 14 & 30 & 65 & 87 & 100 \\
\hline $04-19-76$ & 0 & 0 & 0.6 & 8 & 12 & 13 & 14 & 14 & 24 & 55 & 91 & 100 \\
\hline $04-21-76$ & 0 & 0 & 1 & 17 & 27 & 29 & 31 & 31 & 33 & 49 & 85 & 100 \\
\hline $05-05-76$ & 0 & 0.1 & 5 & 48 & 65 & 70 & 76 & 76 & 93 & 95 & 100 & \\
\hline $05-13-76^{2}$ & 0.2 & .4 & 7 & 43 & 61 & 63 & 65 & 65 & 67 & 70 & 72 & 100 \\
\hline $05-26-76$ & .1 & .3 & 6 & 43 & 63 & 72 & 77 & 77 & 83 & 88 & 89 & 100 \\
\hline $05-27-76$ & 0 & .2 & 7 & 47 & 64 & 69 & 73 & 73 & 82 & 92 & 100 & \\
\hline $06-08-76$ & 0.2 & .6 & 5 & 28 & 53 & 55 & 55 & 55 & 62 & 65 & 67 & 100 \\
\hline $06-10-76$ & .1 & .4 & 7 & 34 & 70 & 75 & 81 & 81 & 92 & 100 & & \\
\hline $04-04-78^{2}$ & 0 & .1 & 1 & 14 & 18 & 20 & 21 & 21 & 29 & 57 & 86 & 100 \\
\hline $04-05-78^{2}$ & 0.1 & .1 & 0.8 & 10 & 16 & 17 & 18 & 20 & 29 & 51 & 78 & 100 \\
\hline $04-29-78^{2}$ & 0.1 & .2 & 2 & 13 & 18 & 20 & 21 & 23 & 28 & 41 & 67 & 100 \\
\hline $05-02-78^{2}$ & 0 & .1 & 1 & 15 & 31 & 32 & 33 & 33 & 36 & 49 & 69 & 100 \\
\hline $05-03-78^{2}$ & 0.1 & .1 & 2 & 23 & 33 & 35 & 37 & 38 & 42 & 51 & 72 & 100 \\
\hline $05-16-78^{2}$ & 0.1 & 0.3 & 4 & 25 & 47 & 49 & 50 & 51 & 52 & 56 & 73 & 100 \\
\hline $05-17-78^{2}$ & 0.1 & .2 & 2 & 18 & 27 & 33 & 38 & 41 & 43 & 55 & 72 & 100 \\
\hline $06-07-78$ & .4 & 2 & 13 & 70 & 82 & 84 & 84 & 84 & 84 & 84 & 100 & \\
\hline $06-13-78$ & .3 & 0.8 & 6 & 61 & 89 & 93 & 96 & 98 & 100 & & & \\
\hline $06-20-78^{2}$ & 0.1 & .2 & 2 & 32 & 63 & 69 & 73 & 76 & 79 & 84 & 90 & 100 \\
\hline $05-02-79^{2}$ & 0.1 & .3 & 6 & 51 & 64 & 66 & 67 & 67 & 68 & 78 & 83 & 100 \\
\hline $05-15-79$ & .1 & .2 & 8 & 71 & 90 & 94 & 97 & 100 & & & & \\
\hline $05-22-79$ & .1 & .2 & 3 & 38 & 77 & 78 & 79 & 79 & 79 & 84 & 89 & 100 \\
\hline $05-24-79$ & .2 & .7 & 8 & 69 & 95 & 97 & 98 & 100 & & & & \\
\hline $06-05-79^{2}$ & 0 & .2 & 4 & 53 & 74 & 76 & 77 & 78 & 78 & 90 & 94 & 100 \\
\hline $06-07-79$ & 0 & .1 & 3 & 44 & 89 & 95 & 97 & 98 & 100 & & & \\
\hline
\end{tabular}

11. Toutle River at Tower Road near Silver Lake, Wash. ${ }^{3}$

\begin{tabular}{|c|c|c|c|c|c|c|c|c|c|c|c|c|}
\hline $\begin{array}{l}01-17-85 \\
01-31-85 \\
01-31-85 \\
01-31-85 \\
02-21-85\end{array}$ & $\begin{array}{r}0.2 \\
.1 \\
.2 \\
.1 \\
13\end{array}$ & $\begin{array}{r}0.9 \\
.2 \\
.4 \\
.6 \\
13\end{array}$ & $\begin{array}{r}5 \\
4 \\
4 \\
7 \\
14\end{array}$ & $\begin{array}{l}25 \\
25 \\
23 \\
31 \\
19\end{array}$ & $\begin{array}{l}47 \\
49 \\
53 \\
55 \\
28\end{array}$ & $\begin{array}{l}58 \\
60 \\
64 \\
76 \\
41\end{array}$ & $\begin{array}{l}65 \\
65 \\
71 \\
85 \\
51\end{array}$ & $\begin{array}{l}71 \\
74 \\
79 \\
91 \\
64\end{array}$ & $\begin{array}{l}83 \\
88 \\
89 \\
97 \\
87\end{array}$ & $\begin{array}{r}95 \\
96 \\
100 \\
100 \\
95\end{array}$ & $\begin{array}{l}100 \\
100\end{array}$ & \\
\hline $\begin{array}{l}02-21-85 \\
02-21-85 \\
02-21-85 \\
03-06-85 \\
03-06-85\end{array}$ & $\begin{array}{l}0.1 \\
.1 \\
0 \\
0.3 \\
.5\end{array}$ & $\begin{array}{r}0.3 \\
.2 \\
.2 \\
1 \\
2\end{array}$ & $\begin{array}{r}4 \\
3 \\
16 \\
8 \\
11\end{array}$ & $\begin{array}{l}25 \\
19 \\
64 \\
26 \\
41\end{array}$ & $\begin{array}{l}47 \\
38 \\
79 \\
40 \\
60\end{array}$ & $\begin{array}{l}60 \\
49 \\
82 \\
48 \\
68\end{array}$ & $\begin{array}{l}67 \\
55 \\
84 \\
53 \\
71\end{array}$ & $\begin{array}{l}75 \\
62 \\
86 \\
62 \\
78\end{array}$ & $\begin{array}{l}84 \\
73 \\
90 \\
78 \\
88\end{array}$ & $\begin{array}{l}95 \\
88 \\
97 \\
91 \\
96\end{array}$ & $\begin{array}{l}100 \\
100 \\
100 \\
100 \\
100\end{array}$ & \\
\hline $\begin{array}{l}03-23-85 \\
03-23-85 \\
04-02-85 \\
04-02-85 \\
04-02-85\end{array}$ & $\begin{array}{l}1 \\
1 \\
0.1 \\
.2 \\
.5\end{array}$ & $\begin{array}{l}6 \\
4 \\
0.4 \\
2 \\
6\end{array}$ & $\begin{array}{r}25 \\
19 \\
2 \\
20 \\
37\end{array}$ & $\begin{array}{l}51 \\
43 \\
13 \\
95 \\
89\end{array}$ & $\begin{array}{l}64 \\
66 \\
22 \\
99 \\
94\end{array}$ & $\begin{array}{r}72 \\
77 \\
29 \\
100 \\
96\end{array}$ & $\begin{array}{l}76 \\
81 \\
37\end{array}$ & $\begin{array}{l}83 \\
85 \\
50\end{array}$ & $\begin{array}{l}89 \\
90 \\
67 \\
98\end{array}$ & $\begin{array}{l}95 \\
96 \\
86\end{array}$ & $\begin{array}{r}96 \\
100 \\
100\end{array}$ & 100 \\
\hline $\begin{array}{l}04-02-85 \\
04-11-85 \\
04-11-85 \\
04-30-85 \\
04-30-85\end{array}$ & $\begin{array}{l}.2 \\
.4 \\
0.1 \\
.1\end{array}$ & $\begin{array}{l}0.7 \\
1 \\
8 \\
0.5 \\
.3\end{array}$ & $\begin{array}{r}5 \\
6 \\
30 \\
3 \\
3\end{array}$ & $\begin{array}{l}23 \\
25 \\
75 \\
21 \\
16\end{array}$ & $\begin{array}{l}41 \\
42 \\
84 \\
38 \\
32\end{array}$ & $\begin{array}{l}48 \\
49 \\
92 \\
43 \\
41\end{array}$ & $\begin{array}{r}53 \\
55 \\
100 \\
46 \\
48\end{array}$ & $\begin{array}{l}59 \\
62\end{array}$ & $\begin{array}{l}69 \\
75\end{array}$ & $\begin{array}{l}86 \\
87\end{array}$ & $\begin{array}{l}100 \\
100\end{array}$ & 100 \\
\hline
\end{tabular}


Table 3.--Bedload particle-size distributions--Continued

\begin{tabular}{|c|c|c|c|c|c|c|c|c|c|c|c|c|}
\hline \multirow{2}{*}{ Date } & \multicolumn{12}{|c|}{ Percent by weight finer than size (millimeters) indicated } \\
\hline & 0.062 & 0.12 & 0.25 & 0.5 & 1 & 2 & 4 & 8 & 16 & 32 & 64 & 128 \\
\hline \multicolumn{13}{|c|}{ 11. Toutle River at Tower Road near Silver Lake, Wash. ${ }^{3}-$-Continued } \\
\hline 06-07-85 & .2 & .3 & 0.8 & 2 & 5 & 15 & 23 & 33 & 45 & 68 & 98 & 100 \\
\hline $06-07-85$ & .1 & .2 & .6 & 1 & 2 & 7 & 12 & 20 & 41 & 65 & 98 & 100 \\
\hline $06-08-85$ & .2 & .4 & 2 & 6 & 13 & 26 & 35 & 46 & 59 & 75 & 98 & 100 \\
\hline $08-23-85$ & .4 & 2 & 18 & 54 & 73 & 80 & 85 & 91 & 96 & 100 & & \\
\hline $08-23-85$ & .3 & 2 & 15 & 50 & 66 & 74 & 82 & 91 & 95 & 99 & 100 & \\
\hline $10-25-85$ & 1.3 & 5 & 18 & 53 & 66 & 69 & 70 & 71 & 80 & 82 & 100 & \\
\hline $10-25-85$ & 0.6 & 2 & 9 & 36 & 48 & 52 & 55 & 62 & 71 & 80 & 100 & \\
\hline $10-25-85$ & .8 & 4 & 16 & 50 & 64 & 68 & 70 & 73 & 79 & 86 & 100 & \\
\hline $10-25-85$ & 1.3 & 5 & 17 & 52 & 70 & 75 & 78 & 83 & 89 & 97 & 100 & \\
\hline $11-06-85$ & 0.1 & 0.2 & 0.7 & 2 & 6 & 19 & 25 & 30 & 44 & 66 & 98 & 100 \\
\hline $11-06-85$ & 0 & .1 & .7 & 5 & 17 & 36 & 49 & 65 & 93 & 98 & 100 & \\
\hline $11-08-85$ & 0.1 & .2 & 1 & 8 & 22 & 38 & 46 & 54 & 68 & 82 & 99 & 100 \\
\hline $11-08-85$ & .1 & .2 & 1 & 6 & 17 & 32 & 43 & 57 & 74 & 89 & 100 & \\
\hline $12-09-85$ & 17 & 41 & 51 & 51 & 51 & 51 & 51 & 51 & 51 & 100 & & \\
\hline $12-09-85$ & & & & & & & 0 & 32 & 59 & 84 & 94 & 100 \\
\hline $12-09-85$ & 0.1 & 0.1 & 0.8 & 7 & 30 & 50 & 54 & 60 & 79 & 96 & 100 & \\
\hline $12-09-85$ & 0 & .1 & 1 & 12 & 38 & 49 & 55 & 65 & 76 & 86 & 100 & \\
\hline $12-09-85$ & 2 & 2 & 3 & 13 & 34 & 65 & 76 & 84 & 94 & 99 & 100 & \\
\hline $01-03-86$ & 0 & 0.1 & 1 & 6 & 19 & 52 & 65 & 74 & 86 & 96 & 100 & \\
\hline $01-03-86$ & & & 0 & 0.2 & 3 & 29 & 37 & 45 & 59 & 94 & 100 & \\
\hline $01-03-86$ & & & 0 & .4 & 5 & 30 & 53 & 67 & 82 & 95 & 100 & \\
\hline $01-03-86$ & & 0 & 0.1 & 3 & 15 & 42 & 52 & 60 & 72 & 87 & 94 & 100 \\
\hline $01-19-86$ & 0 & 0.1 & .2 & 0.6 & 2 & 10 & 24 & 41 & 60 & 82 & 100 & \\
\hline $01-19-86$ & 0 & .1 & .2 & .5 & 2 & 13 & 27 & 43 & 63 & 82 & 100 & \\
\hline $01-19-86$ & & 0 & .2 & .5 & 2 & 11 & 20 & 32 & 48 & 68 & 100 & \\
\hline $01-19-86$ & & 0 & .1 & .4 & 1 & 9 & 17 & 31 & 52 & 75 & 100 & \\
\hline $01-29-86$ & 0.1 & 0.2 & 3 & 40 & 86 & 94 & 96 & 97 & 98 & 99 & 100 & \\
\hline $01-29-86$ & 0 & .1 & 2 & 21 & 49 & 69 & 79 & 87 & 95 & 98 & 100 & \\
\hline $01-29-86$ & 0.1 & .2 & 2 & 28 & 61 & 74 & 78 & 82 & 87 & 94 & 98 & 100 \\
\hline $01-29-86$ & .1 & .2 & 2 & 14 & 30 & 47 & 59 & 71 & 84 & 91 & 100 & \\
\hline $02-11-86$ & .1 & .1 & 0.6 & 4 & 12 & 25 & 31 & 37 & 44 & 48 & 49 & 49 \\
\hline $02-11-86$ & & 0 & .2 & 0.6 & 4 & 33 & 59 & 81 & 95 & 99 & 100 & \\
\hline $02-11-86$ & 0 & 0.1 & 2 & 8 & 22 & 51 & 67 & 79 & 91 & 99 & 100 & \\
\hline $02-11-86$ & 0 & $\therefore 1$ & 0.2 & 0.8 & 3 & 67 & 80 & 87 & 95 & 100 & & \\
\hline $02-16-86$ & 0 & .1 & .5 & 3 & 8 & 21 & 30 & 43 & 71 & 87 & 100 & \\
\hline $02-16-86$ & 0 & .1 & .6 & 4 & 12 & 29 & 40 & 53 & 71 & 87 & 96 & 100 \\
\hline $02-16-86$ & 0 & .1 & .5 & 3 & 8 & 22 & 32 & 43 & 60 & 74 & 97 & 100 \\
\hline $02-16-86$ & 0 & .1 & .6 & 5 & 13 & 29 & 40 & 51 & 65 & 83 & 99 & 100 \\
\hline $05-22-86$ & 0.1 & 0.2 & 2 & 9 & 24 & 56 & 63 & 69 & 79 & 90 & 100 & \\
\hline $05-22-86$ & .1 & .4 & 6 & 20 & 35 & 70 & 79 & 85 & 94 & 98 & 100 & \\
\hline $05-22-86$ & .1 & .3 & 3 & 14 & 35 & 73 & 79 & 82 & 87 & 94 & 100 & \\
\hline $05-22-86$ & .1 & .6 & 5 & 15 & 33 & 61 & 70 & 77 & 87 & 96 & 100 & \\
\hline $07-02-86$ & 0 & .4 & 7 & 40 & 64 & 85 & 91 & 95 & 98 & 100 & & \\
\hline $07-02-86$ & 0.1 & .4 & 8 & 51 & 75 & 90 & 94 & 97 & 99 & 100 & & \\
\hline $07-02-86$ & 0 & .5 & 8 & 45 & 69 & 90 & 95 & 98 & 100 & & & \\
\hline $07-02-86$ & 0 & .4 & 5 & 27 & 47 & 78 & 86 & 92 & 98 & 100 & & \\
\hline $07-22-86$ & 0 & .7 & 9 & 43 & 67 & 85 & 90 & 94 & 97 & 100 & & \\
\hline $07-22-86$ & 0 & .4 & 6 & 36 & 69 & 90 & 94 & 97 & 100 & & & \\
\hline $08-07-86$ & & 0 & 1 & 24 & 63 & 86 & 93 & 98 & 100 & & & \\
\hline $08-07-86$ & 0 & 0.1 & 3 & 31 & 65 & 88 & 92 & 96 & 99 & 100 & & \\
\hline
\end{tabular}


Table 3.--Bedload particle-size distributions--Continued

Date

Percent by weight finer than size (millineters) indicated

\begin{tabular}{lllllllllllll}
\hline 0.062 & 0.12 & 0.25 & 0.5 & 1 & 2 & 4 & 8 & 16 & 32 & 64 & 128
\end{tabular}

11. Toutle River at Tower Road near Silver Lake, Wash. ${ }^{3}$--Continued

\begin{tabular}{|c|c|c|c|c|c|c|c|c|c|c|c|c|}
\hline $10-31-86$ & 0 & .1 & 0.2 & 0.8 & 6 & 33 & 49 & 63 & 80 & 90 & 100 & \\
\hline $11-14-86$ & 0.1 & .1 & .9 & 3 & 12 & 58 & 70 & 78 & 89 & 94 & 100 & \\
\hline $11-14-86$ & 0 & .1 & .9 & 5 & 25 & 51 & 65 & 78 & 95 & 99 & 100 & \\
\hline $11-14-86$ & & 0 & .2 & 1 & 6 & 38 & 52 & 63 & 82 & 90 & 92 & 100 \\
\hline $11-14-86$ & 0 & 0.4 & 4 & 13 & 18 & 66 & 80 & 88 & 96 & 98 & 100 & \\
\hline $11-21-86$ & 0 & .1 & 0.1 & 0.4 & 2 & 7 & 10 & 18 & 41 & 62 & 82 & 100 \\
\hline $11-21-86$ & 0.2 & 1 & 6 & 14 & 26 & 37 & 41 & 46 & 55 & 70 & 85 & 100 \\
\hline $11-21-86$ & 0 & 0.1 & 0.2 & 0.4 & 1 & 4 & 9 & 21 & 48 & 68 & 90 & 100 \\
\hline $11-21-86$ & 0 & .1 & .4 & 1 & 4 & 11 & 21 & 33 & 57 & 72 & 88 & 100 \\
\hline $11-22-86$ & 0.1 & .4 & 2 & 5 & 13 & 47 & 56 & 61 & 70 & 79 & 96 & 100 \\
\hline $11-22-86$ & .1 & .6 & 3 & 7 & 12 & 24 & 33 & 42 & 50 & 66 & 85 & 100 \\
\hline $11-22-86$ & .1 & .4 & 2 & 4 & 10 & 19 & 24 & 29 & 37 & 54 & 75 & 100 \\
\hline $11-22-86$ & 0 & .1 & 0.5 & 2 & 4 & 12 & 17 & 22 & 33 & 60 & 88 & 100 \\
\hline $01-14-87$ & 0.1 & .1 & 1 & 10 & 32 & 54 & 61 & 69 & 79 & 90 & 97 & 100 \\
\hline $01-14-87$ & & 0 & 0.6 & 9 & 39 & 65 & 69 & 77 & 85 & 93 & 98 & 100 \\
\hline $01-14-87$ & 0 & 0.1 & .8 & 13 & 49 & 71 & 75 & 81 & 87 & 93 & 99 & 100 \\
\hline $01-14-87$ & 0.2 & .2 & 2 & 17 & 39 & 58 & 64 & 71 & 80 & 92 & 100 & \\
\hline $01-28-87$ & 0 & 0.1 & 0.7 & 6 & 20 & 48 & 54 & 62 & 71 & 78 & 86 & 89 \\
\hline $01-28-87$ & 0 & .1 & .3 & 3 & 11 & 21 & 23 & 35 & 54 & 80 & 99 & 100 \\
\hline $01-28-87$ & 0 & .3 & 1 & 9 & 27 & 38 & 40 & 51 & 68 & 86 & 97 & 100 \\
\hline $01-28-87$ & 0.3 & .3 & 2 & 7 & 14 & 36 & 47 & 61 & 77 & 92 & 100 & \\
\hline $02-01-87$ & .3 & .8 & 2 & 5 & 11 & 28 & 36 & 44 & 52 & 66 & 84 & 100 \\
\hline
\end{tabular}

12. North Fork Toutle River near Kid Valley, Wash. ${ }^{3}$

\begin{tabular}{|c|c|c|c|c|c|c|c|c|c|c|c|c|}
\hline $06-07-85$ & 0.3 & 0.8 & 3 & 5 & 11 & 31 & 42 & 52 & 65 & 82 & 100 & \\
\hline $06-07-85$ & .2 & .4 & 1 & 3 & 7 & 23 & 33 & 43 & 61 & 81 & 100 & \\
\hline $06-08-85$ & .1 & .5 & 2 & 6 & 14 & 29 & 40 & 56 & 80 & 89 & 98 & 100 \\
\hline $06-08-85$ & 0 & .4 & 1 & 3 & 8 & 18 & 27 & 38 & 62 & 79 & 99 & 100 \\
\hline $12-18-85$ & & 0 & 0.1 & 1 & 11 & 31 & 41 & 53 & 72 & 86 & 100 & \\
\hline $12-18-85$ & & & 0 & 0.5 & 4 & 13 & 22 & 60 & 82 & 92 & 95 & 100 \\
\hline $12-18-85$ & & 0 & 0.1 & 1 & 8 & 33 & 50 & 68 & 80 & 88 & 100 & \\
\hline $12-18-85$ & & 0 & .1 & 0.9 & 6 & 23 & 37 & 56 & 75 & 90 & 100 & \\
\hline $01-19-86$ & 0.1 & 0.5 & 2 & 9 & 23 & 44 & 49 & 53 & 61 & 76 & 95 & 100 \\
\hline $01-19-86$ & .1 & .2 & 0.9 & 4 & 12 & 27 & 38 & 50 & 67 & 89 & 100 & \\
\hline $01-19-86$ & .1 & .2 & .9 & 5 & 11 & 19 & 31 & 47 & 70 & 87 & 98 & 100 \\
\hline $01-19-86$ & .1 & .2 & .9 & 5 & 13 & 24 & 44 & 72 & 89 & 96 & 100 & \\
\hline $02-13-86$ & 0 & .1 & .9 & 6 & 17 & 35 & 52 & 65 & 77 & 88 & 95 & 100 \\
\hline $02-13-86$ & 0.1 & .2 & 2 & 8 & 22 & 47 & 66 & 78 & 84 & 90 & 99 & 100 \\
\hline $02-13-86$ & 0.1 & 0.3 & 3 & 12 & 31 & 51 & 60 & 67 & 75 & 87 & 95 & 100 \\
\hline $02-13-86$ & 0 & .1 & 0.6 & 3 & 9 & 22 & 37 & 58 & 76 & 88 & 100 & \\
\hline $02-26-86$ & 0.2 & .6 & 2 & 7 & 15 & 25 & 35 & 51 & 65 & 76 & 90 & 100 \\
\hline $02-26-86$ & .1 & .4 & 1 & 3 & 7 & 18 & 34 & 59 & 77 & 87 & 93 & 100 \\
\hline $02-26-86$ & .1 & .2 & 0.6 & 1 & 3 & 9 & 18 & 39 & 73 & 84 & 94 & 100 \\
\hline $08-07-86$ & & & 0 & 0.2 & 3 & 24 & 34 & 44 & 57 & 72 & 86 & 100 \\
\hline
\end{tabular}


Table 3.--Bedload particle-size distributions--Continued

Date

Percent by weight finer than size (millineters) indicated

$\begin{array}{llllllllllllll}0.062 & 0.12 & 0.25 & 0.5 & 1 & 2 & 4 & 8 & 16 & 32 & 64 & 128\end{array}$

12. North Fork Toutle River near Kid Valley, Wash. ${ }^{3}-$-Continued

$08-07-86$
$08-07-86$
$08-07-86$
$09-09-86$

$09-09-86$
$09-09-86$

09-09-86

09-09-86

10-10-86

$10-10-86$

$10-10-86$

10-10-86

$02-02-87$

$02-02-87$

\begin{tabular}{|c|c|c|c|}
\hline & 0 & .3 & 5 \\
\hline 0 & 0.3 & 5 & 24 \\
\hline 0 & .2 & 3 & 15 \\
\hline 0 & .1 & 2 & 12 \\
\hline 0 & .1 & 0.7 & 3 \\
\hline 0 & .1 & .1 & 0.9 \\
\hline 0 & .2 & 1 & 4 \\
\hline 0 & .5 & 5 & 17 \\
\hline & 0 & 0.3 & 5 \\
\hline 0 & 0.1 & 4 & 13 \\
\hline
\end{tabular}

$\begin{array}{ll}34 & 46 \\ 51 & 62 \\ 36 & 5 \\ 35 & 44 \\ 27 & 44\end{array}$

$\begin{array}{rrrrr}46 & 62 & 80 & 93 & 100 \\ 62 & 71 & 85 & 96 & 100 \\ 51 & 63 & 78 & 95 & 100 \\ 44 & 58 & 72 & 92 & 100 \\ 44 & 62 & 85 & 100 & \end{array}$

$n^{2}+n^{2}$

0.3

$\begin{array}{rr}0 & \\ 0.4 & 1 \\ .2 & 0.8\end{array}$

$\begin{array}{ll}15 & 2 \\ 18 & 2 \\ 68 & 8 \\ 49 & 69 \\ 53 & 7\end{array}$

$\begin{array}{ll}29 & 4 \\ 29 & 4 \\ 87 & 95 \\ 69 & 79 \\ 70 & 8\end{array}$

$\begin{array}{ll}44 & 57 \\ 40 & 6 \\ 95 & 98 \\ 79 & 89 \\ 88 & 99\end{array}$

$57 \quad 61$

$\begin{array}{lll}61 & 81 & 100\end{array}$

$98 \quad 100$

$89 \quad 100$

$\begin{array}{llllll}.1 & 3 & 13 & 54 & 74 & 84\end{array}$

1

$\begin{array}{rrrr}3 & 13 & 54 & 74 \\ 5 & 13 & 28 & 39 \\ 3 & 9 & 23 & 35\end{array}$

49
49

$89 \quad 93$

$\begin{array}{ll}59 & 70 \\ 61 & 71\end{array}$

100

$\begin{array}{rr}83 & 93 \\ 83 & 100\end{array}$

\section{Oak Creek near Corvallis, Oreg. 4}

\begin{tabular}{|c|c|c|c|c|c|c|c|c|c|c|c|c|}
\hline $01-08-71$ & 0.8 & 2 & 5 & 25 & 46 & 61 & 76 & 84 & 97 & 100 & & \\
\hline $01-10-71$ & 1 & 3 & 9 & 27 & 56 & 72 & 84 & 92 & 98 & 100 & & \\
\hline $01-10-71$ & 0.8 & 3 & 10 & 23 & 47 & 65 & 78 & 90 & 99 & 100 & & \\
\hline $01-11-71$ & 14 & 21 & 29 & 42 & 62 & 77 & 85 & 88 & 92 & 99 & 100 & \\
\hline $01-11-71$ & 0.5 & 2 & 6 & 18 & 43 & 64 & 80 & 92 & 99 & 100 & & \\
\hline $01-12-71$ & 1 & 4 & 14 & 32 & 57 & 75 & 87 & 93 & 98 & 100 & & \\
\hline $01-13-71$ & 1 & 4 & 10 & 25 & 47 & 62 & 76 & 82 & 86 & 100 & & \\
\hline $01-14-71$ & 3 & 5 & 13 & 33 & 61 & 78 & 88 & 95 & 99 & 100 & & \\
\hline $01-16-71$ & 0.1 & 0.1 & 0.4 & 1 & 2 & 5 & 10 & 20 & 37 & 71 & 95 & 100 \\
\hline $01-16-71$ & .1 & .3 & .4 & 1 & 3 & 7 & 14 & 22 & 32 & 60 & 90 & 100 \\
\hline $01-18-71$ & .6 & .9 & 2 & 3 & 7 & 14 & 25 & 38 & 56 & 86 & 98 & 100 \\
\hline $01-20-71$ & .1 & .4 & 1 & 5 & 15 & 36 & 56 & 71 & 84 & 99 & 100 & \\
\hline $01-21-71$ & .4 & 1 & 4 & 10 & 24 & 50 & 67 & 80 & 89 & 98 & 100 & \\
\hline $01-23-71$ & .9 & 2 & 4 & 10 & 26 & 54 & 71 & 82 & 91 & 97 & 100 & \\
\hline $01-25-71$ & .2 & 0.6 & 1 & 4 & 13 & 32 & 52 & 67 & 78 & 92 & 99 & 100 \\
\hline $01-25-71$ & .2 & .7 & 2 & 4 & 15 & 40 & 60 & 74 & 84 & 94 & 100 & \\
\hline $01-26-71$ & .6 & .9 & 2 & 6 & 30 & 51 & 72 & 84 & 90 & 95 & 98 & 100 \\
\hline $01-26-71$ & .2 & .7 & 3 & 11 & 35 & 64 & 84 & 93 & 96 & 100 & & \\
\hline $01-27-71$ & .7 & 2 & 5 & 14 & 37 & 74 & 90 & 96 & 98 & 100 & & \\
\hline $01-28-71$ & 0.5 & 1 & 6 & 19 & 46 & 78 & 93 & 98 & 99 & 100 & & \\
\hline $02-04-71$ & .8 & 3 & 10 & 28 & 58 & 84 & 95 & 97 & 100 & & & \\
\hline $02-05-71$ & .8 & 2 & 8 & 24 & 52 & 82 & 93 & 97 & 100 & & & \\
\hline $02-09-71$ & .8 & 2 & 5 & 20 & 47 & 72 & 86 & 89 & 99 & 100 & & \\
\hline $02-14-71$ & .1 & 0.5 & 3 & 14 & 36 & 62 & 83 & 96 & 100 & & & \\
\hline $02-15-71$ & .4 & 1 & 3 & 12 & 30 & 52 & 68 & 86 & 99 & 100 & & \\
\hline $02-18-71$ & 2 & 3 & 9 & 22 & 44 & 67 & 79 & 88 & 98 & 100 & & \\
\hline $02-22-71$ & 0.6 & 1 & 3 & 15 & 40 & 69 & 83 & 93 & 100 & & & \\
\hline $02-25-71$ & .5 & 3 & 6 & 14 & 27 & 45 & 61 & 77 & 86 & 100 & & \\
\hline $02-26-71$ & .8 & 2 & 5 & 15 & 35 & 57 & 77 & 91 & 99 & 100 & & \\
\hline $02-27-71$ & .8 & 2 & 6 & 17 & 35 & 52 & 63 & 75 & 87 & -- & -- & -- \\
\hline
\end{tabular}


Table 3.--Bedload particle-size distributions--Continued

Date

Percent by weight finer than size (millimeters) indicated

$\begin{array}{llllllllllll}0.062 & 0.12 & 0.25 & 0.5 & 1 & 2 & 4 & 8 & 16 & 32 & 64 & 128\end{array}$

13. Oak Creek near Corvallis, Oreg. ${ }^{4}$--Continued

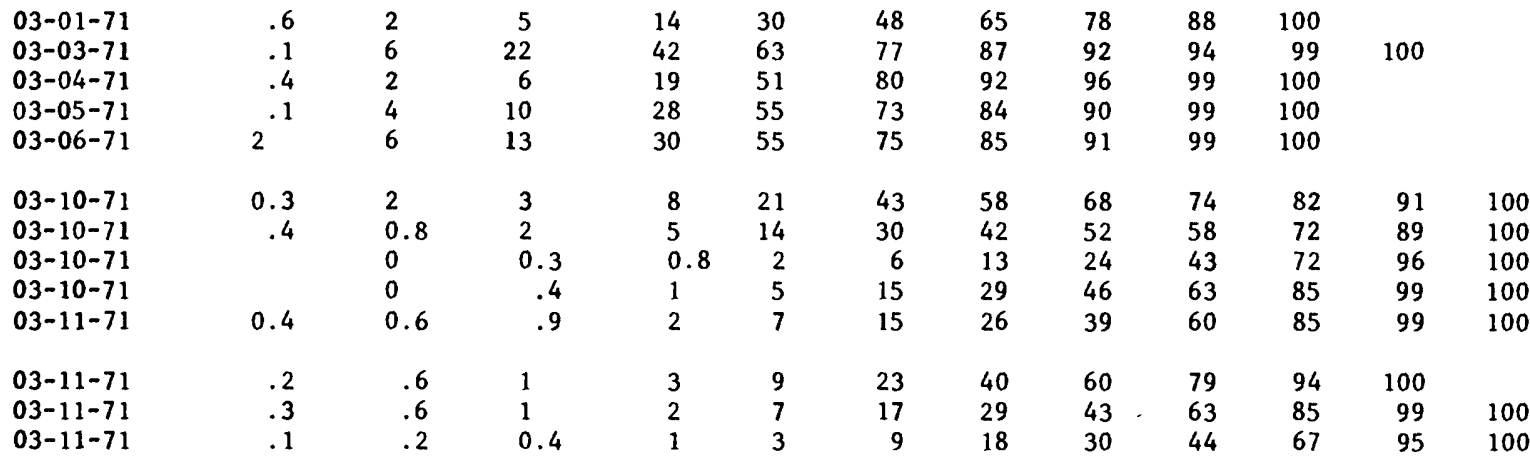

14. San Antonio River near Lockwood, Calif.

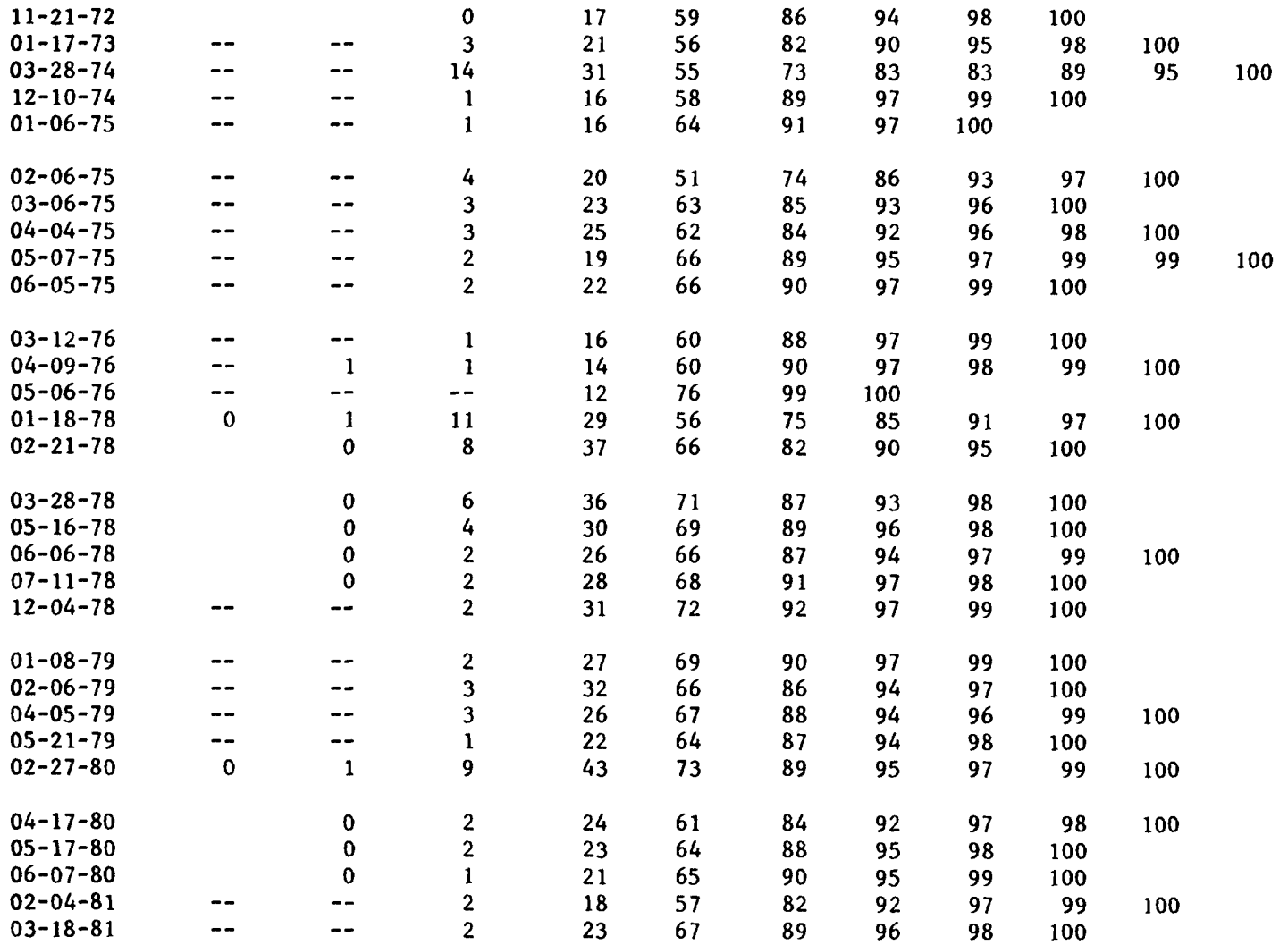


Table 3.--Bedload particle-size distributions--Continued

Percent by weight finer than size (millimeters) indicated

Date

\begin{tabular}{lllllllllllll}
\hline 0.062 & 0.12 & 0.25 & 0.5 & 1 & 2 & 4 & 8 & 16 & 32 & 64 & 128
\end{tabular}

14. San Antonio River near Lockwood, Calif.--Continued

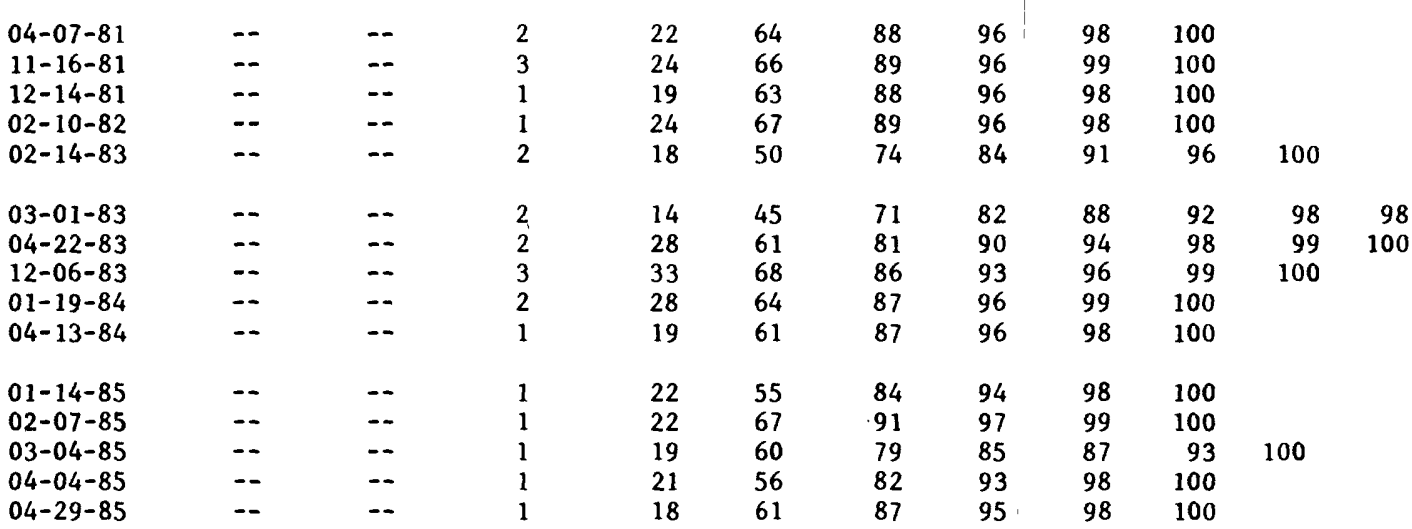

15. Chamise Creek near Island Mountain, Calif.

\begin{tabular}{|c|c|c|c|c|c|c|c|c|c|c|}
\hline $11-07-72$ & -- & -- & 3 & 16 & 32 & 58 & 84 & 97 & 100 & \\
\hline $02-28-72$ & & & 0 & 6 & 15 & 29 & 41 & 54 & 100 & \\
\hline $11-14-73$ & & & 0 & 4 & 12 & 30 & 53 & 73 & 99 & 100 \\
\hline $12-21-73$ & -- & -- & 4 & 9 & 18 & 25 & 33 & 47 & 83 & 100 \\
\hline $\begin{array}{l}04-11-74 \\
03-03-76\end{array}$ & -- & 1 & $\begin{array}{l}0 \\
3\end{array}$ & $\begin{array}{r}3 \\
11\end{array}$ & $\begin{array}{r}6 \\
21\end{array}$ & $\begin{array}{l}15 \\
45\end{array}$ & $\begin{array}{l}40 \\
71\end{array}$ & $\begin{array}{l}62 \\
86\end{array}$ & $\begin{array}{l}67 \\
96\end{array}$ & $\begin{array}{l}100 \\
100\end{array}$ \\
\hline
\end{tabular}

16. Redwood Creek near Blue Lake, Calif.

\begin{tabular}{|c|c|c|c|c|c|c|c|c|c|c|c|}
\hline $01-12-74$ & -- & -- & -- & 2 & 9 & 40 & 76 & 93 & 100 & & \\
\hline $04-01-75$ & - & -- & 1 & 3 & 12 & 30 & 49 & 69 & 86 & 95 & 100 \\
\hline $03-10-78$ & & & 0 & 2 & 6 & 15 & 36 & 63 & 88 & 100 & \\
\hline $01-27-81$ & -- & 1 & 7 & 18 & 18 & 28 & 34 & 42 & 53 & 80 & 100 \\
\hline $01-27-82$ & & & 0 & 2 & 11 & 31 & 57 & 78 & 92 & 98 & 100 \\
\hline $11-30-82$ & -- & -- & 2 & 11 & 28 & 48 & 69 & 84 & 97 & 100 & \\
\hline $01-27-83$ & -- & 1 & 9 & 26 & 38 & 47 & 53 & 61 & 74 & 100 & \\
\hline $11-17-83$ & - & -- & 2 & 11 & 25 & 43 & 57 & 69 & 82 & 92 & 100 \\
\hline $12-14-83^{1}$ & -- & - & 3 & 11 & 17 & 23 & 30 & 41 & 62 & 81 & 92 \\
\hline $03-01-84$ & -- & - & $\sim$ & 2 & 8 & 34 & 74 & 96 & 100 & & \\
\hline
\end{tabular}

17. Redwood Creek above Panther Creek near Orick, Calif.

$\begin{array}{rrrrrrrrrrrr}12-03-80 & -- & -- & 2 & 14 & 24 & 34 & 48 & 63 & 78 & 94 & 100 \\ 01-23-81 & -- & -- & 1 & 8 & 18 & 32 & 49 & 66 & 80 & 94 & 100 \\ 01-28-81 & -- & -- & 1 & 7 & 21 & 37 & 53 & 69 & 81 & 98 & 100 \\ 02-05-81 & -- & -- & -- & 2 & 8 & 29 & 53 & 78 & 93 & 100 & \\ 11-02-81 & -- & -- & -- & 8 & 42 & 69 & 81 & 88 & 97 & 100 & \end{array}$


Table 3.--Bedload particle-size distributions=-Continued

Date Percent by weight finer than size (millimeters) indicated

\begin{tabular}{llllllllllll}
\hline 0.062 & 0.12 & 0.25 & 0.5 & 1 & 2 & 4 & 8 & 16 & 32 & 64 & 128
\end{tabular}

17. Redwood Creek above Panther Creek near Orick, Calif.---Continued

\begin{tabular}{|c|c|c|c|c|c|c|c|c|c|c|c|}
\hline $12-09-81$ & -- & $\cdots$ & 3 & 6 & 13 & 25 & 48 & 77 & 90 & 100 & \\
\hline $02-10-82$ & -- & - & 1 & 2 & 11 & 35 & 60 & 79 & 92 & 100 & \\
\hline $03-19-82$ & -- & - & -- & 2 & 7 & 20 & 45 & 71 & 89 & 100 & \\
\hline $12-01-82$ & -- & - & 1 & 4 & 13 & 26 & 45 & 67 & 87 & 97 & 100 \\
\hline $12-22-82$ & - & - & 1 & 4 & 10 & 17 & 27 & 42 & 61 & 84 & 100 \\
\hline $01-26-83$ & -- & 1 & 4 & 12 & 21 & 32 & 45 & 60 & 81 & 95 & 100 \\
\hline $03-08-84$ & - & $\cdots$ & -- & 1 & 7 & 29 & 66 & 91 & 100 & & \\
\hline $04-03-84$ & -. & -. & - & 2 & 24 & 77 & 98 & 100 & & & \\
\hline
\end{tabular}

18. Redwood Creek at Orick, Calif.

\begin{tabular}{|c|c|c|c|c|c|c|c|c|c|c|c|}
\hline $11-14-79$ & $\cdots$ & -- & 1 & 20 & 44 & 69 & 91 & 99 & 100 & & \\
\hline $02-01-80$ & $\cdots$ & -- & 1 & 7 & 32 & 58 & 81 & 96 & 100 & & \\
\hline $02-21-80$ & $\cdots$ & $\cdots$ & 1 & 7 & 19 & 44 & 69 & 87 & 96 & 99 & 100 \\
\hline $12-11-80$ & $\cdots$ & -- & -- & 14 & 52 & 83 & 96 & 99 & 100 & & \\
\hline $01-29-81$ & - & -- & 1 & 6 & 16 & 34 & 62 & 84 & 96 & 100 & \\
\hline $02-24-81$ & -- & $\cdots$ & 1 & 10 & 28 & 54 & 74 & 89 & 98 & 100 & \\
\hline $04-11-81$ & -- & $\cdots$ & $\cdots$ & 2 & 10 & 35 & 69 & 90 & 100 & & \\
\hline $11-13-81$ & & 0 & 1 & 5 & 17 & 40 & 69 & 91 & 98 & 100 & \\
\hline $12-14-81$ & & 0 & 1 & 4 & 14 & 31 & 49 & 71 & 90 & 97 & 100 \\
\hline $03-03-82$ & & 0 & 1 & 5 & 16 & 31 & 49 & 70 & 88 & 98 & 100 \\
\hline $12-22-82$ & -- & $\cdots$ & 1 & 10 & 26 & 43 & 60 & 79 & 94 & 99 & 100 \\
\hline $01-24-83$ & $\cdots$ & $\cdots$ & 1 & 6 & 17 & 36 & 55 & 73 & 89 & 100 & \\
\hline $02-29-84$ & -. & $\cdots$ & -- & 3 & 10 & 32 & 57 & 79 & 93 & 100 & \\
\hline
\end{tabular}

19. Hayes Creek near Orick, Calif.

$\begin{array}{rrrrrrrrrrr}12-14-77 & 1 & 1 & 6 & 18 & 37 & 60 & 73 & 82 & 88 & 100 \\ 12-14-77 & 1 & 1 & 5 & 16 & 29 & 42 & 59 & 76 & 100 & \end{array}$

20. Lacks Creek near Orick, Calif.

\begin{tabular}{|c|c|c|c|c|c|c|c|c|c|c|c|}
\hline $02-03-78$ & & 0 & 2 & 8 & 17 & 28 & 45 & 69 & 90 & 100 & \\
\hline $03-09-78$ & & 0 & 1 & 4 & 11 & 24 & 46 & 70 & 94 & 100 & \\
\hline $01-22-81$ & & 0 & 2 & 12 & 27 & 38 & 45 & 51 & 58 & 71 & 100 \\
\hline $01-23-81$ & 0 & 1 & 5 & 22 & 47 & 73 & 91 & 98 & 100 & & \\
\hline $02-17-82$ & -- & -- & 1 & 3 & 9 & 19 & 29 & 40 & 57 & 85 & 100 \\
\hline $03-01-82$ & $\cdots$ & 1 & 6 & 30 & 54 & 72 & 86 & 98 & 100 & & \\
\hline $01-26-83$ & - & - & 1 & 8 & 21 & 37 & 55 & 74 & 92 & 100 & \\
\hline $03-29-83$ & - & 1 & 5 & 21 & 38 & 53 & 68 & 82 & 91 & 100 & \\
\hline
\end{tabular}

21. Sacramento River above Bend Bridge near Red Bluff, Calif.

12-01-77

01-03-78

02-02-78

03-01-78

05-01-78

$\begin{array}{rrrrrrrrr}0 & 1 & 15 & 49 & 79 & 92 & 95 & 100 & \\ 0 & 2 & 17 & 49 & 71 & 79 & 85 & 92 & 100 \\ 0 & 3 & 12 & 20 & 26 & 36 & 50 & 83 & 85 \\ 0 & 3 & 35 & 61 & 76 & 85 & 88 & 90 & 100 \\ 0 & 2 & 36 & 62 & 66 & 67 & 68 & 72 & 85\end{array}$

100

100 
Table 3.--Bedload particle-size distributions--Continued

Date Percent by weight finer than size (millimeters) indicated

\begin{tabular}{lllllllllllllll}
\hline & 0.062 & 0.12 & 0.25 & 0.5 & 1 & 2 & 4 & 8 & 16
\end{tabular}

21. Sacramento River above Bend Bridge near Red Bluff, Calif.--Continued

\begin{tabular}{|c|c|c|c|c|c|c|c|c|c|}
\hline $06-02-78$ & 0 & 1 & 25 & 65 & 86 & 92 & 94 & 96 & 100 \\
\hline $07-05-78$ & 0 & 1 & 30 & 92 & 97 & 98 & 100 & & \\
\hline $07-31-78$ & 0 & 1 & 29 & 74 & 81 & 82 & 83 & 84 & 86 \\
\hline $11-07-78$ & 0 & 2 & 16 & 54 & 65 & 67 & 73 & 90 & 100 \\
\hline $01-02-79$ & 0 & 1 & 17 & 76 & 92 & 94 & 96 & 100 & \\
\hline $03-01-79$ & 0 & 5 & 26 & 37 & 51 & 66 & 80 & 91 & 100 \\
\hline $05-02-79$ & 0 & 6 & 38 & 69 & 82 & 86 & 90 & 95 & 100 \\
\hline $12-03-79$ & & 0 & 12 & 46 & 67 & 69 & 75 & 77 & 77 \\
\hline $12-26-79$ & 0 & 1 & 16 & 56 & 72 & 80 & 86 & 91 & 98 \\
\hline $01-02-80$ & 0 & 1 & 14 & 57 & 76 & 84 & 89 & 92 & 100 \\
\hline $02-01-80$ & 0 & 1 & 15 & 31 & 57 & 73 & 82 & 93 & 100 \\
\hline $04-01-80$ & 0 & 6 & 32 & 58 & 62 & 63 & 63 & 67 & 100 \\
\hline $05-01-80$ & 0 & 3 & 36 & 58 & 63 & 66 & 66 & 66 & 100 \\
\hline $06-03-80$ & 0 & 2 & 35 & 53 & 55 & 56 & 58 & 82 & 100 \\
\hline
\end{tabular}

22. Clearwater River at Spalding, Idaho

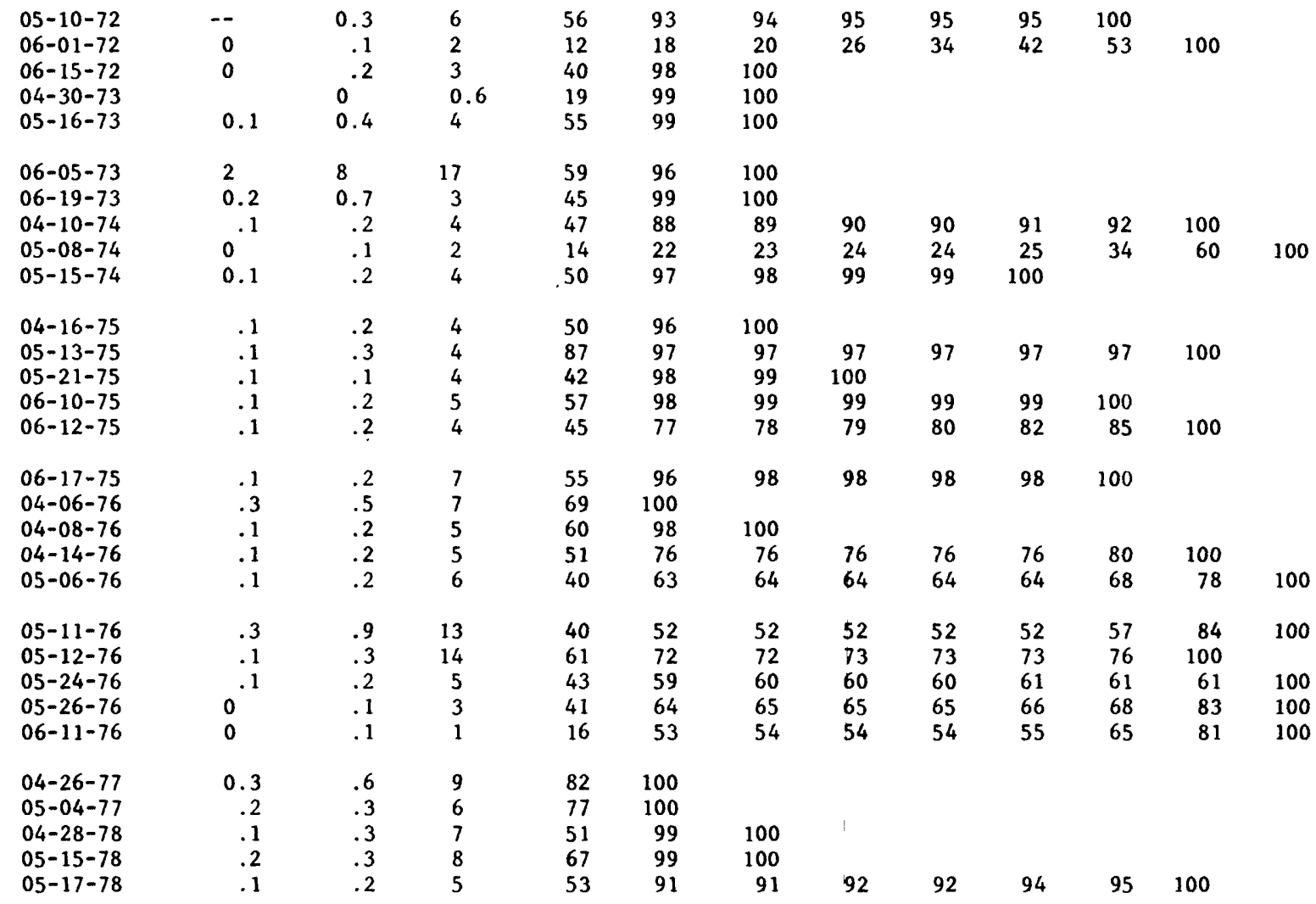


Table 3.--Bedload particle-size distributions--Continued

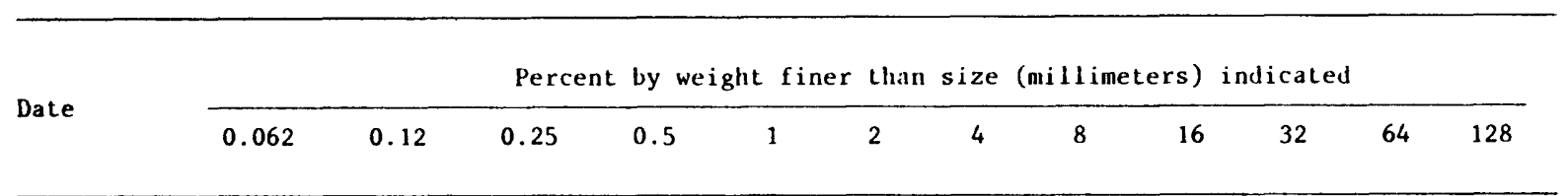

22. Clearwater River at Spalding, Idaho--Continued

\begin{tabular}{|c|c|c|c|c|c|c|c|c|c|c|c|c|}
\hline $06-05-78$ & .2 & .6 & 6 & 58 & 93 & 94 & 94 & 95 & 98 & 100 & & \\
\hline $06-06-78$ & .3 & .8 & 9 & 74 & 98 & 99 & 99 & 99 & 99 & 100 & & \\
\hline $06-08-78$ & .2 & .3 & 7 & 62 & 87 & 87 & 88 & 88 & 89 & 90 & 100 & \\
\hline $06-12-78$ & .1 & .2 & 3 & 53 & 99 & 100 & & & & & & \\
\hline $06-14-78$ & 0 & .1 & 2 & 30 & 90 & 91 & 91 & 91 & 91 & 92 & 100 & \\
\hline $06-19-78$ & 0 & .1 & 3 & 42 & 95 & 95 & 95 & 95 & 96 & 100 & & \\
\hline $05-01-79$ & 0.3 & .7 & 8 & 68 & 99 & 100 & & & & & & \\
\hline $05-03-79$ & .2 & .4 & 7 & 66 & 98 & 99 & 99 & 99 & 99 & 100 & & \\
\hline $05-08-79$ & 1 & 2 & 14 & 78 & 97 & 99 & 100 & & & & & \\
\hline $05-11-79$ & 0.1 & 0.3 & 4 & 49 & 99 & 100 & & & & & & \\
\hline $05-14-79$ & .1 & .1 & 3 & 41 & 99 & 100 & & & & & & \\
\hline $05-16-79$ & 0.1 & 0.2 & 5 & 53 & 98 & 98 & 98 & 98 & 98 & 98 & 100 & \\
\hline $05-21-79$ & .1 & .1 & 2 & 42 & 85 & 86 & 86 & 86 & 86 & 88 & 92 & 100 \\
\hline $05-23-79$ & 0 & .1 & 2 & 29 & 58 & 64 & 68 & 72 & 77 & 96 & 100 & \\
\hline $06-04-79$ & 0 & .1 & 2 & 34 & 99 & 100 & & & & & & \\
\hline $06-06-79$ & 0 & .1 & 1 & 24 & 98 & 99 & 100 & & & & & \\
\hline $06-18-79$ & 0.1 & .1 & 2 & 35 & 99 & 100 & & & & & & \\
\hline
\end{tabular}

23. North Fork of Big Lost River at Wild Horse, Idaho

\begin{tabular}{|c|c|c|c|c|c|c|c|c|c|c|c|c|}
\hline $04-27-81$ & -- & -- & 8 & 51 & 86 & 95 & 99 & 100 & & & & \\
\hline $04-30-81$ & -- & -- & 1 & 7 & 23 & 46 & 84 & 97 & 100 & & & \\
\hline $05-07-81$ & - & -- & 1 & 10 & 26 & 38 & 53 & 62 & 76 & 100 & & \\
\hline $05-15-81$ & -- & -- & 1 & 19 & 39 & 55 & 80 & 91 & 100 & & & \\
\hline $05-27-81$ & -- & - & 1 & 7 & 22 & 33 & 52 & 66 & 81 & 83 & 100 & \\
\hline $05-28-81$ & -- & -- & 2 & 13 & 37 & 56 & 81 & 90 & 99 & 100 & & \\
\hline $05-30-81$ & & & 0 & 1 & 4 & 9 & 27 & 46 & 70 & 88 & 97 & 100 \\
\hline $06-01-81$ & -- & -- & 3 & 15 & 32 & 48 & 75 & 86 & 91 & 100 & & \\
\hline $06-03-81$ & -- & -- & 4 & 18 & 36 & 46 & 62 & 74 & 91 & 100 & & \\
\hline $06-05-81$ & -- & -- & 1 & 12 & 28 & 40 & 62 & 74 & 91 & 100 & & \\
\hline $06-09-81$ & -- & -- & 1 & 12 & 31 & 42 & 52 & 62 & 71 & 83 & 100 & \\
\hline $06-10-81$ & & & 0 & 1 & 5 & 10 & 26 & 46 & 71 & 91 & 94 & 100 \\
\hline $06-10-81$ & & & 0 & 4 & 12 & 21 & 41 & 59 & 79 & 89 & 100 & \\
\hline $06-11-81$ & & & 0 & 3 & 10 & 14 & 20 & 32 & 65 & 88 & 100 & \\
\hline $06-16-81$ & & & 0 & 17 & 34 & 45 & 67 & 84 & 95 & 100 & & \\
\hline $06-17-81$ & -- & -- & 1 & 14 & 27 & 46 & 84 & 96 & 100 & & & \\
\hline $06-18-81$ & - & -- & 1 & 41 & 69 & 78 & 89 & 96 & 100 & & & \\
\hline $06-23-81$ & -- & -- & 1 & 10 & 17 & 20 & 23 & 30 & 57 & 100 & & \\
\hline $06-25-81$ & -- & -- & 1 & 17 & 42 & 59 & 82 & 93 & 100 & & & \\
\hline $07-01-81$ & & & 0 & 12 & 27 & 39 & 60 & 69 & 78 & 100 & & \\
\hline $07-09-81$ & -- & -- & 4 & 44 & 67 & 100 & & & & & & \\
\hline $07-20-81$ & -- & -- & 19 & 69 & 88 & 100 & & & & & & \\
\hline
\end{tabular}

24. Big Lost River at Howel1 Ranch, Idaho

$\begin{array}{rrrrrrrrrrrr}04-30-81 & -- & -- & 1 & 8 & 35 & 51 & 68 & 77 & 90 & 100 & \\ 05-07-81 & & & 0 & 6 & 30 & 48 & 69 & 79 & 95 & 100 & 100 \\ 05-15-81 & -- & -- & 4 & 20 & 36 & 45 & 53 & 60 & 84 & 100 \\ 05-27-81 & -- & -- & 1 & 8 & 22 & 27 & 32 & 37 & 59 & 89 & 100 \\ 05-28-81 & -- & -- & 5 & 29 & 48 & 57 & 73 & 83 & 89 & 100 & \end{array}$


Table 3.--Bedload particle-size distributions--Continued

Date

Percent by weight finer than size (millimeters) indicated

$\begin{array}{llllllllllll}0.062 & 0.12 & 0.25 & 0.5 & 1 & 2 & 4 & 8 & 16 & 32 & 64 & 128\end{array}$

24. Big Lost River at Howell Ranch, Idaho--Continued

\begin{tabular}{|c|c|c|c|c|c|c|c|c|c|c|c|}
\hline $05-30-81$ & -- & -- & 2 & 13 & 24 & 29 & 35 & 39 & 48 & 71 & 100 \\
\hline $06-01-81$ & -- & - & 3 & 18 & 37 & 52 & 75 & 94 & 100 & & \\
\hline $06-03-81$ & -- & -- & 1 & 5 & 13 & 17 & 21 & 27 & 35 & 44 & 100 \\
\hline $06-04-81$ & & & 0 & 24 & 54 & 69 & 82 & 89 & 92 & 100 & \\
\hline $06-05-81$ & & & 0 & 19 & 59 & 74 & 82 & 88 & 92 & 100 & \\
\hline $06-09-81$ & & & 0 & 1 & 3 & 13 & 21 & 44 & 74 & 94 & 100 \\
\hline $06-09-81$ & & & 0 & 3 & 14 & 33 & 55 & 72 & 86 & 93 & 100 \\
\hline $06-10-81$ & & & 0 & 28 & 60 & 74 & 84 & 87 & 90 & 100 & \\
\hline $06-11-81$ & & & 0 & 3 & 10 & 15 & 31 & 53 & 81 & 99 & 100 \\
\hline $06-16-81$ & & & 0 & 20 & 51 & 61 & 72 & 77 & 83 & 92 & 100 \\
\hline $06-17-81$ & & & 0 & 16 & 33 & 46 & 71 & 85 & 88 & 100 & \\
\hline $06-18-81$ & -- & -- & 1 & 25 & 57 & 69 & 87 & 97 & 100 & & \\
\hline $06-23-81$ & & & 0 & 27 & 57 & 65 & 71 & 80 & 87 & 100 & \\
\hline $06-25-81$ & & & 0 & 8 & 20 & 32 & 60 & 78 & 91 & 100 & \\
\hline $07-01-81$ & -- & - & 1 & 22 & 63 & 77 & 90 & 98 & 100 & & \\
\hline
\end{tabular}

\section{Big Lost River at Chilly Bridge, Idaho}

\begin{tabular}{|c|c|c|c|c|c|c|c|c|c|c|c|c|}
\hline $04-30-81$ & -- & 1 & 4 & 20 & 43 & 55 & 74 & 89 & 97 & 100 & & \\
\hline $05-07-81$ & & 0 & 4 & 61 & 97 & 100 & & & & & & \\
\hline $05-11-81$ & & 0 & 7 & 29 & 59 & 76 & 93 & 100 & & & & \\
\hline $05-14-81$ & & 0 & 7 & 15 & 78 & 90 & 100 & & & & & \\
\hline $05-15-81$ & & 0 & 4 & 35 & 70 & 83 & 95 & 100 & & & & \\
\hline $05-27-81$ & & 0 & 4 & 29 & 65 & 76 & 86 & 90 & 92 & 100 & & \\
\hline $05-28-81$ & & 0 & 1 & 4 & 15 & 24 & 38 & 50 & 63 & 82 & 100 & \\
\hline $05-30-81$ & & & 0 & 5 & 14 & 21 & 31 & 40 & 53 & 73 & 89 & 100 \\
\hline $06-01-81$ & & 0 & 2 & 16 & 28 & 32 & 38 & 41 & 45 & 53 & 100 & \\
\hline $06-03-81$ & -- & 1 & 5 & 34 & 60 & 70 & 83 & 92 & 100 & & & \\
\hline $06-04-81$ & & 0 & 1 & 7 & 25 & 38 & 56 & 68 & 80 & 91 & 100 & \\
\hline $06-05-81$ & & 0 & 1 & 12 & 33 & 42 & 51 & 57 & 71 & 92 & 100 & \\
\hline $06-09-81$ & & 0 & 1 & 13 & 28 & 36 & 49 & 59 & 73 & 84 & 92 & 100 \\
\hline $06-10-81$ & & 0 & 1 & 29 & 68 & 82 & 89 & 92 & 94 & 100 & & \\
\hline $06-11-81$ & & & 0 & 2 & 7 & 10 & 15 & 25 & 47 & 77 & 100 & \\
\hline $06-11-81$ & & & 0 & 4 & 16 & 20 & 28 & 37 & 50 & 74 & 100 & \\
\hline $06-16-81$ & & 0 & 1 & 37 & 69 & 81 & 92 & 97 & 100 & & & \\
\hline $06-16-81$ & & 0 & 1 & 27 & 56 & 69 & 84 & 92 & 100 & & & \\
\hline $06-17-81$ & & 0 & 1 & 21 & 51 & 62 & 75 & 82 & 94 & 100 & & \\
\hline $06-18-81$ & & 0 & 1 & 22 & 54 & 68 & 85 & 90 & 100 & & & \\
\hline $06-23-81$ & & & 0 & 11 & 36 & 48 & 63 & 73 & 87 & 100 & & \\
\hline $06-25-81$ & & 0 & 1 & 24 & 45 & 52 & 59 & 63 & 71 & 78 & 100 & \\
\hline $07-01-81$ & & & 0 & 10 & 31 & 40 & 45 & 46 & 49 & 51 & 100 & \\
\hline $07-01-81$ & & 0 & 1 & 16 & 42 & 53 & 66 & 73 & 85 & 100 & & \\
\hline $07-09-81$ & & 0 & 2 & 32 & 64 & 77 & 94 & 100 & & & & \\
\hline
\end{tabular}

26. Big Lost River below Chilly Sinks, Idaho

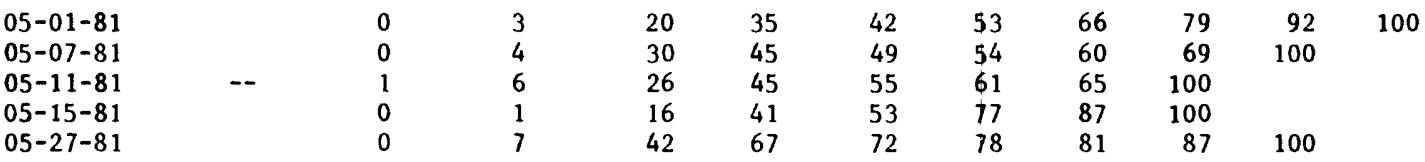


Table 3,--Bedload particle-size distributions--Continued

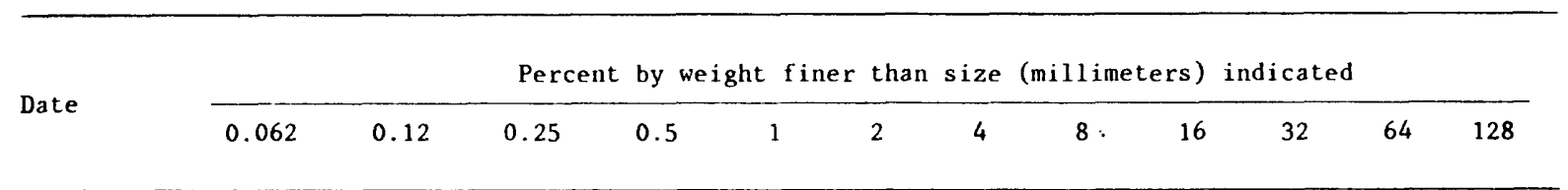

26. Big Lost River below Chilly Sinks, Idaho--Continued

\begin{tabular}{|c|c|c|c|c|c|c|c|c|c|c|c|}
\hline \multirow{3}{*}{$\begin{array}{l}05-28-81 \\
05-30-81 \\
06-01-81 \\
06-03-81 \\
06-03-81\end{array}$} & & 0 & 4 & 22 & 35 & 37 & 41 & 42 & 44 & 46 & 100 \\
\hline & & 0 & 2 & 13 & 40 & 54 & 64 & 70 & 79 & 100 & \\
\hline & & 0 & 9 & 51 & 78 & 84 & 90 & 95 & 100 & & \\
\hline $06-04-81$ & & 0 & 1 & 15 & 31 & 37 & 46 & 55 & 68 & 83 & 100 \\
\hline $06-05-81$ & & 0 & 1 & 17 & 50 & 65 & 86 & 91 & 97 & 100 & \\
\hline $06-10-81$ & & 0 & 1 & 6 & 20 & 24 & 32 & 42 & 60 & 87 & 100 \\
\hline $06-10-81$ & & 0 & 1 & 6 & 20 & 24 & 32 & 42 & 60 & 87 & 100 \\
\hline $06-11-81$ & & 0 & 1 & 20 & 40 & 47 & 57 & 66 & 78 & 84 & 100 \\
\hline $06-12-81$ & & 0 & 1 & 16 & 29 & 54 & 66 & 72 & 78 & 82 & 100 \\
\hline $06-23-81$ & & 0 & 1 & 22 & 58 & 68 & 75 & 80 & 88 & 100 & \\
\hline $06-25-81$ & & 0 & 1 & 9 & 26 & 40 & 57 & 64 & 74 & 100 & \\
\hline $07-01-81$ & & 0 & 1 & 14 & 39 & 54 & 70 & 78 & 87 & 90 & 100 \\
\hline $07-09-81$ & & 0 & 1 & 19 & 52 & 67 & 82 & 93 & 100 & & \\
\hline $07-23-81$ & & 0 & 14 & 62 & 88 & 100 & & & & & \\
\hline
\end{tabular}

27. Big Lost River above East and West Channels, Idaho

\begin{tabular}{|c|c|c|c|c|c|c|c|c|c|c|c|}
\hline $05-01-81$ & -- & 1 & 3 & 12 & 25 & 31 & 42 & 54 & 70 & 100 & \\
\hline $05-07-81$ & -- & 1 & 2 & 25 & 59 & 68 & 75 & 85 & 100 & & \\
\hline $05-11-81$ & -- & 1 & 1 & 8 & 37 & 55 & 72 & 86 & 100 & & \\
\hline $05-15-81$ & & 0 & 1 & 16 & 33 & 42 & 56 & 67 & 82 & 100 & \\
\hline $05-27-81$ & & 0 & 4 & 35 & 60 & 66 & 74 & 77 & 79 & 82 & 100 \\
\hline $05-28-81$ & & 0 & 8 & 51 & 85 & 92 & 98 & 99 & 100 & & \\
\hline $05-30-81$ & & 0 & 1 & 2 & 6 & 10 & 21 & 36 & 58 & 82 & 100 \\
\hline $06-01-81$ & & 0 & 2 & 15 & 29 & 36 & 48 & 58 & 70 & 81 & 100 \\
\hline $06-04-81$ & & 0 & 1 & 6 & 25 & 37 & 57 & 70 & 83 & 100 & \\
\hline $06-05-81$ & & 0 & 1 & 9 & 28 & 40 & 57 & 69 & 83 & 93 & 100 \\
\hline $06-10-81$ & & 0 & 2 & 13 & 24 & 31 & 42 & 53 & 80 & 100 & \\
\hline $06-10-81$ & & 0 & 1 & 3 & 9 & 12 & 21 & 37 & 66 & 95 & 100 \\
\hline $06-11-81$ & & 0 & 1 & 27 & 56 & 67 & 79 & 85 & 96 & 100 & \\
\hline $06-11-81$ & & 0 & 10 & 36 & 44 & 47 & 50 & 51 & 66 & 100 & \\
\hline $06-16-81$ & & 0 & 1 & 13 & 45 & 65 & 84 & 92 & 100 & & \\
\hline $06-17-81$ & & 0 & 1 & 2 & 4 & 5 & 9 & 15 & 32 & 88 & 100 \\
\hline $06-18-81$ & & 0 & 1 & 11 & 31 & 49 & 65 & 84 & 100 & & \\
\hline $06-23-81$ & & 0 & 1 & 23 & 71 & 91 & 99 & 100 & & & \\
\hline $06-25-81$ & & 0 & 1 & 15 & 45 & 74 & 92 & 100 & & & \\
\hline $07-01-81$ & & 0 & 1 & 2 & 8 & 14 & 29 & 43 & 67 & 87 & 100 \\
\hline $07-09-81$ & & 0 & 1 & 9 & 40 & 54 & 72 & 82 & 100 & & \\
\hline $07-22-81$ & & 0 & 1 & 16 & 50 & 69 & 90 & 100 & & & \\
\hline
\end{tabular}


Table 3.--Bedload particle-size distributions--Continued

Percent by weight finer than size (millimeters) indicated

Date

\begin{tabular}{llllllllllll}
\hline 0.062 & 0.12 & 0.25 & 0.5 & 1 & 2 & 4 & 8 & 16 & 32 & 64 & 128
\end{tabular}

31. Muddy Creek near Pinedale, Wyo.

\begin{tabular}{|c|c|c|c|c|c|c|c|c|c|c|c|}
\hline $\begin{array}{l}04-21-75 \\
04-22-75 \\
04-23-75 \\
04-24-75 \\
04-25-75\end{array}$ & $\begin{array}{l}1 \\
2 \\
2 \\
2 \\
1\end{array}$ & $\begin{array}{l}3 \\
5 \\
4 \\
5 \\
3\end{array}$ & $\begin{array}{l}25 \\
28 \\
23 \\
26 \\
20\end{array}$ & $\begin{array}{l}62 \\
74 \\
60 \\
56 \\
57\end{array}$ & $\begin{array}{l}86 \\
94 \\
86 \\
85 \\
70\end{array}$ & $\begin{array}{l}98 \\
99 \\
98 \\
96 \\
90\end{array}$ & $\begin{array}{r}100 \\
100 \\
100 \\
100 \\
99\end{array}$ & -- & -- & - & -- \\
\hline $\begin{array}{l}04-26-75 \\
04-27-75 \\
04-28-75 \\
04-29-75 \\
04-30-75\end{array}$ & $\begin{array}{l}0.5 \\
1 \\
0.4 \\
.3 \\
.7\end{array}$ & $\begin{array}{l}2 \\
3 \\
0.8 \\
.5\end{array}$ & $\begin{array}{r}17 \\
26 \\
9 \\
5 \\
14\end{array}$ & $\begin{array}{l}55 \\
70 \\
43 \\
39 \\
49\end{array}$ & $\begin{array}{l}79 \\
89 \\
79 \\
81 \\
84\end{array}$ & $\begin{array}{l}93 \\
97 \\
95 \\
97 \\
97\end{array}$ & $\begin{array}{r}99 \\
100 \\
100 \\
100 \\
100\end{array}$ & -- & -- & - & -- \\
\hline $\begin{array}{l}05-01-75 \\
05-02-75 \\
05-03-75 \\
05-03-75 \\
05-04-75\end{array}$ & $\begin{array}{l}.2 \\
.3 \\
.2 \\
.3 \\
.1\end{array}$ & $\begin{array}{l}0.5 \\
.7 \\
.3 \\
2 \\
0.1\end{array}$ & $\begin{array}{r}7 \\
10 \\
7 \\
14 \\
3\end{array}$ & $\begin{array}{l}41 \\
48 \\
35 \\
67 \\
30\end{array}$ & $\begin{array}{l}78 \\
80 \\
65 \\
82 \\
63\end{array}$ & $\begin{array}{l}95 \\
95 \\
86 \\
96 \\
87\end{array}$ & $\begin{array}{r}100 \\
100 \\
98 \\
100 \\
98\end{array}$ & 100 & & & \\
\hline $\begin{array}{l}05-06-75 \\
05-08-75 \\
05-09-75 \\
05-23-75 \\
06-20-75 \\
07-11-75\end{array}$ & $\begin{array}{l}.1 \\
.2 \\
.1 \\
.2 \\
.1 \\
.1\end{array}$ & $\begin{array}{l}.2 \\
.5 \\
.3 \\
.3 \\
.1 \\
.1\end{array}$ & $\begin{array}{l}1 \\
9 \\
9 \\
5 \\
3 \\
3\end{array}$ & $\begin{array}{l}18 \\
28 \\
41 \\
37 \\
29 \\
32\end{array}$ & $\begin{array}{l}56 \\
63 \\
72 \\
74 \\
62 \\
67\end{array}$ & $\begin{array}{l}86 \\
90 \\
92 \\
94 \\
86 \\
89\end{array}$ & $\begin{array}{r}98 \\
99 \\
99 \\
100 \\
98 \\
99\end{array}$ & $\begin{array}{l}100 \\
100 \\
100\end{array}$ & & . & \\
\hline
\end{tabular}

32. East Fork River near Pinedale, Wyo.

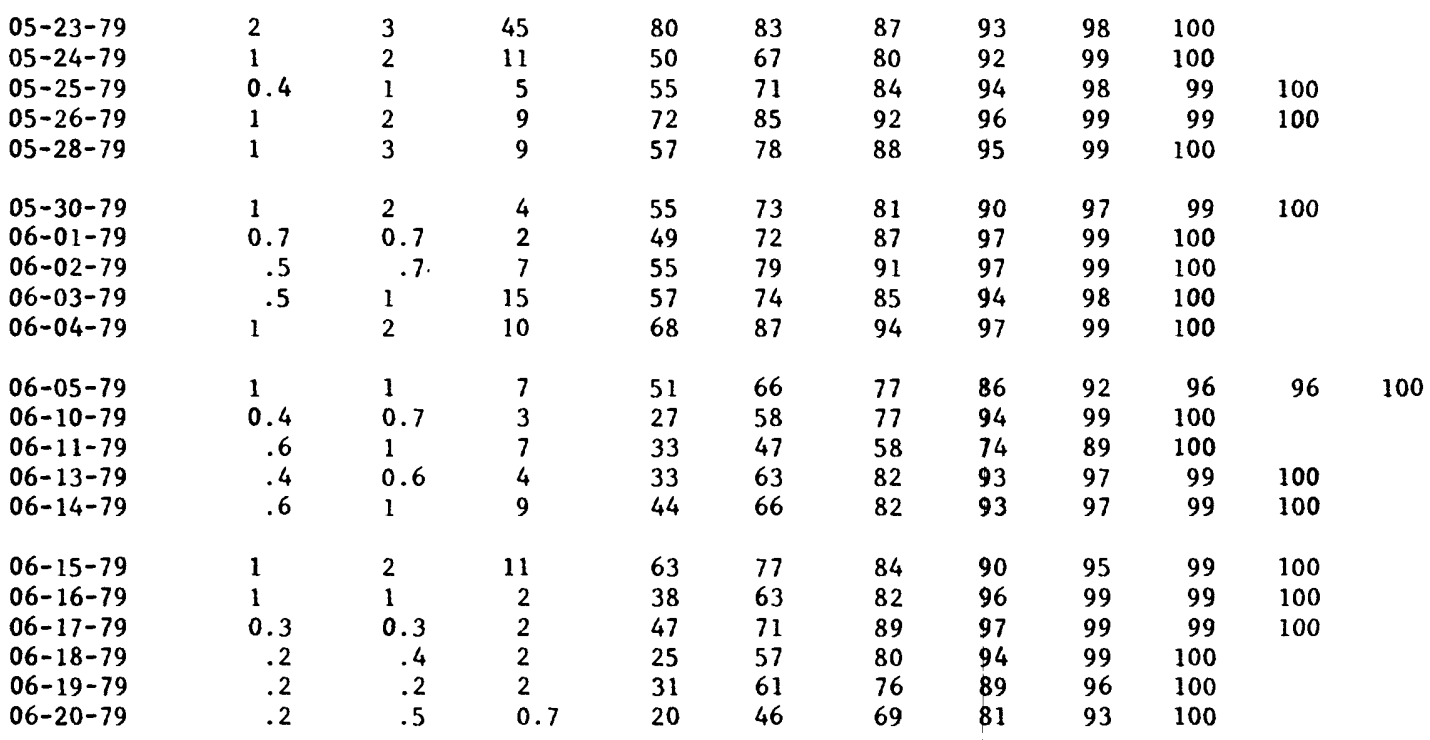


Table 3.--Bedload particle-size distributions--Continued

\begin{tabular}{|c|c|c|c|c|c|c|c|c|c|c|c|c|}
\hline & & & Percent & by weight & $t$ finer & than & size & (millime & ters) & indicated & & \\
\hline & 0.062 & 0.12 & 0.25 & 0.5 & 1 & 2 & 4 & 8 & 16 & 32 & 64 & 128 \\
\hline
\end{tabular}

33. Yampa River at Deerlodge Park, Colo.

\begin{tabular}{|c|c|c|c|c|c|c|c|c|c|c|}
\hline $04-27-82$ & 0 & 1 & 6 & 47 & 87 & 96 & 98 & 99 & 100 & \\
\hline $05-12-82$ & & 0 & 7 & 49 & 85 & 94 & 97 & 99 & 100 & \\
\hline $05-13-82$ & & 0 & 4 & 36 & 72 & 89 & 95 & 98 & 100 & \\
\hline $05-24-82$ & 0 & 1 & 15 & 82 & 96 & 99 & 99 & 100 & & \\
\hline $05-25-82$ & 0 & 1 & 14 & 77 & 94 & 98 & 98 & 99 & 100 & \\
\hline $06-08-82$ & & 0 & 7 & 63 & 95 & 99 & 100 & & & \\
\hline $06-24-82$ & & 0 & 3 & 49 & 85 & 96 & 99 & 100 & & \\
\hline $06-25-82$ & & 0 & 2 & 39 & 82 & 96 & 99 & 100 & & \\
\hline $07-07-82$ & & 0 & 1 & 18 & 71 & 92 & 98 & 100 & & \\
\hline $07-08-82$ & & 0 & 1 & 16 & 62 & 86 & 94 & 98 & 100 & \\
\hline $07-29-82$ & & 0 & 4 & 30 & 76 & 93 & 98 & 100 & & \\
\hline $04-07-83$ & & 0 & 7 & 64 & 92 & 99 & 100 & & & \\
\hline $04-08-83$ & & 0 & 3 & 53 & 88 & 98 & 100 & & & \\
\hline $04-19-83$ & & 0 & 4 & 51 & 88 & 97 & 99 & 100 & & \\
\hline $04-21-83$ & 0 & 1 & 7 & 50 & 87 & 95 & 98 & 100 & & \\
\hline $04-22-83$ & 0 & 1 & 8 & 52 & 85 & 95 & 98 & 99 & 100 & \\
\hline $05-07-83$ & & 0 & 2 & 35 & 87 & 97 & 98 & 99 & 100 & \\
\hline $05-09-83$ & & 0 & 2 & 35 & 86 & 97 & 99 & 99 & 100 & \\
\hline $05-12-83$ & 0 & 1 & 3 & 27 & 79 & 96 & 98 & 99 & 100 & \\
\hline $05-23-83$ & & 0 & 4 & 29 & 65 & 89 & 94 & 97 & 98 & 100 \\
\hline $05-26-83$ & 0 & 2 & 6 & 50 & 90 & 96 & 98 & 100 & & \\
\hline $05-27-83$ & 0 & 1 & 4 & 39 & 91 & 97 & 99 & 100 & & \\
\hline $05-28-83$ & 0 & 2 & 8 & 62 & 95 & 99 & 100 & & & \\
\hline $06-08-83$ & 0 & 1 & 8 & 50 & 79 & 90 & 94 & 95 & 98 & 100 \\
\hline $06-10-83$ & & 0 & 4 & 33 & 90 & 98 & 99 & 100 & & \\
\hline $06-21-83$ & 0 & 1 & 4 & 40 & 92 & 98 & 99 & 100 & & \\
\hline $06-23-83$ & 0 & 1 & 5 & 44 & 92 & 99 & 100 & & & \\
\hline $07-12-83$ & & 0 & 4 & 36 & 89 & 98 & 99 & 100 & & \\
\hline $07-14-83$ & & 0 & 4 & 42 & 87 & 98 & 100 & & & \\
\hline
\end{tabular}

34. Rock Creek near Toponas, Colo.

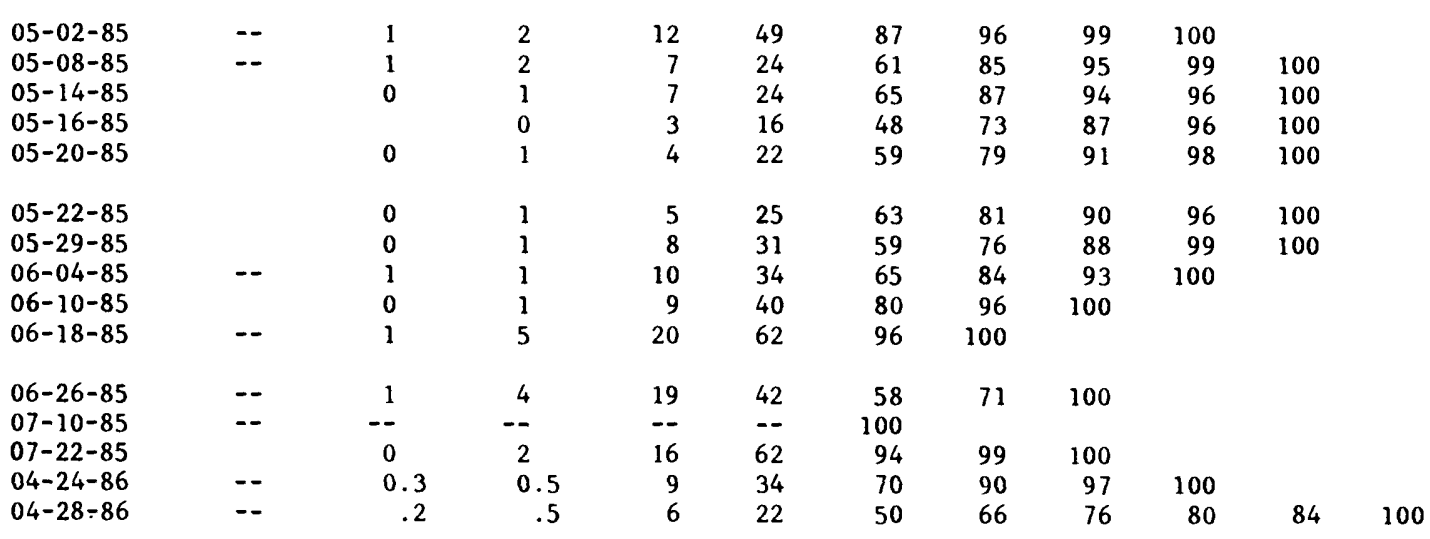


Table 3.--Bedload particle-size distributions--Continued

\begin{tabular}{ccccccccccccc}
\hline & \multicolumn{4}{c}{ Percent by weight finer than size (millimeters) } & indicated \\
\cline { 2 - 10 } & 0.062 & 0.12 & 0.25 & 0.5 & 1 & 2 & 4 & 8 & 16 & 32 & 64 & 128 \\
\hline
\end{tabular}

34. Rock Creek near Toponas, Colo.

$\begin{array}{rrrrrrrrrrrr}05-12-86 & -- & .2 & .4 & 9 & 34 & 58 & 70 & 77 & 85 & 100 & 65 \\ 05-15-86 & -- & .1 & .2 & 6 & 23 & 43 & 55 & 61 & 64 & 68 & 100 \\ 05-20-86 & -- & 1.1 & 6 & 14 & 25 & 37 & 66 & 80 & 90 & 100 & \\ 05-21-86 & -- & 0.1 & 0.3 & 8 & 34 & 59 & 72 & 81 & 92 & 94 & 100 \\ 05-27-86 & -- & .2 & .3 & 6 & 26 & 51 & 69 & 79 & 89 & 98 & 100 \\ & & & .1 & .2 & 5 & 28 & 56 & 72 & 81 & 88 & 95 \\ 05-29-86 & -- & .3 & .5 & 12 & 38 & 57 & 69 & 78 & 86 & 95 & 100 \\ 06-09-86 & -- & .8 & 2 & 12 & 35 & 70 & 92 & 96 & 100 & & \end{array}$

\section{McIntyre Creek near Glendevey Colo. ${ }^{4}, 5$}

$\begin{array}{rrrrrrrrrrr}05-12-77 & -- & 10 & 23 & 38 & 57 & 76 & 95 & 100 & & \\ 05-27-77 & -- & 12 & 28 & 43 & 61 & 78 & 94 & 100 & & \\ 05-31-77 & -- & 9 & 21 & 35 & 55 & 75 & 95 & 100 & & \\ 06-06-77 & -- & 5 & 13 & 24 & 45 & 65 & 86 & 94 & 98 & 100 \\ 06-14-77 & -- & 2 & 6 & 14 & 33 & 52 & 71 & 89 & 100 & \\ 06-22-77 & -- & 8 & 19 & 31 & 49 & 65 & 82 & 93 & 100 \\ 06-28-77 & -- & 13 & 30 & 46 & 63 & 79 & 96 & 100 & \\ 07-06-77 & -- & 12 & 27 & 43 & 60 & 78 & 95 & 100 & \\ 07-12-77 & -- & 14 & 33 & 50 & 66 & 81 & 96 & 100 & \\ 07-19-77 & -- & 12 & 28 & 45 & 62 & 79 & 96 & 100 & \end{array}$

39. Cabin Creek at Mouth near Granby, Colo. ${ }^{4}, 5$

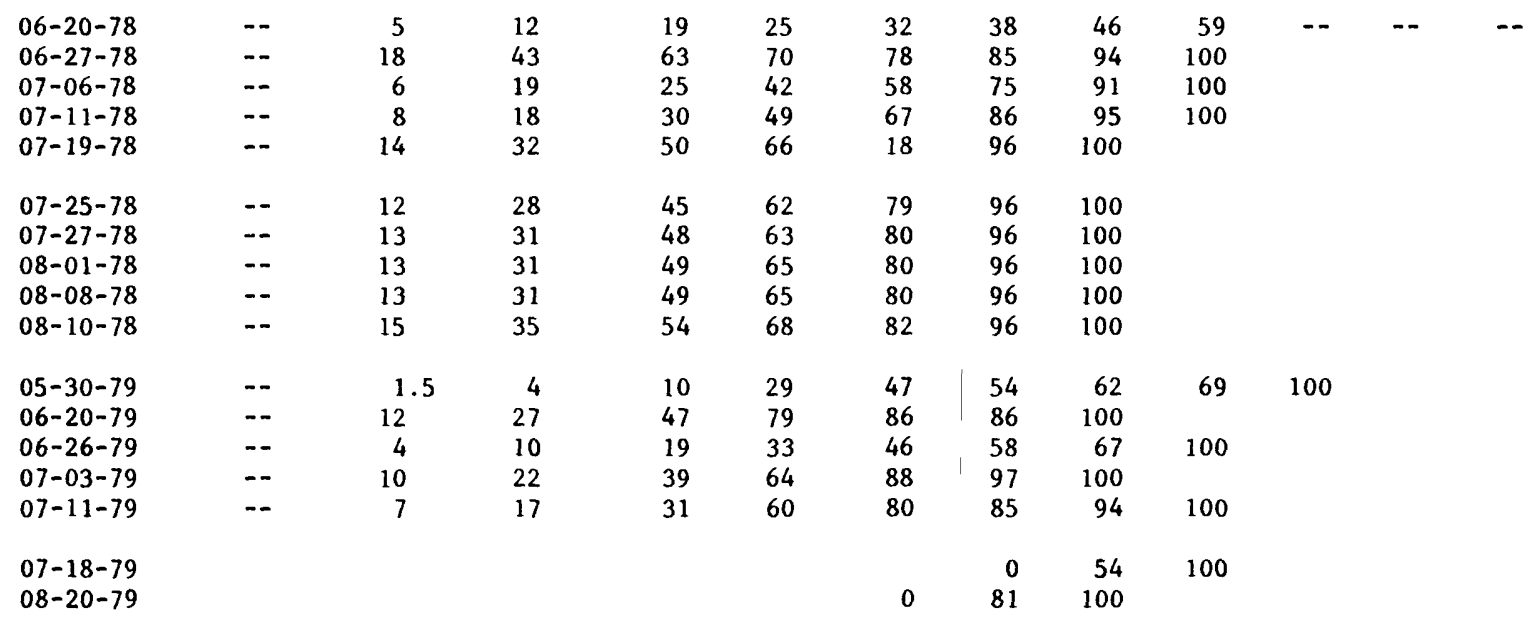

40. Lower North Fork of Cabin Creek near Granby, Colo. ${ }^{4}, 5$

$\begin{array}{rrrrrrrrrr}06-22-78 & -- & 3 & 8 & 15 & 31 & 46 & 62 & 73 & 81 \\ 06-27-78 & -- & 10 & 25 & 39 & 52 & 66 & 80 & 92 & 100 \\ 07-07-78 & -- & 9 & 22 & 35 & 50 & 66 & 81 & 93 & 100 \\ 07-11-78 & -- & 13 & 32 & 48 & 65 & 80 & 95 & 100 & \\ 07-20-78 & -- & 15 & 36 & 54 & 68 & 82 & 96 & 100 & \end{array}$


Table 3.--Bedload particle-size distributions--Continued

\begin{tabular}{|c|c|c|c|c|c|c|c|c|c|c|c|c|}
\hline \multirow{2}{*}{ Date } & \multicolumn{4}{|c|}{ Percent by weight } & it finer & \multicolumn{2}{|c|}{ than size } & \multicolumn{2}{|c|}{ (millimeters) } & \multicolumn{3}{|l|}{ indicated } \\
\hline & 0.062 & 0.12 & 0.25 & 0.5 & 1 & 2 & 4 & 8 & 16 & 32 & 64 & 128 \\
\hline
\end{tabular}

40. Lower North Fork of Cabin Creek near Granby, Colo, 4,5--Continued

$\begin{array}{rrrrrrrrrrrr}07-25-78 & -- & 15 & 36 & 54 & 68 & 82 & 96 & 100 & 58 & -- & -- \\ 06-19-79 & -- & 3 & 6 & 11 & 22 & 33 & 42 & 49 & -- \\ 06-26-79 & -- & 3 & 6 & 18 & 59 & 83 & 97 & 99 & 100 \\ 07-03-79 & -- & 12 & 28 & 46 & 75 & 87 & 97 & 100 & 100 \\ 07-10-79 & -- & 4 & 9 & 18 & 43 & 59 & 92 & 100 \\ 07-16-79 & -- & 8 & 19 & 32 & 51 & 86 & 97 & 100 & \end{array}$

41. Upper North Fork of Cabin Creek near Granby, Colo.4,5

\begin{tabular}{|c|c|c|c|c|c|c|c|c|c|c|c|}
\hline $06-20-78$ & -- & 5 & 12 & 21 & 35 & 49 & 63 & 84 & 100 & & \\
\hline $06-28-78$ & -- & 5 & 11 & 19 & 30 & 42 & 53 & 64 & 84 & -- & - \\
\hline $07-06-78$ & -- & 9 & 20 & 33 & 51 & 69 & 88 & 96 & 100 & & \\
\hline $07-11-78$ & -- & 14 & 33 & 50 & 65 & 81 & 96 & 100 & & & \\
\hline $07-20-78$ & -- & 13 & 30 & 47 & 63 & 79 & 95 & 100 & & & \\
\hline $07-25-78$ & -- & 10 & 23 & 38 & 57 & 76 & 95 & 100 & & & \\
\hline $07-27-78$ & -- & 12 & 28 & 45 & 62 & 79 & 95 & 100 & & & \\
\hline $08-10-78$ & -- & 15 & 35 & 54 & 68 & 82 & 96 & 100 & & & \\
\hline $06-25-79$ & -- & 3 & 7 & 19 & 60 & 71 & 86 & 95 & 100 & & \\
\hline
\end{tabular}

42. Lower Left Fork of North Fork of Cabin Creek near Granby, Colo. ${ }^{4,5}$

\begin{tabular}{|c|c|c|c|c|c|c|c|c|c|c|c|}
\hline $06-21-78$ & -- & 4 & 10 & 17 & 31 & 44 & 58 & 74 & 94 & 100 & \multirow{5}{*}{--} \\
\hline $06-28-78$ & - & 3 & 7 & 13 & 22 & 32 & 41 & 47 & 53 & -- & \\
\hline $07-06-78$ & - & 4 & 11 & 18 & 33 & 48 & 63 & 80 & 97 & 100 & \\
\hline $07-20-78$ & -- & 5 & 11 & 19 & 32 & 45 & 58 & 82 & 100 & & \\
\hline $07-25-78$ & -- & 9 & 23 & 36 & 56 & 75 & 95 & 100 & & & \\
\hline $08-03-78$ & -- & 6 & 14 & 24 & 41 & 57 & 75 & 90 & 100 & & \\
\hline $08-08-78$ & -- & 4 & 10 & 17 & 27 & 36 & 46 & 76 & 100 & & \\
\hline $06-18-79$ & -- & 7 & 18 & 37 & 88 & 100 & & & & & \\
\hline $06-25-79$ & & & 0 & 6 & 38 & 45 & 69 & 88 & 100 & & \\
\hline $07-09-79$ & -- & 10 & 24 & 41 & 70 & 100 & & & & & \\
\hline $07-16-79$ & & & & & & & 0 & 53 & 100 & & \\
\hline
\end{tabular}

43. Upper Left Fork of North Fork of Cabin Creek near Granby, Colo. 4,5

\begin{tabular}{|c|c|c|c|c|c|c|c|c|c|c|c|c|}
\hline $06-21-78$ & -- & 2 & 4 & 7 & 14 & 21 & 28 & 33 & 39 & -- & -- & -- \\
\hline $06-28-78$ & -- & 5 & 11 & 19 & 31 & 43 & 56 & 67 & 83 & -- & -- & -- \\
\hline $07-06-78$ & -- & 7 & 16 & 27 & 41 & 56 & 70 & 88 & 100 & & & \\
\hline $07-11-78$ & -- & 6 & 14 & 23 & 38 & 53 & 68 & 86 & 100 & & & \\
\hline $07-20-78$ & -- & 18 & 43 & 65 & 75 & 86 & 97 & 100 & & & & \\
\hline $07-25-78$ & -- & 12 & 28 & 44 & 61 & 78 & 95 & 100 & & & & \\
\hline $07-27-78$ & -- & 13 & 31 & 47 & 64 & 80 & 95 & 100 & & & & \\
\hline $08-08-78$ & -- & 14 & 32 & 49 & 65 & 80 & 96 & 100 & & & & \\
\hline $06-18-79$ & -- & 4 & 9 & 20 & 48 & 58 & 73 & 89 & 99 & -- & -- & -- \\
\hline $06-25-79$ & -- & 8 & 17 & 33 & 70 & 79 & 93 & 98 & 100 & & & \\
\hline
\end{tabular}


Table 3.--Bedload particle-size distributions--Continued

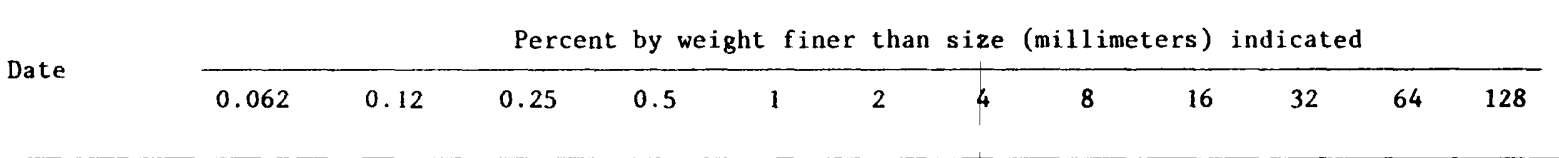

\begin{tabular}{|c|c|c|c|c|c|c|c|c|c|c|c|c|}
\hline & & & 44. & Upper E1k & Creek near & Granby & & $.4,5$ & & & & \\
\hline $06-19-78$ & -- & 3 & 7 & 10 & 11 & 12 & 13 & 18 & 25 & -- & -- & -- \\
\hline $06-26-78$ & -- & 5 & 12 & 21 & 40 & 58 & 76 & 91 & 100 & & & \\
\hline $07-05-78$ & -- & 4 & 10 & 19 & 37 & 56 & 74 & 84 & 95 & 100 & & \\
\hline $07-10-78$ & -- & 5 & 12 & 22 & 41 & 60 & 79 & 92 & 100 & & & \\
\hline $07-13-78$ & -- & 4 & 10 & 19 & 38 & 57 & 76 & 91 & 100 & & & \\
\hline $07-17-78$ & -- & 5 & 11 & 21 & 41 & 60 & 79 & 93 & 100 & & & \\
\hline $06-20-79$ & -- & 4 & 8 & 17 & 44 & 74 & 95 & 100 & & & & \\
\hline $06-26-79$ & -- & 10 & 24 & 41 & 75 & 83 & 93 & 98 & 100 & & & \\
\hline $07-05-79$ & - & 5 & 12 & 24 & 58 & 87 & 95 & 99 & 100 & & & \\
\hline $07-10-79$ & -- & 5 & 12 & 24 & 49 & 70 & 86 & 95 & 100 & & & \\
\hline $07-18-79$ & $-\cdot$ & 1.5 & 3 & 10 & 33 & 50 & 85 & 97 & 100 & & & \\
\hline
\end{tabular}

50. Fourmile Creek near Fairplay, Colo.

\begin{tabular}{|c|c|c|c|c|c|c|c|c|c|c|c|}
\hline $05-16-84$ & 0 & 0.3 & 2 & 12 & 55 & 85 & 92 & 100 & & & \\
\hline $05-24-84$ & 0 & .1 & 2 & 9 & 25 & 38 & 41 & 43 & 47 & 47 & 100 \\
\hline $05-29-84$ & 0 & .7 & 4 & 10 & 29 & 54 & 63 & 69 & 92 & 100 & \\
\hline $05-31-84$ & 0 & .3 & 3 & 13 & 42 & 64 & 69 & 71 & 75 & 100 & \\
\hline $06-06-84$ & 0 & 4 & 17 & 38 & 69 & 93 & 100 & & & & \\
\hline $06-12-84$ & 0 & 0.1 & 0.5 & 1 & 5 & 16 & 18 & 19 & 23 & 23 & 100 \\
\hline $06-14-84$ & 0 & .1 & 1 & 5 & 18 & 37 & 43 & 50 & 60 & 100 & \\
\hline $06-18-84$ & 0 & .5 & 1 & 4 & 19 & 24 & 32 & 34 & 44 & 100 & \\
\hline $06-22-84$ & 0 & .5 & 3 & 9 & 27 & 54 & 61 & 64 & 80 & 100 & \\
\hline $06-26-84$ & 0 & .2 & 1 & 3 & 11 & 23 & 25 & 28 & 37 & 74 & 100 \\
\hline $06-29-84$ & 0 & .3 & 1 & 4 & 15 & 28 & 31 & 32 & 55 & 100 & \\
\hline $07-03-84$ & 0 & 1 & 8 & 22 & 54 & 77 & 87 & 94 & 100 & & \\
\hline $07-06-84$ & & 0 & 1 & 6 & 11 & 85 & 93 & 100 & & & \\
\hline $07-10-84$ & 0 & 0.2 & 0.5 & 3 & 19 & 41 & 54 & 66 & 100 & & \\
\hline $07-17-84$ & 0 & 0.1 & 0.3 & 0.6 & 2 & 5 & 6 & 7 & 14 & 58 & 100 \\
\hline $07-24-84$ & 0 & .1 & .7 & 4 & 18 & 42 & 48 & 54 & 54 & 78 & 100 \\
\hline $08-03-84$ & 0 & .9 & 3 & 6 & 20 & 29 & 32 & 36 & 41 & 100 & \\
\hline $08-08-84$ & 0 & 3 & 9 & 20 & 74 & 93 & 96 & 100 & & & \\
\hline
\end{tabular}

51. Mad Creek (Site 1) near Empire, Colo. 5

$05-29-84$

06-06-84

06-18-84

$06-25-84$

$07-03-84$

$\begin{array}{rrrrrrr}0 & 22 & 44 & 67 & 78 & 89 & 100 \\ 17 & 33 & 50 & 67 & 83 & 100 & \\ 0 & 11 & 22 & 44 & 67 & 89 & 100 \\ & 0 & 14 & 43 & 71 & 86 & 100 \\ 0 & 11 & 22 & 44 & 78 & 100 & \end{array}$


Table 3.--Bedload particle-size distributions--Continued

\begin{tabular}{|c|c|c|c|c|c|c|c|c|c|c|c|c|}
\hline & & & Percent & by weight & t finer & than & size & (millimet & ers) & indicated & & \\
\hline & 0.062 & 0.12 & 0.25 & 0.5 & 1 & 2 & 4 & 8 & 16 & 32 & 64 & 128 \\
\hline
\end{tabular}

52. Mad Creek (Site 3) near Empire, Colo. 5

06-06-84 06-18-84 06-25-84 07-03-84 07-12-84

05-14-84 05-17-84 05-23-84 06-04-84 06-08-84

06-15-84 06-20-84 06-27-84 07-02-84 07-11-84

07-19-84 07-27-84

05-16-84 05-26-84 06-07-84 06-13-84 06-28-84

07-10-84 07-25-84 09-19-84
0 0

2
0
2
0
0

53. Middle Fork of Boulder Creek at Nederland, Colo.

$\begin{array}{rrrrrrr}0 & 9 & 32 & 55 & 82 & 95 & 100 \\ 0 & 22 & 56 & 78 & 100 & & \\ 0 & 20 & 50 & 70 & 90 & 100 & \\ 0 & 10 & 30 & 60 & 90 & 100 & \\ 0 & -- & -- & 100 & & & \\ 0 & 11 & 28 & 44 & 67 & 83 & 100 \\ 0 & 15 & 38 & 54 & 69 & 85 & 100 \\ 0 & 11 & 44 & 67 & 89 & 100 & \\ 0 & 7 & 29 & 62 & 73 & 91 & 100 \\ 0 & 9 & 27 & 45 & 73 & 82 & 100 \\ 0 & 10 & 25 & 55 & 85 & 100 & \\ 0 & 11 & 39 & 72 & 89 & 100 & \end{array}$

54. Jefferson Creek near Jefferson, Colo.

0

$\begin{array}{rrrrrrr}4 & 19 & 41 & 63 & 81 & 93 & 100 \\ 0 & 13 & 33 & 50 & 67 & 83 & 100 \\ 0 & 9 & 27 & 45 & 64 & 82 & 100 \\ 0 & 18 & 45 & 64 & 82 & 100 & \\ 0 & 15 & 38 & 54 & 77 & 92 & 100 \\ 0 & 20 & 47 & 60 & 73 & 87 & 100 \\ 0 & 10 & 25 & 47 & 73 & 92 & 100 \\ 0 & 17 & 33 & 50 & 67 & 83 & 100\end{array}$

55. Craig Creek near Bailey, Colo.

04-16-84 04-26-84 04-30-84 05-03-84 05-10-84

05-15-84 05-17-84 05-24-84 05-29-84 05-31-84

06-04-84 06-06-84 06-11-84 06-19-84 06-26-84

$\begin{array}{rrrrrrr}7 & 24 & 49 & 71 & 87 & 96 & 100 \\ 10 & 38 & 62 & 81 & 95 & 100 & \\ 8 & 28 & 52 & 62 & 72 & 78 & 100 \\ 7 & 26 & 48 & 63 & 70 & 83 & 100 \\ 7 & 30 & 57 & 77 & 87 & 100 & \end{array}$

100 
Table 3.--Bedload particle-size distributions--Continued

\begin{tabular}{|c|c|c|c|c|c|c|c|c|c|c|c|c|}
\hline \multirow{2}{*}{ Date } & \multicolumn{5}{|c|}{ Percent by weight finer } & \multicolumn{5}{|c|}{ than size (millimeters) indicated } & & \\
\hline & 0.062 & 0.12 & 0.25 & 0.5 & 1 & 2 & 4 & 8 & 16 & 32 & 64 & 128 \\
\hline
\end{tabular}

55. Craig Creek near Bailey, Colo.--Continued

07-03-84

$07-12-84$

$07-16-84$

$08-01-84$

$08-15-84$

$08-21-84$

09-05-84

0

$\begin{array}{lrrrrrr}0 & 5 & 19 & 85 & 85 & 99 & 100 \\ 0 & 4 & 26 & 92 & 92 & 100 & \\ 0 & 16 & 48 & 95 & 95 & 100 & \\ 0 & 2 & 18 & 77 & 77 & 100 & \\ 0 & 4 & 26 & 82 & 82 & 99 & 100 \\ & & & & & & \\ 0.2 & 4 & 35 & 91 & 91 & 99 & 100 \\ 0 & 12 & 48 & 95 & 95 & 100 & \end{array}$

05-10-84

05-26-84

06-07-84

06-13-84

06-21-84

06-28-84

07-10-84

$07-18-84$

$07-25-84$

08-02-84

08-20-84

\section{Geneva Creek near Grant, Colo.}

$\begin{array}{rrrrrrrrrr}0 & 16 & 49 & 66 & 67 & 84 & 100 & & \\ 0 & 2 & 23 & 44 & 61 & 79 & 93 & 100 & \\ 0 & 5 & 24 & 48 & 67 & 81 & 90 & 100 & \\ & 0 & 11 & 28 & 50 & 72 & 83 & 100 & \\ 0 & 2 & 16 & 32 & 50 & 66 & 80 & 92 & 100 \\ 0.8 & 3 & 14 & 35 & 60 & 77 & 92 & 100 & \\ 0 & 2 & 21 & 44 & 65 & 79 & 88 & 93 & 100 \\ & 0 & 27 & 45 & 64 & 82 & 91 & 100 & \\ 0 & 0 & 24 & 52 & 76 & 90 & 95 & 100 & \\ 0 & 3 & 17 & 37 & 60 & 80 & 90 & 100 & \\ & 0 & 8 & 25 & 46 & 63 & 71 & 79 & 100\end{array}$

57. Pony Creek near Antero Reservoir, Colo.

$\begin{array}{rrrrrrrrrr}05-16-84 & 0 & 4 & 11 & 33 & 57 & 57 & 81 & 93 & 100 \\ 05-24-84 & 0 & 6 & 38 & 63 & 81 & 81 & 88 & 88 & 100 \\ 06-06-84 & 0 & 24 & 49 & 73 & 98 & 98 & 100 & & \\ 06-12-84 & 0 & 11 & 21 & 47 & 68 & 68 & 84 & 95 & 100 \\ 06-14-84 & 0 & 10 & 20 & 40 & 60 & 60 & 80 & 90 & 100 \\ 06-19-84 & 0 & 7 & 13 & 33 & 53 & 53 & 80 & 93 & 100 \\ 06-22-84 & 0 & 9 & 18 & 36 & 55 & 55 & 64 & 64 & 100 \\ 06-26-84 & 0 & 6 & 13 & 31 & 50 & 50 & 75 & 81 & 100 \\ 06-29-84 & 0 & 4 & 16 & 40 & 64 & 64 & 76 & 76 & 100 \\ 07-03-84 & 0 & 9 & 18 & 35 & 61 & 61 & 88 & 96 & 100 \\ 07-06-84 & 0 & 3 & 21 & 52 & 70 & 70 & 88 & 94 & 100 \\ 07-10-84 & 0 & 0.9 & 10 & 26 & 45 & 45 & 72 & 82 & 100 \\ 07-24-84 & 0 & 6 & 19 & 32 & 44 & 44 & 57 & 62 & 100\end{array}$

58. North Fork of South Platte River at Shawnee, Colo.

05-03-85

05-06-85

05-09-85

05-14-85

$05-17-85$

05-21-85

05-24-85

05-28-85

05-31-85

06-10-85

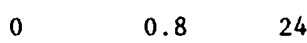

\begin{tabular}{rr}
0.1 & 0.8 \\
.1 & .7 \\
.1 & .5 \\
0 & .3 \\
\hline
\end{tabular}

24
14
16
14
21
14
17
14
14
12

24
14
16
14
21
14
17
14
14
12

64
41
38
39
57
40
48
42
46
33

$\begin{array}{ll}64 & 89 \\ 41 & 7 \\ 38 & 69 \\ 39 & 70 \\ 57 & 88 \\ & \\ 40 & 7 \\ 48 & 7 \\ 42 & 68 \\ 46 & \\ 33 & 54\end{array}$

0

0.1

$\begin{array}{ll}0.1 & .3 \\ 0 & .7 \\ 0.1 & .3 \\ 0 & .5 \\ 0.1 & .4\end{array}$

12

89
70
69
70
88
70
77
68
72
54


Table 3.--Bedload particle-size distributions--Cuntinued

Date

Percent by weight finer than size (millimeters) indicated

\begin{tabular}{llllllllllll}
\hline 0.062 & 0.12 & 0.25 & 0.5 & 1 & 2 & 4 & 8 & 16 & 32 & 64 & 128
\end{tabular}

58. North Fork of South Platte River at Shawnee, Colo.--Continued

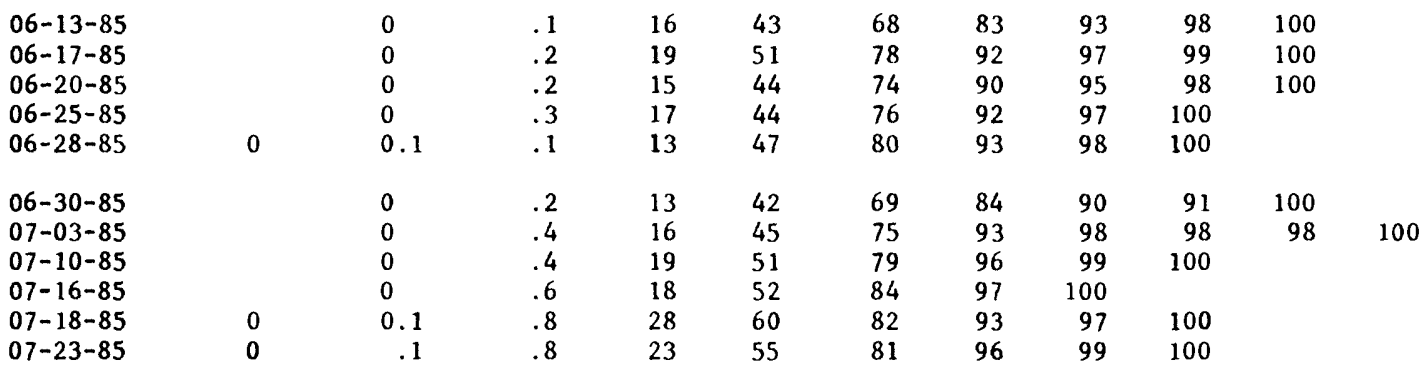

59. North Fork of South Platte River at Crossons, Colo.

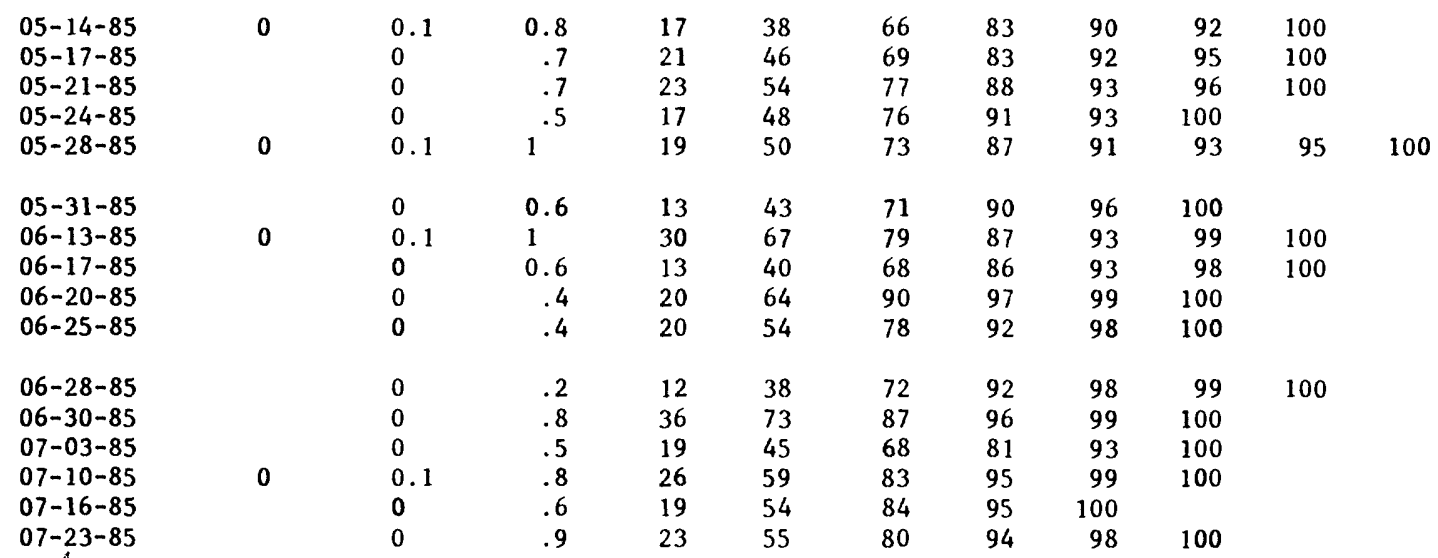

60. North Fork of South Platte River at Buffalo, Colo.

05-03-85

05-06-85

05-09-85

$05-15-85$

05-17-85

$05-21-85$

$05-23-85$

05-28-85

05-30-85

06-10-85

$\begin{array}{lcccc}0 & 0.4 & 11 & 32 & 57 \\ 0 & .3 & 12 & 31 & 51 \\ & 0 & 0.5 & 13 & 30 \\ 0 & 0.1 & 3 & 20 & 46 \\ 0 & .1 & 5 & 29 & 59 \\ & & & & \\ 0 & .3 & 31 & 68 & 86 \\ 0 & .3 & 30 & 66 & 82 \\ 0 & .2 & 15 & 43 & 64 \\ 0 & .3 & 12 & 30 & 49 \\ 0 & .6 & 19 & 40 & 61\end{array}$

82
72
51
75
84
96
93
81
73
80

$\begin{array}{rrr}95 & 100 & \\ 93 & 99 & 100 \\ 80 & 99 & 100 \\ 94 & 100 & \\ 96 & 100 & \end{array}$

06-12-85

06-17-85

06-19-85

06-26-85

$06-27-85$

$\begin{array}{lll}0.1 & .5 & 12 \\ 0 & .2 & 26 \\ 0 & .4 & 24 \\ 0 & .3 & 27 \\ 0.1 & .5 & 27\end{array}$

$\begin{array}{ll}28 & 48 \\ 54 & 69 \\ 50 & 93 \\ 57 & 82 \\ 62 & 78\end{array}$

71
80
90
96
85

100
98

$98 \quad 100$

$92 \quad 94 \quad 97$

$92 \quad 99-100$

100

100

$98 \quad 100$

0

$\begin{array}{rr}90 & 97 \\ 90 & 98 \\ 98 & 100 \\ 99 & 100 \\ 98 & 100\end{array}$

100 
Table 3.--Bedload particle-size distributions--Continued

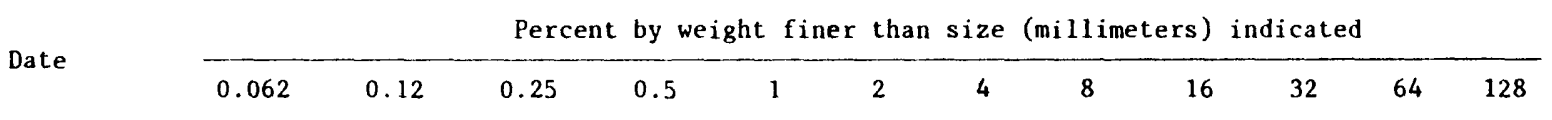

60. North Fork of South Platte River at Buffalo, Colo.--Continued

$06-30-85$

$07-02-85$

07-11-85

$07-16-85$

$07-24-85$

05-15-85

05-16-85

05-22-85

05-23-85

05-29-85

05-30-85

06-11-85

06-12-85

06-18-85

06-19-85

$06-27-85$

07-01-85

07-02-85

07-12-85

07-17-85

07-18-85

$07-25-85$

05-02-85

05-07-85

05-08-85

05-15-85

05-16-85

05-22-85

05-23-85

05-29-85

05-30-85

06-11-85

06-12-85

06-18-85

06-26-85

07-01-85

07-02-85

07-11-85

$07-17-85$

$07-24-85$

$\begin{array}{lrrrrrrr}0 & .8 & 45 & 71 & 81 & 88 & 98 & 100 \\ 0 & .5 & 16 & 52 & 85 & 96 & 99 & 100 \\ 0.1 & .5 & 32 & 76 & 92 & 97 & 100 & \\ .3 & 16 & 34 & 50 & 73 & 90 & 100 & \\ 0 & 0.3 & 44 & 84 & 97 & 100 & & \end{array}$

61. South Fork of South Platte River above Vermillion Creek, Colo.

$\begin{array}{rrrrrrrrr}0.2 & 3 & 19 & 34 & 55 & 78 & 96 & 99 & 100 \\ .2 & 5 & 30 & 47 & 65 & 87 & 99 & 100 & \\ .1 & 3 & 28 & 54 & 73 & 89 & 98 & 100 & \\ .1 & 2 & 21 & 52 & 71 & 86 & 97 & 100 & \end{array}$

$\begin{array}{llll}1 & 3 & 42 & 62 \\ 2 & 3 & 20 & 32\end{array}$

$34-51-75$

$\begin{array}{rrrr}1 & 1 & 8 & 18 \\ 1 & 3 & 21 & 36\end{array}$

$\begin{array}{llll}.1 & 1 & 17 & 36\end{array}$

$\begin{array}{llll}1 & 1 & 13 & 27 \\ 1 & 2 & 12 & 29\end{array}$

$0 \quad 0.6 \quad 11 \quad 26$

$0.1 \quad 130$

$\begin{array}{llllllll}.1 & 1 & 16 & 29 & 50 & 78 & 95 & 100 \\ .1 & 3 & 22 & 39 & 55 & 77 & 97 & 100\end{array}$

62. South Fork of South Platte River at Trumbull, Colo.

$\begin{array}{lllllllll}0 & 0.4 & 7 & 24 & 45 & 69 & 84 & 95 & 100\end{array}$

$\begin{array}{rrrrrrrrrr}0 & 0.4 & 7 & 24 & 45 & 69 & 84 & 95 & 100 & \\ 0 & .2 & 5 & 20 & 38 & 61 & 87 & 97 & 100 & 100 \\ 0 & .1 & 3 & 15 & 35 & 64 & 89 & 98 & 99 & 100 \\ 0 & .4 & 9 & 18 & 27 & 44 & 67 & 87 & 95 & 100 \\ 0 & .4 & 9 & 24 & 50 & 82 & 97 & 100\end{array}$

$\begin{array}{rlrrrrrrrrrr} & 0 & .4 & 9 & 24 & 50 & 82 & 97 & 100 & \\ 0 & 0.1 & .7 & 15 & 24 & 31 & 39 & 49 & 60 & 63 & -- \\ & 0 & 2 & 27 & 50 & 69 & 85 & 97 & 100 & \\ 0 & 0.1 & 0.3 & 7 & 22 & 44 & 67 & 85 & 96 & 100 & -- \\ 0 & .2 & 3 & 16 & 34 & 52 & 68 & 77 & 84 & 87 & -- & -- \\ 0 & .1 & 1 & 29 & 65 & 80 & 88 & 94 & 96 & 100 & \end{array}$

$\begin{array}{lllllllll}0 & 1 & 26 & 45 & 60 & 75 & 91 & 100\end{array}$

$\begin{array}{rrrrrrrr}0 & 1 & 26 & 45 & 60 & 75 & 91 & 100 \\ 0 & 0.5 & 11 & 24 & 42 & 65 & 81 & 92 \\ 0 & .1 & 1 & 3 & 6 & 9 & 12 & 12\end{array}$

$\begin{array}{rllllllrl}0 & 0.2 & 2 & 33 & 67 & 83 & 92 & 100 & \\ & 0 & 0.1 & 11 & 33 & 50 & 76 & 91 & 100\end{array}$

$\begin{array}{rrrrrrrr}0 & .3 & 6 & 18 & 36 & 64 & 91 & 100\end{array}$

$\begin{array}{rrrrrrrrr}0 & .2 & 8 & 24 & 49 & 74 & 91 & 97 & 100 \\ 0 & 0.4 & 2 & 30 & 64 & 88 & 97 & 100 & \end{array}$ 
Table 3.--Bedload particle-size distributions--Continued

\begin{tabular}{|c|c|c|c|c|c|c|c|c|c|c|c|c|}
\hline \multirow{2}{*}{ Date } & \multicolumn{3}{|r|}{ Percent } & by weight & $t$ finer & \multirow{2}{*}{$\frac{\text { than }}{2}$} & \multirow{2}{*}{$\frac{\text { size }}{4}$} & \multicolumn{2}{|c|}{ (millimeters) } & \multicolumn{3}{|l|}{ indicated } \\
\hline & 0.062 & 0.12 & 0.25 & 0.5 & 1 & & & 8 & 16 & 32 & 64 & 128 \\
\hline
\end{tabular}

63. Buffalo Creek at Buffalo, Colo.

$\begin{array}{rlllllllll}05-03-85 & & 0 & 0.2 & 6 & 23 & 47 & 75 & 95 & 100 \\ 05-06-85 & 0 & 0.1 & 1 & 11 & 23 & 46 & 78 & 98 & 100 \\ 05-08-85 & & 0 & 0.8 & 10 & 32 & 57 & 82 & 96 & 100 \\ 05-14-85 & 0 & 0.1 & 1 & 13 & 40 & 66 & 88 & 98 & 100 \\ 05-17-85 & 0 & .1 & 2 & 21 & 44 & 63 & 81 & 96 & 100 \\ 05-21-85 & 0 & .2 & 2 & 20 & 39 & 58 & 79 & 95 & 100 \\ 05-23-85 & 0 & .2 & 3 & 27 & 51 & 68 & 85 & 97 & 100 \\ 05-28-85 & & 0 & 0.5 & 4 & 18 & 47 & 80 & 99 & 100 \\ 05-30-85 & & 0 & .8 & 12 & 33 & 59 & 83 & 97 & 100 \\ 06-10-85 & 0 & 0.1 & 1 & 9 & 27 & 52 & 82 & 99 & 100 \\ 06-12-85 & 0 & .1 & 1 & 13 & 36 & 65 & 89 & 99 & 100 \\ 06-17-85 & & 0 & 0.5 & 10 & 34 & 63 & 88 & 99 & 100 \\ 06-19-85 & 0 & 0.1 & 2 & 20 & 50 & 73 & 91 & 98 & 100 \\ 06-26-85 & 0 & .3 & 4 & 23 & 43 & 61 & 83 & 100 & \\ 06-30-85 & 0 & .3 & 3 & 18 & 33 & 54 & 80 & 96 & 100 \\ & & & & & & & & & \\ 07-02-85 & 0 & .4 & 4 & 22 & 38 & 57 & 79 & 95 & 100 \\ 07-11-85 & 0 & .1 & 2 & 20 & 40 & 62 & 84 & 98 & 100 \\ 07-16-85 & 0 & .2 & 2 & 19 & 50 & 72 & 90 & 99 & 100 \\ 07-19-85 & 0 & .1 & 2 & 22 & 42 & 60 & 80 & 97 & 100 \\ 07-23-85 & 0 & .3 & 4 & 41 & 67 & 79 & 93 & 99 & 100\end{array}$

64. Trail Creek near Westcreek, Colo.

$\begin{array}{lrrrrrrrrr}05-02-84 & 0 & 0.7 & 5 & 27 & 53 & -- & 76 & 79 & 100 \\ 05-08-84 & 0 & .1 & 4 & 15 & 44 & -- & 72 & 83 & 100 \\ 05-11-84 & 0 & .3 & 5 & 27 & 60 & -- & 80 & 89 & 100 \\ 05-15-84 & 0 & .6 & 3 & 15 & 45 & -- & 73 & 89 & 100 \\ 05-26-84 & 0 & .9 & 10 & 43 & 76 & -- & 81 & 91 & 100 \\ 06-01-84 & 0 & .6 & 6 & 25 & 50 & -- & 72 & 81 & 100 \\ 06-08-84 & 0 & .3 & 6 & 29 & 57 & -- & 77 & 89 & 100 \\ 06-16-84 & 0 & .7 & 4 & 30 & 67 & -- & 90 & 97 & 100 \\ 06-26-84 & 0 & .5 & 6 & 27 & 49 & -- & 84 & 95 & 100 \\ 07-10-84 & 0 & .8 & 8 & 31 & 54 & -- & 85 & 92 & 100 \\ 07-27-84 & 0 & .7 & 14 & 49 & 70 & -- & 90 & 93 & 100 \\ 08-13-84 & 0 & .4 & 2 & 11 & 29 & -- & 83 & 96 & 100 \\ 09-04-84 & 0 & .1 & 2 & 10 & 43 & -- & 85 & 96 & 100\end{array}$

65. Horse Creek near Westcreek, Colo.

\begin{tabular}{|c|c|c|c|c|c|c|c|c|c|c|c|c|}
\hline $05-02-85$ & & 0 & 0.2 & 3 & 11 & 26 & 50 & 77 & 93 & 98 & -- & -- \\
\hline $05-07-85$ & & 0 & .2 & 6 & 20 & 38 & 59 & 77 & 90 & 97 & -- & -- \\
\hline $05-08-85$ & 0 & 0.1 & .3 & 6 & 20 & 40 & 60 & 76 & 86 & 100 & & \\
\hline $05-15-85$ & & 0 & .5 & 10 & 26 & 49 & 74 & 93 & 100 & & & \\
\hline $05-16-85$ & 0 & 0.1 & .6 & 12 & 27 & 42 & 55 & 73 & 85 & 90 & -- & -- \\
\hline $05-22-85$ & & 0 & .2 & 4 & 13 & 28 & 50 & 73 & 90 & 95 & -- & -- \\
\hline $05-23-85$ & & 0 & .2 & 6 & 16 & 32 & 54 & 78 & 97 & 100 & & \\
\hline $05-29-85$ & & 0 & .2 & 3 & 17 & 45 & 71 & 90 & 98 & 99 & -- & -- \\
\hline $05-30-85$ & & 0 & .2 & 4 & 13 & 33 & 63 & 85 & 98 & 100 & & \\
\hline $06-11-85$ & 0 & 0.1 & .4 & 15 & 53 & 83 & 95 & 99 & 100 & & & \\
\hline
\end{tabular}


Table 3.--Bedload particle-size distributions--Continued

Date

Percent by weight finer than size (millimeters) indicated

\begin{tabular}{|c|c|c|c|c|c|c|c|c|c|c|c|}
\hline 0.062 & 0.12 & 0.25 & 0.5 & 1 & 2 & 4 & 8 & 16 & 32 & 64 & 128 \\
\hline
\end{tabular}

65. Horse Creek near Westcreek, Colo.--Continued

06-12-85

06-18-85

06-19-85

06-27-85

07-01-85

07-02-85

07-12-85

07-17-85

07-19-85

07-24-85

07-25-85

$\begin{array}{lrrrrrrr} & 0 & 0.2 & 0.6 & 5 & 49 & 88 & 100 \\ 0 & 0.3 & 5 & 25 & 57 & 82 & 98 & 99 \\ 0 & .1 & 4 & 20 & 50 & 75 & 90 & 98 \\ 0 & .9 & 3 & 11 & 28 & 66 & 96 & 100 \\ 0 & .2 & 5 & 20 & 47 & 74 & 95 & 100 \\ 0 & .1 & 6 & 14 & 35 & 67 & 94 & 100 \\ 0.1 & 1 & 17 & 44 & 64 & 77 & 91 & 100 \\ 0 & 0.4 & 8 & 26 & 46 & 69 & 91 & 100 \\ 0.1 & .7 & 9 & 33 & 63 & 87 & 99 & 100 \\ 0 & .1 & 2 & 7 & 18 & 47 & 85 & 100 \\ 0 & .1 & 3 & 14 & 34 & 63 & 90 & 100\end{array}$

66. Blue River below Green Mountain Reservoir, Colo.

\begin{tabular}{|c|c|c|c|c|c|c|c|c|c|c|}
\hline $05-30-85$ & 0 & 0.4 & 2 & 31 & 74 & 90 & 95 & 97 & 100 & \\
\hline $06-04-85$ & 0 & .2 & 1 & 28 & 77 & 89 & 91 & 93 & 100 & \\
\hline $06-06-85$ & 0 & .5 & 3 & 41 & 86 & 97 & 99 & 100 & & \\
\hline $06-10-85$ & 0 & .6 & 3 & 35 & 58 & 62 & 64 & 67 & 80 & 100 \\
\hline $06-17-85$ & 0 & .2 & 1 & 30 & 66 & 85 & 95 & 99 & 100 & \\
\hline $06-18-85$ & 0 & 3 & 5 & 41 & 71 & 79 & 82 & 84 & 86 & 100 \\
\hline $06-25-85$ & 0 & 0.4 & 2 & 34 & 70 & 79 & 82 & 83 & 83 & 100 \\
\hline $06-26-85$ & 0 & .1 & 0.6 & 23 & 65 & 82 & 90 & 95 & 100 & \\
\hline $06-30-85$ & 0 & .2 & 1 & 28 & 63 & 74 & 77 & 77 & 78 & 100 \\
\hline $07-10-85$ & & 0 & 3 & 55 & 94 & 100 & & & & \\
\hline $07-17-85$ & 0 & 0.6 & 4 & 56 & 95 & 99 & 100 & & & \\
\hline $07-31-85$ & 0 & .5 & 3 & 58 & 86 & 91 & 94 & 98 & 100 & \\
\hline
\end{tabular}

67. Williams Fork near Leal, Colo.

\begin{tabular}{|c|c|c|c|c|c|c|c|c|c|c|c|c|}
\hline $05-15-85$ & 0 & 0.2 & 0.7 & 18 & 40 & 55 & 66 & 89 & 100 & & & \\
\hline $05-21-85$ & & 0 & .6 & 20 & 47 & 73 & 86 & 91 & 100 & & & \\
\hline $05-22-85$ & 0 & .0 .1 & .4 & 15 & 40 & 60 & 69 & 79 & 100 & & & \\
\hline $05-22-85$ & 0 & .1 & .3 & 14 & 44 & 64 & 75 & 91 & 97 & 100 & & \\
\hline $05-28-85$ & 0 & .1 & .6 & 9 & 36 & 62 & 75 & 84 & 95 & 100 & & \\
\hline $05-29-85$ & 0 & .1 & .4 & 7 & 26 & 42 & 51 & 60 & 73 & 95 & 100 & \\
\hline $06-05-85$ & 0 & .1 & .2 & 4 & 15 & 24 & 31 & 37 & 49 & 67 & -. & -- \\
\hline $06-06-85$ & & 0 & .2 & 5 & 12 & 16 & 18 & 20 & 24 & 27 & -- & -- \\
\hline $06-10-85$ & 0 & 0.2 & 1 & 19 & 48 & 66 & 73 & 79 & 89 & 100 & & \\
\hline $06-11-85$ & 0 & .1 & 0.8 & 14 & 36 & 56 & 70 & 77 & 78 & 100 & & \\
\hline $06-18-85$ & & 0 & .2 & 9 & 40 & 79 & 91 & 94 & 95 & 100 & & \\
\hline $06-18-85$ & & 0 & .1 & 8 & 44 & 83 & 95 & 98 & 100 & & & \\
\hline $06-26-85$ & 0 & 0.1 & .4 & 4 & 26 & 52 & 67 & 77 & 90 & 100 & & \\
\hline $06-26-85$ & 0 & .1 & .5 & 6 & 24 & 47 & 55 & 61 & 68 & 100 & & \\
\hline $07-01-85$ & 0 & .1 & .7 & 16 & 48 & 77 & 91 & 99 & 100 & & & \\
\hline $07-01-85$ & 0 & .1 & .4 & 6 & 24 & 42 & 52 & 58 & 64 & 100 & & \\
\hline $07-11-85$ & & 0 & .2 & 6 & 19 & 38 & 50 & 58 & 61 & 64 & -- & -- \\
\hline $07-18-85$ & 0 & 0.1 & .5 & 12 & 34 & 62 & 81 & 92 & 100 & & & \\
\hline $08-01-85$ & 0 & .1 & 1 & 22 & 51 & 72 & 83 & 93 & 100 & & & \\
\hline
\end{tabular}


Table 3.--Bedload particle-size distributions--Continued

\begin{tabular}{|c|c|c|c|c|c|c|c|c|c|c|c|c|}
\hline & & & Percent & by weight & finer & than & size & (millimete & rs ) & indicated & & \\
\hline & 0.062 & 0.12 & 0.25 & 0.5 & 1 & 2 & 4 & 8 & 16 & 32 & 64 & 128 \\
\hline
\end{tabular}

68. Upper South Fork of Williams Fork near Lea1,Colo.

$\begin{array}{rrrrrrrrrrrr}05-16-85 & & 0 & 0.2 & 3 & 9 & 15 & 20 & 24 & 27 & 33 \\ 05-23-85 & & 0 & .3 & 3 & 12 & 24 & 35 & 45 & 61 & 90 & 100 \\ 05-30-85 & 0 & 0.1 & .7 & 13 & 31 & 57 & 77 & 87 & 94 & 100 & \\ 06-04-85 & 0 & .1 & .4 & 8 & 22 & 36 & 47 & 56 & 66 & 83 & 100 \\ 06-05-85 & 0 & .1 & .3 & 7 & 21 & 39 & 53 & 61 & 69 & 77 & 100 \\ 06-10-85 & 0 & .1 & .8 & 10 & 22 & 37 & 51 & 65 & 79 & 94 & 100 \\ 06-11-85 & 0 & .1 & .8 & 10 & 24 & 37 & 49 & 59 & 74 & 96 & 100 \\ 06-17-85 & 0 & .1 & .9 & 9 & 18 & 30 & 47 & 61 & 77 & 94 & 100 \\ 06-18-85 & 0 & .1 & .5 & 8 & 20 & 31 & 42 & 51 & 60 & 80 & 100 \\ 07-01-85 & 0 & .1 & .4 & 10 & 23 & 51 & 81 & 92 & 95 & 100 & \\ 07-18-85 & & 0 & .1 & 7 & 23 & 46 & 67 & 74 & 77 & 100 & \end{array}$

69. Lower South Fork of Williams Fork near Leal, Colo.

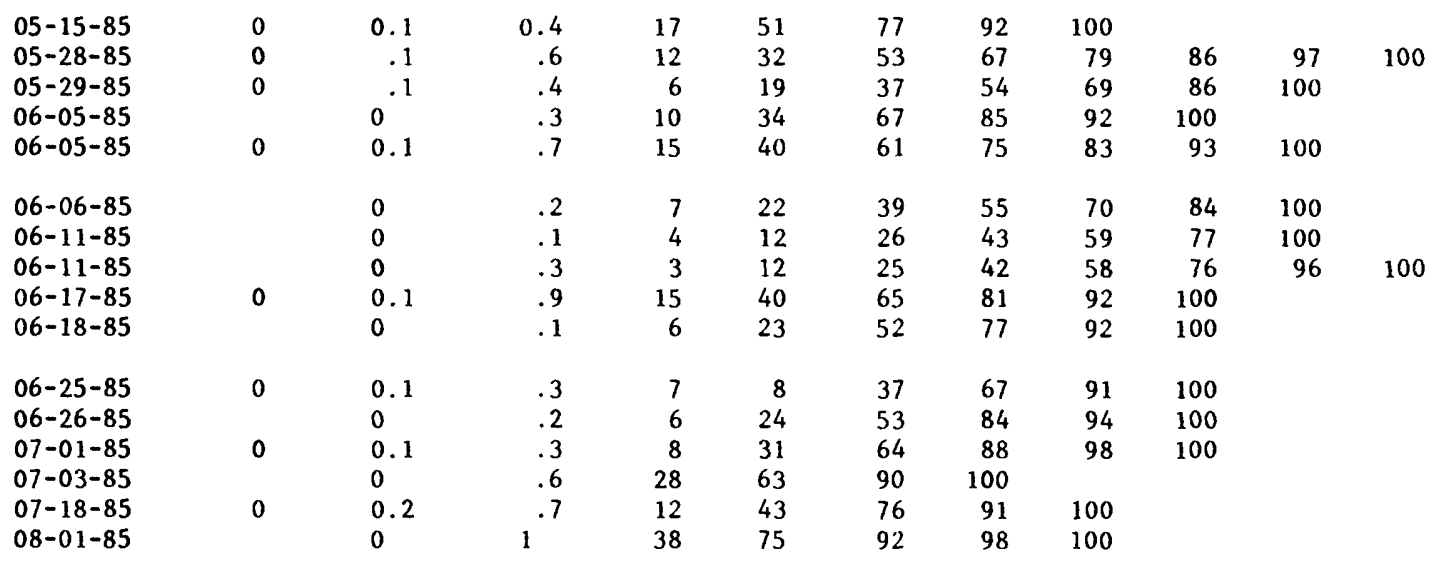

70. Williams Fork below Steelman Creek near Leal, Colo.

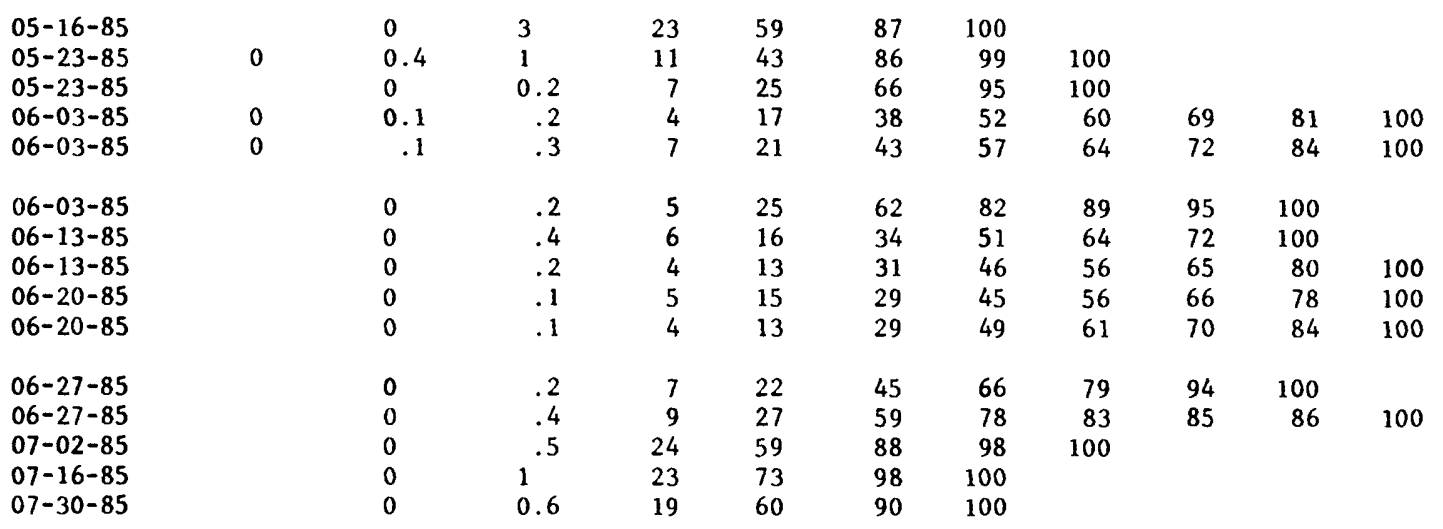


Table 3.--Bedload particle-size distributions--Continued

Date

Percent by weight finer than size (millimeters) indicated

$\begin{array}{llllllllllll}0.062 & 0.12 & 0.25 & 0.5 & 1 & 2 & 4 & 8 & 16 & 32 & 64 & 128\end{array}$

71. Kinney Creek near Leal, Colo.

\begin{tabular}{|c|c|c|c|c|c|c|c|c|c|c|c|}
\hline $\begin{array}{l}05-15-85 \\
05-16-85 \\
05-22-85 \\
05-28-85 \\
05-29-85\end{array}$ & $\begin{array}{l}0 \\
0\end{array}$ & $\begin{array}{l}0.3 \\
0 \\
0 \\
0.1 \\
.3\end{array}$ & $\begin{array}{l}1 \\
0.7 \\
.2 \\
.5 \\
1\end{array}$ & $\begin{array}{r}25 \\
8 \\
2 \\
7 \\
16\end{array}$ & $\begin{array}{l}55 \\
31 \\
12 \\
17 \\
39\end{array}$ & $\begin{array}{l}76 \\
69 \\
27 \\
31 \\
57\end{array}$ & $\begin{array}{l}90 \\
99 \\
52 \\
45 \\
08\end{array}$ & $\begin{array}{r}100 \\
100 \\
78 \\
62 \\
82\end{array}$ & $\begin{array}{r}100 \\
82 \\
89\end{array}$ & $\begin{array}{l}100 \\
100\end{array}$ & \\
\hline $\begin{array}{l}06-05-85 \\
06-06-85 \\
06-11-85 \\
06-11-85 \\
06-18-85\end{array}$ & $\begin{array}{l}0 \\
0 \\
0 \\
0 \\
0\end{array}$ & $\begin{array}{l}0 \\
0.1 \\
0.1 \\
.2 \\
.1\end{array}$ & $\begin{array}{r}0.2 \\
.3 \\
.5 \\
1 \\
0.3\end{array}$ & $\begin{array}{r}5 \\
4 \\
8 \\
12 \\
5\end{array}$ & $\begin{array}{r}13 \\
9 \\
16 \\
24 \\
13\end{array}$ & $\begin{array}{l}26 \\
15 \\
26 \\
36 \\
24\end{array}$ & $\begin{array}{l}41 \\
23 \\
40 \\
50 \\
38\end{array}$ & $\begin{array}{l}52 \\
33 \\
57 \\
67 \\
55\end{array}$ & $\begin{array}{l}64 \\
52 \\
74 \\
92 \\
66\end{array}$ & $\begin{array}{r}87 \\
90 \\
78 \\
100 \\
77\end{array}$ & $\begin{array}{l}100 \\
100 \\
100\end{array}$ \\
\hline $\begin{array}{l}06-26-85 \\
06-26-85 \\
07-01-85 \\
07-03-85 \\
07-11-85 \\
08-01-85\end{array}$ & & $\begin{array}{l}0 \\
0 \\
0\end{array}$ & $\begin{array}{c}.3 \\
.4 \\
0 \\
10 \\
0.8 \\
.8\end{array}$ & $\begin{array}{r}14 \\
8 \\
32 \\
29 \\
16 \\
8\end{array}$ & $\begin{array}{l}32 \\
20 \\
61 \\
53 \\
34 \\
20\end{array}$ & $\begin{array}{l}55 \\
38 \\
76 \\
76 \\
57 \\
37\end{array}$ & $\begin{array}{l}71 \\
60 \\
86 \\
95 \\
77 \\
54\end{array}$ & $\begin{array}{r}79 \\
89 \\
100 \\
100 \\
100 \\
69\end{array}$ & $\begin{array}{l}100 \\
100\end{array}$ & & \\
\hline
\end{tabular}

72. West Fork of San Juan River near Pagosa Springs, Colo.

\begin{tabular}{|c|c|c|c|c|c|c|c|c|c|c|c|c|}
\hline $04-17-85$ & 0 & 1 & 2 & 23 & 43 & 67 & 86 & 97 & 99 & 100 & & \\
\hline $04-23-85$ & 0 & 1 & 2 & 44 & 71 & 92 & 100 & & & & & \\
\hline $05-02-85$ & 0 & 1 & 1 & 24 & 51 & 69 & 76 & 82 & 92 & 100 & & \\
\hline $05-05-85$ & 0 & 1 & 8 & 55 & 70 & 73 & 74 & 75 & 75 & 83 & 100 & \\
\hline $05-08-85$ & 1 & 2 & 8 & 45 & 62 & 69 & 72 & 75 & 82 & 100 & & \\
\hline $05-12-85$ & & 0 & 1 & 19 & 45 & 68 & 78 & 84 & 89 & 90 & 100 & \\
\hline $05-15-85$ & 1 & 1 & 5 & 40 & 67 & 84 & 91 & 93 & 93 & 100 & & \\
\hline $05-20-85$ & & 0 & 1 & 19 & 46 & 74 & 87 & 93 & 99 & 100 & & \\
\hline $05-26-85$ & 1 & 3 & 22 & 65 & 72 & 74 & 76 & 78 & 82 & 100 & & \\
\hline $05-30-85$ & 0 & 1 & 3 & 11 & 16 & 20 & 24 & 28 & 40 & 63 & 88 & 100 \\
\hline $06-03-85$ & & 0 & 2 & 8 & 12 & 15 & 18 & 23 & 31 & 38 & 40 & -- \\
\hline $06-06-85$ & & 0 & 2 & 11 & 16 & 17 & 18 & 20 & 27 & 35 & 100 & \\
\hline $06-18-85$ & & & 0 & 1 & 2 & 2 & 3 & 7 & 20 & 50 & 81 & 100 \\
\hline $07-18-85$ & 1 & 2 & 14 & 42 & 81 & 96 & 99 & 100 & & & & \\
\hline
\end{tabular}

\section{Rich Creek near Weston Pass, Colo.}

\begin{tabular}{|c|c|c|c|c|c|c|c|c|c|c|c|c|}
\hline $05-16-84$ & 0 & 0.4 & 4 & 16 & -- & 76 & 100 & & & & & \\
\hline $05-24-84$ & 0 & .1 & 2 & 17 & -- & 64 & 89 & 93 & 100 & & & \\
\hline $05-29-84$ & 0 & .1 & 3 & 21 & -- & 73 & 88 & 93 & 100 & & & \\
\hline $05-31-84$ & 0 & 3 & 10 & 28 & -- & 72 & 95 & 97 & 100 & & & \\
\hline $06-06-84$ & 0 & 0.1 & 0.8 & 4 & -- & 19 & 28 & 28 & 30 & 30 & -- & -- \\
\hline $06-12-84$ & 0 & .2 & 2 & 13 & -- & 51 & 77 & 83 & 87 & 100 & & \\
\hline $06-14-84$ & 0 & .1 & 2 & 9 & - & 33 & 54 & 58 & 62 & 62 & -- & -- \\
\hline $06-18-84$ & 0 & 1 & 4 & 15 & -- & 68 & 92 & 94 & 100 & & & \\
\hline $06-23-84$ & 0 & 0.6 & 7 & 19 & -- & 75 & 94 & 100 & & & & \\
\hline $06-26-84$ & 0 & .6 & 4 & 16 & -- & 79 & 98 & 100 & & & & \\
\hline $06-29-84$ & 0 & 2 & 8 & 23 & $\cdots$ & 69 & 100 & & & & & \\
\hline $07-03-84$ & 0 & 0.4 & 2 & 10 & - & 40 & 90 & 96 & 100 & & & \\
\hline $07-06-84$ & 0 & .4 & 2 & 11 & - & 51 & 91 & 96 & 100 & & & \\
\hline $07-10-84$ & 0 & 2 & 12 & 33 & $\ldots$ & 74 & 95 & 97 & 100 & & & \\
\hline $07-17-84$ & 0 & 0.5 & 5 & 18 & -. & 58 & 99 & 100 & & & & \\
\hline $08-08-84$ & 0 & 6 & 11 & 33 & - & 89 & 100 & & & & & \\
\hline
\end{tabular}


Table 3.--Bedload particle-size distributions--Continued

\begin{tabular}{lllllllllllll}
\hline & & \multicolumn{4}{c}{ Percent } & by weight & finer than size (millimeters) indicated \\
\cline { 2 - 11 } & 0.062 & 0.12 & 0.25 & 0.5 & 1 & 2 & 4 & 8 & 16 & 32 & 64 & 128 \\
\hline
\end{tabular}

74. Trout Creek near Bayfield, Colo. ${ }^{4}$

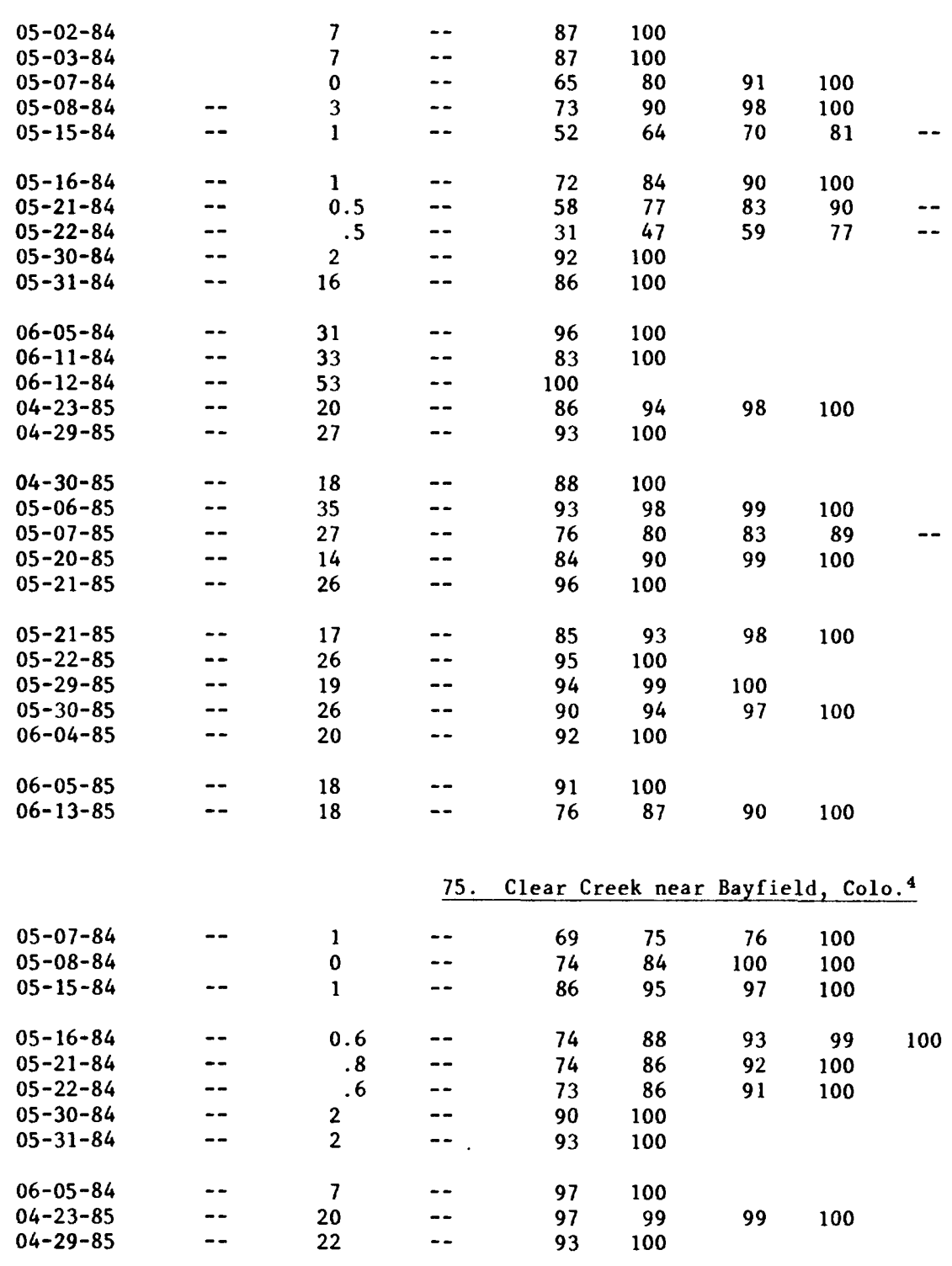


Table 3.--Bedload particle-size distributions--Continued

Percent by weight finer than size (millimeters) indicated

Date

\begin{tabular}{lllllllllllll}
\hline 0.062 & 0.12 & 0.25 & 0.5 & 1 & 2 & 4 & 8 & 16 & 32 & 64 & 128
\end{tabular}

75. Clear Creek near Bayfield, Colo.4--Continued

$\begin{array}{rrrrrrrrr}04-30-85 & -- & 22 & -- & 91 & 100 & & & \\ 05-06-85 & -- & 7 & -- & 64 & 73 & 78 & 85 & 100 \\ 05-07-85 & -- & 5 & -- & 21 & 66 & 78 & 100 & 100 \\ 05-20-85 & -- & 10 & -- & 56 & 61 & 61 & 67 & 100 \\ 05-21-85 & -- & 11 & -- & 55 & 64 & 74 & 98 & \\ 05-21-85 & -- & 11 & -- & 91 & 100 & & & \\ 05-22-85 & -- & 9 & -- & 91 & 94 & 96 & 100 & \\ 05-29-85 & -- & 8 & -- & 48 & 53 & 54 & 67 & 100 \\ 05-30-85 & -- & 15 & -- & 90 & 95 & 96 & 100 & \\ 06-04-85 & -- & 12 & -- & 93 & 97 & 100 & & \\ 06-05-85 & -- & 48 & -- & 55 & 66 & 76 & 92 & 100\end{array}$

76. West Prong Creek near Bayfield, Colo. 4

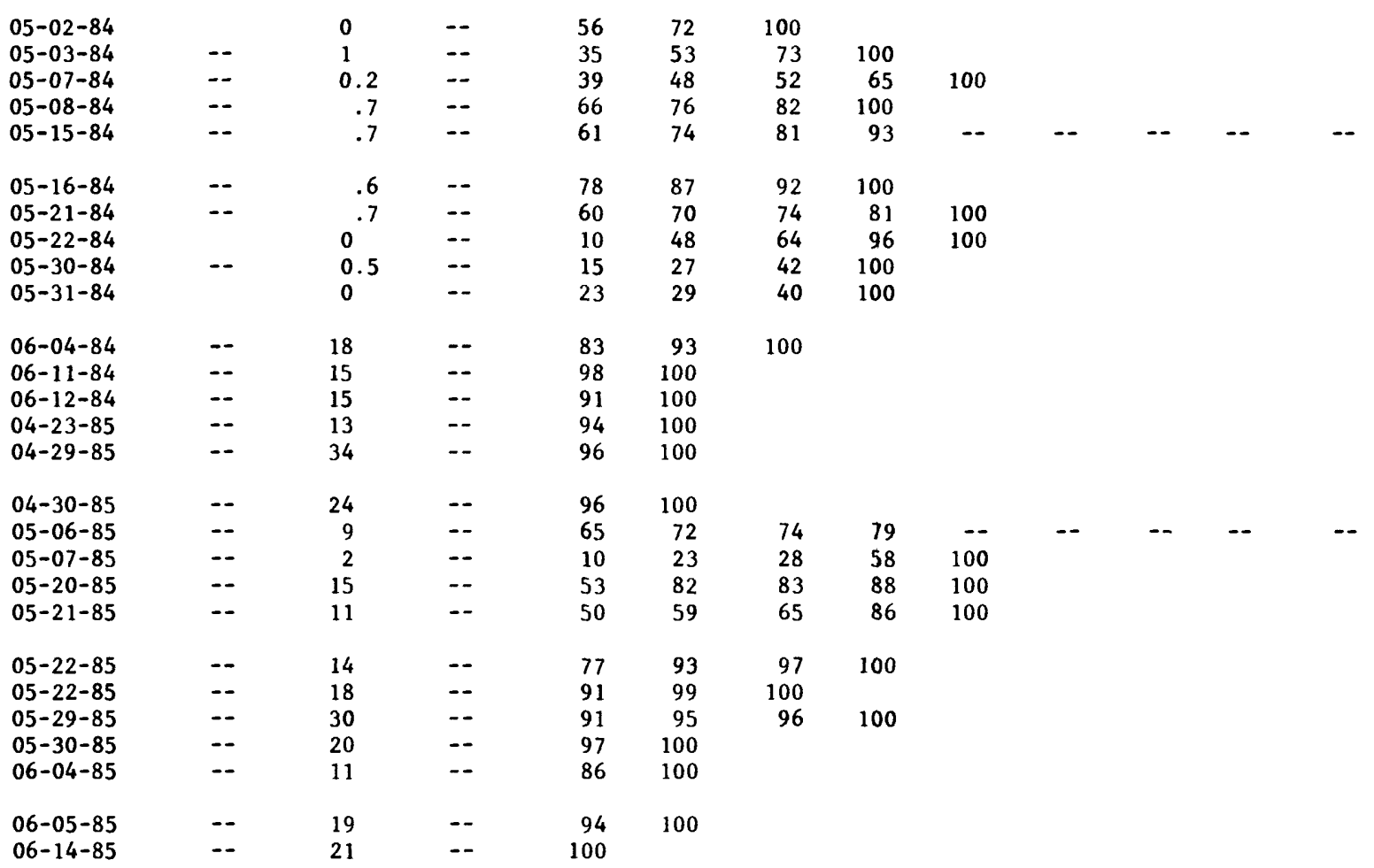


Table 3,--Bedload particle-size distributions--Continued

Percent by weight finer than size (millimeters) indicated

Date

\begin{tabular}{llllllllllll}
\hline 0.062 & 0.12 & 0.25 & 0.5 & 1 & 2 & 4 & 8 & 16 & 32 & 64 & 128
\end{tabular}

\section{Red Creek near Bayfield, Colo. 4}

$\begin{array}{rrrrrrrrr}05-07-84 & -- & 2 & -- & 61 & 72 & 82 & 95 & 100 \\ 05-08-84 & -- & 0.3 & -- & 42 & 52 & 58 & 67 & 100 \\ 05-15-84 & -- & 1 & -- & 67 & 77 & 81 & 85 & 100 \\ 05-16-84 & -- & 2 & -- & 72 & 86 & 96 & 100 & \\ 05-21-84 & -- & 0.5 & -- & 72 & 81 & 85 & 94 & 100 \\ & & & & & & & & \\ 05-22-84 & -- & -3 & -- & 80 & 89 & 93 & 100 & \\ 05-30-84 & -- & 0 & -- & 36 & 100 & & & \\ 05-31-84 & -- & 8 & -- & 85 & 100 & & & \\ 06-05-84 & -- & 25 & -- & 97 & 100 & & & \\ 06-11-84 & -- & 33 & -- & 100 & & & & \\ & & & & & & & & \\ 06-12-84 & -- & 46 & -- & 100 & & & & \\ 04-23-85 & -- & 26 & -- & 100 & & & \\ 04-29-85 & -- & 20 & -- & 91 & 98 & 100 & & \\ 04-30-85 & -- & 16 & -- & 91 & 100 & & & \\ 05-06-85 & -- & 23 & -- & 93 & 97 & 98 & 100 & \\ & & & & & & & & \\ 05-07-85 & -- & 23 & -- & 95 & 98 & 100 & & \\ 05-20-85 & -- & 21 & -- & 90 & 94 & 95 & 100 & \\ 05-21-85 & -- & 20 & -- & 97 & 99 & 100 & & \\ 05-22-85 & -- & 10 & -- & 76 & 88 & 93 & 97 & 100 \\ 05-22-85 & -- & 23 & -- & 95 & 99 & 100 & & \\ 05-29-85 & -- & 23 & -- & 100 & & & & \\ 05-30-85 & -- & 16 & -- & 90 & 96 & 99 & 100 & \\ 06-04-85 & -- & 15 & -- & 93 & 99 & 100 & & \\ 06-05-85 & -- & 6 & -- & 83 & 99 & 100 & & \\ 06-14-85 & -- & 18 & -- & 100 & & & & \\ & & & & & & & & \end{array}$

78. Sheep Creek at Guard Station near Saguache, Colo.

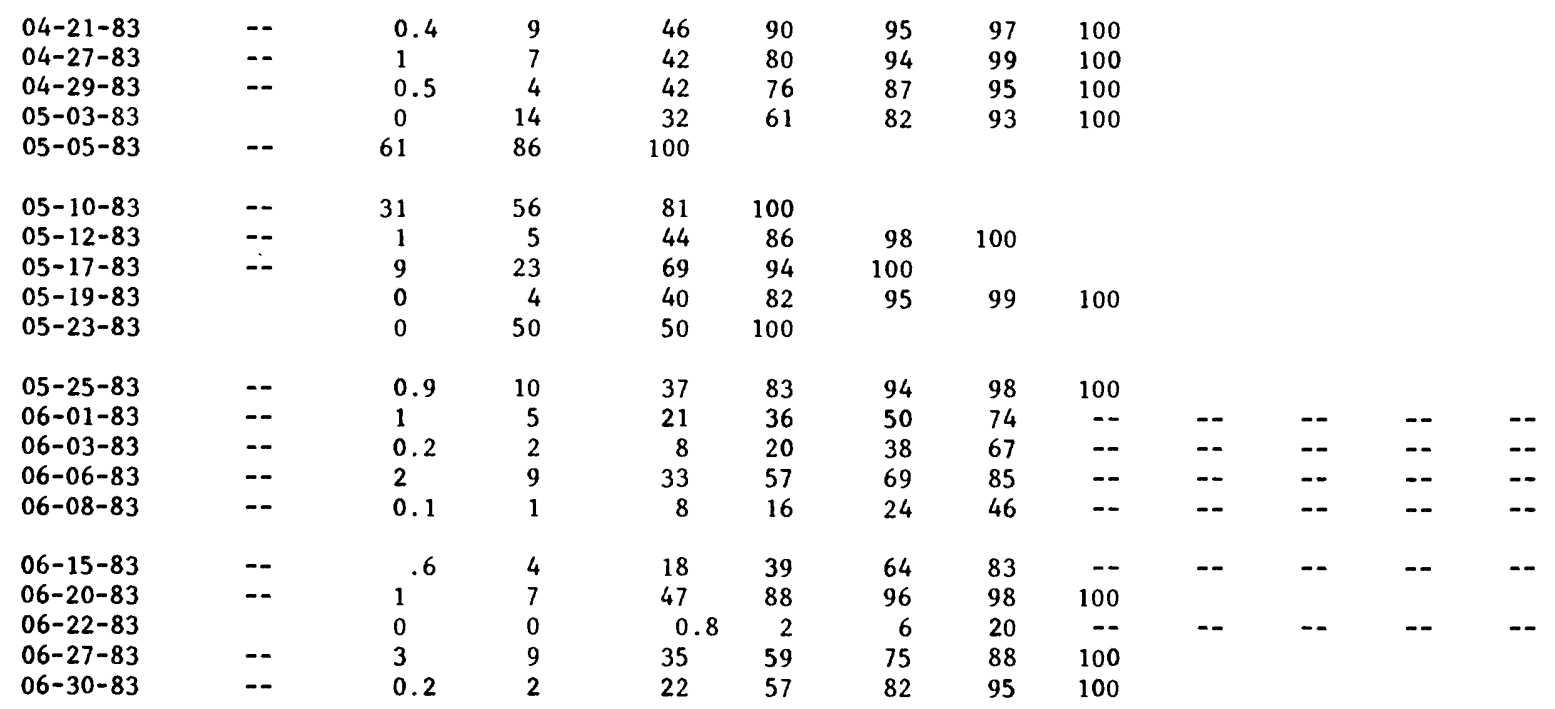


Table 3.--Bedload particle-size distributions--Continued

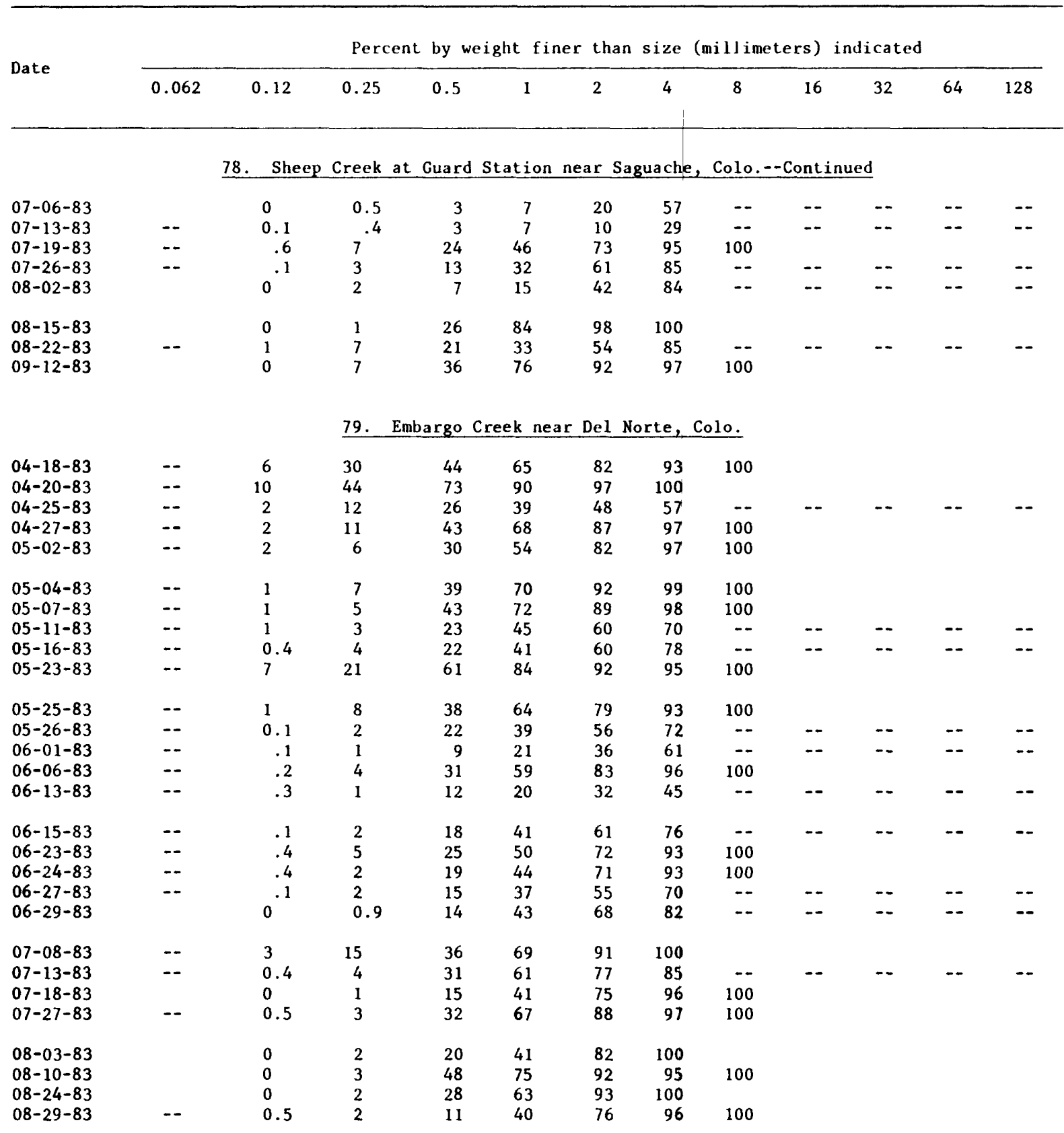

80. Pinos Creek near Del Norte, Colo.

\begin{tabular}{|c|c|c|c|c|c|c|c|c|c|c|c|c|}
\hline $04-20-83$ & -- & 3 & 31 & 69 & 84 & 91 & 99 & 100 & & & & \\
\hline $\begin{array}{l}04-25-83 \\
04-27-83\end{array}$ & $\cdots$ & $\begin{array}{l}2 \\
0\end{array}$ & $\begin{array}{r}12 \\
2\end{array}$ & $\begin{array}{l}26 \\
19\end{array}$ & 40 & $\begin{array}{l}48 \\
69\end{array}$ & $\begin{array}{l}57 \\
83\end{array}$ & -- & $=-$ & -- & -- & -- \\
\hline $05-02-83$ & -- & 0.3 & 2 & 20 & 53 & 80 & 94 & 100 & & & & \\
\hline $05-04-83$ & -- & .2 & 1 & 14 & 40 & 68 & 87 & -- & -- & -- & -- & -- \\
\hline
\end{tabular}


Table 3.--Bedload particle-size distributions--Continued

\begin{tabular}{|c|c|c|c|c|c|c|c|c|c|c|c|c|}
\hline & & & Percent & by weight & t finer & than & size & (millimet & ers) & indicated & & \\
\hline Date & 0.062 & 0.12 & 0.25 & 0.5 & 1 & 2 & 4 & 8 & 16 & 32 & 64 & 128 \\
\hline
\end{tabular}

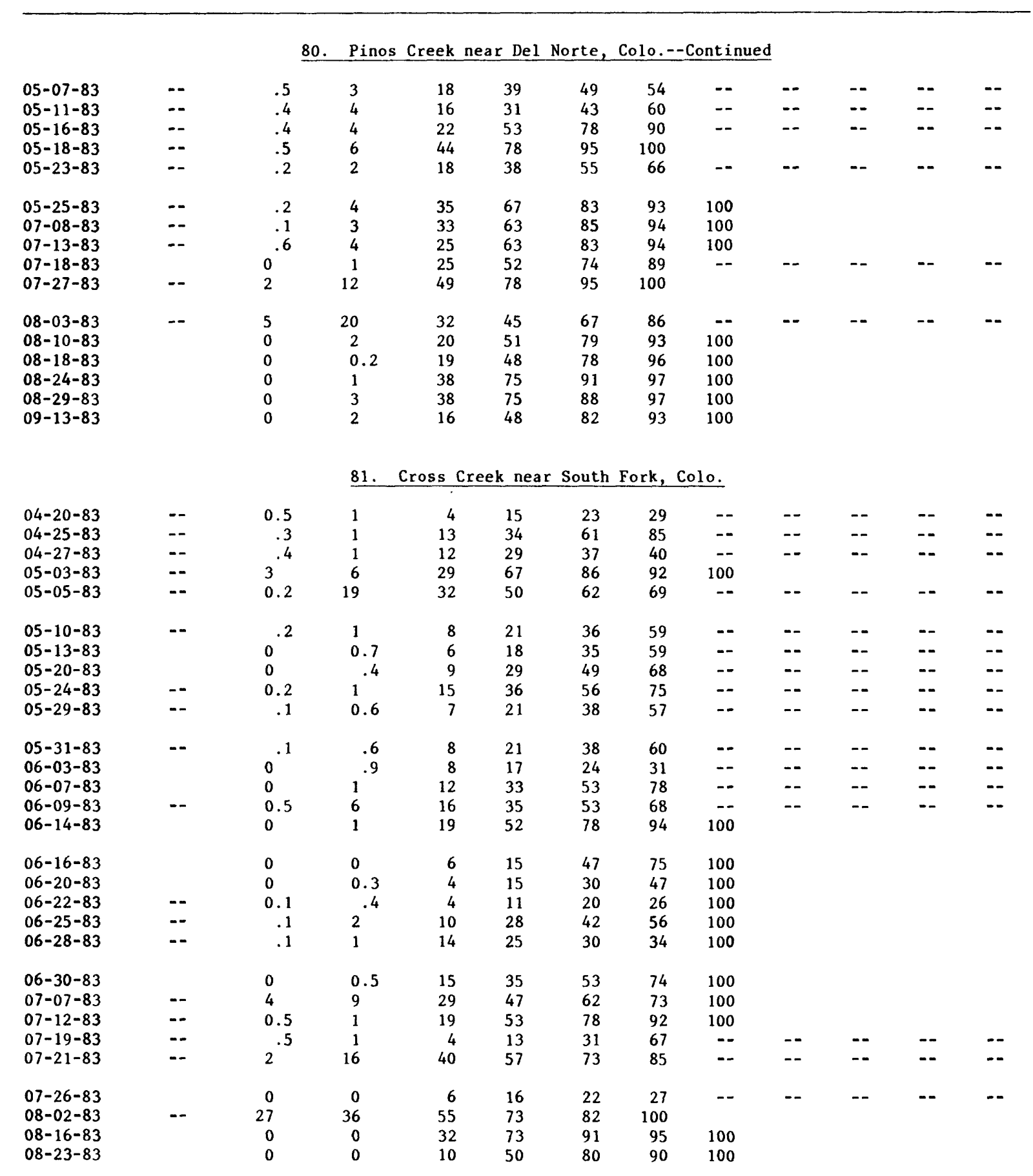


Table 3.--Bedload particle-size distributions--Continued

Percent by weight finer than size (millimeters) indicated

Date

\begin{tabular}{llllllllllll}
\hline 0.062 & 0.12 & 0.25 & 0.5 & 1 & 2 & 4 & 8 & 16 & 32 & 64 & 128
\end{tabular}

82. La Garita Creek near La Garita, Colo.

\begin{tabular}{|c|c|c|c|c|c|c|c|c|c|c|c|c|}
\hline $04-22-83$ & -- & 4 & 24 & 47 & 65 & 79 & 95 & 100 & & & & \\
\hline $04-26-83$ & -- & 1 & 5 & 36 & 67 & 88 & 97 & 100 & & & & \\
\hline $04-28-83$ & -- & 1 & 5 & 25 & 50 & 71 & 87 & -- & $\cdots$ & -- & -- & $\cdots$ \\
\hline $05-02-83$ & -- & 0.6 & 3 & 24 & 62 & 93 & 100 & & & & & \\
\hline $05-04-83$ & $\cdots$ & .5 & 4 & 26 & 67 & 93 & 100 & & & & & \\
\hline $05-07-83$ & -- & 1 & 5 & 35 & 66 & 90 & 97 & 100 & & & & \\
\hline $05-11-83$ & -- & 0.6 & 4 & 27 & 62 & 85 & 94 & 100 & & & & \\
\hline $05-16-83$ & -- & .5 & 4 & 30 & 62 & 85 & 95 & 100 & & & & \\
\hline $05-23-83$ & -- & .7 & 4 & 21 & 56 & 86 & 96 & 100 & & & & \\
\hline $05-25-83$ & -- & .6 & 3 & 14 & 45 & 82 & 97 & 100 & & & & \\
\hline $06-01-83$ & -- & .2 & 3 & 22 & 48 & 66 & 75 & -- & -- & -- & -- & -. \\
\hline $06-03-83$ & -- & .1 & 1 & 6 & 18 & 55 & 89 & -- & -- & -- & -- & -- \\
\hline $06-08-83$ & -- & .2 & 3 & 22 & 54 & 84 & 96 & 100 & & & & \\
\hline $06-13-83$ & -- & .4 & 3 & 24 & 57 & 85 & 94 & 100 & & & & \\
\hline $06-15-83$ & -- & .5 & 3 & 28 & 72 & 93 & 98 & 100 & & & & \\
\hline $06-21-83$ & -- & .4 & 7 & 44 & 82 & 96 & 100 & & & & & \\
\hline $06-22-83$ & $\cdots$ & .6 & 4 & 29 & 65 & 89 & 98 & 100 & & & & \\
\hline $06-26-83$ & -- & .6 & 4 & 27 & 48 & 64 & 76 & -- & -- & -- & -- & -- \\
\hline $06-27-83$ & -- & .3 & 2 & 11 & 36 & 74 & 94 & 100 & & & & \\
\hline $06-29-83$ & -- & 0.4 & 3 & 23 & 48 & 63 & 79 & -- & -- & -- & -- & $\cdots$ \\
\hline $07-06-83$ & -- & 1 & 6 & 72 & 98 & 98 & 98 & 100 & & & & \\
\hline $07-13-83$ & -- & 0.9 & 3 & 32 & 65 & 80 & 88 & -- & -- & -- & -- & $=-$ \\
\hline $07-27-83$ & -- & 2 & 15 & 64 & 91 & 98 & 100 & & & & & \\
\hline $08-03-83$ & -- & 2 & 15 & 57 & 72 & 100 & & & & & & \\
\hline $08-04-83$ & -- & 0.6 & 22 & 59 & 75 & 96 & 99 & 100 & & & & \\
\hline $08-10-83$ & -- & .3 & 5 & 29 & 49 & 68 & 89 & -- & -- & -- & -- & -- \\
\hline $08-15-83$ & -- & .4 & 4 & 28 & 58 & 75 & 90 & 100 & & & & \\
\hline $08-24-83$ & -- & 2 & 17 & 33 & 50 & 74 & 95 & 100 & & & & \\
\hline $08-29-83$ & & 0 & 3 & 23 & 37 & 57 & 80 & -- & -- & -- & -- & -- \\
\hline $09-08-83$ & -- & 0.2 & 3 & 31 & 48 & 63 & 79 & -- & - & -- & -- & - \\
\hline
\end{tabular}

83. House Canyon Creek near Creede, Colo.

\begin{tabular}{|c|c|c|c|c|c|c|c|c|c|c|c|c|}
\hline $04-19-83$ & -- & 13 & 64 & 90 & 97 & 99 & 100 & & & & & \\
\hline $04-26-83$ & -- & 12 & 60 & 91 & 98 & 99 & 100 & & & & & \\
\hline $04-28-83$ & -- & 11 & 27 & 52 & 82 & 94 & 100 & & & & & \\
\hline $05-03-83$ & $=$ & 0.5 & 4 & 30 & 59 & 78 & 93 & 100 & & & & \\
\hline $05-05-83$ & -- & .8 & 4 & 31 & 58 & 76 & 90 & 100 & & & & \\
\hline $05-10-83$ & -- & .3 & 2 & 20 & 41 & 61 & 78 & -- & $\infty$ & - & -- & -- \\
\hline $05-12-83$ & -- & .7 & 3 & 14 & 28 & 38 & 73 & -- & - & - & $=$ & -- \\
\hline $05-17-83$ & -- & 5 & 20 & 72 & 96 & 97 & 98 & 100 & & & & \\
\hline $05-19-83$ & - & .7 & 4 & 20 & 44 & 66 & 87 & -. & -- & -- & - & -- \\
\hline $05-24-83$ & -- & .2 & 2 & 14 & 34 & 54 & 76 & - & - & - & - & $=$ \\
\hline $05-26-83$ & -- & .1 & 2 & 17 & 42 & 65 & 85 & -- & $=-$ & - & -- & -- \\
\hline $05-31-83$ & -- & .1 & 1 & 14 & 36 & 53 & 71 & -- & -- & - & -- & - \\
\hline $06-02-83$ & -- & .1 & 0.8 & 5 & 13 & 19 & 27 & -- & -- & -- & -- & -- \\
\hline $06-07-83$ & -- & .3 & .9 & 11 & 33 & 57 & 77 & -- & -- & -- & - & -- \\
\hline $06-09-83$ & $=$ & .1 & 2 & 12 & 30 & 43 & 56 & -- & -- & - & - & -- \\
\hline
\end{tabular}


Table 3.--Bedload particle-size distributions--Continued

Date

Percent by weight finer than size (millimeters) indicated

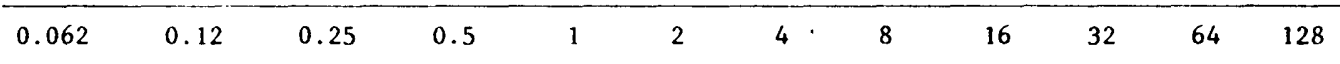

83. House Canyon Creek near Creede, Colo.--Continued

\begin{tabular}{|c|c|c|c|c|c|c|c|c|c|c|c|c|}
\hline $06-14-83$ & -- & .2 & 1 & 7 & 15 & 22 & 35 & -- & $=-$ & - & - & -- \\
\hline $06-16-83$ & -- & 1 & 4 & 19 & 34 & 46 & 56 & -- & -- & -- & -- & -- \\
\hline $06-21-83$ & -- & 3 & 15 & 51 & 76 & 90 & 100 & & & & & \\
\hline $06-23-83$ & -- & 2 & 5 & 35 & 67 & 86 & 94 & 100 & & & & \\
\hline $06-28-83$ & -- & 0.6 & 3 & 25 & 57 & 74 & 84 & -- & -- & -- & -- & -- \\
\hline $06-30-83$ & $\cdots$ & .7 & 2 & 12 & 26 & 37 & 46 & -- & -- & -- & $\cdots$ & -- \\
\hline $07-07-83$ & -- & .8 & 3 & 7 & 9 & 11 & 11 & -- & -- & -- & -- & -- \\
\hline $07-12-83$ & -- & .3 & 2 & 39 & 72 & 95 & 99 & 100 & & & & \\
\hline $07-19-83$ & -- & .7 & 4 & 24 & 55 & 90 & 99 & 100 & & & & \\
\hline $07-26-83$ & -- & .1 & 0.8 & 9 & 29 & 50 & 63 & -- & $\cdots$ & -- & -- & -- \\
\hline $08-02-83$ & -- & .8 & 4 & 41 & 79 & 93 & 98 & 100 & & & & \\
\hline $08-11-83$ & -- & 3 & 3 & 27 & 67 & 90 & 97 & 100 & & & & \\
\hline $08-16-83$ & & & 0 & 18 & 32 & 68 & 100 & & & & & \\
\hline $08-23-83$ & -- & 3 & 10 & 37 & 72 & 93 & 100 & & & & & \\
\hline $09-01-83$ & -- & 3 & 11 & 51 & 82 & 96 & 100 & & & & & \\
\hline $09-06-83$ & - & 0.3 & 2 & 18 & 46 & 59 & 66 & -- & -- & -- & -- & -- \\
\hline
\end{tabular}

84. Crooked Creek near Creede, Colo.

\begin{tabular}{|c|c|c|c|c|c|c|c|c|c|c|c|c|}
\hline $04-19-83$ & -- & 14 & 64 & 92 & 98 & 100 & & & & & & \\
\hline $04-26-83$ & -- & 2 & 6 & 28 & 44 & 60 & 71 & - & -- & - & -- & -- \\
\hline $04-28-83$ & & 0 & 4 & 21 & 46 & 67 & 83 & -- & -- & -- & -- & -- \\
\hline $05-03-83$ & & 0 & 8 & 25 & 48 & 56 & 62 & -- & -- & -- & -- & -- \\
\hline $05-05-83$ & & 0 & 7 & 29 & 52 & 67 & 79 & -- & -- & - & -- & -- \\
\hline $05-10-83$ & -- & 0.9 & 4 & 28 & 51 & 68 & 84 & -- & -- & -- & -- & -- \\
\hline $05-12-83$ & -- & .2 & 1 & 21 & 44 & 59 & 71 & -- & -- & - & -- & -- \\
\hline $05-17-83$ & & 0 & 4 & 24 & 47 & 64 & 86 & -- & -- & - & -- & -. \\
\hline $05-19-83$ & -- & 2 & 10 & 39 & 61 & 82 & 100 & & & & & \\
\hline $05-24-83$ & -- & 1 & 6 & 33 & 52 & 64 & 92 & 100 & & & & \\
\hline $05-26-83$ & -- & 0.1 & 2 & 15 & 33 & 48 & 68 & -- & -- & -- & -- & -- \\
\hline $05-31-83$ & -- & .2 & 2 & 25 & 39 & 52 & 62 & -- & -- & -- & -- & -- \\
\hline $06-02-83$ & -- & 2. & 11 & 32 & 50 & 65 & 81 & -- & -- & -- & -- & -- \\
\hline $06-07-83$ & & 0 & 0.4 & 8 & 22 & 43 & 67 & -- & -- & -- & -- & - \\
\hline $06-09-83$ & & 0 & .8 & 8 & 22 & 39 & 68 & -- & -- & -- & -- & -- \\
\hline $06-14-83$ & & 0 & 4 & 17 & 96 & 100 & & & & & & \\
\hline $06-16-83$ & -- & 13 & 13 & 25 & 63 & 75 & 100 & & & & & \\
\hline $06-21-83$ & -- & 80 & 80 & 100 & & & & & & & & \\
\hline $06-23-83$ & & 0 & 8 & 23 & 85 & 92 & 100 & & & & & \\
\hline $06-28-83$ & -- & 13 & 13 & 50 & 63 & 75 & 100 & & & & & \\
\hline $06-30-83$ & -- & 67 & 67 & 100 & & & & & & & & \\
\hline $07-07-83$ & -- & 0.9 & 4 & 16 & 28 & 39 & 43 & -- & -- & -- & -- & - \\
\hline $07-19-83$ & -- & 33 & 67 & 100 & & & & & & & & \\
\hline $07-26-83$ & & 0 & 4 & 49 & 81 & 95 & 98 & 100 & & & & \\
\hline $08-11-83$ & -- & 2 & 7 & 32 & 57 & 78 & 100 & & & & & \\
\hline $09-01-83$ & -- & 0.4 & 2 & 13 & 25 & 35 & 52 & -- & -- & -- & -- & -- \\
\hline
\end{tabular}


Table 3.--Bedload particle-size distributions--Continued

Date

Percent by weight finer than size (millimeters) indicated

$\begin{array}{lllllllllll}0.062 & 0.12 & 0.25 & 0.5 & 1 & 2 & 4 & 8 & 16 & 32 & 64\end{array}$

85. Rough Creek near Antonito, Colo.

\begin{tabular}{|c|c|c|c|c|c|c|c|c|c|c|c|c|}
\hline $04-28-83$ & & 0 & 8 & 15 & 15 & 15 & 15 & -- & -- & -- & -- & -- \\
\hline $05-02-83$ & -- & 25 & 38 & 50 & 50 & 50 & 75 & $\cdots$ & -- & $\cdots$ & - & $-\infty$ \\
\hline $05-09-83$ & & 0 & 13 & 25 & 50 & 75 & 100 & & & & & \\
\hline $05-11-83$ & & 0 & 0.8 & 0.8 & 2 & 2 & 6 & -- & $=-$ & -- & -- & -- \\
\hline $05-16-83$ & -- & 2 & 7 & 9 & 10 & 12 & 16 & -- & -- & -- & -- & -- \\
\hline $05-18-83$ & & & 0 & 33 & 100 & & & & & & & \\
\hline $05-22-83$ & & & 0 & 3 & 5 & 5 & 8 & -- & -- & -- & -- & -- \\
\hline $05-24-83$ & & 0 & 6 & 7 & 8 & 10 & 14 & -- & -- & -- & -- & -- \\
\hline $05-31-83$ & & 0 & 0.1 & 1 & 5 & 11 & 22 & -- & -- & -- & -- & -- \\
\hline $06-02-83$ & -- & 0.1 & .4 & 1 & 3 & 7 & 15 & -- & -- & -- & -- & -- \\
\hline $06-07-83$ & -- & .1 & .8 & 3 & 7 & 12 & 28 & -- & -- & -- & -- & -- \\
\hline $06-09-83$ & & 0 & .3 & 4 & 12 & 26 & 42 & -- & -- & -- & -- & -- \\
\hline $06-14-83$ & -- & 0.1 & .4 & 3 & 8 & 15 & 26 & -- & -- & -- & -- & -- \\
\hline $06-16-83$ & -- & .2 & .6 & 5 & 13 & 30 & 48 & -- & -- & -- & -- & -- \\
\hline $06-21-83$ & & 0 & .3 & 1 & 3 & 6 & 10 & -- & -- & -- & -- & -- \\
\hline $06-23-83$ & & 0 & .2 & 0.4 & 0.8 & 1 & 2 & -- & -- & -- & -- & -- \\
\hline $06-29-83$ & - & 0.2 & $: 4$ & 1 & 5 & 14 & 36 & -- & -- & -- & -- & -- \\
\hline $07-05-83$ & & & $0^{\circ}$ & 100 & & & & & & & & \\
\hline $07-20-83$ & & 0 & 8 & 17 & 33 & 50 & 67 & -- & -- & -- & -- & $=-$ \\
\hline $08-03-83$ & & & 0 & 0.5 & 5 & 9 & 17 & -- & -- & -- & -- & -- \\
\hline $08-10-83$ & -- & 13 & 25 & 63 & 75 & 88 & 100 & & & & & \\
\hline $08-17-83$ & & 0 & 2 & 6 & 14 & 37 & 63 & -- & -- & -- & -- & -- \\
\hline $08-23-83$ & & 0 & 0.2 & 2 & 5 & 10 & 19 & -- & -- & -- & -- & -- \\
\hline
\end{tabular}

86. Grouse Creek near Osier, Colo.

\begin{tabular}{|c|c|c|c|c|c|c|c|c|c|c|c|c|}
\hline $\begin{array}{l}05-04-83 \\
05-00-83\end{array}$ & -- & 3 & $\begin{array}{r}10 \\
5\end{array}$ & 41 & 72 & $\begin{array}{l}97 \\
98\end{array}$ & 100 & & & & & \\
\hline $05-09-83$ & -- & 0.4 & 5 & 48 & 91 & 98 & 100 & & & & & \\
\hline $05-11-83$ & -- & .6 & 5 & 30 & 48 & 57 & 62 & -- & -- & -- & -- & -- \\
\hline $05-16-83$ & -- & 29 & 57 & 100 & & & & & & & & \\
\hline $05-18-83$ & -- & 3 & 8 & 36 & 65 & 81 & 100 & & & & & \\
\hline $05-22-83$ & & 0 & 4 & 25 & 65 & 100 & & & & & & \\
\hline $05-24-83$ & -- & 1 & 3 & 36 & 68 & 85 & 95 & 100 & & & & \\
\hline $05-31-83$ & -- & 0.1 & 1 & 17 & 31 & 41 & 48 & -- & -- & -- & -- & -- \\
\hline $06-02-83$ & -- & .2 & 1 & 16 & 41 & 63 & 74 & -- & -- & $-\infty$ & -- & $\cdots$ \\
\hline $06-07-83$ & -- & .6 & 3 & 37 & 76 & 93 & 100 & & & & & \\
\hline $06-09-83$ & & 0 & 0.1 & 2 & 6 & 13 & 24 & -- & -- & -- & -- & - \\
\hline $06-14-83$ & & 0 & .5 & 8 & 27 & 51 & 75 & -- & $-\infty$ & -- & -- & - \\
\hline $06-16-83$ & & 0 & .6 & 10 & 28 & 46 & 59 & -- & -- & -- & -- & - \\
\hline $06-21-83$ & -- & 0.3 & 3 & 24 & 61 & 87 & 97 & 100 & & & & \\
\hline $06-29-83$ & -- & .7 & 7 & 36 & 65 & 83 & 97 & 100 & & & & \\
\hline $07-05-83$ & - & .1 & 0.4 & 4 & 15 & 30 & 48 & $\cdots$ & -- & -- & $\cdots$ & -- \\
\hline $07-11-83$ & -- & 44 & 75 & 94 & 100 & & & & & & & \\
\hline $08-03-83$ & -- & 17 & 67 & 100 & & & & & & & & \\
\hline $08-23-83$ & & 0 & 50 & 50 & 50 & 100 & & & & & & \\
\hline
\end{tabular}


Table 3.--Bedload particle-size distributions--Continued

\begin{tabular}{ccccccccccccc}
\hline & \multicolumn{4}{c}{ Percent by weight finer } & \multicolumn{1}{c}{ than size (millimeters) indicated } \\
\cline { 2 - 9 } & 0.062 & 0.12 & 0.25 & 0.5 & 1 & 2 & 4 & 8 & 16 & 32 & 64 & 128
\end{tabular}

87. Wapsipinicon River near Dewitl, Iowa

\begin{tabular}{|c|c|c|c|c|c|c|c|c|}
\hline $\begin{array}{l}04-13-78 \\
06-22-78 \\
03-21-79 \\
04-26-79 \\
08-22-79\end{array}$ & $\begin{array}{l}-- \\
-- \\
-- \\
--\end{array}$ & $\begin{array}{l}-- \\
-- \\
-- \\
--\end{array}$ & $\begin{array}{r}3 \\
12 \\
7 \\
19 \\
8\end{array}$ & $\begin{array}{l}38 \\
69 \\
29 \\
62 \\
42\end{array}$ & $\begin{array}{l}75 \\
95 \\
64 \\
89 \\
82\end{array}$ & $\begin{array}{l}90 \\
98 \\
86 \\
97 \\
95\end{array}$ & $\begin{array}{l}98 \\
99 \\
97 \\
99 \\
99\end{array}$ & $\begin{array}{l}100 \\
100 \\
100 \\
100 \\
100\end{array}$ \\
\hline $\begin{array}{l}07-02-80 \\
08-25-80 \\
04-14-81 \\
06-17-81 \\
09-04-81\end{array}$ & $\begin{array}{l}-- \\
-- \\
--\end{array}$ & $\begin{array}{l}-- \\
-- \\
-\end{array}$ & $\begin{array}{r}12 \\
8 \\
2 \\
0 \\
0\end{array}$ & $\begin{array}{l}60 \\
42 \\
38 \\
33 \\
49\end{array}$ & $\begin{array}{l}90 \\
82 \\
80 \\
82 \\
92\end{array}$ & $\begin{array}{l}97 \\
95 \\
93 \\
96 \\
98\end{array}$ & $\begin{array}{r}99 \\
99 \\
98 \\
99 \\
100\end{array}$ & $\begin{array}{r}99 \\
100 \\
100 \\
100\end{array}$ \\
\hline
\end{tabular}

88. Iowa River at Wapello, Iowa

\begin{tabular}{|c|c|c|c|c|c|c|c|c|c|c|}
\hline $03-28-78$ & -- & -- & 2 & 39 & 85 & 98 & 100 & & & \\
\hline $04-25-78$ & -- & $-\infty$ & 2 & 33 & 75 & 92 & 99 & 100 & & \\
\hline $06-21-78$ & -- & -- & 3 & 34 & 69 & 87 & 97 & 99 & 100 & \\
\hline $07-25-78$ & -- & -- & 2 & 14 & 55 & 80 & 93 & 99 & 100 & \\
\hline $06-13-79$ & -- & -- & 6 & 47 & 85 & 96 & 99 & 100 & & \\
\hline $04-03-80$ & -- & -- & 1 & 21 & 71 & 92 & 94 & 100 & & \\
\hline $06-02-80$ & -- & -- & 4 & 35 & 73 & 91 & 98 & 100 & & \\
\hline $09-03-80$ & $=-$ & -- & 3 & 31 & 73 & 88 & 95 & 98 & 99 & 100 \\
\hline $06-18-81$ & & & 0 & 43 & 88 & 96 & 99 & 100 & & \\
\hline $07-02-81$ & & & 0 & 37 & 73 & 90 & 98 & 100 & & \\
\hline
\end{tabular}

89. Wisconsin River at Muscoda, Wis.

\begin{tabular}{|c|c|c|c|c|c|c|c|c|c|}
\hline $03-24-77$ & 0 & 0.1 & 4 & 57 & 87 & 92 & 95 & 98 & 100 \\
\hline $04-19-77$ & 0.1 & .1 & 7 & 65 & 90 & 94 & 97 & 99 & 100 \\
\hline $05-16-77$ & & 0 & 9 & 62 & 88 & 94 & 97 & 99 & 100 \\
\hline $06-15-77$ & 0 & 0.1 & 7 & 61 & 91 & 97 & 99 & 100 & \\
\hline $07-20-77$ & & 0 & 8 & 79 & 97 & 99 & 100 & & \\
\hline $08-24-77$ & & 0 & 0.4 & 24 & 84 & 95 & 99 & 100 & \\
\hline $09-27-77$ & & 0 & 1 & 34 & 90 & 97 & 99 & 100 & \\
\hline $10-18-77$ & & 0 & 5 & 63 & 95 & 99 & 100 & & \\
\hline $11-14-77$ & & 0 & 23 & 64 & 92 & 97 & 100 & & \\
\hline $04-17-78$ & 0 & 0.1 & 7 & 72 & 96 & 99 & 100 & & \\
\hline $05-08-78$ & 0 & .1 & 6 & 74 & 98 & 99 & 100 & & \\
\hline $06-06-78$ & 0 & .1 & 4 & 70 & 95 & 98 & 99 & 100 & \\
\hline $07-11-78$ & 0 & .1 & 6 & 66 & 93 & 97 & 98 & 99 & 100 \\
\hline $08-15-78$ & 0.1 & .2 & 6 & 65 & 94 & 98 & 100 & & \\
\hline $11-14-78$ & & 0 & 5 & 72 & 95 & 99 & 100 & & \\
\hline $03-28-79$ & & 0 & 5 & 60 & 90 & 96 & 99 & 100 & \\
\hline $04-24-79$ & & 0 & 5 & 66 & 97 & 99 & 100 & & \\
\hline $07-05-79$ & & 0 & 4 & 52 & 88 & 96 & 99 & 100 & \\
\hline $08-25-79$ & & 0 & 5 & 64 & 95 & 98 & 100 & & \\
\hline
\end{tabular}


Table 3.--Bedload particle-size distributions--Continued

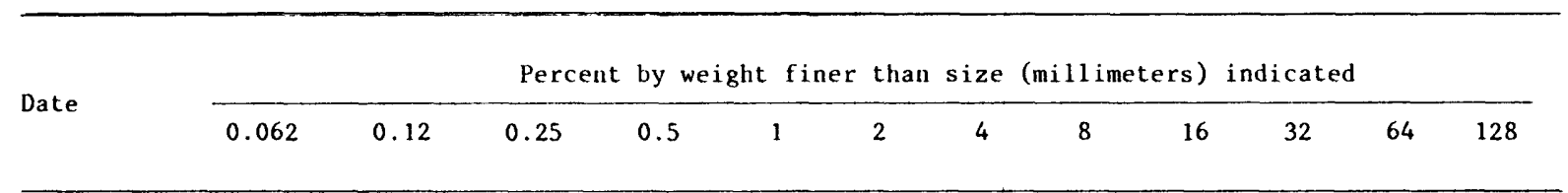

90. Black River near Galesville, Wis.

\begin{tabular}{|c|c|c|c|c|c|c|c|c|c|c|}
\hline $04-23-77$ & 0.4 & 0.7 & 9 & 51 & 93 & 98 & 100 & & & \\
\hline $05-27-77$ & .2 & .2 & 8 & 62 & 96 & 99 & 100 & & & \\
\hline $06-29-77$ & .1 & .1 & 5 & 50 & 92 & 98 & 100 & & & \\
\hline $07-12-77$ & .1 & .1 & 4 & 44 & 91 & 98 & 100 & & & \\
\hline $08-02-77$ & & 0 & 6 & 65 & 96 & 99 & 100 & & & \\
\hline $09-27-77$ & .2 & .4 & 6 & 48 & 88 & 94 & 96 & 97 & 99 & 100 \\
\hline $10-19-77$ & 0 & .1 & 4 & 47 & 93 & 99 & 100 & & & \\
\hline $11-08-77$ & & 0 & 5 & 56 & 95 & 99 & 100 & & & \\
\hline $03-31-78$ & 0.3 & 0.6 & 10 & 65 & 96 & 99 & 100 & & & \\
\hline $04-10-78$ & .2 & .5 & 20 & 74 & 96 & 98 & 99 & 100 & & \\
\hline $05-10-78$ & 0 & .1 & 5 & 66 & 97 & 100 & & & & \\
\hline $06-13-78$ & 0.1 & .2 & 10 & 69 & 97 & 100 & & & & \\
\hline $08-09-78$ & & 0 & 12 & 77 & 97 & 99 & 99 & 100 & & \\
\hline $10-28-78$ & & 0 & 4 & 65 & 97 & 99 & 100 & & & \\
\hline $04-25-79$ & & 0 & 4 & 30 & 84 & 95 & 98 & 99 & 100 & \\
\hline $05-31-79$ & & 0 & 7 & 32 & 88 & 97 & 99 & 100 & & \\
\hline $07-23-79$ & & 0 & 16 & 52 & 94 & 99 & 100 & & & \\
\hline
\end{tabular}

91. Chippewa River near Caryville, Wis.

$\begin{array}{llllllllllll}10-12-77 & 0 & 0.1 & 4 & 29 & 76 & 91 & 95 & 98 & 100 & \\ 04-05-78 & 0 & .1 & 7 & 29 & 69 & 88 & 94 & 98 & 100 & \\ 04-14-78 & & 0 & 1 & 40 & 81 & 94 & 97 & 99 & 100 & \\ 06-06-78 & 0 & 0.1 & 2 & 31 & 88 & 96 & 98 & 100 & & \\ 07-06-78 & & 0 & 2 & 42 & 75 & 86 & 93 & 98 & 100 & \\ & & & & & & & & & & & \\ 09-19-78 & 0 & 0.1 & 1 & 19 & 47 & 69 & 85 & 96 & 100 & \\ 04-17-79 & & 0 & 3 & 42 & 74 & 82 & 88 & 93 & 98 & 100 & \ldots 8 \\ 04-23-79 & & 0 & 1 & 16 & 45 & 70 & 84 & 91 & 96 & 98 & \ldots\end{array}$

92. Chippewa River at Durand, Wis.

$\begin{array}{rlllrlrrrrr}11-11-75 & 0.1 & 0.2 & 2 & 46 & 87 & 96 & 98 & 99 & 100 & \\ 04-06-76 & & 0 & 0.7 & 28 & 84 & 92 & 94 & 97 & 99 & 100 \\ 04-14-76 & & 0 & .2 & 6 & 50 & 90 & 97 & 99 & 100 & \\ 06-03-76 & & 0 & .7 & 23 & 75 & 92 & 97 & 99 & 100 & \\ 07-01-76 & & 0 & 3 & 51 & 91 & 98 & 99 & 100 & & \\ & & & & & & & & & \\ 08-31-76 & 0 & 0.1 & 7 & 62 & 91 & 93 & 97 & 99 & 100 & \\ 10-18-76 & 0 & .1 & 4 & 50 & 90 & 98 & 100 & & & \\ 11-16-76 & & 0 & 5 & 63 & 93 & 98 & 99 & 100 & & \\ 02-02-77 & 0 & 0.1 & 2 & 44 & 87 & 95 & 99 & 100 & & \\ 03-17-77 & 0 & .1 & 5 & 38 & 77 & 90 & 95 & 99 & 100 & \\ 04-13-77 & 0.1 & .3 & 3 & 53 & 91 & 97 & 99 & 100 & & \\ 05-10-77 & & 0 & 2 & 48 & 81 & 91 & 98 & 100 & & \\ 06-15-77 & 0 & .1 & 1 & 35 & 80 & 95 & 99 & 100 & & \\ 07-06-77 & 0 & .1 & 2 & 39 & 78 & 89 & 93 & 97 & 99 & 100 \\ 08-24-77 & & 0 & 0.5 & 20 & 57 & 88 & 97 & 100 & & \end{array}$


Table 3.--Bedload particle-size distributions--Cont inued

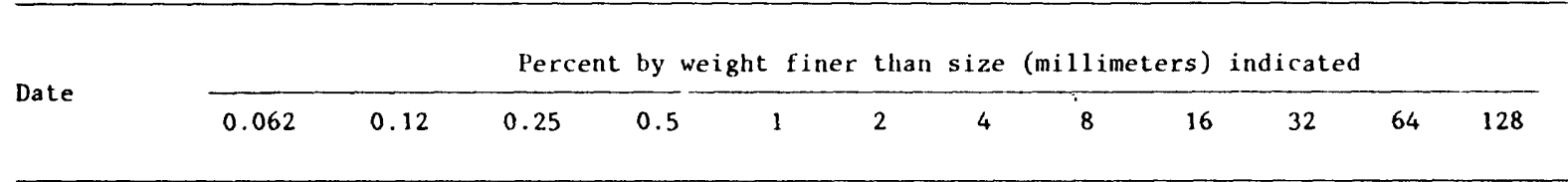

92. Chippewa River at Durand, Wis.--Continued.

$\begin{array}{rlllllllrrr}09-13-77 & & 0 & 2 & 43 & 90 & 97 & 100 & \\ 10-12-77 & 0 & 0.1 & 3 & 44 & 82 & 94 & 97 & 99 & 100 \\ 11-16-77 & & 0 & 4 & 53 & 92 & 98 & 99 & 100 & 100 \\ 04-05-78 & & 0 & 2 & 39 & 83 & 95 & 98 & 99 & 100 \\ 06-06-78 & 0 & 0.3 & 5 & 48 & 87 & 97 & 99 & 100 & \\ 07-06-78 & 0.1 & .2 & 3 & 57 & 92 & 97 & 99 & 100 & 100 & 98 \\ 10-31-78 & & 0 & 1 & 30 & 75 & 91 & 97 & 99 & 100 \\ 04-17-79 & & 0 & 2 & 45 & 86 & 94 & 96 & 98 & 98 \\ 04-23-79 & & 0 & 4 & 49 & 87 & 95 & 97 & 98 & 100 \\ 05-29-79 & 0 & 1 & 2 & 34 & 83 & 95 & 97 & 99 & 100\end{array}$

93. Chippewa River near Pepin, Wis.

\begin{tabular}{|c|c|c|c|c|c|c|c|c|c|}
\hline $09-01-76$ & & 0 & 2 & 41 & 82 & 93 & 97 & 99 & 100 \\
\hline $09-20-76$ & & 0 & 1 & 37 & 86 & 96 & 100 & & \\
\hline $10-19-76$ & 0 & 0.1 & 2 & 52 & 92 & 97 & 99 & 100 & \\
\hline $11-17-76$ & & 0 & 1 & 36 & 84 & 96 & 99 & 100 & \\
\hline $03-16-77$ & 0.2 & 0.2 & 4 & 56 & 92 & 98 & 99 & 100 & \\
\hline $04-14-77$ & 0 & .1 & 2 & 34 & 86 & 96 & 98 & 100 & \\
\hline $05-11-77$ & 0 & .1 & 3 & 44 & 89 & 96 & 98 & 100 & \\
\hline $07-07-77$ & & 0 & 4 & 53 & 93 & 98 & 100 & & \\
\hline $09-14-77$ & 0 & 0.1 & 2 & 45 & 88 & 97 & 99 & 100 & \\
\hline $10-13-77$ & 0 & .1 & 5 & 51 & 83 & 93 & 96 & 98 & 100 \\
\hline $11-17-77$ & & 0 & 2 & 46 & 88 & 96 & 99 & 100 & \\
\hline $04-06-78$ & 0 & 0.1 & 7 & 62 & 94 & 99 & 99 & 100 & \\
\hline $05-16-78$ & & 0 & 7 & 65 & 92 & 98 & 99 & 100 & \\
\hline $06-07-78$ & 0 & 0.2 & 3 & 42 & 83 & 93 & 97 & 99 & 100 \\
\hline $07-07-78$ & 0 & .1 & 0.2 & 3 & 50 & 87 & 96 & 98 & 100 \\
\hline $09-20-78$ & 0.1 & .3 & 3 & 45 & 84 & 94 & 98 & 99 & 100 \\
\hline $11-01-78$ & & 0 & 2 & 40 & 82 & 94 & 98 & 100 & \\
\hline $04-18-79$ & & 0 & 5 & 50 & 85 & 93 & 96 & 98 & 99 \\
\hline $04-24-79$ & & 0 & 4 & 53 & 90 & 97 & 98 & 99 & 100 \\
\hline $05-30-79$ & & 0 & 2 & 49 & 86 & 96 & 99 & 100 & \\
\hline $07-24-79$ & & 0 & 2 & 42 & 87 & 97 & 99 & 100 & \\
\hline $09-11-79$ & & 0 & 1 & 42 & 88 & 96 & 98 & 99 & 100 \\
\hline
\end{tabular}

\footnotetext{
${ }^{1} 100$ percent is less than 76 millimeters.

2100 percent is less than 90 millimeters.
}

${ }^{3}$ Where more than one size analysis is listed for a given date, each analysis represents a different transect. Bedload transport rates (Table 1) are composites of all transects.

${ }^{4}$ All percentages for this station interpolated from dry-sieve analysis that involved sieve classes different from those listed here.

${ }^{5}$ Sediment samples wet-sieved; values then adjusted by original investigators such that percentages listed here approximate dry-sieved percentages. 
Table 4.--Bed-material particle-size distributions

[--, no data available]

Date

Percent by weight finer than size (millimeters) indicated

Sampling location,

in meters from
reference point

\section{Susitna River near Talkeetna, Alaska ${ }^{1}$}

$08-04-82$

08-04-82

05-16-83

05-16-83

05-16-83

05-16-83

05-16-83

05-16-83

05-16-83

$05-16-83$

05-16-83

$05-17-84$

05-17-84

05-17-84

05-17-84

05-31-85

05-31-85

07-28-82

$07-28-82$

09-20-82

09-20-82

09-20-82

09-20-82

05-18-83

05-18-83

$05-18-83$

05-18-83

05-18-83

05-18-83

05-18-83

05-18-83

09-13-83

09-13-83

$09-13-83$

09-13-83

09-13-83

05-15-84

05-15-84

05-15-84

05-15-84

05-15-84

05-15-84

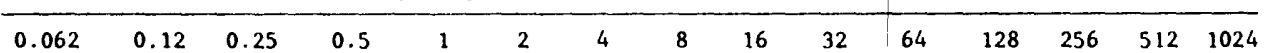

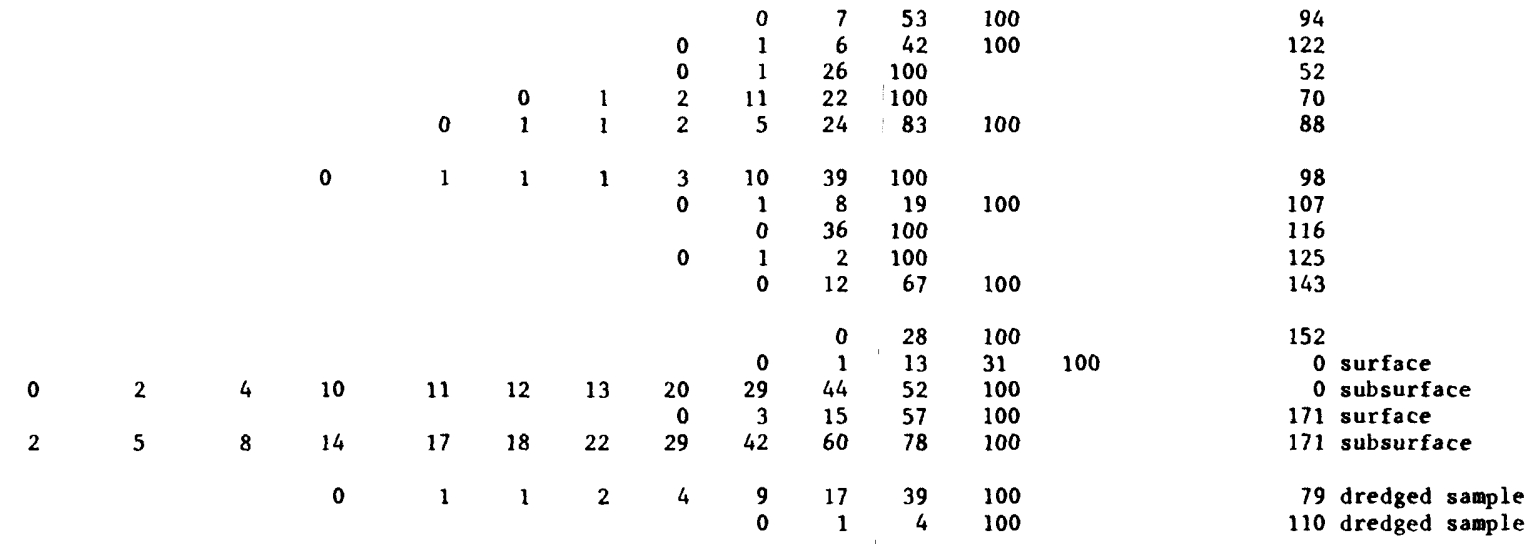

2. Talkeetna River near Talkeetna, Alaskal

2.090 .100

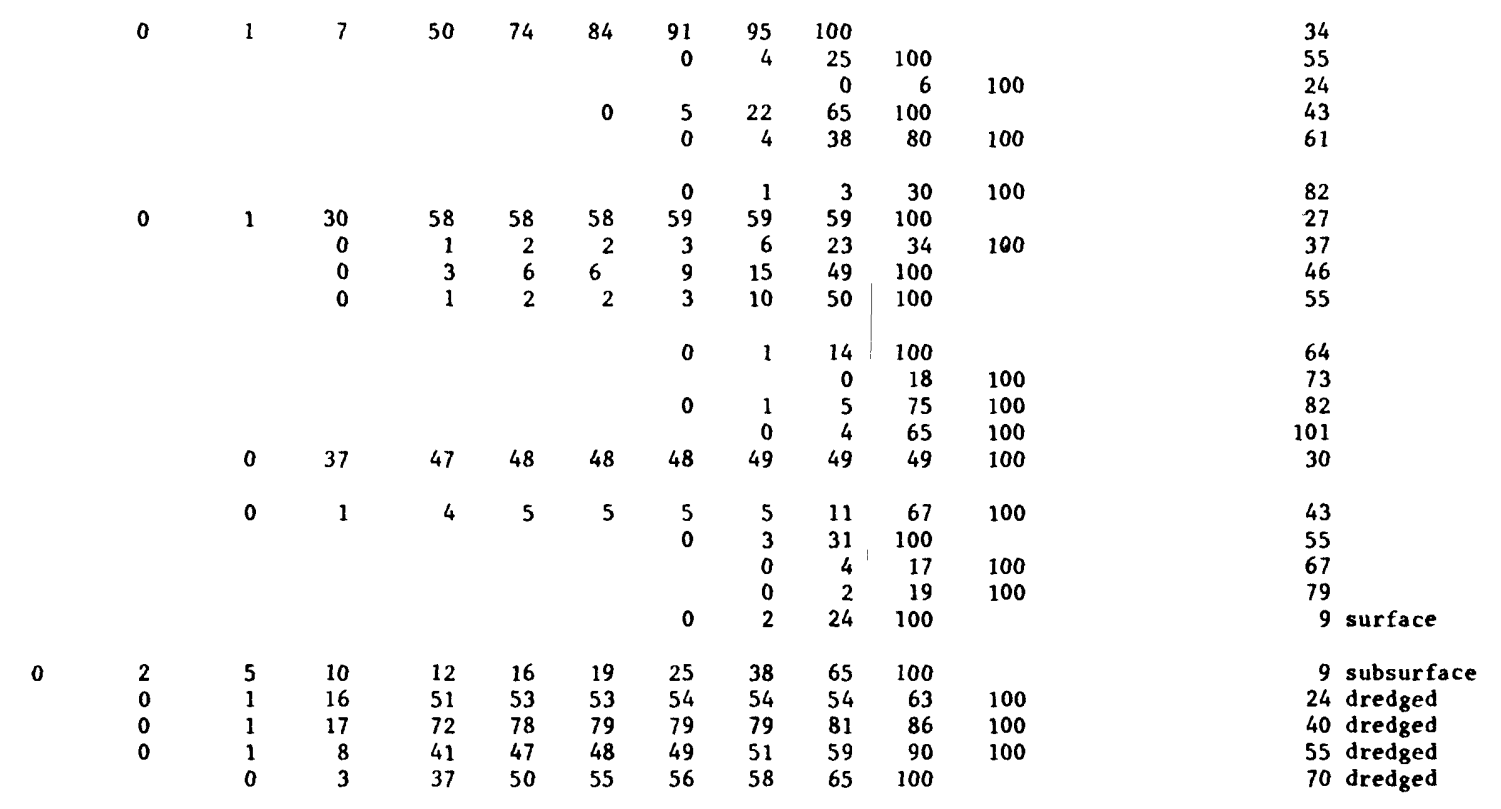


Table 4.--Bed-material particle-size distributions--Continued

\begin{tabular}{|c|c|c|c|c|c|c|c|c|c|c|c|c|c|c|c|c|}
\hline \multirow{2}{*}{ Date } & \multicolumn{5}{|c|}{ Percent by weight } & \multirow{2}{*}{$\frac{\text { finer }}{2}$} & \multirow{2}{*}{$\frac{\text { than }}{4}$} & \multirow{2}{*}{$\frac{\text { size }}{8}$} & \multicolumn{7}{|c|}{ (millimeters) indicated } & \multirow{2}{*}{$\begin{array}{l}\text { Sampling location, } \\
\text { in meters from } \\
\text { reference point }\end{array}$} \\
\hline & 0.062 & 0.12 & 0.25 & 0.5 & 1 & & & & 16 & 32 & 64 & 128 & 256 & 512 & 1024 & \\
\hline
\end{tabular}

\section{Talkeetna River near Talkeetna, Alaska ${ }^{1}-$-Continued}

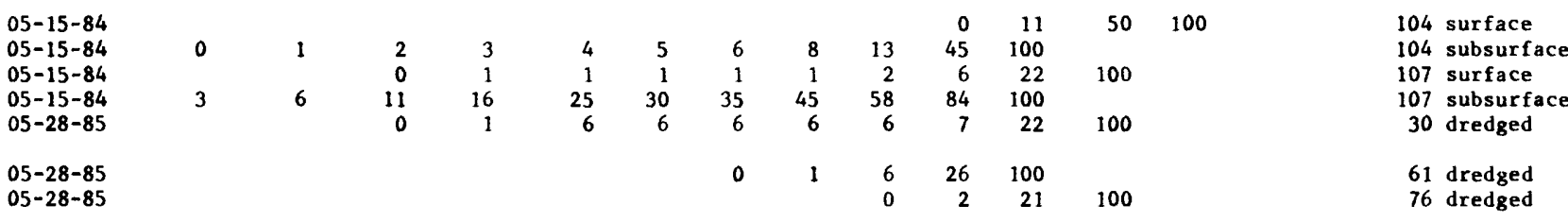

3. Chulitna River below Canyon near Talkeetna, Alaska

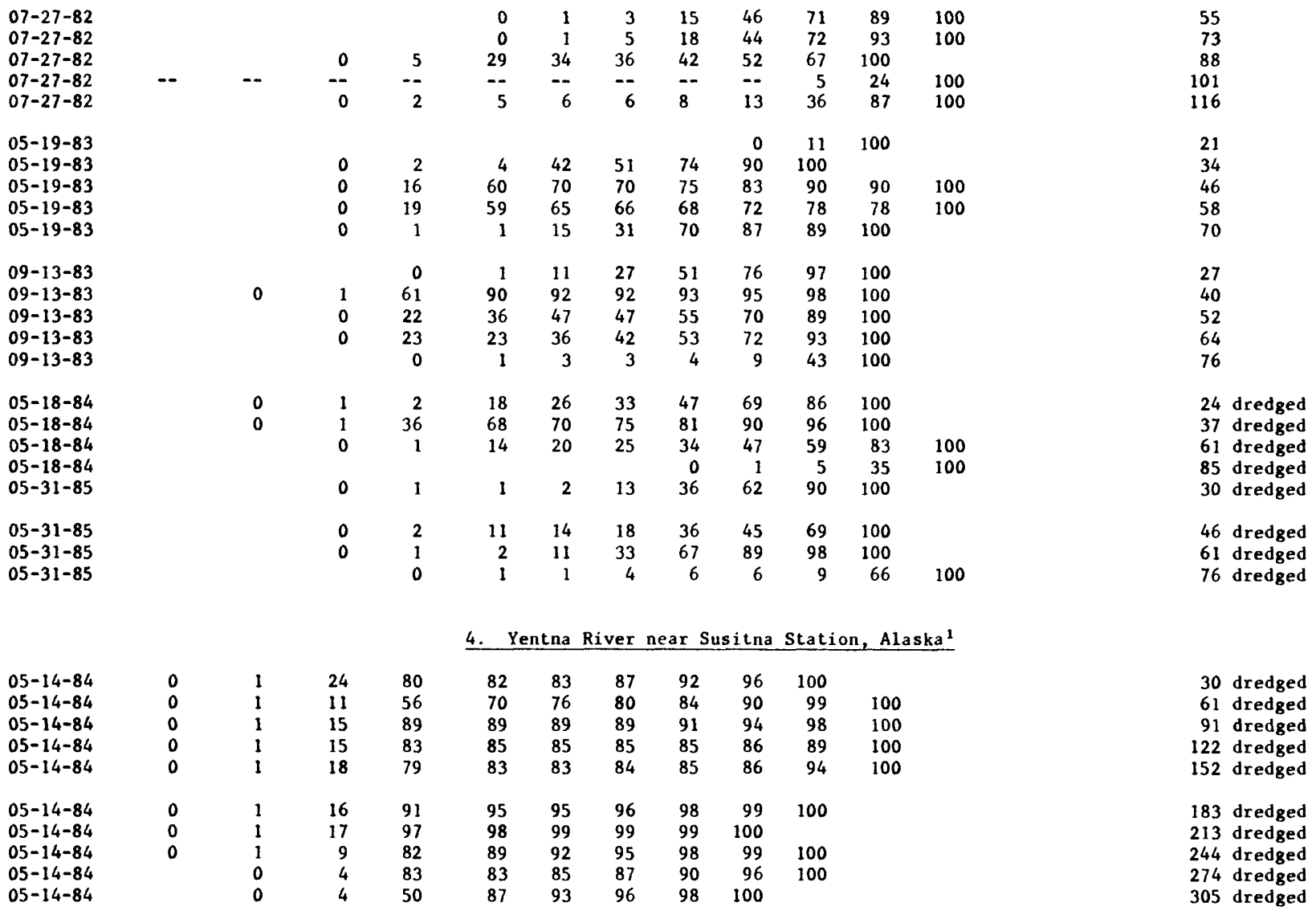


Table 4.--Bed-material particle-size distributions--Continued

Percent by weight finer than size (millimeters) indicated

Sampling location,

Date

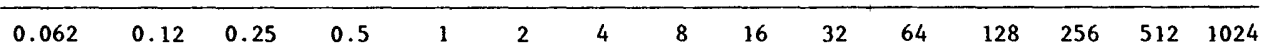

in meters from

reference point

4. Yentna River near Susitna Station, Alaska ${ }^{1}-$-Continued

05-14-84

05-14-84

08-14-85

08-14-85

08-14-85

08-14-85

08-14-85

05-17-84

05-17-84

05-17-84

05-17-84

05-30-85

05-30-85

05-30-85

05-17-84

05-17-84

05-17-84

05-17-84

05-17-84

05-17-84

05-17-84

05-17-84

05-17-84

05-17-84

05-30-85

05-30-85

06-26-85

$06-26-85$

06-26-85

06-26-85

06-26-85

06-26-85

06-26-85

06-26-85

06-26-85

06-26-85

06-26-85

06-26-85

06-26-85

06-26-85 06-26-85 $\begin{array}{llllll}46 & 56 & 68 & 82 & 95 & 100\end{array}$

\begin{tabular}{rrrrrrrrr|r}
0 & 2 & 46 & 56 & 68 & 82 & 95 & 100 & & \\
0 & 1 & 50 & 51 & 61 & 79 & 94 & 100 & & \\
0 & 2 & 38 & 88 & 90 & 94 & 94 & 99 & 100 & \\
0 & 5 & 64 & 90 & 92 & 93 & 95 & 98 & 100 & \\
0 & 6 & 65 & 92 & 95 & 96 & 97 & 99 & 100 & \\
0 & 4 & 51 & 93 & 93 & 94 & 96 & 98 & 100 & \\
0 & 3 & 40 & 60 & 62 & 64 & 66 & 70 & 78 & 100
\end{tabular}

5. Susitna River (Right Channel) below Chulitna River near Talkeetna, Alaska ${ }^{1}$

\begin{tabular}{|c|c|c|c|c|c|c|c|c|c|}
\hline 0 & 1 & 3 & 3 & $\begin{array}{l}4 \\
0\end{array}$ & $\begin{array}{l}6 \\
1\end{array}$ & $\begin{array}{r}12 \\
2\end{array}$ & $\begin{array}{l}29 \\
15\end{array}$ & $\begin{array}{l}64 \\
15\end{array}$ & $\begin{array}{l}100 \\
100\end{array}$ \\
\hline 0 & 2 & 3 & 9 & 29 & 48 & 72 & $\begin{array}{r}87 \\
0\end{array}$ & $\begin{array}{r}100 \\
25\end{array}$ & 100 \\
\hline & & & 0 & 1 & 2 & 6 & 12 & 42 & 100 \\
\hline & & & 0 & 1 & 2 & 4 & 11 & 47 & 100 \\
\hline 0 & 1 & 3 & 6 & 10 & 23 & 47 & 85 & 95 & 100 \\
\hline
\end{tabular}

6. Susitna River (Left Channel) below Chulitna River near Talkeetna, Alaska ${ }^{1}$

1,150 dredged

1,180 dredged

1,210 dredged

1,240 dredged

1,130 dredged

1,160 dredged

1,190 dredged $\begin{array}{lllllll}0 & 2 & 8 & 44 & 100\end{array}$

$\begin{array}{rrrrrrr} & & & 0 & 2 & 8 & 4 \\ & & 0 & 1 & 4 & 18 & 6 \\ 3 & 3 & 4 & 7 & 35 & 100 & \\ & & 1 & 2 & 10 & 35 & 7 \\ 22 & 24 & 29 & 38 & 60 & 88 & 10 \\ & & 0 & 1 & 5 & 28 & 6 \\ 20 & 22 & 27 & 38 & 55 & 75 & 10 \\ 5 & 5 & 5 & 6 & 10 & 29 & 10 \\ 62 & 64 & 68 & 74 & 84 & 97 & 10\end{array}$

65100

$74 \quad 100$

$\begin{array}{llll}0 & 1 & 2 & 3\end{array}$

1

$\begin{array}{rrrr}1 & 2 & 5 & 16 \\ 1 & 2 & 3 & 5\end{array}$

25

2
1
1
0
0
2
1

$\begin{array}{rr}2 & \\ 9 & 27 \\ 45 & 92 \\ 1 & \\ 2 & \end{array}$

$\begin{array}{ll} & \\ 0 & \\ 4 & \\ 2 & \\ 4 & \\ 2 & \\ 1 & \\ 5 & \\ 2 & \end{array}$

$\begin{array}{rr}4 & 8 \\ 27 & 79 \\ 92 & 99 \\ 2 & \\ 4 & \end{array}$

$\begin{array}{rr}8 & 15 \\ 79 & 98 \\ 99 & 100 \\ 3 & \\ 9 & \\ & \end{array}$

2
1
12
5
19
83
2
18
4
15
98
100
3
14

1

$$
\begin{array}{r}
3 \\
1 \\
18 \\
5 \\
\\
26 \\
100 \\
3 \\
22
\end{array}
$$$$
4
$$$$
\begin{array}{rr}
4 & 4 \\
& 0 \\
1 & 1 \\
27 & 34 \\
6 & 7
\end{array}
$$$$
\begin{array}{r}
5 \\
1 \\
2 \\
47
\end{array}
$$$$
\begin{array}{r|r}
16 & 7 \\
29 & 100 \\
9 & 32 \\
68 & 88
\end{array}
$$

71
100
32
88

$\begin{array}{lllllll}27 & 28 & 34 & 45 & 61 & 80 & 100\end{array}$

$\begin{array}{rrrrrrr}3 & 3 & 4 & 4 & 9 & 19 & 100\end{array}$

$\begin{array}{rrrr}16 & 18 & 22 & 29 \\ 1 & 1 & 1 & \\ 18 & 20 & 24 & 35\end{array}$
100

100
100

100

100

00

100

100
100

100

100
30 dredged

183 dredged

213 dredged

244 dredged

274 surface

274 subsurface

305 surface

305 subsurface

840 surface

840 subsurface

213 dredged

244 dredged

335 surface

335 subsurface

400 surface

400 subsurface

460 surface, subsur.

520 surface

520 subsurface

580 surface

580 subsurface

640 surface, subsur.

725 surface, subsur

780 surface

780 subsurface

845 surface

845 subsurface 
T.uble 4,--Bed-material particle-size distributions--Continued

\begin{tabular}{|c|c|c|c|c|c|c|c|c|c|c|c|c|c|c|c|c|}
\hline \multirow{2}{*}{ Date } & \multicolumn{5}{|c|}{ Percent by weigh } & it finer & \multicolumn{9}{|c|}{ than size (millimeters) indicated } & \multirow{2}{*}{$\begin{array}{l}\text { Sampling location } \\
\text { in meters from } \\
\text { reference point }\end{array}$} \\
\hline & 0.062 & 0.12 & 0.25 & 0.5 & 1 & 2 & 4 & 8 & 16 & 32 & 64 & 128 & 256 & 512 & 1024 & \\
\hline \multicolumn{17}{|c|}{ 7. Susitna River at Sunshine, Alaska ${ }^{1}$} \\
\hline $\begin{array}{l}07-26-82 \\
07-26-82 \\
07-26-82 \\
07-26-82 \\
07-26-82\end{array}$ & & & 0 & 1 & 3 & 5 & 11 & $\begin{array}{r}23 \\
0\end{array}$ & $\begin{array}{r}2 \\
0 \\
0 \\
38 \\
1\end{array}$ & $\begin{array}{r}18 \\
8 \\
4 \\
53 \\
15\end{array}$ & $\begin{array}{r}100 \\
54 \\
31 \\
62 \\
100\end{array}$ & $\begin{array}{l}100 \\
100 \\
100\end{array}$ & & & & $\begin{array}{r}70 \\
162 \\
198 \\
244 \\
253\end{array}$ \\
\hline $\begin{array}{l}07-26-82 \\
05-18-83 \\
05-18-83 \\
05-18-83 \\
05-18-83\end{array}$ & & & 0 & $\begin{array}{l}0 \\
3 \\
1 \\
1\end{array}$ & $\begin{array}{l}2 \\
6 \\
1 \\
1 \\
1\end{array}$ & $\begin{array}{r}4 \\
14 \\
2 \\
2 \\
1\end{array}$ & $\begin{array}{r}6 \\
15 \\
2 \\
2 \\
1\end{array}$ & $\begin{array}{r}12 \\
30 \\
5 \\
4 \\
3\end{array}$ & $\begin{array}{r}23 \\
51 \\
13 \\
9 \\
9\end{array}$ & $\begin{array}{l}64 \\
81 \\
43 \\
20 \\
43\end{array}$ & $\begin{array}{r}100 \\
100 \\
100 \\
84 \\
78\end{array}$ & $\begin{array}{l}100 \\
100\end{array}$ & & & & $\begin{array}{r}274 \\
61 \\
91 \\
122 \\
152\end{array}$ \\
\hline $\begin{array}{l}05-18-83 \\
10-04-83 \\
10-04-83 \\
10-04-83 \\
10-04-83\end{array}$ & & & $\begin{array}{l}0 \\
0\end{array}$ & $\begin{array}{l}9 \\
2 \\
0\end{array}$ & $\begin{array}{r}20 \\
6 \\
1 \\
0\end{array}$ & $\begin{array}{r}23 \\
9 \\
1 \\
1\end{array}$ & $\begin{array}{r}24 \\
14 \\
2 \\
1\end{array}$ & $\begin{array}{r}32 \\
31 \\
6 \\
1\end{array}$ & $\begin{array}{r}0 \\
45 \\
61 \\
14 \\
5\end{array}$ & $\begin{array}{r}1 \\
57 \\
84 \\
28 \\
18\end{array}$ & $\begin{array}{r}11 \\
100 \\
100 \\
41 \\
100\end{array}$ & 100 & & & & $\begin{array}{r}183 \\
61 \\
76 \\
91 \\
107\end{array}$ \\
\hline $\begin{array}{l}10-04-83 \\
05-16-84 \\
05-16-84 \\
05-16-84 \\
05-16-84\end{array}$ & & $\begin{array}{l}0 \\
0 \\
0\end{array}$ & $\begin{array}{l}1 \\
0 \\
1 \\
2\end{array}$ & $\begin{array}{r}46 \\
1 \\
2 \\
5\end{array}$ & $\begin{array}{r}87 \\
2 \\
3 \\
5\end{array}$ & $\begin{array}{r}88 \\
4 \\
3 \\
5\end{array}$ & $\begin{array}{r}88 \\
8 \\
4 \\
6\end{array}$ & $\begin{array}{r}89 \\
15 \\
5 \\
6\end{array}$ & $\begin{array}{r}0 \\
91 \\
44 \\
7 \\
9\end{array}$ & $\begin{array}{r}5 \\
92 \\
61 \\
25 \\
23\end{array}$ & $\begin{array}{r}28 \\
100 \\
95 \\
49 \\
100\end{array}$ & $\begin{array}{l}100 \\
100 \\
100\end{array}$ & & & & $\begin{array}{r}122 \text { dredged } \\
61 \text { dredged } \\
91 \text { dredged } \\
122 \text { dredged } \\
206 \text { surface }\end{array}$ \\
\hline $\begin{array}{l}05-16-84 \\
05-16-84 \\
05-16-84 \\
05-16-84 \\
07-23-85\end{array}$ & $\begin{array}{r}1 \\
1 \\
1 \\
41\end{array}$ & $\begin{array}{r}2 \\
4 \\
2 \\
76\end{array}$ & $\begin{array}{r}2 \\
19 \\
11 \\
97\end{array}$ & $\begin{array}{r}5 \\
94 \\
41 \\
100\end{array}$ & $\begin{array}{r}11 \\
100 \\
41\end{array}$ & $\begin{array}{l}15 \\
44\end{array}$ & $\begin{array}{l}18 \\
44\end{array}$ & $\begin{array}{l}22 \\
45\end{array}$ & $\begin{array}{l}29 \\
47\end{array}$ & $\begin{array}{r}40 \\
57 \\
0\end{array}$ & $\begin{array}{l}69 \\
88 \\
11\end{array}$ & $\begin{array}{l}100 \\
100 \\
100\end{array}$ & & & & $\begin{aligned} 206 & \text { subsurface } \\
221 & \text { surface } \\
221 & \text { subsurface } \\
244 & \text { surface } \\
91 & \text { dredged }\end{aligned}$ \\
\hline $\begin{array}{l}07-23-85 \\
07-23-85 \\
07-23-85 \\
07-23-85 \\
07-23-85 \\
07-23-85\end{array}$ & 11 & 52 & $\begin{array}{r}0 \\
97 \\
0\end{array}$ & $\begin{array}{r}2 \\
99 \\
13\end{array}$ & $\begin{array}{r}3 \\
100 \\
57\end{array}$ & $\begin{array}{l}0 \\
3\end{array}$ & $\begin{array}{l}1 \\
3\end{array}$ & $\begin{array}{l}2 \\
0 \\
4\end{array}$ & $\begin{array}{l}3 \\
1 \\
4 \\
0\end{array}$ & $\begin{array}{l}9 \\
3 \\
8 \\
8\end{array}$ & $\begin{array}{r}39 \\
27 \\
100 \\
100 \\
100\end{array}$ & $\begin{array}{l}100 \\
100\end{array}$ & & & & $\begin{array}{l}122 \text { dredged } \\
152 \text { dredged } \\
183 \text { dredged } \\
213 \text { dredged } \\
244 \text { dredged } \\
274 \text { dredged }\end{array}$ \\
\hline & & & & 8. & Tanana & River & at $\mathrm{l}$ & er E & d of & oose & [s land & Alask & & & & \\
\hline $\begin{array}{l}07-18-80 \\
07-18-80 \\
07-18-80 \\
07-18-80 \\
07-18-80\end{array}$ & $\begin{array}{l}1 \\
1 \\
0.2 \\
.4 \\
.1\end{array}$ & $\begin{array}{l}4 \\
7 \\
3 \\
2 \\
0.2\end{array}$ & $\begin{array}{c}62 \\
93 \\
39 \\
9 \\
0.3\end{array}$ & $\begin{array}{r}98 \\
100 \\
90 \\
15 \\
1\end{array}$ & $\begin{array}{r}96 \\
15 \\
1\end{array}$ & $\begin{array}{r}97 \\
15 \\
1\end{array}$ & $\begin{array}{r}98 \\
17 \\
5\end{array}$ & $\begin{array}{l}98 \\
36 \\
21\end{array}$ & $\begin{array}{l}100 \\
99 \\
86 \\
64\end{array}$ & $\begin{array}{r}100 \\
100 \\
96\end{array}$ & 100 & & & & & $\begin{array}{l}17 \\
34 \\
51 \\
68 \\
85\end{array}$ \\
\hline $\begin{array}{l}07-18-80 \\
07-18-80 \\
07-18-80 \\
07-18-80 \\
07-18-80\end{array}$ & $\begin{array}{l}.3 \\
0.1 \\
0\end{array}$ & $\begin{array}{l}1 \\
0.2 \\
0.2\end{array}$ & $\begin{array}{l}2 \\
1 \\
1\end{array}$ & $\begin{array}{l}2 \\
0 \\
1 \\
0 \\
2\end{array}$ & $\begin{array}{l}2 \\
0.5 \\
1 \\
0.3 \\
3\end{array}$ & $\begin{array}{l}3 \\
1 \\
1 \\
0.4 \\
4\end{array}$ & $\begin{array}{r}8 \\
4 \\
2 \\
2 \\
10\end{array}$ & $\begin{array}{l}22 \\
22 \\
10 \\
12 \\
27\end{array}$ & $\begin{array}{l}56 \\
55 \\
38 \\
46 \\
59\end{array}$ & $\begin{array}{l}91 \\
98 \\
84 \\
91 \\
87\end{array}$ & $\begin{array}{l}100 \\
100 \\
100 \\
100 \\
100\end{array}$ & & & & & $\begin{array}{l}102 \\
119 \\
136 \\
153 \\
170\end{array}$ \\
\hline $\begin{array}{l}07-18-80 \\
07-18-80 \\
07-18-80 \\
07-18-80 \\
07-18-80\end{array}$ & $\begin{array}{l}0.1 \\
.3 \\
1 \\
0.1 \\
.3\end{array}$ & $\begin{array}{l}1 \\
3 \\
1 \\
0.2 \\
1\end{array}$ & $\begin{array}{c}13 \\
36 \\
3 \\
0.4 \\
26\end{array}$ & $\begin{array}{r}19 \\
40 \\
4 \\
1 \\
38\end{array}$ & $\begin{array}{r}20 \\
40 \\
4 \\
1 \\
39\end{array}$ & $\begin{array}{r}20 \\
40 \\
4 \\
1 \\
39\end{array}$ & $\begin{array}{r}20 \\
40 \\
4 \\
1 \\
42\end{array}$ & $\begin{array}{r}22 \\
41 \\
5 \\
2 \\
49\end{array}$ & $\begin{array}{r}28 \\
46 \\
9 \\
7 \\
65\end{array}$ & $\begin{array}{l}50 \\
56 \\
36 \\
20 \\
85\end{array}$ & $\begin{array}{r}100 \\
100 \\
100 \\
46 \\
92\end{array}$ & $\begin{array}{l}100 \\
100\end{array}$ & & & & $\begin{array}{l}187 \\
204 \\
221 \\
238 \\
255\end{array}$ \\
\hline
\end{tabular}


Table 4.--Bed-material particle-size distributions--Continued

Date

Percent by weight finer than size (millimeters) indicated

$\begin{array}{lllllllllllllllll}0.062 & 0.12 & 0.25 & 0.5 & 1 & 2 & 4 & 8 & 16 & 32 & 64 & 128 & 256 & 512 & 1024 & \text { reference point }\end{array}$

8. Tanana River at Upper End of Goose Island, Alaska ${ }^{2}$--Continued

\begin{tabular}{|c|c|c|c|c|c|c|c|c|c|c|c|c|}
\hline $\begin{array}{l}07-18-80 \\
07-18-80 \\
07-18-80 \\
08-03-81 \\
08-03-81\end{array}$ & $\begin{array}{r}1 \\
3 \\
16 \\
0 \\
0\end{array}$ & $\begin{array}{r}3 \\
65 \\
82 \\
0.1 \\
.1\end{array}$ & $\begin{array}{l}18 \\
99 \\
98 \\
1 \\
0.4\end{array}$ & $\begin{array}{r}21 \\
100 \\
100 \\
3 \\
1\end{array}$ & $\begin{array}{l}3 \\
2\end{array}$ & $\begin{array}{l}3 \\
2\end{array}$ & $\begin{array}{l}3 \\
5\end{array}$ & $\begin{array}{l}4 \\
22\end{array}$ & $\begin{array}{l}10 \\
60\end{array}$ & $\begin{array}{l}36 \\
84\end{array}$ & $\begin{array}{l}100 \\
100\end{array}$ & $\begin{array}{r}272 \\
289 \\
306 \\
15 \\
30\end{array}$ \\
\hline $\begin{array}{l}08-03-81 \\
08-03-81 \\
08-03-81 \\
08-03-81 \\
08-03-81\end{array}$ & $\begin{array}{l}0.1 \\
0.1 \\
0\end{array}$ & $\begin{array}{l}0 \\
1 \\
0.3 \\
.1\end{array}$ & $\begin{array}{l}.2 \\
2 \\
1 \\
0.2\end{array}$ & $\begin{array}{l}0.4 \\
0 \\
5 \\
2 \\
1\end{array}$ & $\begin{array}{l}1 \\
0.1 \\
6 \\
3 \\
1\end{array}$ & $\begin{array}{l}1 \\
0.2 \\
7 \\
5 \\
2\end{array}$ & $\begin{array}{r}2 \\
1 \\
13 \\
13 \\
8\end{array}$ & $\begin{array}{r}10 \\
6 \\
38 \\
33 \\
20\end{array}$ & $\begin{array}{l}33 \\
23 \\
79 \\
68 \\
50\end{array}$ & $\begin{array}{r}70 \\
71 \\
100 \\
95 \\
87\end{array}$ & $\begin{array}{l}100 \\
100 \\
100 \\
100\end{array}$ & $\begin{array}{r}45 \\
60 \\
75 \\
90 \\
105\end{array}$ \\
\hline $\begin{array}{l}08-03-81 \\
08-03-81 \\
08-03-81 \\
08-03-81 \\
08-03-81\end{array}$ & $\begin{array}{l}0 \\
0\end{array}$ & $\begin{array}{r}0.1 \\
.1\end{array}$ & $\begin{array}{l}0.2 \\
1\end{array}$ & $\begin{array}{l}0 \\
0 \\
0 \\
0.3 \\
1\end{array}$ & $\begin{array}{l}0.1 \\
.1 \\
1 \\
1 \\
1\end{array}$ & $\begin{array}{l}0.3 \\
1 \\
1 \\
1\end{array}$ & $\begin{array}{l}1 \\
4 \\
2 \\
4 \\
6\end{array}$ & $\begin{array}{r}4 \\
24 \\
10 \\
16 \\
21\end{array}$ & $\begin{array}{l}9 \\
61 \\
38 \\
47 \\
46\end{array}$ & $\begin{array}{l}21 \\
92 \\
84 \\
84 \\
82\end{array}$ & $\begin{array}{l}100 \\
100 \\
100 \\
100 \\
100\end{array}$ & $\begin{array}{l}120 \\
135 \\
150 \\
165 \\
180\end{array}$ \\
\hline $\begin{array}{l}08-03-81 \\
08-03-81 \\
08-03-81 \\
08-03-81 \\
08-03-81\end{array}$ & $\begin{array}{l}0 \\
0 \\
0.4 \\
.4\end{array}$ & $13^{.2}$ & $\begin{array}{c}0.3 \\
1 \\
78 \\
89\end{array}$ & $\begin{array}{c}0.4 \\
0 \\
1 \\
89 \\
100\end{array}$ & $\begin{array}{l}1 \\
0.4 \\
1 \\
89\end{array}$ & $\begin{array}{r}1 \\
1 \\
1 \\
89\end{array}$ & $\begin{array}{r}3 \\
1 \\
2 \\
91\end{array}$ & $\begin{array}{r}15 \\
2 \\
5 \\
95\end{array}$ & $\begin{array}{r}43 \\
10 \\
18 \\
100\end{array}$ & $\begin{array}{l}91 \\
40 \\
43\end{array}$ & $\begin{array}{l}100 \\
100 \\
100\end{array}$ & $\begin{array}{l}195 \\
210 \\
225 \\
240 \\
255\end{array}$ \\
\hline $\begin{array}{l}08-03-81 \\
08-03-81 \\
08-03-81\end{array}$ & $\begin{array}{r}0 \\
1 \\
53\end{array}$ & $\begin{array}{l}0.1 \\
22 \\
98\end{array}$ & $\begin{array}{l}0.4 \\
74 \\
100\end{array}$ & $\begin{array}{r}1 \\
100\end{array}$ & 1 & 1 & 1 & 3 & 19 & 66 & 100 & $\begin{array}{l}270 \\
285 \\
300\end{array}$ \\
\hline
\end{tabular}

9. Tanana River at Fairbanks, Alaska ${ }^{3}$

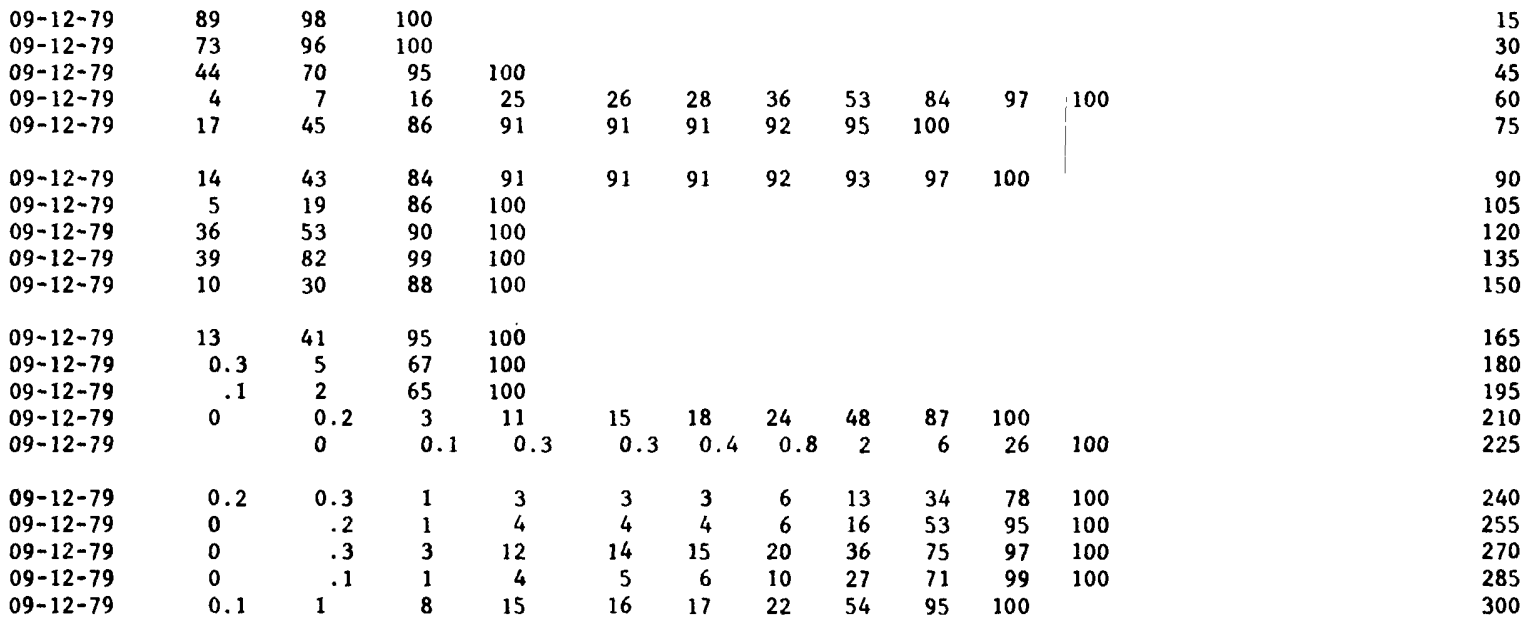


Table 4.--Bed-material particle-size distributions--Continued

\begin{tabular}{|c|c|c|c|c|c|c|c|c|c|c|c|c|c|c|c|}
\hline \multirow{2}{*}{ Date } & \multicolumn{5}{|c|}{ Percent by weight } & \multirow{2}{*}{$\frac{\text { finer }}{2}$} & \multirow{2}{*}{\multicolumn{2}{|c|}{$\frac{\text { than size }}{4}$}} & \multicolumn{6}{|c|}{ (millimeters) indicated } & \multirow{2}{*}{$\begin{array}{l}\text { Sampling location, } \\
\text { in meters from } \\
\text { reference point }\end{array}$} \\
\hline & 0.062 & 0.12 & 0.25 & 0.5 & 1 & & & & 16 & 32 & 128 & 256 & 512 & 1024 & \\
\hline
\end{tabular}

\section{Tanana River at Fairbanks, Alaska ${ }^{3}-$-Continued}

\begin{tabular}{|c|c|c|c|c|c|c|c|c|c|c|c|c|c|}
\hline $\begin{array}{l}09-12-79 \\
09-12-79 \\
09-12-79 \\
09-12-79 \\
09-12-79\end{array}$ & $\begin{array}{r}10^{.4} \\
11 \\
1\end{array}$ & $\begin{array}{r}2 \\
27 \\
3 \\
33 \\
13\end{array}$ & $\begin{array}{r}9 \\
54 \\
10 \\
90 \\
89\end{array}$ & $\begin{array}{r}14 \\
59 \\
17 \\
100 \\
100\end{array}$ & $\begin{array}{l}15 \\
59 \\
19\end{array}$ & $\begin{array}{l}17 \\
59 \\
21\end{array}$ & $\begin{array}{l}22 \\
60 \\
27\end{array}$ & $\begin{array}{l}49 \\
68 \\
40\end{array}$ & $\begin{array}{l}85 \\
87 \\
65\end{array}$ & $\begin{array}{r}100 \\
100 \\
89\end{array}$ & 100 & & $\begin{array}{l}315 \\
330 \\
345 \\
360 \\
375\end{array}$ \\
\hline $\begin{array}{l}09-12-79 \\
07-17-80 \\
07-17-80 \\
07-17-80 \\
07-17-80\end{array}$ & $\begin{array}{l}80 \\
81 \\
16 \\
78\end{array}$ & $\begin{array}{r}0 \\
99 \\
99 \\
48 \\
100\end{array}$ & $\begin{array}{r}1 \\
100 \\
100 \\
95\end{array}$ & 100 & 4 & 4 & 5 & 11 & 60 & 91 & 100 & & $\begin{array}{r}390 \\
15 \\
30 \\
45 \\
60\end{array}$ \\
\hline $\begin{array}{l}07-17-80 \\
07-17-80 \\
07-17-80 \\
07-17-80 \\
07-17-80\end{array}$ & $\begin{array}{r}0.3 \\
2^{.4} \\
11 \\
2\end{array}$ & $\begin{array}{r}1 \\
2 \\
13 \\
40 \\
18\end{array}$ & $\begin{array}{l}12 \\
10 \\
83 \\
93 \\
87\end{array}$ & $\begin{array}{r}18 \\
15 \\
98 \\
100 \\
100\end{array}$ & $\begin{array}{l}18 \\
15 \\
98\end{array}$ & $\begin{array}{l}20 \\
17 \\
98\end{array}$ & $\begin{array}{l}25 \\
23 \\
98\end{array}$ & $\begin{array}{l}43 \\
39 \\
99\end{array}$ & $\begin{array}{r}66 \\
60 \\
100\end{array}$ & $\begin{array}{r}100 \\
86\end{array}$ & 100 & & $\begin{array}{r}75 \\
90 \\
105 \\
120 \\
135\end{array}$ \\
\hline $\begin{array}{l}07-17-80 \\
07-17-80 \\
07-17-80 \\
07-17-80 \\
07-17-80\end{array}$ & $\begin{array}{r}58 \\
0 \\
0 \\
0 \\
0\end{array}$ & $\begin{array}{r}79 \\
0.2 \\
.2 \\
.1 \\
.1\end{array}$ & $\begin{array}{r}97 \\
1 \\
2 \\
1 \\
1\end{array}$ & $\begin{array}{r}100 \\
3 \\
4 \\
3 \\
2\end{array}$ & $\begin{array}{l}4 \\
4 \\
4 \\
2\end{array}$ & $\begin{array}{l}5 \\
4 \\
5 \\
2\end{array}$ & $\begin{array}{l}9 \\
5 \\
7 \\
5\end{array}$ & $\begin{array}{r}17 \\
8 \\
18 \\
17\end{array}$ & $\begin{array}{l}29 \\
20 \\
48 \\
44\end{array}$ & $\begin{array}{l}64 \\
43 \\
86 \\
76\end{array}$ & $\begin{array}{r}85 \\
100 \\
100 \\
100\end{array}$ & 100 & $\begin{array}{l}150 \\
165 \\
180 \\
195 \\
210\end{array}$ \\
\hline $\begin{array}{l}07-17-80 \\
07-17-80 \\
07-17-80 \\
07-17-80 \\
07-17-80\end{array}$ & $\begin{array}{l}0.1 \\
0 \\
0.1 \\
.2 \\
.1\end{array}$ & $\begin{array}{l}1 \\
0.2 \\
1 \\
1 \\
0.3\end{array}$ & $\begin{array}{r}11 \\
3 \\
18 \\
4 \\
1\end{array}$ & $\begin{array}{r}40 \\
5 \\
31 \\
6 \\
1\end{array}$ & $\begin{array}{r}43 \\
5 \\
32 \\
6 \\
1\end{array}$ & $\begin{array}{r}44 \\
5 \\
32 \\
6 \\
1\end{array}$ & $\begin{array}{r}46 \\
8 \\
35 \\
7 \\
2\end{array}$ & $\begin{array}{r}56 \\
21 \\
45 \\
11 \\
5\end{array}$ & $\begin{array}{l}82 \\
46 \\
63 \\
20 \\
21\end{array}$ & $\begin{array}{l}99 \\
64 \\
74 \\
51 \\
64\end{array}$ & $\begin{array}{r}100 \\
100 \\
100 \\
80 \\
100\end{array}$ & 100 & $\begin{array}{l}225 \\
240 \\
255 \\
270 \\
285\end{array}$ \\
\hline $\begin{array}{l}07-17-80 \\
07-17-80 \\
07-17-80 \\
07-17-80 \\
07-17-80\end{array}$ & $\begin{array}{l}.1 \\
.1 \\
0 \\
1 \\
0.2\end{array}$ & $\begin{array}{l}.3 \\
0.2 \\
3 \\
1\end{array}$ & $\begin{array}{l}1 \\
3 \\
1 \\
6 \\
3\end{array}$ & $\begin{array}{l}1 \\
4 \\
2 \\
7 \\
4\end{array}$ & $\begin{array}{l}1 \\
5 \\
2 \\
7 \\
4\end{array}$ & $\begin{array}{l}1 \\
5 \\
2 \\
8 \\
4\end{array}$ & $\begin{array}{l}1 \\
5 \\
2 \\
8 \\
5\end{array}$ & $\begin{array}{r}6 \\
13 \\
9 \\
21 \\
12\end{array}$ & $\begin{array}{l}50 \\
65 \\
64 \\
84 \\
59\end{array}$ & $\begin{array}{l}100 \\
100 \\
100 \\
100 \\
100\end{array}$ & & & $\begin{array}{l}300 \\
315 \\
330 \\
345 \\
360\end{array}$ \\
\hline $\begin{array}{l}07-17-80 \\
07-17-80 \\
08-05-81 \\
08-05-81 \\
08-05-81\end{array}$ & $\begin{array}{l}.2 \\
.1 \\
0 \\
1\end{array}$ & $\begin{array}{c}1 \\
1 \\
56 \\
0.4 \\
24\end{array}$ & $\begin{array}{r}4 \\
6 \\
86 \\
1 \\
75\end{array}$ & $\begin{array}{r}5 \\
10 \\
87 \\
1 \\
87\end{array}$ & $\begin{array}{r}5 \\
10 \\
87 \\
1 \\
87\end{array}$ & $\begin{array}{r}5 \\
12 \\
87 \\
1 \\
88\end{array}$ & $\begin{array}{r}6 \\
16 \\
87 \\
2 \\
88\end{array}$ & $\begin{array}{r}12 \\
40 \\
87 \\
5 \\
88\end{array}$ & $\begin{array}{l}43 \\
87 \\
87 \\
31 \\
88\end{array}$ & $\begin{array}{r}100 \\
100 \\
92 \\
95 \\
100\end{array}$ & $\begin{array}{l}100 \\
100\end{array}$ & & $\begin{array}{r}375 \\
390 \\
0 \\
15 \\
30\end{array}$ \\
\hline $\begin{array}{l}08-05-81 \\
08-05-81 \\
08-05-81 \\
08-05-81 \\
08-05-81\end{array}$ & $\begin{array}{l}34 \\
1 \\
0.1 \\
0 \\
0.1\end{array}$ & $\begin{array}{c}89 \\
16 \\
1 \\
0.1 \\
1\end{array}$ & $\begin{array}{r}99 \\
93 \\
10 \\
1 \\
15\end{array}$ & $\begin{array}{r}100 \\
100 \\
20 \\
3 \\
21\end{array}$ & $\begin{array}{r}26 \\
4 \\
21\end{array}$ & $\begin{array}{r}30 \\
4 \\
21\end{array}$ & $\begin{array}{r}39 \\
7 \\
22\end{array}$ & $\begin{array}{l}59 \\
20 \\
26\end{array}$ & $\begin{array}{l}84 \\
60 \\
45\end{array}$ & $\begin{array}{r}100 \\
100 \\
89\end{array}$ & 100 & & $\begin{array}{r}90 \\
105 \\
120 \\
135 \\
150\end{array}$ \\
\hline $\begin{array}{l}08-05-81 \\
08-05-81 \\
08-05-81 \\
08-05-81 \\
08-05-81\end{array}$ & $\begin{array}{l}.1 \\
.1 \\
.2 \\
.3 \\
.4\end{array}$ & $\begin{array}{r}2 \\
4 \\
14 \\
17 \\
12\end{array}$ & $\begin{array}{l}36 \\
77 \\
97 \\
97 \\
96\end{array}$ & $\begin{array}{r}49 \\
98 \\
100 \\
100 \\
100\end{array}$ & $\begin{array}{l}49 \\
98\end{array}$ & $\begin{array}{l}49 \\
98\end{array}$ & $\begin{array}{l}49 \\
99\end{array}$ & $\begin{array}{l}51 \\
99\end{array}$ & $\begin{array}{r}62 \\
100\end{array}$ & 100 & & & $\begin{array}{l}165 \\
180 \\
195 \\
210 \\
225\end{array}$ \\
\hline
\end{tabular}


Table 4.--Bed-material particle-size distributions--Cont inued

Percent by weight finer than size (millimeters) indicated

Date

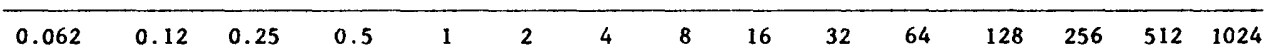

in meters from

reference point

9. Tanana River at Fairbanks, Alaska ${ }^{3}$--Continued

\begin{tabular}{|c|c|c|c|c|c|c|c|c|c|c|c|c|}
\hline $08-05-81$ & 0 & 0.2 & 1 & 1 & 1 & 1 & 2 & 3 & 7 & 34 & 100 & 240 \\
\hline $08-05-81$ & 0 & .2 & 1 & 2 & 2 & 2 & 3 & 7 & 30 & 91 & 100 & 255 \\
\hline $08-05-81$ & 0 & .2 & 1 & 1 & 1 & 1 & 1 & 3 & 14 & 56 & 100 & 270 \\
\hline $08-05-81$ & 0 & .2 & 2 & 6 & 6 & 7 & 10 & 20 & 50 & 92 & 100 & 285 \\
\hline $08-05-81$ & 0 & .2 & 1 & 3 & 3 & 4 & 9 & 28 & 61 & 88 & 100 & 300 \\
\hline $08-05-81$ & 0 & .1 & 1 & 1 & 2 & 2 & 6 & 24 & 72 & 100 & & 315 \\
\hline $08-05-81$ & 0 & .1 & 1 & 2 & 3 & 4 & 9 & 39 & 85 & 100 & & 330 \\
\hline $08-05-81$ & 0 & .3 & 5 & 9 & 10 & 11 & 15 & 30 & 79 & 99 & 100 & 345 \\
\hline $08-05-81$ & 0 & .1 & 1 & 3 & 3 & 3 & 4 & 10 & 31 & 80 & 100 & 360 \\
\hline $08-05-81$ & 0 & .4 & 4 & 10 & 10 & 11 & 11 & 16 & 34 & 100 & & 375 \\
\hline $\begin{array}{l}08-05-81 \\
08-05-81\end{array}$ & $\begin{array}{l}0 \\
0.2\end{array}$ & $4^{.4}$ & $\begin{array}{r}6 \\
81\end{array}$ & $\begin{array}{r}10 \\
100\end{array}$ & 10 & 11 & 11 & 14 & 29 & 84 & 100 & $\begin{array}{l}390 \\
405\end{array}$ \\
\hline
\end{tabular}

11. Toutle River at Tower Road near Silver Lake, Wash. 2

\begin{tabular}{|c|c|c|c|c|c|c|c|c|c|c|c|}
\hline $\begin{array}{l}01-17-85 \\
01-17-85 \\
01-17-85 \\
01-17-85 \\
01-17-85\end{array}$ & $\begin{array}{l}0 \\
0 \\
0 \\
0 \\
0\end{array}$ & $\begin{array}{l}0.1 \\
.3 \\
.1 \\
.1 \\
.3\end{array}$ & $\begin{array}{l}0.5 \\
3 \\
2 \\
1 \\
5\end{array}$ & $\begin{array}{r}2 \\
17 \\
15 \\
9 \\
42\end{array}$ & $\begin{array}{r}9 \\
68 \\
47 \\
25 \\
97\end{array}$ & $\begin{array}{r}29 \\
89 \\
61 \\
42 \\
100\end{array}$ & $\begin{array}{l}50 \\
94 \\
68 \\
54\end{array}$ & $\begin{array}{l}68 \\
97 \\
74 \\
70\end{array}$ & $\begin{array}{r}91 \\
100 \\
87 \\
90\end{array}$ & $\begin{array}{l}100 \\
100\end{array}$ & $\begin{array}{l}11 \\
13 \\
15 \\
17 \\
20\end{array}$ \\
\hline $\begin{array}{l}01-31-85 \\
01-31-85 \\
01-31-85 \\
01-31-85 \\
02-21-85\end{array}$ & $\begin{array}{l}0 \\
0 \\
0 \\
0 \\
0\end{array}$ & $\begin{array}{l}.2 \\
.3 \\
.1 \\
.2 \\
.1\end{array}$ & $\begin{array}{l}4 \\
3 \\
3 \\
5 \\
1\end{array}$ & $\begin{array}{r}34 \\
19 \\
15 \\
35 \\
6\end{array}$ & $\begin{array}{l}56 \\
75 \\
29 \\
64 \\
24\end{array}$ & $\begin{array}{l}61 \\
94 \\
49 \\
84 \\
45\end{array}$ & $\begin{array}{l}63 \\
97 \\
61 \\
94 \\
59\end{array}$ & $\begin{array}{r}66 \\
100 \\
70 \\
98 \\
74\end{array}$ & $\begin{array}{r}83 \\
100 \\
93\end{array}$ & 100 & $\begin{array}{l}-- \\
-- \\
-- \\
11\end{array}$ \\
\hline $\begin{array}{l}02-21-85 \\
02-21-85 \\
02-21-85 \\
02-21-85 \\
03-06-85\end{array}$ & $\begin{array}{l}0 \\
0 \\
0 \\
0 \\
0\end{array}$ & $\begin{array}{l}.1 \\
.1 \\
.1 \\
.2 \\
.1\end{array}$ & $\begin{array}{l}1 \\
2 \\
2 \\
3 \\
0.8\end{array}$ & $\begin{array}{r}8 \\
19 \\
18 \\
16 \\
6\end{array}$ & $\begin{array}{l}34 \\
59 \\
35 \\
36 \\
33\end{array}$ & $\begin{array}{l}52 \\
85 \\
46 \\
64 \\
60\end{array}$ & $\begin{array}{l}62 \\
93 \\
53 \\
82 \\
75\end{array}$ & $\begin{array}{l}74 \\
98 \\
59 \\
91 \\
88\end{array}$ & $\begin{array}{r}94 \\
100 \\
73 \\
94 \\
97\end{array}$ & $\begin{array}{l}100 \\
100 \\
100\end{array}$ & $\begin{array}{l}13 \\
15 \\
18 \\
21 \\
10\end{array}$ \\
\hline $\begin{array}{l}03-06-85 \\
03-06-85 \\
03-06-85 \\
03-23-85 \\
03-23-85\end{array}$ & $\begin{array}{l}0 \\
0 \\
0 \\
0.1 \\
.1\end{array}$ & $\begin{array}{l}.2 \\
.2 \\
.2 \\
.5 \\
.5\end{array}$ & $\begin{array}{l}2 \\
4 \\
3 \\
2 \\
2\end{array}$ & $\begin{array}{r}21 \\
37 \\
19 \\
5 \\
10\end{array}$ & $\begin{array}{l}70 \\
79 \\
39 \\
10 \\
33\end{array}$ & $\begin{array}{l}85 \\
91 \\
58 \\
16 \\
45\end{array}$ & $\begin{array}{l}89 \\
95 \\
75 \\
24 \\
52\end{array}$ & $\begin{array}{l}93 \\
98 \\
89 \\
34 \\
61\end{array}$ & $\begin{array}{r}98 \\
100 \\
100 \\
60 \\
78\end{array}$ & $\begin{array}{l}100 \\
100\end{array}$ & $\begin{array}{l}13 \\
15 \\
18 \\
11 \\
13\end{array}$ \\
\hline $\begin{array}{l}03-23-85 \\
03-23-85 \\
03-23-85 \\
04-02-85 \\
04-02-85\end{array}$ & $\begin{array}{l}.1 \\
0 \\
0.3 \\
0 \\
0\end{array}$ & $\begin{array}{l}.7 \\
.4 \\
3 \\
0.2 \\
.1\end{array}$ & $\begin{array}{r}6 \\
3 \\
27 \\
1 \\
2\end{array}$ & $\begin{array}{r}35 \\
9 \\
93 \\
10 \\
15\end{array}$ & $\begin{array}{r}72 \\
14 \\
100 \\
29 \\
51\end{array}$ & $\begin{array}{l}43 \\
76\end{array}$ & $\begin{array}{l}88 \\
29\end{array}$ & $\begin{array}{l}93 \\
48 \\
\\
62 \\
91\end{array}$ & $\begin{array}{l}73 \\
94\end{array}$ & $\begin{array}{l}100 \\
100\end{array}$ & $\begin{array}{l}16 \\
20 \\
27 \\
16 \\
21\end{array}$ \\
\hline $\begin{array}{l}04-02-85 \\
04-11-85 \\
04-11-85 \\
04-11-85 \\
04-30-85\end{array}$ & $\begin{array}{l}0.2 \\
0 \\
0.1 \\
.1 \\
0.1\end{array}$ & $\begin{array}{l}3 \\
0.2 \\
.5 \\
1 \\
0.2\end{array}$ & $\begin{array}{c}46 \\
0.9 \\
4 \\
11 \\
4\end{array}$ & $\begin{array}{r}98 \\
5 \\
21 \\
60 \\
26\end{array}$ & $\begin{array}{r}100 \\
16 \\
54 \\
99 \\
60\end{array}$ & $\begin{array}{r}28 \\
75 \\
100 \\
73\end{array}$ & $\begin{array}{l}43 \\
83\end{array}$ & $\begin{array}{l}68 \\
89\end{array}$ & $\begin{array}{l}92 \\
96\end{array}$ & $\begin{array}{l}100 \\
100 \\
100\end{array}$ & $\begin{array}{l}32 \\
14 \\
17 \\
22 \\
16\end{array}$ \\
\hline
\end{tabular}




\begin{tabular}{|c|c|c|c|c|c|c|c|c|c|c|c|c|c|c|c|c|}
\hline \multirow{2}{*}{ Date } & \multicolumn{5}{|c|}{ Percent by weight } & \multirow{2}{*}{$\frac{\text { finer }}{2}$} & \multirow{2}{*}{ than } & \multirow{2}{*}{$\frac{\text { size }}{8}$} & \multicolumn{7}{|c|}{ (millimeters) indicated } & \multirow{2}{*}{$\begin{array}{l}\text { Sampling location } \\
\text { in meters from } \\
\text { reference point }\end{array}$} \\
\hline & 0.062 & 0.12 & 0.25 & 0.5 & 1 & & & & 16 & 32 & 6 & 128 & 256 & 512 & 1024 & \\
\hline
\end{tabular}

11. Toutle River at Tower Road near Silver Lake, Wash. ${ }^{2}=-$ Cont inued

\begin{tabular}{|c|c|c|c|c|c|c|c|c|c|c|c|c|}
\hline $\begin{array}{l}04-30-85 \\
04-30-85 \\
06-07-85 \\
06-07-85 \\
06-07-85\end{array}$ & $\begin{array}{l}0 \\
0 \\
0.2 \\
.2 \\
.3\end{array}$ & $\begin{array}{l}.1 \\
.6 \\
1 \\
0.9 \\
.8\end{array}$ & $\begin{array}{r}2 \\
15 \\
4 \\
5 \\
3\end{array}$ & $\begin{array}{r}11 \\
90 \\
6 \\
15 \\
6\end{array}$ & $\begin{array}{r}39 \\
100 \\
8 \\
27 \\
14\end{array}$ & $\begin{array}{l}15 \\
47 \\
27\end{array}$ & $\begin{array}{l}30 \\
68 \\
41\end{array}$ & $\begin{array}{l}57 \\
89 \\
52\end{array}$ & $\begin{array}{r}80 \\
100 \\
77\end{array}$ & 100 & & $\begin{array}{l}19 \\
23 \\
11 \\
17 \\
23\end{array}$ \\
\hline $\begin{array}{l}06-07-85 \\
06-08-85 \\
06-08-85 \\
06-08-85 \\
06-08-85\end{array}$ & $\begin{array}{l}.4 \\
.2 \\
.2 \\
.1 \\
.1\end{array}$ & $\begin{array}{l}1 \\
1 \\
1 \\
0.3 \\
1\end{array}$ & $\begin{array}{c}3 \\
7 \\
11 \\
0.9 \\
5\end{array}$ & $\begin{array}{r}5 \\
28 \\
43 \\
3 \\
11\end{array}$ & $\begin{array}{r}13 \\
56 \\
72 \\
9 \\
18\end{array}$ & $\begin{array}{l}39 \\
66 \\
85 \\
22 \\
27\end{array}$ & $\begin{array}{l}61 \\
72 \\
92 \\
56 \\
39\end{array}$ & $\begin{array}{l}75 \\
80 \\
97 \\
88 \\
58\end{array}$ & $\begin{array}{r}93 \\
92 \\
100 \\
97 \\
69\end{array}$ & $\begin{array}{l}100 \\
100\end{array}$ & & $\begin{array}{l}52 \\
11 \\
16 \\
26 \\
39\end{array}$ \\
\hline $\begin{array}{l}08-23-85 \\
08-23-85 \\
08-23-85 \\
08-23-85 \\
08-23-85\end{array}$ & $\begin{array}{l}0.2 \\
.2 \\
.2 \\
2 \\
0.1\end{array}$ & $\begin{array}{l}0.4 \\
2 \\
2 \\
13 \\
0.4\end{array}$ & $\begin{array}{r}4 \\
15 \\
17 \\
61 \\
6\end{array}$ & $\begin{array}{l}29 \\
52 \\
63 \\
96 \\
20\end{array}$ & $\begin{array}{r}61 \\
85 \\
90 \\
100 \\
33\end{array}$ & $\begin{array}{l}73 \\
97 \\
97\end{array}$ & $\begin{array}{l}78 \\
99 \\
98\end{array}$ & $\begin{array}{r}82 \\
100 \\
98\end{array}$ & 100 & 100 & & $\begin{array}{l}10 \\
12 \\
13 \\
15 \\
18\end{array}$ \\
\hline $\begin{array}{l}12-09-85 \\
01-19-86 \\
01-19-86 \\
01-19-86 \\
01-29-86\end{array}$ & $\begin{array}{l}0 \\
0.1 \\
0 \\
0.1\end{array}$ & $2^{.8}$ & $\begin{array}{r}6 \\
2 \\
25\end{array}$ & $\begin{array}{r}45 \\
9 \\
97\end{array}$ & $\begin{array}{r}89 \\
15 \\
100\end{array}$ & $\begin{array}{l}98 \\
98 \\
21\end{array}$ & $\begin{array}{r}100 \\
0 \\
100 \\
29\end{array}$ & 59 & $\begin{array}{r}17 \\
100\end{array}$ & 100 & & $\begin{array}{l}18 \\
20 \\
26 \\
30 \\
19\end{array}$ \\
\hline $\begin{array}{l}01-29-86 \\
02-11-86 \\
02-16-86 \\
11-21-86 \\
11-21-86\end{array}$ & $\begin{array}{l}0^{.6} \\
0.1 \\
.2\end{array}$ & $\begin{array}{l}9 \\
0.2 \\
.4 \\
3 \\
0.3\end{array}$ & $\begin{array}{c}63 \\
0.7 \\
5 \\
26 \\
4\end{array}$ & $\begin{array}{r}99 \\
1 \\
28 \\
90 \\
33\end{array}$ & $\begin{array}{r}100 \\
2 \\
61 \\
100 \\
85\end{array}$ & $\begin{array}{l}10 \\
74 \\
96\end{array}$ & $\begin{array}{l}43 \\
79 \\
98\end{array}$ & $\begin{array}{r}74 \\
83 \\
100\end{array}$ & $\begin{array}{l}88 \\
88\end{array}$ & $\begin{array}{l}100 \\
100\end{array}$ & & $\begin{array}{l}24 \\
13 \\
24 \\
14 \\
24\end{array}$ \\
\hline $\begin{array}{l}11-21-86 \\
11-21-86 \\
11-22-86 \\
11-22-86 \\
11-22-86\end{array}$ & $\begin{array}{l}0 \\
0 \\
0.1 \\
.1 \\
.1\end{array}$ & $\begin{array}{l}.1 \\
2^{.2} \\
1 \\
0.5\end{array}$ & $\begin{array}{r}0.8 \\
16 \\
9 \\
3\end{array}$ & $\begin{array}{r}4 \\
2 \\
82 \\
37 \\
7\end{array}$ & $\begin{array}{r}13 \\
3 \\
100 \\
91 \\
16\end{array}$ & $\begin{array}{l}99 \\
51\end{array}$ & $\begin{array}{r}53 \\
19 \\
\\
100 \\
82\end{array}$ & $\begin{array}{l}66 \\
38\end{array}$ & $\begin{array}{l}82 \\
73\end{array}$ & $\begin{array}{l}100 \\
100\end{array}$ & & $\begin{array}{l}36 \\
46 \\
12 \\
24 \\
36\end{array}$ \\
\hline $\begin{array}{l}11-22-86 \\
11-22-86 \\
01-14-87 \\
01-14-87 \\
01-28-87\end{array}$ & $\begin{array}{l}0 \\
0 \\
0.1 \\
0.1\end{array}$ & $\begin{array}{l}2^{.2} \\
1 \\
0.1 \\
.5\end{array}$ & $\begin{array}{l}0.6 \\
6 \\
12 \\
0.3 \\
7\end{array}$ & $\begin{array}{r}1 \\
9 \\
73 \\
2 \\
46\end{array}$ & $\begin{array}{r}2 \\
10 \\
99 \\
6 \\
97\end{array}$ & $\begin{array}{r}7 \\
10 \\
100 \\
10 \\
99\end{array}$ & $\begin{array}{r}28 \\
11 \\
14 \\
100\end{array}$ & $\begin{array}{l}68 \\
15\end{array}$ & $\begin{array}{l}97 \\
34\end{array}$ & $\begin{array}{l}100 \\
100\end{array}$ & 100 & $\begin{array}{l}48 \\
59 \\
26 \\
34 \\
26\end{array}$ \\
\hline $\begin{array}{l}01-28-87 \\
01-28-87\end{array}$ & $\begin{array}{l}0 \\
0\end{array}$ & $\begin{array}{l}.2 \\
.1\end{array}$ & $\begin{array}{l}2 \\
0.4\end{array}$ & $\begin{array}{r}15 \\
\cdot 2\end{array}$ & $\begin{array}{r}54 \\
2\end{array}$ & $\begin{array}{r}81 \\
3\end{array}$ & $\begin{array}{r}89 \\
7\end{array}$ & $\begin{array}{l}94 \\
16\end{array}$ & $\begin{array}{l}98 \\
36\end{array}$ & $\begin{array}{r}100 \\
70\end{array}$ & 100 & $\begin{array}{l}35 \\
43\end{array}$ \\
\hline
\end{tabular}

12. North Fork of Toutle River near Kid Valley, Wash. ${ }^{2}$

\begin{tabular}{|c|c|c|c|c|c|c|c|c|c|c|c|c|}
\hline $\begin{array}{l}06-07-85 \\
06-07-85 \\
06-07-85 \\
06-07-85 \\
06-07-85\end{array}$ & $\begin{array}{r}0.4 \\
.4 \\
.2 \\
.4 \\
.2\end{array}$ & $\begin{array}{l}2 \\
2 \\
0.8 \\
2 \\
0.5\end{array}$ & $\begin{array}{r}8 \\
9 \\
3 \\
12 \\
1\end{array}$ & $\begin{array}{r}20 \\
21 \\
9 \\
36 \\
2\end{array}$ & $\begin{array}{r}27 \\
38 \\
23 \\
64 \\
4\end{array}$ & $\begin{array}{l}30 \\
55 \\
54 \\
79 \\
26\end{array}$ & $\begin{array}{l}33 \\
66 \\
81 \\
86 \\
75\end{array}$ & $\begin{array}{l}37 \\
71 \\
96 \\
91 \\
91\end{array}$ & $\begin{array}{r}57 \\
80 \\
100 \\
97 \\
94\end{array}$ & $\begin{array}{l}100 \\
100\end{array}$ & & $\begin{array}{l}16 \\
44 \\
52 \\
57 \\
60\end{array}$ \\
\hline $\begin{array}{l}06-08-85 \\
06-08-85 \\
06-08-85 \\
06-08-85 \\
06-08-85\end{array}$ & $\begin{array}{l}.5 \\
.2 \\
.1 \\
.1 \\
.1\end{array}$ & $\begin{array}{l}3 \\
1 \\
1 \\
0.6 \\
.4\end{array}$ & $\begin{array}{c}9 \\
9 \\
13 \\
3 \\
0.9\end{array}$ & $\begin{array}{r}26 \\
29 \\
39 \\
7 \\
1\end{array}$ & $\begin{array}{r}64 \\
46 \\
62 \\
13 \\
3\end{array}$ & $\begin{array}{l}83 \\
58 \\
78 \\
23 \\
14\end{array}$ & $\begin{array}{l}90 \\
66 \\
87 \\
35 \\
36\end{array}$ & $\begin{array}{l}96 \\
73 \\
95 \\
49 \\
58\end{array}$ & $\begin{array}{r}100 \\
81 \\
100 \\
66 \\
83\end{array}$ & $\begin{array}{l}100 \\
100\end{array}$ & 100 & $\begin{array}{l}17 \\
27 \\
45 \\
54 \\
60\end{array}$ \\
\hline
\end{tabular}


Table 4.--Bed-material particle-size distributions--Continued

Date

Percent by weight finer than size (millimeters) indicated

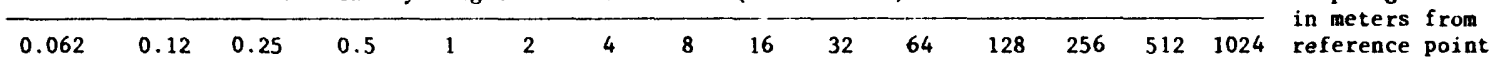

12. North Fork of Toutle River near Kid Valley, Wash. ${ }^{2}-$ - Continued

\begin{tabular}{|c|c|c|c|c|c|c|c|c|c|c|c|c|}
\hline $\begin{array}{l}12-18-85 \\
01-19-86 \\
01-19-86 \\
01-19-86 \\
01-19-86\end{array}$ & $\begin{array}{l}0 \\
0.3 \\
.8 \\
.1 \\
.2\end{array}$ & $\begin{array}{l}0.1 \\
.8 \\
3 \\
0.4 \\
.8\end{array}$ & $\begin{array}{r}2 \\
2 \\
12 \\
2 \\
3\end{array}$ & $\begin{array}{r}5 \\
4 \\
38 \\
7 \\
8\end{array}$ & $\begin{array}{r}14 \\
7 \\
62 \\
16 \\
13\end{array}$ & $\begin{array}{l}38 \\
14 \\
78 \\
25 \\
23\end{array}$ & $\begin{array}{l}67 \\
24 \\
87 \\
30 \\
32\end{array}$ & $\begin{array}{l}93 \\
43 \\
92 \\
34 \\
40\end{array}$ & $\begin{array}{r}98 \\
67 \\
100 \\
41 \\
59\end{array}$ & $\begin{array}{l}100 \\
100\end{array}$ & $\begin{array}{l}100 \\
100\end{array}$ & $\begin{array}{l}62 \\
17 \\
29 \\
53 \\
58\end{array}$ \\
\hline $\begin{array}{l}01-19-86 \\
02-13-86 \\
02-13-86 \\
02-13-86 \\
02-13-86\end{array}$ & $\begin{array}{l}.4 \\
0 \\
0.1 \\
0 \\
0.1\end{array}$ & $\begin{array}{l}2 \\
3 \\
0.2 \\
.2 \\
.8\end{array}$ & $\begin{array}{c}7 \\
14 \\
0.9 \\
3 \\
8\end{array}$ & $\begin{array}{r}10 \\
38 \\
5 \\
26 \\
34\end{array}$ & $\begin{array}{l}12 \\
62 \\
12 \\
80 \\
74\end{array}$ & $\begin{array}{l}17 \\
83 \\
21 \\
95 \\
89\end{array}$ & $\begin{array}{l}29 \\
93 \\
36 \\
99 \\
94\end{array}$ & $\begin{array}{r}62 \\
100 \\
55 \\
100 \\
97\end{array}$ & 82 & 100 & 1 & $\begin{array}{l}63 \\
52 \\
56 \\
58 \\
61\end{array}$ \\
\hline $\begin{array}{l}02-13-86 \\
02-26-86 \\
02-26-86 \\
02-26-86 \\
02-26-86\end{array}$ & $\begin{array}{l}0 \\
0.1 \\
.3 \\
.1 \\
.1\end{array}$ & $\begin{array}{l}1 \\
1 \\
0.5 \\
1 \\
0.6\end{array}$ & $\begin{array}{l}0.2 \\
6 \\
1 \\
6 \\
2\end{array}$ & $\begin{array}{r}1 \\
18 \\
2 \\
13 \\
4\end{array}$ & $\begin{array}{r}12 \\
38 \\
4 \\
18 \\
9\end{array}$ & $\begin{array}{r}31 \\
59 \\
5 \\
22 \\
24\end{array}$ & $\begin{array}{r}55 \\
74 \\
6 \\
28 \\
45\end{array}$ & $\begin{array}{r}73 \\
88 \\
6 \\
33 \\
68\end{array}$ & $\begin{array}{l}87 \\
97 \\
15 \\
37 \\
89\end{array}$ & $\begin{array}{r}87 \\
100 \\
100 \\
100 \\
100\end{array}$ & 100 & $\begin{array}{l}64 \\
16 \\
22 \\
32 \\
62\end{array}$ \\
\hline $\begin{array}{l}08-07-86 \\
08-07-86\end{array}$ & 0 & $0^{.1}$ & $\begin{array}{r}0.8 \\
.5\end{array}$ & $\begin{array}{l}9 \\
7\end{array}$ & $\begin{array}{l}23 \\
38\end{array}$ & $\begin{array}{l}34 \\
75\end{array}$ & $\begin{array}{l}42 \\
90\end{array}$ & $\begin{array}{l}54 \\
97\end{array}$ & $\begin{array}{l}74 \\
98\end{array}$ & $\begin{array}{l}100 \\
100\end{array}$ & & $\begin{array}{l}61 \\
64\end{array}$ \\
\hline
\end{tabular}

13. Oak Creek near Corvallis, Oreg.

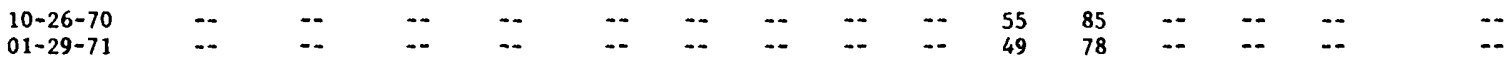

14. San Antonio River near Lockwood, Calif. ${ }^{4}$

$\begin{array}{rrrrrrrrrrr}01-10-73 & - & - & 3 & 23 & 47 & 70 & 82 & 91 & 97 & 100\end{array}$

$11-20-74$

16. Redwood Creek near Blue Lake, Calif.

$02-28-83$

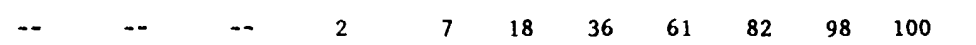

17. Redwood Creek above Panther Creek near Orick, Calif.

$\begin{array}{rrrrrrrrrrrr}01-20-83 & -- & -- & 1 & 3 & 7 & 13 & 31 & 63 & 89 & 100 & 100 \\ 02-23-83 & -- & -- & -- & 6 & 23 & 39 & 53 & 67 & 83 & 98 & 100\end{array}$

$\cdots$

18. Redwood Creek at Orick, Calif. 4

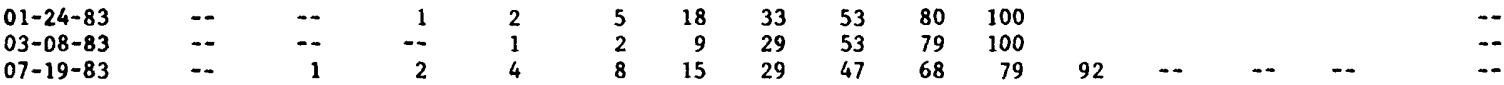

21. Sacramento River above Bend Bridge near Red Bluff, Calif. ${ }^{4}$

01-05-77

01-05-77

$05-04-77$

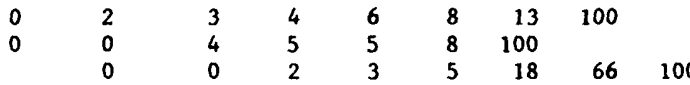

$\cdots$

$-$ 
Table 4.--Bed-material particle-size distributions--Continued

\begin{tabular}{|c|c|c|c|c|c|c|c|c|c|c|c|c|c|c|c|c|}
\hline \multirow{2}{*}{ Date } & \multicolumn{6}{|c|}{ Percent by weight } & \multicolumn{9}{|c|}{ finer than size (millimeters) indicated } & \multirow{2}{*}{$\begin{array}{l}\text { Sampling location, } \\
\text { in meters from } \\
\text { reference point }\end{array}$} \\
\hline & 0.062 & 0.12 & 0.25 & 0.5 & 1 & 2 & 4 & 8 & 16 & 32 & 64 & 128 & 256 & 512 & 1024 & \\
\hline & \multicolumn{16}{|c|}{ 25. Big Lost River at Chilly Bridge, Idaho 4,5} \\
\hline \multirow[t]{2}{*}{$06-81$} & -- & -- & -- & 2 & 6 & 10 & 15 & 22 & 40 & 68 & 94 & 100 & & & & -- \\
\hline & \multicolumn{16}{|c|}{ 26. Big Lost River below Chilly Sinks, Idaho ${ }^{4}$} \\
\hline \multirow[t]{2}{*}{$06-81$} & -- & -- & 2 & 8 & 18 & 22 & 26 & 36 & 53 & 68 & 89 & 100 & & & & $-\cdot$ \\
\hline & \multicolumn{15}{|c|}{ 27. Big Lost River above East and West Channels, Idaho ${ }^{4}$} & \\
\hline \multirow[t]{2}{*}{$06-81$} & -- & 1 & 5 & 22 & 29 & 33 & 39 & 49 & 69 & 90 & 99 & 100 & & & & -- \\
\hline & \multicolumn{16}{|c|}{ 31. Muddy Creek near Pinedale, Wyo. ${ }^{4}$} \\
\hline \multirow[t]{2}{*}{$07-31-75$} & 0.1 & 0.1 & 3 & 33 & 62 & 85 & 97 & 100 & & & & & & & & -- \\
\hline & \multicolumn{16}{|c|}{ 32. East Fork River near Pinedale, Wyo. 4} \\
\hline \multirow[t]{2}{*}{$05-18-79$} & 1 & 3 & 8 & 19 & 23 & 29 & 40 & 53 & 79 & 92 & 100 & & & & & -- \\
\hline & \multicolumn{15}{|c|}{ 33. Yampa River near Deerlodge Park, Colo. } & \\
\hline $04-27-82$ & 0 & 1 & 1 & 9 & 40 & 83 & 98 & 99 & 100 & & & & & & & -- \\
\hline $05-12-82$ & & 0 & 3 & 36 & 78 & 88 & 91 & 94 & 97 & 100 & & & & & & -- \\
\hline $05-13-82$ & & 0 & 6 & 46 & 85 & 97 & 98 & 99 & 100 & & & & & & & -- \\
\hline $05-24-82$ & 0 & 1 & 18 & 74 & 90 & 96 & 99 & 100 & 100 & & & & & & & -- \\
\hline $05-25-82$ & 0 & 2 & 22 & 74 & 89 & 94 & 95 & 96 & 96 & 100 & & & & & & -- \\
\hline $06-08-82$ & 0 & 1 & 8 & 56 & 91 & 98 & 100 & & & & & & & & & -- \\
\hline $06-24-82$ & & 0 & 3 & 38 & 77 & 92 & 97 & 99 & 100 & & & & & & & -- \\
\hline $07-07-82$ & & 0 & 1 & 16 & 52 & 75 & 89 & 97 & 100 & & & & & & & -- \\
\hline $07-08-82$ & & 0 & 1 & 11 & 50 & 78 & 91 & 97 & 99 & 100 & & & & & & -- \\
\hline $07-29-82$ & & 0 & 5 & 26 & 69 & 89 & 96 & 99 & 100 & & & & & & & -- \\
\hline $04-07-83$ & & 0 & 8 & 40 & 76 & 93 & 98 & 100 & & & & & & & & -- \\
\hline $04-08-83$ & & 0 & 10 & 46 & 82 & 94 & 98 & 99 & 100 & & & & & & & -- \\
\hline $04-19-83$ & & 0 & 5 & 40 & 80 & 94 & 98 & 100 & & & & & & & & -- \\
\hline $04-21-83$ & 0 & 2 & 10 & 53 & 94 & 99 & 100 & & & & & & & & & -- \\
\hline $04-22-83$ & 0 & 2 & 15 & 49 & 87 & 96 & 98 & 100 & & & & & & & & -- \\
\hline $05-07-83$ & & 0 & 2 & 29 & 76 & 89 & 95 & 99 & 100 & & & & & & & -- \\
\hline $05-09-83$ & & 0 & 3 & 42 & 88 & 97 & 99 & 100 & & & & & & & & -- \\
\hline $05-12-83$ & 0 & 1 & 3 & 26 & 76 & 95 & 98 & 99 & 100 & & & & & & & -- \\
\hline $05-23-83$ & 0 & 1 & 8 & 54 & 78 & 89 & 93 & 95 & 98 & 100 & & & & & & -- \\
\hline $05-26-83$ & 0 & 1 & 6 & 57 & 84 & 89 & 93 & 97 & 100 & & & & & & & -- \\
\hline $05-27-83$ & 0 & & 5 & 36 & 91 & 98 & 99 & 100 & & & & & & & & -- \\
\hline $05-28-83$ & 0 & 1 & 6 & 30 & 80 & 91 & 95 & 98 & 100 & & & & & & & -- \\
\hline $06-08-83$ & 0 & 1 & 15 & 56 & 91 & 97 & 99 & 100 & & & & & & & & -- \\
\hline $06-10-83$ & 0 & $i$ & 18 & 55 & 90 & 97 & 98 & 98 & 99 & 100 & & & & & & -- \\
\hline $06-21-83$ & & 0 & 7 & 41 & 92 & 99 & 99 & 100 & & & & & & & & -- \\
\hline $06-23-83$ & & 0 & 6 & 42 & 89 & 98 & 99 & 100 & & & & & & & & -- \\
\hline $07-12-83$ & 0 & 3 & 9 & 32 & 78 & 95 & 98 & 99 & 100 & & & & & & & -- \\
\hline $07-14-83$ & 0 & 4 & 12 & 38 & 86 & 96 & 99 & 99 & 100 & & & & & & & -- \\
\hline
\end{tabular}


Table 4.--Bed-material particle-size distributions--Conlinued

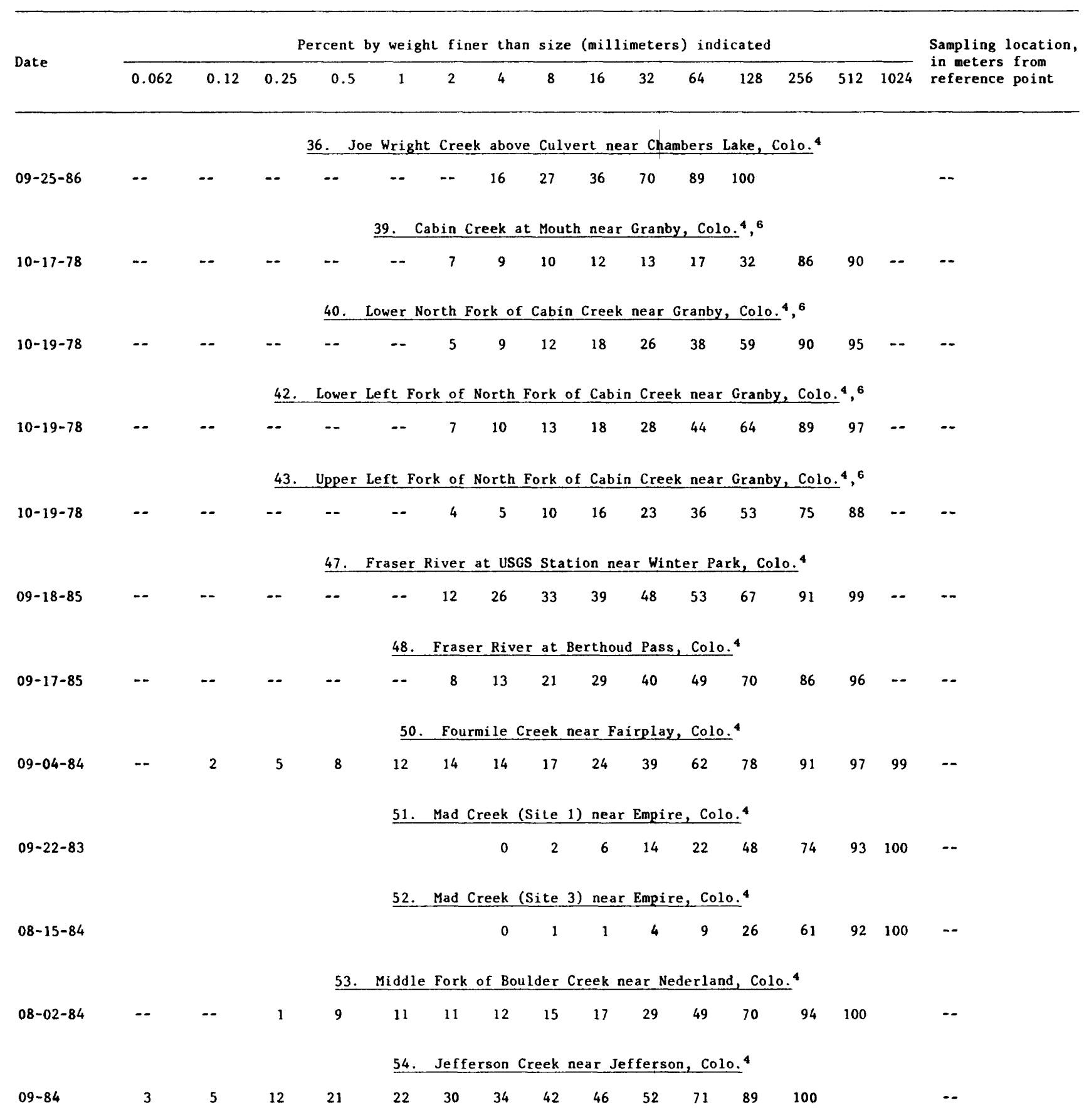


Table 4.--Bed-material particle-size distributions--Continued

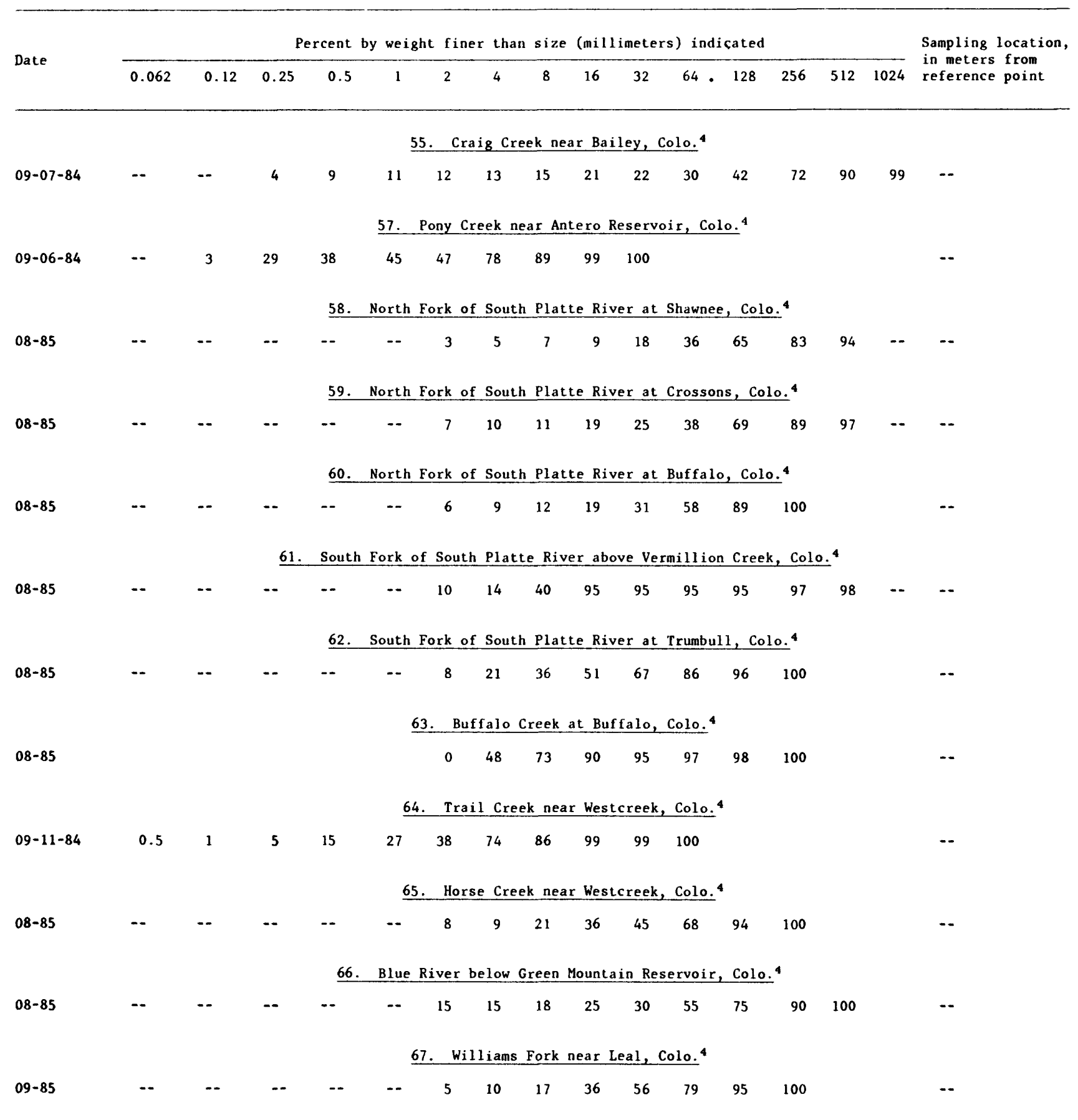




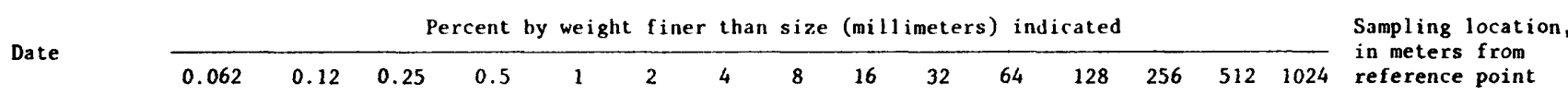

68. Upper South Fork of Williams Fork near Leal, Colo. 4

08-85

69. Lower South Fork of Williams Fork near Leal, Colo. 4

$\begin{array}{lllllllllllllllllllll}09-05-85 & - & - & - & - & - & - & - & 12 & 12 & 13 & 22 & 33 & 47 & 69 & 89 & 99 & -- & -\end{array}$

70. Williams Fork below Steelman Creek near Leal, Colo. 4

$09-18-85$

71. Kinney Creek near Leal, Colo. 4

$08-15-85$

$\begin{array}{lllllllllll}0 & 2 & 11 & 33 & 55 & 75 & 92 & 98 & \ldots & \ldots\end{array}$

73. Rich Creek near Weston Pass, Colo. 4

$09-06-84$

$\begin{array}{llllllllll}12 & 15 & 20 & 22 & 29 & 37 & 54 & 74 & 89 & 100\end{array}$

74. Trout Creek near Bayfield, Colo.

$\begin{array}{llllllllllllllll}08-07-84 & 2 & 2 & 4 & 4 & 4 & 4 & 4 & 8 & 12 & 17 & 44 & 69 & 94 & 100 & -- \\ 08-07-85 & 0 & 2 & 6 & 6 & 6 & 6 & 6 & 6 & 13 & 25 & 50 & 67 & 94 & 100 & --\end{array}$

$08-07-84$

75. Clear Creek near Bayfield, Colo. 4

08-07-85

$08-07-84$

$08-07-85$

$08-07-84$

08-07-85

$\begin{array}{rrrrrrrrrrrr}2 & 3 & 3 & 19 & 20 & 37 & 61 & 83 & 92 & 95 & 100 & \ldots \\ 4 & 6 & 6 & 6 & 18 & 28 & 48 & 62 & 92 & 100 & & -\end{array}$

76. West Prong Creek near Bayfield, Colo. ${ }^{4}$

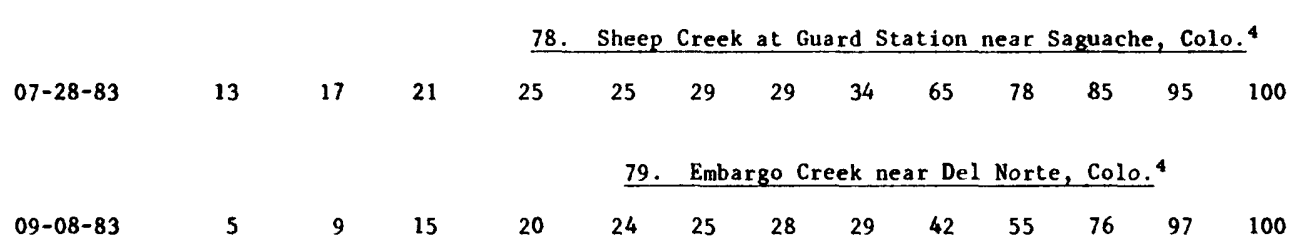


Table 4.--Bed-material particle-size distributions--Continued

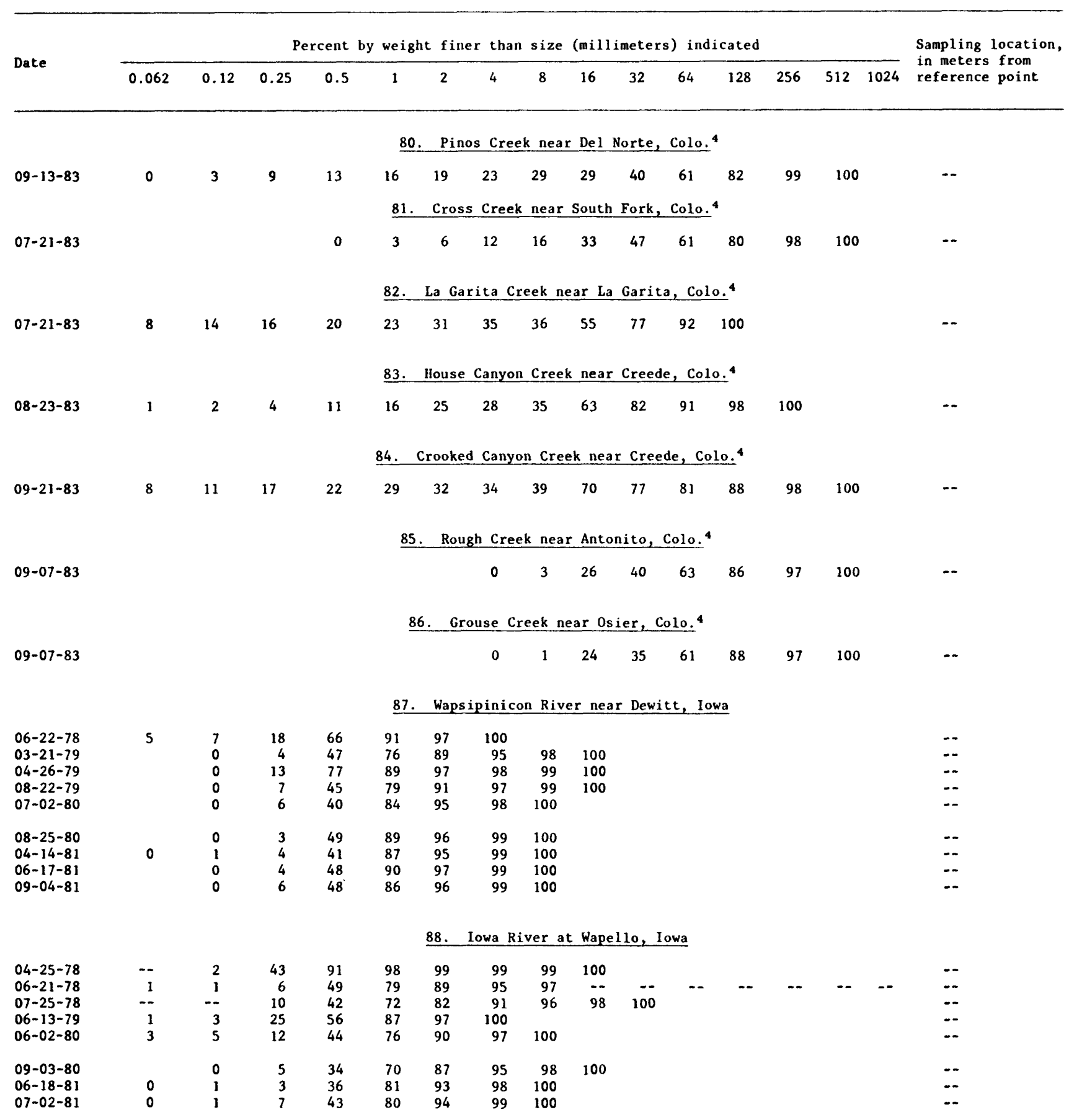


Table 4.--Bed-material particle-size distributions--Continued

Percent by weight finer than size (millimeters) indicated Sampling location,

Date

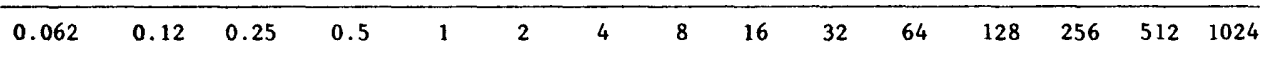

89. Wisconsin River at Muscoda, Wis.

\begin{tabular}{|c|c|c|c|c|c|c|c|c|c|c|c|}
\hline $03-24-77$ & 0 & 0.2 & 7 & 61 & 87 & 92 & 96 & 99 & 100 & & - \\
\hline $04-19-77$ & 0 & .2 & 6 & 62 & 87 & 92 & 95 & 98 & 99 & 100 & $\cdots$ \\
\hline $05-16-77$ & 0 & .4 & 9 & 53 & 76 & 82 & 88 & 95 & 100 & & -- \\
\hline $06-15-77$ & $\cdots$ & $\cdots$ & 6 & 56 & 81 & 88 & 92 & 97 & 99 & 100 & $=-$ \\
\hline $07-20-77$ & 0 & .1 & 12 & 64 & 83 & 87 & 91 & 97 & 100 & & - \\
\hline $08-24-77$ & 0.2 & .3 & 7 & 65 & 92 & 98 & 99 & 100 & & & - \\
\hline $09-27-77$ & .1 & .4 & 7 & 58 & 90 & 96 & 99 & 100 & & & $\cdots$ \\
\hline $10-18-77$ & 0 & .2 & 8 & 61 & 84 & 89 & 93 & 97 & 100 & & -- \\
\hline $11-14-77$ & 0.1 & .2 & 5 & 59 & 87 & 96 & 99 & 99 & 100 & & - \\
\hline $04-17-78$ & 0 & .2 & 8 & 61 & 91 & 95 & 97 & 98 & 99 & 100 & -- \\
\hline $05-08-78$ & 0.1 & .5 & 11 & 67 & 92 & 97 & 99 & 100 & & & - \\
\hline $06-06-78$ & 0 & .1 & 4 & 63 & 94 & 98 & 100 & & & & - \\
\hline $07-11-78$ & 0.1 & .2 & 7 & 68 & 94 & 97 & 98 & 99 & 100 & & -- \\
\hline $08-15-78$ & .1 & .4 & 8 & 62 & 89 & 94 & 96 & 99 & 100 & & - \\
\hline $10-24-78$ & 0 & 12 & 7 & 69 & 95 & 98 & 99 & 100 & & & - \\
\hline $11-14-78$ & & 0 & 8 & 69 & 94 & 98 & 99 & 100 & & & - \\
\hline $03-28-79$ & & 0 & 8 & 60 & 89 & 96 & 98 & 99 & 100 & & - \\
\hline $04-24-79$ & & 0 & 7 & 63 & 96 & 99 & 100 & & & & - \\
\hline $07-05-79$ & & 0 & 11 & 56 & 87 & 94 & 97 & 98 & 100 & & $\cdots$ \\
\hline $08-25-79$ & & 0 & 7 & 63 & 92 & 97 & 99 & 100 & & & $=$ \\
\hline
\end{tabular}

90. Black River near Galesville, Wis.

$\begin{array}{rllrllllll}04-23-77 & 1 & 3 & 12 & 46 & 87 & 96 & 98 & 99 & 100 \\ 05-27-77 & 0 & 0.4 & 8 & 46 & 87 & 97 & 99 & 100 & \\ 06-29-77 & 0.2 & .8 & 5 & 48 & 92 & 98 & 100 & & \\ 07-12-77 & .3 & 2 & 9 & 54 & 95 & 97 & 99 & 100 & \\ 08-02-77 & .5 & 1 & 9 & 53 & 91 & 96 & 98 & 99 & 100 \\ & & & & & & & & & \\ 10-19-77 & .3 & 4 & 20 & 59 & 91 & 96 & 98 & 99 & 100 \\ 11-08-77 & .3 & 3 & 19 & 59 & 90 & 96 & 98 & 99 & 100 \\ 03-31-78 & .3 & 2 & 27 & 71 & 96 & 99 & 99 & 100 & \\ 04-10-78 & .1 & 0.4 & 19 & 69 & 96 & 99 & 100 & & \\ 05-10-78 & 0 & .2 & 9 & 69 & 96 & 98 & 99 & 99 & 100 \\ & & & & & & & & & \\ 06-13-78 & 0.1 & .3 & 7 & 58 & 92 & 97 & 98 & 98 & 100 \\ 08-09-78 & .3 & .7 & 10 & 65 & 91 & 97 & 99 & 100 & \\ 10-28-78 & 0 & 0 & 4 & 62 & 94 & 97 & 98 & 99 & 100 \\ 04-25-79 & 0 & 1 & 10 & 46 & 91 & 98 & 99 & 100 & \\ 05-31-79 & 2 & 3 & 14 & 47 & 92 & 98 & 99 & 100 & \\ 07-23-79 & 0 & 3 & 28 & 56 & 89 & 97 & 99 & 100 & \end{array}$

$-$

$-$

$--$

$-$

91. Chippewa River near Caryville, Wis.

$08-30-76$

$09-20-76$

10-18-76

$11-17-76$

$03-15-77$

$\begin{array}{rll}0.2 & 0.7 & 2 \\ .5 & 2 & 5 \\ .2 & 0.6 & 4 \\ .3 & 1 & 4 \\ .7 & 2 & 5\end{array}$

$\begin{array}{ll}36 & 43 \\ 43 & 47 \\ 26 & 35 \\ 41 & 46 \\ 33 & 38\end{array}$

$\begin{array}{lll}50 & 61 & 87 \\ 53 & 62 & 87 \\ 44 & 62 & 79 \\ 52 & 64 & 90 \\ 43 & 52 & 68\end{array}$

$\begin{array}{rrr}87 & 100 & \\ 87 & 100 & \\ 79 & 85 & 100 \\ 90 & 100 & \\ 68 & 90 & 100\end{array}$

--
--
--
-- 
Table 4.--Bed-material particle-size distributions--Contimud

Percent by weight finer than size (millimeters) indicated

Date

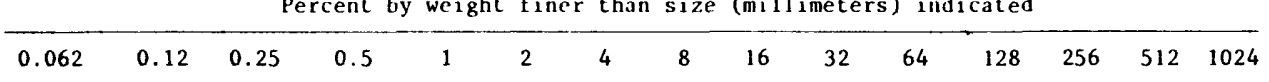

Sampling location,

n meters from

reference point

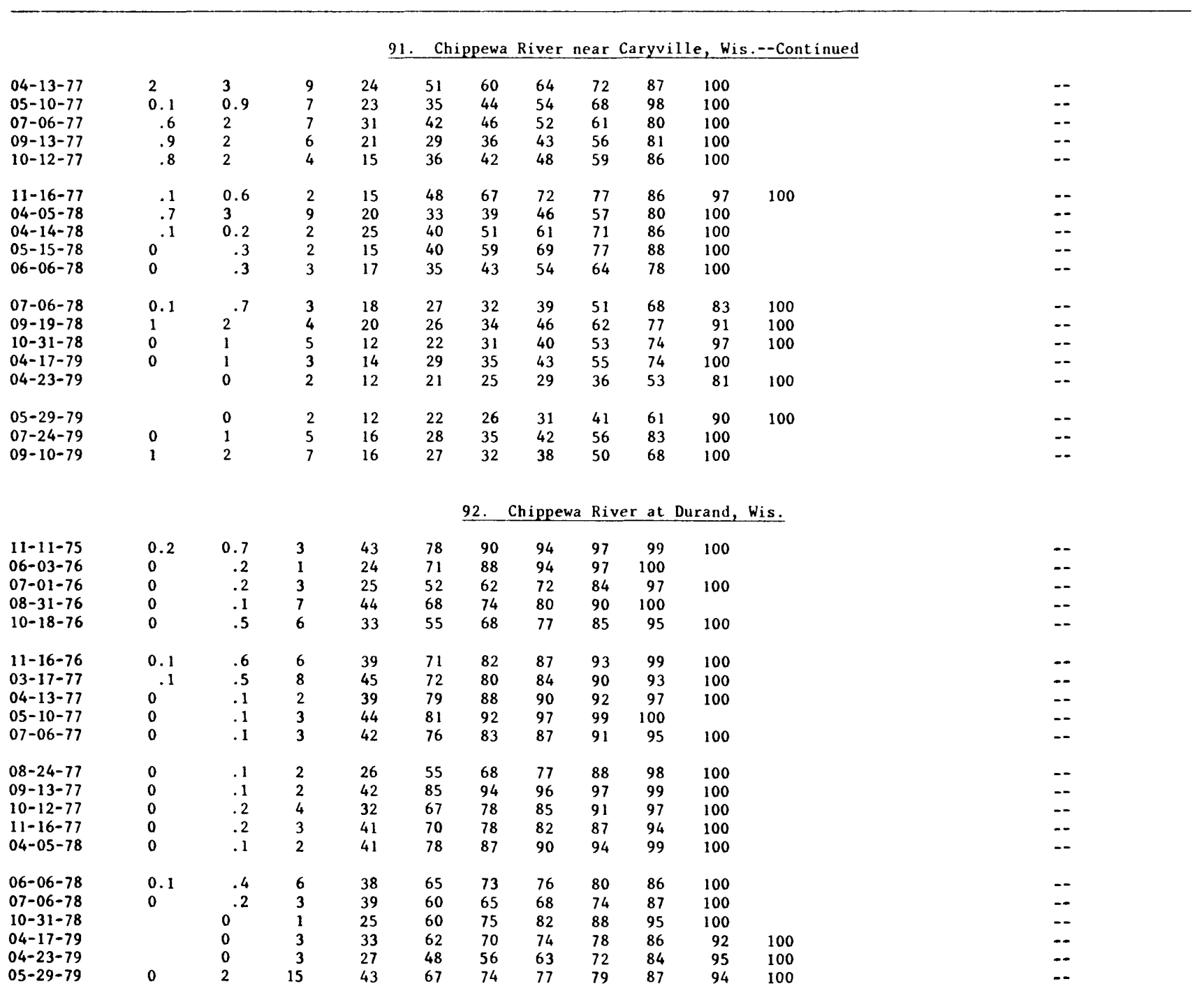


Table 4.--Bed-material particle-size distributions--Continued

\begin{tabular}{|c|c|c|c|c|c|c|c|c|c|c|c|c|c|c|c|c|}
\hline \multirow{2}{*}{ Date } & \multicolumn{15}{|c|}{ Percent by weight finer than size (millimeters) indicated } & \multirow{2}{*}{$\begin{array}{l}\text { Sampling location } \\
\text { in meters from } \\
\text { reference point }\end{array}$} \\
\hline & 0.062 & 0.12 & 0.25 & 0.5 & 1 & 2 & 4 & 8 & 16 & 32 & 64 & 128 & 256 & 512 & 1024 & \\
\hline
\end{tabular}

93. Chippewa River near Pepin, Wis.

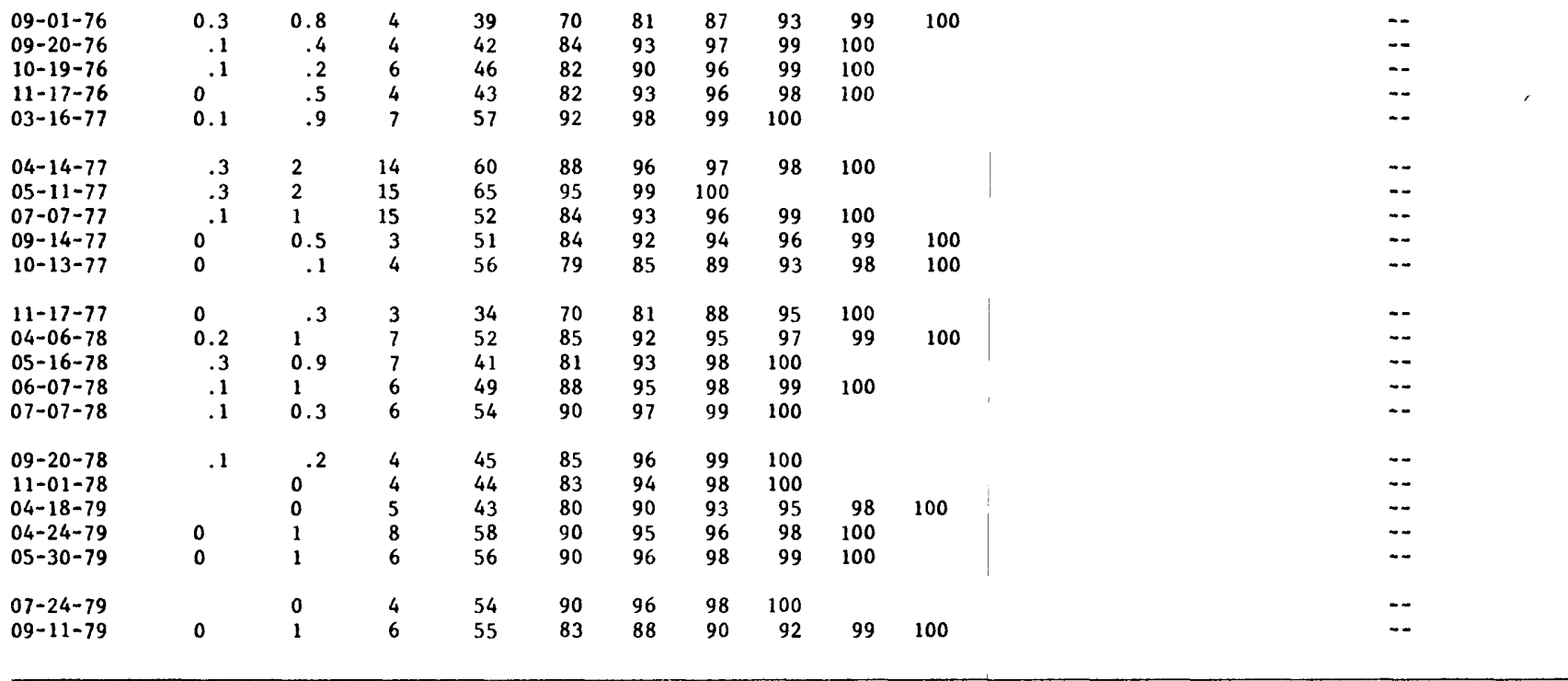

${ }^{1}$ Reference point for sampling location = left bank.

${ }^{2}$ Reference point for sampling location = monument on left-bank floodplain.

${ }^{3}$ Reference point for sampling location = monument on right-bank floodplain.

${ }^{4}$ Few or no bed-material analyses for dates of sediment-transport measurements; available analyses for other dates are given here to indicate general sizes of streambed sediments.

SOptical analysis (Williams and Krupin, 1984).

${ }^{6}$ All percentages for this station interpolated from a size analysis that involved class limits different from those listed here. 